Supporting Information for

\title{
Modular approaches to lankacidin antibiotics
}

\author{
Lingchao Cai, ${ }^{\ddagger}$ Yanmin Yao,${ }^{\ddagger}$ Seul Ki Yeon and Ian B. Seiple* \\ Department of Pharmaceutical Chemistry and Cardiovascular Research Institute, University of California, San \\ Francisco, San Francisco, California 94158, United States \\ ian.seiple@ucsf.edu
}


General experimental, materials, and instrumentation....

page S5

Experimental procedures and characterization for compounds

Compound 30

page $\mathrm{S} 7$

Compound SI-1 and 31..................................................................... page S7

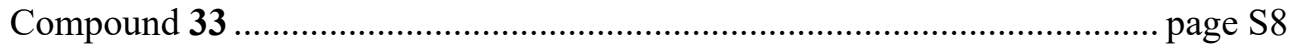

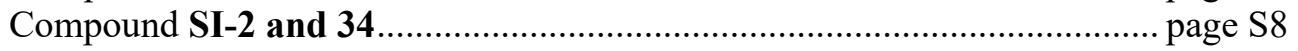

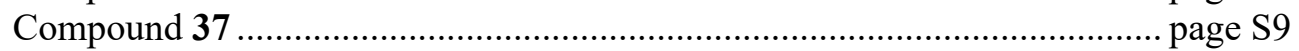

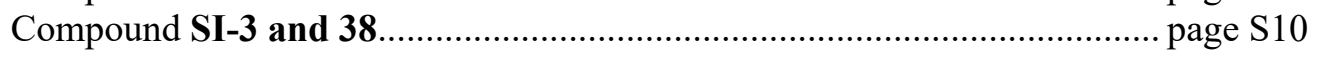

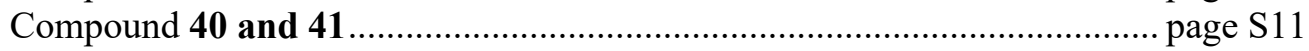

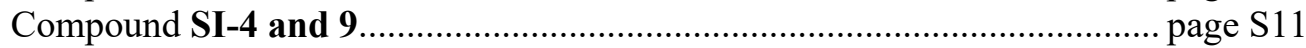

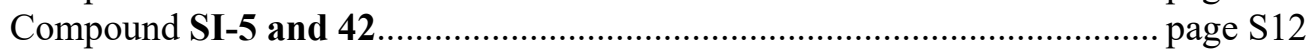

Compound SI-6 and 10................................................................... page S13

Optimization for stille coupling of compound 20 .................................... page S15

NMR spectral data comparison for 2,18-seco-lankacidinol B ...................... page S16

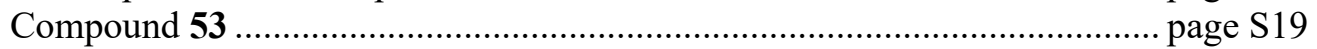

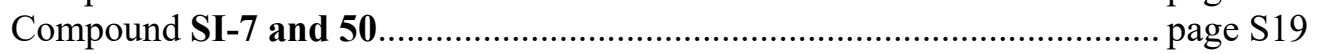

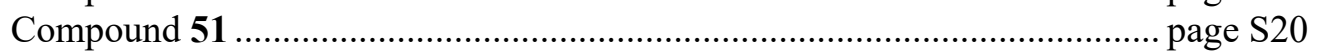

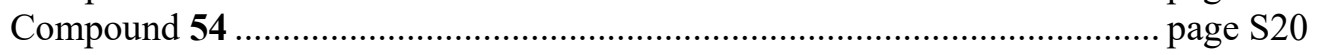

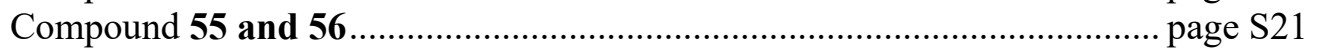

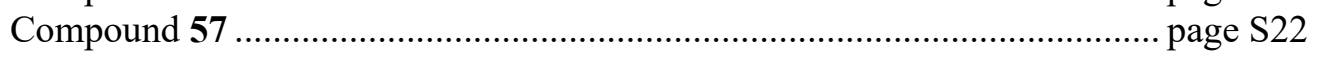

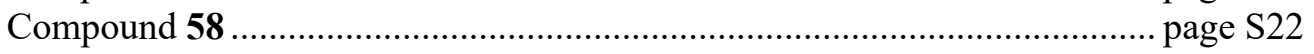

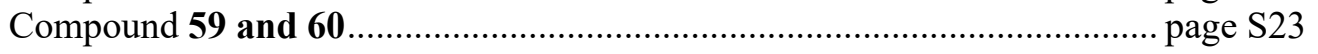

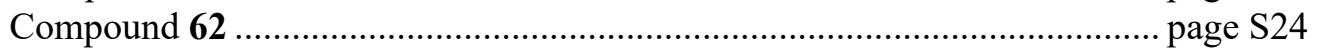

Compound 63 ................................................................................ page S24

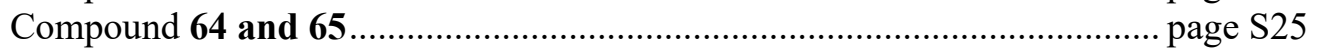

Compound 66 .................................................................................. page S26

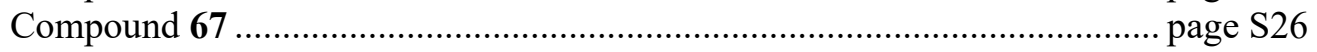

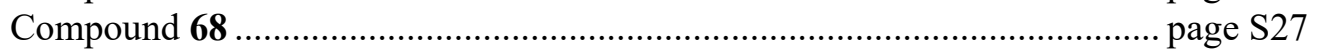

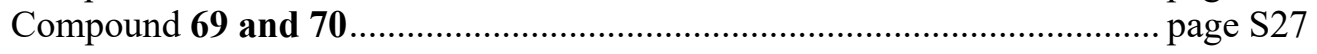

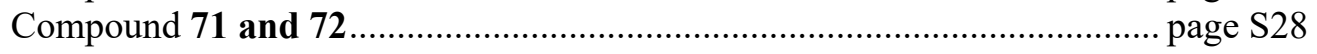

Optimization for Marko's oxidation of compound 63 ............................... page S30

Compound SI-8 and 77..................................................................... page S32

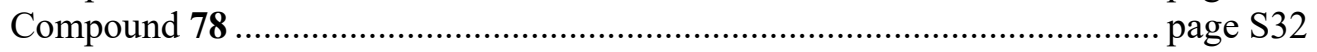

Compound 79 ..................................................................................... page S33

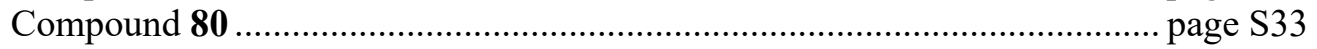

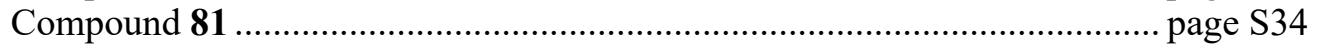

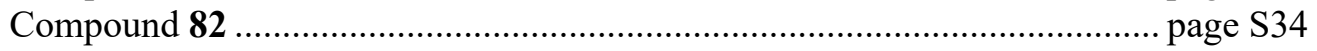

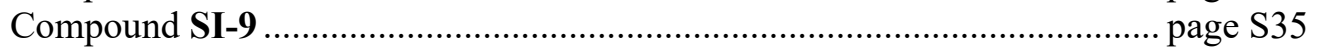

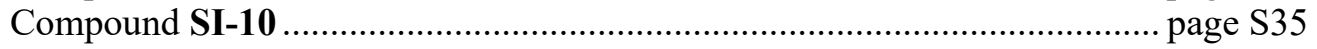

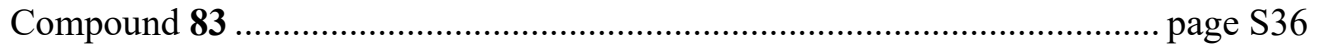

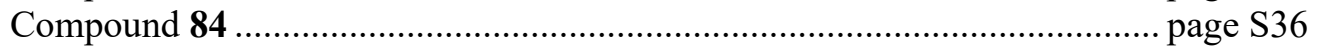

Compound SI-11 ............................................................................. page S37

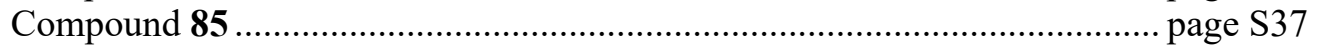

Compound SI-12 and SI-13 ................................................................. page S39

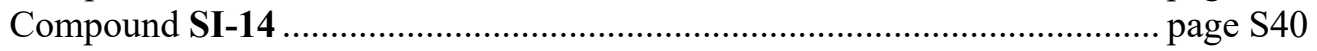

Compound SI-15 ............................................................................ page S40

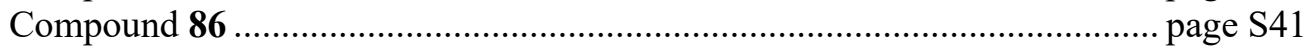

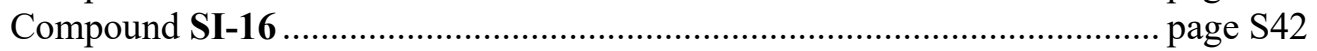

Compound 87 ................................................................................ page S42

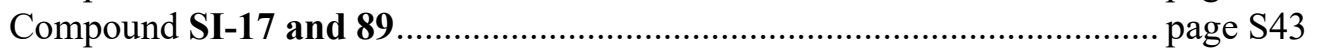

Compound 90 ............................................................................... page S44

Compound SI-18 ............................................................................. page S44

Compound SI-19 .......................................................................... page S45

Compound 91 .................................................................................... page S46

Compound SI-20 and SI-21 .............................................................. page S46 


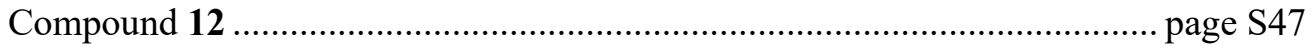

Optimization for Dieckmann cyclization of compounds 83, 86 and 91 ........ page S49

NMR Spectral Data Comparison for iso-lankacidinol .................................. page S52

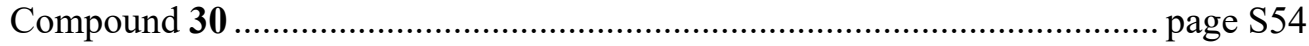

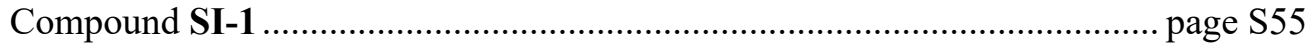

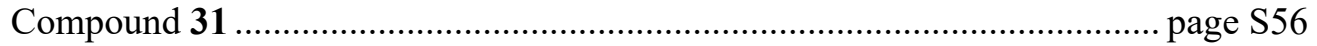

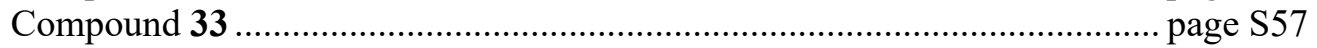

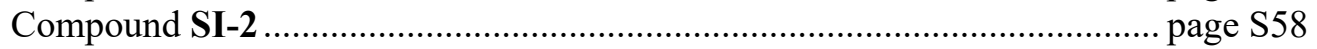

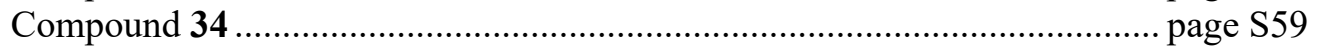

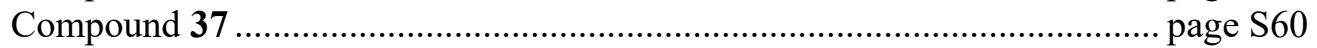

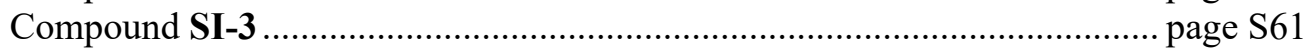

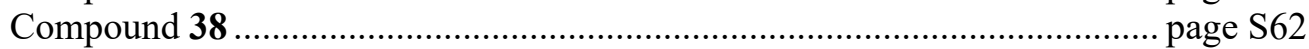

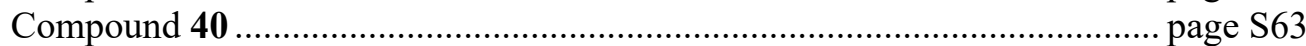

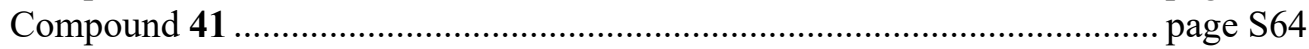

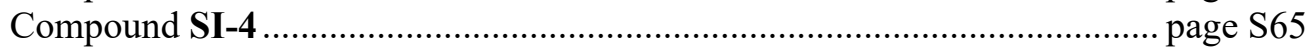

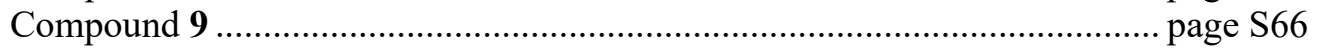

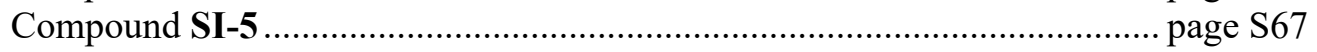

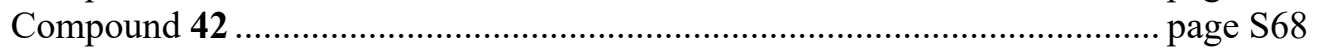

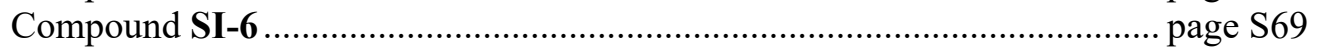

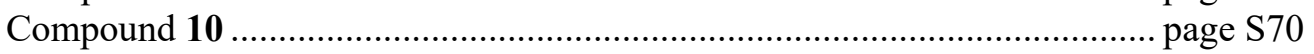

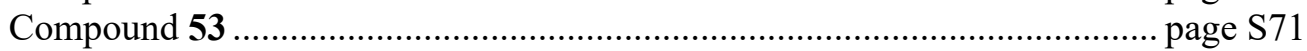

Compound SI-7 ................................................................................ page S $\mathrm{S} 72$

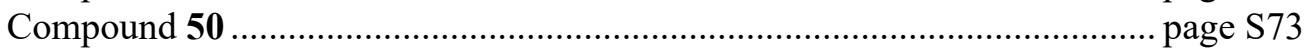

Compound 51 .................................................................................. page S74

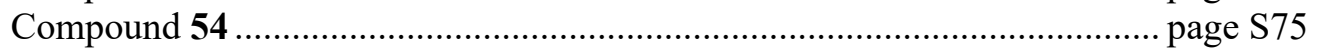

Compound 55 .................................................................................. page S76

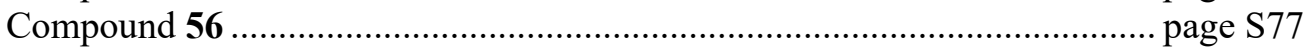

Compound 57 ................................................................................ page S78

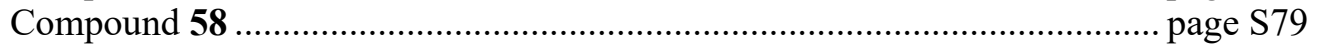

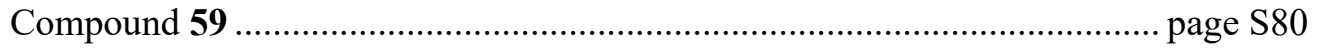

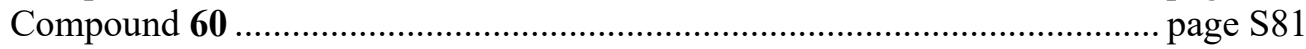

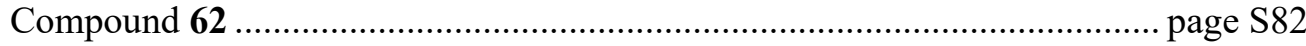

Compound 63 ...................................................................................

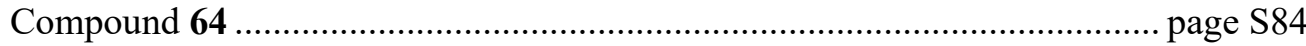

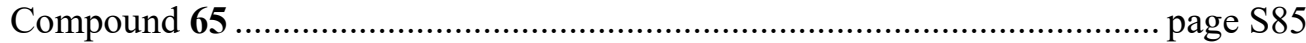

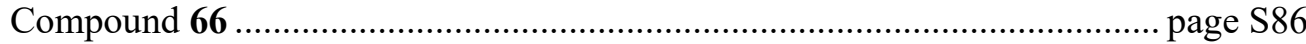

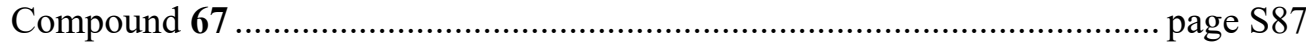

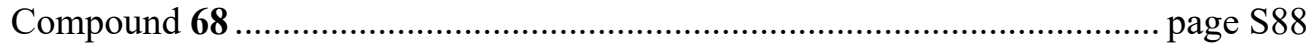

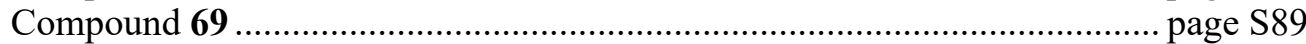

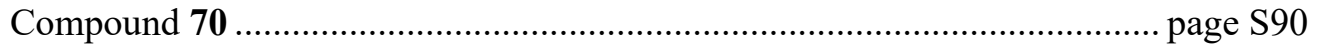

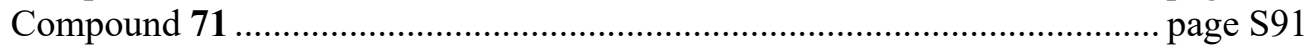

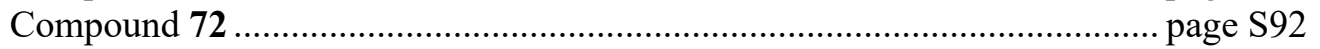

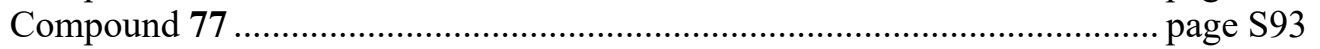

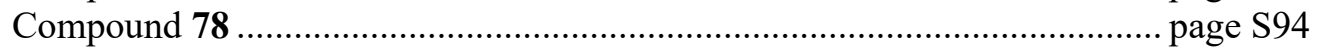

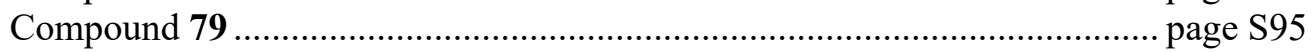

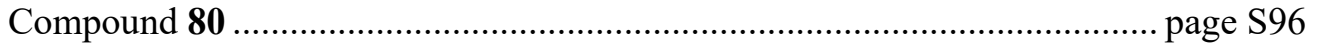

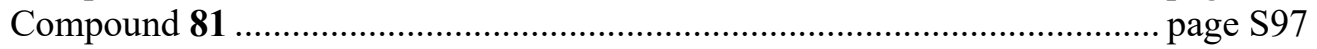

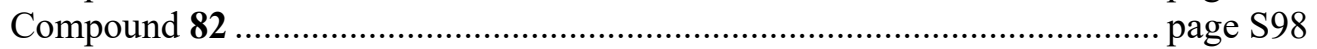

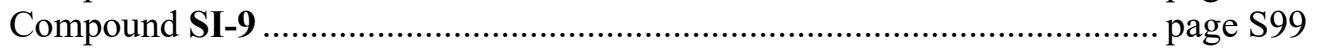

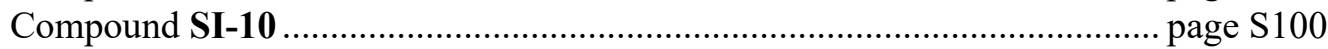

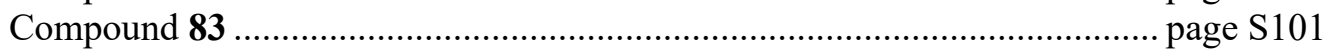

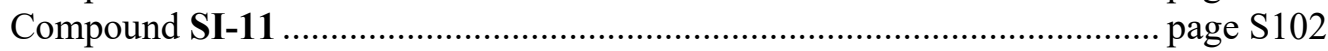

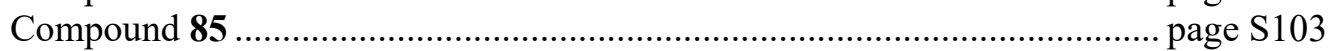

COSY and NOESY Spectra for compound 85 ........................................ page S104

Compound SI-12 ................................................................................. page S105 


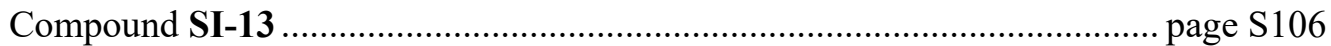

Compound SI-14 ............................................................................... page S107

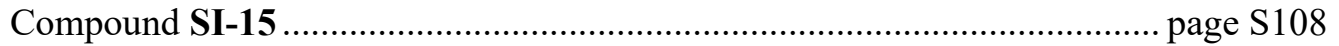

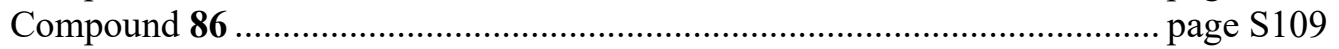

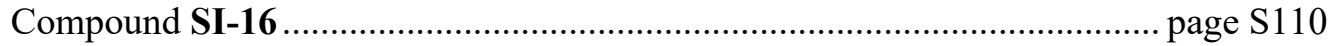

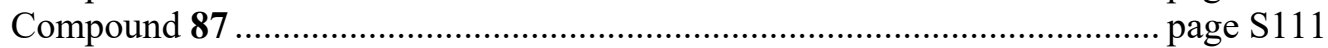

COSY and NOESY Spectra for compound 87........................................ page S112

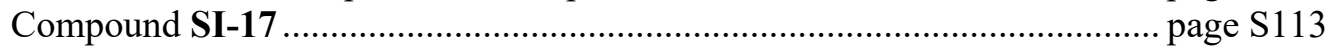

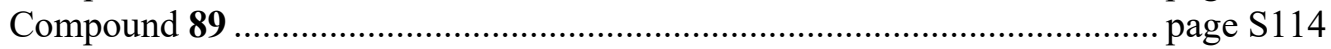

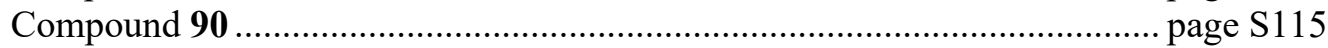

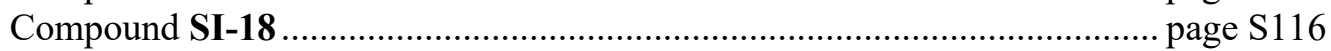

Compound SI-19 ........................................................................... page S 117

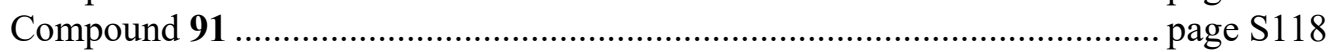

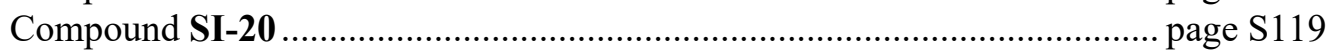

Compound SI-21 …..................................................................... page S 120

Compound 12 ..................................................................................... page $\mathrm{S} 121$

COSY and NOESY Spectra for compound 12 …..................................... page S122

X-Ray Crystallographic Information................................................................. page S123

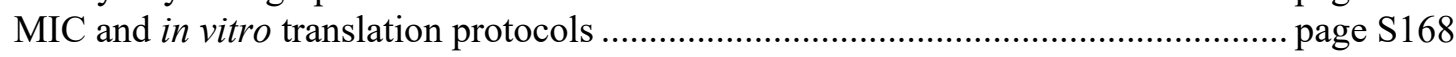

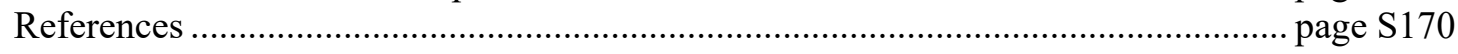


General Experimental Procedures: All reactions were performed in flame- or oven-dried glassware fitted with rubber septa under a positive pressure of nitrogen or argon, unless otherwise noted. All reaction mixtures were stirred throughout the course of each procedure using Teflon-coated magnetic stir bars. Air- and moisture-sensitive liquids were transferred via syringe or stainless steel cannula. Solutions were concentrated by rotary evaporation at or below $35{ }^{\circ} \mathrm{C}$. Analytical thin-layer chromatography (TLC) was performed using glass plates pre-coated with silica gel $(0.25-\mathrm{mm}, 60-\AA ̊$ pore size, $230-400 \mathrm{mesh}$, SILICYCLE INC) impregnated with a fluorescent indicator $(254 \mathrm{~nm})$. TLC plates were visualized by exposure to ultraviolet light (UV), and then were stained by submersion in a basic aqueous solution of potassium permanganate or with an acidic ethanolic solution of anisaldehyde, followed by brief heating.

Materials: DCM, DMF, THF, and ethyl ether to be used in anhydrous reaction mixtures were dried by passage through activated alumina columns immediately prior to use. Hexanes used were $\geq 85 \% n$-hexane. Other commercial solvents and reagents were used as received, unless otherwise noted.

Instrumentation: Unless otherwise noted, proton nuclear magnetic resonance ( ${ }^{1} \mathrm{H}$ NMR) spectra and carbon nuclear magnetic resonance $\left({ }^{13} \mathrm{C}\right.$ NMR) spectra were recorded on a Bruker Avance III HD 2-channel instrument NMR spectrometer $\left(400 \mathrm{MHz},{ }^{1} \mathrm{H} ; 100 \mathrm{MHz},{ }^{13} \mathrm{C}\right)$ at $23{ }^{\circ} \mathrm{C}$. Proton chemical shifts are reported in parts per million (ppm, $\delta$ scale) and are referenced to residual protium in the NMR solvent $\left(\mathrm{CHCl}_{3}: \delta 7.26, \mathrm{CHD}_{2} \mathrm{OD}: \delta 3.31\right)$. Carbon chemical shifts are expressed in parts per million (ppm, $\delta$ scale) and are referenced to the carbon resonance of the NMR solvent $\left(\mathrm{CDC1}_{3}: \delta 77.0, \mathrm{CD}_{3} \mathrm{OD}: \delta 49.0\right)$. Data are represented as follows: chemical shift, multiplicity ( $\mathrm{s}=$ singlet, $\mathrm{d}=$ doublet, $\mathrm{t}=$ triplet, $\mathrm{q}=$ quartet, $\mathrm{dd}=$ doublet of doublets, $\mathrm{dq}=$ doublet of quartets, $\mathrm{dt}=$ doublet of triplets, $\mathrm{qd}=$ quartet of doublets, $\mathrm{tq}=$ triplet of quartets, $\mathrm{ddd}=$ doublet of doublet of doublets, $\mathrm{ddq}=\mathrm{doublet}$ of doublet of quartets, $\mathrm{ddt}=$ doublet of doublet of triplets, $\mathrm{dtd}=$ doublet of triplet of doublets, $\mathrm{dddd}=$ doublet of doublet of doublet of doublets, $\mathrm{m}=$ multiplet, $\mathrm{br}=$ broad), integration, and coupling constant $(J)$ in hertz $(\mathrm{Hz})$. High-resolution mass spectra were obtained on a Waters Acquity UPLC/Xevo G2-XS QTOF mass spectrometer with ESI ionization (special thanks to Ziyang Zhang in the Shokat Laboratory for assistance). Melting points were recorded on an Electrothermal IA6304 Melting Point Apparatus. 


\section{Scheme 1. Synthesis and structural reassignment of 2,18-seco-Lankacidinol B}

A. Synthesis of right half $\mathbf{3 1}$

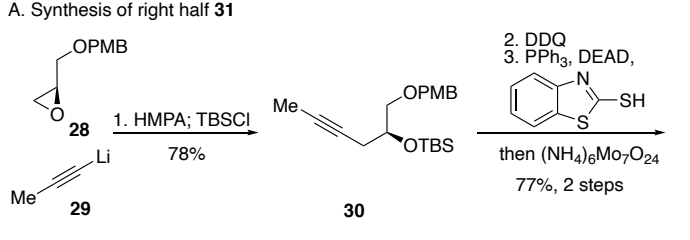

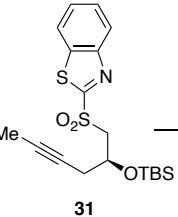

31

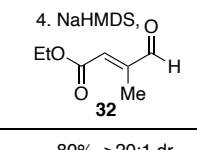

$80 \%,>20: 1 \mathrm{dr}$

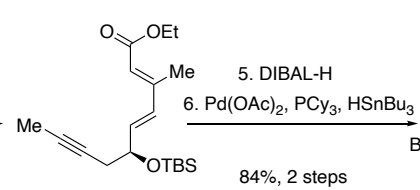

33

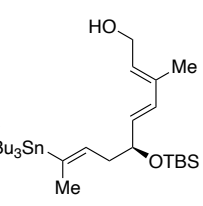

right half $\mathbf{3 4}$

B. Synthesis of $4(R), 5(R)$-left half $(\mathbf{3 7})$ and $4(S), 5(S)$-left half $(\mathbf{3 8})$ by means of an anti-selective aldol-lactonization cascade.

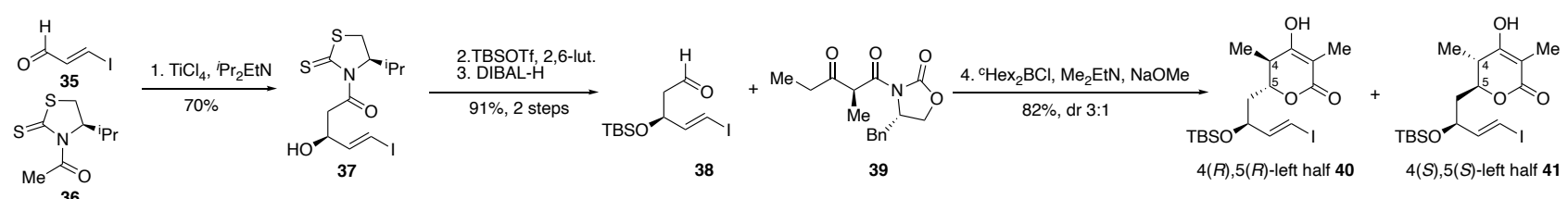

C. Convergent coupling and structural reassignment of 2,18-seco-lankacidinol B (8).
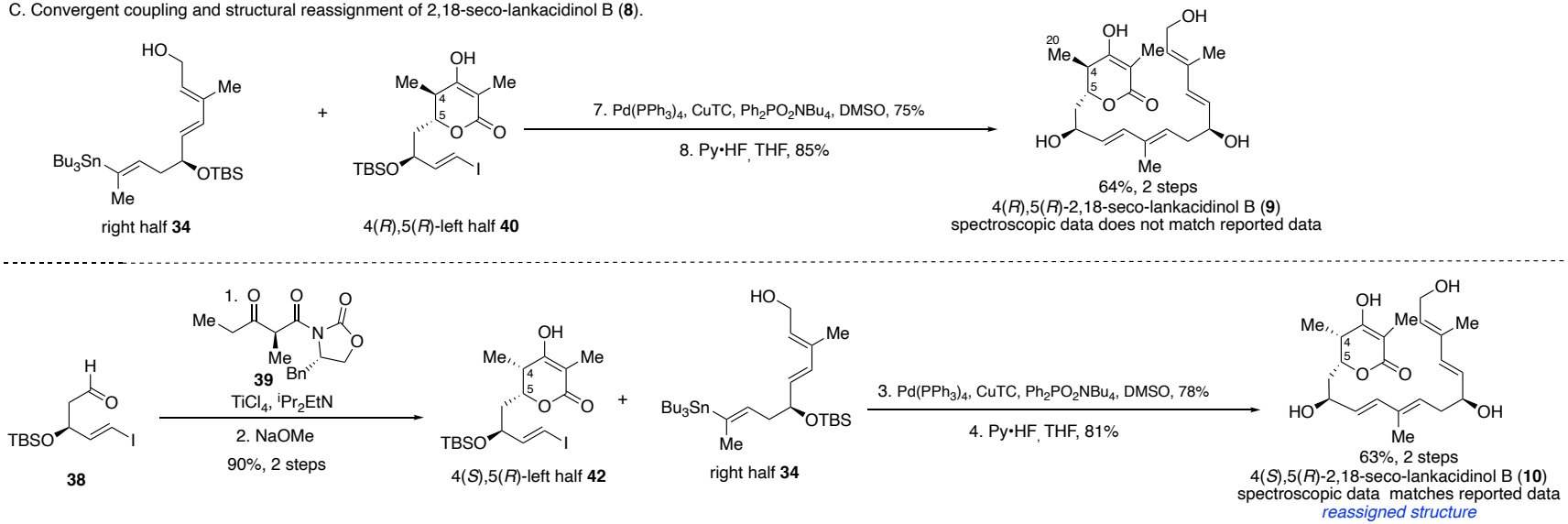


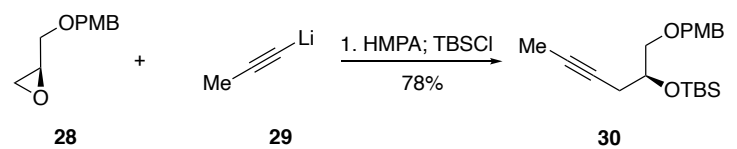

A solution of $n$-butyllithium (2.50 M in hexanes, $102 \mathrm{~mL}, 255 \mathrm{mmol}, 3.90$ equiv) was added to a solution of (Z/E)1-bromopropene $\left(11.20 \mathrm{~mL}, 131 \mathrm{mmol}, 2.00\right.$ equiv) in THF $(120 \mathrm{~mL})$ at $-78^{\circ} \mathrm{C}$, resulting in a white suspension. ${ }^{1}$ After $2 \mathrm{~h}$, HMPA (44.40 mL, $255 \mathrm{mmol}, 3.90$ equiv) was added dropwise, resulting in a colorless solution. After $10 \mathrm{~min}$, a solution of epoxide $\mathbf{2 8}^{2}(12.70 \mathrm{~g}, 65.40 \mathrm{mmol}, 1$ equiv) in THF (15 mL) was added dropwise over $10 \mathrm{~min}$. After $1 \mathrm{~h}$, the vessel was removed from the cooling bath and the system was allowed to warm to $23{ }^{\circ} \mathrm{C}$. After $16 \mathrm{~h}$, TBSCl (21.70 g, $144 \mathrm{mmol}, 2.20$ equiv) was added in one portion to the white reaction suspension, resulting in a colorless solution. After $3 \mathrm{~h}$, water $(100 \mathrm{~mL})$ was added. The biphasic mixture was transferred to a separatory funnel and the layers were separated. The aqueous layer was extracted with ethyl acetate $(2 \times 150 \mathrm{~mL})$. The combined organic layers were washed successively with water $(2 \times 30 \mathrm{~mL})$ and saturated aqueous sodium chloride solution $(50 \mathrm{~mL})$, and the washed solution was dried $\left(\mathrm{Na}_{2} \mathrm{SO}_{4}\right)$. The dried solution was filtered, and the filtrate was concentrated. The resulting crude residue was purified by flash chromatography (silica gel, eluent: EtOAc:hexanes $=1: 50$ to $1: 30)$ to afford TBS ether $\mathbf{3 0}(22.3 \mathrm{~g}, 78 \%)$ as a colorless oil.

TLC $($ EtOAc:hexanes $=1: 25): \mathrm{R}_{f}=0.38\left(\mathrm{UV}, \mathrm{KMnO}_{4}\right)$.

${ }^{1} \mathbf{H}$ NMR $\left(400 \mathrm{MHz}, \mathrm{CDCl}_{3}\right): \delta 7.32-7.21(\mathrm{~m}, 2 \mathrm{H}), 6.91-6.83(\mathrm{~m}, 2 \mathrm{H}), 4.48(\mathrm{~s}, 2 \mathrm{H}), 3.97-3.87(\mathrm{~m}, 1 \mathrm{H}), 3.80$ (s, 3H), $3.48(\mathrm{dd}, J=9.7,5.0 \mathrm{~Hz}, 1 \mathrm{H}), 3.42(\mathrm{dd}, J=9.8,5.5 \mathrm{~Hz}, 1 \mathrm{H}), 2.40(\mathrm{ddq}, J=16.5,6.2,2.6 \mathrm{~Hz}, 1 \mathrm{H}), 2.27$ (ddq, $J=16.5,6.3,2.6 \mathrm{~Hz}, 1 \mathrm{H}), 1.76$ (t, $J=2.6 \mathrm{~Hz}, 3 \mathrm{H}), 0.90$ (s, 9H), 0.10 (s, 3H), 0.08 (s, 3H).

${ }^{13} \mathrm{C} \mathrm{NMR}\left(100 \mathrm{MHz}, \mathrm{CDCl}_{3}\right): \delta 159.1,130.5,129.1,113.6,76.96,76.1,73.6,72.9,70.9,55.2,25.8,25.0,18.2,3.5$, $-4.6,-4.8$.

HRMS-ESI m/z cacld for $\mathrm{C}_{20} \mathrm{H}_{32} \mathrm{NaO}_{3} \mathrm{Si}[\mathrm{M}+\mathrm{Na}]^{+}$371.2013, found 371.2021.
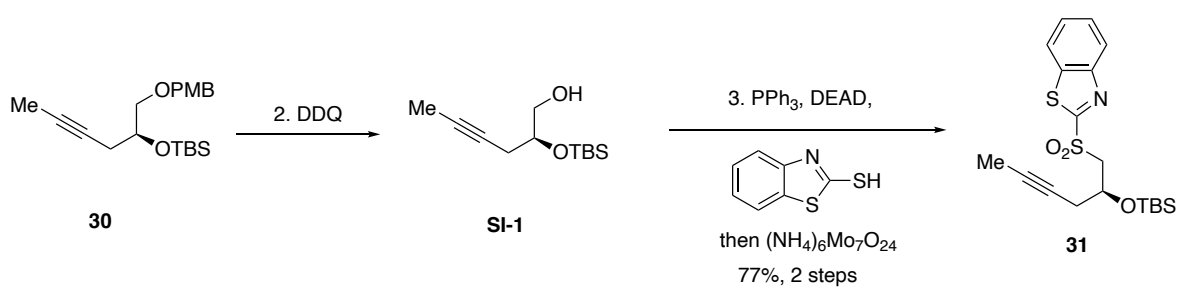

A $250-\mathrm{mL}$ round-bottom flask containing TBS ether 30 (13.60 g, $39.00 \mathrm{mmol}, 1$ equiv), DCM (130 mL) and water $(13.00 \mathrm{~mL})$ was cooled to $0{ }^{\circ} \mathrm{C}$ by means of an ice-water bath. DDQ (10.60 g, $46.80 \mathrm{mmol}, 1.20$ equiv) was added in one portion, resulting a deep green solution. After $2 \mathrm{~h}$, silica gel $(27.20 \mathrm{~g})$ was added, and the mixture was concentrated. The residue was purified by flash chromatography ( silica gel, eluent: EtOAc:hexanes $=1: 20$ ) to afford alcohol SI-1 (7.60 g, 85\%) as a colorless oil.

TLC $($ EtOAc:hexanes $=1: 8): \mathrm{R}_{f}=0.32\left(\mathrm{KMnO}_{4}\right)$.

${ }^{1}$ H NMR $\left(400 \mathrm{MHz}, \mathrm{CDCl}_{3}\right): \delta 3.89-3.77(\mathrm{~m}, 1 \mathrm{H}), 3.64(\mathrm{dd}, J=11.1,3.8 \mathrm{~Hz}, 1 \mathrm{H}), 3.55(\mathrm{dd}, J=11.1,4.9 \mathrm{~Hz}$, $1 \mathrm{H}), 2.52-2.20(\mathrm{~m}, 1 \mathrm{H}), 1.96(\mathrm{~s}, 1 \mathrm{H}), 1.74(\mathrm{t}, J=2.6 \mathrm{~Hz}, 3 \mathrm{H}), 0.88(\mathrm{~s}, 9 \mathrm{H}), 0.09(\mathrm{~s}, 3 \mathrm{H}), 0.08(\mathrm{~s}, 3 \mathrm{H})$.

${ }^{13}$ C NMR $\left(100 \mathrm{MHz}, \mathrm{CDCl}_{3}\right): \delta 77.6,75.4,71.8,65.8,25.7,24.1,18.0,3.4,-4.7,-4.8$.

HRMS-ESI m/z cacld for $\mathrm{C}_{12} \mathrm{H}_{25} \mathrm{O}_{2} \mathrm{Si}[\mathrm{M}+\mathrm{H}]^{+} 229.1618$, found 229.1627 .

A 500-mL round-bottom flask containing SI-1 (3.00 g, $13.1 \mathrm{mmol}, 1$ equiv), benzo[d]thiazole-2-thiol (2.40 g, 14.4 mmol, 1.10 equiv) and $\mathrm{PPh}_{3}(5.17 \mathrm{~g}, 19.7 \mathrm{mmol}, 1.50$ equiv) was evacuated and flushed with nitrogen (this process was repeated a total of 3 times) and was then sealed with a rubber septum. THF ( $60 \mathrm{~mL})$ was added, and the vessel was cooled to $0{ }^{\circ} \mathrm{C}$ by means of an ice-water bath. DEAD $(3.12 \mathrm{~mL}, 19.70 \mathrm{mmol}, 1.50$ equiv) was added dropwise over $10 \mathrm{~min}$. The vessel was removed from the cooling bath and the system was allowed to warm to $23^{\circ} \mathrm{C}$. After 1 h, EtOH $(120 \mathrm{~mL})$ was added. The vessel was cooled to $0{ }^{\circ} \mathrm{C}$ by means of an ice-water bath and $\left(\mathrm{NH}_{4}\right)_{6} \mathrm{Mo}_{7} \mathrm{O}_{24} \bullet 4$ $\mathrm{H}_{2} \mathrm{O}$ (1.60 g, 1.40 mmol, 0.10 equiv) was added followed by $\mathrm{H}_{2} \mathrm{O}_{2}(30 \%$ solution in water, $16.1 \mathrm{~mL}, 158 \mathrm{mmol}$, 12.0 equiv). The vessel was removed from the cooling bath and the system was allowed to warm to $23^{\circ} \mathrm{C}$. After 48 $\mathrm{h}$, the mixture was transferred to a separatory funnel and was extracted with ethyl acetate $(3 \times 50 \mathrm{~mL})$. The 
combined organic layers were washed with saturated aqueous sodium chloride solution $(2 \times 30 \mathrm{~mL})$ and the washed solution was dried $\left(\mathrm{Na}_{2} \mathrm{SO}_{4}\right)$. The dried solution was filtered, and the filtrate was concentrated. The resulting crude residue was purified by flash chromatography (silica gel, eluent: EtOAc:hexanes =1:4) to afford sulfone 31 (4.89 $\mathrm{g}, 91 \%)$ as a colorless oil.

TLC $\left(\right.$ EtOAc:hexanes = 1:8): $\mathrm{R}_{f}=0.39\left(\mathrm{UV}, \mathrm{KMnO}_{4}\right)$.

${ }^{1} \mathbf{H}$ NMR $\left(400 \mathrm{MHz}, \mathrm{CDCl}_{3}\right): \delta 8.21(\mathrm{dd}, J=7.4,1.0 \mathrm{~Hz}, 1 \mathrm{H}), 8.09-7.99(\mathrm{~m}, 1 \mathrm{H}), 7.73-7.54(\mathrm{~m}, 2 \mathrm{H}), 4.51(\mathrm{qd}$, $J=6.2,4.4 \mathrm{~Hz}, 1 \mathrm{H}), 3.98(\mathrm{dd}, J=14.6,4.2 \mathrm{~Hz}, 1 \mathrm{H}), 3.82(\mathrm{dd}, J=14.6,6.7 \mathrm{~Hz}, 1 \mathrm{H}), 2.51(\mathrm{dq}, J=5.1,2.4 \mathrm{~Hz}, 2 \mathrm{H})$, $1.73(\mathrm{t}, J=2.5 \mathrm{~Hz}, 3 \mathrm{H}), 0.81(\mathrm{~s}, 9 \mathrm{H}), 0.07(\mathrm{~s}, 6 \mathrm{H})$.

${ }^{13} \mathrm{C}$ NMR $\left(100 \mathrm{MHz}, \mathrm{CDCl}_{3}\right): \delta 166.7,152.6,136.6,127.9,127.5,125.3,122.3,79.1,73.8,66.4,60.2,27.9,25.5$, $17.8,3.4,-4.7,-4.9$.

HRMS-ESI $\mathrm{m} / \mathrm{z}$ cacld for $\mathrm{C}_{19} \mathrm{H}_{27} \mathrm{NNaO}_{3} \mathrm{~S}_{2} \mathrm{Si}[\mathrm{M}+\mathrm{Na}]^{+}$432.1094, found 432.1096.

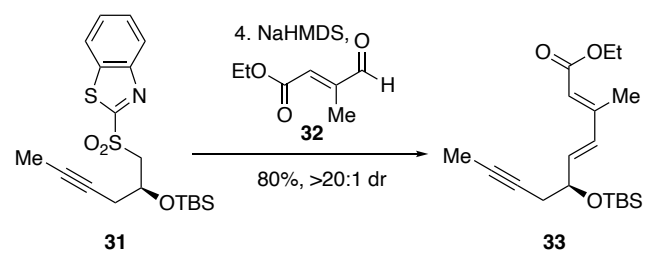

A 100-mL round-bottom flask containing 31 (3.00 g, $7.32 \mathrm{mmol}, 1$ equiv) and $\mathbf{3 2}$ (1.99 mL, $14.6 \mathrm{mmol}, 2.00$ equiv) was evacuated and flushed with nitrogen (this process was repeated a total of 3 times) and was then sealed with a rubber septum. DMF $(50 \mathrm{~mL})$ was added, resulting a colorless solution, and the vessel was cooled to $-40{ }^{\circ} \mathrm{C}$ in a dry ice-acetonitrile bath. NaHMDS (2.00 M in THF, $7.30 \mathrm{~mL}, 14.60 \mathrm{mmol}, 2.00$ equiv) was added dropwise over $10 \mathrm{~min}$. After $2 \mathrm{~h}$, saturated aqueous ammonium chloride solution $(10 \mathrm{~mL})$ and $\mathrm{H}_{2} \mathrm{O}(150 \mathrm{~mL})$ were added successively. The vessel was removed from the cooling bath and the system was allowed to warm to $23{ }^{\circ} \mathrm{C}$. The biphasic mixture was transferred to a separatory funnel and the layers were separated. The aqueous layer was extracted with ethyl acetate $(2 \times 50 \mathrm{~mL})$. The combined organic layers were washed with water $(2 \times 20 \mathrm{~mL})$ and saturated aqueous sodium chloride solution $(20 \mathrm{~mL})$ and the washed solution was dried $\left(\mathrm{Na}_{2} \mathrm{SO}_{4}\right)$. The dried solution was filtered and the filtrate was concentrated. The crude residue was purified by flash chromatography (silica gel, eluent: EtOAc:hexanes = 1:10) to afford dienoate $\mathbf{3 3}(1.97 \mathrm{~g}, 80 \%)$ as a colorless oil.

TLC $($ EtOAc:hexanes $=1: 30): \mathrm{R}_{f}=0.40\left(\mathrm{UV}, \mathrm{KMnO}_{4}\right)$.

${ }^{1}$ H NMR $\left(400 \mathrm{MHz}, \mathrm{CDCl}_{3}\right): \delta 6.29(\mathrm{~d}, J=15.8,1 \mathrm{H}), 6.17(\mathrm{dd}, J=15.6,5.2 \mathrm{~Hz}, 1 \mathrm{H}), 5.76(\mathrm{~d}, J=1.4 \mathrm{~Hz}, 1 \mathrm{H})$, $4.34(\mathrm{q}, J=1.4 \mathrm{~Hz}, 1 \mathrm{H}), 4.17(\mathrm{q}, J=7.1 \mathrm{~Hz}, 2 \mathrm{H}), 2.39(\mathrm{~m}, 1 \mathrm{H}), 2.32-2.20(\mathrm{~m}, 4 \mathrm{H}), 1.77(\mathrm{t}, J=2.6 \mathrm{~Hz}, 3 \mathrm{H}), 1.28$ (t, $J=7.1 \mathrm{~Hz}, 3 \mathrm{H}), 0.90(\mathrm{~s}, 9 \mathrm{H}), 0.08(\mathrm{~s}, 3 \mathrm{H}), 0.05(\mathrm{~s}, 3 \mathrm{H})$.

${ }^{13}$ C NMR (100 MHz, $\left.\mathrm{CDCl}_{3}\right): \delta 167.0,151.7,137.8,132.8,119.3,77.7,75.6,72.0,59.7,28.7,25.8,18.2,14.3$, $13.9,3.5,-4.7,-4.8$.

HRMS-ESI m/z cacld for $\mathrm{C}_{19} \mathrm{H}_{33} \mathrm{O}_{3} \mathrm{Si}[\mathrm{M}+\mathrm{H}]^{+}$337.2193, found 337.2188.

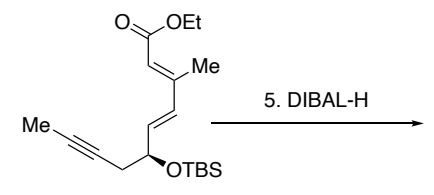

33

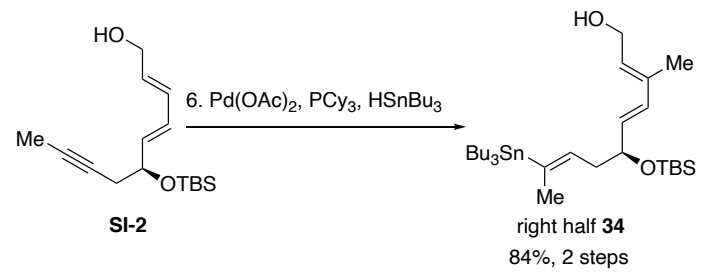

A $250-\mathrm{mL}$ round-bottom flask containing $33(4.10 \mathrm{~g}, 12.20 \mathrm{mmol}, 1$ equiv) was evacuated and flushed with nitrogen (this process was repeated a total of 3 times) and was then sealed with a rubber septum. THF (60 mL) was added, resulting a colorless solution, and the vessel was cooled to $-78^{\circ} \mathrm{C}$ in a dry ice-acetone bath. DIBAL-H (1.00 M in hexane, $25.00 \mathrm{~mL}, 25.00 \mathrm{mmol}, 2.05$ equiv) was added dropwise over $10 \mathrm{~min}$. The vessel was removed from the cooling bath and was allowed to warm to $23{ }^{\circ} \mathrm{C}$. After $1 \mathrm{~h}, \mathrm{MeOH}(4.10 \mathrm{~mL})$ was added followed by saturated aqueous potassium sodium tartrate solution $(150 \mathrm{~mL})$. After $2 \mathrm{~h}$ of very rapid stirring, the biphasic mixture was transferred to a separatory funnel and the layers were separated. The aqueous layer was extracted with ethyl acetate 
$(2 \times 50 \mathrm{~mL})$. The combined organic layers were washed with saturated aqueous sodium chloride solution $(20 \mathrm{~mL})$ and the washed solution was dried $\left(\mathrm{Na}_{2} \mathrm{SO}_{4}\right)$. The dried solution was filtered and the filtrate was concentrated. The crude residue was purified by flash chromatography (silica gel, eluent: EtOAc:hexanes =1:6) to afford alcohol SI$2(3.55 \mathrm{~g}, 99 \%)$ as a colorless oil.

TLC (EtOAc:hexanes = 1:4): $\mathrm{R}_{f}=0.34\left(\mathrm{UV}, \mathrm{KMnO}_{4}\right)$.

${ }^{1} \mathbf{H}$ NMR $\left(400 \mathrm{MHz}, \mathrm{CDCl}_{3}\right): \delta 6.23(\mathrm{dt}, J=15.7,1.0 \mathrm{~Hz}, 1 \mathrm{H}), 5.73(\mathrm{dd}, J=15.7,5.9 \mathrm{~Hz}, 1 \mathrm{H}), 5.64(\mathrm{t}, J=6.7 \mathrm{~Hz}$, $1 \mathrm{H}), 4.27(\mathrm{~d}, J=7.0 \mathrm{~Hz}, 3 \mathrm{H}), 2.52-2.30(\mathrm{~m}, 1 \mathrm{H}), 2.30-2.16(\mathrm{~m}, 1 \mathrm{H}), 1.78(\mathrm{~s}, 3 \mathrm{H}), 1.76(\mathrm{t}, J=2.6 \mathrm{~Hz}, 3 \mathrm{H}), 0.89$ (s, 9H), 0.07 (s, 3H), 0.05 (s, 3H).

${ }^{13} \mathrm{C}$ NMR $\left(100 \mathrm{MHz}, \mathrm{CDCl}_{3}\right): \delta 135.7,133.6,131.4,129.8,77.2,76.1,72.5,59.4,29.0,25.8,18.3,12.7,3.5,-4.6$, -4.8 .

HRMS-ESI m/z cacld for $\mathrm{C}_{18} \mathrm{H}_{31} \mathrm{O}_{4} \mathrm{Si}[\mathrm{M}+\mathrm{HCO} 2]^{-3} 39.1997$, found 339.1991.

A 50-mL round-bottom flask containing $\mathrm{Pd}(\mathrm{OAc})_{2}(122 \mathrm{mg}, 0.543 \mathrm{mmol}, 0.100$ equiv) and tricyclohexylphosphine (305 mg, $1.09 \mathrm{mmol}, 0.200$ equiv) was evacuated and flushed with nitrogen (this process was repeated a total of 3 times) and was then sealed with a rubber septum. THF $(15 \mathrm{~mL})$ was added at $23{ }^{\circ} \mathrm{C}$. After 30 min, a solution of SI2 (1.60 g, $5.43 \mathrm{mmol}, 1$ equiv) in THF $(5 \mathrm{~mL})$ was added, and a solution of $\mathrm{HSnBu}_{3}(2.90 \mathrm{~mL}, 10.9 \mathrm{mmol}, 2.00$ equiv) in THF (6 mL) was added via syringe pump over $2 \mathrm{~h}$. The mixture was concentrated, and the crude residue was purified by flash chromatography (silica gel, eluent: EtOAc:hexanes $=1: 15$ to 1:8) to afford right half 34 (2.70 $\mathrm{g}, 85 \%)$ as a colorless oil.

TLC (EtOAc:hexanes = 1:6): $\mathrm{R}_{f}=0.28\left(\mathrm{UV}, \mathrm{KMnO}_{4}\right)$.

${ }^{1} \mathbf{H}$ NMR $\left(400 \mathrm{MHz}, \mathrm{CDCl}_{3}\right): \delta 6.17(\mathrm{~d}, J=15.7 \mathrm{~Hz}, 1 \mathrm{H}), 5.69(\mathrm{dd}, J=15.7,6.3 \mathrm{~Hz}, 1 \mathrm{H}), 5.62(\mathrm{t}, J=6.8 \mathrm{~Hz}, 1 \mathrm{H})$, 5.57 (ddd, $J=8.7,5.1,1.8 \mathrm{~Hz}, 1 \mathrm{H}), 4.28(\mathrm{t}, J=6.2 \mathrm{~Hz}, 2 \mathrm{H}), 4.21(\mathrm{q}, J=6.4 \mathrm{~Hz}, 1 \mathrm{H}), 2.34(\mathrm{tq}, J=13.7,6.4 \mathrm{~Hz}$, $2 \mathrm{H}), 1.82(\mathrm{~d}, J=1.9 \mathrm{~Hz}, 3 \mathrm{H}), 1.77(\mathrm{~s}, 3 \mathrm{H}), 1.55-1.41(\mathrm{~m}, 6 \mathrm{H}), 1.36-1.20(\mathrm{~m}, 6 \mathrm{H}), 0.94-0.77(\mathrm{~m}, 24 \mathrm{H}), 0.05(\mathrm{~s}$, $3 \mathrm{H}), 0.03(\mathrm{~s}, 3 \mathrm{H})$.

${ }^{13}$ C NMR (100 MHz, $\left.\mathrm{CDCl}_{3}\right): \delta 139.9,136.4,135.9,132.8,132.8,129.3,73.4,59.4,37.3,29.2,29.1,27.4,25.93$, $25.90,19.4,18.3,13.7,12.6,9.1,-4.3,-4.7$.

HRMS-ESI The compound failed to ionize under electrospray ionization in both positive and negative modes.

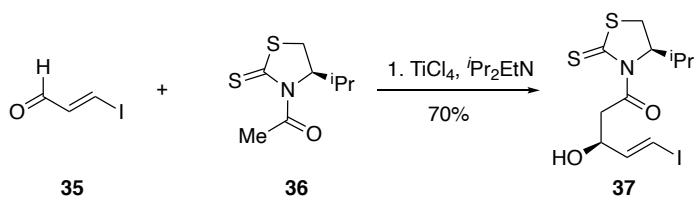

A 1000-mL round-bottom flask containing 36 (18.90 g, $93.10 \mathrm{mmol}, 1.10$ equiv) was evacuated and flushed with nitrogen (this process was repeated a total of 3 times) and was then sealed with a rubber septum. DCM $(360 \mathrm{~mL})$ was added, resulting a yellow solution and the vessel was cooled to $-78{ }^{\circ} \mathrm{C}$ in a dry ice-acetone bath. $\mathrm{TiCl}_{4}(1.00 \mathrm{M}$ in DCM, $102 \mathrm{~mL}, 102 \mathrm{mmol}, 1.20$ equiv) was added dropwise, resulting a deep yellow solution. After $5 \mathrm{~min}$, ${ }^{i} \operatorname{Pr}_{2} \operatorname{EtN}$ (17.70 mL, $102 \mathrm{mmol}, 1.20$ equiv) was added by syringe pump over $30 \mathrm{~min}$, and the resulting deep red solution was stirred for $2 \mathrm{~h}$ at $-78^{\circ} \mathrm{C}$. A solution of $\mathbf{3 5}^{3}$ (15.40 g, $84.60 \mathrm{mmol}, 1$ equiv) in DCM (20 mL) was added via syringe pump over $30 \mathrm{~min}$. After $30 \mathrm{~min}, \mathrm{pH} 7.0$ phosphate buffer $(200 \mathrm{~mL})$ was added. The vessel was removed from the cooling bath and the system was allowed to warm to $23{ }^{\circ} \mathrm{C}$ while the mixture was rapidly stirred. The biphasic mixture was transferred to a separatory funnel and the layers were separated. The aqueous layer was extracted with DCM $(2 \times 150 \mathrm{~mL})$. The combined organic layers were washed with saturated aqueous sodium chloride solution $(2 \times 100 \mathrm{~mL})$ and the washed solution was dried $\left(\mathrm{Na}_{2} \mathrm{SO}_{4}\right)$. The dried solution was filtered and the filtrate was concentrated. The crude residue was purified by flash chromatography (silica gel, eluent: EtOAc:hexanes $=1: 6$ to 1:3) to afford ß-hydroxyl amide $37(22.80 \mathrm{~g}, 70 \%)$ as a yellow solid.

m. p. $101-103^{\circ} \mathrm{C}$

TLC (EtOAc:hexanes = 1:2): $\mathrm{R}_{f}=0.39\left(\mathrm{UV}, \mathrm{KMnO}_{4}\right)$. 
${ }^{1}$ H NMR $\left(400 \mathrm{MHz}, \mathrm{CDCl}_{3}\right): \delta 6.62(\mathrm{dd}, J=14.5,5.3 \mathrm{~Hz}, 1 \mathrm{H}), 6.47(\mathrm{dd}, J=14.5,1.5 \mathrm{~Hz}, 1 \mathrm{H}), 5.14(\mathrm{ddd}, J=7.7$, $6.3,1.1 \mathrm{~Hz}, 1 \mathrm{H}), 4.63(\mathrm{~m}, 1 \mathrm{H}), 3.67(\mathrm{dd}, J=17.6,3.1 \mathrm{~Hz}, 1 \mathrm{H}), 3.54(\mathrm{dd}, J=11.5,7.9 \mathrm{~Hz}, 1 \mathrm{H}), 3.29(\mathrm{dd}, J=17.6$, $8.6 \mathrm{~Hz}, 1 \mathrm{H}), 3.16-2.90(\mathrm{~m}, 2 \mathrm{H}), 2.35(\mathrm{dq}, J=13.6,6.8 \mathrm{~Hz}, 1 \mathrm{H}), 1.06(\mathrm{~d}, J=6.8 \mathrm{~Hz}, 3 \mathrm{H}), 0.98(\mathrm{~d}, J=7.0 \mathrm{~Hz}, 3 \mathrm{H})$.

${ }^{13}$ C NMR (100 MHz, $\left.\mathrm{CDCl}_{3}\right): \delta 203.0,171.8,145.8,78.3,71.3,70.4,44.3,30.8,30.7,19.1,17.8$.

HRMS-ESI $\mathrm{m} / \mathrm{z}$ cacld for $\mathrm{C}_{11} \mathrm{H}_{17} \mathrm{INO}_{2} \mathrm{~S}_{2}[\mathrm{M}+\mathrm{H}]^{+}$385.9740, found 385.9733.
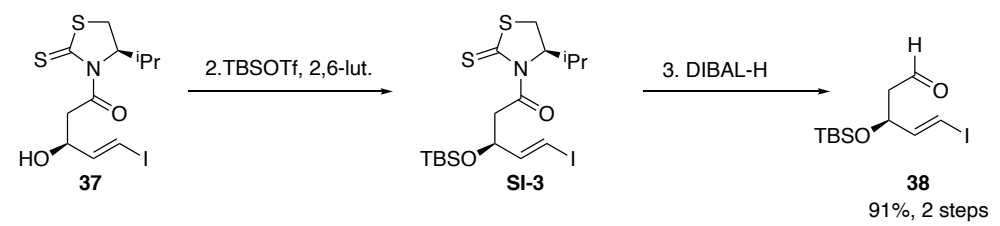

A 250-mL round-bottom flask containing 37 (6.68 g, $17.3 \mathrm{mmol}, 1$ equiv) was evacuated and flushed with nitrogen (this process was repeated a total of 3 times) and was then sealed with a rubber septum. Dry DCM (70 mL) was added followed by 2,6-lutidine $(3.03 \mathrm{~mL}, 26.0 \mathrm{mmol}, 1.50$ equiv), resulting in a yellow solution. The vessel was cooled to $0{ }^{\circ} \mathrm{C}$ by means of an ice-water bath. TBSOTf $(4.38 \mathrm{~mL}, 19.10 \mathrm{mmol}, 1.10$ equiv) was added dropwise over $10 \mathrm{~min}$. After $1 \mathrm{~h}$, the mixture was transferred to a separatory funnel and was washed with water $(2 \times 30 \mathrm{~mL})$ and saturated aqueous sodium chloride solution $(30 \mathrm{~mL})$. The washed solution was dried $\left(\mathrm{Na}_{2} \mathrm{SO}_{4}\right)$. The dried solution was filtered and the filtrate was concentrated. The resulting crude residue was purified by flash chromatography (silica gel, eluent: EtOAc:hexanes $=1: 20$ to $1: 15)$ to afford TBS ether SI-3 (8.73 g, 93\%) as a yellow solid.

m. p. $50-52{ }^{\circ} \mathrm{C}$

TLC $($ EtOAc:hexanes $=1: 16): \mathrm{R}_{f}=0.37\left(\mathrm{UV}, \mathrm{KMnO}_{4}\right)$.

${ }^{1} \mathbf{H}$ NMR $\left(400 \mathrm{MHz}, \mathrm{CDCl}_{3}\right): \delta 6.61(\mathrm{dd}, J=14.4,6.4 \mathrm{~Hz}, 1 \mathrm{H}), 6.35(\mathrm{dd}, J=14.5,1.1 \mathrm{~Hz}, 1 \mathrm{H}), 5.04(\mathrm{ddd}, J=7.6$, $6.3,1.1 \mathrm{~Hz}, 1 \mathrm{H}), 4.70(\mathrm{~m}, 1 \mathrm{H}), 3.60(\mathrm{dd}, J=16.7,7.8 \mathrm{~Hz}, 1 \mathrm{H}), 3.48(\mathrm{dd}, J=11.5,7.8 \mathrm{~Hz}, 1 \mathrm{H}), 3.25(\mathrm{dd}, J=16.7$, $4.6 \mathrm{~Hz}, 1 \mathrm{H}), 3.03(\mathrm{dd}, J=11.5,1.1 \mathrm{~Hz}, 1 \mathrm{H}), 2.37(\mathrm{dq}, J=13.6,6.8 \mathrm{~Hz}, 1 \mathrm{H}), 1.06(\mathrm{~d}, J=6.8 \mathrm{~Hz}, 3 \mathrm{H}), 0.98(\mathrm{~d}, J=$ $6.9 \mathrm{~Hz}, 3 \mathrm{H}), 0.86(\mathrm{~s}, 9 \mathrm{H}), 0.05(\mathrm{~s}, 3 \mathrm{H}), 0.05(\mathrm{~s}, 3 \mathrm{H})$.

${ }^{13}$ C NMR (100 MHz, $\left.\mathrm{CDCl}_{3}\right): \delta 202.8,170.5,147.7,77.4,72.0,71.6,45.5,30.84,30.75,25.7,19.1,18.1,17.8,-$ $4.5,-5.0$.

HRMS-ESI The compound failed to ionize under electrospray ionization in both positive and negative modes.

A 250-mL round-bottom flask containing TBS ether SI-3 (8.20 g, $16.40 \mathrm{mmol}, 1$ equiv) was evacuated and flushed with nitrogen (this process was repeated a total of 3 times) and was then sealed with a rubber septum. Dry DCM $(100 \mathrm{~mL})$ was added, resulting in a yellow solution and the vessel was cooled to $-78^{\circ} \mathrm{C}$ in a dry ice-acetone bath. DIBAL-H (1.00 M in hexane, $33.70 \mathrm{~mL}, 2.05$ equiv) was added dropwise over $10 \mathrm{~min}$. After $1 \mathrm{~h}, \mathrm{MeOH}(8.20 \mathrm{~mL})$ was added followed by saturated aqueous potassium sodium tartrate solution $(100 \mathrm{~mL})$. The vessel was removed from the cooling bath and the system was allowed to warm to $23{ }^{\circ} \mathrm{C}$ and was stirred very rapidly for $2 \mathrm{~h}$. The biphasic mixture was transferred to a separatory funnel and the layers were separated. The aqueous layer was extracted with DCM $(2 \times 50 \mathrm{~mL})$. The combined organic layers were washed with saturated aqueous sodium chloride solution $(30 \mathrm{~mL})$ and the washed solution was dried $\left(\mathrm{Na}_{2} \mathrm{SO}_{4}\right)$. The dried solution was filtered, and the filtrate was concentrated. The resulting crude residue was purified by flash chromatography (silica gel, eluent: EtOAc:hexanes $=1: 10$ to $1: 5)$ to afford aldehyde $\mathbf{3 8}(5.49 \mathrm{~g}, 98 \%)$ as a pale yellow oil.

TLC $($ EtOAc:hexanes $=1: 16): \mathrm{R}_{f}=0.34\left(\mathrm{UV}, \mathrm{KMnO}_{4}\right)$.

${ }^{1} \mathbf{H}$ NMR $\left(400 \mathrm{MHz}, \mathrm{CDCl}_{3}\right): \delta 9.75(\mathrm{t}, J=2.1 \mathrm{~Hz}, 1 \mathrm{H}), 6.58(\mathrm{dd}, J=14.4,5.8 \mathrm{~Hz}, 1 \mathrm{H}), 6.38(\mathrm{dd}, J=14.4,1.3 \mathrm{~Hz}$, $1 \mathrm{H}), 4.62(\mathrm{dtd}, J=6.8,5.4,1.3 \mathrm{~Hz}, 1 \mathrm{H}), 2.63(\mathrm{ddd}, J=16.2,6.7,2.3 \mathrm{~Hz}, 1 \mathrm{H}), 2.53(\mathrm{ddd}, J=16.2,5.2,1.9 \mathrm{~Hz}, 1 \mathrm{H})$, $0.87(\mathrm{~s}, 9 \mathrm{H}), 0.06(\mathrm{~s}, 3 \mathrm{H}), 0.05(\mathrm{~s}, 3 \mathrm{H})$.

${ }^{13}$ C NMR (100 MHz, $\left.\mathrm{CDCl}_{3}\right): \delta 200.3,147.2,77.4,70.7,50.6,25.7,18.0,-4.6,-5.1$.

HRMS-ESI The compound failed to ionize under electrospray ionization in both positive and negative modes. 


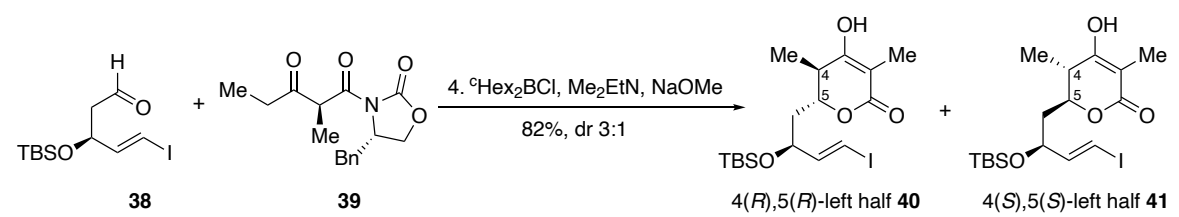

A $250-\mathrm{mL}$ round-bottom flask containing $\beta$-ketoimide $39^{4}(1.66 \mathrm{~g}, 5.73 \mathrm{mmol}, 1.30$ equiv) was evacuated and flushed with nitrogen (this process was repeated a total of 3 times) and was then sealed with a rubber septum. $\mathrm{Et}_{2} \mathrm{O}$ $(70 \mathrm{~mL})$ was added, resulting in a colorless solution and the vessel was cooled to $0{ }^{\circ} \mathrm{C}$ by means of an ice-water bath. Chlorodicyclohexylborane $(1.00 \mathrm{M}$ in hexanes, $6.88 \mathrm{~mL}, 6.88 \mathrm{mmol}, 1.56$ equiv) was added dropwise followed by $\mathrm{N}, \mathrm{N}$-dimethylethanamine $(745 \mu \mathrm{L}, 6.88 \mathrm{mmol}, 1.56$ equiv), resulting a pale yellow suspension. After $4 \mathrm{~h}$, The vessel was cooled to $-78^{\circ} \mathrm{C}$ in a dry ice-acetone bath. A solution of aldehyde $38(1.50 \mathrm{~g}, 4.41 \mathrm{mmol}, 1$ equiv) in $\mathrm{Et}_{2} \mathrm{O}(10 \mathrm{~mL})$ was added dropwise over $10 \mathrm{~min}$. After $20 \mathrm{~h}$, a solution of $\mathrm{MeOH}(0.28 \mathrm{~mL}, 6.88 \mathrm{mmol}$, 1.56 equiv) in THF $(80 \mathrm{~mL})$ was added in a single portion. The vessel was removed from the cooling bath and the system was allowed to warm to $23^{\circ} \mathrm{C}$. NaOMe $(476 \mathrm{mg}, 8.82 \mathrm{mmol}, 2.00$ equiv) was added, resulting in a colorless solution. After $30 \mathrm{~min}$, saturated aqueous ammonium chloride solution $(30 \mathrm{~mL})$ and water $(30 \mathrm{~mL})$ were added, followed by $\mathrm{AcOH}(500 \mu \mathrm{L}, 8.82 \mathrm{mmol}, 2.0$ equiv) to bring the $\mathrm{pH}$ to $\sim 7$ as measured by universal $\mathrm{pH}$ strips. The biphasic mixture was transferred to a separatory funnel and the layers were separated. The aqueous layer was extracted with diethyl ether $(2 \times 80 \mathrm{~mL})$. The combined organic layers were washed with saturated aqueous sodium chloride solution $(30 \mathrm{~mL})$ and the washed solution was dried $\left(\mathrm{Na}_{2} \mathrm{SO}_{4}\right)$. The dried solution was filtered, and the filtrate was concentrated. The resulting crude residue was purified by flash chromatography (silica gel, eluent: EtOAc:hexanes $=1: 5$ to $1: 2)$ to provide a mixture of two diastereomers $\left(\sim 3: 1\right.$ ratio by $\left.{ }^{1} \mathrm{H}-\mathrm{NMR}\right)$. The two diastereomers were separated by flash chromatography (silica gel, eluent: DCM:hexanes $=2: 1$ ) to afford $4(R), 5(R)$ left half $40(1.22 \mathrm{~g}, 61 \%)$ and $4(S), 5(S)$-left half $\mathbf{4 1}(0.41 \mathrm{~g}, 21 \%)$ as pale yellow oils.

\section{$4(R), 5(R)$-left half 40}

TLC (DCM:hexanes $=4: 1): \mathrm{R}_{f}=0.22\left(\mathrm{UV}, \mathrm{KMnO}_{4}\right)$.

${ }^{1}$ H NMR $(400 \mathrm{MHz}, \mathrm{MeOD}) \delta 6.58(\mathrm{dd}, J=14.5,6.7 \mathrm{~Hz}, 1 \mathrm{H}), 6.49(\mathrm{~d}, J=14.6 \mathrm{~Hz}, 1 \mathrm{H}), 4.44-4.33(\mathrm{~m}, 1 \mathrm{H}), 4.14$ $(\mathrm{dt}, J=8.8,4.2 \mathrm{~Hz}, 1 \mathrm{H}), 2.46(\mathrm{qd}, J=6.8,3.0 \mathrm{~Hz}, 1 \mathrm{H}), 2.04(\mathrm{ddd}, J=14.3,9.2,5.2 \mathrm{~Hz}, 1 \mathrm{H}), 1.85-1.73(\mathrm{~m}, 1 \mathrm{H})$, $1.72(\mathrm{~s}, 3 \mathrm{H}), 1.26(\mathrm{~d}, J=7.1 \mathrm{~Hz}, 3 \mathrm{H}), 0.89(\mathrm{~s}, 9 \mathrm{H}), 0.06(\mathrm{~s}, 3 \mathrm{H}), 0.05(\mathrm{~s}, 3 \mathrm{H})$.

${ }^{13}$ C NMR (100 MHz, MeOD) $\delta 171.1,170.4,149.0,98.0,78.6,78.3,73.7,42.0,38.1,26.3,19.0,16.7,8.6,-4.4,-$ 4.8.

HRMS-ESI m/z cacld for $\mathrm{C}_{17} \mathrm{H}_{29} \mathrm{INaO}_{4} \mathrm{Si}[\mathrm{M}+\mathrm{Na}]^{+}$475.0772, found 475.0771.

\section{$4(S), 5(S)$ - left half 41}

TLC $(\mathrm{DCM}:$ hexanes $=4: 1): \mathrm{R}_{f}=0.25\left(\mathrm{UV}, \mathrm{KMnO}_{4}\right)$.

${ }^{1}$ H NMR (400 MHz, MeOD) $\delta 6.56(\mathrm{dd}, J=14.6,6.7 \mathrm{~Hz}, 1 \mathrm{H}), 6.44(\mathrm{~d}, J=14.4 \mathrm{~Hz}, 1 \mathrm{H}), 4.41(\mathrm{ddd}, J=9.7,6.6$, $2.6 \mathrm{~Hz}, 1 \mathrm{H}), 4.25(\mathrm{ddd}, J=10.2,5.2,2.8 \mathrm{~Hz}, 1 \mathrm{H}), 2.44(\mathrm{t}, J=6.2 \mathrm{~Hz}, 1 \mathrm{H}), 1.98-1.79(\mathrm{~m}, 1 \mathrm{H}), 1.72(\mathrm{~m}, 4 \mathrm{H}), 1.23$ (d, $J=7.0 \mathrm{~Hz}, 3 \mathrm{H}), 0.91(\mathrm{~s}, 9 \mathrm{H}), 0.11(\mathrm{~s}, 3 \mathrm{H}), 0.07(\mathrm{~s}, 3 \mathrm{H})$.

${ }^{13}$ C NMR (100 MHz, MeOD) $\delta 171.0,170.4,150.1,98.2,78.0,77.6,72.7,42.4,38.3,26.3,19.0,16.1,8.7,-4.2,-$ 4.8 .

HRMS-ESI m/z cacld for $\mathrm{C}_{17} \mathrm{H}_{29} \mathrm{INaO}_{4} \mathrm{Si}[\mathrm{M}+\mathrm{Na}]^{+}$475.0772, found 475.0771.

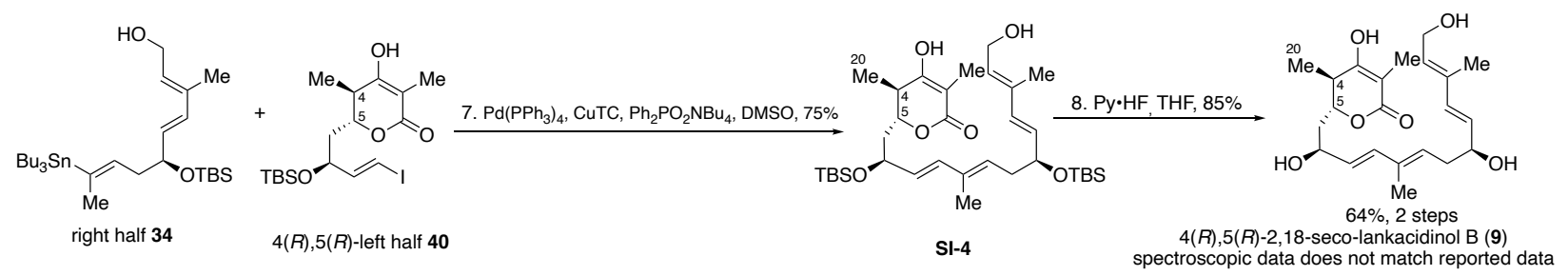

Preparation of tetra-n-butylammonium diphenylphosphinate: $:^{5}$ To a suspension of $\mathrm{Ph}_{2} \mathrm{PO}_{2} \mathrm{H}(1.09 \mathrm{~g}, 5.00 \mathrm{mmol})$ in dry $\mathrm{MeOH}(10.0 \mathrm{~mL})$ at room temperature was added $\mathrm{Bu}_{4} \mathrm{NOH}(1.0 \mathrm{M}$ in $\mathrm{MeOH}, 5.00 \mathrm{~mL}, 5.00 \mathrm{mmol})$, resulting 
colorless solution. After $15 \mathrm{~min}$, the mixture was concentrated. The resulting clear oil was dried under high vacuum for $2 \mathrm{~h}$. The residue was dissolved in DMSO to make $0.500 \mathrm{M}$ stock solution for the Stille coupling reaction.

A $25-\mathrm{mL}$ round-bottom flask containing $40(50.00 \mathrm{mg}, 0.11 \mathrm{mmol}, 1$ equiv) and $34(64.70 \mathrm{mg}, 0.111 \mathrm{mmol}, 1.00$ equiv) was evacuated and flushed with nitrogen (this process was repeated a total of 3 times) and was then sealed with a rubber septum. DMSO $(5 \mathrm{~mL})$ was added, and $\mathrm{Ph}_{2} \mathrm{PO}_{2} \mathrm{NBu}_{4}(0.50 \mathrm{M}$ in DMSO, $0.33 \mathrm{~mL}, 0.17 \mathrm{mmol}, 1.50$ equiv) was added followed by $\mathrm{Pd}\left(\mathrm{Ph}_{3} \mathrm{P}\right)_{4}(6.40 \mathrm{mg}, 5.55$ umol, 0.0500 equiv) and CuTC (23.20 mg, $0.12 \mathrm{mmol}$, 1.10 equiv). After $30 \mathrm{~min}$, the mixture was diluted with $\mathrm{Et}_{2} \mathrm{O}(15 \mathrm{~mL})$ and was poured into an ice-water $(20 \mathrm{~mL})$. The biphasic mixture was transferred to a separatory funnel and the layers were separated. The aqueous layer was extracted with $\mathrm{Et}_{2} \mathrm{O}(2 \times 15 \mathrm{~mL})$. The combined organic layers were washed with water $(10 \mathrm{~mL})$ and saturated aqueous sodium chloride solution $(10 \mathrm{~mL})$ and the washed solution was dried $\left(\mathrm{Na}_{2} \mathrm{SO}_{4}\right)$. The dried solution was filtered and the filtrate was concentrated. The crude residue was purified by flash chromatography (silica gel, eluent: EtOAc:hexanes $=1: 2)$ to afford alcohol SI-4 $(51.5 \mathrm{mg}, 75 \%)$ as a colorless oil.

TLC (EtOAc:hexanes = 1:2): $\mathrm{R}_{f}=0.29$ (UV, anisaldehyde).

${ }^{1}$ H NMR (400 MHz, MeOD): $\delta 6.26(\mathrm{~d}, J=8.2 \mathrm{~Hz}, 1 \mathrm{H}), 6.22(\mathrm{~d}, J=8.2 \mathrm{~Hz}, 1 \mathrm{H}), 5.69(\mathrm{dd}, J=15.6,6.4 \mathrm{~Hz}, 1 \mathrm{H})$, $5.62-5.49(\mathrm{~m}, 3 \mathrm{H}), 4.41(\mathrm{q}, J=7.5 \mathrm{~Hz}, 1 \mathrm{H}), 4.29(\mathrm{q}, J=6.3 \mathrm{~Hz}, 1 \mathrm{H}), 4.21(\mathrm{~d}, J=6.7 \mathrm{~Hz}, 2 \mathrm{H}), 4.13(\mathrm{dt}, J=8.5$, $4.8 \mathrm{~Hz}, 1 \mathrm{H}), 2.53-2.45(\mathrm{~m}, 1 \mathrm{H}), 2.36(\mathrm{tt}, J=14.3,7.3 \mathrm{~Hz}, 2 \mathrm{H}), 2.07(\mathrm{ddd}, J=14.0,8.7,5.3 \mathrm{~Hz}, 1 \mathrm{H}), 1.83-1.73$ $(\mathrm{m}, 10 \mathrm{H}), 1.73-1.70(\mathrm{~m}, 3 \mathrm{H}), 1.24(\mathrm{~d}, J=7.1 \mathrm{~Hz}, 3 \mathrm{H}), 0.90(\mathrm{~s}, 9 \mathrm{H}), 0.89(\mathrm{~s}, 9 \mathrm{H}), 0.06(\mathrm{~s}, 3 \mathrm{H}), 0.04(\mathrm{~s}, 6 \mathrm{H}), 0.02$ $(\mathrm{s}, 3 \mathrm{H})$.

${ }^{13}$ C NMR (100 MHz, MeOD) $\delta 171.1,170.6,137.2,136.0,135.6,134.8,133.1,131.5,130.5,129.8,98.0,79.1$, $74.8,72.4,59.3,43.1,38.6,38.0,26.4,26.1,19.08,19.06,16.7,13.0,12.7,8.6,-4.0,-4.1,-4.4,-4.6$.

HRMS-ESI m/z cacld for $\mathrm{C}_{34} \mathrm{H}_{59} \mathrm{O}_{6} \mathrm{Si}_{2}[\mathrm{M}-\mathrm{H}]^{-}$619.3856, found 619.3878.

A 50-mL plastic vial containing SI-4 $\left(28.00 \mathrm{mg}, 45.1\right.$ umol, 1 equiv) and THF (5 mL) was cooled to $0{ }^{\circ} \mathrm{C}$ by means of an ice-water bath. Py $(\mathrm{HF})_{\mathrm{x}}(\sim 70 \% \mathrm{HF})(400 \mu \mathrm{L})$ was added dropwise, and the vessel was removed from the cooling bath and the system was allowed to warm to $23^{\circ} \mathrm{C}$. After $3 \mathrm{~h}, \mathrm{NaHCO}_{3}(970 \mathrm{mg})$ was added and the mixture was rapidly stirred. After $15 \mathrm{~min}$, the mixture was filtered through a pad of Celite and the filtrate was concentrated. The crude residue was purified by flash chromatography (silica gel, eluent: acetone:hexanes $=2: 1$ ) to afford 4(R),5(R)-2,18-seco-lankacidinol B (9) $(15.00 \mathrm{mg}, 85 \%)$ as a colorless oil.

TLC (EtOAc: $\mathrm{MeOH}=30: 1): \mathrm{R}_{f}=0.40$ (UV, anisaldehyde).

${ }^{1} \mathbf{H}$ NMR (400 MHz, MeOD): $\delta 6.30(\mathrm{~d}, J=15.7 \mathrm{~Hz}, 1 \mathrm{H}), 6.25(\mathrm{~d}, J=15.7 \mathrm{~Hz}, 1 \mathrm{H}), 5.69(\mathrm{dd}, J=15.7,6.7 \mathrm{~Hz}$, $1 \mathrm{H}), 5.62(\mathrm{~d}, J=6.7 \mathrm{~Hz}, 1 \mathrm{H}), 5.59(\mathrm{~d}, J=7.3 \mathrm{~Hz}, 1 \mathrm{H}), 5.55(\mathrm{~d}, J=7.4 \mathrm{~Hz}, 1 \mathrm{H}), 4.28(\mathrm{q}, J=7.1 \mathrm{~Hz}, 1 \mathrm{H}), 4.20(\mathrm{~d}$, $J=6.7 \mathrm{~Hz}, 2 \mathrm{H}), 4.16(\mathrm{~m}, 2 \mathrm{H}), 2.53-2.46(\mathrm{~m}, 1 \mathrm{H}), 2.45-2.35(\mathrm{~m}, 2 \mathrm{H}), 2.08(\mathrm{ddd}, J=14.3,8.7,5.9 \mathrm{~Hz}, 1 \mathrm{H}), 1.81$ $-1.73(\mathrm{~m}, 7 \mathrm{H}), 1.72(\mathrm{~d}, J=0.7 \mathrm{~Hz}, 3 \mathrm{H}), 1.25(\mathrm{~d}, J=7.0 \mathrm{~Hz}, 3 \mathrm{H})$.

${ }^{13}$ C NMR (100 MHz, MeOD) $\delta 171.2,170.6,137.4,136.0,135.9,135.5,132.3,131.8,129.7,129.7,98.0,79.4$, $73.3,70.8,59.3,41.9,38.0,37.5,16.8,12.9,12.7,8.6$.

HRMS-ESI m/z cacld for $\mathrm{C}_{22} \mathrm{H}_{31} \mathrm{O}_{6}[\mathrm{M}-\mathrm{H}]^{-} 391.2126$, found 391.2139.

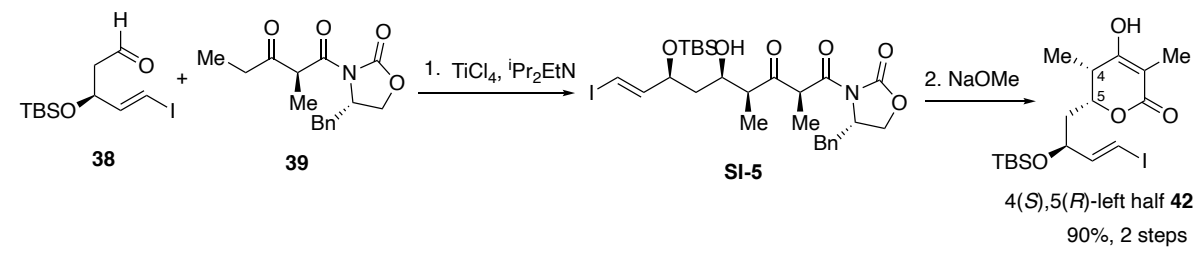

A 100-mL round-bottom flask containing $\beta$-ketoimide $3^{4}$ ( $868 \mathrm{mg}, 3.00 \mathrm{mmol}, 1$ equiv) was evacuated and flushed with nitrogen (this process was repeated a total of 3 times) and was then sealed with a rubber septum. DCM (20 $\mathrm{mL}$ ) was added, resulting a colorless solution, and the vessel was cooled to $-78^{\circ} \mathrm{C}$ in a dry ice-acetone bath. $\mathrm{TiCl}_{4}$ (1.00 M in DCM, $3.30 \mathrm{~mL}, 3.30 \mathrm{mmol}, 1.10$ equiv) was added dropwise followed by ${ }^{\mathrm{i}} \operatorname{Pr}_{2} \mathrm{EtN}(576 \mu \mathrm{L}, 3.30 \mathrm{mmol}$, 1.10 equiv), resulting in a red solution. The cooling bath was removed, and the system was warmed to between -10 to $-5^{\circ} \mathrm{C}$ in an acetone-ice bath. After $1 \mathrm{~h}$, The vessel was cooled to $-78^{\circ} \mathrm{C}$ with a dry ice-acetone bath, and a solution of aldehyde 38 ( $871 \mathrm{mg}, 3.00 \mathrm{mmol}, 1.00$ equiv) in DCM (3 mL) was added dropwise over $10 \mathrm{~min}$. After $30 \mathrm{~min}$, the mixture was gradually warmed to $-40{ }^{\circ} \mathrm{C}$ over $1 \mathrm{~h}$, and $\mathrm{pH} 7.0$ phosphate buffer $(35 \mathrm{~mL})$ was added in a single 
portion. The vessel was removed from the cooling bath and the system was allowed to warm to $0{ }^{\circ} \mathrm{C}$ in an ice-water bath. After $10 \mathrm{~min}$, the mixture was poured into a rapidly stirred mixture of DCM $(210 \mathrm{~mL})$ and water $(35 \mathrm{~mL})$. After $10 \mathrm{~min}$, the biphasic mixture was transferred to a separatory funnel and the layers were separated. The organic layer was washed with saturated aqueous $\mathrm{NaHCO}_{3}$ solution $(50 \mathrm{~mL})$ and saturated aqueous sodium chloride solution $(30 \mathrm{~mL})$ and the washed solution was dried $\left(\mathrm{Na}_{2} \mathrm{SO}_{4}\right)$. The dried solution was filtered and the filtrate was concentrated. The crude residue was purified by flash chromatography (silica gel, eluent: EtOAc:hexanes $=1: 3$ ) to afford $\beta$-hydroxyl amide SI-5 (1.89 g, $100 \%$ ) as a pale yellow oil.

TLC (EtOAc:hexanes = 1:4): $\mathrm{R}_{f}=0.31\left(\mathrm{UV}, \mathrm{KMnO}_{4}\right)$.

${ }^{1}$ H NMR (400 MHz, $\left.\mathrm{CDCl}_{3}\right): \delta 7.41-7.24(\mathrm{~m}, 3 \mathrm{H}), 7.23-7.15(\mathrm{~m}, 2 \mathrm{H}), 6.57(\mathrm{dd}, J=14.5,6.6 \mathrm{~Hz}, 1 \mathrm{H}), 6.30(\mathrm{dd}$, $J=14.5,1.1 \mathrm{~Hz}, 1 \mathrm{H}), 4.85(\mathrm{q}, J=7.2 \mathrm{~Hz}, 1 \mathrm{H}), 4.75(\mathrm{ddt}, J=9.5,8.0,3.2 \mathrm{~Hz}, 1 \mathrm{H}), 4.35(\mathrm{qd}, J=6.5,1.1 \mathrm{~Hz}, 1 \mathrm{H})$, $4.26(\mathrm{ddd}, J=8.7,8.0,0.6 \mathrm{~Hz}, 1 \mathrm{H}), 4.19(\mathrm{dd}, J=9.1,3.0 \mathrm{~Hz}, 1 \mathrm{H}), 4.18-4.09(\mathrm{~m}, 1 \mathrm{H}), 3.29(\mathrm{dd}, J=13.4,3.4 \mathrm{~Hz}$, $1 \mathrm{H}), 3.03(\mathrm{~d}, J=3.2 \mathrm{~Hz}, 1 \mathrm{H}), 2.87(\mathrm{qd}, J=7.0,3.3 \mathrm{~Hz}, 1 \mathrm{H}), 2.78(\mathrm{dd}, J=13.4,9.5 \mathrm{~Hz}, 1 \mathrm{H}), 1.74(\mathrm{ddd}, J=14.0$, 9.3, $6.0 \mathrm{~Hz}, 1 \mathrm{H}), 1.65-1.53(\mathrm{~m}, 1 \mathrm{H}), 1.46(\mathrm{~d}, J=7.3 \mathrm{~Hz}, 3 \mathrm{H}), 1.13(\mathrm{~d}, J=7.0 \mathrm{~Hz}, 3 \mathrm{H}), 0.89(\mathrm{~s}, 8 \mathrm{H}), 0.08(\mathrm{~s}, 3 \mathrm{H})$, $0.05(\mathrm{~s}, 3 \mathrm{H})$.

${ }^{13}$ C NMR $\left(100 \mathrm{MHz}, \mathrm{CDCl}_{3}\right): \delta 170.3,153.7,148.2,134.9,129.4,129.0,127.4,76.8,74.3,68.8,66.2,55.3,51.9$, $49.6,41.1,37.9,25.8,18.1,13.0,10.5,-4.5,-4.9$.

HRMS-ESI m/z cacld for $\mathrm{C}_{27} \mathrm{H}_{39} \mathrm{INO}_{6} \mathrm{Si}[\mathrm{M}-\mathrm{H}]^{-}$628.1597, found 628.1589.

A 100-mL round-bottom flask containing SI-5 (1.50 g, $2.38 \mathrm{mmol}, 1.0$ equiv) was evacuated and flushed with nitrogen (this process was repeated a total of 3 times) and was then sealed with a rubber septum. THF (24 mL) was added, resulting a colorless solution, and the vessel was cooled to $0{ }^{\circ} \mathrm{C}$ by means of an ice-water bath. $\mathrm{NaOMe}(135$ $\mathrm{mg}, 2.50 \mathrm{mmol}, 1.05$ equiv) was added in one portion. After $2 \mathrm{~h}$, saturated aqueous ammonium chloride solution $(20 \mathrm{~mL})$ was added followed by $\mathrm{AcOH}(143 \mathrm{uL}, 2.50 \mathrm{mmol}, 1.05$ equiv). The biphasic mixture was transferred to a separatory funnel and the layers were separated. The aqueous layer was extracted with ethyl acetate $(2 \times 20 \mathrm{~mL})$. The combined organic layers were washed with saturated aqueous sodium chloride solution $(10 \mathrm{~mL})$ and the washed solution was dried $\left(\mathrm{Na}_{2} \mathrm{SO}_{4}\right)$. The dried solution was filtered, and the filtrate was concentrated. The resulting crude residue was purified by flash chromatography ( silica gel, eluent: EtOAc:hexanes $=1: 2$ ) to afford $4(S), 5(R)$-left half $42(0.97 \mathrm{~g}, 90 \%)$ as a pale yellow oil.

TLC (EtOAc:hexanes = 1:2): $\mathrm{R}_{f}=0.42\left(\mathrm{UV}, \mathrm{KMnO}_{4}\right)$.

${ }^{1} \mathbf{H}$ NMR (400 MHz, MeOD) $\delta 6.57(\mathrm{dd}, J=14.5,6.9 \mathrm{~Hz}, 1 \mathrm{H}), 6.48(\mathrm{dd}, J=14.5,0.5 \mathrm{~Hz}, 1 \mathrm{H}), 4.45-4.31(\mathrm{~m}$, $2 \mathrm{H}), 2.38$ (qd, $J=7.4,3.2 \mathrm{~Hz}, 1 \mathrm{H}), 1.99$ (ddd, $J=14.1,8.8,5.5 \mathrm{~Hz}, 1 \mathrm{H}), 1.76-1.67$ (m, 4H), $1.12(\mathrm{~d}, J=7.1 \mathrm{~Hz}$, $3 \mathrm{H}), 0.91(\mathrm{~s}, 9 \mathrm{H}), 0.10(\mathrm{~s}, 3 \mathrm{H}), 0.08(\mathrm{~s}, 3 \mathrm{H})$.

${ }^{13}$ C NMR (100 MHz, MeOD) $\delta 173.9,171.9,149.3,98.0,78.5,75.7,73.7,39.7,37.6,26.3,19.0,11.1,8.7,-4.3,-$ 4.8.

HRMS-ESI m/z cacld for $\mathrm{C}_{17} \mathrm{H}_{29} \mathrm{INaO}_{4} \mathrm{Si}[\mathrm{M}+\mathrm{Na}]^{+}$475.0772, found 475.0771.

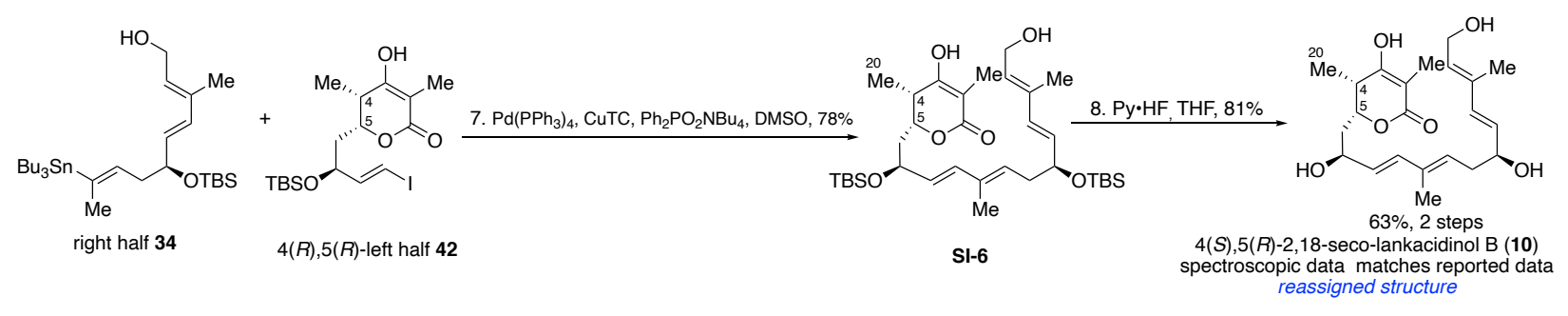

A 25-mL round-bottom flask containing $42(100 \mathrm{mg}, 0.222 \mathrm{mmol}, 1$ equiv) and $\mathbf{3 4}(129.40 \mathrm{mg}, 0.22 \mathrm{mmol}, 1.00$ equiv) was evacuated and flushed with nitrogen (this process was repeated a total of 3 times) and was then sealed with a rubber septum. DMSO $(10 \mathrm{~mL})$ was added, and $\mathrm{Ph}_{2} \mathrm{PO}_{2} \mathrm{NBu}_{4}(0.50 \mathrm{M}$ in DMSO, $666 \mu \mathrm{L}, 0.33 \mathrm{mmol}, 1.50$ equiv) was added followed by $\mathrm{Pd}\left(\mathrm{Ph}_{3} \mathrm{P}\right)_{4}(12.8 \mathrm{mg}, 0.0111 \mathrm{mmol}, 0.0500$ equiv) and CuTC (46.4 $\mathrm{mg}, 0.244 \mathrm{mmol}$, 1.10 equiv). After $30 \mathrm{~min}$, the mixture was diluted with $\mathrm{Et}_{2} \mathrm{O}(30 \mathrm{~mL})$ and was poured into an ice-water $(40 \mathrm{~mL})$. The biphasic mixture was transferred to a separatory funnel and the layers were separated. The aqueous layer was extracted with $\mathrm{Et}_{2} \mathrm{O}(2 \times 30 \mathrm{~mL})$. The combined organic layers were washed with water $(10 \mathrm{~mL})$ and saturated 
aqueous sodium chloride solution $(10 \mathrm{~mL})$ and the washed solution was dried $\left(\mathrm{Na}_{2} \mathrm{SO}_{4}\right)$. The dried solution was filtered and the filtrate was concentrated. The crude residue was purified by flash chromatography (silica gel, eluent: EtOAc:hexanes $=1: 2)$ to afford alcohol SI-6 $(100 \mathrm{mg}, 78 \%)$ as a colorless oil.

TLC (EtOAc:hexanes = 1:2): $\mathrm{R}_{f}=0.20(\mathrm{UV}$, anisaldehyde).

${ }^{1}$ H NMR (400 MHz, MeOD): $\delta 6.24(\mathrm{~s}, 1 \mathrm{H}), 6.20(\mathrm{~s}, 1 \mathrm{H}), 5.69(\mathrm{dd}, J=15.7,6.3 \mathrm{~Hz}, 1 \mathrm{H}), 5.55$ (m, 3H), 4.43 (q, $J$ $=7.2 \mathrm{~Hz}, 1 \mathrm{H}), 4.38-4.31(\mathrm{~m}, 1 \mathrm{H}), 4.28(\mathrm{q}, J=6.6 \mathrm{~Hz}, 1 \mathrm{H}), 4.21(\mathrm{~d}, J=6.7 \mathrm{~Hz}, 2 \mathrm{H}), 2.36(\mathrm{~m}, 3 \mathrm{H}), 2.04(\mathrm{~m}, 1 \mathrm{H})$, $1.76(\mathrm{~s}, 3 \mathrm{H}), 1.75(\mathrm{~s}, 3 \mathrm{H}), 1.70(\mathrm{~m}, 4 \mathrm{H}), 1.12(\mathrm{~d}, J=7.0 \mathrm{~Hz}, 3 \mathrm{H}), 0.91(\mathrm{~s}, 9 \mathrm{H}), 0.88(\mathrm{~s}, 9 \mathrm{H}), 0.09(\mathrm{~s}, 3 \mathrm{H}), 0.06(\mathrm{~s}$, $3 \mathrm{H}), 0.02(\mathrm{~s}, 3 \mathrm{H}), 0.01(\mathrm{~s}, 3 \mathrm{H})$.

${ }^{13}$ C NMR (100 MHz, MeOD) $\delta 173.9,172.1,137.4,136.0,135.5,134.8,133.1,131.5,130.7,129.9,98.0,76.2$, $74.8,72.4,59.3,40.7,38.6,37.6,26.4,26.4,19.1,19.0,13.0,12.7,11.1,8.7,-3.8,-4.2,-4.4,-4.6$.

HRMS-ESI m/z cacld for $\mathrm{C}_{34} \mathrm{H}_{59} \mathrm{O}_{6} \mathrm{Si}_{2}[\mathrm{M}-\mathrm{H}]^{-}$619.3856, found 619.3878.

A 50-mL plastic vial containing SI-6 $\left(76.0 \mathrm{mg}, 0.122 \mathrm{mmol}, 1\right.$ equiv) and THF $(5 \mathrm{~mL})$ was cooled to $0{ }^{\circ} \mathrm{C}$ by means of an ice-water bath. Py $(\mathrm{HF})_{\mathrm{x}}(\sim 70 \% \mathrm{HF})(400 \mu \mathrm{L})$ was added dropwise, and the vessel was removed from the cooling bath and the system was allowed to warm to $23{ }^{\circ} \mathrm{C}$. After $3 \mathrm{~h}, \mathrm{NaHCO}_{3}(970 \mathrm{mg})$ was added and the mixture was rapidly stirred. After $15 \mathrm{~min}$, the mixture was filtered through a pad of Celite and the filtrate was concentrated. The crude residue was purified by flash chromatography (silica gel, eluent: acetone:hexanes $=2: 1$ ) to afford 4(S),5(R)-2,18-seco-lankacidinol B (10) $(39.0 \mathrm{mg}, 81 \%)$ as a colorless oil.

TLC (EtOAc: $\mathrm{MeOH}=30: 1): \mathrm{R}_{f}=0.31$ (UV, anisaldehyde).

${ }^{1}$ H NMR (400 MHz, MeOD): $\delta 6.28(\mathrm{~d}, J=16.0 \mathrm{~Hz}, 1 \mathrm{H}), 6.24(\mathrm{~d}, J=16.3 \mathrm{~Hz}, 1 \mathrm{H}), 5.69(\mathrm{dd}, J=15.7,6.6 \mathrm{~Hz}$, $1 \mathrm{H}), 5.64-5.51(\mathrm{~m}, 3 \mathrm{H}), 4.38(\mathrm{ddd}, J=8.5,5.3,3.2 \mathrm{~Hz}, 1 \mathrm{H}), 4.29(\mathrm{q}, J=7.0 \mathrm{~Hz}, 1 \mathrm{H}), 4.20(\mathrm{~d}, J=6.7 \mathrm{~Hz}, 2 \mathrm{H})$, $4.17(\mathrm{dd}, J=13.0,6.4 \mathrm{~Hz}, 1 \mathrm{H}), 2.53-2.31(\mathrm{~m}, 3 \mathrm{H}), 2.05$ (ddd, $J=14.2,8.5,6.4 \mathrm{~Hz}, 1 \mathrm{H}), 1.76(\mathrm{~s}, 6 \mathrm{H}), 1.70(\mathrm{~m}$, $4 \mathrm{H}), 1.12(\mathrm{~d}, J=7.0 \mathrm{~Hz}, 3 \mathrm{H})$.

${ }^{13}$ C NMR (100 MHz, MeOD) $\delta$ 174.7, 172.3, 137.5, 136.0, 135.9, 135.5, 132.3, 131.7, 129.8, 129.7, 97.7, 76.4, $73.3,70.6,59.3,39.6,37.7,37.5,12.9,12.7,11.0,8.7$.

HRMS-ESI m/z cacld for $\mathrm{C}_{22} \mathrm{H}_{31} \mathrm{O}_{6}[\mathrm{M}-\mathrm{H}]^{-} 391.2126$, found 391.2139. 


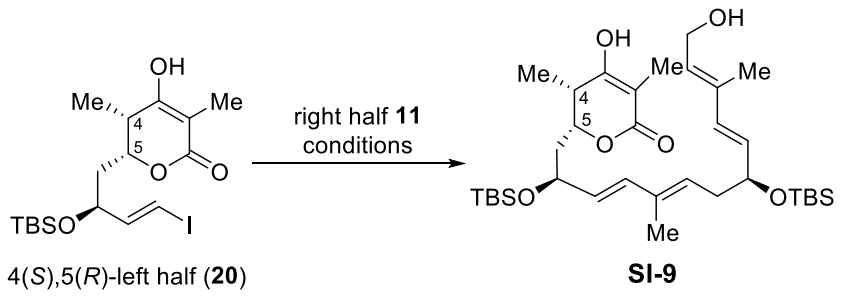

\begin{tabular}{|c|c|c|}
\hline Entry & Conditions & Yield \\
\hline 1 & $\mathrm{Pd}_{2}(\mathrm{dba})_{3}(10 \mathrm{~mol} \%), \mathrm{Ph}_{3} \mathrm{As}(40 \mathrm{~mol} \%), \mathrm{DMF}, 35^{\circ} \mathrm{C}$ & $39 \%$ \\
\hline 2 & $\mathrm{Pd}_{2}(\mathrm{dba})_{3}(10 \mathrm{~mol} \%), \mathrm{Ph}_{3} \mathrm{As}(40 \mathrm{~mol} \%),{ }^{\mathrm{i}} \operatorname{Pr}_{2} \mathrm{EtN}$ (10 eq.), DMF, $35^{\circ} \mathrm{C}$ & $0 \%$ \\
\hline 3 & $\mathrm{CuTc}$ (2.0 eq.), NMP, rt & $<5 \%$ \\
\hline 4 & $\mathrm{CuCl}$ (2.0 eq.), DMF, $\mathrm{rt}$ & $<5 \%$ \\
\hline 5 & $\mathrm{Pd}_{2}(\mathrm{dba})_{3}(10 \mathrm{~mol} \%), \mathrm{Ph}_{3} \mathrm{As}(40 \mathrm{~mol} \%), \mathrm{CuCl}$ (1.2 eq.), DMF, rt & $10 \%$ \\
\hline 6 & $\mathrm{Pd}_{2}(\mathrm{dba})_{3}(10 \mathrm{~mol} \%), \mathrm{Ph}_{3} \mathrm{As}(40 \mathrm{~mol} \%), \mathrm{LiCl}$ (5.0 eq.), DMF, rt & $<5 \%$ \\
\hline 7 & $\mathrm{Pd}_{2}(\mathrm{dba})_{3}(10 \mathrm{~mol} \%), \mathrm{Ph}_{3} \mathrm{As}(40 \mathrm{~mol} \%), \mathrm{CsF}$ (1.0 eq.), DMF, rt & $6 \%$ \\
\hline 8 & $\mathrm{Pd}\left(\mathrm{PPh}_{3}\right)_{4}(5 \mathrm{~mol} \%), \mathrm{CuI}(10 \mathrm{~mol} \%), \mathrm{CsF}$ (2.0 eq. $), \mathrm{DMF}, 45^{\circ} \mathrm{C}$ & $35 \%$ \\
\hline 9 & $\mathrm{Pd}\left(\mathrm{PPh}_{3}\right)_{4}(5 \mathrm{~mol} \%), \mathrm{CuI}(10 \mathrm{~mol} \%), \mathrm{NaF}$ (1.0 eq.), DMF, rt & $40 \%$ \\
\hline 10 & $\mathrm{Pd}\left(\mathrm{PPh}_{3}\right)_{4}(5 \mathrm{~mol} \%), \mathrm{CuI}(10 \mathrm{~mol} \%), \mathrm{DMF}, 45^{\circ} \mathrm{C}$ & $28 \%$ \\
\hline 11 & $\operatorname{Pd}\left(\mathrm{PPh}_{3}\right)_{4}(5 \mathrm{~mol} \%), \mathrm{CuTC}$ (1.1 eq.), $\mathrm{Ph}_{2} \mathrm{PO}_{2} \mathrm{NBu}_{4}, \mathrm{DMSO}, \mathrm{rt}$ & $73 \%$ \\
\hline
\end{tabular}




\begin{tabular}{|c|c|c|c|}
\hline \multicolumn{4}{|c|}{$\begin{array}{l}4(R), 5(R)-2,18-\text {-seco-lankacidinol B }(9) \\
\text { spectroscopic data does not match heported data } \\
\text { reported structure- misasssigned }\end{array}$} \\
\hline \multicolumn{4}{|c|}{${ }^{1} \mathrm{H}$ NMR Comparison (outstanding reassignment peaks highlighted in red) } \\
\hline Position & Originally assigned structure 9 & Reassigned structure $\mathbf{1 0}$ & Isolation $^{5}$ \\
\hline 4 & $2.53-2.46(\mathrm{~m}, 1 \mathrm{H})$ & $2.53-2.31(\mathrm{~m}, 1 \mathrm{H})$ & $2.38(\mathrm{~m}, 1 \mathrm{H})$ \\
\hline 5 & $4.16(\mathrm{~m}, 1 \mathrm{H})$ & $\begin{array}{c}4.38(\mathrm{ddd}, J=8.5,5.3, \\
3.2 \mathrm{~Hz}, 1 \mathrm{H})\end{array}$ & $4.37(\mathrm{~m}, 1 \mathrm{H})$ \\
\hline 6 & $\begin{array}{c}2.08(\mathrm{ddd}, J=14.3,8.7,5.9 \\
\mathrm{Hz}, 1 \mathrm{H}) \\
1.81-1.73(\mathrm{~m}, 1 \mathrm{H})\end{array}$ & $\begin{array}{c}2.05(\mathrm{ddd}, J=14.2,8.5 \\
6.4 \mathrm{~Hz}, 1 \mathrm{H}) \\
1.70(\mathrm{~m}, 1 \mathrm{H})\end{array}$ & $\begin{array}{l}2.05(\mathrm{~m}, 1 \mathrm{H}) \\
1.70(\mathrm{~m}, 1 \mathrm{H})\end{array}$ \\
\hline 7 & $4.28(\mathrm{q}, J=7.1 \mathrm{~Hz}, 1 \mathrm{H})$ & $4.29(\mathrm{q}, J=7.0 \mathrm{~Hz}, 1 \mathrm{H})$ & $4.30(\mathrm{dd}, J=14.0,7.0 \mathrm{~Hz}, 1 \mathrm{H})$ \\
\hline 8 & $5.55(\mathrm{~d}, J=7.4 \mathrm{~Hz}, 1 \mathrm{H})$ & $5.64-5.51(\mathrm{~m}, 1 \mathrm{H})$ & $5.57(\mathrm{~m}, 1 \mathrm{H})$ \\
\hline 9 & $6.30(\mathrm{~d}, J=15.7 \mathrm{~Hz}, 1 \mathrm{H})$ & $6.28(\mathrm{~d}, J=16.0 \mathrm{~Hz}, 1 \mathrm{H})$ & $6.28(\mathrm{~d}, J=16.0 \mathrm{~Hz}, 1 \mathrm{H})$ \\
\hline 11 & $5.59(\mathrm{~d}, J=7.3 \mathrm{~Hz}, 1 \mathrm{H})$ & $5.64-5.51(\mathrm{~m}, 1 \mathrm{H})$ & $5.57(\mathrm{~m}, 1 \mathrm{H})$ \\
\hline 12 & $2.45-2.35(\mathrm{~m}, 2 \mathrm{H})$ & $2.53-2.31(\mathrm{~m}, 2 \mathrm{H})$ & $2.41(\mathrm{~m}, 2 \mathrm{H})$ \\
\hline 13 & $4.16(\mathrm{~m}, 1 \mathrm{H})$ & $\begin{array}{c}4.17(\mathrm{dd}, J=13.0,6.4 \mathrm{~Hz}, \\
1 \mathrm{H})\end{array}$ & $4.18(\mathrm{dd}, J=13.4,6.4 \mathrm{~Hz}, 1 \mathrm{H})$ \\
\hline 14 & $\begin{array}{c}5.69(\mathrm{dd}, J=15.7,6.7 \mathrm{~Hz} \\
1 \mathrm{H})\end{array}$ & $\begin{array}{c}5.69(\mathrm{dd}, J=15.7,6.6 \mathrm{~Hz}, \\
1 \mathrm{H})\end{array}$ & $5.69(\mathrm{dd}, J=15.7,6.6 \mathrm{~Hz}, 1 \mathrm{H})$ \\
\hline 15 & $6.25(\mathrm{~d}, J=15.7 \mathrm{~Hz}, 1 \mathrm{H})$ & $6.24(\mathrm{~d}, J=16.3 \mathrm{~Hz}, 1 \mathrm{H})$ & $6.24(\mathrm{~d}, J=15.7 \mathrm{~Hz}, 1 \mathrm{H})$ \\
\hline 17 & $5.62(\mathrm{~d}, J=6.7 \mathrm{~Hz}, 1 \mathrm{H})$ & $5.64-5.51(\mathrm{~m}, 1 \mathrm{H})$ & $5.61(\mathrm{~m}, 1 \mathrm{H})$ \\
\hline 18 & $4.20(\mathrm{~d}, J=6.7 \mathrm{~Hz}, 2 \mathrm{H})$ & $4.20(\mathrm{~d}, J=6.7 \mathrm{~Hz}, 2 \mathrm{H})$ & $4.21(\mathrm{~d}, J=6.7 \mathrm{~Hz}, 2 \mathrm{H})$ \\
\hline 19 & $1.72(\mathrm{~d}, J=0.7 \mathrm{~Hz}, 3 \mathrm{H})$ & $1.70(\mathrm{~s}, 3 \mathrm{H})$ & $1.70(\mathrm{~s}, 3 \mathrm{H})$ \\
\hline 20 & $1.25(\mathrm{~d}, J=7.0 \mathrm{~Hz}, 3 \mathrm{H})$ & $1.12(\mathrm{~d}, J=7.1 \mathrm{~Hz}, 3 \mathrm{H})$ & $1.12(\mathrm{~d}, J=7.1 \mathrm{~Hz}, 3 \mathrm{H})$ \\
\hline 21 & $1.81-1.73(\mathrm{~s}, 3 \mathrm{H})$ & $1.76(\mathrm{~s}, 3 \mathrm{H})$ & 1.77 (br s, 3H) \\
\hline 22 & $1.81-1.73(\mathrm{~s}, 3 \mathrm{H})$ & $1.76(\mathrm{~s}, 3 \mathrm{H})$ & 1.77 (br s, 3H) \\
\hline
\end{tabular}




\begin{tabular}{|c|c|c|c|}
\hline \multicolumn{5}{|c|}{${ }^{13}$ C NMR Comparison (outstanding reassignment peaks highlighted in red) } \\
\hline Position & Originally assigned structure 9 & Reassigned structure 10 & Isolation $^{5}$ \\
\hline 1 & 171.4 & 172.3 & 172.4 \\
\hline 2 & 97.9 & 97.7 & 97.4 \\
\hline 3 & 170.7 & 174.7 & 175.4 \\
\hline 4 & 38.1 & 37.7 & 37.9 \\
\hline 5 & 79.4 & 76.4 & 76.4 \\
\hline 6 & 41.9 & 39.6 & 39.7 \\
\hline 7 & 70.8 & 70.6 & 70.6 \\
\hline 8 & 129.7 & 129.7 & 129.7 \\
\hline 9 & 137.4 & 137.5 & 137.4 \\
\hline 10 & 136.0 & 136.0 & 136.0 \\
\hline 11 & 129.7 & 129.8 & 129.8 \\
\hline 12 & 37.5 & 37.5 & 37.5 \\
\hline 13 & 73.3 & 73.3 & 73.3 \\
\hline 14 & 132.3 & 132.2 & 132.3 \\
\hline 15 & 135.5 & 135.5 & 135.5 \\
\hline 16 & 135.9 & 135.9 & 135.9 \\
\hline 17 & 131.8 & 131.7 & 131.7 \\
\hline 18 & 59.3 & 59.3 & 59.4 \\
\hline 19 & 8.6 & 8.7 & 8.7 \\
\hline 20 & 16.8 & 11.0 & 11.0 \\
\hline 21 & 12.9 & 12.9 & 12.9 \\
\hline 22 & 12.7 & 12.7 & 12.6 \\
\hline
\end{tabular}




\section{Scheme 2. Synthesis of 4-methyl lankacidins}

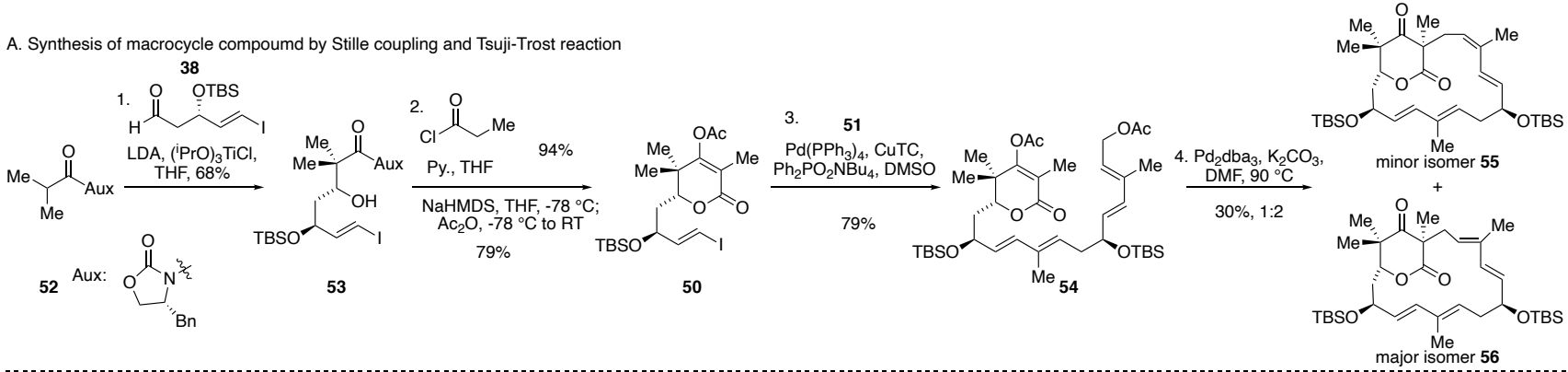

B. Synthesis of 4-Me-lankacidin C from minor macrocycle $\mathbf{5 5}$
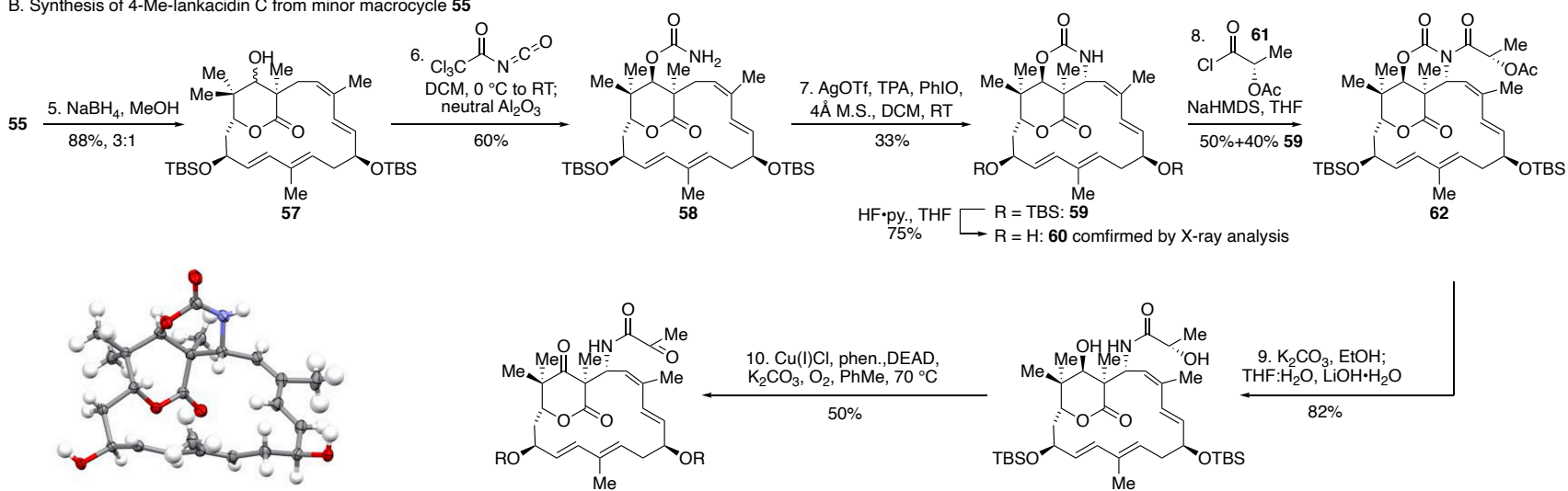

11. HF.py., THF $\square \begin{array}{r}R \\ 90 \%\end{array} \quad$ TBS: 64
$R=H: 65$

X-ray crystallography of compound 60
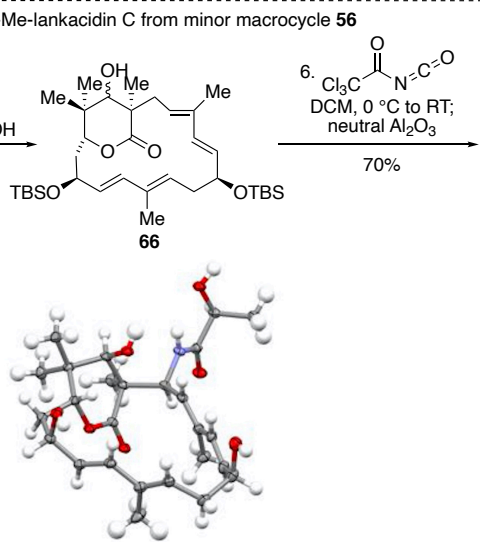

X-ray crystallography of compound $\mathbf{7 0}$

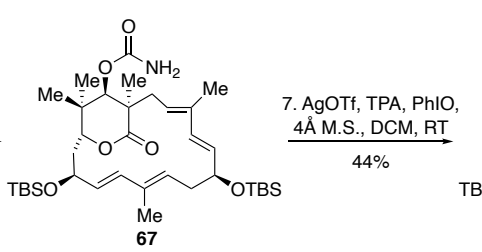

$\mathrm{Me}$
67
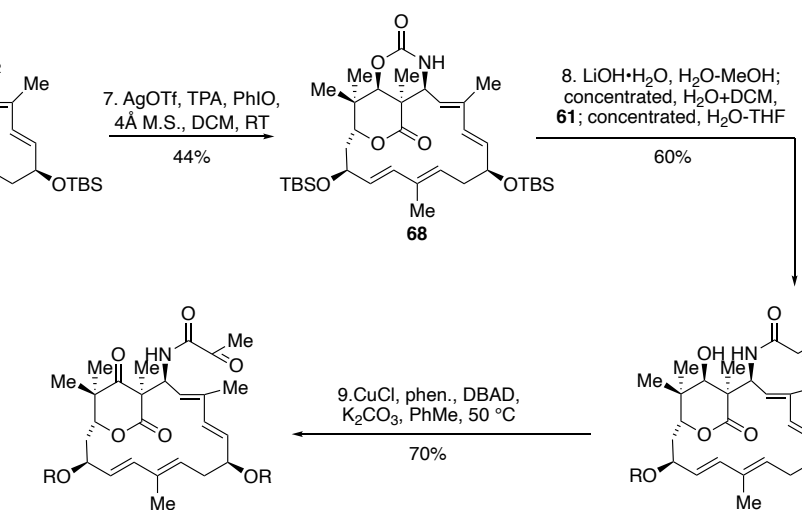

$\begin{array}{rl}\text { 10. HF.py., THF } \square \text { R }=\text { TBS: } 71 \\ 95 \% & R=H: 72\end{array}$
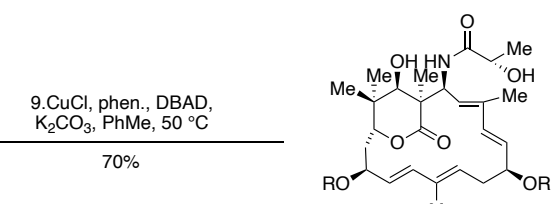

HF.py., THF $\square$ R $=$ TBS: 69

.py., THF $\square$ R $=$ TBS: 69
$9 \mathrm{R}=\mathrm{H}: \mathbf{7 0}$ comfirmed by $\mathrm{X}$-ray analysis 


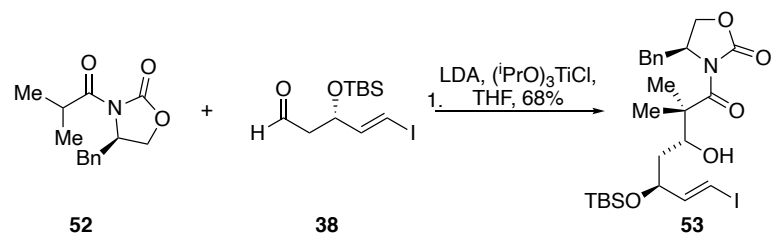

A 500-mL round-bottom flask was evacuated and flushed with nitrogen (this process was repeated a total of 3 times) and was then sealed with a rubber septum. THF $(90 \mathrm{~mL})$ and diisopropylamine $(3.79 \mathrm{~g}, 5.34 \mathrm{~mL}, 37.5 \mathrm{mmol}, 1.50$ equiv) were added, and the mixture was cooled to $0{ }^{\circ} \mathrm{C}$ by means of an ice-water bath. ${ }^{n} \mathrm{BuLi}(734 \mathrm{mg}, 4.58 \mathrm{~mL}$, $37.50 \mathrm{mmol}, 1.30$ equiv) was added dropwise. After stirring for $10 \mathrm{~min}$, the mixture was cooled to $-78^{\circ} \mathrm{C}$ by means of a dry ice-acetone bath, and a solution of $\mathbf{5 2}(6.79 \mathrm{~g}, 27.50 \mathrm{mmol}, 1.10$ equiv $)$ in THF $(60 \mathrm{~mL})$ was added dropwise. After stirring for $30 \mathrm{~min}$, chlorotitanium triisopropoxide (1.00 $\mathrm{M}$ in hexane, $99.90 \mathrm{~mL}, 99.90 \mathrm{mmol}, 4.00$ equiv) was added, and the mixture was warmed to $-40^{\circ} \mathrm{C}$. After stirring for $1 \mathrm{~h}$, the mixture was cooled to $-78^{\circ} \mathrm{C}$, and a solution of $\mathbf{3 8}(8.50 \mathrm{~g}, 25.00 \mathrm{mmol}, 1$ equiv) in THF $(10 \mathrm{~mL})$ was added dropwise. The mixture was slowly warmed to $-40{ }^{\circ} \mathrm{C}$. After stirring for $3 \mathrm{~h}$, the mixture was poured into a stirred mixture of ethyl acetate $(100 \mathrm{~mL})$ and saturated ammonium chloride aqueous $(50 \mathrm{~mL})$. The biphasic mixture was filtered through a pad of celite, and the filtrate was transferred to a separatory funnel and the layers were separated. The organic layer was washed with saturated sodium bicarbonate solution $(2 \times 30 \mathrm{~mL})$ and brine $(30 \mathrm{~mL})$, and the washed solution was dried $\left(\mathrm{Na}_{2} \mathrm{SO}_{4}\right)$. The dried solution was filtered and the filtrate was concentrated. The crude residue was purified by flash chromatography (silica gel, eluent: EtOAc:hexanes $=1: 8$ to $1: 4)$ to afford alcohol $\mathbf{5 3}(10.0 \mathrm{~g}, 68 \%)$ as a colorless oil.

TLC (EtOAc:hexanes = 1:4): $\mathrm{R}_{f}=0.34\left(\mathrm{UV}, \mathrm{KMnO}_{4}\right)$.

${ }^{1}$ H NMR $\left(400 \mathrm{MHz}, \mathrm{CDCl}_{3}\right): \delta 7.36-7.30(\mathrm{~m}, 3 \mathrm{H}), 7.29-7.26(\mathrm{~m}, 1 \mathrm{H}), 7.25-7.22(\mathrm{~m}, 3 \mathrm{H}), 6.56(\mathrm{dd}, J=14.5$, $7.0 \mathrm{~Hz}, 1 \mathrm{H}), 6.32(\mathrm{dd}, J=14.5,0.9 \mathrm{~Hz}, 1 \mathrm{H}), 4.82-4.65(\mathrm{~m}, 1 \mathrm{H}), 4.55(\mathrm{dt}, J=7.1,3.8 \mathrm{~Hz}, 1 \mathrm{H}), 4.39(\mathrm{q}, J=6.6 \mathrm{~Hz}$, $1 \mathrm{H}), 4.22-4.16(\mathrm{dd}, J=9.0,7.1 \mathrm{~Hz}, 1 \mathrm{H}), 4.14(\mathrm{dd}, J=9.0,2.7 \mathrm{~Hz}, 1 \mathrm{H}), 3.27(\mathrm{dd}, J=13.4,3.4 \mathrm{~Hz}, 1 \mathrm{H}), 3.19(\mathrm{~d}$, $J=3.2 \mathrm{~Hz}, 1 \mathrm{H}), 2.73(\mathrm{dd}, J=13.4,9.7 \mathrm{~Hz}, 1 \mathrm{H}), 1.62(\mathrm{~m}, 2 \mathrm{H}), 1.37(\mathrm{~s}, 3 \mathrm{H}), 1.32(\mathrm{~s}, 3 \mathrm{H}), 0.90(\mathrm{~s}, 9 \mathrm{H}), 0.12(\mathrm{~s}, 3 \mathrm{H})$, $0.08(\mathrm{~s}, 3 \mathrm{H})$.

${ }^{13}$ C NMR $\left(100 \mathrm{MHz}, \mathrm{CDCl}_{3}\right): \delta 177.3,152.3,148.4,135.6,129.4,128.9,127.2,77.2,75.8,72.4,66.2,57.7,49.8$, $38.7,37.7,25.8,19.8,19.3,18.1,-4.3,-4.9$.

HRMS-ESI m/z cacld for $\mathrm{C}_{25} \mathrm{H}_{38} \mathrm{INNaO}_{5} \mathrm{Si}[\mathrm{M}+\mathrm{Na}]^{+}$610.1456, found 610.1460 .

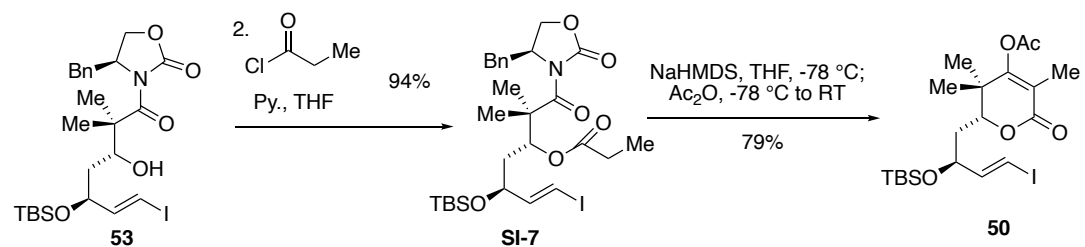

A 250-mL round-bottom flask containing 53 (10.00 g, $17.00 \mathrm{mmol}, 1$ equiv) was evacuated and flushed with nitrogen (this process was repeated a total of 3 times) and sealed with a rubber septum. THF (100 mL) was added, and pyridine $\left(8.08 \mathrm{~g}, 8.24 \mathrm{~mL}, 102 \mathrm{mmol}, 6.00\right.$ equiv). The mixture was cooled to $0{ }^{\circ} \mathrm{C}$ by means of an ice-water bath. Propionyl chloride ( $4.72 \mathrm{~g}, 4.46 \mathrm{~mL}, 51.10 \mathrm{mmol}, 3.00$ equiv) was added, and the cooling bath was removed. After stirring for $16 \mathrm{~h}$ at room temperature, the mixture was poured into a stirred mixture of ethyl acetate $(60 \mathrm{~mL})$ and water $(40 \mathrm{~mL})$. The biphasic mixture was transferred to a separatory funnel and the layers were separated. The aqueous layer was extracted with ethyl acetate $(2 \times 30 \mathrm{~mL})$. The combined organic layers were washed with brine $(30 \mathrm{~mL})$ and the washed solution was dried $\left(\mathrm{Na}_{2} \mathrm{SO}_{4}\right)$. The dried solution was filtered and the filtrate was concentrated. The crude residue was purified by flash chromatography (silica gel, eluent: EtOAc:hexanes $=1: 6$ ) to afford ester SI-7 (10.4 g, 94\%) as a colorless oil.

TLC (EtOAc:hexanes = 1:8): $\mathrm{R}_{f}=0.31\left(\mathrm{UV}, \mathrm{KMnO}_{4}\right)$.

${ }^{1}$ H NMR (400 MHz, $\left.\mathrm{CDCl}_{3}\right): \delta 7.38-7.30(\mathrm{~m}, 2 \mathrm{H}), 7.29-7.21(\mathrm{~m}, 3 \mathrm{H}), 6.59(\mathrm{dd}, J=14.4,5.8 \mathrm{~Hz}, 1 \mathrm{H}), 6.30(\mathrm{dd}$, $J=14.4,1.3 \mathrm{~Hz}, 1 \mathrm{H}), 6.19-6.12(\mathrm{~m}, 1 \mathrm{H}), 4.59(\mathrm{ddt}, J=10.1,6.4,3.1 \mathrm{~Hz}, 1 \mathrm{H}), 4.17-4.06(\mathrm{~m}, 3 \mathrm{H}), 3.25(\mathrm{dd}, J=$ 13.2, 3.1 Hz, 1H), $2.63(\mathrm{dd}, J=13.2,10.8 \mathrm{~Hz}, 1 \mathrm{H}), 2.30(\mathrm{q}, J=7.6 \mathrm{~Hz}, 2 \mathrm{H}), 1.81-1.75(\mathrm{~m}, 2 \mathrm{H}), 1.40(\mathrm{~s}, 3 \mathrm{H}), 1.31$ $(\mathrm{s}, 3 \mathrm{H}), 1.11(\mathrm{t}, J=7.6 \mathrm{~Hz}, 3 \mathrm{H}), 0.89(\mathrm{~s}, 9 \mathrm{H}), 0.05(\mathrm{~s}, 3 \mathrm{H}), 0.04(\mathrm{~s}, 3 \mathrm{H})$. 
${ }^{13}$ C NMR (100 MHz, $\left.\mathrm{CDCl}_{3}\right): \delta 175.6,173.6,152.1,147.4,136.1,129.4,128.9,127.1,77.0,72.5,70.7,66.1,58.0$, $49.3,38.4,37.1,27.6,25.8,20.5,20.2,18.3,9.2,-4.8,-5.0$.

HRMS-ESI $\mathrm{m} / \mathrm{z}$ cacld for $\mathrm{C}_{28} \mathrm{H}_{42} \mathrm{INNaO}_{6} \mathrm{Si}[\mathrm{M}+\mathrm{Na}]^{+}$666.1718, found 666.1744.

A 500-mL round-bottom flask containing SI-7 (10.00 g, $15.50 \mathrm{mmol}, 1$ equiv) was evacuated and flushed with nitrogen (this process was repeated a total of 3 times) and sealed with a rubber septum. THF (150 mL) was added, and the mixture was cooled to $-78^{\circ} \mathrm{C}$ by means of a dry ice-acetone bath. NaHMDS (24.1 mL, 2.0 M in THF, 48.2 mmol, 3.10 equiv) was added dropwise. After stirring for $2 \mathrm{~h}, \mathrm{Ac}_{2} \mathrm{O}(5.55 \mathrm{~g}, 5.14 \mathrm{~mL}, 54.40 \mathrm{mmol}, 3.50$ equiv) was added, and the cooling bath was removed. The mixture was warmed to room temperature and stirred for $12 \mathrm{~h}$. The mixture was poured into a stirred mixture of ethyl acetate $(50 \mathrm{~mL})$ and saturated $\mathrm{NH}_{4} \mathrm{Cl}$ aqueous $(40 \mathrm{~mL})$. The biphasic mixture was transferred to a separatory funnel and the layers were separated. The aqueous layer was extracted with ethyl acetate $(2 \times 40 \mathrm{~mL})$. The combined organic layers were washed with brine $(20 \mathrm{~mL})$ and the washed solution was dried $\left(\mathrm{Na}_{2} \mathrm{SO}_{4}\right)$. The dried solution was filtered and the filtrate was concentrated. The crude residue was purified by flash chromatography (silica gel, eluent: EtOAc:hexanes = 1:5) to afford $\mathbf{5 0}(5.71 \mathrm{~g}, 79 \%)$ as a colorless oil.

TLC (EtOAc:hexanes = 1:6): $\mathrm{R}_{f}=0.31\left(\mathrm{UV}, \mathrm{KMnO}_{4}\right)$.

${ }^{1} \mathbf{H}$ NMR $\left(400 \mathrm{MHz}, \mathrm{CDCl}_{3}\right): \delta 6.51(\mathrm{dd}, J=14.5,6.9 \mathrm{~Hz}, 1 \mathrm{H}), 6.34(\mathrm{dd}, J=14.5,0.7 \mathrm{~Hz}, 1 \mathrm{H}), 4.41(\mathrm{ddd}, J=9.9$, $6.9,3.8 \mathrm{~Hz}, 1 \mathrm{H}), 4.09(\mathrm{dd}, J=10.9,1.6 \mathrm{~Hz}, 1 \mathrm{H}), 2.26(\mathrm{~s}, 3 \mathrm{H}), 1.95(\mathrm{ddd}, J=14.7,11.0,4.0 \mathrm{~Hz}, 1 \mathrm{H}), 1.69(\mathrm{~s}, 3 \mathrm{H})$, $1.67-1.62(\mathrm{~m}, 1 \mathrm{H}), 1.06(\mathrm{~s}, 3 \mathrm{H}), 1.02(\mathrm{~s}, 3 \mathrm{H}), 0.87(\mathrm{~s}, 9 \mathrm{H}), 0.06(\mathrm{~s}, 3 \mathrm{H}), 0.04(\mathrm{~s}, 3 \mathrm{H})$.

${ }^{13}$ C NMR (100 MHz, $\left.\mathrm{CDCl}_{3}\right): \delta 166.4,165.5,164.2,147.3,115.7,79.7,78.0,72.2,38.5,36.8,25.7,20.9,20.3$, $18.6,18.1,11.1,-4.6,-4.9$.

HRMS-ESI m/z cacld for $\mathrm{C}_{20} \mathrm{H}_{33} \mathrm{INaO}_{5} \mathrm{Si}[\mathrm{M}+\mathrm{Na}]^{+}$531.1034, found 531.1017.

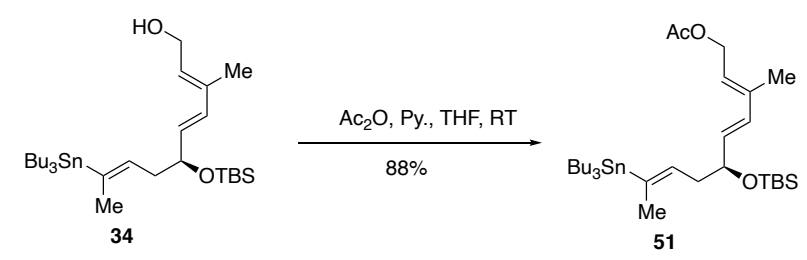

A 500-mL round-bottom flask containing 34 (9.87 g, $16.90 \mathrm{mmol}, 1$ equiv) was evacuated and flushed with nitrogen (this process was repeated a total of 3 times) and was then sealed with a rubber septum. THF $(170 \mathrm{~mL})$ and pyridine ( $10.70 \mathrm{~g}, 10.90 \mathrm{~mL}, 135 \mathrm{mmol}, 8.00$ equiv) were added followed by acetic anhydride ( $6.88 \mathrm{~g}, 6.37 \mathrm{~mL}, 67.4 \mathrm{mmol}$, 4.00 equiv). After stirring for $60 \mathrm{~h}$, the mixture was concentrated. The resulting crude residue was purified by flash chromatography (silica gel, eluent: EtOAc:hexanes =1:20) to afford obtain $\mathbf{5 1}(9.10 \mathrm{~g}, 86 \%)$ as a pale yellow oil.

TLC (EtOAc:hexanes $=1: 10): \mathrm{R}_{f}=0.40\left(\mathrm{UV}, \mathrm{KMnO}_{4}\right)$.

${ }^{1} \mathbf{H}$ NMR $\left(400 \mathrm{MHz}, \mathrm{CDCl}_{3}\right): \delta 6.17(\mathrm{~d}, J=15.7 \mathrm{~Hz}, 1 \mathrm{H}), 5.72(\mathrm{dd}, J=15.7,6.1 \mathrm{~Hz}, 1 \mathrm{H}), 5.59-5.51(\mathrm{~m}, 2 \mathrm{H})$, $4.70(\mathrm{~d}, J=7.1 \mathrm{~Hz}, 2 \mathrm{H}), 4.22(\mathrm{q}, J=6.4 \mathrm{~Hz}, 1 \mathrm{H}), 2.34(\mathrm{tt}, J=14.3,7.2 \mathrm{~Hz}, 2 \mathrm{H}), 2.06(\mathrm{~s}, 3 \mathrm{H}), 1.83-1.80(\mathrm{~m}, 3 \mathrm{H})$, $1.79(\mathrm{~s}, 3 \mathrm{H}), 1.52-1.41(\mathrm{~m}, 6 \mathrm{H}), 1.36-1.24(\mathrm{~m}, 6 \mathrm{H}), 0.91-0.83(\mathrm{~m}, 25 \mathrm{H}), 0.05(\mathrm{~s}, 3 \mathrm{H}), 0.03(\mathrm{~s}, 3 \mathrm{H})$.

${ }^{13} \mathbf{C}$ NMR (100 MHz, $\left.\mathrm{CDCl}_{3}\right): \delta 171.0,140.0,138.1,136.3,133.5,132.4,123.9,73.3,61.2,37.2,29.2,27.4,25.9$, $21.0,19.4,18.2,13.7,12.8,9.1,-4.3,-4.7$.

HRMS-ESI The compound failed to ionize under electrospray ionization in both positive and negative modes.

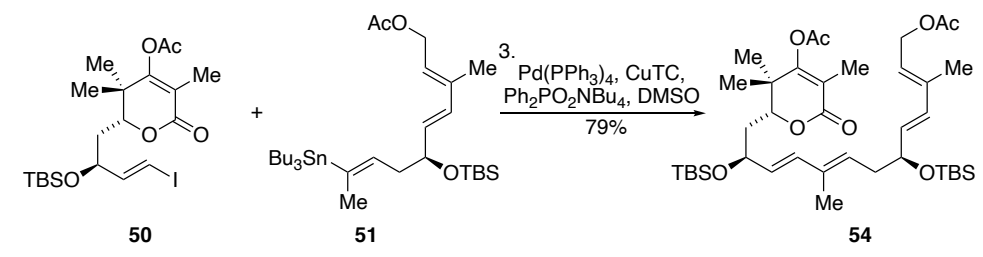

A 500-mL round-bottom flask containing $\mathbf{5 0}$ (7.49 g, $14.7 \mathrm{mmol}, 1$ equiv) and $\mathbf{5 1}$ (10.20 g, $16.20 \mathrm{mmol}, 1.10$ equiv) was evacuated and flushed with nitrogen (this process was repeated a total of 3 times) and sealed with a rubber septum. DMSO (147 mL) was added, and $\mathrm{Ph}_{2} \mathrm{PO}_{2} \mathrm{NBu}_{4}(44.20 \mathrm{~mL}, 0.50 \mathrm{M}$ in DMSO, $22.10 \mathrm{mmol}$, 1.50 equiv) 
was added followed by $\mathrm{Pd}\left(\mathrm{PPh}_{3}\right)_{4}(851 \mathrm{mg}, 0.74 \mathrm{mmol}, 0.050$ equiv) and CuTC (3.09 g, $16.20 \mathrm{mmol}, 1.10$ equiv). After stirring for $30 \mathrm{~min}$, the mixture was poured into a stirred mixture of diethyl ether $(200 \mathrm{~mL})$ and ice-water $(200 \mathrm{~mL})$. The biphasic mixture was transferred to a separatory funnel and the layers were separated. The aqueous layer was extracted with diethyl ether $(2 \times 100 \mathrm{~mL})$. The combined organic layers were washed with water $(50 \mathrm{~mL})$ and brine $(50 \mathrm{~mL})$ and the washed solution was dried $\left(\mathrm{Na}_{2} \mathrm{SO}_{4}\right)$. The dried solution was filtered and the filtrate was concentrated. The crude residue was purified by flash chromatography ( afford diacetate $\mathbf{5 4}(8.37 \mathrm{~g}, 79 \%)$ as a colorless oil.

TLC (EtOAc:hexanes = 1:4): $\mathrm{R}_{f}=0.39$ (UV, anisaldehyde).

${ }^{1} \mathbf{H}$ NMR $\left(400 \mathrm{MHz}, \mathrm{CDCl}_{3}\right): \delta 6.22(\mathrm{~d}, J=9.8 \mathrm{~Hz}, 1 \mathrm{H}), 6.18(\mathrm{~d}, J=9.9 \mathrm{~Hz}, 1 \mathrm{H}), 5.71(\mathrm{dd}, J=15.6,6.2 \mathrm{~Hz}, 1 \mathrm{H})$, $5.55(\mathrm{t}, J=6.9 \mathrm{~Hz}, 1 \mathrm{H}), 5.49(\mathrm{t}, J=7.9 \mathrm{~Hz}, 1 \mathrm{H}), 5.43(\mathrm{dd}, J=15.7,7.8 \mathrm{~Hz}, 1 \mathrm{H}), 4.70(\mathrm{~d}, J=7.1 \mathrm{~Hz}, 2 \mathrm{H}), 4.47(\mathrm{td}$, $J=9.7,3.7 \mathrm{~Hz}, 1 \mathrm{H}), 4.22(\mathrm{q}, J=6.1 \mathrm{~Hz}, 1 \mathrm{H}), 4.08(\mathrm{~d}, J=9.5 \mathrm{~Hz}, 1 \mathrm{H}), 2.39(\mathrm{dt}, J=15.1,7.7 \mathrm{~Hz}, 1 \mathrm{H}), 2.32-2.27$ $(\mathrm{m}, 1 \mathrm{H}), 2.25(\mathrm{~s}, 3 \mathrm{H}), 2.06(\mathrm{~s}, 3 \mathrm{H}), 2.00(\mathrm{ddd}, J=14.3,11.0,4.0 \mathrm{~Hz}, 1 \mathrm{H}), 1.80(\mathrm{~s}, 3 \mathrm{H}), 1.71(\mathrm{~s}, 3 \mathrm{H}), 1.68(\mathrm{~s}, 3 \mathrm{H})$, $1.70-1.65(\mathrm{~m}, 1 \mathrm{H}), 1.07(\mathrm{~s}, 3 \mathrm{H}), 1.00(\mathrm{~s}, 3 \mathrm{H}), 0.88(\mathrm{~s}, 9 \mathrm{H}), 0.87(\mathrm{~s}, 9 \mathrm{H}), 0.06(\mathrm{~s}, 3 \mathrm{H}), 0.03(\mathrm{~s}, 3 \mathrm{H}), 0.01(\mathrm{~s}, 3 \mathrm{H})$, $0.00(\mathrm{~s}, 3 \mathrm{H})$.

${ }^{13}$ C NMR (100 MHz, $\left.\mathrm{CDCl}_{3}\right): \delta 171.0,166.5,165.8,164.4,138.0,136.4,134.4,133.3,132.6,129.3,128.3,124.2$, 115.6, 80.4, 73.2, 71.1, 61.2, 38.5, 37.8, 37.7, 25.87, 25.85, 21.0, 20.8, 20.4, 18.7, 18.19, 18.15, 12.79, 12.77, 11.1, $-4.2,-4.4,-4.7$.

HRMS-ESI m/z cacld for $\mathrm{C}_{39} \mathrm{H}_{66} \mathrm{NaO}_{8} \mathrm{Si}_{2}[\mathrm{M}+\mathrm{Na}]^{+}$741.4188, found 741.4182.

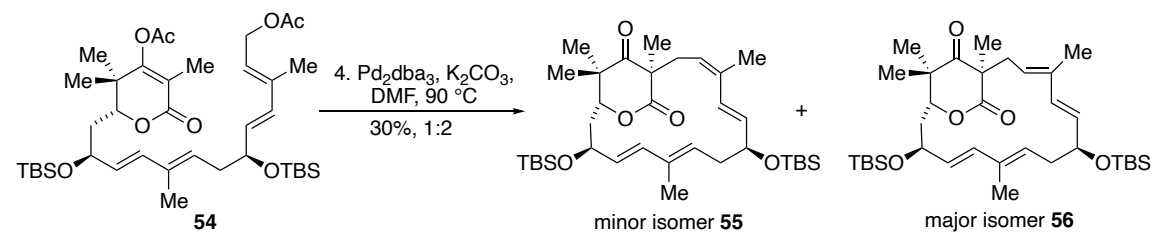

A 500-mL round-bottom flask containing $54(3.65 \mathrm{~g}, 5.08 \mathrm{mmol}, 1$ equiv) was evacuated and flushed with nitrogen (this process was repeated a total of 3 times) and sealed with a rubber septum. DMF $(200 \mathrm{~mL})$ was added, and $\mathrm{K}_{2} \mathrm{CO}_{3}$ (700 mg, $5.08 \mathrm{mmol}, 1.00$ equiv) was added followed by $\mathrm{Pd}\left(\mathrm{PPh}_{3}\right)_{4}(293 \mathrm{mg}, 0.25 \mathrm{mmol}, 0.050$ equiv). The mixture was warmed to $90^{\circ} \mathrm{C}$ by means of an oil bath. After stirring for $3 \mathrm{~h}$, the mixture was poured into a stirred mixture of ethyl acetate $(150 \mathrm{~mL})$ and ice-water $(200 \mathrm{~mL})$. The biphasic mixture was transferred to a separatory funnel and the layers were separated. The aqueous layer was extracted with ethyl acetate $(2 \times 100 \mathrm{~mL})$. The combined organic layers were washed with water $(50 \mathrm{~mL})$ and brine $(50 \mathrm{~mL})$ and the washed solution was dried $\left(\mathrm{Na}_{2} \mathrm{SO}_{4}\right)$. The dried solution was filtered and the filtrate was concentrated. The crude residue was purified by flash chromatography (silica gel, eluent: EtOAc:hexanes = 1:20) to afford minor keto-ester $55(313 \mathrm{mg}, 0.51 \mathrm{mmol}, 10 \%)$ and major keto-ester 56 (626 mg, $20 \%$ ) as a colorless oil.

\section{Minor isomer 55:}

TLC $($ EtOAc:hexanes $=1: 12): \mathrm{R}_{f}=0.35(\mathrm{UV}$, anisaldehyde) .

${ }^{1} \mathbf{H}$ NMR $\left(400 \mathrm{MHz}, \mathrm{CDCl}_{3}\right): \delta 6.21(\mathrm{~d}, J=15.6 \mathrm{~Hz}, 1 \mathrm{H}), 6.00(\mathrm{~d}, J=15.8 \mathrm{~Hz}, 1 \mathrm{H}), 5.52(\mathrm{dd}, J=15.8,6.7 \mathrm{~Hz}$, $1 \mathrm{H}), 5.34-5.24(\mathrm{~m}, 2 \mathrm{H}), 5.12(\mathrm{t}, J=6.0 \mathrm{~Hz}, 1 \mathrm{H}), 4.46(\mathrm{dt}, J=9.0,5.1 \mathrm{~Hz}, 1 \mathrm{H}), 4.26(\mathrm{dt}, J=9.5,4.0 \mathrm{~Hz}, 1 \mathrm{H}), 4.17$ (dd, $J=10.9,1.7 \mathrm{~Hz}, 1 \mathrm{H}), 2.63(\mathrm{dd}, J=16.3,6.1 \mathrm{~Hz}, 1 \mathrm{H}), 2.55-2.42(\mathrm{~m}, 2 \mathrm{H}), 2.31(\mathrm{dt}, J=13.1,4.4 \mathrm{~Hz}, 1 \mathrm{H})$, $2.04(\mathrm{ddd}, J=13.8,11.0,4.5 \mathrm{~Hz}, 1 \mathrm{H}), 1.75-1.71(\mathrm{~m}, 3 \mathrm{H}), 1.66-1.57(\mathrm{~m}, 4 \mathrm{H}), 1.43(\mathrm{~s}, 3 \mathrm{H}), 1.06(\mathrm{~s}, 3 \mathrm{H}), 1.04(\mathrm{~s}$, $3 \mathrm{H}), 0.88$ (s, 9H), 0.87 (s, 9H), 0.07 (s, 3H), 0.06 (s, 3H), 0.04 (s, 3H), 0.02 (s, 3H).

${ }^{13}$ C NMR (100 MHz, $\left.\mathrm{CDCl}_{3}\right): \delta 210.9,173.4,137.5,134.7,134.3,133.3,129.0,127.9,126.3,120.4,74.0,72.0$, $54.6,46.2,36.9,36.8,35.5,25.8,22.0,20.1,19.8,18.2,18.12,18.10,12.9,-4.1,-4.5,-4.7,-4.8$.

HRMS-ESI m/z cacld for $\mathrm{C}_{35} \mathrm{H}_{60} \mathrm{NaO}_{5} \mathrm{Si}_{2}[\mathrm{M}+\mathrm{Na}]^{+}$639.3871, found 639.3865.

\section{Major isomer 56:}

TLC (EtOAc:hexanes $=1: 12): \mathrm{R}_{f}=0.32(\mathrm{UV}$, anisaldehyde). 
${ }^{1} \mathbf{H}$ NMR $\left(400 \mathrm{MHz}, \mathrm{CDCl}_{3}\right): \delta 6.13(\mathrm{~d}, J=15.5 \mathrm{~Hz}, 1 \mathrm{H}), 5.70(\mathrm{~d}, J=15.9 \mathrm{~Hz}, 1 \mathrm{H}), 5.33(\mathrm{dd}, J=15.9,6.8 \mathrm{~Hz}$, $1 \mathrm{H}), 5.28(\mathrm{dd}, J=15.6,7.8 \mathrm{~Hz}, 1 \mathrm{H}), 5.22(\mathrm{t}, J=7.9 \mathrm{~Hz}, 1 \mathrm{H}), 5.12(\mathrm{dd}, J=9.6,7.1 \mathrm{~Hz}, 2 \mathrm{H}), 4.43(\mathrm{ddd}, J=10.0$, 7.9, $4.1 \mathrm{~Hz}, 2 \mathrm{H}), 4.21(\mathrm{q}, J=6.8 \mathrm{~Hz}, 1 \mathrm{H}), 3.79(\mathrm{~d}, J=9.2 \mathrm{~Hz}, 1 \mathrm{H}), 3.10(\mathrm{dd}, J=13.5,10.3 \mathrm{~Hz}, 1 \mathrm{H}), 2.39-2.32$ (m, 3H), $2.00(\mathrm{td}, J=9.9,4.9 \mathrm{~Hz}, 1 \mathrm{H}), 1.68(\mathrm{dd}, J=13.5,10.3 \mathrm{~Hz}, 1 \mathrm{H}), 1.60(\mathrm{~s}, 3 \mathrm{H}), 1.46(\mathrm{~s}, 3 \mathrm{H}), 1.12(\mathrm{~s}, 3 \mathrm{H})$, $1.01(\mathrm{~s}, 3 \mathrm{H}), 0.09(\mathrm{~s}, 3 \mathrm{H}), 0.05(\mathrm{~s}, 3 \mathrm{H}), 0.05(\mathrm{~s}, 3 \mathrm{H}), 0.02(\mathrm{~s}, 3 \mathrm{H})$.

${ }^{13} \mathbf{C}$ NMR $\left(100 \mathrm{MHz}, \mathrm{CDCl}_{3}\right): \delta 211.0,174.2,137.3,136.9,134.3,130.8,129.0,127.9,124.4,77.6,74.5,70.7$, $54.6,46.1,38.3,36.8,27.3,25.87,25.86,19.4,18.23,18.16,17.8,13.3,12.0,-4.2,-4.55,-4.64,-4.8$.

HRMS-ESI m/z cacld for $\mathrm{C}_{35} \mathrm{H}_{60} \mathrm{NaO}_{5} \mathrm{Si}_{2}[\mathrm{M}+\mathrm{Na}]^{+}$639.3871, found 639.3865.

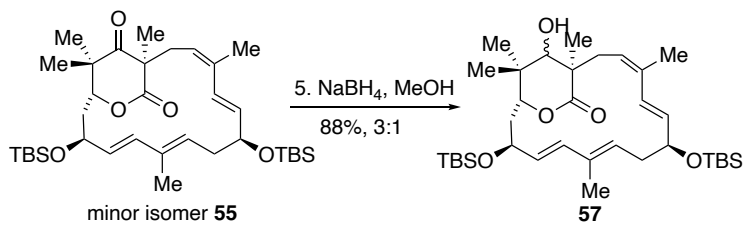

$\mathrm{MeOH}(22.0 \mathrm{~mL})$ was added into a 100-mL round-bottom flask containing 55 (435 mg, $0.71 \mathrm{mmol}, 1$ equiv), and $\mathrm{NaBH}_{4}(1.07 \mathrm{~g}, 28.20 \mathrm{mmol}, 40$ equiv) was added in several portions. The reaction was monitored by TLC until the starting material was disappeared, and the mixture was poured into a stirred mixture of ethyl acetate $(40 \mathrm{~mL})$ and water $(10 \mathrm{~mL})$. The biphasic mixture was transferred to a separatory funnel and the layers were separated. The aqueous layer was extracted with ethyl acetate $(2 \times 20 \mathrm{~mL})$. The combined organic layers were washed with brine $(10 \mathrm{~mL})$ and the washed solution was dried $\left(\mathrm{Na}_{2} \mathrm{SO}_{4}\right)$. The dried solution was filtered and the filtrate was concentrated. The crude residue was purified by flash chromatography (silica gel, eluent: EtOAc:hexanes $=1: 5)$ to afford a pair of inseparable alcohol 57 (384 mg, 3:1 dr, 88\%) as a colorless oil.

\section{Major isomer:}

TLC (EtOAc:hexanes = 1:5): $\mathrm{R}_{f}=0.33$ (UV, anisaldehyde).

${ }^{1}$ H NMR $\left(400 \mathrm{MHz}, \mathrm{CDCl}_{3}\right): \delta 6.22(\mathrm{~d}, J=16.0 \mathrm{~Hz}, 1 \mathrm{H}), 6.14(\mathrm{~d}, J=15.7 \mathrm{~Hz}, 1 \mathrm{H}), 5.57(\mathrm{dd}, J=16.0,5.2 \mathrm{~Hz}$, $1 \mathrm{H}), 5.41(\mathrm{t}, J=5.5 \mathrm{~Hz}, 1 \mathrm{H}), 5.36-5.26(\mathrm{~m}, 2 \mathrm{H}), 4.44-4.36(\mathrm{~m}, 3 \mathrm{H}), 3.34(\mathrm{~d}, J=3.9 \mathrm{~Hz}, 1 \mathrm{H}), 2.56-2.39(\mathrm{~m}$, $3 \mathrm{H}), 2.28$ (ddd, $J=13.8,9.7,5.1 \mathrm{~Hz}, 1 \mathrm{H}), 2.07(\mathrm{~d}, J=4.0 \mathrm{~Hz}, 1 \mathrm{H}), 1.91$ (ddd, $J=13.8,11.3,4.4 \mathrm{~Hz}, 1 \mathrm{H}), 1.78(\mathrm{~s}$, $3 \mathrm{H}), 1.69(\mathrm{~s}, 3 \mathrm{H}), 1.54-1.45(\mathrm{~m}, 1 \mathrm{H}), 1.38(\mathrm{~s}, 3 \mathrm{H}), 0.89(\mathrm{~s}, 3 \mathrm{H}), 0.88(\mathrm{~s}, 9 \mathrm{H}), 0.86(\mathrm{~s}, 9 \mathrm{H}), 0.05(\mathrm{~s}, 3 \mathrm{H}), 0.04(\mathrm{~s}$, $3 \mathrm{H}), 0.02(\mathrm{~s}, 3 \mathrm{H}), 0.02(\mathrm{~s}, 3 \mathrm{H})$.

${ }^{13}$ C NMR (100 MHz, $\left.\mathrm{CDCl}_{3}\right): \delta 176.9,136.6,134.6,133.9,133.1,128.5,128.4,127.1,122.2,81.0,77.3,72.9$, $72.1,46.4,39.3,37.2,36.4,33.1,25.9,25.0,21.4,20.6,19.8,18.2,18.1,13.0,-4.1,-4.6,-4.7,-4.9$.

HRMS-ESI m/z cacld for $\mathrm{C}_{35} \mathrm{H}_{62} \mathrm{NaO}_{5} \mathrm{Si}_{2}[\mathrm{M}+\mathrm{Na}]^{+}$641.4028, found 641.4017.

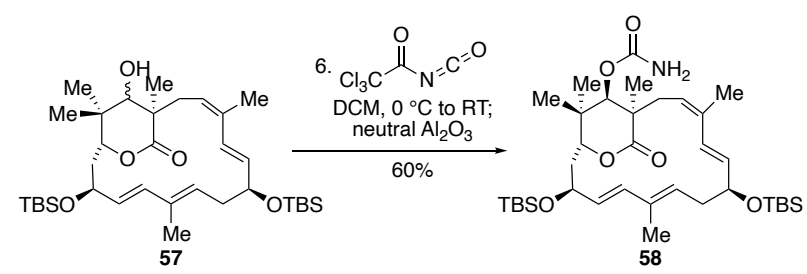

A 100-mL round-bottom flask containing $57(384 \mathrm{mg}, 0.62 \mathrm{mmol}, 1$ equiv) was evacuated and flushed with nitrogen (this process was repeated a total of 3 times) and sealed with a rubber septum. DCM $(30.0 \mathrm{~mL})$ was added, and the resulting solution was cooled to $0^{\circ} \mathrm{C}$ by means of an ice-water bath. Trichloroacetyl isocyanate $(111 \mu \mathrm{L}, 0.93 \mathrm{mmol}$, 1.50 equiv) was added dropwise, and the cooling bath was removed. The resulting solution was stirred at room temperature for $3 \mathrm{~h}$, and aluminum oxide (neutral, $4.85 \mathrm{~g}$ ) was added. The mixture was stirred for additional $2 \mathrm{~h}$. The mixture was filtered through a pad of aluminum oxide, and was washed with ethyl acetate $(50 \mathrm{~mL})$. The filtrate was concentrated and the crude residue was purified by flash chromatography (silica gel, eluent: EtOAc:hexanes = 1:5) to afford carbamate $\mathbf{5 8}(246.40 \mathrm{mg}, 60 \%)$ as a colorless oil.

TLC (EtOAc:hexanes = 1:3): $\mathrm{R}_{f}=0.31(\mathrm{UV}$, anisaldehyde). 
${ }^{1}$ H NMR $\left(400 \mathrm{MHz}, \mathrm{CDCl}_{3}\right): \delta 6.23(\mathrm{~d}, J=11.2 \mathrm{~Hz}, 1 \mathrm{H}), 6.19(\mathrm{~d}, J=10.9 \mathrm{~Hz}, 1 \mathrm{H}), 5.57(\mathrm{dd}, J=16.0,5.0 \mathrm{~Hz}$, $1 \mathrm{H}), 5.36(\mathrm{~d}, J=7.8 \mathrm{~Hz}, 1 \mathrm{H}), 5.33(\mathrm{t}, J=8.0 \mathrm{~Hz}, 1 \mathrm{H}), 5.29(\mathrm{t}, J=5.8 \mathrm{~Hz}, 1 \mathrm{H}), 4.56(\mathrm{~s}, 1 \mathrm{H}), 4.48-4.40(\mathrm{~m}, 2 \mathrm{H})$, $4.33(\mathrm{dd}, J=10.8,1.4 \mathrm{~Hz}, 1 \mathrm{H}), 2.52(\mathrm{ddd}, J=14.2,9.8,7.3 \mathrm{~Hz}, 1 \mathrm{H}), 2.44(\mathrm{dd}, J=15.8,6.3 \mathrm{~Hz}, 1 \mathrm{H}), 2.36-2.22$ $(\mathrm{m}, 2 \mathrm{H}), 1.90(\mathrm{ddd}, J=13.9,10.9,4.4 \mathrm{~Hz}, 1 \mathrm{H}), 1.77(\mathrm{~d}, J=1.0 \mathrm{~Hz}, 3 \mathrm{H}), 1.70(\mathrm{~s}, 3 \mathrm{H}), 1.52(\mathrm{ddd}, J=13.7,9.9,1.5$ $\mathrm{Hz}, 1 \mathrm{H}), 1.43$ (s, 3H), 0.96 (s, 3H), $0.90(\mathrm{~s}, 9 \mathrm{H}), 0.87(\mathrm{~s}, 9 \mathrm{H}), 0.85(\mathrm{~s}, 3 \mathrm{H}), 0.07(\mathrm{~s}, 3 \mathrm{H}), 0.05(\mathrm{~s}, 3 \mathrm{H}), 0.04(\mathrm{~s}, 3 \mathrm{H})$, $0.03(\mathrm{~s}, 3 \mathrm{H})$.

${ }^{13}$ C NMR $\left(100 \mathrm{MHz}, \mathrm{CDCl}_{3}\right): \delta 175.5,156.0,136.8,134.4,132.5,132.3,128.9,128.2,127.1,122.6,81.5,77.7$, $72.6,71.9,45.6,39.2,37.0,36.3,33.6,25.9,25.9,24.9,20.9,20.5,18.9,18.3,18.1,13.0,-4.2,-4.5,-4.6,-4.9$.

HRMS-ESI m/z cacld for $\mathrm{C}_{36} \mathrm{H}_{63} \mathrm{NNaO}_{6} \mathrm{Si}_{2}[\mathrm{M}+\mathrm{Na}]^{+}$684.4086, found 684.4099.

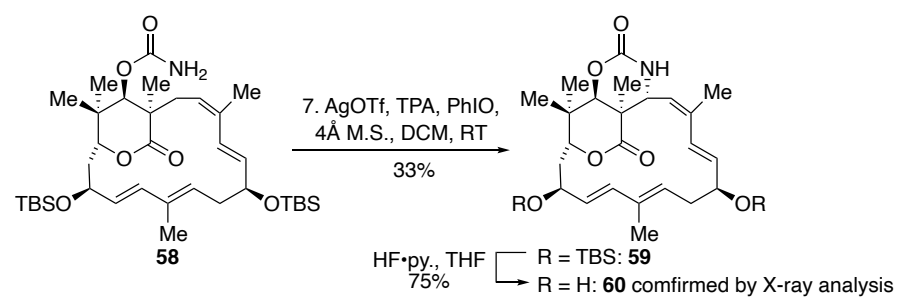

A $50-\mathrm{mL}$ round-bottom flask containing $\operatorname{AgOTf}(9.50 \mathrm{mg}, 0.037 \mathrm{mmol}, 0.10$ equiv) and TPA (16.10 $\mathrm{mg}, 0.056$ mmol, 0.15 equiv) was evacuated and flushed with nitrogen (this process was repeated a total of 3 times) and sealed with a rubber septum. DCM $(15 \mathrm{~mL})$ was added, and the resulting solution was stirred at room temperature for 30

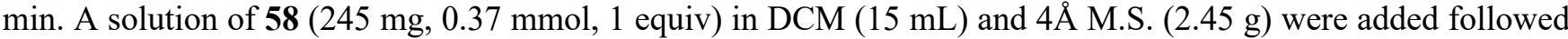
by $\mathrm{PhIO}$ (187 $\mathrm{mg}, 0.85 \mathrm{mmol}, 2.30$ equiv). The mixture was filtered through a pad of celite and the filtrate was concentrated. The crude residue was purified by flash chromatography ( silica gel, eluent: EtOAc:hexanes $=1: 5)$ to afford carbamate $\mathbf{5 9}(80.60 \mathrm{mg}, 33 \%)$ as a colorless oil.

TLC (EtOAc:hexanes $=1: 2): \mathrm{R}_{f}=0.34(\mathrm{UV}$, anisaldehyde).

${ }^{1} \mathbf{H}$ NMR $\left(400 \mathrm{MHz}, \mathrm{CDCl}_{3}\right): \delta 6.36(\mathrm{~d}, J=15.5 \mathrm{~Hz}, 1 \mathrm{H}), 6.18(\mathrm{~d}, J=15.7 \mathrm{~Hz}, 1 \mathrm{H}), 5.88(\mathrm{dd}, J=15.4,3.8 \mathrm{~Hz}$, $1 \mathrm{H}), 5.41(\mathrm{~d}, J=8.7 \mathrm{~Hz}, 1 \mathrm{H}), 5.37(\mathrm{dd}, J=15.6,8.4 \mathrm{~Hz}, 1 \mathrm{H}), 5.22-5.15(\mathrm{~m}, 1 \mathrm{H}), 4.71(\mathrm{~s}, 1 \mathrm{H}), 4.60(\mathrm{~d}, J=3.4$ $\mathrm{Hz}, 1 \mathrm{H}), 4.49-4.40(\mathrm{~m}, 2 \mathrm{H}), 4.14(\mathrm{~d}, J=8.4 \mathrm{~Hz}, 1 \mathrm{H}), 3.69(\mathrm{~s}, 1 \mathrm{H}), 2.61$ (ddd, $J=14.2,9.9,4.6 \mathrm{~Hz}, 1 \mathrm{H}), 2.21$ (ddd, $J=14.0,6.2,3.2 \mathrm{~Hz}, 1 \mathrm{H}), 2.03-1.93(\mathrm{~m}, 1 \mathrm{H}), 1.87(\mathrm{~s}, 3 \mathrm{H}), 1.76(\mathrm{~s}, 3 \mathrm{H}), 1.62-1.55(\mathrm{~m}, 1 \mathrm{H}), 1.47(\mathrm{~s}, 3 \mathrm{H})$, $1.11(\mathrm{~s}, 3 \mathrm{H}), 0.98(\mathrm{~s}, 3 \mathrm{H}), 0.89(\mathrm{~s}, 9 \mathrm{H}), 0.87(\mathrm{~s}, 9 \mathrm{H}), 0.06(\mathrm{~s}, 3 \mathrm{H}), 0.04(\mathrm{~s}, 3 \mathrm{H}), 0.02(\mathrm{~s}, 3 \mathrm{H}), 0.01(\mathrm{~s}, 3 \mathrm{H})$.

${ }^{13} \mathrm{C}$ NMR $\left(100 \mathrm{MHz}, \mathrm{CDCl}_{3}\right): \delta 171.4,154.6,140.3,137.1,135.2,135.2,128.4,127.3,125.8,118.0,88.7,77.4$, $71.9,70.7,50.9,48.6,38.7,36.7,34.2,25.9,25.7,21.5,20.54,20.45,18.6,18.2,18.1,13.0,-4.1,-4.6,-4.7,-4.9$.

HRMS-ESI $\mathrm{m} / \mathrm{z}$ cacld for $\mathrm{C}_{36} \mathrm{H}_{61} \mathrm{NNaO}_{6} \mathrm{Si}_{2}[\mathrm{M}+\mathrm{Na}]^{+} 682.3930$, found 682.3923 .

A 50-mL plastic vial containing $59\left(10.00 \mathrm{mg}, 0.015 \mathrm{mmol}, 1\right.$ equiv) and THF $(4.00 \mathrm{~mL})$ was cooled to $0{ }^{\circ} \mathrm{C}$ by means of an ice-water bath. Py $\bullet(\mathrm{HF})_{\mathrm{x}}(\sim 70 \% \mathrm{HF})(0.400 \mathrm{~mL})$ was added dropwise, and the vessel was removed from the cooling bath and the system was allowed to warm to $23{ }^{\circ} \mathrm{C}$. After stirring for $18 \mathrm{~h}, \mathrm{NaHCO}_{3}$ solid $(450$ $\mathrm{mg}$ ) was added and the mixture was vigorously stirred. After stirring for $30 \mathrm{~min}$, the mixture was filtered through a pad of silica gel and the filtrate was concentrated. The crude residue was purified by flash chromatography (silica gel, eluent: ethyl acetate: $\mathrm{MeOH}=20: 1)$ to afford diol $\mathbf{6 0}(4.90 \mathrm{mg}, 75 \%)$ as a white solid.

m. p. $173-175^{\circ} \mathrm{C}(\mathrm{DCM})$

TLC (EtOAc: $\mathrm{MeOH}=20: 1): \mathrm{R}_{f}=0.37$ (UV, anisaldehyde).

${ }^{1}$ H NMR (400 MHz, CD $\left.{ }_{3} \mathrm{OD}\right): \delta 6.41(\mathrm{~d}, J=16.0 \mathrm{~Hz}, 1 \mathrm{H}), 6.20(\mathrm{~d}, J=15.6 \mathrm{~Hz}, 1 \mathrm{H}), 5.98(\mathrm{dd}, J=15.8,3.8 \mathrm{~Hz}$, $1 \mathrm{H}), 5.49-5.39(\mathrm{~m}, 2 \mathrm{H}), 5.24(\mathrm{dd}, J=9.5,6.4 \mathrm{~Hz}, 1 \mathrm{H}), 4.64-4.57(\mathrm{~m}, 2 \mathrm{H}), 4.36(\mathrm{td}, J=10.1,4.7 \mathrm{~Hz}, 1 \mathrm{H}), 4.21$ $(\mathrm{d}, J=9.1 \mathrm{~Hz}, 1 \mathrm{H}), 3.79(\mathrm{~s}, 1 \mathrm{H}), 2.66(\mathrm{ddd}, J=14.7,9.9,5.1 \mathrm{~Hz}, 1 \mathrm{H}), 2.29(\mathrm{ddd}, J=13.8,5.5,3.3 \mathrm{~Hz}, 1 \mathrm{H}), 2.06$ (ddd, $J=13.3,12.0,4.7 \mathrm{~Hz}, 1 \mathrm{H}), 1.90(\mathrm{~d}, J=1.0 \mathrm{~Hz}, 3 \mathrm{H}), 1.81(\mathrm{~s}, 3 \mathrm{H}), 1.60$ (ddd, $J=13.2,10.3,2.7 \mathrm{~Hz}, 1 \mathrm{H}), 1.45$ (s, 3H), $1.11(\mathrm{~s}, 3 \mathrm{H}), 0.99(\mathrm{~s}, 3 \mathrm{H})$.

${ }^{13}$ C NMR (100 MHz, $\left.\mathrm{CD}_{3} \mathrm{OD}\right): \delta 173.9,158.2,141.0,139.0,136.4,135.7,129.9,127.7,126.6,119.6,89.7,78.9$, 71.9, 71.2, 52.0, 50.2, 39.8, 36.7, 34.5, 21.7, 20.8, 20.7, 18.4, 12.8. 
HRMS-ESI $\mathrm{m} / \mathrm{z}$ cacld for $\mathrm{C}_{24} \mathrm{H}_{33} \mathrm{NNaO}_{6}[\mathrm{M}+\mathrm{Na}]^{+}$454.2200, found 454.2212.

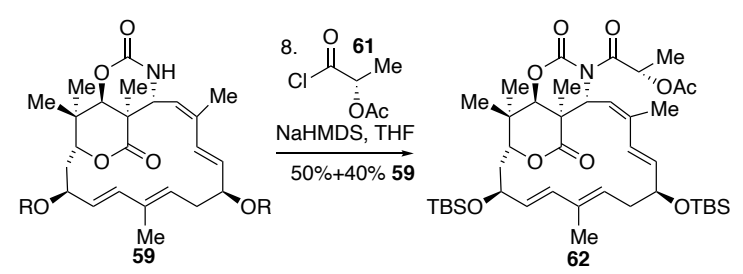

A 50-mL round-bottom flask containing $59(76.00 \mathrm{mg}, 0.115 \mathrm{mmol}, 1$ equiv) was evacuated and flushed with nitrogen (this process was repeated a total of 3 times) and was then sealed with a rubber septum. Dry THF (10 mL) was added, and the vessel was cooled to $-78^{\circ} \mathrm{C}$ by means of a dry ice-acetone bath. NaHMDS $(115 \mu \mathrm{L}, 2.00 \mathrm{M}$ in hexane, $0.23 \mathrm{mmol}, 2.00$ equiv) was added dropwise. After stirring for $10 \mathrm{~min}, \mathbf{6 1}(29.20 \mu \mathrm{L}, 0.23 \mathrm{mmol}, 2.00$ equiv) was added. After stirring for $10 \mathrm{~min}$, saturated ammonium chloride aqueous $(5 \mathrm{~mL})$ and ethyl acetate $(20$ $\mathrm{mL}$ ) were added, and the vessel was removed from the cooling bath and the system was allowed to warm to $23{ }^{\circ} \mathrm{C}$. The biphasic mixture was transferred to a separatory funnel and the layers were separated. The aqueous layer was extracted with ethyl acetate $(2 \times 10 \mathrm{~mL})$. The combined organic layers were washed with saturated aqueous sodium chloride solution $(10 \mathrm{~mL})$ and the washed solution was dried $\left(\mathrm{Na}_{2} \mathrm{SO}_{4}\right)$. The dried solution was filtered, and the filtrate was concentrated. The resulting crude residue was purified by flash chromatography (silica gel, eluent: EtOAc:hexanes $=1: 6$ to $1: 1)$ to afford $\mathbf{6 2}(44.20 \mathrm{mg}, 0.057 \mathrm{mmol}, 50 \%)$ as a colorless oil and recover starting material 59 (30.5 mg, 40\%).

TLC (EtOAc:hexanes = 1:10): $\mathrm{R}_{f}=0.30(\mathrm{UV}$, anisaldehyde).

${ }^{1}$ H NMR $\left(400 \mathrm{MHz}, \mathrm{CDCl}_{3}\right): \delta 6.47(\mathrm{~d}, J=16.4 \mathrm{~Hz}, 1 \mathrm{H}), 6.26(\mathrm{~d}, J=15.6 \mathrm{~Hz}, 1 \mathrm{H}), 5.84(\mathrm{dd}, J=16.4,3.0 \mathrm{~Hz}$, $1 \mathrm{H}), 55.39(\mathrm{dd}, J=10.1,2.0 \mathrm{~Hz}, 1 \mathrm{H}), 5.35(\mathrm{q}, J=6.6 \mathrm{~Hz}, 1 \mathrm{H}), 5.29(\mathrm{dd}, J=15.7,8.9 \mathrm{~Hz}, 1 \mathrm{H}), 5.01(\mathrm{~d}, J=10.1$ $\mathrm{Hz}, 1 \mathrm{H}), 4.89(\mathrm{~d}, J=10.1 \mathrm{~Hz}, 1 \mathrm{H}), 4.45(\mathrm{~m}, 2 \mathrm{H}), 4.16(\mathrm{dd}, J=11.5,3.6 \mathrm{~Hz}, 1 \mathrm{H}), 3.91(\mathrm{~s}, 1 \mathrm{H}), 2.73(\mathrm{dt}, J=15.2$, $10.2 \mathrm{~Hz}, 1 \mathrm{H}), 2.42(\mathrm{~d}, J=15.4 \mathrm{~Hz}, 1 \mathrm{H}), 2.11(\mathrm{~s}, 3 \mathrm{H}), 2.02-1.93(\mathrm{~m}, 1 \mathrm{H}), 1.85(\mathrm{ddd}, J=13.3,9.9,3.6 \mathrm{~Hz}, 1 \mathrm{H})$, $1.80(\mathrm{~d}, J=0.9 \mathrm{~Hz}, 3 \mathrm{H}), 1.76(\mathrm{~s}, 3 \mathrm{H}), 1.48(\mathrm{~d}, J=6.6 \mathrm{~Hz}, 3 \mathrm{H}), 1.40(\mathrm{~s}, 3 \mathrm{H}), 1.16(\mathrm{~s}, 3 \mathrm{H}), 1.10(\mathrm{~s}, 3 \mathrm{H}), 0.90(\mathrm{~s}, 9 \mathrm{H})$, $0.87(\mathrm{~s}, 9 \mathrm{H}), 0.08(\mathrm{~s}, 6 \mathrm{H}), 0.04(\mathrm{~s}, 3 \mathrm{H}), 0.02(\mathrm{~s}, 3 \mathrm{H})$.

${ }^{13}$ C NMR (100 MHz, $\left.\mathrm{CDCl}_{3}\right): \delta 171.7,171.5,170.4,151.1,138.8,137.3,137.0,134.0,129.3,127.2,124.4,120.9$, 89.6, 79.1, 72.7, 71.4, 70.7, 53.9, 45.2, 40.0, 37.1, 35.4, 25.9, 25.9, 23.8, 23.2, 20.7, 20.5, 19.2, 18.2, 18.1, 16.6, $12.6,-4.0,-4.6,-4.7,-4.8$.

HRMS-ESI m/z cacld for $\mathrm{C}_{41} \mathrm{H}_{67} \mathrm{NNaO}_{9} \mathrm{Si}_{2}[\mathrm{M}+\mathrm{Na}]^{+}$796.4247, found 796.4260.
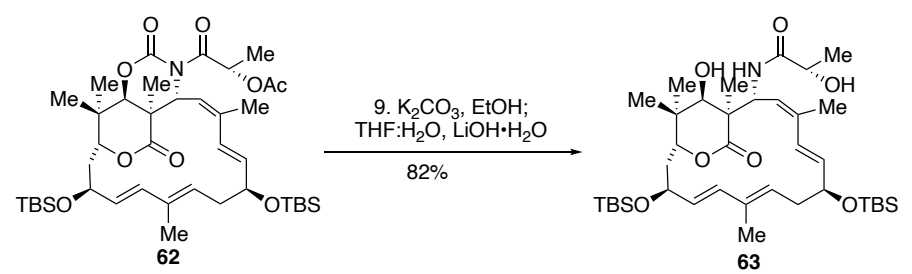

A $25-\mathrm{mL}$ round-bottom flask containing $62(42.00 \mathrm{mg}, 0.054 \mathrm{mmol}, 1$ equiv) was evacuated and flushed with nitrogen (this process was repeated a total of 3 times) and was then sealed with a rubber septum. Dry EtOH (10 $\mathrm{mL}$ ) was added followed by anhydrous $\mathrm{K}_{2} \mathrm{CO}_{3}(30.00 \mathrm{mg}, 0.22 \mathrm{mmol}, 4$ equiv). After stirring for $4 \mathrm{~h}$, THF (10 mL) and $\mathrm{H}_{2} \mathrm{O}(15 \mathrm{~mL})$ was added followed by $\mathrm{LiOH} \cdot \mathrm{H}_{2} \mathrm{O}(34.20 \mathrm{mg}, 0.81 \mathrm{mmol}, 15$ equiv). After stirring for $40 \mathrm{~h}$, the mixture was poured into a stirred mixture of saturated ammonium chloride aqueous $(5 \mathrm{~mL})$ and ethyl acetate $(30$ $\mathrm{mL}$ ). The biphasic mixture was transferred to a separatory funnel and the layers were separated. The aqueous layer was extracted with ethyl acetate $(2 \times 10 \mathrm{~mL})$. The combined organic layers were washed with saturated aqueous sodium chloride solution $(10 \mathrm{~mL})$ and the washed solution was dried $\left(\mathrm{Na}_{2} \mathrm{SO}_{4}\right)$. The dried solution was filtered, and the filtrate was concentrated. The resulting crude residue was purified by flash chromatography (silica gel, eluent: EtOAc:hexanes $=1: 3)$ to afford diol $63(31.40 \mathrm{mg}, 82 \%)$ as a pale yellow oil.

TLC (EtOAc:hexanes = 1:2): $\mathrm{R}_{f}=0.27$ (UV, anisaldehyde).

${ }^{1} \mathbf{H}$ NMR $\left(400 \mathrm{MHz}, \mathrm{CDCl}_{3}\right): \delta 6.51(\mathrm{~m}, 2 \mathrm{H}), 6.30(\mathrm{~d}, J=4.5 \mathrm{~Hz}, 1 \mathrm{H}), 6.18(\mathrm{~d}, J=15.6 \mathrm{~Hz}, 1 \mathrm{H}), 5.71(\mathrm{dd}, J=15.2$, $3.2 \mathrm{~Hz}, 1 \mathrm{H}), 5.65(\mathrm{dd}, J=10.3,8.5 \mathrm{~Hz}, 1 \mathrm{H}), 5.54(\mathrm{~d}, J=10.2 \mathrm{~Hz}, 1 \mathrm{H}), 5.36(\mathrm{dd}, J=15.6,7.7 \mathrm{~Hz}, 1 \mathrm{H}), 5.23(\mathrm{t}, J=$ 
$8.0 \mathrm{~Hz}, 1 \mathrm{H}), 4.61(\mathrm{~m}, 2 \mathrm{H}), 4.44(\mathrm{td}, J=8.9,5.1 \mathrm{~Hz}, 1 \mathrm{H}), 4.20(\mathrm{~m}, 1 \mathrm{H}), 3.08(\mathrm{~d}, J=4.5 \mathrm{~Hz}, 1 \mathrm{H}), 2.55(\mathrm{ddd}, J=$ $13.5,9.8,3.8 \mathrm{~Hz}, 1 \mathrm{H}), 2.45-2.39(\mathrm{~m}, 1 \mathrm{H}), 2.19(\mathrm{ddd}, J=13.4,7.0,2.8 \mathrm{~Hz}, 1 \mathrm{H}), 1.91(\mathrm{ddd}, J=13.6,12.0,5.0 \mathrm{~Hz}$, $1 \mathrm{H}), 1.84(\mathrm{~s}, 3 \mathrm{H}), 1.71(\mathrm{~s}, 3 \mathrm{H}), 1.52(\mathrm{ddd}, J=11.9,9.5,2.2 \mathrm{~Hz}, 1 \mathrm{H}), 1.43(\mathrm{~d}, J=6.8 \mathrm{~Hz}, 3 \mathrm{H}), 1.28(\mathrm{~s}, 3 \mathrm{H}), 1.06(\mathrm{~s}$, $3 \mathrm{H}), 0.98(\mathrm{~s}, 3 \mathrm{H}), 0.91(\mathrm{~s}, 9 \mathrm{H}), 0.87(\mathrm{~s}, 9 \mathrm{H}), 0.05(\mathrm{~s}, 3 \mathrm{H}), 0.03(\mathrm{~s}, 3 \mathrm{H}), 0.02(\mathrm{~s}, 3 \mathrm{H}), 0.00(\mathrm{~s}, 3 \mathrm{H})$.

${ }^{13} \mathbf{C}$ NMR $\left(100 \mathrm{MHz}, \mathrm{CDCl}_{3}\right): \delta 173.9,173.7,137.0,135.4,133.8,133.5,127.4,127.0,124.7,123.9,78.8,78.0$, $71.9,71.0,68.5,52.2,47.5,38.8,37.0,34.5,25.9,24.0,23.0,21.6,20.8,20.2,18.3,18.2,13.0,-4.2,-4.6,-4.8$, 5.1.

HRMS-ESI m/z cacld for $\mathrm{C}_{38} \mathrm{H}_{68} \mathrm{NO}_{7} \mathrm{Si}_{2}[\mathrm{M}+\mathrm{H}]^{+}$706.4529, found 706.4509.

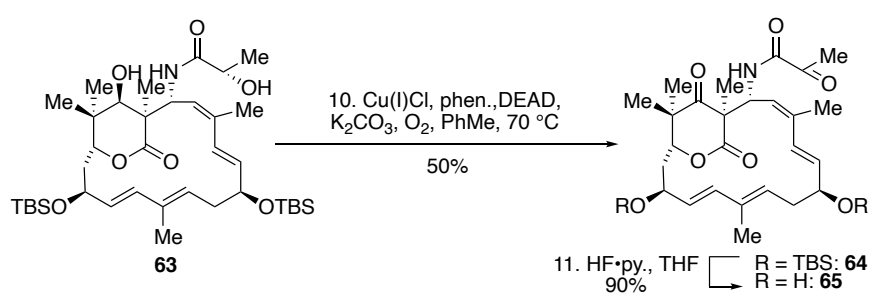

A $25-\mathrm{mL}$ round-bottom flask containing $63(20.00 \mathrm{mg}, 0.028 \mathrm{mmol}, 1$ equiv), $\mathrm{Cu}(\mathrm{I}) \mathrm{Cl}(0.84 \mathrm{mg}, 0.0085 \mathrm{mmol}$, 0.30 equiv), 1,10 -phenanthroline ( $1.53 \mathrm{mg}, 0.0085 \mathrm{mmol}, 0.30$ equiv), DEAD (1.48 $\mathrm{mg}, 0.0085 \mathrm{mmol}, 0.30$ equiv) and $\mathrm{K}_{2} \mathrm{CO}_{3}(15.70 \mathrm{mg}, 0.113 \mathrm{mmol}, 4.00$ equiv) was evacuated and flushed with oxygen (this process was repeated a total of 3 times) and was then sealed with a rubber septum. PhMe $(5.00 \mathrm{~mL})$ was added. The resulting solution was warmed to $70{ }^{\circ} \mathrm{C}$ by means of an oil bath. After stirring for $5 \mathrm{~h}$, the mixture was purified directly by flash chromatography (silica gel, eluent: EtOAc:hexanes $=1: 10$ to $1: 5)$ to afford ketone $64(10.0 \mathrm{mg}, 50 \%)$ as a pale yellow oil.

TLC (EtOAc:hexanes = 1:8): $\mathrm{R}_{f}=0.36$ (UV, anisaldehyde).

${ }^{1} \mathbf{H}$ NMR $\left(400 \mathrm{MHz}, \mathrm{CDCl}_{3}\right): \delta 7.48(\mathrm{~d}, J=10.0 \mathrm{~Hz}, 1 \mathrm{H}), 6.43(\mathrm{~d}, J=15.7 \mathrm{~Hz}, 1 \mathrm{H}), 6.00(\mathrm{~d}, J=15.8 \mathrm{~Hz}, 1 \mathrm{H}), 5.55$ $(\mathrm{dd}, J=15.8,8.1 \mathrm{~Hz}, 1 \mathrm{H}), 5.40-5.32(\mathrm{~m}, 2 \mathrm{H}), 5.26(\mathrm{dd}, J=15.6,8.7 \mathrm{~Hz}, 1 \mathrm{H}), 4.95(\mathrm{~d}, J=9.0 \mathrm{~Hz}, 1 \mathrm{H}), 4.49(\mathrm{td}$, $J=9.7,4.9 \mathrm{~Hz}, 1 \mathrm{H}), 4.16(\mathrm{td}, J=8.9,5.8 \mathrm{~Hz}, 1 \mathrm{H}), 4.06(\mathrm{dd}, J=10.9,2.8 \mathrm{~Hz}, 1 \mathrm{H}), 2.48(\mathrm{~s}, 3 \mathrm{H}), 2.46-2.38(\mathrm{~m}$, $2 \mathrm{H}), 2.14-2.03(\mathrm{~m}, 1 \mathrm{H}), 1.72(\mathrm{~d}, J=0.9 \mathrm{~Hz}, 3 \mathrm{H}), 1.66-1.58(\mathrm{~m}, 1 \mathrm{H}), 1.61(\mathrm{~s}, 3 \mathrm{H}), 1.41(\mathrm{~s}, 3 \mathrm{H}), 1.12(\mathrm{~s}, 3 \mathrm{H})$, $1.03(\mathrm{~s}, 3 \mathrm{H}), 0.88(\mathrm{~s}, 9 \mathrm{H}), 0.88(\mathrm{~s}, 9 \mathrm{H}), 0.08(\mathrm{~s}, 3 \mathrm{H}), 0.07(\mathrm{~s}, 3 \mathrm{H}), 0.05(\mathrm{~s}, 3 \mathrm{H}), 0.04(\mathrm{~s}, 3 \mathrm{H})$.

${ }^{13} \mathbf{C}$ NMR $\left(100 \mathrm{MHz}, \mathrm{CDCl}_{3}\right): \delta 212.8,196.6,172.1,158.9,138.2,137.2,136.8,134.5,128.9,127.0,125.3,122.3$, $77.2,76.6,75.1,72.2,58.1,49.2,46.8,37.5,36.2,25.9,24.6,21.8,20.3,19.4,18.8,18.2,18.1,12.9,-4.1,-4.4,-$ 4.66, -4.68 .

HRMS-ESI m/z cacld for $\mathrm{C}_{38} \mathrm{H}_{63} \mathrm{NNaO}_{7} \mathrm{Si}_{2}[\mathrm{M}+\mathrm{Na}]^{+}$724.4035, found 724.4046.

A 50-mL plastic vial containing $64\left(10.00 \mathrm{mg}, 0.014 \mathrm{mmol}, 1\right.$ equiv) and THF $(4.00 \mathrm{~mL})$ was cooled to $0{ }^{\circ} \mathrm{C}$ by means of an ice-water bath. Py• $(\mathrm{HF})_{\mathrm{x}}(\sim 70 \% \mathrm{HF})(0.400 \mathrm{~mL})$ was added dropwise, and the vessel was removed from the cooling bath and the system was allowed to warm to $23{ }^{\circ} \mathrm{C}$. After stirring for $4 \mathrm{~h}, \mathrm{NaHCO}_{3}$ solid $(600 \mathrm{mg})$ was added and the mixture was vigorously stirred. After stirring for $30 \mathrm{~min}$, the mixture was filtered through a pad of silica gel and the filtrate was concentrated. The crude residue was purified by flash chromatography (silica gel, eluent: ethyl acetate:hexanes $=2: 1)$ to afford $\mathbf{6 5}(6.10 \mathrm{mg}, 90 \%)$ as a colorless oil.

TLC (EtOAc:hexanes = 3:1): $\mathrm{R}_{f}=0.32$ (UV, anisaldehyde).

${ }^{1}$ H NMR $\left(400 \mathrm{MHz}, \mathrm{CDCl}_{3}\right): \delta 7.63(\mathrm{~d}, J=9.9 \mathrm{~Hz}, 1 \mathrm{H}), 6.51(\mathrm{~d}, J=15.5 \mathrm{~Hz}, 1 \mathrm{H}), 6.14(\mathrm{~d}, J=15.6 \mathrm{~Hz}, 1 \mathrm{H}), 5.56$ $(\mathrm{dd}, J=15.7,8.6 \mathrm{~Hz}, 1 \mathrm{H}), 5.42(\mathrm{dd}, J=11.6,4.8 \mathrm{~Hz}, 1 \mathrm{H}), 5.39-5.33(\mathrm{~m}, 2 \mathrm{H}), 5.33-5.27(\mathrm{~m}, 1 \mathrm{H}), 4.93(\mathrm{~d}, J=$ $9.4 \mathrm{~Hz}, 1 \mathrm{H}), 4.56(\mathrm{td}, J=10.0,5.0 \mathrm{~Hz}, 2 \mathrm{H}), 4.31-4.21(\mathrm{~m}, 1 \mathrm{H}), 3.97(\mathrm{dd}, J=11.4,3.2 \mathrm{~Hz}, 1 \mathrm{H}), 2.54-2.41(\mathrm{~m}$, $2 \mathrm{H}), 2.46(\mathrm{~s}, 3 \mathrm{H}), 2.25(\mathrm{ddd}, J=13.5,11.6,5.1 \mathrm{~Hz}, 2 \mathrm{H}), 1.72(\mathrm{~s}, 3 \mathrm{H}), 1.63(\mathrm{~s}, 3 \mathrm{H}), 1.60(\mathrm{dd}, J=10.6,3.2 \mathrm{~Hz}, 2 \mathrm{H})$, $1.42(\mathrm{~s}, 3 \mathrm{H}), 1.15(\mathrm{~s}, 3 \mathrm{H}), 1.05(\mathrm{~s}, 3 \mathrm{H})$.

${ }^{13}$ C NMR (100 MHz, $\left.\mathrm{CDCl}_{3}\right): \delta 213.1,196.4,172.2,159.1,140.0,136.6,135.5,134.6,129.5,126.6,125.4,123.2$, $77.2,76.4,74.4,71.6,57.8,49.7,46.9,36.1,34.3,24.5,22.9,20.4,19.1,18.7,12.9$.

HRMS-ESI $\mathrm{m} / \mathrm{z}$ cacld for $\mathrm{C}_{26} \mathrm{H}_{36} \mathrm{NO}_{7}[\mathrm{M}+\mathrm{H}]^{+} 474.2486$, found 474.2491. 


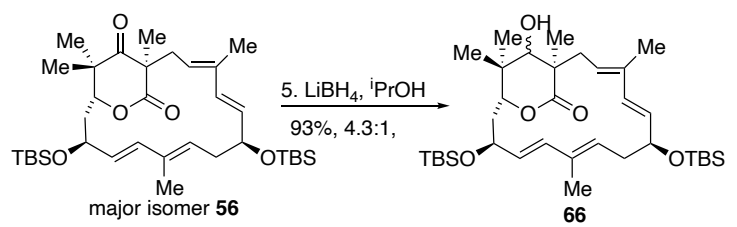

A 100-mL round-bottom flask containing $\mathbf{5 6}(520 \mathrm{mg}, 0.84 \mathrm{mmol}, 1$ equiv) was evacuated and flushed with nitrogen (this process was repeated a total of 3 times) and sealed with a rubber septum. ${ }^{i} \mathrm{PrOH}(35.00 \mathrm{~mL})$ was added followed by $\mathrm{LiBH}_{4}(2.11 \mathrm{~mL}, 4.00 \mathrm{M}$ in THF, $8.44 \mathrm{mmol}, 10$ equiv). The resulting solution was stirred at room temperature for $12 \mathrm{~h}$. Saturated ammonium chloride aqueous $(10 \mathrm{~mL})$ was added dropwise. The mixture was transferred to a separatory funnel, and the aqueous layer was extracted with ethyl acetate $(2 \times 10 \mathrm{~mL})$. The combined organic layers were washed with brine $(10 \mathrm{~mL})$ and the washed solution was dried $\left(\mathrm{Na}_{2} \mathrm{SO}_{4}\right)$. The dried solution was filtered and the filtrate was concentrated. The crude residue was purified by flash chromatography (silica gel, eluent: EtOAc:hexanes = 1:5) to afford alcohol $66(485 \mathrm{mg}, 93 \%)$ as a colorless oil.

TLC (EtOAc:hexanes = 1:5): $\mathrm{R}_{f}=0.31$ (UV, anisaldehyde).

${ }^{1}$ H NMR $\left(400 \mathrm{MHz}, \mathrm{CDCl}_{3}\right): \delta 6.19(\mathrm{~d}, J=15.5 \mathrm{~Hz}, 1 \mathrm{H}), 5.75(\mathrm{~d}, J=16.2 \mathrm{~Hz}, 1 \mathrm{H}), 5.36(\mathrm{dd}, J=16.2,6.8 \mathrm{~Hz}$, $1 \mathrm{H}), 5.32-5.22(\mathrm{~m}, 3 \mathrm{H}), 4.44(\mathrm{td}, J=9.7,4.5 \mathrm{~Hz}, 1 \mathrm{H}), 4.22(\mathrm{q}, J=7.6,7.0 \mathrm{~Hz}, 1 \mathrm{H}), 4.12(\mathrm{~d}, J=10.3 \mathrm{~Hz}, 1 \mathrm{H})$, $3.34(\mathrm{~d}, J=8.0 \mathrm{~Hz}, 1 \mathrm{H}), 2.79(\mathrm{dd}, J=14.1,11.1 \mathrm{~Hz}, 1 \mathrm{H}), 2.42-230(\mathrm{~m}, 3 \mathrm{H}), 2.05(\mathrm{~d}, J=7.6 \mathrm{~Hz}, 1 \mathrm{H}), 1.93$ (ddd, $J=14.1,11.0,4.5 \mathrm{~Hz}, 1 \mathrm{H}), 1.73(\mathrm{~s}, 3 \mathrm{H}), 1.59(\mathrm{~s}, 3 \mathrm{H}), 1.51(\mathrm{dd}, J=13.3,10.2 \mathrm{~Hz}, 1 \mathrm{H}), 1.41(\mathrm{~s}, 3 \mathrm{H}), 0.96(\mathrm{~s}, 3 \mathrm{H})$, $0.94(\mathrm{~s}, 3 \mathrm{H}), 0.89$ (s, 9H), 0.87 (s, 9H), 0.07 (s, 3H), 0.06 (s, 3H), 0.05 (s, 3H), 0.04 (s, 3H).

${ }^{13}$ C NMR (100 MHz, $\left.\mathrm{CDCl}_{3}\right): \delta 175.9,137.3,135.8,134.5,134.2,130.6,129.1,128.5,127.4,81.9,77.6,74.6$, $71.5,47.0,38.8,38.2,37.8,37.4,30.5,25.9,23.5,19.2,18.3,18.2,13.1,12.5,-4.1,-4.5,-4.6,-4.8$.

HRMS-ESI m/z cacld for $\mathrm{C}_{35} \mathrm{H}_{62} \mathrm{NaO}_{5} \mathrm{Si}_{2}[\mathrm{M}+\mathrm{Na}]^{+}$641.4028, found 641.4017.
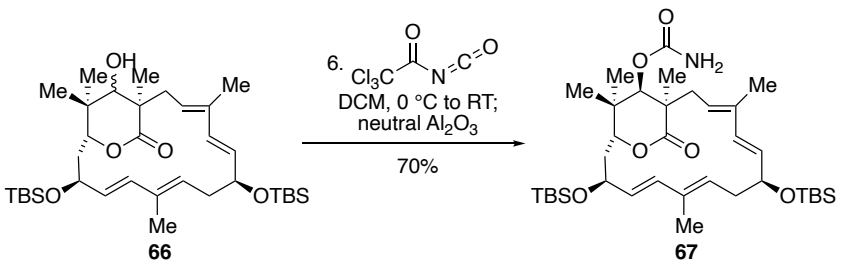

A 100-mL round-bottom flask containing $66(485 \mathrm{mg}, 0.79 \mathrm{mmol}, 1$ equiv) was evacuated and flushed with nitrogen (this process was repeated a total of 3 times) and sealed with a rubber septum. DCM $(30.00 \mathrm{~mL})$ was added, and the resulting solution was cooled to $0{ }^{\circ} \mathrm{C}$ by means of an ice-water bath. Trichloroacetyl isocyanate $(140 \mu \mathrm{L}, 1.18$ mmol, 1.50 equiv) was added dropwise, and the cooling bath was removed. The resulting solution was stirred at room temperature for $3 \mathrm{~h}$, and aluminum oxide (neutral, $4.85 \mathrm{~g}$ ) was added. The mixture was stirred for additional $2 \mathrm{~h}$. The mixture was filtered through a pad of aluminum oxide, and was washed with ethyl acetate $(50 \mathrm{~mL})$. The filtrate was concentrated and the crude residue was purified by flash chromatography (silica gel, eluent: EtOAc:hexanes $=1: 5)$ to afford carbamate $67(363 \mathrm{mg}, 70 \%)$ as a colorless oil.

TLC (EtOAc:hexanes = 1:3): $\mathrm{R}_{f}=0.25$ (UV, anisaldehyde).

${ }^{1} \mathbf{H}$ NMR $\left(400 \mathrm{MHz}, \mathrm{CDCl}_{3}\right): \delta 6.27(\mathrm{~d}, J=15.6 \mathrm{~Hz}, 1 \mathrm{H}), 5.86(\mathrm{~d}, J=16.1 \mathrm{~Hz}, 1 \mathrm{H}), 5.37(\mathrm{ddd}, J=16.5,10.5,6.2$ $\mathrm{Hz}, 3 \mathrm{H}), 5.17(\mathrm{t}, J=7.3 \mathrm{~Hz}, 1 \mathrm{H}), 4.65(\mathrm{~s}, 1 \mathrm{H}), 4.55(\mathrm{br}, 1 \mathrm{H}), 4.49(\mathrm{td}, J=7.7,4.3 \mathrm{~Hz}, 1 \mathrm{H}), 4.38(\mathrm{q}, J=6.1,5.6 \mathrm{~Hz}$, $1 \mathrm{H}), 4.30(\mathrm{~d}, J=9.3 \mathrm{~Hz}, 1 \mathrm{H}), 2.48-2.34(\mathrm{~m}, 4 \mathrm{H}), 1.89(\mathrm{ddd}, J=14.3,10.2,4.2 \mathrm{~Hz}, 1 \mathrm{H}), 1.65(\mathrm{~s}, 3 \mathrm{H}), 1.61(\mathrm{~s}, 3 \mathrm{H})$, $1.61-1.56(\mathrm{~m}, 1 \mathrm{H}), 1.46(\mathrm{~s}, 3 \mathrm{H}), 1.02(\mathrm{~s}, 3 \mathrm{H}), 0.90(\mathrm{~m}, 21 \mathrm{H}), 0.07(\mathrm{~s}, 6 \mathrm{H}), 0.05(\mathrm{~s}, 3 \mathrm{H}), 0.04(\mathrm{~s}, 3 \mathrm{H})$.

${ }^{13}$ C NMR $\left(100 \mathrm{MHz}, \mathrm{CDCl}_{3}\right): \delta 174.9,156.1,136.4,135.3,134.3,128.8,128.7,128.1,127.3,82.0,77.9,72.8$, $70.8,46.5,39.1,37.8,37.6,36.9,29.8,25.92,25.88,22.4,18.9,18.3,18.2,13.1,12.7,-4.4,-4.59,-4.64,-4.8$.

HRMS-ESI m/z cacld for $\mathrm{C}_{36} \mathrm{H}_{63} \mathrm{NNaO}_{6} \mathrm{Si}_{2}[\mathrm{M}+\mathrm{Na}]^{+}$684.4086, found 684.4099. 


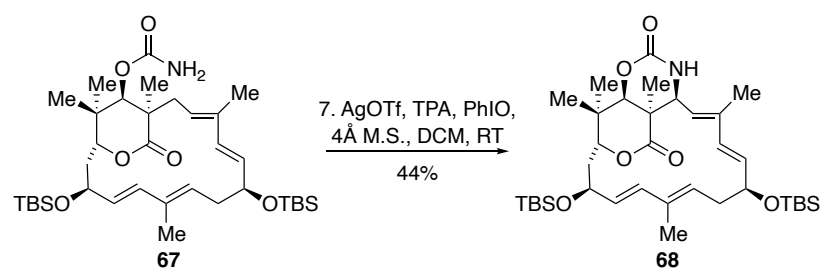

A 100-mL round-bottom flask containing AgOTf (11.60 mg, $0.045 \mathrm{mmol}, 0.10$ equiv) and TPA (19.70 mg, 0.068 mmol, 0.15 equiv) was evacuated and flushed with nitrogen (this process was repeated a total of 3 times) and sealed with a rubber septum. DCM $(20 \mathrm{~mL})$ was added, and the resulting solution was stirred at room temperature for 30

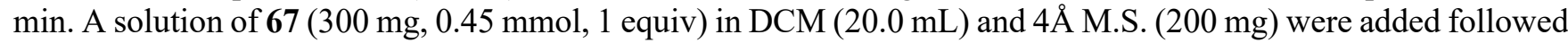
by PhIO (229 mg, $1.04 \mathrm{mmol}, 2.30$ equiv). After stirring for $16 \mathrm{~h}$, the mixture was filtered through a pad of celite and the filtrate was concentrated. The crude residue was purified by flash chromatography (silica gel, eluent: EtOAc:hexanes $=1: 5)$ to afford carbamate $\mathbf{6 8}(132 \mathrm{mg}, 44 \%)$ as a colorless oil.

TLC (EtOAc:hexanes = 1:2): $\mathrm{R}_{f}=0.29(\mathrm{UV}$, anisaldehyde).

${ }^{1} \mathbf{H}$ NMR $\left(400 \mathrm{MHz}, \mathrm{CDCl}_{3}\right): \delta 6.25(\mathrm{dd}, J=15.6,0.8 \mathrm{~Hz}, 1 \mathrm{H}), 5.91(\mathrm{~d}, J=15.7 \mathrm{~Hz}, 1 \mathrm{H}), 5.56(\mathrm{dd}, J=12.0,3.7$ $\mathrm{Hz}, 1 \mathrm{H}), 5.52(\mathrm{dd}, J=11.8,3.7 \mathrm{~Hz}, 1 \mathrm{H}), 5.37(\mathrm{t}, J=7.7 \mathrm{~Hz}, 1 \mathrm{H}), 5.24(\mathrm{~d}, J=4.3 \mathrm{~Hz}, 1 \mathrm{H}), 5.17(\mathrm{~d}, J=10.4 \mathrm{~Hz}$, $1 \mathrm{H}), 4.57-4.48(\mathrm{~m}, 3 \mathrm{H}), 4.02(\mathrm{dd}, J=10.4,4.3 \mathrm{~Hz}, 1 \mathrm{H}), 3.72(\mathrm{~s}, 1 \mathrm{H}), 2.39(\mathrm{dt}, J=12.9,6.3 \mathrm{~Hz}, 1 \mathrm{H}), 2.30(\mathrm{ddd}, J$ $=13.5,9.1,2.9 \mathrm{~Hz}, 1 \mathrm{H}), 1.87(\mathrm{ddd}, J=12.9,9.2,3.6 \mathrm{~Hz}, 1 \mathrm{H}), 1.77(\mathrm{ddd}, J=14.3,5.8,1.0 \mathrm{~Hz}, 1 \mathrm{H}), 1.66(\mathrm{~d}, J=$ $1.1 \mathrm{~Hz}, 3 \mathrm{H}), 1.62(\mathrm{~s}, 3 \mathrm{H}), 1.56(\mathrm{~s}, 3 \mathrm{H}), 1.14(\mathrm{~s}, 3 \mathrm{H}), 0.97(\mathrm{~s}, 3 \mathrm{H}), 0.92(\mathrm{~s}, 9 \mathrm{H}), 0.90(\mathrm{~s}, 9 \mathrm{H}), 0.07(\mathrm{~s}, 3 \mathrm{H}), 0.07(\mathrm{~s}$, $3 \mathrm{H}), 0.06(\mathrm{~s}, 3 \mathrm{H}), 0.01(\mathrm{~s}, 3 \mathrm{H})$.

${ }^{13}$ C NMR (100 MHz, $\left.\mathrm{CDCl}_{3}\right): \delta 171.2,157.5,138.6,135.2,134.3,134.1,131.1,127.7,126.7,124.9,87.4,76.8$, $70.9,69.5,59.2,48.5,38.0,37.1,34.9,28.0,25.91,25.90,22.6,18.8,18.3,18.2,13.2,12.9,-4.6,-4.7,-4.9,-5.0$.

HRMS-ESI m/z cacld for $\mathrm{C}_{36} \mathrm{H}_{61} \mathrm{NNaO}_{6} \mathrm{Si}_{2}[\mathrm{M}+\mathrm{Na}]^{+}$682.3930, found 682.3923 .
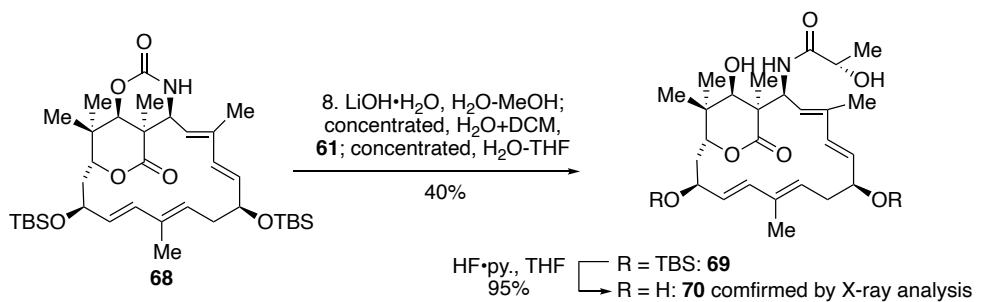

A 25-mL round-bottom flask containing $68\left(45.00 \mathrm{mg}, 0.068 \mathrm{mmol}, 1\right.$ equiv) and $\mathrm{LiOH} \cdot \mathrm{H}_{2} \mathrm{O}(675 \mathrm{mg}, 16.10 \mathrm{mmol}$, 236 equiv) equipped with a reflux condenser was evacuated and flushed with nitrogen (this process was repeated a total of 3 times) and was then sealed with a rubber septum. $\mathrm{H}_{2} \mathrm{O}(9.00 \mathrm{~mL})$ and $\mathrm{MeOH}(13.50 \mathrm{~mL})$ were added. The mixture was warmed to $68{ }^{\circ} \mathrm{C}$ by means of oil bath. After stirring for $12 \mathrm{~h}$, the oil bath was removed, and the mixture was cooled to room temperature. the mixture was concentrated, and then the residue was dissolved with $\mathrm{H}_{2} \mathrm{O}(3.00 \mathrm{~mL})$ and DCM $(9.00 \mathrm{~mL})$. The resulting biphasic mixture was vigorously stirred and acyl chloride 61 $(2 \times 50 \mu \mathrm{L})$ was added dropwise. The mixture was stirred for additional $10 \mathrm{~min}$, and then the mixture was concentrated. THF $(3.00 \mathrm{~mL})$ was added into the aqueous solution. After stirring for $2 \mathrm{~h}$, the mixture was transferred to a separatory funnel, and was extracted with ethyl acetate $(3 \times 10 \mathrm{~mL})$. The combined organic layers were washed brine $(10 \mathrm{~mL})$ and the washed solution was dried $\left(\mathrm{Na}_{2} \mathrm{SO}_{4}\right)$. The dried solution was filtered and the filtrate was concentrated. The crude residue was purified by flash chromatography (silica gel, eluent: EtOAc:hexanes $=1: 1$ to $100 \%$ EtOAc) to afford carbamate $\mathbf{6 9}(18.7 \mathrm{mg}, 40 \%)$ as a colorless oil.

TLC (EtOAc: $\mathrm{MeOH}=10: 1): \mathrm{R}_{f}=0.28$ (UV, anisaldehyde).

${ }^{1} \mathbf{H}$ NMR (400 MHz, $\left.\mathrm{CDCl}_{3}\right): \delta 7.62(\mathrm{~d}, J=10.4 \mathrm{~Hz}, 1 \mathrm{H}), 6.25(\mathrm{~d}, J=15.4 \mathrm{~Hz}, 1 \mathrm{H}), 5.93(\mathrm{~d}, J=15.7 \mathrm{~Hz}, 1 \mathrm{H}), 5.64$ $(\mathrm{d}, J=10.0 \mathrm{~Hz}, 1 \mathrm{H}), 5.50(\mathrm{dd}, J=13.4,4.7 \mathrm{~Hz}, 1 \mathrm{H}), 5.46(\mathrm{dd}, J=13.2,4.5 \mathrm{~Hz}, 1 \mathrm{H}), 5.36(\mathrm{t}, J=7.7 \mathrm{~Hz}, 1 \mathrm{H}), 5.17$ $(\mathrm{t}, J=10.2 \mathrm{~Hz}, 1 \mathrm{H}), 4.57-4.49(\mathrm{~m}, 3 \mathrm{H}), 4.23(\mathrm{q}, J=6.7 \mathrm{~Hz}, 1 \mathrm{H}), 3.52-3.47(\mathrm{~m}, 2 \mathrm{H}), 2.42(\mathrm{dd}, J=13.7,6.6 \mathrm{~Hz}$, $1 \mathrm{H}), 2.34(\mathrm{ddt}, J=13.2,8.5,5.0 \mathrm{~Hz}, 1 \mathrm{H}), 1.83(\mathrm{ddd}, J=13.2,9.3,3.4 \mathrm{~Hz}, 1 \mathrm{H}), 1.71(\mathrm{~s}, 3 \mathrm{H}), 1.67(\mathrm{dd}, J=14.3,6.2$ $\mathrm{Hz}, 1 \mathrm{H}), 1.59(\mathrm{~s}, 3 \mathrm{H}), 1.39(\mathrm{~s}, 3 \mathrm{H}), 1.37(\mathrm{~d}, J=6.7 \mathrm{~Hz}, 3 \mathrm{H}), 0.99(\mathrm{~s}, 3 \mathrm{H}), 0.92(\mathrm{~s}, 3 \mathrm{H}), 0.90(\mathrm{~s}, 18 \mathrm{H}), 0.07(\mathrm{~s}, 3 \mathrm{H})$, $0.05(\mathrm{~s}, 3 \mathrm{H}), 0.04(\mathrm{~s}, 3 \mathrm{H}), 0.03(\mathrm{~s}, 3 \mathrm{H})$. 
${ }^{13}$ C NMR (100 MHz, $\left.\mathrm{CDCl}_{3}\right): \delta 174.4,174.3,137.4,135.2,134.8,134.7,129.3,128.0,127.3,80.0,76.2,71.1$, $70.0,68.2,55.5,50.8,38.3,37.9,35.1,27.7,25.9,22.9,21.3,19.1,18.3,12.92,12.87,-4.7,-4.8,-4.9$.

HRMS-ESI m/z cacld for $\mathrm{C}_{38} \mathrm{H}_{68} \mathrm{NO}_{7} \mathrm{Si}_{2}[\mathrm{M}+\mathrm{H}]^{+}$706.4529, found 706.4509.

A 50-mL plastic vial containing $69\left(8.00 \mathrm{mg}, 0.011 \mathrm{mmol}, 1\right.$ equiv) and THF $(3.00 \mathrm{~mL})$ was cooled to $0{ }^{\circ} \mathrm{C}$ by means of an ice-water bath. Py $\bullet(\mathrm{HF})_{\mathrm{x}}(\sim 70 \% \mathrm{HF})(0.300 \mathrm{~mL})$ was added dropwise, and the vessel was removed from the cooling bath and the system was allowed to warm to $23{ }^{\circ} \mathrm{C}$. After stirring for $18 \mathrm{~h}, \mathrm{NaHCO}_{3}$ solid (450 $\mathrm{mg}$ ) was added and the mixture was vigorously stirred. After stirring for $30 \mathrm{~min}$, the mixture was filtered through a pad of silica gel and the filtrate was concentrated. The crude residue was purified by flash chromatography (silica gel, eluent: ethyl acetate: $\mathrm{MeOH}=10: 1)$ to afford tetra-ol $70(5.10 \mathrm{mg}, 95 \%)$ as a white solid.

m. p. $204-206^{\circ} \mathrm{C}(\mathrm{DCM})$

TLC (EtOAc: $\mathrm{MeOH}=10: 1): \mathrm{R}_{f}=0.28$ (UV, anisaldehyde).

${ }^{1} \mathbf{H}$ NMR $\left(400 \mathrm{MHz}, \mathrm{CDCl}_{3}\right): \delta 6.30(\mathrm{~d}, J=15.6 \mathrm{~Hz}, 1 \mathrm{H}), 5.97(\mathrm{~d}, J=16.1 \mathrm{~Hz}, 1 \mathrm{H}), 5.76(\mathrm{~d}, J=10.0 \mathrm{~Hz}, 1 \mathrm{H}), 5.63$ $(\mathrm{dd}, J=16.0,4.8 \mathrm{~Hz}, 1 \mathrm{H}), 5.54(\mathrm{dd}, J=15.6,5.8 \mathrm{~Hz}, 1 \mathrm{H}), 5.36(\mathrm{t}, J=7.7 \mathrm{~Hz}, 1 \mathrm{H}), 5.11(\mathrm{~d}, J=10.0 \mathrm{~Hz}, 1 \mathrm{H}), 4.60$ $(\mathrm{dd}, J=9.9,1.3 \mathrm{~Hz}, 1 \mathrm{H}), 4.52-4.42(\mathrm{~m}, 2 \mathrm{H}), 4.15(\mathrm{q}, J=6.8 \mathrm{~Hz}, 1 \mathrm{H}), 3.48(\mathrm{~s}, 1 \mathrm{H}), 2.54-2.40(\mathrm{~m}, 2 \mathrm{H}), 1.96-$ $1.86(\mathrm{~m}, 1 \mathrm{H}), 1.78-1.72(\mathrm{~m}, 1 \mathrm{H}), 1.72(\mathrm{~d}, J=1.2 \mathrm{~Hz}, 3 \mathrm{H}), 1.67(\mathrm{~s}, 3 \mathrm{H}), 1.39(\mathrm{~s}, 3 \mathrm{H}), 1.29(\mathrm{~d}, J=6.8 \mathrm{~Hz}, 3 \mathrm{H})$, $0.99(\mathrm{~s}, 3 \mathrm{H}), 0.94(\mathrm{~s}, 3 \mathrm{H})$.

${ }^{13}$ C NMR (100 MHz, $\left.\mathrm{CDCl}_{3}\right): \delta 177.1,177.0,138.2,136.6,136.5,136.1,130.5,130.1,128.7,128.4,80.4,78.5$, $71.3,70.4,69.1,56.5,52.0,39.7,37.9,34.9,28.1,23.4,21.1,19.4,13.2,13.0$.

HRMS-ESI m/z cacld for $\mathrm{C}_{26} \mathrm{H}_{39} \mathrm{NNaO}_{7}[\mathrm{M}+\mathrm{Na}]^{+} 500.2619$, found 500.2611.

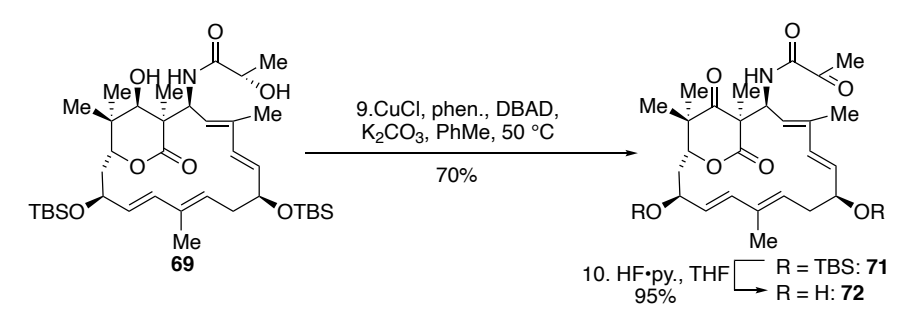

A 25-mL round-bottom flask containing 69 (15.00 mg, $0.021 \mathrm{mmol}, 1$ equiv), $\mathrm{Cu}(\mathrm{I}) \mathrm{Cl}(0.63 \mathrm{mg}, 0.0064 \mathrm{mmol}$, 0.30 equiv), 1,10 -phenanthroline ( $1.15 \mathrm{mg}, 0.0064 \mathrm{mmol}, 0.30$ equiv), DBAD ( $1.47 \mathrm{mg}, 0.0064 \mathrm{mmol}, 0.30$ equiv) and $\mathrm{K}_{2} \mathrm{CO}_{3}$ (11.80 mg, $0.085 \mathrm{mmol}, 4.00$ equiv) was evacuated and flushed with oxygen (this process was repeated a total of 3 times) and was then sealed with a rubber septum. PhMe $(5.00 \mathrm{~mL})$ was added. The resulting solution was warmed to $50{ }^{\circ} \mathrm{C}$ by means of an oil bath. After stirring for $3 \mathrm{~h}$, the mixture was purified directly by flash chromatography (silica gel, eluent: EtOAc:hexanes $=1: 10$ to $1: 5)$ to afford aldehyde $71(10.50 \mathrm{mg}, 70 \%)$ as a pale yellow oil.

TLC (EtOAc:hexanes = 1:4): $\mathrm{R}_{f}=0.27$ (UV, anisaldehyde).

${ }^{1} \mathbf{H}$ NMR (400 MHz, $\left.\mathrm{CDCl}_{3}\right): \delta 8.35(\mathrm{~d}, J=10.1 \mathrm{~Hz}, 1 \mathrm{H}), 6.18(\mathrm{~d}, J=15.5 \mathrm{~Hz}, 1 \mathrm{H}), 5.87(\mathrm{~d}, J=15.6 \mathrm{~Hz}, 1 \mathrm{H}), 5.61$ $(\mathrm{dd}, J=15.4,3.4 \mathrm{~Hz}, 1 \mathrm{H}), 5.37(\mathrm{dd}, J=15.5,5.9 \mathrm{~Hz}, 1 \mathrm{H}), 5.31-5.24(\mathrm{~m}, 2 \mathrm{H}), 4.83(\mathrm{~d}, J=10.6 \mathrm{~Hz}, 1 \mathrm{H}), 4.63-$ $4.58(\mathrm{~m}, 1 \mathrm{H}), 4.54-4.47(\mathrm{~m}, 1 \mathrm{H}), 3.95(\mathrm{dd}, J=9.1,1.4 \mathrm{~Hz}, 1 \mathrm{H}), 2.47(\mathrm{~s}, 3 \mathrm{H}), 2.45-2.39(\mathrm{~m}, 1 \mathrm{H}), 2.27(\mathrm{ddd}, J=$ 13.2, 8.8, 3.4 Hz, 1H), $2.01(\mathrm{ddd}, J=13.5,9.2,3.8 \mathrm{~Hz}, 1 \mathrm{H}), 1.83(\mathrm{~s}, 3 \mathrm{H}), 1.81-1.73(\mathrm{~m}, 1 \mathrm{H}), 1.60(\mathrm{~s}, 3 \mathrm{H}), 1.52$ $(\mathrm{s}, 3 \mathrm{H}), 1.19(\mathrm{~s}, 3 \mathrm{H}), 1.11(\mathrm{~s}, 3 \mathrm{H}), 0.89(\mathrm{~s}, 18 \mathrm{H}), 0.06(\mathrm{~s}, 3 \mathrm{H}), 0.05(\mathrm{~s}, 3 \mathrm{H}), 0.04(\mathrm{~s}, 3 \mathrm{H}),-0.01(\mathrm{~s}, 3 \mathrm{H})$.

${ }^{13} \mathbf{C}$ NMR (100 MHz, $\left.\mathrm{CDCl}_{3}\right): \delta 214.1,196.7,172.1,159.5,139.8,136.6,134.9,132.3,128.0,126.3,122.8,77.2$, $71.1,70.1,56.6,54.2,47.2,37.8,34.8,25.90,25.88,24.9,24.6,19.2,18.5,18.3,18.2,13.4,12.9,-4.5,-4.7,-4.8,-$ 5.0 .

HRMS-ESI $\mathrm{m} / \mathrm{z}$ cacld for $\mathrm{C}_{38} \mathrm{H}_{63} \mathrm{NNaO}_{7} \mathrm{Si}_{2}[\mathrm{M}+\mathrm{Na}]^{+}$724.4035, found 724.4046.

A 50-mL plastic vial containing $71\left(10.00 \mathrm{mg}, 0.014 \mathrm{mmol}, 1\right.$ equiv) and THF $(2 \mathrm{~mL})$ was cooled to $0{ }^{\circ} \mathrm{C}$ by means of an ice-water bath. Py $\bullet(\mathrm{HF})_{\mathrm{x}}(\sim 70 \% \mathrm{HF})(0.20 \mathrm{~mL})$ was added dropwise, and the vessel was removed from the cooling bath and the system was allowed to warm to $23{ }^{\circ} \mathrm{C}$. After stirring for $18 \mathrm{~h}, \mathrm{NaHCO}_{3}$ solid $(1.00 \mathrm{~g})$ was added and the mixture was vigorously stirred. After stirring for $30 \mathrm{~min}$, the mixture was filtered through a pad of 
silica gel and the filtrate was concentrated. The crude residue was purified by flash chromatography (silica gel, eluent: ethyl acetate:hexanes $=2: 1)$ to afford $72(6.40 \mathrm{mg}, 95 \%)$ as a colorless oil.

TLC (EtOAc:hexanes $=3: 2): \mathrm{R}_{f}=0.25$ (UV, anisaldehyde).

${ }^{1}$ H NMR (400 MHz, $\left.\mathrm{CDCl}_{3}\right): \delta 8.37(\mathrm{~d}, J=9.8 \mathrm{~Hz}, 1 \mathrm{H}), 6.24(\mathrm{~d}, J=15.6 \mathrm{~Hz}, 1 \mathrm{H}), 5.90(\mathrm{~d}, J=15.9 \mathrm{~Hz}, 1 \mathrm{H}), 5.74$ $(\mathrm{dd}, J=15.8,3.3 \mathrm{~Hz}, 1 \mathrm{H}), 5.48(\mathrm{dd}, J=15.6,6.5 \mathrm{~Hz}, 1 \mathrm{H}), 5.33(\mathrm{t}, J=8.3 \mathrm{~Hz}, 1 \mathrm{H}), 5.25(\mathrm{t}, J=10.3 \mathrm{~Hz}, 1 \mathrm{H}), 4.91$ $(\mathrm{d}, J=10.6 \mathrm{~Hz}, 1 \mathrm{H}), 4.68(\mathrm{br}, 1 \mathrm{H}), 4.60-4.53(\mathrm{~m}, 1 \mathrm{H}), 3.96(\mathrm{dd}, J=10.1,1.9 \mathrm{~Hz}, 1 \mathrm{H}), 2.59$ (ddd, $J=12.2,8.6$, $3.1 \mathrm{~Hz}, 1 \mathrm{H}), 2.46$ (s, 3H), 2.37 (ddd, $J=12.8,8.1,3.6 \mathrm{~Hz}, 1 \mathrm{H}), 2.17$ (ddd, $J=14.1,10.2,4.1 \mathrm{~Hz}, 1 \mathrm{H}), 1.86(\mathrm{~s}, 3 \mathrm{H})$, 1.80-1.72 (m, 1H), $1.68(\mathrm{~s}, 3 \mathrm{H}), 1.53(\mathrm{~s}, 3 \mathrm{H}), 1.21(\mathrm{~s}, 3 \mathrm{H}), 1.14(\mathrm{~s}, 3 \mathrm{H})$.

${ }^{13}$ C NMR $\left(100 \mathrm{MHz}, \mathrm{CDCl}_{3}\right): \delta 213.6,196.5,172.0,159.5,139.5,137.0,135.1,132.2,132.1,127.8,126.4,123.8$, $77.1,70.7,69.5,56.6,54.2,47.2,36.1,33.7,24.8,24.6,19.0,18.3,13.4,12.8$.

HRMS-ESI m/z cacld for $\mathrm{C}_{26} \mathrm{H}_{36} \mathrm{NO}_{7}[\mathrm{M}+\mathrm{H}]^{+} 474.2486$, found 474.2491. 


\section{Optimization for Marko's oxidation of compound 63}

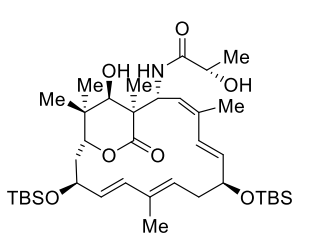

63

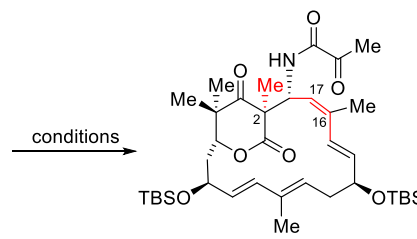

64

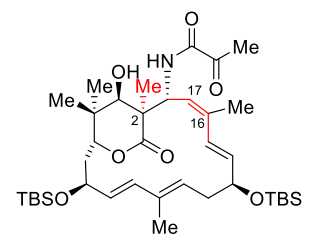

$64^{\prime}$

\begin{tabular}{|c|c|c|}
\hline entry & condition & Result \\
\hline 1 & DMP, DCM, RT & 64 \\
\hline 2 & DMP, wet DCM, RT & $63+64$ \\
\hline 3 & IBX, EtOAc, $80^{\circ} \mathrm{C}$ & complex mixture \\
\hline 4 & IBX, DMSO, RT & $64 '$ \\
\hline 5 & IBX, DMSO, $50{ }^{\circ} \mathrm{C}$ & 64 ' \\
\hline 6 & IBX, DMSO, $80^{\circ} \mathrm{C}$ & complex mixture \\
\hline 7 & 2-IBA, Oxone, $\mathrm{ACN}: \mathrm{H}_{2} \mathrm{O}(2: 1), 70{ }^{\circ} \mathrm{C}$ & complex mixture \\
\hline 8 & $\mathrm{SO}_{3} \bullet$ py., DMSO, Et 3 N, DCM, RT & NR \\
\hline 9 & TFAA, DMSO, $\mathrm{Et}_{3} \mathrm{~N}, \mathrm{DCM},-78^{\circ} \mathrm{C}$ & NR \\
\hline 10 & Burgess reagent, DMSO, RT & NR \\
\hline 11 & PCC, AcONa, DCM, RT & complex mixture \\
\hline 12 & PDC, $4 \AA \AA$ M.S., DCM, RT & complex mixture \\
\hline 13 & $\mathrm{Pr}_{4 \mathrm{NRuO}}, \mathrm{NMO}, 4 \AA$ M.S., DCM, RT & complex mixture \\
\hline 14 & $\mathrm{RuCl}_{2}\left(\mathrm{PPh}_{3}\right)_{3}, \mathrm{PhIO}, \mathrm{DCM}, \mathrm{RT}$ & complex mixture \\
\hline 15 & $\mathrm{RuCl}_{2}\left(\mathrm{PPh}_{3}\right)_{3}, \mathrm{~K}_{2} \mathrm{CO}_{3}, \mathrm{PhH}, \mathrm{RT}$ & NR \\
\hline 16 & TCICA, py., acetone, RT & complex mixture \\
\hline 17 & TCICA, py., acetone, RT & complex mixture \\
\hline 18 & $\begin{array}{c}\text { TEMPO, } \mathrm{NaClO}, \mathrm{NaBr}^{\mathrm{Bu}} \mathrm{Bu}_{4} \mathrm{NBr}, \mathrm{DCM}- \\
\text { sat. } \mathrm{NaHCO}_{3} \text { aq, RT }\end{array}$ & complex mixture \\
\hline 19 & $\mathrm{NaClO}, \mathrm{AcOH}, \mathrm{RT}$ & complex mixture \\
\hline 20 & $\mathrm{Cu}(\mathrm{I}) \mathrm{Cl}$, phen., DEAD, $\mathrm{K}_{2} \mathrm{CO}_{3}, \mathrm{O}_{2}, \mathrm{PhMe}, 70^{\circ} \mathrm{C}$ & $64(50 \%)$ \\
\hline
\end{tabular}


Scheme 3. Synthesis of the reported structure of iso-lankacidinol
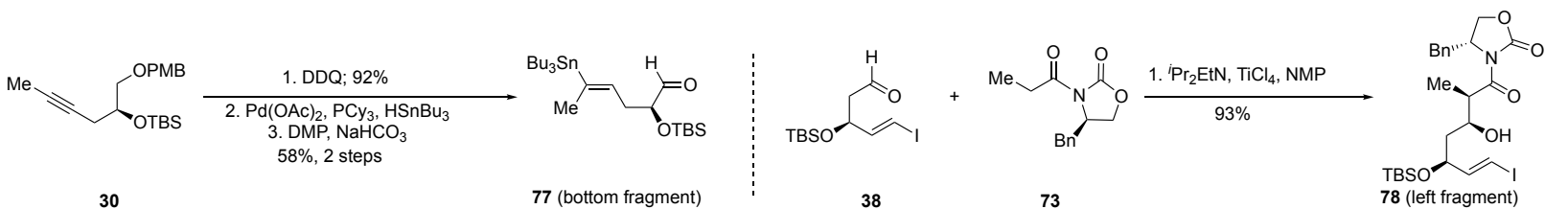

30

77 (bottom fragment)

38

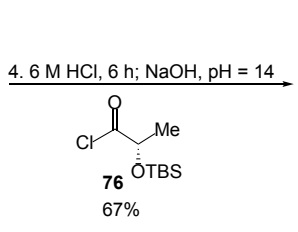

TBSO,
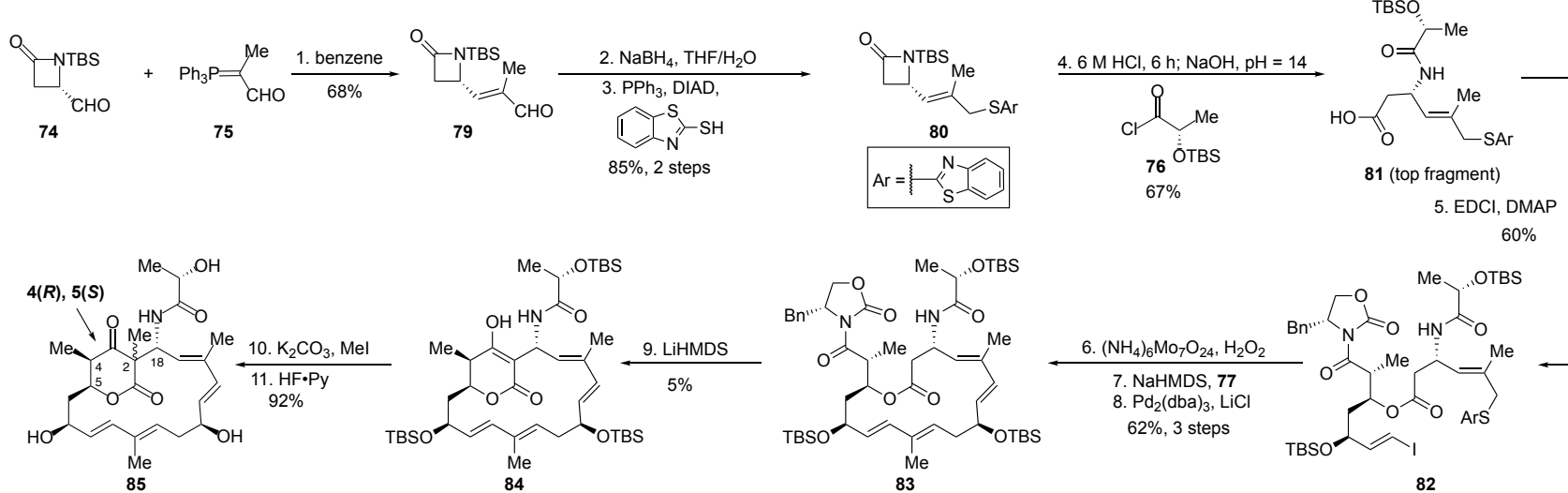

spectroscopic data does not match reported data

83

82 


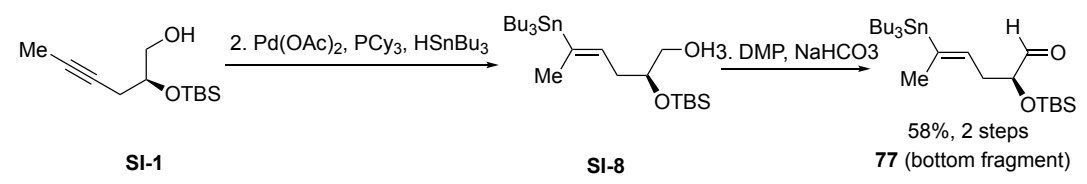

A $250-\mathrm{mL}$ round-bottom flask containing $\mathrm{Pd}(\mathrm{OAc})_{2}(491 \mathrm{mg}, 2.19 \mathrm{mmol}, 0.05$ equiv) and tricyclohexylphosphine ( $1.23 \mathrm{~g}, 4.38 \mathrm{mmol}, 0.10$ equiv) was evacuated and flushed with nitrogen (this process was repeated a total of 3 times). THF $(100 \mathrm{~mL})$ was added at room temperature. After stirring for $30 \mathrm{~min}$, a solution of starting material SI$1\left(10.0 \mathrm{~g}, 43.8 \mathrm{mmol}, 1\right.$ equiv) in THF $(25 \mathrm{~mL})$ was added, followed by a solution of $\mathrm{HSnBu}_{3}(23.4 \mathrm{~mL}, 87.6 \mathrm{mmol}$, 2.0 equiv) in THF $(25 \mathrm{~mL})$ was added via syringe pump over $2 \mathrm{~h}$. After finishing addition, The mixture was concentrated, and the crude residue was purified by flash chromatography (silica gel, eluent: EtOAc:hexanes = 1:30) to afford vinyltin SI-8 (14.51 g, 64\%) as a colorless liquid.

TLC $($ EtOAc:hexanes $=1: 15): \mathrm{R}_{f}=0.50\left(\mathrm{KMnO}_{4}\right)$.

${ }^{1} \mathbf{H}$ NMR $\left(400 \mathrm{MHz}, \mathrm{CDCl}_{3}\right): \delta 5.56(\mathrm{ttd}, J=34.5,7.2,2.3 \mathrm{~Hz}, 1 \mathrm{H}), 3.84-3.78(\mathrm{~m}, 1 \mathrm{H}), 3.57(\mathrm{ddd}, J=11.5,7.0$, $3.8 \mathrm{~Hz}, 1 \mathrm{H}), 3.45(\mathrm{dt}, J=11.0,6.0 \mathrm{~Hz}, 1 \mathrm{H}), 2.38-2.34(\mathrm{~m}, 2 \mathrm{H}), 1.86-1.85(\mathrm{~m}, 3 \mathrm{H}), 1.52-1.46(\mathrm{~m}, 6 \mathrm{H}), 1.35-$ $1.29(\mathrm{~m}, 6 \mathrm{H}), 0.93-0.86(\mathrm{~m}, 24 \mathrm{H}), 0.125(\mathrm{~s}, 3 \mathrm{H}), 0.122(\mathrm{~s}, 3 \mathrm{H})$.

${ }^{13}$ C NMR $\left(100 \mathrm{MHz}, \mathrm{CDCl}_{3}\right): \delta 141.0,135.5,72.8,66.1,32.8,29.27,29.13,27.3,19.3,18.0,13.7,9.0,-4.3,-4.6$.

HRMS-ESI The compound failed to ionize under electrospray in both positive and negative modes.

A 100-mL round-bottom flask containing vinyltin $\mathbf{S I - 8}$ (3.00 g, $5.78 \mathrm{mmol}, 1$ equiv) and DCM (70 mL) was added sodium bicarbonate (970 mg, $11.6 \mathrm{mmol}, 2.0$ equiv.) and Dess-Martin periodinane (2.94 g, $6.93 \mathrm{mmol}, 1.2 \mathrm{equiv})$. The resulting mixture was stirred for 30 min and checked by TLC. After starting material was consumed, the mixture was concentrated, and the crude residue was purified by flash chromatography (silica gel, eluent: EtOAc:hexanes = 1:50) to afford aldehyde $77(2.73 \mathrm{~g}, 91 \%)$ as a colorless liquid.

TLC $($ EtOAc:hexanes $=1: 15): \mathrm{R}_{f}=0.80\left(\mathrm{KMnO}_{4}\right)$.

${ }^{1} \mathbf{H}$ NMR $\left(400 \mathrm{MHz}, \mathrm{CDCl}_{3}\right): \delta 9.62(\mathrm{~d}, J=1.7 \mathrm{~Hz}, 1 \mathrm{H}), 5.56(\mathrm{tdt}, J=34.5,7.2,2.3 \mathrm{~Hz}, 1 \mathrm{H}), 4.03(\mathrm{dt}, J=6.0,1.6$ $\mathrm{Hz}, 1 \mathrm{H}), 2.48-2.47(\mathrm{~m}, 2 \mathrm{H}), 1.86-1.85(\mathrm{~m}, 3 \mathrm{H}), 3.57(\mathrm{ddd}, J=11.5,7.0,3.8 \mathrm{~Hz}, 1 \mathrm{H}), 3.45(\mathrm{dt}, J=11.0,6.0 \mathrm{~Hz}$, $1 \mathrm{H}), 2.38-2.34(\mathrm{~m}, 2 \mathrm{H}), 1.86-1.85(\mathrm{~m}, 2 \mathrm{H}), 1.53-1.45(\mathrm{~m}, 6 \mathrm{H}), 1.36-1.27(\mathrm{~m}, 6 \mathrm{H}), 0.94-0.86(\mathrm{~m}, 24 \mathrm{H})$, $0.10(\mathrm{~s}, 3 \mathrm{H}), 0.09(\mathrm{~s}, 3 \mathrm{H})$.

${ }^{13}$ C NMR (100 MHz, $\left.\mathrm{CDCl}_{3}\right): \delta 203.8,142.0,133.8,77.6,31.4,29.1,27.3,25.7,19.3,18.1,13.6,9.0,-4.6,-4.8$.

HRMS-ESI $\mathrm{m} / \mathrm{z}$ cacld for $\mathrm{C}_{24} \mathrm{H}_{51} \mathrm{O}_{2} \mathrm{SiSn}[\mathrm{M}+\mathrm{H}]^{+}$519.2680, found 519.2681.

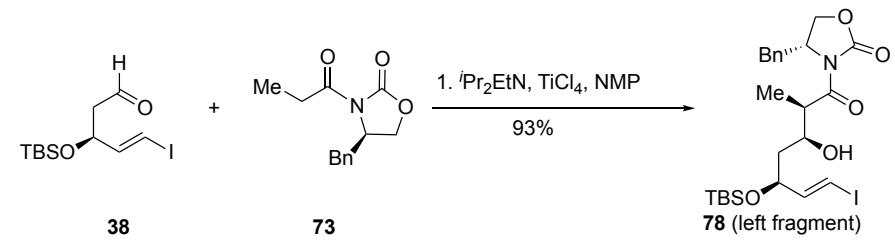

A 250-mL round-bottom flask containing imide 73 (4.90 g, $21.0 \mathrm{mmol}, 1.1$ equiv.) in $70 \mathrm{~mL}$ DCM was cooled to $0{ }^{\circ} \mathrm{C}$ by means of an ice-water bath. $\mathrm{TiCl}_{4}(1.0 \mathrm{M}$ solution in DCM, $22.9 \mathrm{~mL}, 22.9 \mathrm{mmol}, 1.2$ equiv. $)$ was added dropwise at $0{ }^{\circ} \mathrm{C}$, resulting in an orange solution. After $15 \mathrm{~min}$, diisopropylethylamine $(4.16 \mathrm{~mL}, 23.9 \mathrm{mmol}, 1.2$ equiv.) was added dropwise. The resulting deep red reaction mixture was allowed to stir 40 min. 1-Methyl-2pyrrolidinone $(2.02 \mathrm{~mL}, 21.0 \mathrm{mmol}, 1.1$ equiv.) was added and the reaction mixture was stirred for an additional 10 min. Aldehyde 38 (6.50 g, 19.1 mmol, 1 equiv.) was added dropwise. After $2 \mathrm{~h}$, saturated aqueous $\mathrm{NH}_{4} \mathrm{Cl}$ solution $(50 \mathrm{~mL})$ was added in one portion. After thorough mixing, the biphasic mixture was transferred to a separatory funnel and the layers were separated. The aqueous layer was extracted with DCM $(2 \times 50 \mathrm{~mL})$. The combined organic layers were washed with water $(2 \times 20 \mathrm{~mL})$ and saturated aqueous sodium chloride solution $(20$ $\mathrm{mL})$ and the washed solution was dried $\left(\mathrm{Na}_{2} \mathrm{SO}_{4}\right)$. The dried solution was filtered and the filtrate was concentrated. The crude residue was purified by flash chromatography (silica gel, eluent: EtOAc:hexanes $=1: 5$ ) to afford amide $\mathbf{7 8}(10.2 \mathrm{~g}, 93 \%)$ as a colorless oil.

TLC (EtOAc:hexanes $=1: 5): \mathrm{R}_{f}=0.45(\mathrm{UV})$. 
${ }^{1} \mathbf{H}$ NMR $\left(400 \mathrm{MHz}, \mathrm{CDCl}_{3}\right) \delta 7.37-7.28(\mathrm{~m}, 3 \mathrm{H}), 7.23-7.21(\mathrm{~m}, 2 \mathrm{H}), 6.58(\mathrm{dd}, J=14.4,7.0 \mathrm{~Hz}, 1 \mathrm{H}), 6.31(\mathrm{~d}$, $J=14.4 \mathrm{~Hz}, 1 \mathrm{H}), 4.75-4.70(\mathrm{~m}, 1 \mathrm{H}), 4.46-4.42(\mathrm{~m}, 1 \mathrm{H}), 4.26-4.17(\mathrm{~m}, 3 \mathrm{H}), 3.76$ (dddd, $J=8.8,6.0,2.9,1.2$ $\mathrm{Hz}, 1 \mathrm{H}), 3.29-3.25(\mathrm{~m}, 2 \mathrm{H}), 2.80(\mathrm{dd}, J=14.0,9.3 \mathrm{~Hz}, 1 \mathrm{H}), 1.71(\mathrm{ddd}, J=13.4,8.8,3.5 \mathrm{~Hz}, 1 \mathrm{H}), 1.54(\mathrm{dd}, J=$ 14.0, 7.3 Hz, 1H), 1.27 (d, J=7.0 Hz, 3H), 0.89 (s, 9H), $0.11(\mathrm{~s}, 3 \mathrm{H}), 0.07$ (s, 3H).

${ }^{13}$ C NMR $\left(100 \mathrm{MHz}, \mathrm{CDCl}_{3}\right): \delta 176.8,153.1,148.5,135.0,129.4,128.9,127.4,76.3,72.6,67.9,66.1,55.1,42.6$, $40.6,37.8,25.8,18.1,11.2,-4.5,-5.0$.

HRMS-ESI m/z cacld for $\mathrm{C}_{24} \mathrm{H}_{36} \mathrm{INO}_{5} \mathrm{SiNa}[\mathrm{M}+\mathrm{Na}]^{+}$596.1305, found 596.1337.

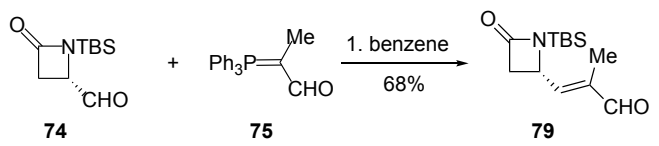

A 250-mL round-bottom flask containing lactam $74(2.00 \mathrm{~g}, 9.37 \mathrm{mmol}, 1$ equiv. $)$ and benzene $(200 \mathrm{~mL})$ was added ylide 75 (4.18 g, $13.1 \mathrm{mmol}, 1.4$ equiv.) in one portion. The resulting mixture was stirred for 48 . After starting material was consumed as indicated by TLC analysis, the solution was concentrated. The residue was purified by flash chromatography (silica gel, eluent: EtOAc:hexanes =1:3) to afford enal $79(1.43 \mathrm{~g}, 68 \%)$ as a white solid.

MP: $89^{\circ} \mathrm{C}$

TLC (EtOAc:hexanes $=1: 3): \mathrm{R}_{f}=0.18(\mathrm{UV})$.

${ }^{1} \mathbf{H}$ NMR $\left(400 \mathrm{MHz}, \mathrm{CDCl}_{3}\right): \delta 9.43(\mathrm{~s}, 1 \mathrm{H}), 6.43(\mathrm{dd}, J=9.7,1.4 \mathrm{~Hz}, 1 \mathrm{H}), 4.49(\mathrm{ddd}, J=8.5,5.7,2.7 \mathrm{~Hz}, 1 \mathrm{H})$, $3.43(\mathrm{dd}, J=15.3,5.7 \mathrm{~Hz}, 1 \mathrm{H}), 2.83(\mathrm{dd}, J=15.3,2.7 \mathrm{~Hz}, 1 \mathrm{H}), 1.79(\mathrm{~d}, J=1.4 \mathrm{~Hz}, 3 \mathrm{H}), 0.92(\mathrm{~s}, 9 \mathrm{H}), 0.17$ (s, $3 \mathrm{H}), 0.12(\mathrm{~s}, 3 \mathrm{H})$.

${ }^{13}$ C NMR (100 MHz, $\left.\mathrm{CDCl}_{3}\right): \delta 194.0,171.1,152.3,139.9,45.8,45.5,26.1,18.3,9.5,-5.7,-5.8$.

HRMS-ESI m/z cacld for $\mathrm{C}_{13} \mathrm{H}_{24} \mathrm{NO}_{2} \mathrm{Si}[\mathrm{M}+\mathrm{H}]^{+} 254.1576$, found 254.1581.
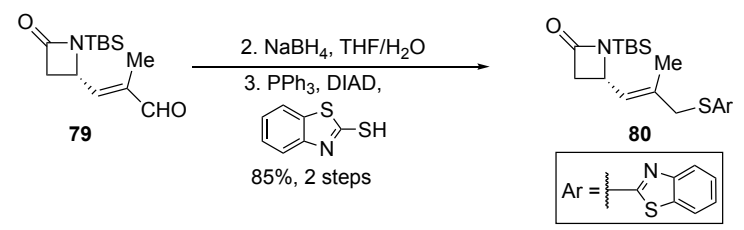

A $250-\mathrm{mL}$ round-bottom flask containing aldehyde $79(10.0 \mathrm{~g}, 39.5 \mathrm{mmol}, 1$ equiv) and THF (130 mL) was added $\mathrm{NaBH}_{4}(2.24 \mathrm{~g}, 59.15 \mathrm{mmol}, 1.5$ equiv. $)$ at $0{ }^{\circ} \mathrm{C}$ by means of an ice-water bath. Water $(8 \mathrm{~mL})$ was added dropwise. After starting material was consumed as indicated by TLC analysis, saturated aqueous $\mathrm{NH}_{4} \mathrm{Cl}$ solution $(50 \mathrm{~mL})$ was added in one portion. After thorough mixing, the mixture was transferred to a separatory funnel and was extracted with ethyl acetate $(3 \times 50 \mathrm{~mL})$. The combined organic layers were washed with saturated aqueous sodium chloride solution $(50 \mathrm{~mL})$ and the washed solution was dried $\left(\mathrm{Na}_{2} \mathrm{SO}_{4}\right)$. The dried solution was filtered, and the filtrate was concentrated. The resulting colorless oil was used directly for the next step without further purification.

A 500-mL round-bottom flask containing the crude allyl alcohol from the previous step (10.1g, $39.5 \mathrm{mmol}, 1$ equiv), benzo[d]thiazole-2-thiol (8.58 g, $51.3 \mathrm{mmol}, 1.3$ equiv) and $\mathrm{PPh}_{3}(15.5 \mathrm{~g}, 59.2 \mathrm{mmol}, 1.5$ equiv) was evacuated and flushed with nitrogen (this process was repeated a total of 3 times). DCM (200 mL) was added, and solution was cooled to $0{ }^{\circ} \mathrm{C}$ by means of an ice-water bath. DIAD $(11.7 \mathrm{~mL}, 59.2 \mathrm{mmol}, 1.5$ equiv) was added dropwise over $20 \mathrm{~min}$. The vessel was removed from the cooling bath and the system was allowed to warm to $23^{\circ} \mathrm{C}$. After 1 $\mathrm{h}$, the reaction mixture was concentrated. The resulting crude residue was purified by flash chromatography (silica gel, eluent: EtOAc:hexanes = 1:5) to afford thioether $\mathbf{8 0}(13.6 \mathrm{~g}, 85 \%$ for 2 steps $)$ as a colorless oil.

TLC (EtOAc:hexanes = 1:3): $\mathrm{R}_{f}=0.45(\mathrm{UV})$.

${ }^{1} \mathbf{H}$ NMR $\left(400 \mathrm{MHz}, \mathrm{CDCl}_{3}\right): \delta 7.86(\mathrm{~d}, J=8.2 \mathrm{~Hz}, 1 \mathrm{H}), 7.74(\mathrm{~d}, J=7.8 \mathrm{~Hz}, 1 \mathrm{H}), 7.42(\mathrm{td}, J=7.8,1.0 \mathrm{~Hz}, 1 \mathrm{H})$, $7.30(\mathrm{td}, J=7.8,1.0 \mathrm{~Hz}, 1 \mathrm{H}), 5.63(\mathrm{dd}, J=9.7,1.0 \mathrm{~Hz}, 1 \mathrm{H}), 4.24(\mathrm{ddd}, J=8.5,5.7,2.7 \mathrm{~Hz}, 1 \mathrm{H}), 4.04(\mathrm{~d}, J=13.8$ $\mathrm{Hz}, 1 \mathrm{H}), 3.99(\mathrm{~d}, J=13.8 \mathrm{~Hz}, 1 \mathrm{H}), 3.26(\mathrm{dd}, J=15.3,5.7 \mathrm{~Hz}, 1 \mathrm{H}), 2.65(\mathrm{dd}, J=15.3,2.7 \mathrm{~Hz}, 1 \mathrm{H}), 1.86(\mathrm{~d}, J=$ $1.4 \mathrm{~Hz}, 3 \mathrm{H}), 0.90(\mathrm{~s}, 9 \mathrm{H}), 0.11(\mathrm{~s}, 3 \mathrm{H}), 0.06(\mathrm{~s}, 3 \mathrm{H})$. 
${ }^{13}$ C NMR (100 MHz, $\left.\mathrm{CDCl}_{3}\right): \delta$ 172.2, 165.9, 153.0, 135.3, 133.1, 131.3, 126.1, 124.4, 121.6, 120.9, 46.2, 45.5, $41.9,26.2,18.2,15.8,9.5,-5.85,-5.87$.

HRMS-ESI m/z cacld for $\mathrm{C} 20 \mathrm{H} 29 \mathrm{~N}_{2} \mathrm{OS}_{2} \mathrm{Si}[\mathrm{M}+\mathrm{H}]^{+}$405.1491, found 405.1493.

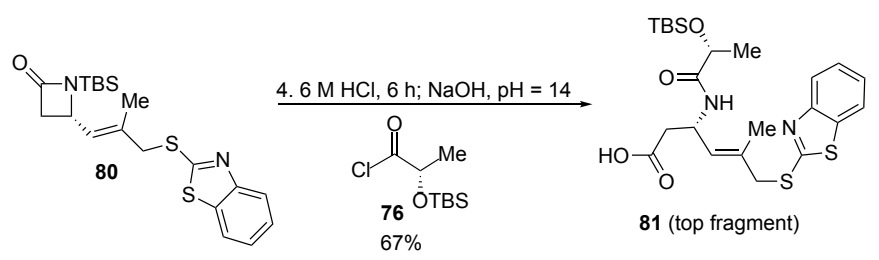

A $100-\mathrm{mL}$ round-bottom flask containing thioether $80(15.0 \mathrm{~g}, 37.1 \mathrm{mmol}, 1$ equiv.) was added $6.0 \mathrm{M}$ aquous hydrochloric acid solution $(15 \mathrm{~mL})$. The solution was stirred for $6 \mathrm{~h}$ at $23{ }^{\circ} \mathrm{C}$, and was then cooled to $0{ }^{\circ} \mathrm{C}$ by means of an ice-water bath. $\mathrm{NaOH}$ pellet was added until the reaction mixture reached $\mathrm{pH}=14$ as indicated by colormetric pH strips. Acyl chloride 76 (9.91 g, $44.5 \mathrm{mmol}, 1.5$ equiv.) was added in one portion. After $15 \mathrm{~min}, 6.0 \mathrm{M}$ aqueous hydrochloric acid solution was added until the $\mathrm{pH}$ reached 3 . The resulting mixture was transferred to a separatory funnel and was extracted with DCM $(3 \times 50 \mathrm{~mL})$. The combined organic layers were washed with saturated aqueous sodium chloride solution $(50 \mathrm{~mL})$ and the washed solution was dried $\left(\mathrm{Na}_{2} \mathrm{SO}_{4}\right)$. The dried solution was filtered, and the filtrate was concentrated. The crude residue was purified by flash chromatography (silica gel, eluent: EtOAc:hexanes $=1: 1)$ to afford acid $81(12.3 \mathrm{~g}, 67 \%)$ as a colorless oil.

TLC (EtOAc:hexanes $=1: 1): \mathrm{R}_{f}=0.50(\mathrm{UV})$.

${ }^{1}$ H NMR $\left(400 \mathrm{MHz}, \mathrm{CDCl}_{3}\right): \delta 9.6(\mathrm{~s}, 1 \mathrm{H}), 7.86(\mathrm{~d}, J=8.2 \mathrm{~Hz}, 1 \mathrm{H}), 7.74(\mathrm{~d}, J=7.8 \mathrm{~Hz}, 1 \mathrm{H}), 7.40(\mathrm{td}, J=7.8,1.0$ $\mathrm{Hz}, 1 \mathrm{H}), 7.36(\mathrm{~d}, J=7.8 \mathrm{~Hz}, 1 \mathrm{H}), 7.31-7.27(\mathrm{~m}, 1 \mathrm{H}), 5.69(\mathrm{~d}, J=9.7 \mathrm{~Hz}, 1 \mathrm{H}), 5.04-4.99(\mathrm{~m}, 1 \mathrm{H}), 4.19(\mathrm{q}, J=$ $6.8 \mathrm{~Hz}, 1 \mathrm{H}), 4.01(\mathrm{~d}, J=13.8 \mathrm{~Hz}, 1 \mathrm{H}), 3.96(\mathrm{~d}, J=13.8 \mathrm{~Hz}, 1 \mathrm{H}), 2.64(\mathrm{~d}, J=6.0 \mathrm{~Hz}, 1 \mathrm{H}), 1.98(\mathrm{~d}, J=1.4 \mathrm{~Hz}$, $3 \mathrm{H}), 1.35(\mathrm{~d}, J=6.8 \mathrm{~Hz}, 1 \mathrm{H}), 0.88(\mathrm{~s}, 9 \mathrm{H}), 0.06(\mathrm{~s}, 3 \mathrm{H}), 0.04(\mathrm{~s}, 3 \mathrm{H})$.

${ }^{13}$ C NMR (100 MHz, $\left.\mathrm{CDCl}_{3}\right): \delta 175.3,173.9,166.5,152.9,135.1,134.7,127.8,126.1,124.3,121.5,120.9,69.7$, $43.2,42.0,38.9,25.6,21.7,17.9,16.1,-4.7,-5.3$.

HRMS-ESI m/z cacld for $\mathrm{C}_{23} \mathrm{H}_{35} \mathrm{~N}_{2} \mathrm{O}_{4} \mathrm{~S}_{2} \mathrm{Si}[\mathrm{M}+\mathrm{H}]^{+} 495.1808$, found 495.1826 .

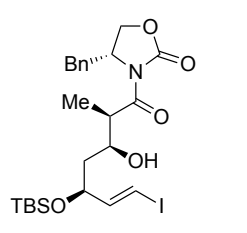

78
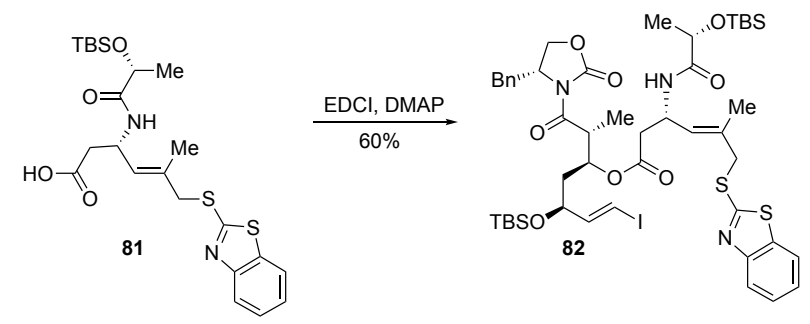

A 100-mL round-bottom flask containing acid 81 (2.80 g, $5.66 \mathrm{mmol}, 1$ equiv.) and alcohol 78 (3.90 g, $6.79 \mathrm{mmol}$, 1.2 equiv.) was added $50 \mathrm{~mL}$ chloroform. DMAP (3.46 g, $28.3 \mathrm{mmol}, 5$ equiv.) and EDCI (5.42 g, $28.3 \mathrm{mmol}, 5$ equiv.) was added in sequence. The reaction mixture was heated at $50{ }^{\circ} \mathrm{C}$ for $3 \mathrm{~h}$, and was then concentrated. The crude residue was purified by flash chromatography (silica gel, eluent: EtOAc:hexanes $=1: 3$ ) to afford ester 82 $(3.57 \mathrm{~g} 60 \%)$ as a colorless oil.

TLC (EtOAc:hexanes $=1: 3): \mathrm{R}_{f}=0.35(\mathrm{UV})$.

${ }^{1} \mathbf{H}$ NMR $\left(400 \mathrm{MHz}, \mathrm{CDCl}_{3}\right) \delta 7.87(\mathrm{~d}, J=8.4 \mathrm{~Hz}, 1 \mathrm{H}), 7.76(\mathrm{~d}, J=8.2 \mathrm{~Hz}, 1 \mathrm{H}), 7.43(\mathrm{td}, J=7.5,1.6 \mathrm{~Hz}, 1 \mathrm{H})$, $7.33-7.28(\mathrm{~m}, 3 \mathrm{H}), 7.25-7.17(\mathrm{~m}, 3 \mathrm{H}), 6.50(\mathrm{dd}, J=14.8,6.8 \mathrm{~Hz}, 1 \mathrm{H}), 6.30(\mathrm{~d}, J=14.4 \mathrm{~Hz}, 1 \mathrm{H}), 5.70(\mathrm{dd}, J=$ 9.5, $0.8 \mathrm{~Hz}, 1 \mathrm{H}), 5.40(\mathrm{dt}, J=9.5,2.8 \mathrm{~Hz}, 1 \mathrm{H}), 5.02-4.95(\mathrm{~m}, 1 \mathrm{H}), 4.59-4.53(\mathrm{~m}, 1 \mathrm{H}), 4.27(\mathrm{t}, J=8.9 \mathrm{~Hz}, 1 \mathrm{H})$, $4.21-4.14(\mathrm{~m}, 2 \mathrm{H}), 4.08-4.00(\mathrm{~m}, 4 \mathrm{H}), 3.21(\mathrm{dd}, J=14.0,3.5 \mathrm{~Hz}, 1 \mathrm{H}), 2.81(\mathrm{dd}, J=14.0,9.3 \mathrm{~Hz}, 1 \mathrm{H}), 2.66(\mathrm{dd}$, $J=16.5,5.0, \mathrm{~Hz}, 1 \mathrm{H}), 2.57(\mathrm{dd}, J=16.5,5.0, \mathrm{~Hz}, 1 \mathrm{H}), 1.90(\mathrm{~d}, J=1.4 \mathrm{~Hz}, 3 \mathrm{H}), 1.83-1.73(\mathrm{~m}, 2 \mathrm{H}), 1.38(\mathrm{~d}, J$ $=7.0 \mathrm{~Hz}, 3 \mathrm{H}), 1.20(\mathrm{~d}, J=7.0 \mathrm{~Hz}, 3 \mathrm{H}), 0.89(\mathrm{~s}, 9 \mathrm{H}), 0.86(\mathrm{~s}, 9 \mathrm{H}), 0.03(\mathrm{~s}, 6 \mathrm{H}), 0.01(\mathrm{~s}, 6 \mathrm{H})$. 
${ }^{13} \mathrm{C}$ NMR $\left(100 \mathrm{MHz}, \mathrm{CDCl}_{3}\right): \delta 173.5,173.4,170.6,166.4,153.5,153.1,148.5,135.29,135.25,134.7,129.4$, $128.9,127.9,127.2,126.0,124.3,121.5,120.9,72.1,70.7,70.0,66.2,55.8,43.1,41.9,41.3,40.2,38.9,37.8,25.76$, $25.71,21.9,18.07,18.03,16.2,10.1,-4.0,-4.8,-5.0,-5.3$.

HRMS-ESI $\mathrm{m} / \mathrm{z}$ cacld for $\mathrm{C}_{47} \mathrm{H}_{69} \mathrm{IN}_{3} \mathrm{O}_{8} \mathrm{~S}_{2} \mathrm{Si}_{2}[\mathrm{M}+\mathrm{H}]^{+} 1050.3109$, found 1050.3110 .

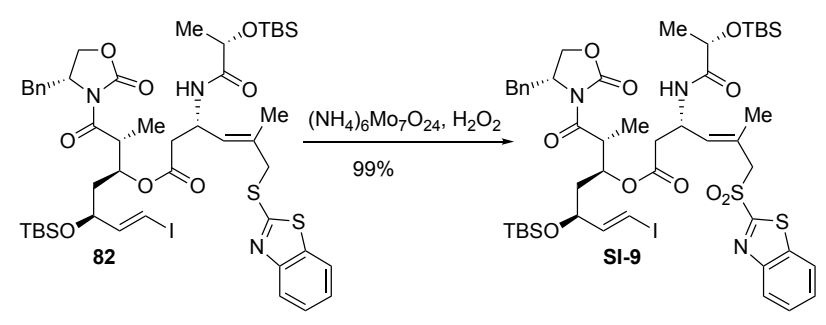

A $250-\mathrm{mL}$ round-bottom flask containing thioether $82(3.00 \mathrm{~g}, 2.86 \mathrm{mmol}, 1$ equiv.), ethanol $(50 \mathrm{~mL})$, and acetonitrile $(50 \mathrm{~mL})$ was cooled to $0{ }^{\circ} \mathrm{C}$ by means of an ice-water bath. $\left(\mathrm{NH}_{4}\right)_{6} \mathrm{Mo}_{7} \mathrm{O}_{24} \bullet 4 \mathrm{H}_{2} \mathrm{O}(1.06 \mathrm{~g}, 0.86 \mathrm{mmol}$, 0.1 equiv.) dissolved in $\mathrm{H}_{2} \mathrm{O}_{2}(30 \%$ solution in water, $10 \mathrm{~mL})$ was added to the reaction mixture. The vessel was removed from the cooling bath and the system was allowed to warm to $23{ }^{\circ} \mathrm{C}$. After $1 \mathrm{~h}$, the organic phase was removed under reduced pressure and the resulting solution was transferred to a separatory funnel and was extracted with DCM $(3 \times 50 \mathrm{~mL})$. The combined organic layers were washed with saturated aqueous sodium chloride solution $(50 \mathrm{~mL})$ and the washed solution was dried $\left(\mathrm{Na}_{2} \mathrm{SO}_{4}\right)$. The dried solution was filtered, and the filtrate was concentrated. The resulting crude residue was purified by flash chromatography (silica gel, eluent: EtOAc:hexanes $=1: 2)$ to afford sulfone SI-9 (3.09 g, 99\%) as a colorless oil.

TLC (EtOAc:hexanes = 1:3): $\mathrm{R}_{f}=0.25(\mathrm{UV})$.

${ }^{1} \mathbf{H}$ NMR $\left(400 \mathrm{MHz}, \mathrm{CDCl}_{3}\right) \delta 8.21(\mathrm{~d}, J=8.2 \mathrm{~Hz}, 1 \mathrm{H}), 8.02(\mathrm{~d}, J=8.2 \mathrm{~Hz}, 1 \mathrm{H}), 7.66-7.57(\mathrm{~m}, 2 \mathrm{H}), 7.31-7.27$ $(\mathrm{m}, 3 \mathrm{H}), 7.25-7.16(\mathrm{~m}, 2 \mathrm{H}), 6.47(\mathrm{dd}, J=14.8,6.8 \mathrm{~Hz}, 1 \mathrm{H}), 6.32(\mathrm{~d}, J=14.4 \mathrm{~Hz}, 1 \mathrm{H}), 5.61(\mathrm{~d}, J=9.5 \mathrm{~Hz}, 1 \mathrm{H})$, $5.36(\mathrm{dt}, J=9.5,2.8 \mathrm{~Hz}, 1 \mathrm{H}), 4.98-4.91(\mathrm{~m}, 1 \mathrm{H}), 4.56-4.51(\mathrm{~m}, 1 \mathrm{H}), 4.26(\mathrm{t}, J=8.9 \mathrm{~Hz}, 1 \mathrm{H}), 4.23-4.01(\mathrm{~m}$, $6 \mathrm{H}), 3.2(\mathrm{dd}, J=14.0,3.5 \mathrm{~Hz}, 1 \mathrm{H}), 2.79(\mathrm{dd}, J=14.0,9.3 \mathrm{~Hz}, 1 \mathrm{H}), 2.56(\mathrm{dd}, J=16.5,5.0, \mathrm{~Hz}, 1 \mathrm{H}), 2.45(\mathrm{dd}, J$ $=16.5,5.0, \mathrm{~Hz}, 1 \mathrm{H}), 1.95(\mathrm{~s}, 3 \mathrm{H}), 1.79-1.75(\mathrm{~m}, 1 \mathrm{H}), 1.34(\mathrm{~d}, J=7.0 \mathrm{~Hz}, 3 \mathrm{H}), 1.17(\mathrm{~d}, J=7.0 \mathrm{~Hz}, 3 \mathrm{H}), 0.88(\mathrm{~s}$, 9H), $0.87(\mathrm{~s}, 9 \mathrm{H}), 0.05(\mathrm{~s}, 3 \mathrm{H}), 0.04(\mathrm{~s}, 3 \mathrm{H}), 0.006(\mathrm{~s}, 3 \mathrm{H}), 0.003(\mathrm{~s}, 3 \mathrm{H})$.

${ }^{13}$ C NMR $\left(100 \mathrm{MHz}, \mathrm{CDCl}_{3}\right): \delta 173.59,173.56,170.4,165.9,153.4,152.6,148.5,136.8,135.4,135.3,129.4$, $128.9,128.0,127.7,127.3,126.1,125.4,122.4,72.0,70.9,69.9,66.3,55.8,43.3,41.2,40.1,38.5,37.8,25.77$, $25.75,21.8,18.07,18.02,17.8,10.3,-4.0,-4.8,-5.0,-5.2$.

HRMS-ESI m/z cacld for $\mathrm{C}_{47} \mathrm{H}_{69} \mathrm{IN}_{3} \mathrm{O}_{8} \mathrm{~S}_{2} \mathrm{Si}_{2}[\mathrm{M}+\mathrm{H}]^{+} 1082.3008$, found 1082.3035.

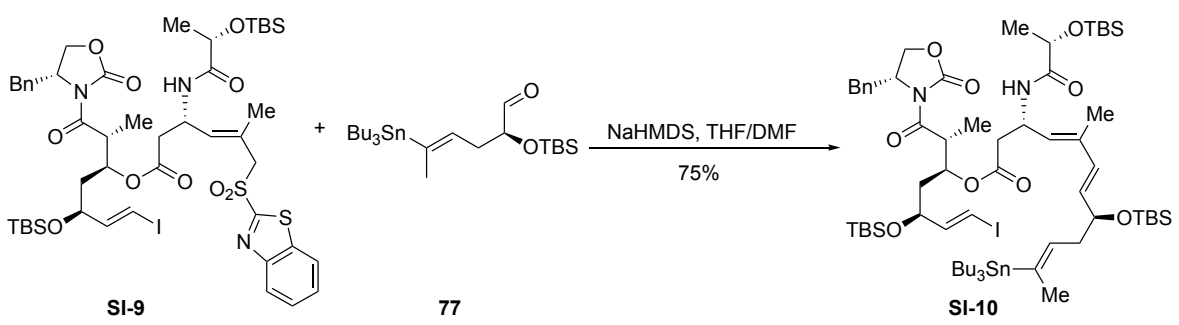

A 100-mL round-bottom flask containing sulfone SI-9 (1.32 g, $1.22 \mathrm{mmol}, 1$ equiv.) and aldehyde 77 (757 mg, $1.46 \mathrm{mmol}, 1.2$ equiv.) was evacuated and flushed with nitrogen (this process was repeated a total of 3 times) and was then sealed with a rubber septum. DMF $(35 \mathrm{~mL})$ and THF $(35 \mathrm{~mL})$ were added, resulting a colorless solution, and the system was cooled to $-78^{\circ} \mathrm{C}$ in a dry ice-acetone bath. NaHMDS (2.0 M in THF, $0.73 \mathrm{~mL}, 1.46 \mathrm{mmol}, 1.20$ equiv.) was added dropwise over $20 \mathrm{~min}$. After $1 \mathrm{~h}$, saturated aqueous $\mathrm{NH}_{4} \mathrm{Cl}(50 \mathrm{~mL})$ were added. The vessel was removed from the cooling bath and the system was allowed to warm to $23{ }^{\circ} \mathrm{C}$. The biphasic mixture was transferred to a separatory funnel and the layers were separated. The aqueous layer was extracted with ethyl acetate $(2 \times 50$ $\mathrm{mL})$. The combined organic layers were washed with water $(30 \mathrm{~mL})$ and saturated aqueous sodium chloride solution $(50 \mathrm{~mL})$ and the washed solution was dried $\left(\mathrm{Na}_{2} \mathrm{SO}_{4}\right)$. The dried solution was filtered and the filtrate was concentrated. The crude residue was purified by flash chromatography (silica gel, eluent: EtOAc:hexanes $=1: 10$ ) to afford diene SI-10 (1.27 g, 75\%) as a colorless oil. 
TLC (EtOAc:hexanes $=1: 3): \mathrm{R}_{f}=0.70(\mathrm{UV})$.

${ }^{1} \mathbf{H}$ NMR $\left(400 \mathrm{MHz}, \mathrm{CDCl}_{3}\right) \delta 7.34-7.27(\mathrm{~m}, 2 \mathrm{H}), 7.21-7.14(\mathrm{~m}, 3 \mathrm{H}), 6.50(\mathrm{dd}, J=14.8,6.8 \mathrm{~Hz}, 1 \mathrm{H}), 6.27(\mathrm{dd}$, $J=14.4,1.2 \mathrm{~Hz}, 1 \mathrm{H}), 6.10(\mathrm{~d}, J=16.4 \mathrm{~Hz}, 1 \mathrm{H}), 5.73(\mathrm{dd}, J=16.4,6.5 \mathrm{~Hz}, 1 \mathrm{H}), 5.62(\mathrm{td}, J=7.0,2.8 \mathrm{~Hz}, 1 \mathrm{H}), 5.50$ $(\mathrm{d}, J=9.0 \mathrm{~Hz}, 1 \mathrm{H}), 5.41(\mathrm{td}, J=9.0,2.8 \mathrm{~Hz}, 1 \mathrm{H}), 5.11-5.04(\mathrm{~m}, 1 \mathrm{H}), 4.57-4.53(\mathrm{~m}, 1 \mathrm{H}), 4.27(\mathrm{t}, J=8.9 \mathrm{~Hz}$, $1 \mathrm{H}), 4.20-4.13(\mathrm{~m}, 3 \mathrm{H}), 4.04-3.98(\mathrm{~m}, 2 \mathrm{H}), 3.21(\mathrm{dd}, J=14.0,3.5 \mathrm{~Hz}, 1 \mathrm{H}), 2.81(\mathrm{dd}, J=14.0,9.3 \mathrm{~Hz}, 1 \mathrm{H})$, $2.64(\mathrm{qd}, J=16.7,5.8 \mathrm{~Hz}, 2 \mathrm{H}), 2.39-2.24(\mathrm{~m}, 2 \mathrm{H}), 1.82-1.80(\mathrm{~m}, 7 \mathrm{H}), 1.51-1.45(\mathrm{~m}, 6 \mathrm{H}), 1.39-1.29(\mathrm{~m}$, $9 \mathrm{H}), 1.19(\mathrm{~d}, J=7.0 \mathrm{~Hz}, 3 \mathrm{H}), 0.90-0.97(\mathrm{~m}, 43 \mathrm{H}), 0.04-0.005(\mathrm{~m}, 18 \mathrm{H})$,

${ }^{13} \mathbf{C}$ NMR (100 MHz, $\left.\mathrm{CDCl}_{3}\right): \delta 173.5,173.1,170.6,153.5,148.6,139.9,137.2,136.4,135.3,133.6,132.4,129.4$, $128.89,128.80,127.2,73.7,72.0,70.7,70.1,66.2,55.8,42.8,41.3,40.2,39.1,37.8,37.4,29.1,27.4,25.9,25.7$, $25.6,21.9,19.4,18.2,18.04,18.01,13.7,13.1,10.2,9.0,-4.02,-4.07,-4.6,-4.8,-5.0,-5.4$.

HRMS-ESI m/z cacld for $\mathrm{C}_{64} \mathrm{H}_{114} \mathrm{IN}_{2} \mathrm{O}_{9} \mathrm{Si}_{3} \mathrm{Sn}[\mathrm{M}+\mathrm{H}]^{+} 1385.5899$, found 1385.5862 .
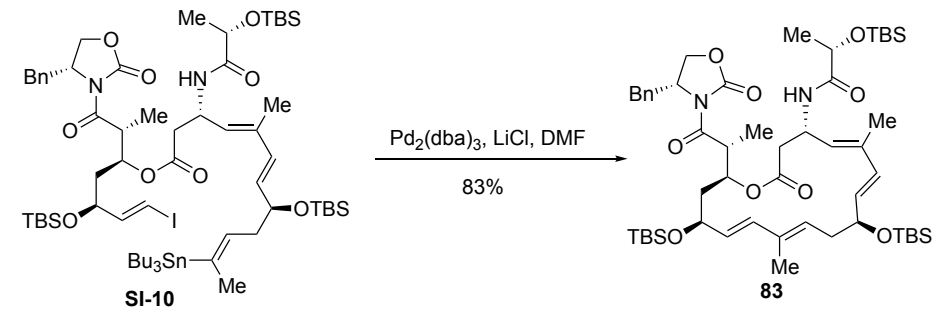

A 500-mL round-bottom flask containing $\mathrm{LiCl}(0.92 \mathrm{~g}, 21.67 \mathrm{mmol}, 30$ equiv. $)$ was flame dried under vacumm for 5 min. After cooling to the $23{ }^{\circ} \mathrm{C}$, DMF $(350 \mathrm{~mL})$ and Stille Coupling precursor SI-10 (1.00 g, $0.72 \mathrm{mmol}, 1$ equiv) was added. The vessel was evacuated and filled with nitrogen (this process was repeated a total of 3 times). $\mathrm{Pd}_{2}(\mathrm{dba})_{3}$ (132 $\mathrm{mg}, 0.14 \mathrm{mmol}, 0.2$ equiv) was added in one portion. A stream of argon was passed through the solution for $10 \mathrm{~min}$. After $1 \mathrm{~h}$, the mixture was concentrated and resulting crude residue was purified by flash chromatography (silica gel, eluent: EtOAc:hexanes $=1: 4)$ to afford product $\mathbf{8 3}(580 \mathrm{mg}, 83 \%)$ as a light yellow foam.

TLC (EtOAc:hexanes $=1: 3): \mathrm{R}_{f}=0.60(\mathrm{UV})$.

${ }^{1} \mathbf{H}$ NMR $\left(400 \mathrm{MHz}, \mathrm{CDCl}_{3}\right) \delta 7.53(\mathrm{~d}, J=9.6 \mathrm{~Hz}, 1 \mathrm{H}), 7.33-7.27(\mathrm{~m}, 3 \mathrm{H}), 7.18-7.16(\mathrm{~m}, 2 \mathrm{H}), 6.06(\mathrm{~d}, J=16.0$ $\mathrm{Hz}, 1 \mathrm{H}), 5.62(\mathrm{~d}, J=16.0 \mathrm{~Hz}, 1 \mathrm{H}), 5.43-5.27(\mathrm{~m}, 4 \mathrm{H}), 5,16(\mathrm{dd}, J=8.8,1.8 \mathrm{~Hz}, 1 \mathrm{H}), 5.06-5.00(\mathrm{~m}, 1 \mathrm{H}), 4.60-$ $4.54(\mathrm{~m}, 1 \mathrm{H}), 4.29(\mathrm{t}, J=9.0 \mathrm{~Hz}, 1 \mathrm{H}), 4.20-4.11(\mathrm{~m}, 3 \mathrm{H}), 4.05(\mathrm{dt}, J=8.8,2.9 \mathrm{~Hz}, 1 \mathrm{H}), 3.91(\mathrm{dq}, J=6.0,1.2 \mathrm{~Hz}$, $1 \mathrm{H}), 3.15$ (dd, $J=14.0,3.5 \mathrm{~Hz}, 1 \mathrm{H}), 2.82(\mathrm{dd}, J=14.0,9.3 \mathrm{~Hz}, 1 \mathrm{H}), 2.72(\mathrm{dd}, J=16.7,5.8 \mathrm{~Hz}, 2 \mathrm{H}), 2.42-2.22$ $(\mathrm{m}, 3 \mathrm{H}), 2.06-1.98(\mathrm{~m}, 1 \mathrm{H}), 1.91-1.87(\mathrm{~m}, 1 \mathrm{H}), 1.75(\mathrm{~s}, 3 \mathrm{H}), 1.44(\mathrm{~s}, 3 \mathrm{H}), 1.41(\mathrm{~d}, J=7.0 \mathrm{~Hz}, 3 \mathrm{H}), 1.17(\mathrm{~d}, J=$ $7.0 \mathrm{~Hz}, 3 \mathrm{H}), 0.90(\mathrm{~s}, 9 \mathrm{H}), 0.85(\mathrm{~s}, 9 \mathrm{H}), 0.83(\mathrm{~s}, 9 \mathrm{H}), 0.07$ (s, 3H), $0.06(\mathrm{~s}, 3 \mathrm{H}), 0.05(\mathrm{~s}, 6 \mathrm{H}),-0.006(\mathrm{~s}, 3 \mathrm{H}),-0.02$ $(\mathrm{s}, 3 \mathrm{H})$.

${ }^{13} \mathrm{C}$ NMR $\left(100 \mathrm{MHz}, \mathrm{CDCl}_{3}\right): \delta 173.38,173.33,171.4,153.6,137.5,136.1,135.0,134.2 .133 .2,130.5,129.5$, $128.9,128.75,128.71,127.3,127.2,75.0,74.3,71.5,70.1,66.1,55.5,43.0,42.3,41.5,39.1,38.3,37.7,25.8,25.6$, $22.1,18.2,18.1,18.0,12.6,12.1,10.0,-3.9,-4.4,-4.5,-4.7,-5.0,-5.2$.

HRMS-ESI m/z cacld for $\mathrm{C}_{52} \mathrm{H}_{87} \mathrm{~N}_{2} \mathrm{O}_{9} \mathrm{Si}_{3}[\mathrm{M}+\mathrm{H}]^{+}$967.5719, found 967.5776 .

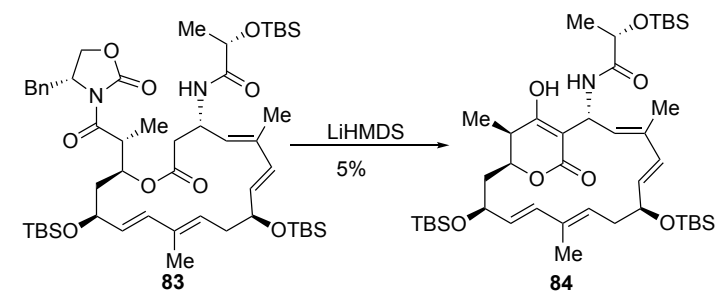

A $50-\mathrm{mL}$ round-bottom flask containing macrocycle 83 (100 mg, $0.1 \mathrm{mmol}, 1$ equiv) and THF (20 mL) was evacuated and filled with nitrogen (this process was repeated a total of 3 times). LiHMDS (1.0 M in THF, $1.0 \mathrm{~mL}$, $1.0 \mathrm{mmol}, 10$ equiv.) was added slowly to the reaction mixture. The resulting solution was stirred for 24 hours. Saturated aqueous $\mathrm{NH}_{4} \mathrm{Cl}$ solution $(20 \mathrm{~mL})$ was added. The biphasic mixture was transferred to a separatory funnel and the layers were separated. The aqueous layer was extracted with ethyl acetate $(2 \times 20 \mathrm{~mL})$. The combined 
organic layers were washed with water $(20 \mathrm{~mL})$ and saturated aqueous sodium chloride solution $(30 \mathrm{~mL})$ and the washed solution was dried $\left(\mathrm{Na}_{2} \mathrm{SO}_{4}\right)$. The dried solution was filtered and the filtrate was concentrated. The crude residue was purified by short quick flash chromatography (silica gel, eluent: EtOAc:hexanes $=1: 10$ ) to afford 84 (5.10 $\mathrm{mg}, 5 \%)$ as a colorless oil (not stable, used immediately for the next step).

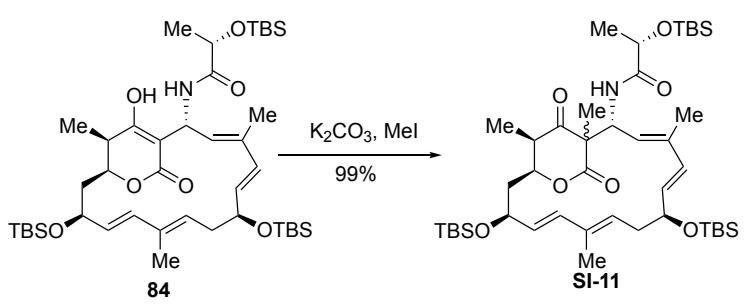

The enol 84 from previous steps was dissolved in $2 \mathrm{~mL}$ acetone. $\mathrm{K}_{2} \mathrm{CO}_{3}(8.7 \mathrm{mg}, 63 \mu \mathrm{mol}, 10$ equiv.) and $\mathrm{MeI}(9.0$ $\mathrm{mg}, 63 \mu \mathrm{mol}, 10$ equiv.) were added to the solution sequentially. After $30 \mathrm{~min}$, the mixture was concentrated, and the crude residus was purified by flash chromatography (silica gel, eluent: EtOAc:hexanes $=1: 8$ ) to afford desired product SI-11 (5.19 $\mathrm{mg}, 99 \%)$ as a colorless oil

TLC (EtOAc:hexanes $=1: 3): \mathrm{R}_{f}=0.75(\mathrm{UV})$.

${ }^{1} \mathbf{H}$ NMR $\left(400 \mathrm{MHz}, \mathrm{CDCl}_{3}\right) \delta 7.57(\mathrm{~d}, J=10.2 \mathrm{~Hz}, 1 \mathrm{H}), 6.02(\mathrm{~d}, J=16.2 \mathrm{~Hz}, 1 \mathrm{H}), 5.76(\mathrm{t}, J=10.0 \mathrm{~Hz}, 1 \mathrm{H}), 5.58$ $(\mathrm{d}, J=16.2 \mathrm{~Hz}, 1 \mathrm{H}), 5.44(\mathrm{dd}, J=16.2,8.0 \mathrm{~Hz}, 1 \mathrm{H}), 5.38(\mathrm{dd}, J=16.0,6.0 \mathrm{~Hz}, 1 \mathrm{H}), 5.10(\mathrm{t}, J=9.8 \mathrm{~Hz}, 1 \mathrm{H}), 4.93$ $(\mathrm{d}, J=10.5 \mathrm{~Hz}, 1 \mathrm{H}), 4.60(\mathrm{dd}, J=8.0,5.2 \mathrm{~Hz}, 1 \mathrm{H}), 4.41(\mathrm{dd}, J=5.2,2.0 \mathrm{~Hz}, 1 \mathrm{H}), 4.20(\mathrm{q}, J=6.8 \mathrm{~Hz}, 1 \mathrm{H}), 4.05-$ $3.99(\mathrm{~m}, 1 \mathrm{H}), 3.45(\mathrm{q}, J=6.2 \mathrm{~Hz}, 1 \mathrm{H}), 2.46(\mathrm{q}, J=7.0 \mathrm{~Hz}, 1 \mathrm{H}), 2.26-2.18(\mathrm{~m}, 1 \mathrm{H}), 1.91(\mathrm{~s}, 3 \mathrm{H}), 1.62(\mathrm{~s}, 3 \mathrm{H})$, $1.54(\mathrm{~s}, 3 \mathrm{H}), 1.40(\mathrm{~d}, J=7.0 \mathrm{~Hz}, 3 \mathrm{H}), 1.10(\mathrm{~d}, J=7.0 \mathrm{~Hz}, 3 \mathrm{H}), 0.98-0.96(\mathrm{~m}, 1 \mathrm{H}), 0.94(\mathrm{~s}, 9 \mathrm{H}), 0.90(\mathrm{~s}, 9 \mathrm{H}), 0.88$ (s, 9H), $0.12(\mathrm{~s}, 3 \mathrm{H}), 0.09(\mathrm{~s}, 3 \mathrm{H}), 0.07(\mathrm{~s}, 3 \mathrm{H}), 0.06(\mathrm{~s}, 3 \mathrm{H}), 0.04(\mathrm{~s}, 3 \mathrm{H}), 0.03(\mathrm{~s}, 3 \mathrm{H})$.

${ }^{13}$ C NMR $\left(100 \mathrm{MHz}, \mathrm{CDCl}_{3}\right): \delta 203.6,174.7,173.5,137.9,135.2,134.8,133.9,133.1,130.7,128.0,126.5,75.8$, $70.1,68.2,60.9,47.1,42.6,41.9,36.4,25.87,25.81,25.7,24.8,22.1,18.2,18.08,18.05,13.8,12.9,9.5,0.005$, $3.9,-4.4,-4.7,-4.8,-4.9,-5.3$.

HRMS-ESI m/z cacld for $\mathrm{C}_{43} \mathrm{H}_{78} \mathrm{NO}_{7} \mathrm{Si}_{3}[\mathrm{M}+\mathrm{H}]^{+}$804.5086, found 804.5072.

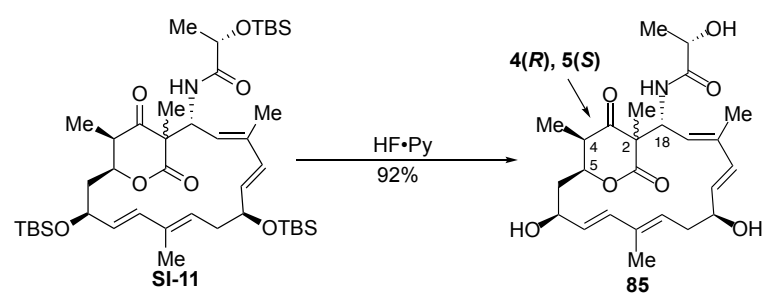

A 25-mL plastic vial containing a solution of SI-11 $(5.0 \mathrm{mg}, 6.2 \mu \mathrm{mol}, 1$ equiv.) and THF (3 mL) was cooled to 0 ${ }^{\circ} \mathrm{C}$ by means of an ice-water bath. Py•HF $(\sim 70 \% \mathrm{HF})(300 \mu \mathrm{L})$ was added dropwise, and the vessel was removed from the cooling bath and the system was allowed to warm to $23{ }^{\circ} \mathrm{C}$. After $5 \mathrm{~h}, \mathrm{NaHCO}_{3}(300 \mathrm{mg})$ was added and the mixture was rapidly stirred. After $15 \mathrm{~min}$, the mixture was filtered through a pad of Celite and the filtrate was concentrated. The crude residue was purified by flash chromatography (silica gel, eluent: acetone:hexanes $=2: 1$ ) to afford $85(2.6 \mathrm{mg}, 92 \%)$ as a white solid.

TLC (EtOAc: $\mathrm{MeOH}=30: 1): \mathrm{R}_{f}=0.40(\mathrm{UV})$.

${ }^{1} \mathbf{H}$ NMR $\left(400 \mathrm{MHz}, \mathrm{CD}_{3} \mathrm{OD}\right) \delta 6.09(\mathrm{~d}, J=16.0 \mathrm{~Hz}, 1 \mathrm{H}), 5.77(\mathrm{~d}, J=16.0 \mathrm{~Hz}, 1 \mathrm{H}), 5.69(\mathrm{~d}, J=10.0 \mathrm{~Hz}, 1 \mathrm{H})$, $5.56-5.48(\mathrm{~m}, 2 \mathrm{H}), 5.16(\mathrm{t}, J=8.0 \mathrm{~Hz}, 1 \mathrm{H}), 5.00(\mathrm{~d}, J=10.0 \mathrm{~Hz}, 1 \mathrm{H}), 4.79(\mathrm{dd}, J=12.0,6.0 \mathrm{~Hz}, 1 \mathrm{H}), 4.38(\mathrm{dd}, J$ $=11.2,6.4 \mathrm{~Hz}, 1 \mathrm{H}), 4.10(\mathrm{q}, J=7.5 \mathrm{~Hz}, 1 \mathrm{H}), 4.04-3.98(\mathrm{~m}, 1 \mathrm{H}), 3.74-3.73(\mathrm{~m}, 1 \mathrm{H}), 2.61-2.58(\mathrm{~m}, 1 \mathrm{H}), 2.19$ $-2.08(\mathrm{~m}, 1 \mathrm{H}), 1.92(\mathrm{~s}, 3 \mathrm{H}), 1.68(\mathrm{~s}, 3 \mathrm{H}), 1.63-1.57(\mathrm{~m}, 1 \mathrm{H}), 1.56(\mathrm{~s}, 3 \mathrm{H}), 1.37(\mathrm{~d}, J=7.0 \mathrm{~Hz}, 3 \mathrm{H}), 1.09(\mathrm{~d}, J=$ $7.0 \mathrm{~Hz}, 3 \mathrm{H}), 1.09-1.02(\mathrm{~m}, 1 \mathrm{H})$.

${ }^{13}$ C NMR (100 MHz, $\left.\mathrm{CD}_{3} \mathrm{OD}\right): \delta 204.4,175.1,175.0,137.6,135.6,135.2,133.7,132.1,130.7,127.5,126.6,77.1$, 74.1, 67.7, 66.0, 60.4, 42.1, 40.4, 34.6, 23.8, 19.8, 12.6, 11.7, 8.2.

HRMS-ESI $\mathrm{m} / \mathrm{z}$ cacld for $\mathrm{C}_{25} \mathrm{H}_{35} \mathrm{NO}_{7} \mathrm{Na}[\mathrm{M}+\mathrm{Na}]^{+}$484.2311, found 484.2296. 
Scheme 4. Reassignment of iso-lankacidinol by means of modular chemical synthesis
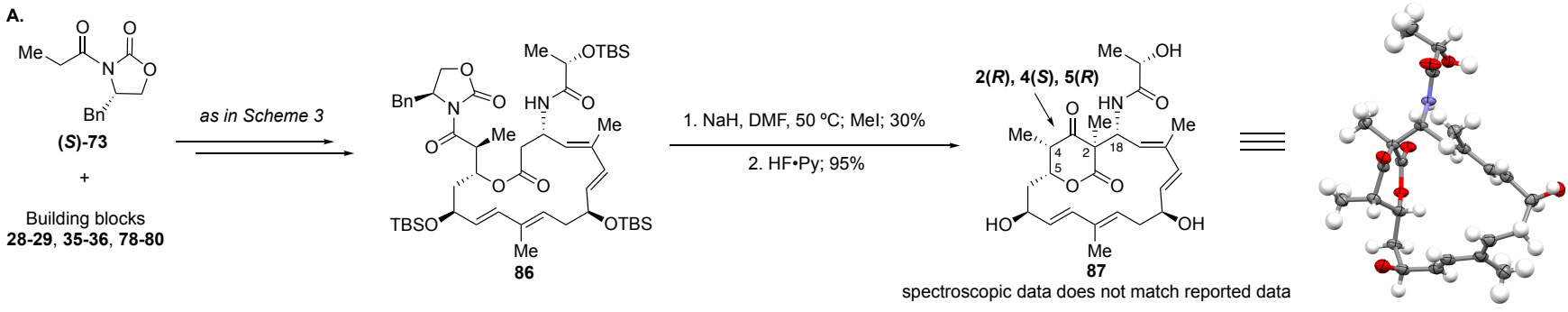

B.

38

88

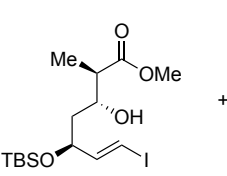

89 (left fragment)

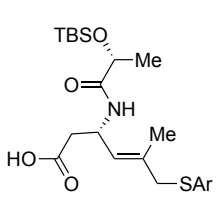

82 (top fragment)
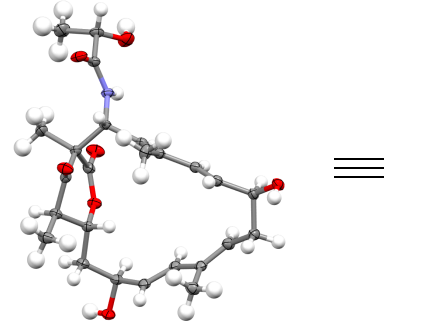

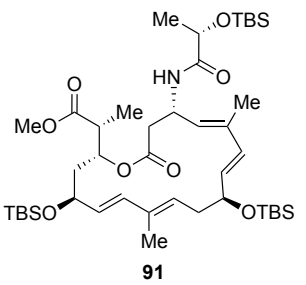

4. $\left(\mathrm{NH}_{4}\right)_{6} \mathrm{Mo}_{7} \mathrm{O}_{24}, \mathrm{H}_{2} \mathrm{O}_{2}$

6. $\mathrm{Pd}_{2}(\mathrm{dba})_{3}$, LiCl

$64 \%$, 3 steps 


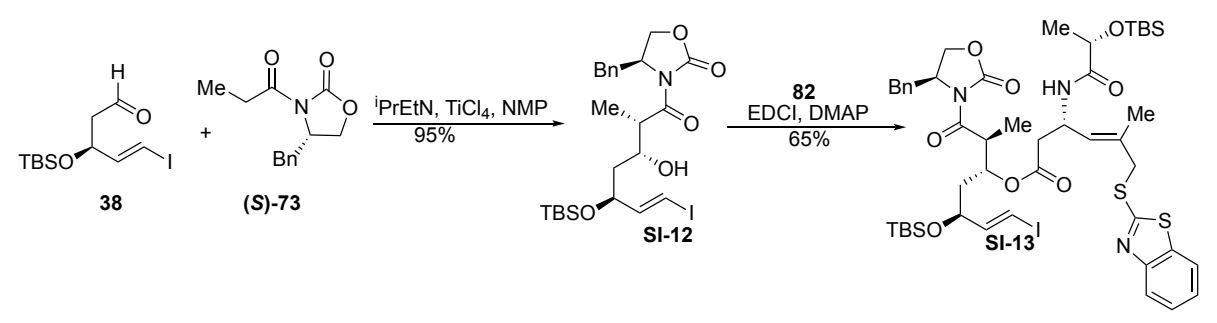

A 250-mL round-bottom flask containing imide $(S)-73$ (4.90 g, $21.0 \mathrm{mmol}, 1.1$ equiv.) in $70 \mathrm{~mL}$ DCM was cooled to $0{ }^{\circ} \mathrm{C}$ by means of an ice-water bath. $\mathrm{TiCl}_{4}(1.0 \mathrm{M}$ in DCM, $22.9 \mathrm{~mL}, 22.9 \mathrm{mmol}, 1.2$ equiv.) was added dropwise at $0{ }^{\circ} \mathrm{C}$, resulting in an organge solution. After $15 \mathrm{~min}$, diisopropylethylamine $(4.16 \mathrm{~mL}$, 23.9 mmol, 1.2 equiv.) was added dropwise. The resulting deep red reaction mixture was allowed to stir 40 min. 1-Methyl-2-pyrrolidinone ( $2.02 \mathrm{~mL}, 21.0 \mathrm{mmol}, 1.1$ equiv.) was added and the reaction mixture was stirred for an additional $10 \mathrm{~min}$. Aldehyde $38(6.50 \mathrm{~g}, 19.1 \mathrm{mmol}, 1$ equiv.) was added dropwise. After $2 \mathrm{~h}$, saturated aqueous $\mathrm{NH}_{4} \mathrm{Cl}$ solution $(50 \mathrm{~mL})$ was added in one portion. After thorough mixing, the biphasic mixture was transferred to a separatory funnel and the layers were separated. The aqueous layer was extracted with DCM $(2 \times 50 \mathrm{~mL})$. The combined organic layers were washed with water $(30 \mathrm{~mL})$ and saturated aqueous sodium chloride solution $(50.0 \mathrm{~mL})$ and the washed solution was dried $\left(\mathrm{Na}_{2} \mathrm{SO}_{4}\right)$. The dried solution was filtered and the filtrate was concentrated. The crude residue was purified by flash chromatography (silica gel, eluent: EtOAc:hexanes = 1:5) to afford syn-aldol product SI-12 (10.4 g, 95\%) as a colorless oil.

TLC (EtOAc:hexanes = 1:5): $\mathrm{R}_{f}=0.45(\mathrm{UV})$.

${ }^{1}$ H NMR $\left(400 \mathrm{MHz}, \mathrm{CDCl}_{3}\right) \delta 7.35-7.25(\mathrm{~m}, 3 \mathrm{H}), 7.22-7.19(\mathrm{~m}, 2 \mathrm{H}), 6.54(\mathrm{dd}, J=14.4,7.0 \mathrm{~Hz}, 1 \mathrm{H})$, $6.31(\mathrm{~d}, J=14.4 \mathrm{~Hz}, 1 \mathrm{H}), 4.72-4.67(\mathrm{~m}, 1 \mathrm{H}), 4.37$ (q, $J=6.4 \mathrm{~Hz}, 1 \mathrm{H}), 4.20$ (q, $J=8.6 \mathrm{~Hz}, 1 \mathrm{H}), 4.17$ (dd, $J=8.7,2.8 \mathrm{~Hz}, 1 \mathrm{H})$, ), $4.07-4.02(\mathrm{~m}, 1 \mathrm{H}), 3.76$ (dddd, $J=8.8,6.0,2.9,1.2 \mathrm{~Hz}, 1 \mathrm{H}), 3.30(\mathrm{~d}, J=1.6 \mathrm{~Hz}$, 1H), 3.25 (dd, $J=14.0,3.5 \mathrm{~Hz}, 1 \mathrm{H}), 2.79$ (dd, $J=14.0,9.3 \mathrm{~Hz}, 1 \mathrm{H}), 1.85-1.77$ (m, 1H), 1.58 (ddd, $J=$ 13.5, 6.3, $2.5 \mathrm{~Hz}, 1 \mathrm{H}), 1.27$ (d, $J=7.0 \mathrm{~Hz}, 3 \mathrm{H}), 0.89$ (s, 9H), 0.09 (s, 3H), 0.07 (s, 3H).

${ }^{13} \mathbf{C}$ NMR (100 MHz, $\left.\mathrm{CDCl}_{3}\right): \delta 176.6,152.9,148.3,135.1,129.4,128.9,127.4,77.2,74.5,69.3,66.1,55.1$, $42.6,41.2,37.7,25.8,18.1,11.2,-4.2,-4.8$.

HRMS-ESI $\mathrm{m} / \mathrm{z}$ cacld for $\mathrm{C}_{24} \mathrm{H}_{36} \mathrm{INO}_{5} \mathrm{SiNa}[\mathrm{M}+\mathrm{Na}]^{+}$596.1305, found 596.1337.

A 100-mL round-bottom flask containing acid $82(2.80 \mathrm{~g}, 5.66 \mathrm{mmol}, 1$ equiv.) and alcohol SI-12 (3.90 g, 6.79 mmol, 1.2 equiv.) was added $50 \mathrm{~mL}$ chloroform. DMAP (3.46 g, $28.3 \mathrm{mmol}, 5$ equiv.) and EDCI (5.42 g, $28.3 \mathrm{mmol}, 5$ equiv) was added in sequence. The reaction mixture was heated at $50{ }^{\circ} \mathrm{C}$ for $3 \mathrm{~h}$ and was then concentrated. The crude residue was purified by flash chromatography (silica gel, eluent: EtOAc:hexanes = 1:3) to afford ester SI-13 (3.86 g 65\%) as a colorless oil.

TLC (EtOAc:hexanes = 1:3): $\mathrm{R}_{f}=0.35(\mathrm{UV})$.

${ }^{1}$ H NMR $\left(400 \mathrm{MHz}, \mathrm{CDCl}_{3}\right) \delta 7.86(\mathrm{~d}, J=8.2 \mathrm{~Hz}, 1 \mathrm{H}), 7.75(\mathrm{~d}, J=8.2 \mathrm{~Hz}, 1 \mathrm{H}), 7.42(\mathrm{td}, J=7.5,1.6 \mathrm{~Hz}$, $1 \mathrm{H}), 7.32-7.28(\mathrm{~m}, 3 \mathrm{H}), 7.25-7.18(\mathrm{~m}, 3 \mathrm{H}), 6.50(\mathrm{dd}, J=14.8,6.8 \mathrm{~Hz}, 1 \mathrm{H}), 6.22(\mathrm{dd}, J=14.4,1.2 \mathrm{~Hz}$, $1 \mathrm{H}), 5.67(\mathrm{dd}, J=9.5,0.8 \mathrm{~Hz}, 1 \mathrm{H}), 5.38(\mathrm{dt}, J=9.5,2.8 \mathrm{~Hz}, 1 \mathrm{H}), 5.04-4.99(\mathrm{~m}, 1 \mathrm{H}), 4.61-4.56(\mathrm{~m}, 1 \mathrm{H})$, $4.26(\mathrm{t}, J=8.9 \mathrm{~Hz}, 1 \mathrm{H}), 4.21-4.14(\mathrm{~m}, 3 \mathrm{H}), 4.06(\mathrm{~s}, 2 \mathrm{H}), 3.98$ (dddd, $J=8.8,6.0,2.9,1.2 \mathrm{~Hz}, 1 \mathrm{H}), 3.23$ $(\mathrm{dd}, J=14.0,3.5 \mathrm{~Hz}, 1 \mathrm{H}), 2.82(\mathrm{dd}, J=14.0,9.3 \mathrm{~Hz}, 1 \mathrm{H}), 2.61(\mathrm{dtd}, J=22.0,16.5,5.0, \mathrm{~Hz}, 2 \mathrm{H}), 1.96-$ 1.89 (m, 4H), 1.80 (ddd, $J=13.5,6.3,2.5 \mathrm{~Hz}, 1 \mathrm{H}), 1.37$ (d, $J=7.0 \mathrm{~Hz}, 3 \mathrm{H}), 1.19$ (d, $J=7.0 \mathrm{~Hz}, 3 \mathrm{H}), 0.90$ (s, 9H), $0.88(\mathrm{~s}, 9 \mathrm{H}), 0.08(\mathrm{~s}, 3 \mathrm{H}), 0.06(\mathrm{~s}, 3 \mathrm{H}), 0.04(\mathrm{~s}, 3 \mathrm{H}), 0.03(\mathrm{~s}, 3 \mathrm{H})$.

${ }^{13}$ C NMR (100 MHz, $\left.\mathrm{CDCl}_{3}\right): \delta 173.5,173.3,170.6,166.8,153.4,153.0,147.8,135.2,135.1,134.5,129.4$, $128.9,128.3,127.3,126.0,124.2,121.5,120.9,76.1,72.0,70.0,69.9,66.2,55.7,42.9,42.0,41.5,39.7$, $38.9,37.8,25.79,25.71,21.9,18.1,18.0,16.1,10.5,-4.7,-4.9,-5.3$. 
HRMS-ESI m/z cacld for $\mathrm{C}_{47} \mathrm{H}_{69} \mathrm{IN}_{3} \mathrm{O}_{8} \mathrm{~S}_{2} \mathrm{Si}_{2}[\mathrm{M}+\mathrm{H}]^{+} 1050.3109$, found 1050.3110 .

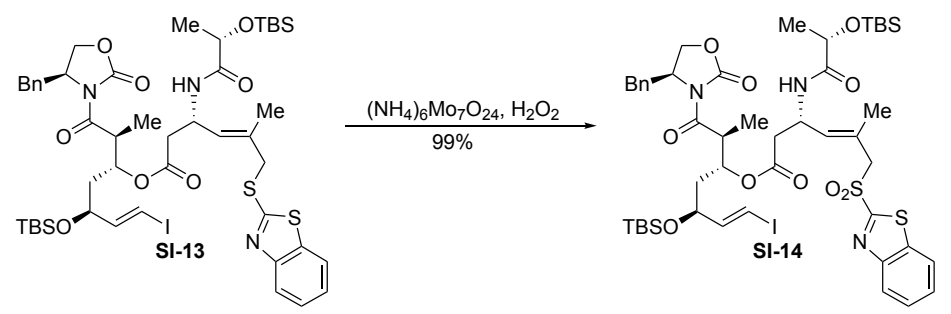

A 250-mL round-bottom flask containing thioether $\mathbf{S I - 1 3}$ (3.00 g, $2.86 \mathrm{mmol}, 1$ equiv.), ethanol (50 $\mathrm{mL})$, and acetonitrile $(50 \mathrm{~mL})$ was cooled to $0{ }^{\circ} \mathrm{C}$ by means of an ice-water bath. $\left(\mathrm{NH}_{4}\right)_{6} \mathrm{Mo}_{7} \mathrm{O}_{24} \bullet 4 \mathrm{H}_{2} \mathrm{O}(1.06 \mathrm{~g}$, 0.86 mmol, 0.10 equiv.) dissolved in $\mathrm{H}_{2} \mathrm{O}_{2}(30 \%$ solution in water, $10.0 \mathrm{~mL})$ was added to the reaction mixture. The vessel was removed from the cooling bath and the system was allowed to warm to $23{ }^{\circ} \mathrm{C}$. After $1 \mathrm{~h}$, the organic solvent was removed under reduced pressure and the resulting solution was transferred to a separatory funnel and was extracted with DCM $(3 \times 50 \mathrm{~mL})$. The combined organic layers were washed with saturated aqueous sodium chloride solution $(2 \times 30 \mathrm{~mL})$ and the washed solution was dried $\left(\mathrm{Na}_{2} \mathrm{SO}_{4}\right)$. The dried solution was filtered, and the filtrate was concentrated. The resulting crude residue was purified by flash chromatography ( silica gel, eluent: EtOAc:hexanes $=1: 2$ ) to afford sulfone SI-14 (3.09 g, 99\%) as a colorless oil.

TLC (EtOAc:hexanes = 1:3): $\mathrm{R}_{f}=0.25(\mathrm{UV})$.

${ }^{1} \mathbf{H}$ NMR $\left(400 \mathrm{MHz}, \mathrm{CDCl}_{3}\right) \delta 8.18(\mathrm{~d}, J=8.2 \mathrm{~Hz}, 1 \mathrm{H}), 8.00(\mathrm{~d}, J=8.2 \mathrm{~Hz}, 1 \mathrm{H}), 7.64-7.56(\mathrm{~m}, 2 \mathrm{H}), 7.36$ $(\mathrm{d}, J=8.2 \mathrm{~Hz}, 1 \mathrm{H}), 7.32-7.28(\mathrm{~m}, 2 \mathrm{H}), 7.25-7.18(\mathrm{~m}, 3 \mathrm{H}), 6.47(\mathrm{dd}, J=14.8,6.8 \mathrm{~Hz}, 1 \mathrm{H}), 6.19(\mathrm{dd}, J=$ $14.4,1.2 \mathrm{~Hz}, 1 \mathrm{H}), 5.57(\mathrm{~d}, J=9.5 \mathrm{~Hz}, 1 \mathrm{H}), 5.34(\mathrm{dt}, J=9.5,2.8 \mathrm{~Hz}, 1 \mathrm{H}), 4.96-4.91(\mathrm{~m}, 1 \mathrm{H}), 4.64-4.58$ $(\mathrm{m}, 1 \mathrm{H}), 4.27(\mathrm{t}, J=8.9 \mathrm{~Hz}, 1 \mathrm{H}), 4.23-4.14(\mathrm{~m}, 4 \mathrm{H}), 4.09$ (q, $J=8.2 \mathrm{~Hz}, 1 \mathrm{H}), 3.96$ (dddd, $J=8.8,6.0$, 2.9, $1.2 \mathrm{~Hz}, 1 \mathrm{H}), 3.19$ (dd, $J=14.0,3.5 \mathrm{~Hz}, 1 \mathrm{H}), 2.86(\mathrm{dd}, J=14.0,9.3 \mathrm{~Hz}, 1 \mathrm{H}), 2.57$ (dd, $J=16.5,5.0$, $\mathrm{Hz}, 1 \mathrm{H}), 2.43(\mathrm{dd}, J=16.5,5.0, \mathrm{~Hz}, 1 \mathrm{H}), 2.89(\mathrm{~s}, 3 \mathrm{H}), 1.91-1.86(\mathrm{~m}, 1 \mathrm{H}), 1.78(\mathrm{ddd}, J=13.5,6.3,2.5$ $\mathrm{Hz}, 1 \mathrm{H}), 1.34(\mathrm{~d}, J=7.0 \mathrm{~Hz}, 3 \mathrm{H}), 1.14(\mathrm{~d}, J=7.0 \mathrm{~Hz}, 3 \mathrm{H}), 0.89(\mathrm{~s}, 9 \mathrm{H}), 0.87(\mathrm{~s}, 9 \mathrm{H}), 0.07$ (s, 3H), 0.05 (s, $36 \mathrm{H}), 0.01(\mathrm{~s}, 3 \mathrm{H})$.

${ }^{13}$ C NMR (100 MHz, $\left.\mathrm{CDCl}_{3}\right): \delta 173.5,173.3,170.4,165.8,153.4,152.6,147.8,136.9,135.7,135.2,129.4$, $128.9,128.0,127.6,127.2,126.2,125.4,122.4,75.9,71.8,70.1,69.7,66.3,63.8,55.5,42.8,41.4,39.4$, $38.4,37.8,25.79,25.75,21.8,18.1,17.9,17.7,10.6,-4.8,-4.9,-5.1$.

HRMS-ESI $\mathrm{m} / \mathrm{z}$ cacld for $\mathrm{C}_{47} \mathrm{H}_{69} \mathrm{IN}_{3} \mathrm{O}_{8} \mathrm{~S}_{2} \mathrm{Si}_{2}[\mathrm{M}+\mathrm{H}]^{+}$1082.3008, found 1082.3035.

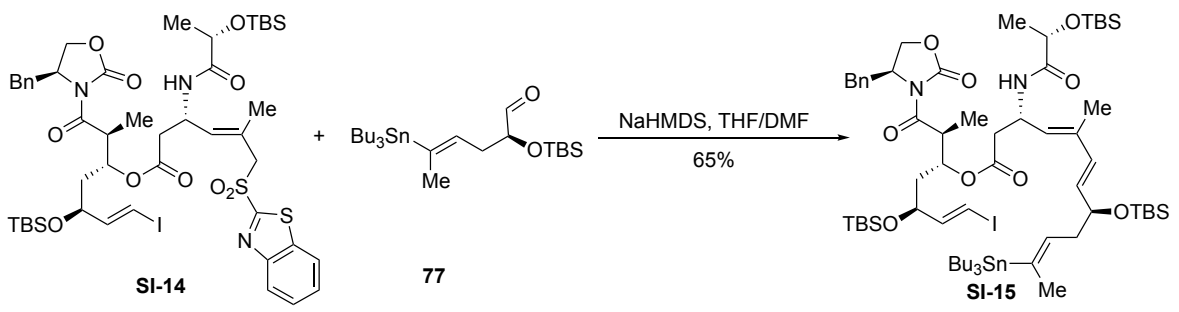

A 100-mL round-bottom flask containing sulfone SI-14 (1.32 g, 1.22 mmol, 1 equiv.) and aldehyde 77 (757 $\mathrm{mg}, 1.46 \mathrm{mmol}, 1.20$ equiv.) was evacuated and flushed with nitrogen (this process was repeated a total of 3 times) and was then sealed with a rubber septum. DMF $(35 \mathrm{~mL})$ and THF $(35 \mathrm{~mL})$ was added, resulting a colorless solution, and the system was cooled to $-78^{\circ} \mathrm{C}$ in a dry ice-acetone bath. NaHMDS (2.0 M in THF, $0.73 \mathrm{~mL}, 1.46 \mathrm{mmol}, 1.20$ equiv.) was added dropwise over $20 \mathrm{~min}$. After $1 \mathrm{~h}$, saturated aqueous $\mathrm{NH}_{4} \mathrm{Cl}(50 \mathrm{~mL})$ were added. The vessel was removed from the cooling bath and the system was allowed to warm to $23^{\circ} \mathrm{C}$. The biphasic mixture was transferred to a separatory funnel and the layers were separated. The aqueous layer was extracted with ethyl acetate $(2 \times 50 \mathrm{~mL})$. The combined organic layers were washed 
with water $(30 \mathrm{~mL})$ and saturated aqueous sodium chloride solution $(50 \mathrm{~mL})$ and the washed solution was dried $\left(\mathrm{Na}_{2} \mathrm{SO}_{4}\right)$. The dried solution was filtered and the filtrate was concentrated. The crude residue was purified by flash chromatography (silica gel, eluent: EtOAc:hexanes =1:10) to afford diene SI-15 (1.10 g, $65 \%$ ) as a colorless oil.

TLC (EtOAc:hexanes = 1:3): $\mathrm{R}_{f}=0.65(\mathrm{UV})$.

${ }^{1}$ H NMR $\left(400 \mathrm{MHz}, \mathrm{CDCl}_{3}\right) \delta 7.35-7.27(\mathrm{~m}, 2 \mathrm{H}), 7.21-7.14(\mathrm{~m}, 3 \mathrm{H}), 6.50(\mathrm{dd}, J=14.8,6.8 \mathrm{~Hz}, 1 \mathrm{H})$, $6.21(\mathrm{dd}, J=14.4,1.2 \mathrm{~Hz}, 1 \mathrm{H}), 6.13(\mathrm{~d}, J=16.4 \mathrm{~Hz}, 1 \mathrm{H}), 5.73(\mathrm{dd}, J=16.4,6.5 \mathrm{~Hz}, 1 \mathrm{H}), 5.62(\mathrm{td}, J=7.0$, $2.8 \mathrm{~Hz}, 1 \mathrm{H}), 5.45(\mathrm{~d}, J=9.0 \mathrm{~Hz}, 1 \mathrm{H}), 5.34(\mathrm{td}, J=9.0,2.8 \mathrm{~Hz}, 1 \mathrm{H}), 5.13-5.09(\mathrm{~m}, 1 \mathrm{H}), 4.59-4.56(\mathrm{~m}$, $1 \mathrm{H}), 4.26(\mathrm{t}, J=8.9 \mathrm{~Hz}, 1 \mathrm{H}), 4.21-4.15(\mathrm{~m}, 4 \mathrm{H}), 3.96(\mathrm{dddd}, J=8.8,6.0,2.9,1.2 \mathrm{~Hz}, 1 \mathrm{H}), 3.23(\mathrm{dd}, J=$ 14.0, 3.5 Hz, 1H), $2.81(\mathrm{dd}, J=14.0,9.3 \mathrm{~Hz}, 1 \mathrm{H}), 2.64(\mathrm{~d}, J=5.8 \mathrm{~Hz}, 1 \mathrm{H}), 2.37-2.30(\mathrm{~m}, 1 \mathrm{H}), 2.02-$ $1.88(\mathrm{~m}, 1 \mathrm{H}), 1.83(\mathrm{~s}, 6 \mathrm{H}), 1.86-1.85(\mathrm{~m}, 2 \mathrm{H}), 1.53-1.45(\mathrm{~m}, 6 \mathrm{H}), 1.38(\mathrm{~d}, J=7.0 \mathrm{~Hz}, 3 \mathrm{H}), 1.35-1.27$ $(\mathrm{m}, 6 \mathrm{H}), 1.18(\mathrm{~d}, J=7.0 \mathrm{~Hz}, 3 \mathrm{H}), 0.94-0.86(\mathrm{~m}, 46 \mathrm{H}), 0.08(\mathrm{~s}, 3 \mathrm{H}), 0.07$ (s, 3H), 0.05 (s, 3H), 0.03 (s, $6 \mathrm{H}), 0.01(\mathrm{~s}, 3 \mathrm{H})$.

${ }^{13}$ C NMR $\left(100 \mathrm{MHz}, \mathrm{CDCl}_{3}\right): \delta 173.5,173.1,170.4,153.4,147.8,139.9,137.2,136.5,135.2,133.5,132.4$, $129.4,128.9,128.8,127.3,73.6,72.1,70.2,69.9,66.2,55.6,43.0,41.6,39.9,39.3,37.8,37.3,29.1,27.4$, $25.9,25.7,25.6,21.9,19.4,18.2,18.1,18.0,13.7,13.1,10.6,9.0,-4.1,-4.6,-4.72,-4.76,-4.9,-5.4$.

HRMS-ESI m/z cacld for $\mathrm{C}_{64} \mathrm{H}_{114} \mathrm{IN}_{2} \mathrm{O}_{9} \mathrm{Si}_{3} \mathrm{Sn}[\mathrm{M}+\mathrm{H}]^{+} 1385.5899$, found 1385.5862 .

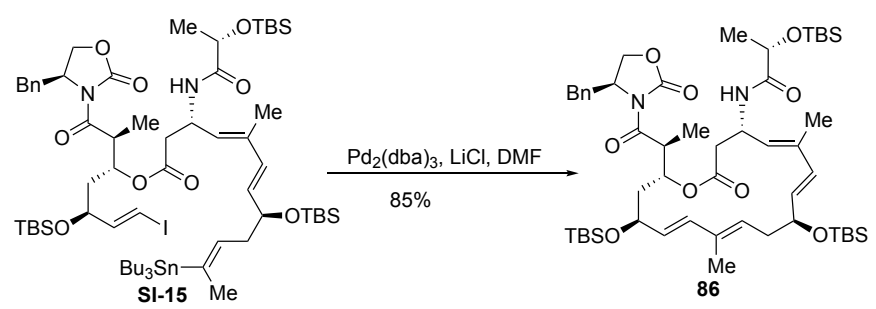

A 500-mL round-bottom flask containing $\mathrm{LiCl}(0.92 \mathrm{~g}, 21.67 \mathrm{mmol}, 30$ equiv. $)$ was flame dried under vacumm for $5 \mathrm{~min}$. After cooling to the $23^{\circ} \mathrm{C}$, DMF $(350 \mathrm{~mL})$ and Stille Coupling precursor SI-15 (1.00 g, $0.72 \mathrm{mmol}, 1$ equiv) was added. The vessel was evacuated and filled with nitrogen (this process was repeated a total of 3 times). $\mathrm{Pd}_{2}(\mathrm{dba})_{3}(132 \mathrm{mg}, 0.14 \mathrm{mmol}, 0.2$ equiv) was added in one portion. A stream of argon was passed through the solution for $10 \mathrm{~min}$. After $1 \mathrm{~h}$, the mixture was concentrated and resulting crude residue was purified by flash chromatography (silica gel, eluent: EtOAc:hexanes $=1: 4$ ) to afford product 86 (594 $\mathrm{mg}, 85 \%)$ as a light yellow foam.

TLC (EtOAc:hexanes = 1:3): $\mathrm{R}_{f}=0.60(\mathrm{UV})$.

${ }^{1} \mathbf{H}$ NMR $\left(400 \mathrm{MHz}, \mathrm{CDCl}_{3}\right) \delta 7.35-7.30(\mathrm{~m}, 2 \mathrm{H}), 7.29-7.23(\mathrm{~m}, 3 \mathrm{H}), 6.69(\mathrm{~d}, J=7.2 \mathrm{~Hz}, 1 \mathrm{H}), 5.96$ $(\mathrm{d}, J=16.0 \mathrm{~Hz}, 1 \mathrm{H}), 5.69(\mathrm{~d}, J=16.0 \mathrm{~Hz}, 1 \mathrm{H}), 5.55(\mathrm{dd}, J=16.0,6.5 \mathrm{~Hz}, 1 \mathrm{H}), 5.38(\mathrm{dd}, J=16.0,6.5 \mathrm{~Hz}$, $1 \mathrm{H}), 5.32-5.30(\mathrm{~m}, 1 \mathrm{H}), 5.14-5.10(\mathrm{~m}, 1 \mathrm{H}), 5.00-4.98(\mathrm{~m}, 2 \mathrm{H}), 4.60-4.56(\mathrm{~m}, 1 \mathrm{H}), 4.23-4.08(\mathrm{~m}$, $5 \mathrm{H}), 3.94(\mathrm{dd}, J=8.8,6.0,2.9,1.2 \mathrm{~Hz}, 1 \mathrm{H}), 3.25(\mathrm{dd}, J=14.0,3.5 \mathrm{~Hz}, 1 \mathrm{H}), 2.82-2.74(\mathrm{~m}, 2 \mathrm{H}), 2.44-$ $2.32(\mathrm{~m}, 1 \mathrm{H}), 2.81(\mathrm{dd}, J=14.0,9.3 \mathrm{~Hz}, 1 \mathrm{H}), 2.64(\mathrm{~d}, J=5.8 \mathrm{~Hz}, 1 \mathrm{H}), 2.37-2.30(\mathrm{~m}, 1 \mathrm{H}), 2.02-1.88$ $(\mathrm{m}, 3 \mathrm{H}), 1.95(\mathrm{t}, J=5.0 \mathrm{~Hz}, 2 \mathrm{H}), 1.79(\mathrm{~s}, 3 \mathrm{H}), 1.55(\mathrm{~s}, 3 \mathrm{H}), 1.38(\mathrm{~d}, J=7.0 \mathrm{~Hz}, 3 \mathrm{H}), 1.21(\mathrm{~d}, J=7.0 \mathrm{~Hz}$, $3 \mathrm{H}), 0.91(\mathrm{~s}, 9 \mathrm{H}), 0.90(\mathrm{~s}, 9 \mathrm{H}), 0.88(\mathrm{~s}, 9 \mathrm{H}), 0.11(\mathrm{~s}, 3 \mathrm{H}), 0.08(\mathrm{~s}, 3 \mathrm{H}), 0.07(\mathrm{~s}, 3 \mathrm{H}), 0.06(\mathrm{~s}, 6 \mathrm{H}), 0.02(\mathrm{~s}$, $3 \mathrm{H})$.

${ }^{13}$ C NMR (100 MHz, $\left.\mathrm{CDCl}_{3}\right): \delta 173.8,173.5,169.5,153.6,137.4,136.8,135.4,135.1 .133 .6,131.7,129.5$, 128.9, 128.6, 127.8, 127.6, 127.2, 75.4, 71.7, 70.6, 66.3, 55.8, 43.6, 42.1, 41.0, 39.3, 38.0, 37.4, 25.89, $25.84,25.7,21.8,18.2,18.1,18.0,12.9,12.8,10.3,-4.0,-4.4,-4.61,-4.66,-4.7,-5.4$.

HRMS-ESI m/z cacld for $\mathrm{C}_{52} \mathrm{H}_{87} \mathrm{~N}_{2} \mathrm{O}_{9} \mathrm{Si}_{3}[\mathrm{M}+\mathrm{H}]^{+}$967.5719, found 967.5776. 


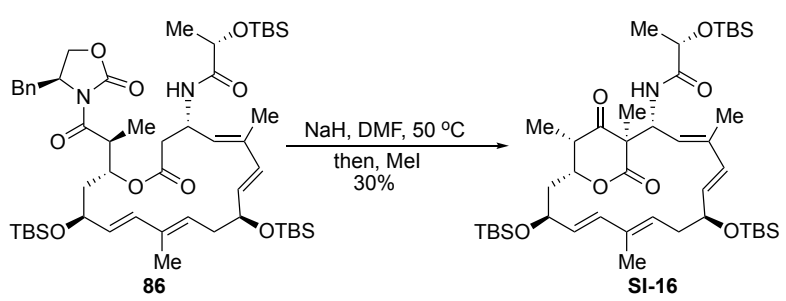

A $100-\mathrm{mL}$ round-bottom flask containing $60 \% \mathrm{NaH}(0.95 \mathrm{~g}, 2.58 \mathrm{mmol}, 5$ equiv.) and macrocycle 86 (500 $\mathrm{mg}, 0.52 \mathrm{mmol}, 1$ equiv.) was evacuated and filled with nitrogen (this process was repeated a total of 3 times) and was sealed with a rubber septum. DMF $(60 \mathrm{~mL})$ was added and the resulting solution was stirred at $50{ }^{\circ} \mathrm{C}$, resulting in a pink solution. After $1 \mathrm{~h}$, the reaction mixture was cooled to $23^{\circ} \mathrm{C}$. MeI $(1.47 \mathrm{~g}, 10.34$ mmol, 20 equiv.) was added to the reaction mixture. The reaction mixture was further stirred for $3 \mathrm{~h}$. Saturated aqueous $\mathrm{NH}_{4} \mathrm{Cl}$ solution $(50 \mathrm{~mL})$ was added. The biphasic mixture was transferred to a separatory funnel and the layers were separated. The aqueous layer was extracted with ethyl acetate $(2 \times 50 \mathrm{~mL})$. The combined organic layers were washed with water $(30 \mathrm{~mL})$ and saturated aqueous sodium chloride solution $(50 \mathrm{~mL})$ and the washed solution was dried $\left(\mathrm{Na}_{2} \mathrm{SO}_{4}\right)$. The dried solution was filtered and the filtrate was concentrated. The crude residue was purified by flash chromatography (silica gel, eluent: EtOAc:hexanes $=1: 10)$ to afford desired product SI-16 (122 $\mathrm{mg}, 30 \%)$ as a colorless oil.

TLC (EtOAc:hexanes = 1:3): $\mathrm{R}_{f}=0.70(\mathrm{UV})$.

${ }^{1}$ H NMR $\left(400 \mathrm{MHz}, \mathrm{CDCl}_{3}\right) \delta 7.49(\mathrm{~d}, J=10.2 \mathrm{~Hz}, 1 \mathrm{H}), 6.08(\mathrm{~d}, J=16.2 \mathrm{~Hz}, 1 \mathrm{H}), 5.60(\mathrm{~d}, J=16.0 \mathrm{~Hz}$, $1 \mathrm{H}), 5.55-5.45(\mathrm{~m}, 2 \mathrm{H}), 5.35(\mathrm{dd}, J=16.0,6.0 \mathrm{~Hz}, 1 \mathrm{H}), 5.25(\mathrm{dd}, J=9.8,6.0 \mathrm{~Hz}, 1 \mathrm{H}), 4.88(\mathrm{~d}, J=10.5$ $\mathrm{Hz}, 1 \mathrm{H}), 4.58(\mathrm{q}, J=5.2 \mathrm{~Hz}, 1 \mathrm{H}), 4.33(\mathrm{td}, J=5.2,2.0 \mathrm{~Hz}, 1 \mathrm{H}), 4.21(\mathrm{q}, J=6.8 \mathrm{~Hz}, 1 \mathrm{H}), 4.09-4.03(\mathrm{~m}$, $1 \mathrm{H}), 2.77(\mathrm{qd}, J=7.0,2.3 \mathrm{~Hz}, 1 \mathrm{H}), 2.45-2.41(\mathrm{~m}, 1 \mathrm{H}), 2.38-2.30(\mathrm{~m}, 1 \mathrm{H}), 2.12-2.06(\mathrm{~m}, 1 \mathrm{H}), 2.01-$ $1.94(\mathrm{~m}, 1 \mathrm{H}), 1.84(\mathrm{~s}, 3 \mathrm{H}), 1.62(\mathrm{~d}, J=7.0 \mathrm{~Hz}, 3 \mathrm{H}), 1.43(\mathrm{~s}, 3 \mathrm{H}), 1.42(\mathrm{~d}, J=7.0 \mathrm{~Hz}, 3 \mathrm{H}), 1.25(\mathrm{~d}, J=7.0$ $\mathrm{Hz}, 3 \mathrm{H}), 0.93$ (s, 9H), 0.89 (s, 9H), 0.88 (s, 9H), 0.10 (s, 3H), 0.073 (s, 3H), 0.070 (s, 3H), 0.05 (s, 3H), $0.04(\mathrm{~s}, 3 \mathrm{H}), 0.03(\mathrm{~s}, 3 \mathrm{H})$.

${ }^{13} \mathbf{C}$ NMR $\left(100 \mathrm{MHz}, \mathrm{CDCl}_{3}\right): \delta 207.9,174.0,173.9,138.1,135.0,134.6,133.6,133.3,128.3,127.4,125.6$, $75.2,73.8,70.1,69.5,56.9,49.7,46.2,39.1,36.9,25.85,25.82,25.7,23.3,22.1,18.2,18.1,18.0,13.4$, $12.5,9.9,-4.4,-4.5,-4.7,-4.8,-4.9,-5.2$.

HRMS-ESI m/z cacld for $\mathrm{C}_{43} \mathrm{H}_{78} \mathrm{NO}_{7} \mathrm{Si}_{3}[\mathrm{M}+\mathrm{H}]^{+} 804.5086$, found 804.5072.
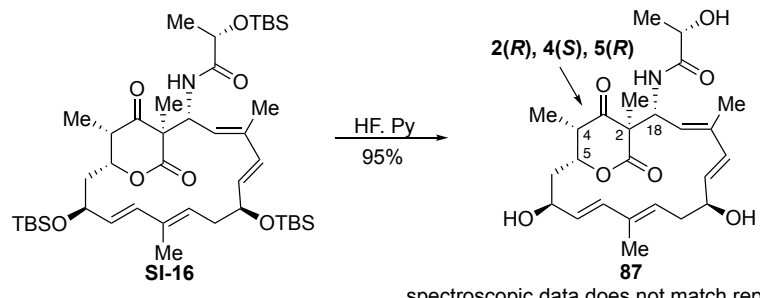

spectroscopic data does not match reported data

A 25-mL plastic vial containing a solution of SI-16 (30.0 mg, $0.037 \mathrm{mmol}, 1$ equiv.) and THF (8 mL) was cooled to $0^{\circ} \mathrm{C}$ by means of an ice-water bath. Py•HF $(\sim 70 \% \mathrm{HF})(800 \mu \mathrm{L})$ was added dropwise, and the vessel was removed from the cooling bath and the system was allowed to warm to $23^{\circ} \mathrm{C}$. After $5 \mathrm{~h}, \mathrm{NaHCO}_{3}$ $(970 \mathrm{mg}$ ) was added and the mixture was rapidly stirred. After $15 \mathrm{~min}$, the mixture was filtered through a pad of Celite and the filtrate was concentrated. The crude residue was purified by flash chromatography ( silica gel, eluent: acetone:hexanes $=2: 1)$ to afford desired product $87(16.7 \mathrm{mg}, 95 \%$ ) as a white solid.

TLC (EtOAc: $\mathrm{MeOH}=30: 1): \mathrm{R}_{f}=0.40(\mathrm{UV})$.

${ }^{1}$ H NMR $\left(400 \mathrm{MHz}, \mathrm{CD}_{3} \mathrm{OD}\right) \delta 6.23(\mathrm{~d}, J=16.4 \mathrm{~Hz}, 1 \mathrm{H}), 5.79(\mathrm{~d}, J=16.0 \mathrm{~Hz}, 1 \mathrm{H}), 5.55-5.42(\mathrm{~m}, 3 \mathrm{H})$, $5.35(\mathrm{dd}, J=16.0,6.0 \mathrm{~Hz}, 1 \mathrm{H}), 4.99(\mathrm{~d}, J=11.0 \mathrm{~Hz}, 1 \mathrm{H}), 4.45(\mathrm{td}, J=8.0,3.0 \mathrm{~Hz}, 1 \mathrm{H}), 4.26(\mathrm{td}, J=5.2$, 
$2.4 \mathrm{~Hz}, 1 \mathrm{H}), 4.15-4.07(\mathrm{~m}, 2 \mathrm{H}), 2.73(\mathrm{qd}, J=7.0,2.3 \mathrm{~Hz}, 1 \mathrm{H}), 2.50(\mathrm{td}, J=11.0,3.2 \mathrm{~Hz}, 1 \mathrm{H}), 2.31(\mathrm{q}$, $J=12.0 \mathrm{~Hz}, 1 \mathrm{H}), 2.14(\mathrm{ddd}, J=12.8,7.8,4.8 \mathrm{~Hz}, 1 \mathrm{H}), 1.85$ (ddd, $J=18.2,10.2,5.4 \mathrm{~Hz}, 1 \mathrm{H}), 1.77(\mathrm{~s}, 3 \mathrm{H})$, $1.65(\mathrm{~s}, 3 \mathrm{H}), 1.42(\mathrm{~s}, 3 \mathrm{H}), 1.38(\mathrm{~d}, J=7.0 \mathrm{~Hz}, 3 \mathrm{H}), 1.27(\mathrm{~d}, J=7.0 \mathrm{~Hz}, 3 \mathrm{H})$.

${ }^{13} \mathrm{C}$ NMR $\left(100 \mathrm{MHz}, \mathrm{CD}_{3} \mathrm{OD}\right): \delta 206.3,174.0,172.3,136.0,134.6,132.9,130.8,126.3,126.1,124.1,72.6$, $72.1,66.9,66.2,55.2,47.9,44.7,36.8,33.8,21.0,18.3,10.5,9.8,7.4$.

HRMS-ESI m/z cacld for $\mathrm{C}_{25} \mathrm{H}_{35} \mathrm{NO}_{7} \mathrm{Na}[\mathrm{M}+\mathrm{Na}]^{+}$484.2311, found 484.2296.

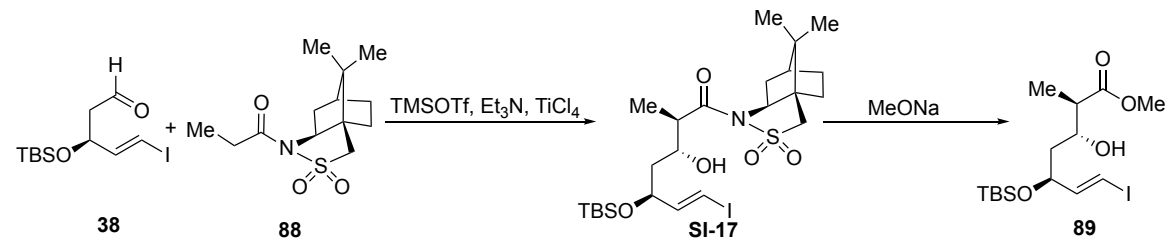

A 50-mL round-bottom flask containing propionated Oppolzer's sultam 88 (10.0 g, $37.0 \mathrm{mmol}, 1.8$ equiv.), triethylamine (7.17 mL, $41.1 \mathrm{mmol}, 2$ equiv.), and DCM $(20.0 \mathrm{~mL})$ was added trimethylsilyl trifluormethanesulfonate $(9.14 \mathrm{~g}, 41.1 \mathrm{mmol}, 2.0$ equiv). The reaction mixture was stirred for 20 hours. $\mathrm{TiCl}_{4}$ (1.0 M in DCM, $20.5 \mathrm{~mL}, 20.5 \mathrm{mmol}, 1$ equiv.) was added to aldehyde $\mathbf{3 8}$ (7.0 g, $20.5 \mathrm{mmol}, 1$ equiv.) in $20 \mathrm{~mL}$ DCM in a separate $150-\mathrm{mL}$ round-bottom flask at $-78^{\circ} \mathrm{C}$ over the period of $5 \mathrm{~min}$. The solution of the preformed ketene acetal was added to this solution via cannula over $10 \mathrm{~min}$. The transfer was quantitated with $10 \mathrm{~mL} \mathrm{DCM}$. The resulting mixture was stirred for $2 \mathrm{~h}$ at $-78{ }^{\circ} \mathrm{C}$ before a saturated aqueous solution of $\mathrm{NH}_{4} \mathrm{Cl}(50 \mathrm{~mL})$ was added and the reaction mixture was allowed to warm to $23^{\circ} \mathrm{C}$. The biphasic mixture was transferred to a separatory funnel and the layers were separated. The aqueous layer was extracted with DCM $(2 \times 50 \mathrm{~mL})$. The combined organic layers were washed with water $(50 \mathrm{~mL})$ and saturated aqueous sodium chloride solution $(50 \mathrm{~mL})$ and the washed solution was dried $\left(\mathrm{Na}_{2} \mathrm{SO}_{4}\right)$. The dried solution was filtered and the filtrate was concentrated. The crude residue was purified by flash chromatography (silica gel, eluent: EtOAc:hexanes = 1:5) to afford anti-aldol product SI-17 (11.3 g, 90\%) as a colorless foam.

TLC (EtOAc:hexanes =1:3): $\mathrm{R}_{f}=0.40(\mathrm{UV})$.

${ }^{1}$ H NMR $\left(400 \mathrm{MHz}, \mathrm{CDCl}_{3}\right) \delta 6.52(\mathrm{dd}, J=14.4,7.0 \mathrm{~Hz}, 1 \mathrm{H}), 6.29(\mathrm{~d}, J=14.4 \mathrm{~Hz}, 1 \mathrm{H}), 4.37(\mathrm{q}, J=6.8$ $\mathrm{Hz}, 1 \mathrm{H}), 3.91(\mathrm{dd}, J=6.0,5.2 \mathrm{~Hz}, 1 \mathrm{H}), 3.77-3.74(\mathrm{~m}, 1 \mathrm{H}), 3.54(\mathrm{~d}, J=15.0 \mathrm{~Hz}, 1 \mathrm{H}), 3.46(\mathrm{~d}, J=15.0$ $\mathrm{Hz}, 1 \mathrm{H}), 3.18$ (q, $J=8.0 \mathrm{~Hz}, 1 \mathrm{H}), 2.92$ (d, $J=8.0 \mathrm{~Hz}, 1 \mathrm{H}), 2.12-2.09$ (m, 2H), $1.94-1.87$ (m, 3H), 1.70 $-1.65(\mathrm{~m}, 2 \mathrm{H}), 1.41-1.36(\mathrm{~m}, 2 \mathrm{H}), 1.24(\mathrm{~d}, J=7.0 \mathrm{~Hz}, 3 \mathrm{H}), 1.18(\mathrm{~s}, 3 \mathrm{H}), 0.99$ (s, 3H), 0.87 (s, 9H), 0.06 $(\mathrm{s}, 3 \mathrm{H}), 0.04(\mathrm{~s}, 3 \mathrm{H})$.

${ }^{13} \mathbf{C}$ NMR (100 MHz, $\left.\mathrm{CDCl}_{3}\right): \delta 175.3,148.1,73.4,72.4,65.3,53.1,48.3,47.7,44.7,44.6,43.1,38.5,32.9$, 26.4, 25.7, 20.9, 19.9, 18.0, 14.0, -4.3, -4.9.

HRMS-ESI $\mathrm{m} / \mathrm{z}$ cacld for $\mathrm{C}_{24} \mathrm{H}_{42} \mathrm{INO}_{5} \mathrm{SSiNa}[\mathrm{M}+\mathrm{Na}]^{+}$634.1495, found 634.1485.

A 250-mL round-bottom flask containing SI-17 (10.0 g, $161.3 \mathrm{mmol}, 1$ equiv.) in $50 \mathrm{~mL}$ THF and $50 \mathrm{~mL}$ $\mathrm{MeOH}$ was evacuated and filled with nitrogen (this process was repeated a total of 3 times) and was sealed with a rubber septum. $\mathrm{MeONa}(5.4 \mathrm{M}$ in $\mathrm{MeOH}, 15.1 \mathrm{~mL}, 81.7 \mathrm{mmol}, 5$ equiv.) was added slowly to the reaction mixture. The resulting solution was stirred for 1 hour. Saturated aqueous $\mathrm{NH}_{4} \mathrm{Cl}$ solution $(50 \mathrm{~mL})$ was added. The biphasic mixture was transferred to a separatory funnel and the layers were separated. The aqueous layer was extracted with ethyl acetate $(2 \times 50 \mathrm{~mL})$. The combined organic layers were washed with water $(30 \mathrm{~mL})$ and saturated aqueous sodium chloride solution $(50 \mathrm{~mL})$ and the washed solution was dried $\left(\mathrm{Na}_{2} \mathrm{SO}_{4}\right)$. The dried solution was filtered and the filtrate was concentrated. The crude residue was purified by flash chromatography (silica gel, eluent: EtOAc:hexanes $=1: 5)$ to afford methyl ester 89 (7.50 g, 94\%) as a colorless oil. 
TLC (EtOAc:hexanes = 1:3): $\mathrm{R}_{f}=0.60(\mathrm{UV})$.

${ }^{1}$ H NMR $\left(400 \mathrm{MHz}, \mathrm{CDCl}_{3}\right) \delta 6.55(\mathrm{dd}, J=14.4,7.0 \mathrm{~Hz}, 1 \mathrm{H}), 6.31(\mathrm{~d}, J=14.4 \mathrm{~Hz}, 1 \mathrm{H}), 4.38(\mathrm{q}, J=6.8$ $\mathrm{Hz}, 1 \mathrm{H}), 3.85-3.82(\mathrm{~m}, 1 \mathrm{H}), 3.71(\mathrm{~s}, 3 \mathrm{H}), 3.19(\mathrm{~d}, J=1.5 \mathrm{~Hz}, 1 \mathrm{H}), 2.56(\mathrm{q}, J=8.0 \mathrm{~Hz}, 1 \mathrm{H}), 1.69-1.66$ $(\mathrm{m}, 2 \mathrm{H}), 1.18(\mathrm{~d}, J=7.0 \mathrm{~Hz}, 3 \mathrm{H}), 0.89(\mathrm{~s}, 9 \mathrm{H}), 0.09$ (s, 3H), $0.06(\mathrm{~s}, 3 \mathrm{H})$.

${ }^{13}$ C NMR (100 MHz, $\left.\mathrm{CDCl}_{3}\right): \delta 175.7,148.3,77.1,74.6,71.0,51.7,45.3,41.4,25.8,18.0,13.4,-4.2,-4.8$.

HRMS-ESI m/z cacld for $\mathrm{C}_{15} \mathrm{H}_{29} \mathrm{IO}_{4} \mathrm{SiNa}[\mathrm{M}+\mathrm{Na}]^{+}$451.0776, found 451.0767.

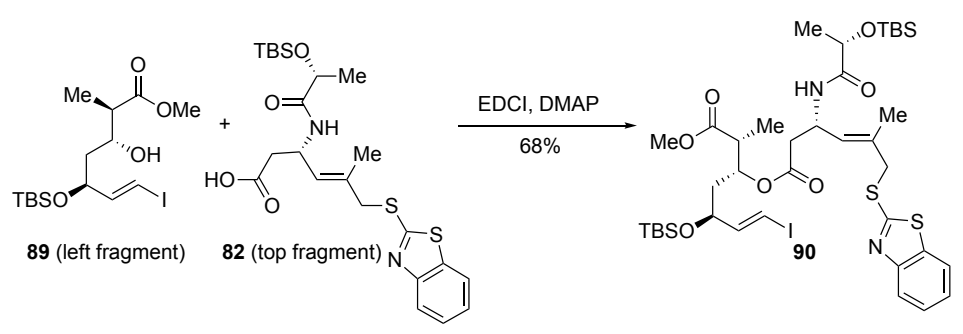

A 100-mL round-bottom flask containing acid 82 (3.46 g, $7.00 \mathrm{mmol}, 1.2$ equiv.) and alcohol 89 (2.50 g, 5.84 mmol, 1 equiv.) was added $50 \mathrm{~mL}$ chloroform. DMAP (3.56 g, $29.2 \mathrm{mmol}, 5$ equiv.) and EDCI (5.59 $\mathrm{g}, 29.2 \mathrm{mmol}, 5$ equiv) was added in sequence. The reaction mixture was heated at $50{ }^{\circ} \mathrm{C}$ for $3 \mathrm{~h}$ and was then concentrated. The crude residue was purified by flash chromatography (silica gel, eluent: EtOAc:hexanes $=1: 3)$ to afford ester $90(3.59 \mathrm{~g} 68 \%)$ as a colorless oil.

TLC (EtOAc:hexanes = 1:3): $\mathrm{R}_{f}=0.38(\mathrm{UV})$.

${ }^{1}$ H NMR $\left(400 \mathrm{MHz}, \mathrm{CDCl}_{3}\right) \delta 7.86(\mathrm{~d}, J=8.2 \mathrm{~Hz}, 1 \mathrm{H}), 7.75(\mathrm{~d}, J=8.2 \mathrm{~Hz}, 1 \mathrm{H}), 7.42(\mathrm{td}, J=7.5,1.6 \mathrm{~Hz}$, $1 \mathrm{H}), 7.32-7.25(\mathrm{~m}, 2 \mathrm{H}), 6.51(\mathrm{dd}, J=14.8,6.8 \mathrm{~Hz}, 1 \mathrm{H}), 6.28(\mathrm{dd}, J=14.4,1.2 \mathrm{~Hz}, 1 \mathrm{H}), 5.66(\mathrm{dd}, J=9.5$, $0.8 \mathrm{~Hz}, 1 \mathrm{H}), 5.21(\mathrm{dt}, J=9.5,2.8 \mathrm{~Hz}, 1 \mathrm{H}), 5.02-4.98(\mathrm{~m}, 1 \mathrm{H}), 4.20-4.14(\mathrm{~m}, 2 \mathrm{H}), 4.02(\mathrm{~s}, 2 \mathrm{H}), 3.69(\mathrm{~s}$, $3 \mathrm{H}), 2.79(\mathrm{dt}, J=14.0,7.0 \mathrm{~Hz}, 1 \mathrm{H}), 2.60(\mathrm{~d}, J=6.0, \mathrm{~Hz}, 2 \mathrm{H}), 1.92(\mathrm{~s}, 3 \mathrm{H}), 1.83-1.80(\mathrm{~m}, 1 \mathrm{H}), 1.77-$ $1.73(\mathrm{~m}, 1 \mathrm{H}), 1.36(\mathrm{~d}, J=7.0 \mathrm{~Hz}, 3 \mathrm{H}), 1.11$ (d, $J=7.0 \mathrm{~Hz}, 3 \mathrm{H}), 0.90(\mathrm{~s}, 9 \mathrm{H}), 0.89$ (s, 9H), 0.07 (s, 3H), $0.054(\mathrm{~s}, 3 \mathrm{H}), 0.051(\mathrm{~s}, 3 \mathrm{H}), 0.04(\mathrm{~s}, 3 \mathrm{H})$.

${ }^{13}$ C NMR (100 MHz, $\left.\mathrm{CDCl}_{3}\right): \delta 173.4,173.1,170.1,166.5,153.1,147.8,135.2,134.7,128.1,126.0,124.2$, $121.5,120.9,76.5,72.0,71.0,69.8,51.9,43.2,42.8,41.8,39.1,38.6,25.77,25.72,21.9,18.1,18.0,16.2$, $12.0,-4.6,-4.7,-4.9,-5.3$.

HRMS-ESI $\mathrm{m} / \mathrm{z}$ cacld for $\mathrm{C}_{38} \mathrm{H}_{61} \mathrm{IN}_{2} \mathrm{O}_{7} \mathrm{~S}_{2} \mathrm{Si}_{2} \mathrm{Na}[\mathrm{M}+\mathrm{Na}]^{+}$927.2401, found 927.2421.

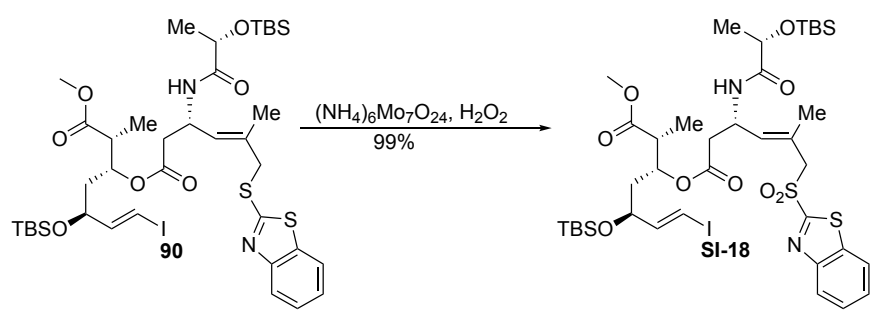

A $250-\mathrm{mL}$ round-bottom flask containing thioether 90 (3.00 g, $3.31 \mathrm{mmol}, 1$ equiv.), ethanol (50 $\mathrm{mL})$, and acetonitrile $(50 \mathrm{~mL})$ was cooled to $0{ }^{\circ} \mathrm{C}$ by means of an ice-water bath. $\left(\mathrm{NH}_{4}\right)_{6} \mathrm{Mo}_{7} \mathrm{O}_{24} \bullet 4 \mathrm{H}_{2} \mathrm{O}(410 \mathrm{mg}, 0.33$ mmol, 0.1 equiv.) dissolved in $\mathrm{H}_{2} \mathrm{O}_{2}(30 \%$ solution in water, $10.0 \mathrm{~mL})$ was added to the reaction mixture. The vessel was removed from the cooling bath and the system was allowed to warm to $23{ }^{\circ} \mathrm{C}$. After $1 \mathrm{~h}$, the organic phase was removed under reduced pressure and the resulting solution was transferred to a separatory funnel and was extracted with DCM $(3 \times 50 \mathrm{~mL})$. The combined organic layers were washed with saturated aqueous sodium chloride solution $(50 \mathrm{~mL})$ and the washed solution was dried $\left(\mathrm{Na}_{2} \mathrm{SO}_{4}\right)$. The dried solution was filtered, and the filtrate was concentrated. The resulting crude residue was purified by 
flash chromatography (silica gel, eluent: EtOAc:hexanes = 1:2) to afford sulfone SI-18 (3.11 g, 99\%) as a colorless oil.

TLC (EtOAc:hexanes = 1:3): $\mathrm{R}_{f}=0.25(\mathrm{UV})$.

${ }^{1} \mathbf{H}$ NMR $\left(400 \mathrm{MHz}, \mathrm{CDCl}_{3}\right) \delta 8.22(\mathrm{~d}, J=8.2 \mathrm{~Hz}, 1 \mathrm{H}), 8.02(\mathrm{~d}, J=8.2 \mathrm{~Hz}, 1 \mathrm{H}), 7.66-7.58(\mathrm{~m}, 2 \mathrm{H}), 7.32$ $(\mathrm{d}, J=8.2 \mathrm{~Hz}, 1 \mathrm{H}), 6.48(\mathrm{dd}, J=14.8,6.8 \mathrm{~Hz}, 1 \mathrm{H}), 6.24(\mathrm{dd}, J=14.4,1.2 \mathrm{~Hz}, 1 \mathrm{H}), 5.67(\mathrm{dd}, J=9.5,0.8$ $\mathrm{Hz}, 1 \mathrm{H}), 5.18(\mathrm{dt}, J=9.5,2.8 \mathrm{~Hz}, 1 \mathrm{H}), 4.96-4.91(\mathrm{~m}, 1 \mathrm{H}), 4.15(\mathrm{~s}, 3 \mathrm{H}), 4.09$ (q, $J=6.0, \mathrm{~Hz}, 1 \mathrm{H}), 3.69$ $(\mathrm{s}, 3 \mathrm{H}), 2.79(\mathrm{dt}, J=14.0,7.0 \mathrm{~Hz}, 1 \mathrm{H}), 2.55(\mathrm{~d}, J=6.0, \mathrm{~Hz}, 1 \mathrm{H}), 2.46(\mathrm{~d}, J=6.0, \mathrm{~Hz}, 1 \mathrm{H}), 1.94(\mathrm{~s}, 3 \mathrm{H})$, $1.84-1.77(\mathrm{~m}, 1 \mathrm{H}), 1.75-1.68(\mathrm{~m}, 1 \mathrm{H}), 1.32(\mathrm{~d}, J=7.0 \mathrm{~Hz}, 3 \mathrm{H}), 1.11(\mathrm{~d}, J=7.0 \mathrm{~Hz}, 3 \mathrm{H}), 0.91(\mathrm{~s}, 9 \mathrm{H})$, $0.88(\mathrm{~s}, 9 \mathrm{H}), 0.07(\mathrm{~s}, 3 \mathrm{H}), 0.06(\mathrm{~s}, 3 \mathrm{H}), 0.03(\mathrm{~s}, 3 \mathrm{H}), 0.02(\mathrm{~s}, 3 \mathrm{H})$.

${ }^{13}$ C NMR (100 MHz, $\left.\mathrm{CDCl}_{3}\right): \delta 173.5,173.1,169.9,165.8,152.6,147.8,136.9,135.5,128.0,127.6,126.1$, 125.4, 122.4, 76.4, 71.9, 71.0, 69.7, 63.8, 51.9, 43.3, 42.8, 38.6, 38.5, 25.76, 25.75, 21.8, 18.1, 18.0, 17.7, $11.9,-4.6,-4.7,-4.9,-5.2$.

HRMS-ESI m/z cacld for $\mathrm{C}_{38} \mathrm{H}_{61} \mathrm{IN}_{2} \mathrm{O}_{9} \mathrm{~S}_{2} \mathrm{Si}_{2} \mathrm{Na}[\mathrm{M}+\mathrm{Na}]^{+}$959.2299, found 959.2335.

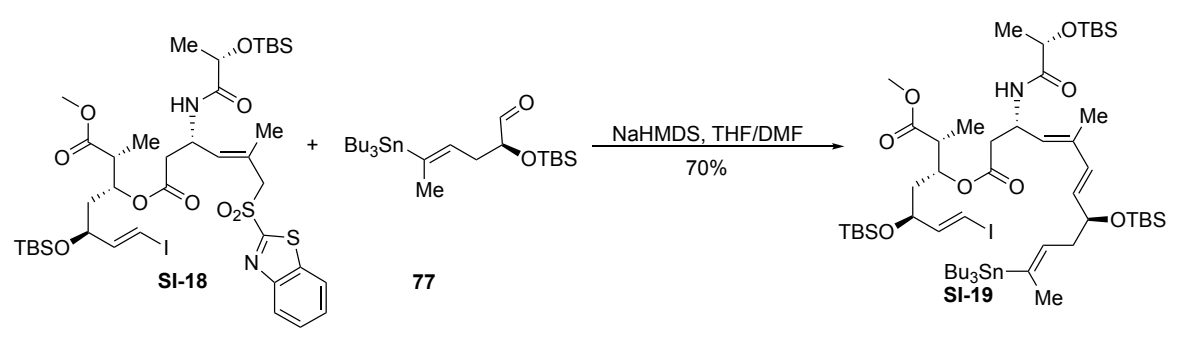

A 100-mL round-bottom flask containing sulfone SI-18 (2.00 g, $2.13 \mathrm{mmol}, 1$ equiv.) and aldehyde 77 $(1.55 \mathrm{~g}, 2.99 \mathrm{mmol}, 1.4$ equiv.) was evacuated and flushed with nitrogen (this process was repeated a total of 3 times $)$ and was then sealed with a rubber septum. DMF $(50 \mathrm{~mL})$ and THF $(50 \mathrm{~mL})$ were added, resulting a colorless solution, and the system was cooled to $-78^{\circ} \mathrm{C}$ in a dry ice-acetone bath. NaHMDS (2.0 M in THF, $1.49 \mathrm{~mL}, 2.99 \mathrm{mmol}, 1.40$ equiv.) was added dropwise over $20 \mathrm{~min}$. After $1 \mathrm{~h}$, saturated aqueous $\mathrm{NH}_{4} \mathrm{Cl}(50 \mathrm{~mL})$ were added. The vessel was removed from the cooling bath and the system was allowed to warm to $23{ }^{\circ} \mathrm{C}$. The biphasic mixture was transferred to a separatory funnel and the layers were separated. The aqueous layer was extracted with ethyl acetate $(2 \times 50 \mathrm{~mL})$. The combined organic layers were washed with water $(30 \mathrm{~mL})$ and saturated aqueous sodium chloride solution $(50 \mathrm{~mL})$ and the washed solution was dried $\left(\mathrm{Na}_{2} \mathrm{SO}_{4}\right)$. The dried solution was filtered and the filtrate was concentrated. The crude residue was purified by flash chromatography (silica gel, eluent: EtOAc:hexanes = 1:10) to afford diene SI-19 (1.85 g, $70 \%)$ as a colorless oil.

TLC (EtOAc:hexanes = 1:3): $\mathrm{R}_{f}=0.72(\mathrm{UV})$.

${ }^{1}$ H NMR $\left(400 \mathrm{MHz}, \mathrm{CDCl}_{3}\right) \delta 7.23(\mathrm{~d}, J=8.0 \mathrm{~Hz}, 1 \mathrm{H}), 6.51(\mathrm{dd}, J=14.8,6.8 \mathrm{~Hz}, 1 \mathrm{H}), 6.29(\mathrm{dd}, J=14.4$, $1.2 \mathrm{~Hz}, 1 \mathrm{H}), 6.11(\mathrm{~d}, J=16.4 \mathrm{~Hz}, 1 \mathrm{H}), 5.72(\mathrm{dd}, J=16.4,6.5 \mathrm{~Hz}, 1 \mathrm{H}), 5.61(\mathrm{td}, J=7.0,2.8 \mathrm{~Hz}, 1 \mathrm{H}), 5.44$ $(\mathrm{d}, J=9.0 \mathrm{~Hz}, 1 \mathrm{H}), 5.21(\mathrm{td}, J=9.0,2.8 \mathrm{~Hz}, 1 \mathrm{H}), 5.12-5.08(\mathrm{~m}, 1 \mathrm{H}), 4.21-4.16(\mathrm{~m}, 3 \mathrm{H}), 3.69(\mathrm{~s}, 3 \mathrm{H})$, $2.82(\mathrm{dd}, J=14.0,9.3 \mathrm{~Hz}, 1 \mathrm{H}), 2.64(\mathrm{~d}, J=5.8 \mathrm{~Hz}, 1 \mathrm{H}), 2.38-2.29(\mathrm{~m}, 1 \mathrm{H}), 1.83(\mathrm{~s}, 6 \mathrm{H}), 1.82-1.75(\mathrm{~m}$, 1H), $1.60(\mathrm{~s}, 3 \mathrm{H}), 1.51-1.47(\mathrm{~m}, 6 \mathrm{H}), 1.38(\mathrm{~d}, J=7.0 \mathrm{~Hz}, 3 \mathrm{H}), 1.35-1.29(\mathrm{~m}, 7 \mathrm{H}), 1.13(\mathrm{~d}, J=7.0 \mathrm{~Hz}$, $3 \mathrm{H}), 0.92-0.88(\mathrm{~m}, 43 \mathrm{H}), 0.10(\mathrm{~s}, 3 \mathrm{H}), 0.08(\mathrm{~s}, 3 \mathrm{H}), 0.06(\mathrm{~s}, 3 \mathrm{H}), 0.05(\mathrm{~s}, 3 \mathrm{H}), 0.04(\mathrm{~s}, 3 \mathrm{H}), 0.02(\mathrm{~s}, 3 \mathrm{H})$.

${ }^{13}$ C NMR $\left(100 \mathrm{MHz}, \mathrm{CDCl}_{3}\right): \delta 173.28,173.21,170.2,147.8,139.9,137.1,136.5,135.5,132.4,128.7$, 73.6, 72.0, 70.9, 69.9, 51.8, 43.2, 42.9, 39.4, 38.7, 37.3, 29.1, 27.4, 25.9, 25.7, 25.6, 21.9, 19.3, 18.2, 18.1, $18.0,13.7,13.0,11.9,9.0,-4.1,-4.60,-4.67,-4.74,-4.9,-5.4$.

HRMS-ESI m/z cacld for $\mathrm{C}_{55} \mathrm{H}_{106} \mathrm{NNO}_{8} \mathrm{Si}_{3} \mathrm{SnNa}[\mathrm{M}+\mathrm{Na}]^{+}$1262.5191, found 1262.5201. 

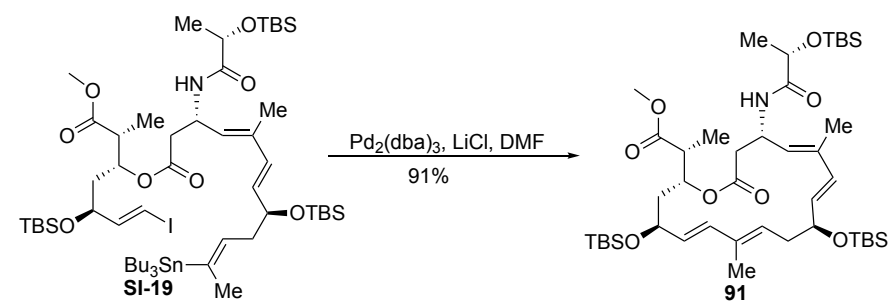

A 500-mL round-bottom flask containing $\mathrm{LiCl}(1.03 \mathrm{~g}, 24.20 \mathrm{mmol}, 30$ equiv.) was flame dried under vacumm for $5 \mathrm{~min}$. After cooling to the $23{ }^{\circ} \mathrm{C}$, DMF $(350 \mathrm{~mL})$ and Stille Coupling precursor SI-19 $(1.00 \mathrm{~g}$, $0.81 \mathrm{mmol}, 1$ equiv) was added. The vessel was evacuated and filled with nitrogen (this process was repeated a total of 3 times). $\operatorname{Pd}_{2}(\mathrm{dba})_{3}(148 \mathrm{mg}, 0.16 \mathrm{mmol}, 0.2$ equiv) was added in one portion. A stream of argon was passed through the solution for $10 \mathrm{~min}$, and was then stirred for 1 hour. The mixture was concentrated and resulting crude residue was purified by flash chromatography (silica gel, eluent: EtOAc:hexanes = 1:4) to afford product $91(602 \mathrm{mg}, 91 \%)$ as a light yellow foam.

TLC (EtOAc:hexanes = 1:3): $\mathrm{R}_{f}=0.60(\mathrm{UV})$.

${ }^{1}$ H NMR $\left(400 \mathrm{MHz}, \mathrm{CDCl}_{3}\right) \delta 6.81(\mathrm{~d}, J=7.2 \mathrm{~Hz}, 1 \mathrm{H}), 5.99(\mathrm{~d}, J=16.0 \mathrm{~Hz}, 1 \mathrm{H}), 5.69(\mathrm{~d}, J=16.0 \mathrm{~Hz}$, $1 \mathrm{H}), 5.54(\mathrm{dd}, J=16.0,6.5 \mathrm{~Hz}, 1 \mathrm{H}), 5.43(\mathrm{dd}, J=16.0,6.5 \mathrm{~Hz}, 1 \mathrm{H}), 5.21-5.17(\mathrm{~m}, 2 \mathrm{H}), 5.10-5.02(\mathrm{~m}$, $2 \mathrm{H}), 4.26-4.21(\mathrm{~m}, 1 \mathrm{H}), 4.18(\mathrm{q}, J=7.2 \mathrm{~Hz}, 1 \mathrm{H}), 4.11-4.05(\mathrm{~m}, 1 \mathrm{H}), 3.69(\mathrm{~s}, 3 \mathrm{H}), 2.91-2.87(\mathrm{~m}, 1 \mathrm{H})$, $2.79(\mathrm{dd}, J=14.0,9.3 \mathrm{~Hz}, 1 \mathrm{H}), 2.46-2.30(\mathrm{~m}, 3 \mathrm{H}), 2.00-1.90(\mathrm{~m}, 2 \mathrm{H}), 1.83(\mathrm{~s}, 3 \mathrm{H}), 1.53(\mathrm{~s}, 3 \mathrm{H}), 1.37$ $(\mathrm{d}, J=7.0 \mathrm{~Hz}, 3 \mathrm{H}), 1.14(\mathrm{~d}, J=7.0 \mathrm{~Hz}, 3 \mathrm{H}), 0.90(\mathrm{~s}, 18 \mathrm{H}), 0.87(\mathrm{~s}, 9 \mathrm{H}), 0.10(\mathrm{~s}, 3 \mathrm{H}), 0.08$ (s, 3H), 0.077 $(\mathrm{s}, 3 \mathrm{H}), 0.070(\mathrm{~s}, 3 \mathrm{H}), 0.05(\mathrm{~s}, 3 \mathrm{H}), 0.01(\mathrm{~s}, 3 \mathrm{H})$.

${ }^{13} \mathbf{C ~ N M R}\left(100 \mathrm{MHz}, \mathrm{CDCl}_{3}\right): \delta 173.7,173.6,169.2,137.5,136.2,135.5,133.4,131.8,128.6,127.8,127.4$, $75.2,71.9,71.7,70.0,51.8,43.7,43.4,39.8,39.7,37.5,25.9,25.8,25.7,21.8,18.3,18.1,18.0,13.0,12.8$, $12.5,-4.0,-4.4,-4.6,-4.7,-5.4$.

HRMS-ESI $\mathrm{m} / \mathrm{z}$ cacld for $\mathrm{C}_{43} \mathrm{H}_{79} \mathrm{NO}_{8} \mathrm{Si}_{3} \mathrm{Na}[\mathrm{M}+\mathrm{Na}]^{+}$844.5011, found 805.5015.

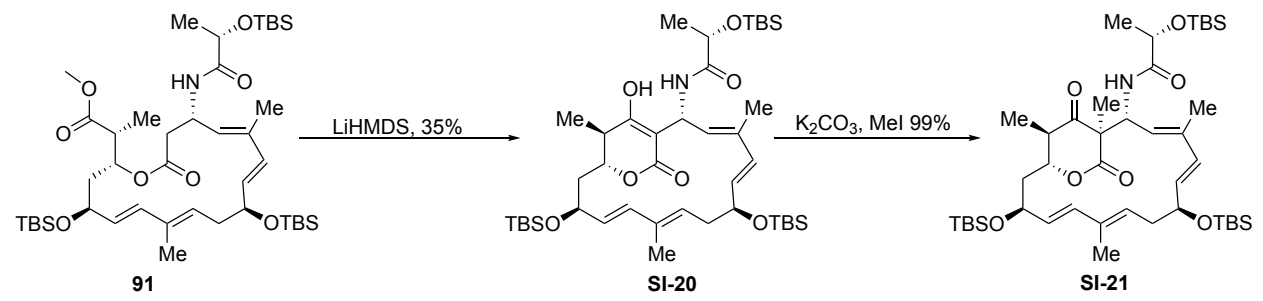

A 100-mL round-bottom flask containing macrocycle 91 (500 mg, $0.68 \mathrm{mmol}, 1$ equiv.) and THF (50 mL) was evacuated and filled with nitrogen (this process was repeated a total of 3 times) and was sealed with a rubber septum. LiHMDS (1.0 M in THF, $3.04 \mathrm{~mL}, 3.04 \mathrm{mmol}, 5$ equiv.) was added slowly to the reaction mixture. The resulting solution was stirred for 30 . Saturated aqueous $\mathrm{NH}_{4} \mathrm{Cl}$ solution $(50 \mathrm{~mL})$ was added. The biphasic mixture was transferred to a separatory funnel and the layers were separated. The aqueous layer was extracted with ethyl acetate $(2 \times 30 \mathrm{~mL})$. The combined organic layers were washed with water $(30 \mathrm{~mL})$ and saturated aqueous sodium chloride solution $(30 \mathrm{~mL})$ and the washed solution was dried $\left(\mathrm{Na}_{2} \mathrm{SO}_{4}\right)$. The dried solution was filtered and the filtrate was concentrated. The crude residue was purified by flash chromatography (silica gel, eluent: EtOAc:hexanes $=1: 10)$ to afford desired product SI-20 (168 $\mathrm{mg}, 35 \%)$ as a colorless oil

${ }^{1}$ H NMR $\left(400 \mathrm{MHz}, \mathrm{CDCl}_{3}\right) \delta 11.09(\mathrm{~s}, 1 \mathrm{H}), 7.71(\mathrm{~d}, J=8.2 \mathrm{~Hz}, 1 \mathrm{H}), 6.07(\mathrm{~d}, J=16.2 \mathrm{~Hz}, 1 \mathrm{H}), 5.95(\mathrm{~d}$, $J=16.0 \mathrm{~Hz}, 1 \mathrm{H}), 5.90(\mathrm{~d}, J=8.0 \mathrm{~Hz}, 1 \mathrm{H}), 5.33(\mathrm{t}, J=8.0 \mathrm{~Hz}, 1 \mathrm{H}), 5.26(\mathrm{dd}, J=16.0,8.0 \mathrm{~Hz}, 1 \mathrm{H}), 5.05$ $(\mathrm{dd}, J=9.8,5.0 \mathrm{~Hz}, 1 \mathrm{H}), 4.48(\mathrm{td}, J=5.2,2.0 \mathrm{~Hz}, 1 \mathrm{H}), 4.27(\mathrm{q}, J=6.8 \mathrm{~Hz}, 1 \mathrm{H}), 4.23-4.19(\mathrm{~m}, 1 \mathrm{H}), 3.39$ $(\mathrm{td}, J=7.0,1.3 \mathrm{~Hz}, 1 \mathrm{H}), 2.54-2.44(\mathrm{~m}, 3 \mathrm{H}), 1.96(\mathrm{td}, J=13.0,5.0 \mathrm{~Hz}, 1 \mathrm{H}), 1.70(\mathrm{~s}, 3 \mathrm{H}), 1.61(\mathrm{~s}, 3 \mathrm{H})$, 
$1.53(\mathrm{~s}, 3 \mathrm{H}), 1.35(\mathrm{~d}, J=7.0 \mathrm{~Hz}, 3 \mathrm{H}), 1.11(\mathrm{~d}, J=7.0 \mathrm{~Hz}, 3 \mathrm{H}), 0.97$ (s, 9H), $0.91(\mathrm{~s}, 9 \mathrm{H}), 0.87$ (s, 9H), $0.13(\mathrm{~s}, 3 \mathrm{H}), 0.12(\mathrm{~s}, 3 \mathrm{H}), 0.10(\mathrm{~s}, 3 \mathrm{H}), 0.07(\mathrm{~s}, 3 \mathrm{H}), 0.06(\mathrm{~s}, 3 \mathrm{H}), 0.03(\mathrm{~s}, 3 \mathrm{H}), 0.02(\mathrm{~s}, 3 \mathrm{H})$.

${ }^{13} \mathbf{C ~ N M R}\left(100 \mathrm{MHz}, \mathrm{CDCl}_{3}\right): \delta 177.2,170.7,166.7,136.7,134.1,133.6,133.1,132.2,129.1,127.8,106.7$, $79.2,74.9,71.9,69.1,44.7,38.6,38.2,37.4,25.9,25.8,25.7,21.4,18.2,18.1,18.0,12.76,12.72,10.8$, $0.001,-4.20,-4.23,-4.5,-4.6,-4.7,-5.2$.

The enol SI-20 (70.0 mg, $88.6 \mu \mathrm{mol}, 1$ equiv.) was dissolved in $10 \mathrm{~mL}$ acetone. $\mathrm{K}_{2} \mathrm{CO}_{3}(122 \mathrm{mg}, 886 \mu \mathrm{mol}$, 10. equiv.) and $\mathrm{MeI}(126 \mathrm{mg}, 886 \mu \mathrm{mol}, 10$. equiv.) were added to the solution sequentially. After $30 \mathrm{~min}$, the mixture was concentrated, and the crude residus was purified by flash chromatography (silica gel, eluent: EtOAc:hexanes $=1: 8)$ to afford desired product SI-21 $(71 \mathrm{mg}, 99 \%)$ as a colorless oil

TLC (EtOAc:hexanes = 1:3): $\mathrm{R}_{f}=0.70(\mathrm{UV})$.

${ }^{1} \mathbf{H}$ NMR $\left(400 \mathrm{MHz}, \mathrm{CDCl}_{3}\right) \delta 7.54(\mathrm{~d}, J=10.2 \mathrm{~Hz}, 1 \mathrm{H}), 6.14(\mathrm{~d}, J=16.2 \mathrm{~Hz}, 1 \mathrm{H}), 5.64(\mathrm{~d}, J=16.0 \mathrm{~Hz}$, $1 \mathrm{H}), 5.62(\mathrm{~d}, J=10.0 \mathrm{~Hz}, 1 \mathrm{H}), 5.39(\mathrm{dd}, J=16.0,6.0 \mathrm{~Hz}, 1 \mathrm{H}), 5.23(\mathrm{dd}, J=15.8,6.0 \mathrm{~Hz}, 1 \mathrm{H}), 5.21(\mathrm{~d}, J$ $=9.8 \mathrm{~Hz}, 1 \mathrm{H}), 4.88(\mathrm{~d}, J=10.5 \mathrm{~Hz}, 1 \mathrm{H}), 4.46(\mathrm{td}, J=5.2,2.0 \mathrm{~Hz}, 1 \mathrm{H}), 4.22(\mathrm{q}, J=5.2 \mathrm{~Hz}, 1 \mathrm{H}), 4.16-4.14$ $(\mathrm{m}, 1 \mathrm{H}), 3.57(\mathrm{t}, J=11.2 \mathrm{~Hz}, 1 \mathrm{H}), 2.73-2.69(\mathrm{~m}, 1 \mathrm{H}), 2.41-2.34(\mathrm{~m}, 2 \mathrm{H}), 2.07-2.00(\mathrm{~m}, 1 \mathrm{H}), 1.85-$ $1.79(\mathrm{~m}, 1 \mathrm{H}), 1.77(\mathrm{~s}, 3 \mathrm{H}), 1.61(\mathrm{~d}, J=7.0 \mathrm{~Hz}, 3 \mathrm{H}), 1.59(\mathrm{~s}, 3 \mathrm{H}), 1.42(\mathrm{~d}, J=7.0 \mathrm{~Hz}, 3 \mathrm{H}), 1.40(\mathrm{~s}, 3 \mathrm{H})$, $1.04(\mathrm{~d}, J=7.0 \mathrm{~Hz}, 3 \mathrm{H}), 0.96(\mathrm{~s}, 9 \mathrm{H}), 0.896(\mathrm{~s}, 9 \mathrm{H}), 0.892(\mathrm{~s}, 9 \mathrm{H}), 0.12(\mathrm{~s}, 3 \mathrm{H}), 0.11(\mathrm{~s}, 3 \mathrm{H}), 0.10(\mathrm{~s}, 3 \mathrm{H})$, $0.06(\mathrm{~s}, 3 \mathrm{H}), 0.05(\mathrm{~s}, 3 \mathrm{H}), 0.04(\mathrm{~s}, 3 \mathrm{H}), 0.01(\mathrm{~s}, 3 \mathrm{H})$.

${ }^{13}$ C NMR $\left(100 \mathrm{MHz}, \mathrm{CDCl}_{3}\right): \delta 204.1,173.8,173.7,137.2,134.0,133.7,132.9,129.4,128.2,126.2,75.3$, $70.8,70.0,59.0,48.1,44.1,41.9,37.0,25.85,25.83,25.7,22.6,22.1,18.2,18.1,18.0,13.1,12.7,9.5,-4.0$, $-4.4,-4.6,-4.7,-5.1$.

HRMS-ESI $\mathrm{m} / \mathrm{z}$ cacld for $\mathrm{C}_{43} \mathrm{H}_{78} \mathrm{NO}_{7} \mathrm{Si}_{3}[\mathrm{M}+\mathrm{H}]^{+}$804.5086, found 804.5072.

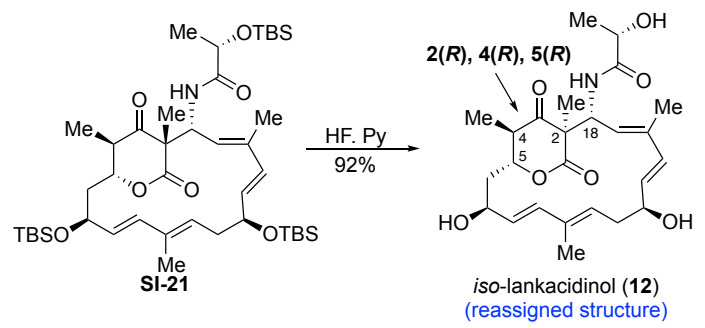

A 25-mL plastic vial containing a solution of SI-21 (30.0 mg, $0.037 \mathrm{mmol}, 1$ equiv) and THF (8 mL) was cooled to $0^{\circ} \mathrm{C}$ by means of an ice-water bath. Py $\bullet \mathrm{HF}(\sim 70 \% \mathrm{HF})(800 \mu \mathrm{L})$ was added dropwise, and the vessel was removed from the cooling bath and the system was allowed to warm to $23^{\circ} \mathrm{C}$. After $5 \mathrm{~h}, \mathrm{NaHCO}_{3}$ $(970 \mathrm{mg}$ ) was added and the mixture was rapidly stirred. After $15 \mathrm{~min}$, the mixture was filtered through a pad of Celite and the filtrate was concentrated. The crude residue was purified by flash chromatography ( silica gel, eluent: acetone:hexanes = 2:1) to afford desired product $12(16.2 \mathrm{mg}, 92 \%)$ as a white solid.

TLC (EtOAc: $\mathrm{MeOH}=30: 1): \mathrm{R}_{f}=0.40$ (UV).

${ }^{1}$ H NMR $\left(400 \mathrm{MHz}, \mathrm{CD}_{3} \mathrm{OD}\right) \delta 6.21(\mathrm{~d}, J=16.0 \mathrm{~Hz}, 1 \mathrm{H}), 5.81(\mathrm{~d}, J=16.0 \mathrm{~Hz}, 1 \mathrm{H}), 5.57(\mathrm{~d}, J=10.0 \mathrm{~Hz}$, $1 \mathrm{H}), 5.42$ (dd, $J=16.0,7.0 \mathrm{~Hz}, 1 \mathrm{H}), 5.33-5.27$ (m, 2H), 4.97 (d, $J=10.4 \mathrm{~Hz}, 1 \mathrm{H}), 4.35$ (ddd, $J=11.5$, 8.0, $4.0 \mathrm{~Hz}, 1 \mathrm{H}), 4.17(\mathrm{td}, J=5.2,2.4 \mathrm{~Hz}, 1 \mathrm{H}), 4.20-4.17(\mathrm{~m}, 1 \mathrm{H}), 4.09$ (q, $J=7.0 \mathrm{~Hz}, 1 \mathrm{H}), 3.61(\mathrm{dd}, J=$ $16.0,8.0 \mathrm{~Hz}, 1 \mathrm{H}), 3.08-3.00(\mathrm{~m}, 1 \mathrm{H}), 2.47(\mathrm{dt}, J=12.0,4.0 \mathrm{~Hz}, 1 \mathrm{H}), 2.31(\mathrm{dt}, J=14.0,11.0 \mathrm{~Hz}, 1 \mathrm{H})$, 2.07 (ddd, $J=14.4,12.0,4.2 \mathrm{~Hz}, 1 \mathrm{H}), 1.84(\mathrm{qd}, J=14.0,12.0 \mathrm{~Hz}, 1 \mathrm{H}), 1.72(\mathrm{~s}, 3 \mathrm{H}), 1.63(\mathrm{~s}, 3 \mathrm{H}), 1.38(\mathrm{~s}$, $3 \mathrm{H}), 1.37(\mathrm{~d}, J=7.0 \mathrm{~Hz}, 3 \mathrm{H}), 1.01(\mathrm{~d}, J=7.0 \mathrm{~Hz}, 3 \mathrm{H})$.

${ }^{13}$ C NMR (100 MHz, $\left.\mathrm{CD}_{3} \mathrm{OD}\right): \delta 206.2,176.8,175.0,139.1,138.2,136.2,135.0,133.4,130.9,128.6,127.7$, 78.7, 75.0, 70.7, 69.1, 60.2, 49.5, 45.0, 41.6, 36.8, 22.8, 21.2, 13.2, 12.9, 9.7. 
HRMS-ESI m/z cacld for $\mathrm{C}_{25} \mathrm{H}_{35} \mathrm{NO}_{7} \mathrm{Na}[\mathrm{M}+\mathrm{Na}]^{+}$484.2311, found 484.2296. 


\section{Optimization for Dieckmann cyclization of compounds 83, 86 and 91}

\section{General procedure}

A 5-mL round-bottom flask containing starting material $(0.005 \mathrm{mmol}, 1$ equiv) and THF (2 $\mathrm{mL})$ was evacuated and filled with nitrogen gas (this process was repeated a total of 3 times). Base was added slowly to the above reaction mixture. Saturated aqueous $\mathrm{NH}_{4} \mathrm{Cl}$ solution $(2 \mathrm{~mL})$ was added after starting material was consumed as monitored by TLC. The biphasic mixture was transferred to a separatory funnel and the layers were separated. The aqueous layer was extracted with ethyl acetate $(2 \times 2 \mathrm{~mL})$. The combined organic layers were washed with water $(2 \mathrm{~mL})$ and saturated aqueous sodium chloride solution $(3 \mathrm{~mL})$ and the washed solution was dried $\left(\mathrm{Na}_{2} \mathrm{SO}_{4}\right)$. The dried solution was filtered and the filtrate was concentrated. The crude residue was purified by flash chromatography.
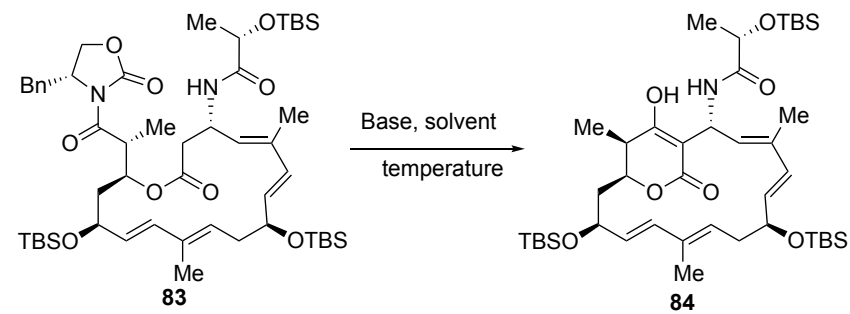

\begin{tabular}{lllll}
\hline entry $^{\boldsymbol{a}, \boldsymbol{b}}$ & base & solvent & temp. & yield (\%) \\
\hline $\mathbf{1}$ & $\mathrm{NaH}$ & $\mathrm{THF}$ & $\mathrm{rt}$ & complex mixture \\
$\mathbf{2}$ & $\mathrm{NaH}$ & diethyl ether & $\mathrm{rt}$ & complex mixture \\
$\mathbf{3}$ & $\mathrm{NaH}$ & toluene & $\mathrm{rt}$ & complex mixture \\
$\mathbf{4}$ & $\mathrm{NaH}$ & benzene & $\mathrm{rt}$ & complex mixture \\
$\mathbf{5}$ & $\mathrm{NaH}$ & $\mathrm{DMF}$ & $\mathrm{rt}$ & complex mixture \\
$\mathbf{6}$ & $\mathrm{LDA}$ & $\mathrm{THF}$ & $-78{ }^{\circ} \mathrm{C}$ to $0{ }^{\circ} \mathrm{C}$ & complex mixture \\
$\mathbf{7}$ & $\mathrm{LDA}$ & diethyl ether & $-78^{\circ} \mathrm{C}$ to $0{ }^{\circ} \mathrm{C}$ & complex mixture \\
$\mathbf{8}$ & $\mathrm{Cs} \mathrm{CO}_{3}$ & $\mathrm{DMF}$ & $\mathrm{rt}$ & complex mixture \\
$\mathbf{9}$ & $\mathrm{Cs}_{2} \mathrm{CO}_{3}$ & $\mathrm{DMF}$ & $50{ }^{\circ} \mathrm{C}$ & complex mixture \\
$\mathbf{1 0}$ & $\mathrm{NaHMDS}$ & $\mathrm{THF}$ & $\mathrm{rt}$ & complex mixture \\
$\mathbf{1 1}$ & $\mathrm{NaHMDS}$ & diethyl ether & $\mathrm{rt}$ & complex mixture \\
$\mathbf{1 2}$ & $\mathrm{NaHMDS}$ & toluene & $\mathrm{rt}$ & complex mixture \\
$\mathbf{1 3}$ & $\mathrm{NaHMDS}$ & benzene & $\mathrm{rt}$ & complex mixture \\
$\mathbf{1 4}$ & $\mathrm{KHMDS}$ & $\mathrm{THF}$ & $\mathrm{rt}$ & complex mixture \\
$\mathbf{1 5}$ & $\mathrm{KHMDS}$ & diethyl ether & $\mathrm{rt}$ & complex mixture \\
$\mathbf{1 6}$ & $\mathrm{KHMDS}$ & toluene & $\mathrm{rt}$ & complex mixture \\
$\mathbf{1 7}$ & $\mathrm{KHMDS}$ & benzene & $\mathrm{rt}$ & complex mixture \\
$\mathbf{1 8}$ & $\mathrm{LiHMDS}$ & diethyl ether & $\mathrm{rt}$ & complex mixture \\
$\mathbf{1 9}$ & LiHMDS & toluene & $\mathrm{rt}$ & complex mixture \\
$\mathbf{2 0}$ & LiHMDS & benzene & $\mathrm{rt}$ & complex mixture \\
$\mathbf{2 1}$ & LiHMDS & THF & $\mathrm{rt}$ & $5 \%$ \\
$\mathbf{2 2}$ & LiHMDS & THF & $\mathrm{rt}$ & $5 \%$ \\
\hline
\end{tabular}

${ }^{a}$ Unless otherwise stated, 5 equivalents of base was used, ${ }^{b}$ Further details: (1) NaH as base, THF, diethyl ether, toluene, benzne, or DMF as solvent, the starting material was slowly decomposed overnight (entries 1-5); (2) LDA as base, the SM was stable at $-78{ }^{\circ} \mathrm{C}$ overnight. However, SM was slowly decomposed warming to the room temperature. Excess base was tried, showing the same result (entries 6 and 7); (3) $\mathrm{Cs}_{2} \mathrm{CO}_{3}$ as base and DMF as solvent, the $\mathrm{SM}$ was decomposed within $1 \mathrm{~h}$ at $50{ }^{\circ} \mathrm{C}$. It takes a few hours to decompose at room temperature (entries 8 and 9); (4) NaHMDS and KHMDS showed silimar results as $\mathrm{NaH}$ (entries 10-17). (5) LiHMDS as base, different solvents was tested for reaction, only THF gave around $5 \%$ of desired product. Other solvents resulted decomposion of starting material (entry 18-21). ${ }^{c}$ For large scale $(>200 \mathrm{mg}$ ) reaction, reproducible result was observed when 10 equivalent of base was used. 

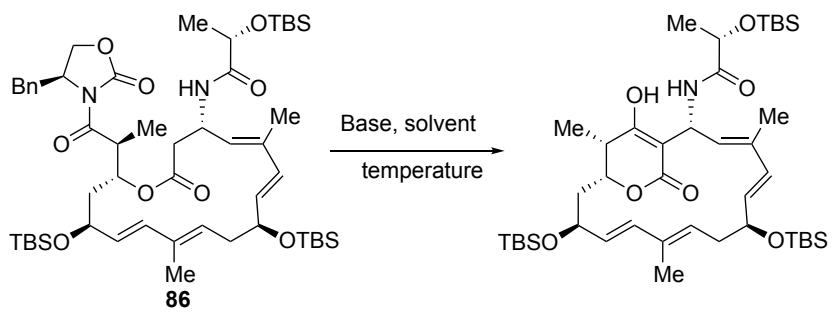

\begin{tabular}{lllll}
\hline entry $^{\boldsymbol{a}}$ & base & solvent & temp. & Yield (\%) \\
\hline $\mathbf{1}$ & LiHMDS & THF & $\mathrm{rt}$ & complex mixture \\
$\mathbf{2}$ & LiHMDS & THF & reflux & complex mixture \\
$\mathbf{3}$ & LiHMDS & diethyl ether & $\mathrm{rt}$ & complex mixture \\
$\mathbf{4}$ & LiHMDS & toluene & $\mathrm{rt}$ & complex mixture \\
$\mathbf{5}$ & LiHMDS & benzene & $\mathrm{rt}$ & complex mixture \\
$\mathbf{6}$ & NaH & THF & $\mathrm{rt}$ & complex mixture \\
$\mathbf{7}$ & NaH & diethyl ether & $\mathrm{rt}$ & complex mixture \\
$\mathbf{8}$ & NaH & toluene & $\mathrm{rt}$ & complex mixture \\
$\mathbf{9}$ & NaH & benzene & $\mathrm{rt}$ & complex mixture \\
$\mathbf{1 0}$ & NaH & DMF & $\mathrm{rt}$ & $25-30 \%$ \\
$\mathbf{1 1}$ & NaH & DMF & $50{ }^{\circ} \mathrm{C}$ & $30-45 \%$ \\
$\mathbf{1 2}$ & NaHMDS & THF & $\mathrm{rt}$ & complex mixture \\
$\mathbf{1 3}$ & NaHMDS & diethyl ether & $\mathrm{rt}$ & complex mixture \\
$\mathbf{1 4}$ & NaHMDS & toluene & $\mathrm{rt}$ & complex mixture \\
$\mathbf{1 5}$ & NaHMDS & benzene & $\mathrm{rt}$ & complex mixture \\
$\mathbf{1 6}$ & KHMDS & THF & $\mathrm{rt}$ & complex mixture \\
$\mathbf{1 7}$ & KHMDS & diethyl ether & $\mathrm{rt}$ & complex mixture \\
$\mathbf{1 8}$ & KHMDS & toluene & $\mathrm{rt}$ & complex mixture \\
$\mathbf{1 9}$ & KHMDS & benzene & $\mathrm{rt}$ & complex mixture \\
\hline
\end{tabular}

${ }^{a}$ (1) When the optimized condition for $\mathbf{8 3}$ was applied to compound $\mathbf{8 6}$ for Dieckmann cyclization, no desired production was observed (entry 1). Higher temperature and shorter reaction time were applied, resulted in apparent decomposition of starting material (entry 2). (2) After screening different conditions, we found $\mathrm{NaH}$ in DMF was effective, providing desired cyclization product. Different temperatures were tested for this condition, $50{ }^{\circ} \mathrm{C}$ was optimal in term of reaction yield.

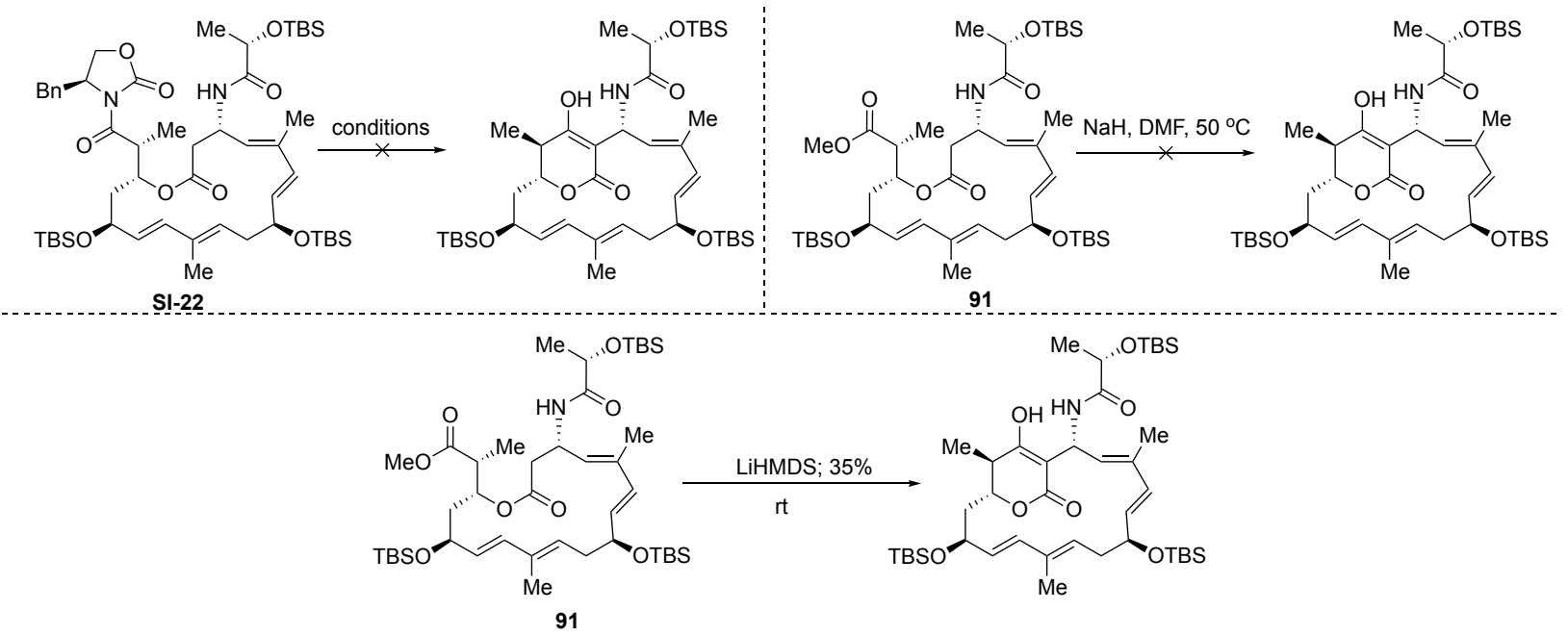


All previous conditions were tested for SI-22, no desired cyclization product was obtained. Then, methyl ester 91 was employed for Dieckmann cyclization. We found only LiHMDS was effective for cyclization in $35 \%$ yield at room temperature. 


\section{NMR Spectral Data Comparison}

\begin{tabular}{|c|c|c|c|c|}
\hline \multicolumn{5}{|c|}{$\begin{array}{cc}\text { iso-Lankacidinol (85) } & \text { iso-Lankacidinol (12) } \\
\text { spectroscopic data does not match reported data } & \begin{array}{c}\text { (12) } \\
\text { spectroscopic data matches reported data } \\
\text { (reassigned structure) }\end{array} \\
\end{array}$} \\
\hline \multicolumn{5}{|c|}{${ }^{1} \mathrm{H}$ NMR Comparison } \\
\hline Position & Compound 85 & Compound 87 & Reassigned structure $\mathbf{1 2}$ & Isolation $^{\mathrm{a}}$ \\
\hline 4 & $3.74-3.73(\mathrm{~m}, 1 \mathrm{H})$ & $\begin{array}{c}2.73(\mathrm{qd}, J=7.0,2.3 \\
\mathrm{Hz}, 1 \mathrm{H}),\end{array}$ & $3.08-3.00(\mathrm{~m}, 1 \mathrm{H})$ & $3.03(\mathrm{~m})$ \\
\hline 5 & $\begin{array}{c}4.79(\mathrm{dd}, J=12.0,6.0 \mathrm{~Hz} \\
1 \mathrm{H})\end{array}$ & $5.55-5.42(\mathrm{~m}, 3 \mathrm{H})$ & $\begin{array}{c}3.61(\mathrm{dd}, J=16.0,8.0 \\
\mathrm{Hz}, 1 \mathrm{H})\end{array}$ & $3.59(\mathrm{~m})$ \\
\hline 6 & $\begin{aligned} & 1.63- 1.57(\mathrm{~m}, 1 \mathrm{H}), 1.09- \\
& 1.02(\mathrm{~m}, 1 \mathrm{H})\end{aligned}$ & $\begin{array}{l}2.14(\mathrm{ddd}, J=12.8,7.8 \\
4.8 \mathrm{~Hz}, 1 \mathrm{H})\end{array}$ & $\begin{array}{c}2.07(\mathrm{ddd}, J=14.4 \\
12.0,4.2 \mathrm{~Hz}, 1 \mathrm{H}), 1.84 \\
(\mathrm{dq}, J=14.0,12.0 \mathrm{~Hz} \\
1 \mathrm{H})\end{array}$ & $1.84,2.06(\mathrm{~m})$ \\
\hline 7 & $\begin{array}{c}4.38(\mathrm{dd}, J=11.2,6.4 \mathrm{~Hz} \\
1 \mathrm{H})\end{array}$ & $\begin{array}{c}4.45(\mathrm{td}, J=8.0,3.0 \mathrm{~Hz}, \\
1 \mathrm{H})\end{array}$ & $\begin{array}{c}4.35(\mathrm{ddd}, J=11.5,8.0, \\
4.0 \mathrm{~Hz}, 1 \mathrm{H})\end{array}$ & $\begin{array}{c}4.35(\mathrm{ddd}, J=4.0,8.9, \\
11.3 \mathrm{~Hz})\end{array}$ \\
\hline 8 & $5.56-5.48(\mathrm{~m}, 1 \mathrm{H})$ & $5.55-5.42(\mathrm{~m}, 3 \mathrm{H})$ & $5.29-5.27(\mathrm{~m}, 1 \mathrm{H})$ & $5.28(\mathrm{~m})$ \\
\hline 9 & $6.09(\mathrm{~d}, J=16.0 \mathrm{~Hz}, 1 \mathrm{H})$ & $\begin{array}{c}6.23(\mathrm{~d}, J=16.4 \mathrm{~Hz} \\
1 \mathrm{H})\end{array}$ & $\begin{array}{c}6.21(\mathrm{~d}, J=16.0 \mathrm{~Hz} \\
1 \mathrm{H})\end{array}$ & $6.20(\mathrm{~d}, J=15.6 \mathrm{~Hz})$ \\
\hline 11 & $5.16(\mathrm{t}, J=8.0 \mathrm{~Hz}, 1 \mathrm{H})$ & $\begin{array}{c}5.35(\mathrm{dd}, J=16.0,6.0 \\
\mathrm{Hz}, 1 \mathrm{H})\end{array}$ & $5.33-5.30(\mathrm{~m}, 1 \mathrm{H})$ & $5.31(\mathrm{~m})$ \\
\hline 12 & $\begin{array}{l}2.61-2.58(\mathrm{~m}, 1 \mathrm{H}), 2.19- \\
2.08(\mathrm{~m}, 1 \mathrm{H})\end{array}$ & $\begin{array}{c}2.50(\mathrm{td}, J=11.0,3.2 \\
\mathrm{Hz}, 1 \mathrm{H}), 2.31(\mathrm{q}, J= \\
12.0 \mathrm{~Hz}, 1 \mathrm{H})\end{array}$ & $\begin{array}{c}2.47(\mathrm{dt}, J=12.0,4.0 \\
\mathrm{Hz}, 1 \mathrm{H}), 2.31(\mathrm{dt}, J= \\
14.0,11.0 \mathrm{~Hz}, 1 \mathrm{H})\end{array}$ & $2.30,2.46(\mathrm{~m})$ \\
\hline 13 & $4.04-3.98(\mathrm{~m}, 1 \mathrm{H})$ & $4.15-4.07(\mathrm{~m}, 2 \mathrm{H})$ & $\begin{array}{c}4.17(\mathrm{td}, J=5.2,2.4 \mathrm{~Hz} \\
1 \mathrm{H})\end{array}$ & $4.16(\mathrm{~m})$ \\
\hline 14 & $5.52-5.48(\mathrm{~m}, 1 \mathrm{H})$ & $\begin{array}{c}5.35(\mathrm{dd}, J=16.0,6.0 \\
\mathrm{Hz}, 1 \mathrm{H})\end{array}$ & $\begin{array}{c}5.42(\mathrm{dd}, J=16.0,7.0 \\
\mathrm{Hz}, 1 \mathrm{H})\end{array}$ & $5.42(\mathrm{dd}, J=7.7,15.8 \mathrm{~Hz})$ \\
\hline 15 & $5.77(\mathrm{~d}, J=16.0 \mathrm{~Hz}, 1 \mathrm{H})$ & $\begin{array}{c}5.79(\mathrm{~d}, J=16.0 \mathrm{~Hz}, \\
1 \mathrm{H})\end{array}$ & $\begin{array}{c}5.81(\mathrm{~d}, J=16.0 \mathrm{~Hz}, \\
1 \mathrm{H})\end{array}$ & $5.81(\mathrm{~d}, J=15.8 \mathrm{~Hz})$ \\
\hline 17 & $5.00(\mathrm{~d}, J=10.0 \mathrm{~Hz}, 1 \mathrm{H})$ & $\begin{array}{c}4.99(\mathrm{~d}, J=11.0 \mathrm{~Hz}, \\
1 \mathrm{H})\end{array}$ & $\begin{array}{c}4.97(\mathrm{~d}, J=10.4 \mathrm{~Hz}, \\
1 \mathrm{H})\end{array}$ & $4.96(\mathrm{~d}, J=10.4 \mathrm{~Hz})$ \\
\hline 18 & $5.69(\mathrm{~d}, J=10.0 \mathrm{~Hz}, 1 \mathrm{H})$ & $5.55-5.42(\mathrm{~m}, 3 \mathrm{H})$ & $\begin{array}{c}5.57(\mathrm{~d}, J=10.0 \mathrm{~Hz} \\
1 \mathrm{H})\end{array}$ & $5.57(\mathrm{t}, J=10.4 \mathrm{~Hz})$ \\
\hline 19 & $1.56(\mathrm{~s}, 3 \mathrm{H})$ & $1.42(\mathrm{~s}, 3 \mathrm{H})$ & $1.38(\mathrm{~s}, 3 \mathrm{H})$ & $1.38(\mathrm{~s})$ \\
\hline 20 & $1.09(\mathrm{~d}, J=7.0 \mathrm{~Hz}, 3 \mathrm{H})$ & $1.27(\mathrm{~d}, J=7.0 \mathrm{~Hz}, 3 \mathrm{H})$ & $1.01(\mathrm{~d}, J=7.0 \mathrm{~Hz}, 3 \mathrm{H})$ & $1.00(\mathrm{~d}, J=6.5 \mathrm{~Hz})$ \\
\hline 21 & $1.68(\mathrm{~s}, 3 \mathrm{H})$ & $1.65(\mathrm{~s}, 3 \mathrm{H})$ & $1.63(\mathrm{~s}, 3 \mathrm{H})$ & $1.62(\mathrm{~s})$ \\
\hline 22 & $1.92(\mathrm{~s}, 3 \mathrm{H})$ & $1.77(\mathrm{~s}, 3 \mathrm{H})$ & $1.72(\mathrm{~s}, 3 \mathrm{H})$ & $1.72(\mathrm{~s})$ \\
\hline 24 & $4.10(\mathrm{q}, J=7.5 \mathrm{~Hz}, 1 \mathrm{H})$ & $\begin{array}{c}4.26(\mathrm{td}, J=5.2,2.4 \mathrm{~Hz}, \\
1 \mathrm{H})\end{array}$ & $4.09(\mathrm{q}, J=7.0 \mathrm{~Hz}, 1 \mathrm{H})$ & $4.09(\mathrm{q}, J=6.7 \mathrm{~Hz})$ \\
\hline 25 & $1.37(\mathrm{~d}, J=7.0 \mathrm{~Hz}, 3 \mathrm{H})$ & $1.38(\mathrm{~d}, J=7.0 \mathrm{~Hz}, 3 \mathrm{H})$ & $1.37(\mathrm{~d}, J=7.0 \mathrm{~Hz}, 3 \mathrm{H})$ & $1.36(\mathrm{~d}, J=6.7 \mathrm{~Hz})$ \\
\hline $\mathrm{N}-\mathrm{H}$ & N.D. in $\mathrm{CD}_{3} \mathrm{OD}$ & & N.D. in $\mathrm{CD}_{3} \mathrm{OD}$ & N.D. in $\mathrm{CD}_{3} \mathrm{OD}$ \\
\hline
\end{tabular}

${ }^{\text {a }}$ Suzuki, T; Mochizuki, S.; Yamamoto, S.;Arakawa, K.; Kinashi, H. Regulation of Lankamycin Biosynthesis in Streptomyces rochei by Two SARP Genes, srrY and srrZ. Bioscience, Biotechnology, and Biochemistry 2010, 74, 819-827. 


\begin{tabular}{|c|c|c|c|c|}
\hline \multicolumn{5}{|c|}{${ }^{13} \mathrm{C}$ NMR Comparison } \\
\hline Position & Compound 85 & Compound 87 & Reassigned structure 12 & Isolation $^{\mathrm{a}}$ \\
\hline 1 & 175.0 & 174.0 & 175.0 & 175.0 \\
\hline 2 & 60.4 & 55.2 & 60.2 & 60.2 \\
\hline 3 & 204.4 & 206.3 & 206.2 & 206.2 \\
\hline 4 & 40.4 & 44.7 & 45.0 & 45.1 \\
\hline 5 & 77.1 & 72.6 & 78.7 & 78.7 \\
\hline 6 & 34.6 & 33.8 & 41.6 & 41.7 \\
\hline 7 & 67.7 & 66.9 & 70.7 & 70.8 \\
\hline 8 & 130.7 & 130.8 & 130.9 & 130.9 \\
\hline 9 & 137.6 & 136.0 & 139.1 & 139.2 \\
\hline 10 & 133.7 & 126.3 & 135.0 & 135.0 \\
\hline 11 & 127.5 & 124.1 & 128.6 & 128.7 \\
\hline 12 & 34.6 & 36.8 & 36.8 & 36.9 \\
\hline 13 & 74.1 & 72.1 & 75.0 & 75.1 \\
\hline 14 & 132.1 & 132.9 & 133.4 & 133.4 \\
\hline 15 & 135.2 & 136.0 & 136.2 & 136.3 \\
\hline 16 & 135.6 & 134.6 & 138.2 & 138.3 \\
\hline 17 & 126.6 & 126.1 & 127.7 & 127.7 \\
\hline 18 & 42.1 & 47.9 & 49.5 & 49.7 \\
\hline 19 & 23.8 & 21.0 & 22.8 & 22.8 \\
\hline 20 & 8.2 & 7.4 & 9.7 & 9.7 \\
\hline 21 & 12.6 & 10.5 & 13.2 & 13.3 \\
\hline 22 & 11.7 & 9.8 & 12.9 & 12.9 \\
\hline 23 & 175.1 & 172.3 & 176.8 & 176.9 \\
\hline 24 & 66.0 & 66.2 & 69.1 & 69.2 \\
\hline 25 & 19.8 & 18.3 & 21.2 & 21.3 \\
\hline
\end{tabular}

${ }^{a}$ Suzuki, T; Mochizuki, S.; Yamamoto, S.;Arakawa, K.; Kinashi, H. Regulation of Lankamycin Biosynthesis in Streptomyces rochei by Two SARP Genes, srrY and srrZ. Bioscience, Biotechnology, and Biochemistry 2010, 74, 819-827. 
${ }^{1} \mathrm{H}$ NMR and ${ }^{13} \mathrm{C}$ NMR Spectra for Compounds $\left({ }^{1} \mathrm{H}\right.$ NMR, $\left.\mathrm{CDCl}_{3}, 400 \mathrm{MHz}\right)$

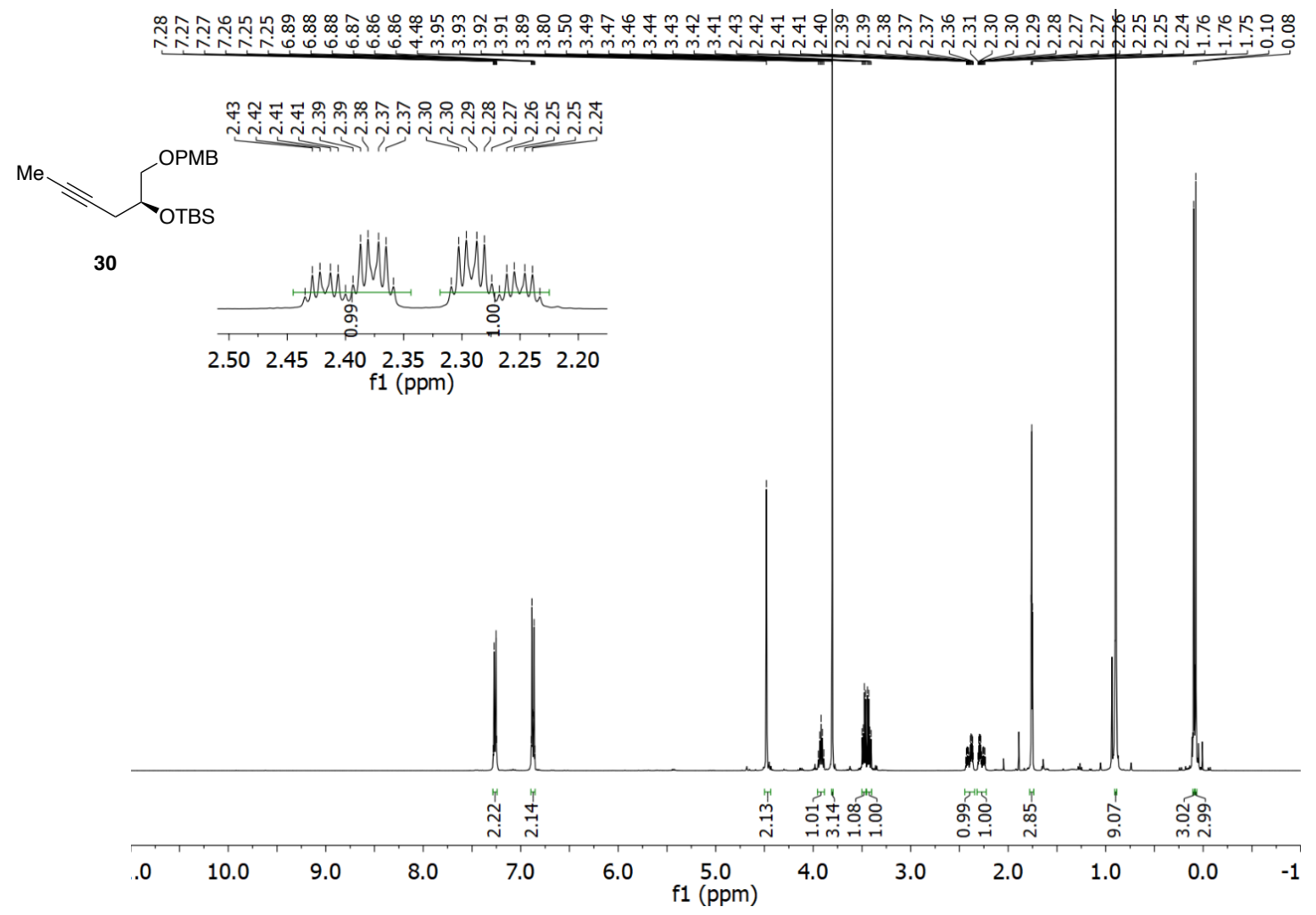

$\left({ }^{13} \mathrm{C} \mathrm{NMR}, \mathrm{CDCl}_{3}, 100 \mathrm{MHz}\right)$
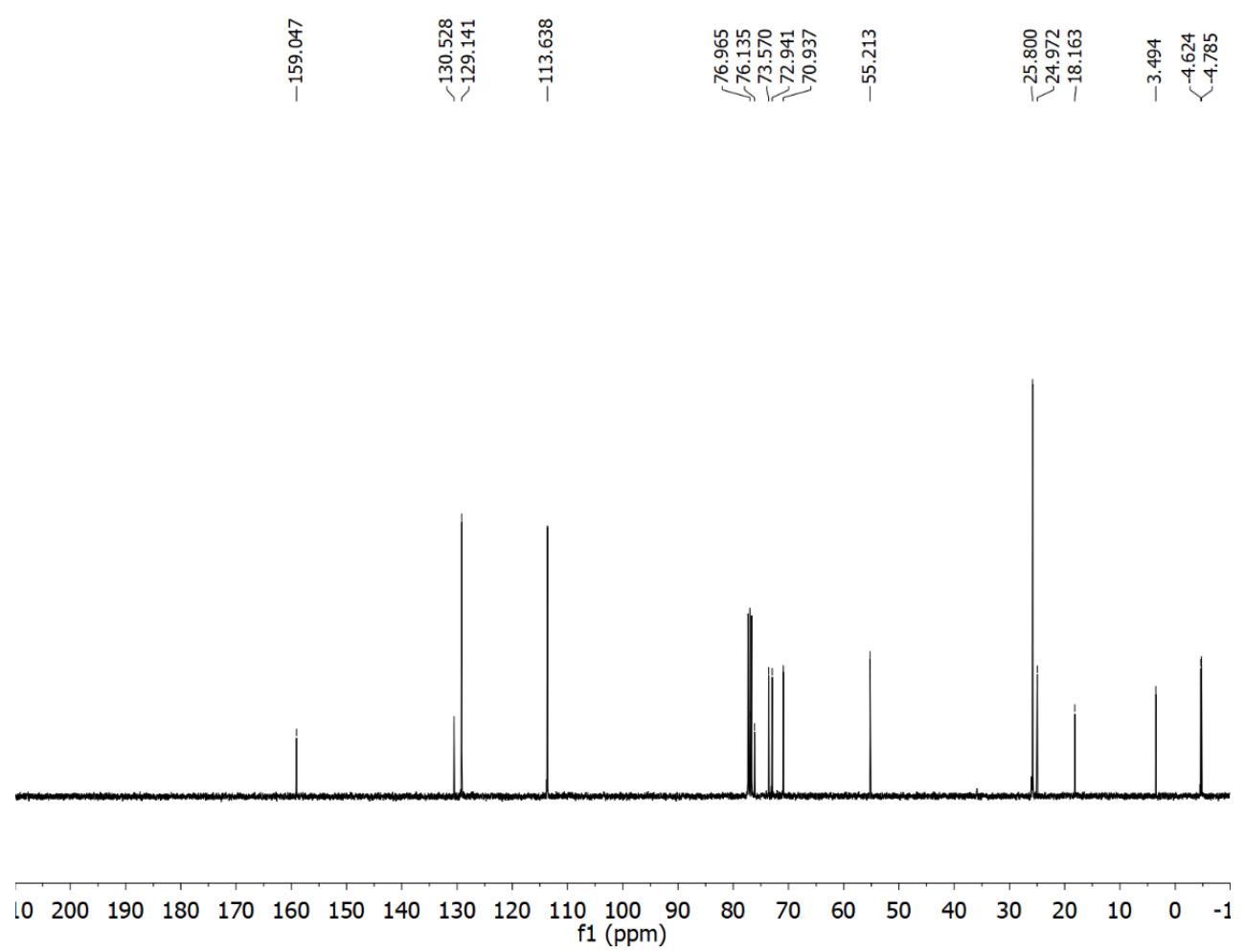
$\left({ }^{1} \mathrm{H} \mathrm{NMR}, \mathrm{CDCl}_{3}, 400 \mathrm{MHz}\right)$

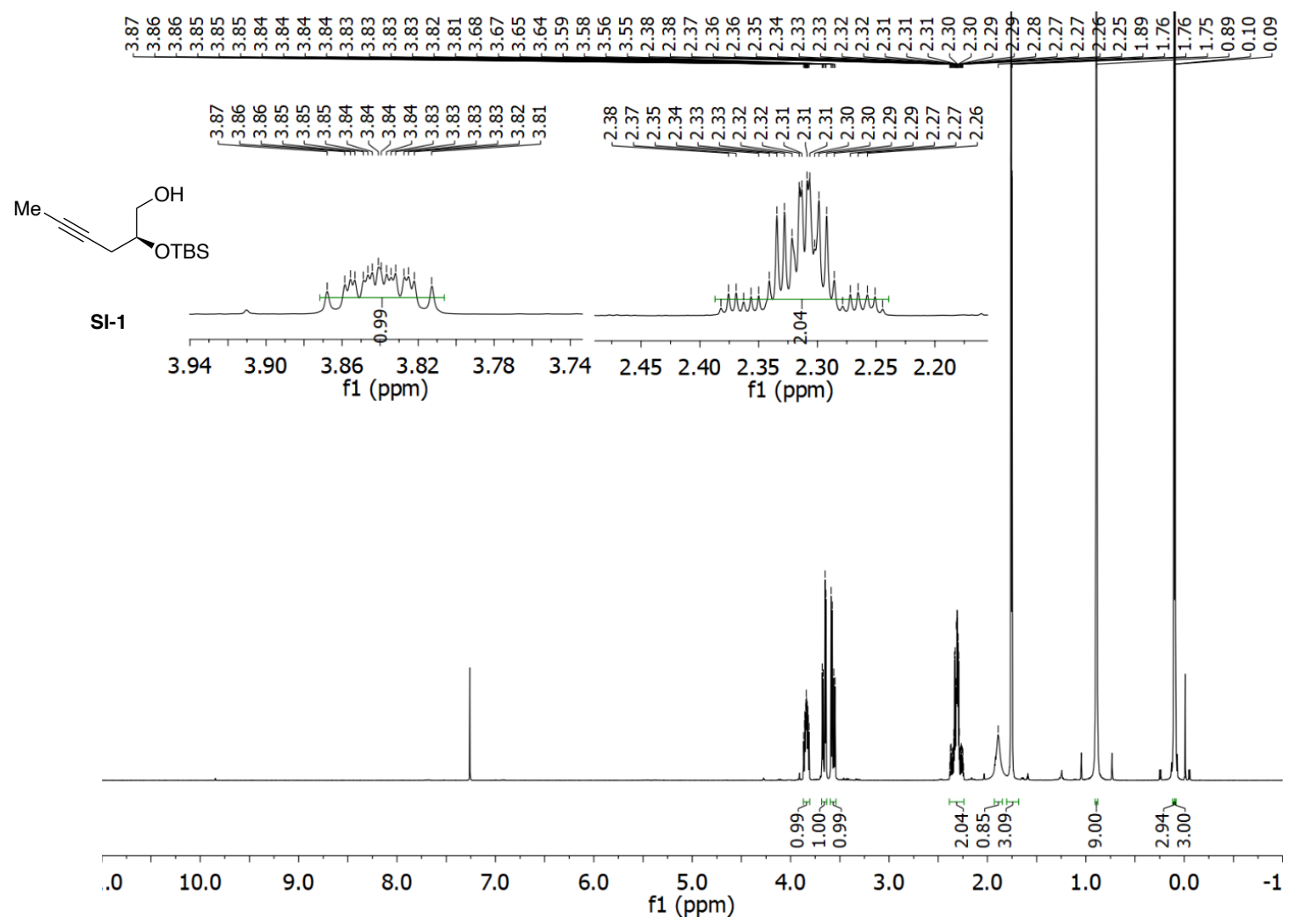

$\left({ }^{13} \mathrm{C} \mathrm{NMR}, \mathrm{CDCl}_{3}, 100 \mathrm{MHz}\right)$

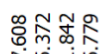

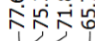

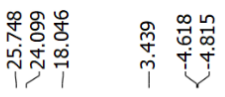

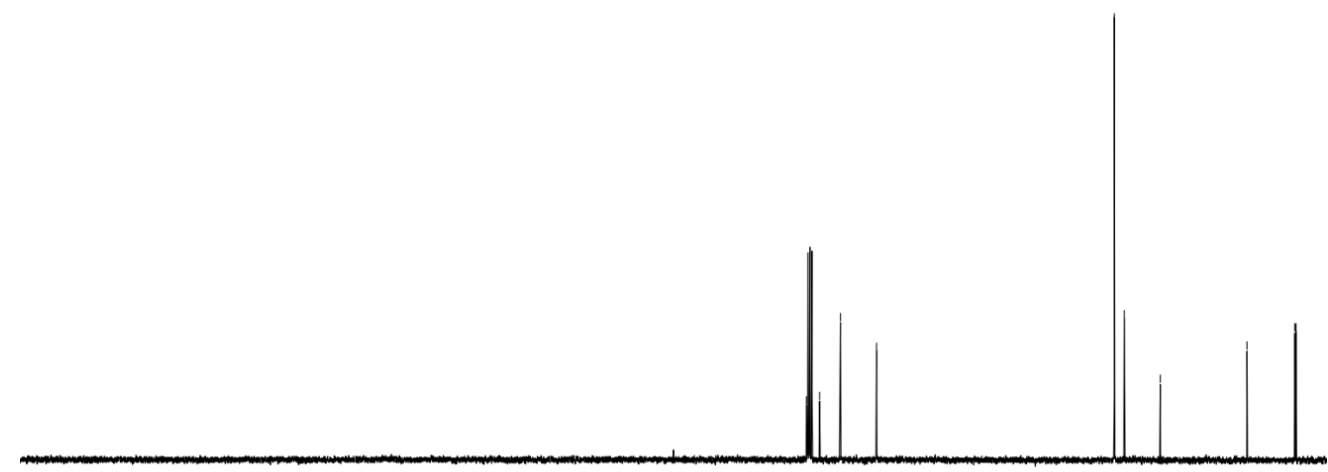

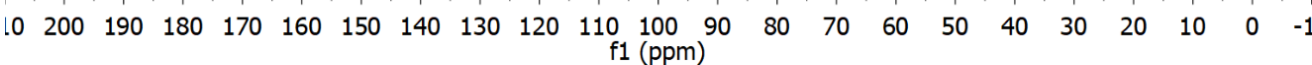


$\left({ }^{1} \mathrm{H} \mathrm{NMR}, \mathrm{CDCl}_{3}, 400 \mathrm{MHz}\right)$

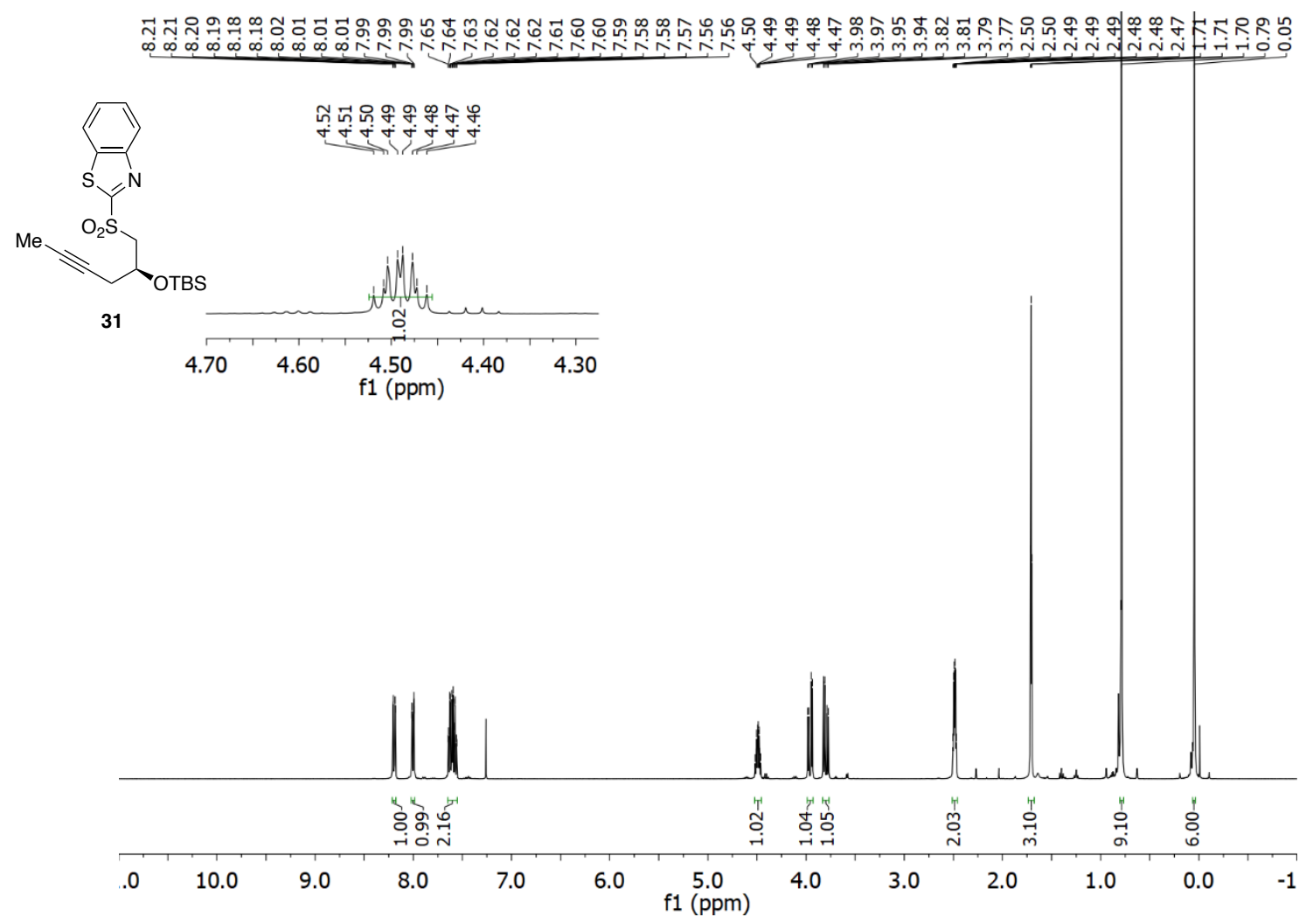

$\left({ }^{13} \mathrm{C}\right.$ NMR, $\left.\mathrm{CDCl}_{3}, 100 \mathrm{MHz}\right)$

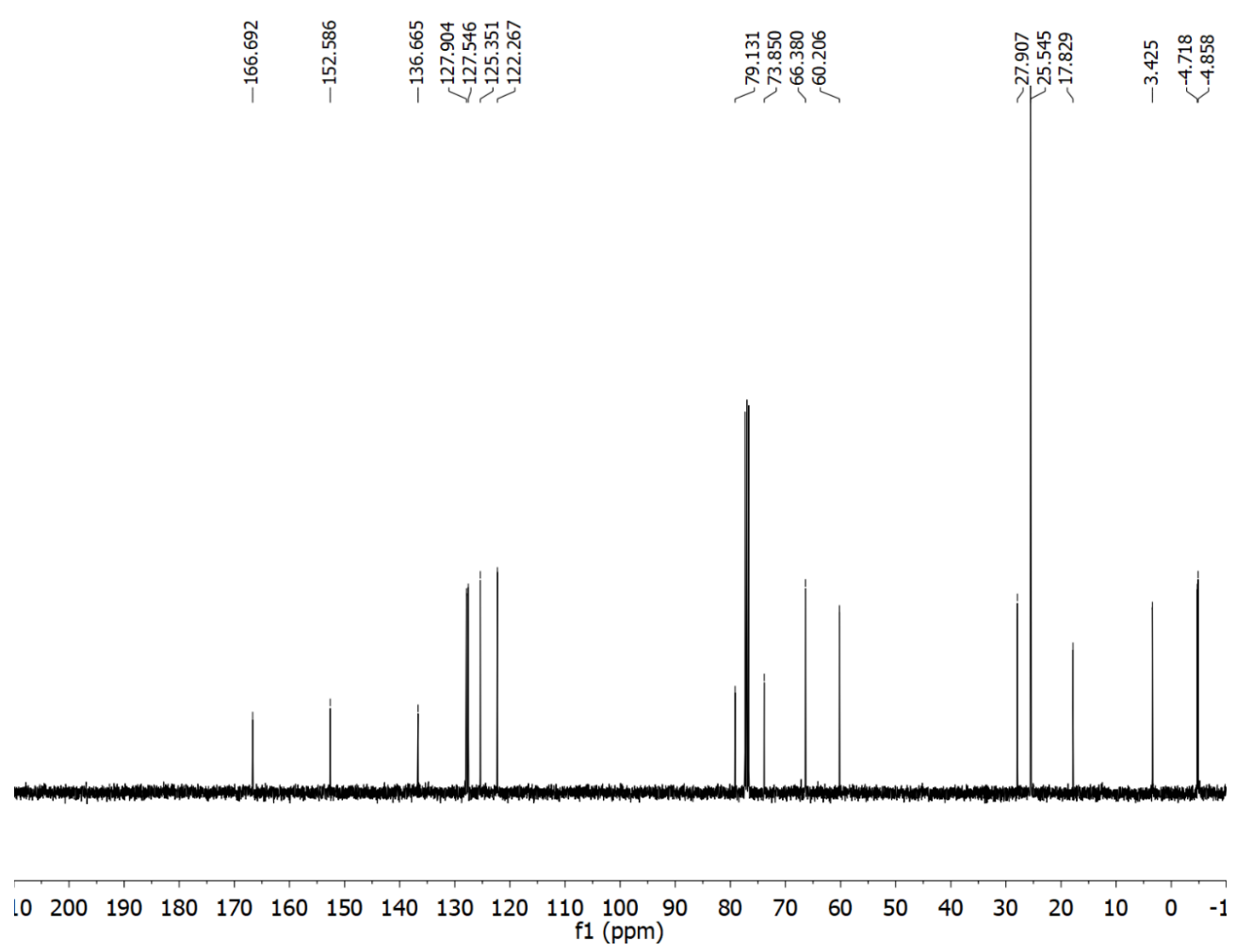


$\left({ }^{1} \mathrm{H} \mathrm{NMR}, \mathrm{CDCl}_{3}, 400 \mathrm{MHz}\right)$

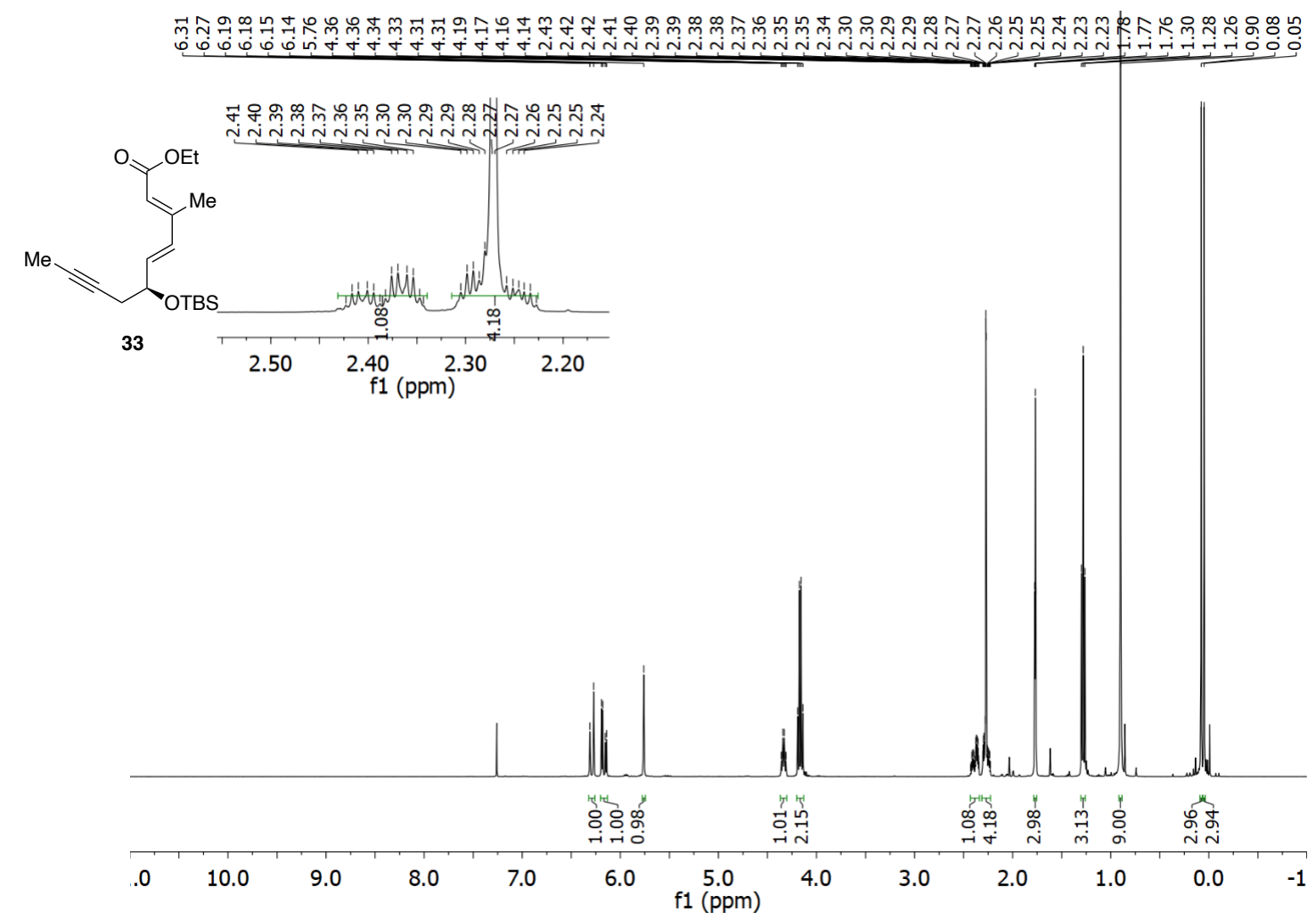

$\left({ }^{13} \mathrm{C} \mathrm{NMR}, \mathrm{CDCl}_{3}, 100 \mathrm{MHz}\right)$

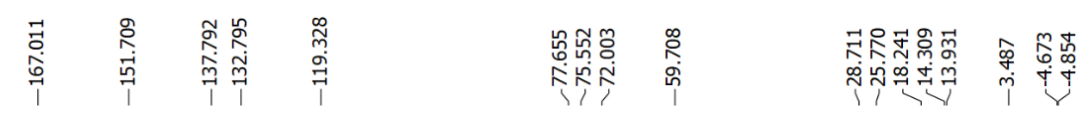

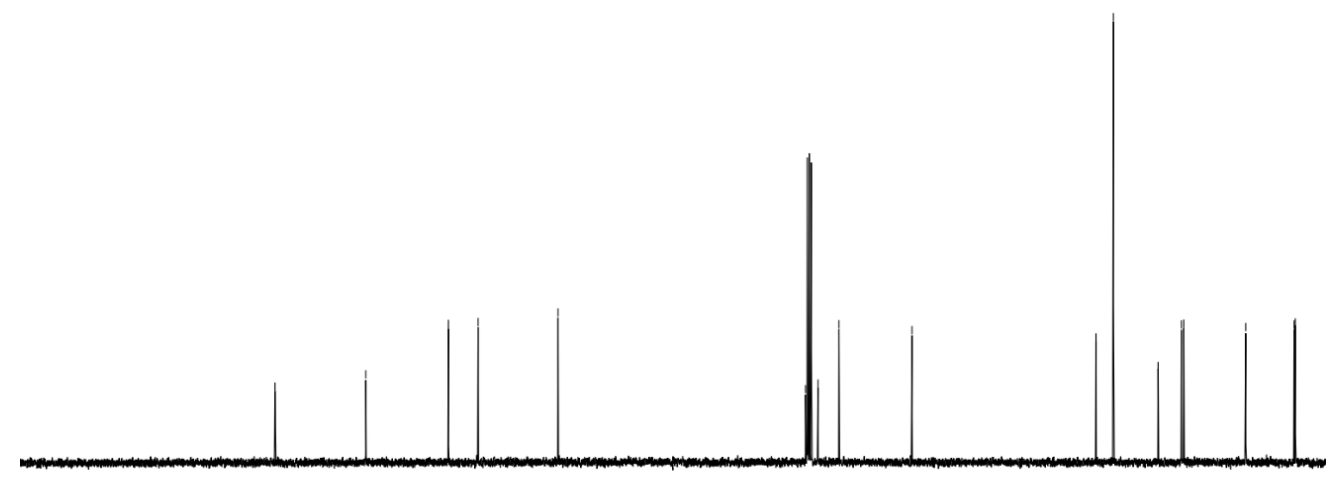

\begin{tabular}{llllllllllllllllllllllll}
\hline 0 & 200 & 190 & 180 & 170 & 160 & 150 & 140 & 130 & 120 & 110 & 100 & 90 & 80 & 70 & 60 & 50 & 40 & 30 & 20 & 10 & 0 & -1
\end{tabular} 
( $\left.{ }^{1} \mathrm{H} \mathrm{NMR}, \mathrm{CDCl}_{3}, 400 \mathrm{MHz}\right)$

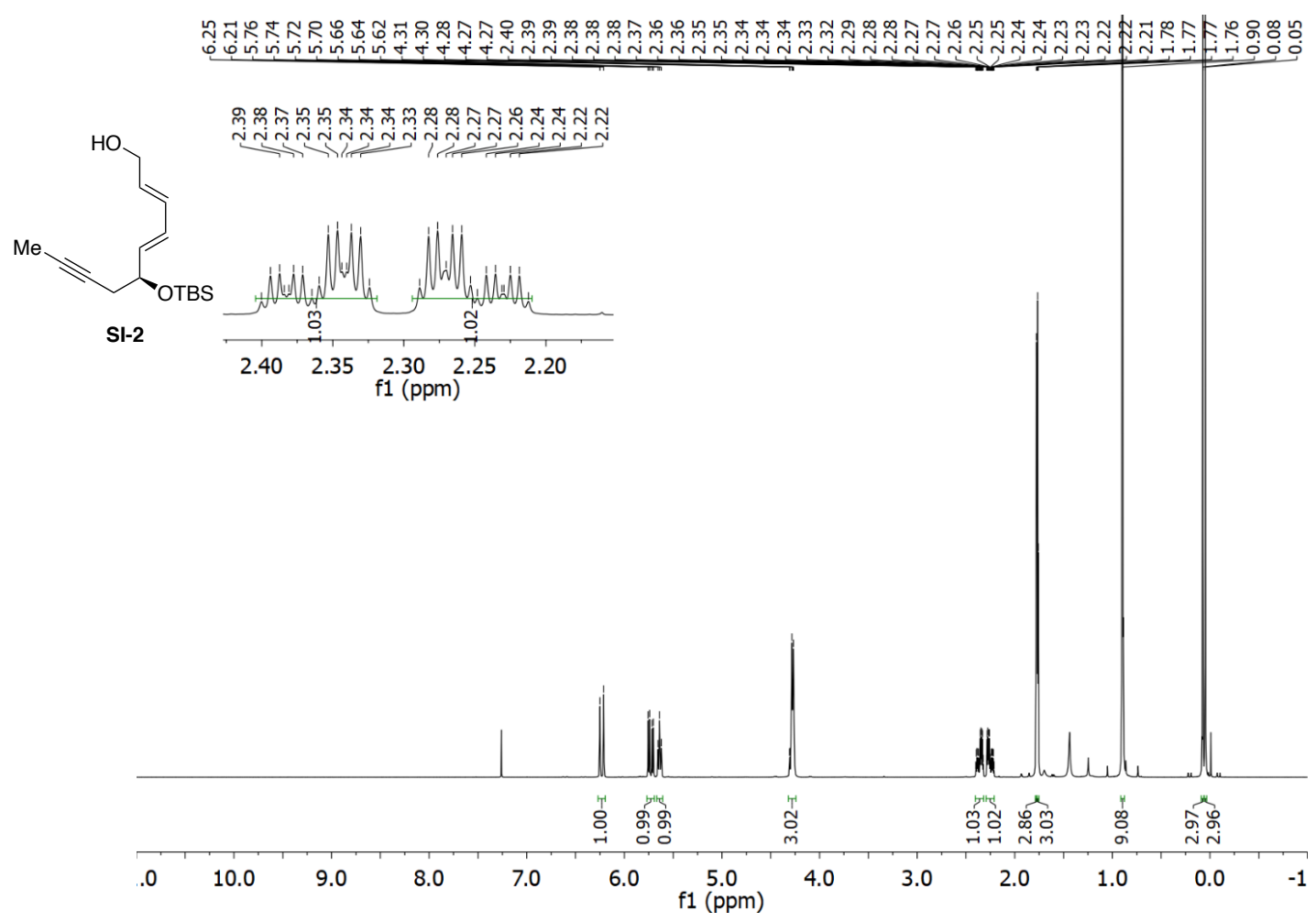

$\left({ }^{13} \mathrm{C} \mathrm{NMR}, \mathrm{CDCl}_{3}, 100 \mathrm{MHz}\right)$

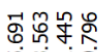

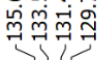

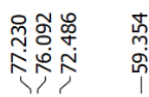

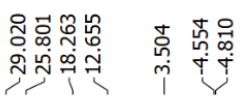

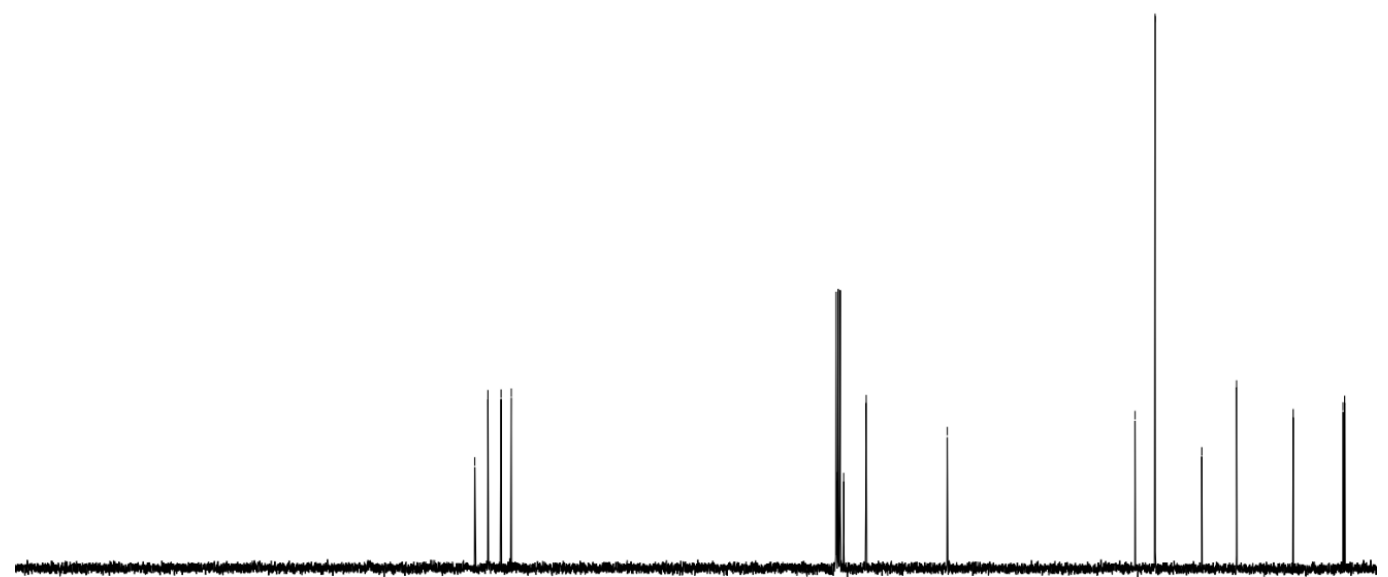

\begin{tabular}{llllllllllllllllllllllll}
\hline 0 & 200 & 190 & 180 & 170 & 160 & 150 & 140 & 130 & 120 & 110 & 100 & 100 & 80 & 70 & 60 & 50 & 40 & 30 & 20 & 10 & 0 & -1
\end{tabular} 
$\left({ }^{1} \mathrm{H} \mathrm{NMR}, \mathrm{CDCl}_{3}, 400 \mathrm{MHz}\right)$

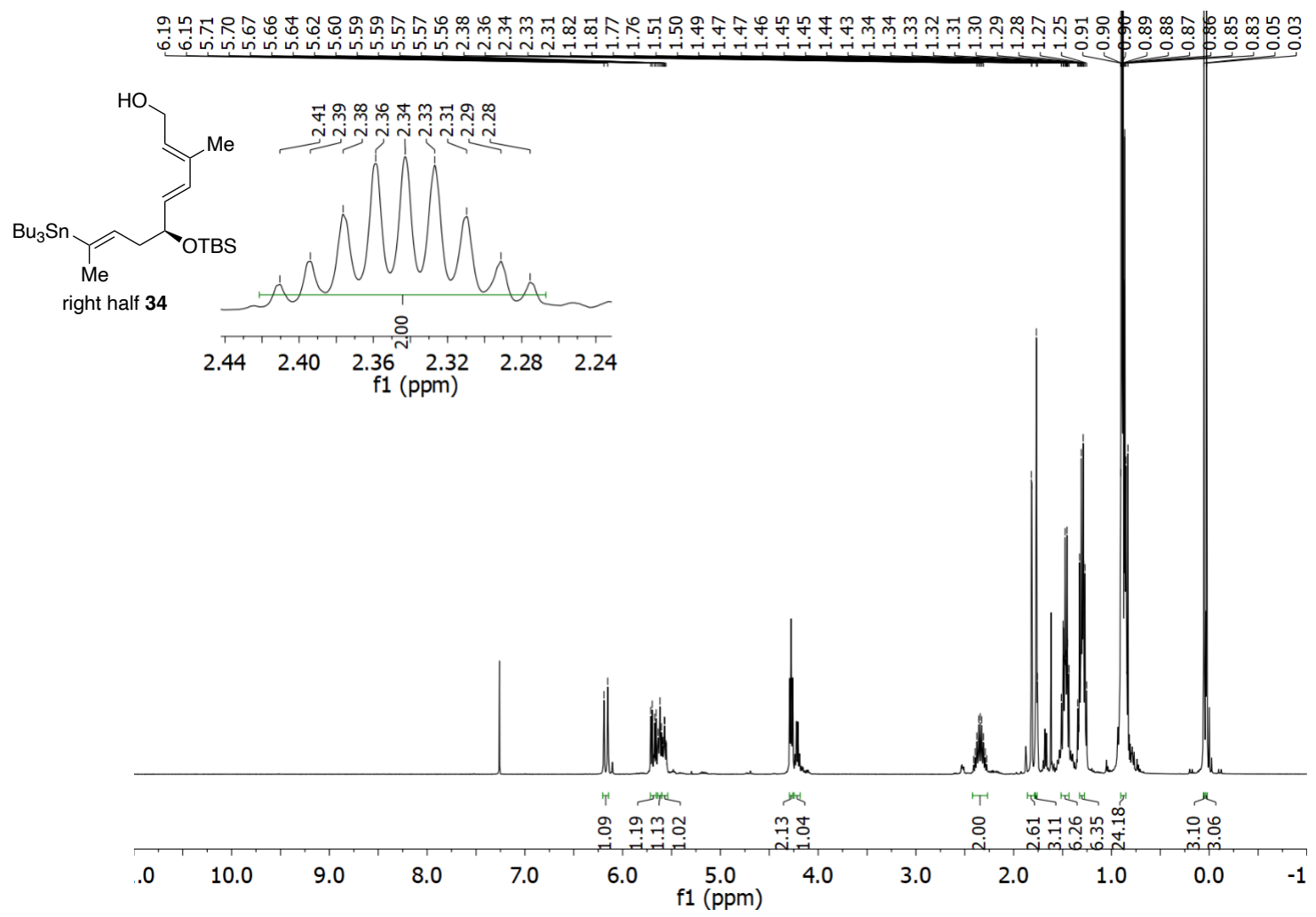

$\left({ }^{13} \mathrm{C} \mathrm{NMR}, \mathrm{CDCl}_{3}, 100 \mathrm{MHz}\right)$
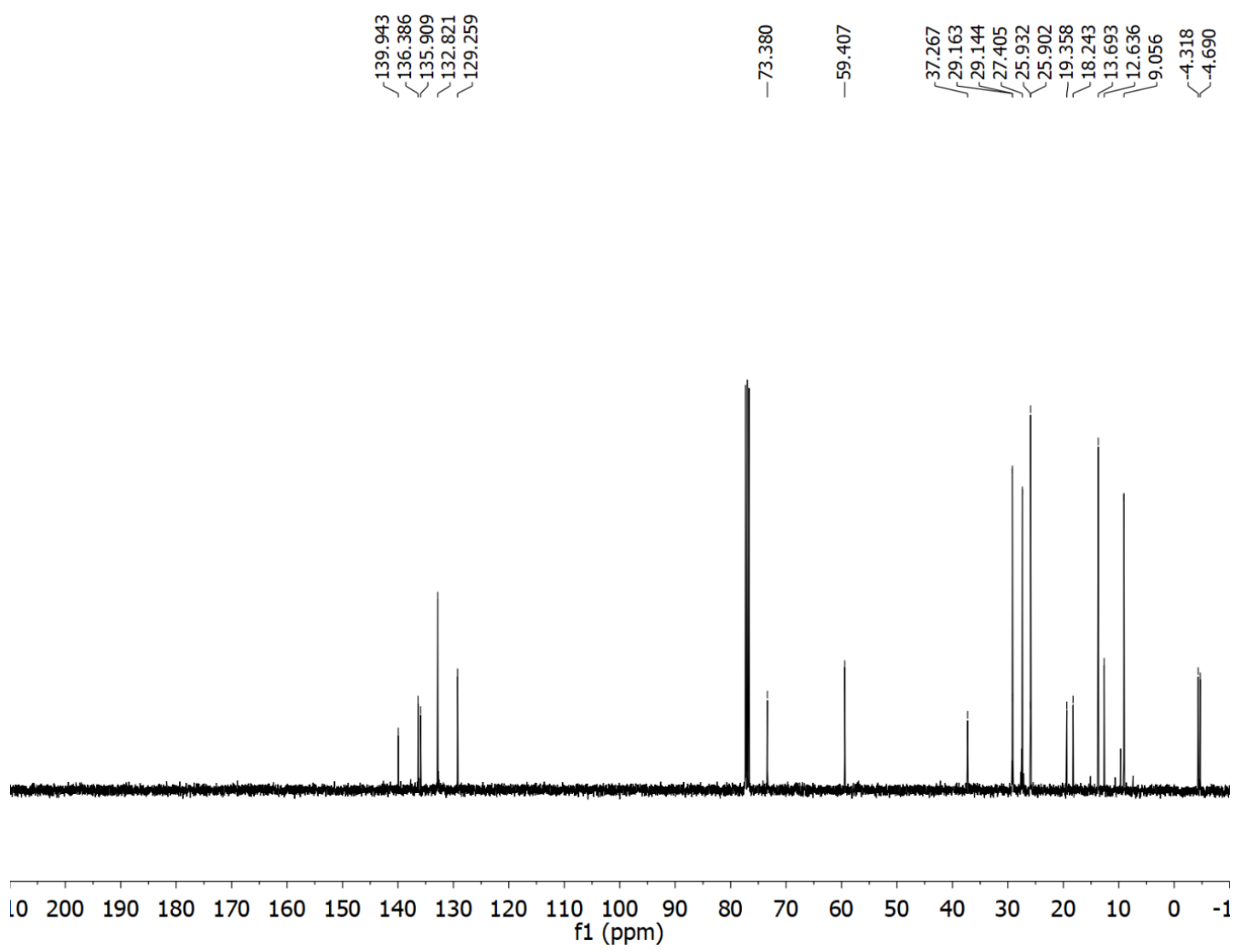
$\left({ }^{1} \mathrm{H} \mathrm{NMR}, \mathrm{CDCl}_{3}, 400 \mathrm{MHz}\right)$
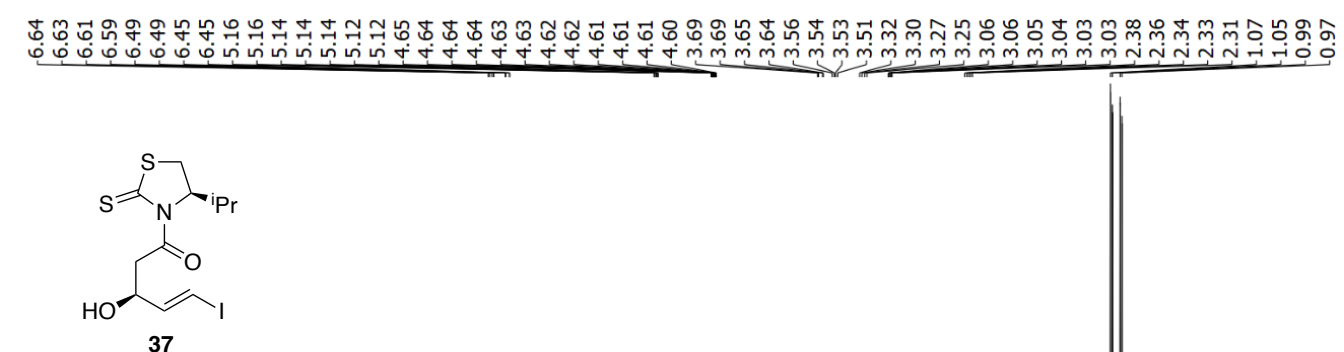

37

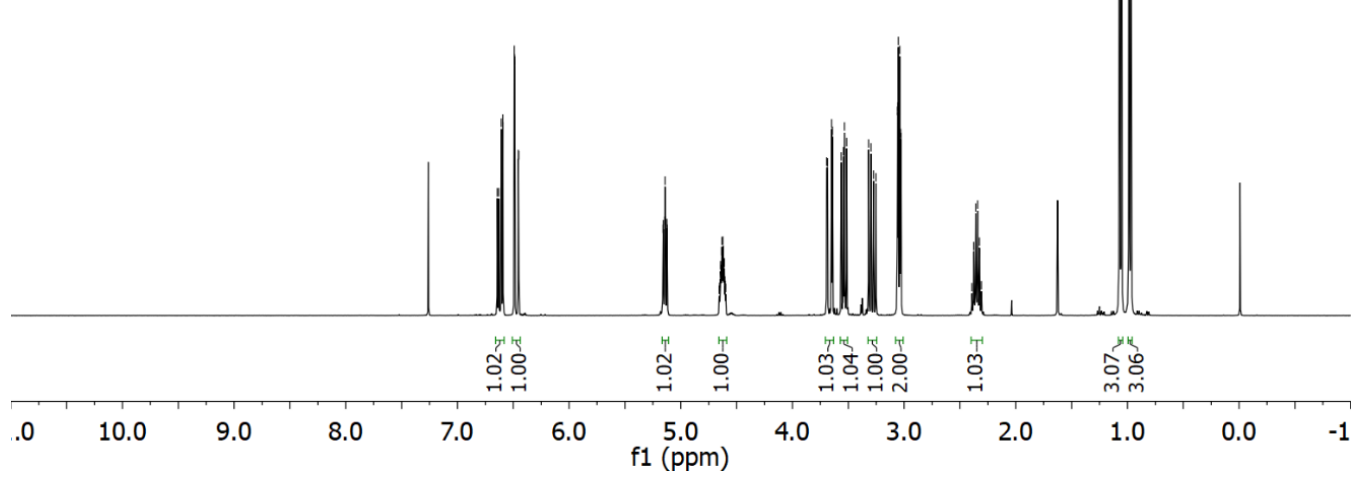

$\left({ }^{13} \mathrm{C} \mathrm{NMR}, \mathrm{CDCl}_{3}, 100 \mathrm{MHz}\right)$

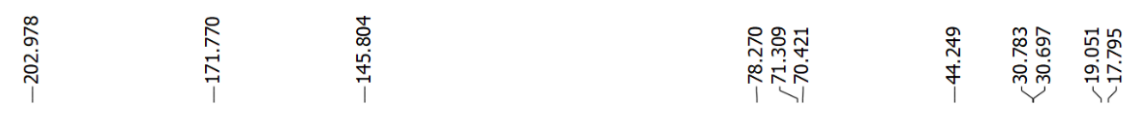

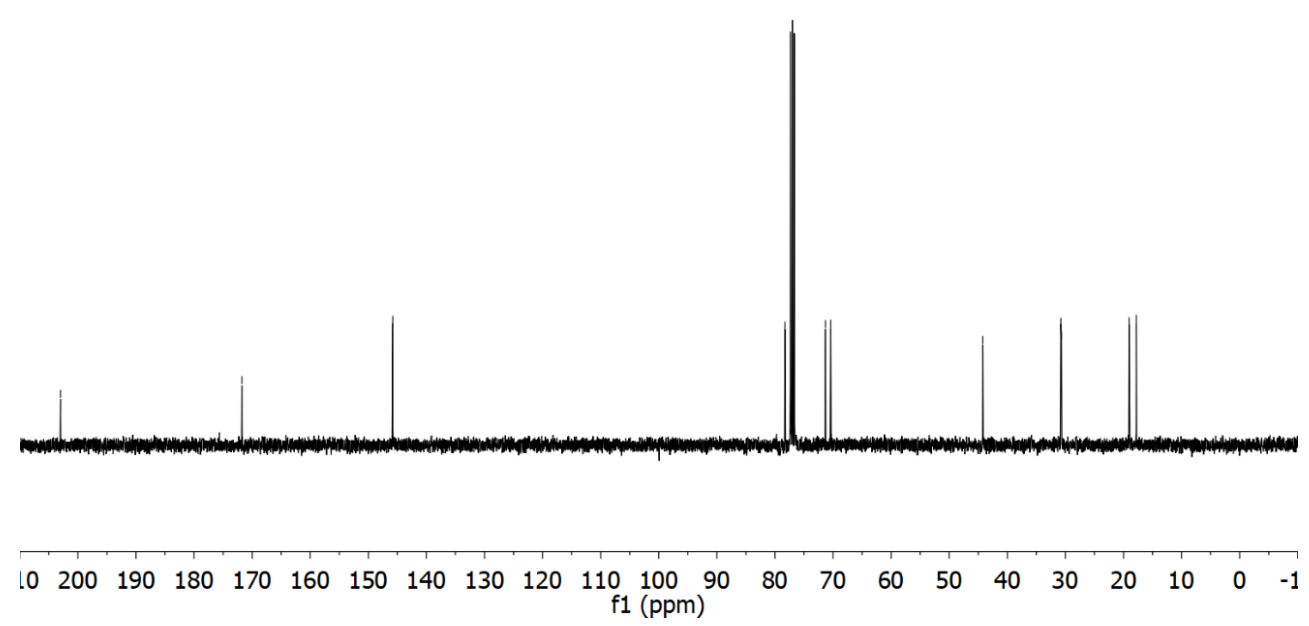


$\left({ }^{1} \mathrm{H} \mathrm{NMR}, \mathrm{CDCl}_{3}, 400 \mathrm{MHz}\right)$

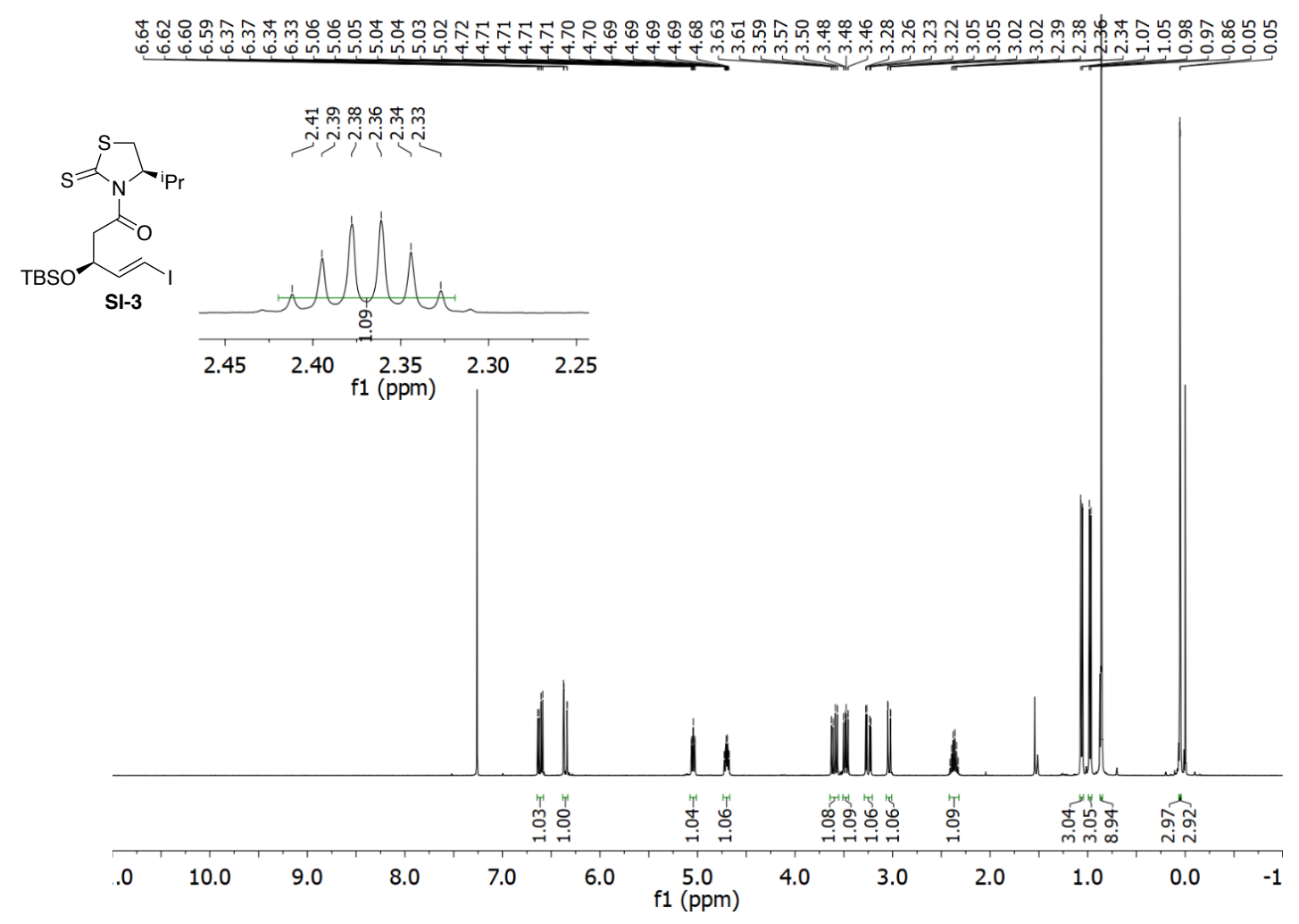

$\left({ }^{13} \mathrm{C} \mathrm{NMR}, \mathrm{CDCl}_{3}, 100 \mathrm{MHz}\right)$
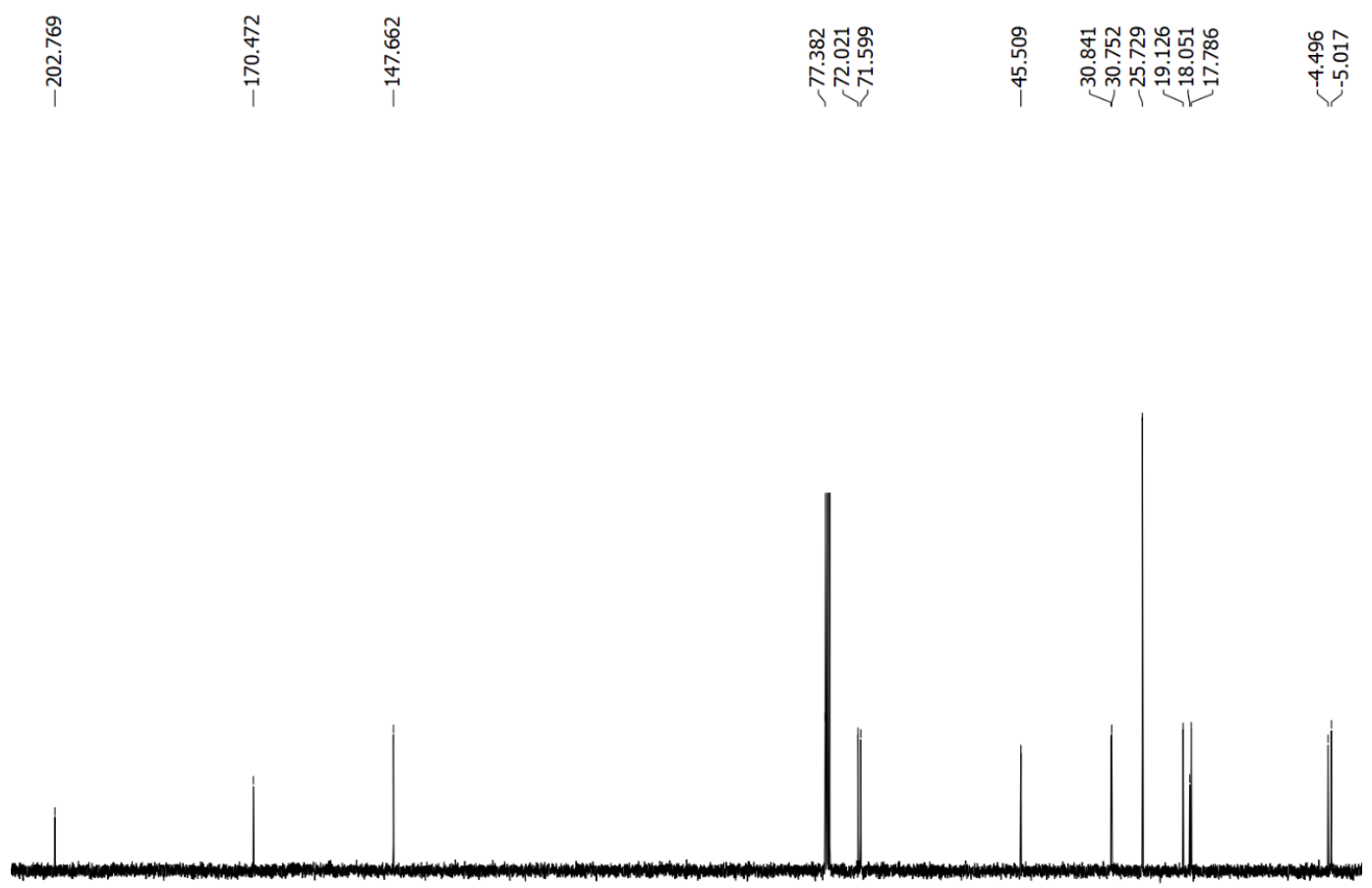

\begin{tabular}{lllllllllllllllllllllll}
\hline 0 & 200 & 190 & 180 & 170 & 160 & 150 & 140 & 130 & 120 & 110 & 100 & 90 & 80 & 70 & 60 & 50 & 40 & 30 & 20 & 10 & 0 & -1
\end{tabular} f1 (ppm) 
$\left({ }^{1} \mathrm{H} \mathrm{NMR}, \mathrm{CDCl}_{3}, 400 \mathrm{MHz}\right)$

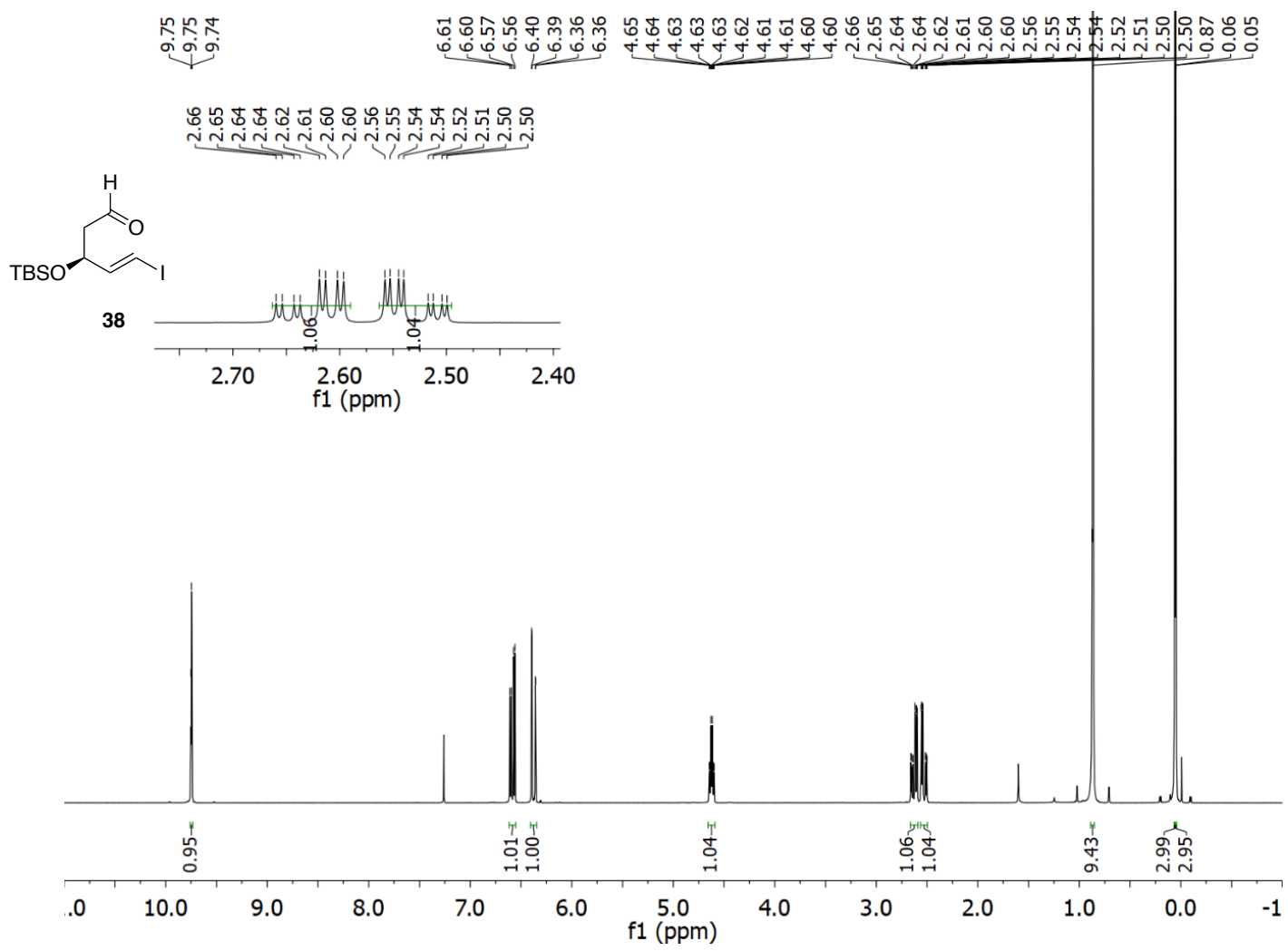

$\left({ }^{13} \mathrm{C} \mathrm{NMR}, \mathrm{CDCl}_{3}, 100 \mathrm{MHz}\right)$
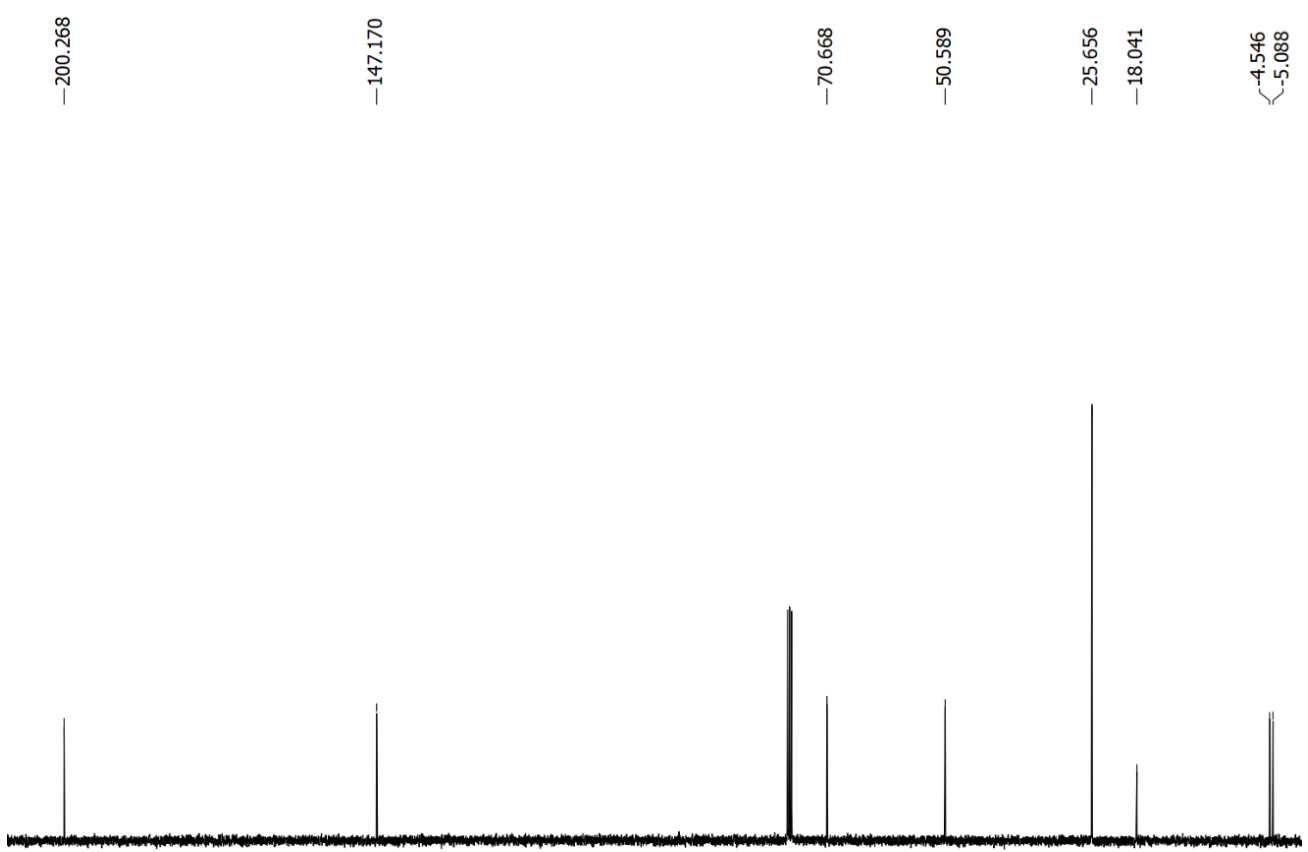

\begin{tabular}{lllllllllllllllllllllllllllll}
\hline 0 & 200 & 190 & 180 & 170 & 160 & 150 & 140 & 130 & 120 & 110 & 100 & 90 & 80 & 70 & 60 & 50 & 40 & 30 & 20 & 10 & 0 & -1
\end{tabular} 
$\left({ }^{1} \mathrm{H} \mathrm{NMR}, \mathrm{CDCl}_{3}, 400 \mathrm{MHz}\right)$

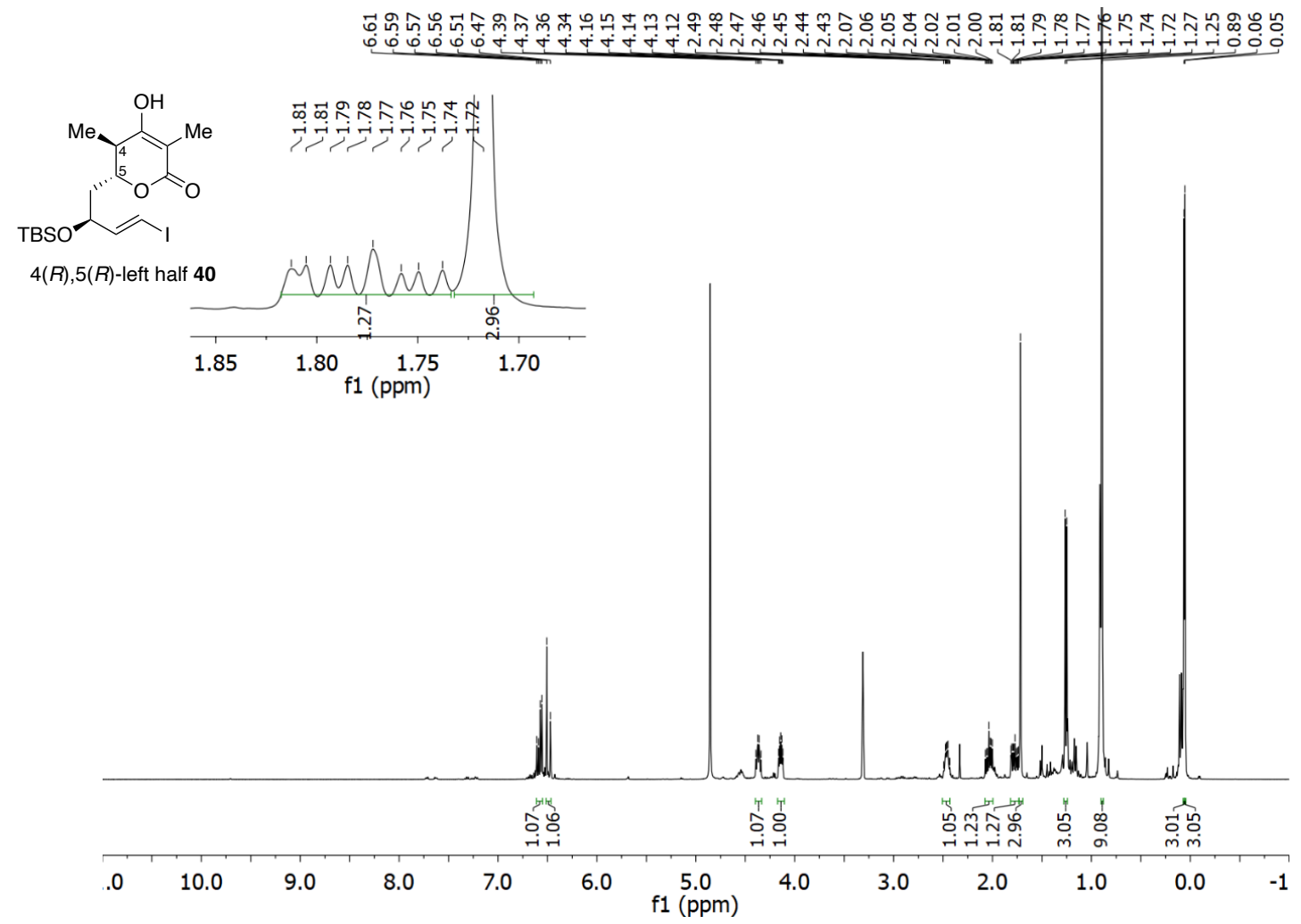

$\left({ }^{13} \mathrm{C} \mathrm{NMR}, \mathrm{CDCl}_{3}, 100 \mathrm{MHz}\right)$

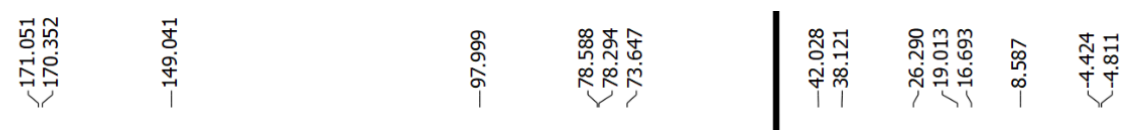

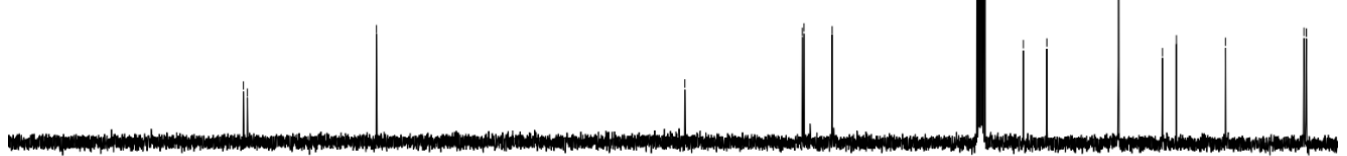

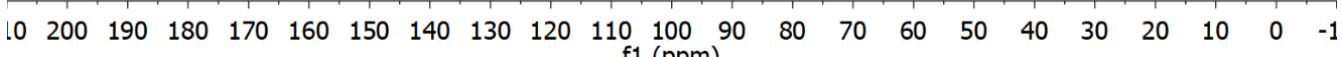
f1 (ppm) 
( $\left.{ }^{1} \mathrm{H} \mathrm{NMR}, \mathrm{CDCl}_{3}, 400 \mathrm{MHz}\right)$

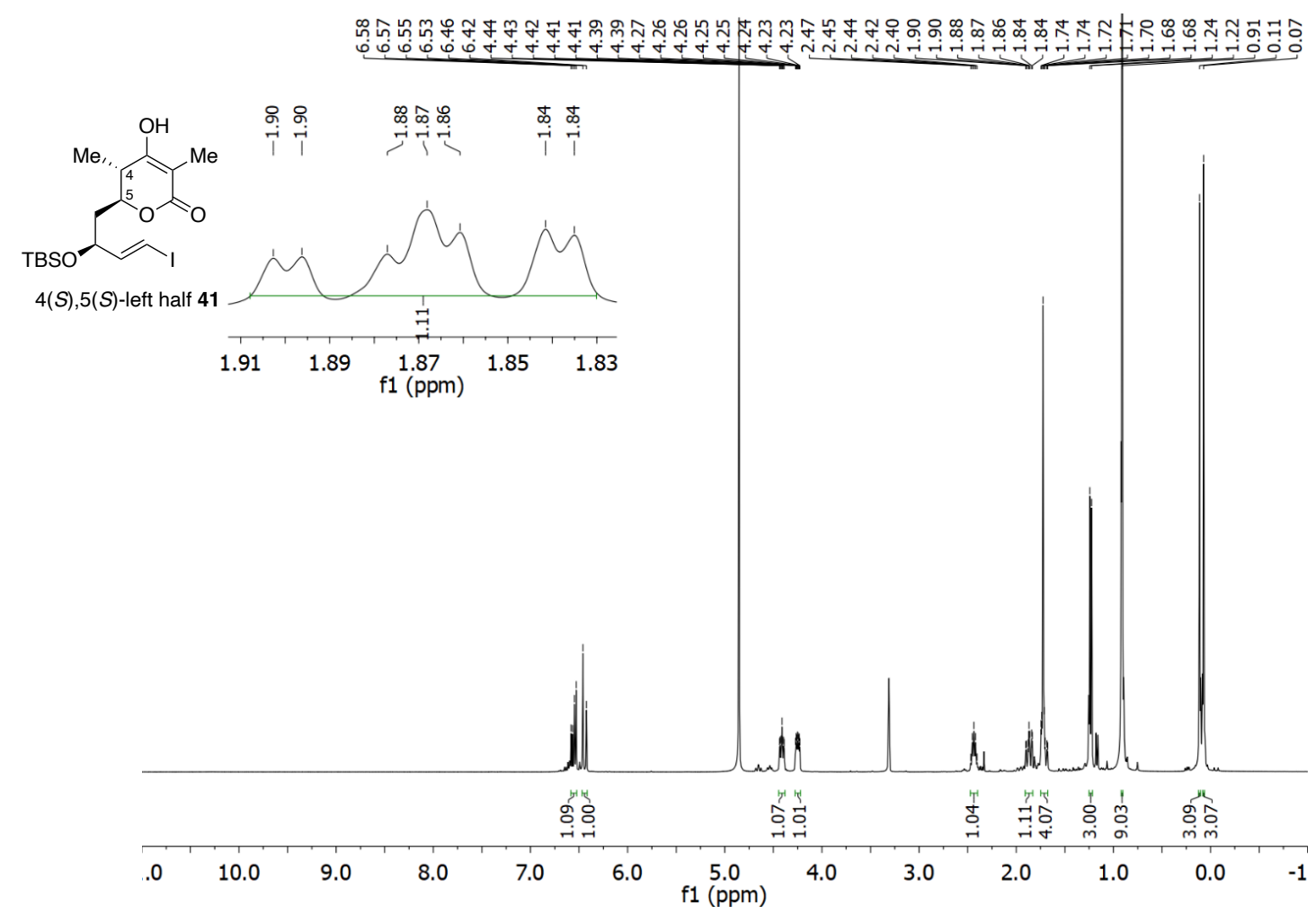

$\left({ }^{13} \mathrm{C} \mathrm{NMR}, \mathrm{CDCl}_{3}, 100 \mathrm{MHz}\right)$

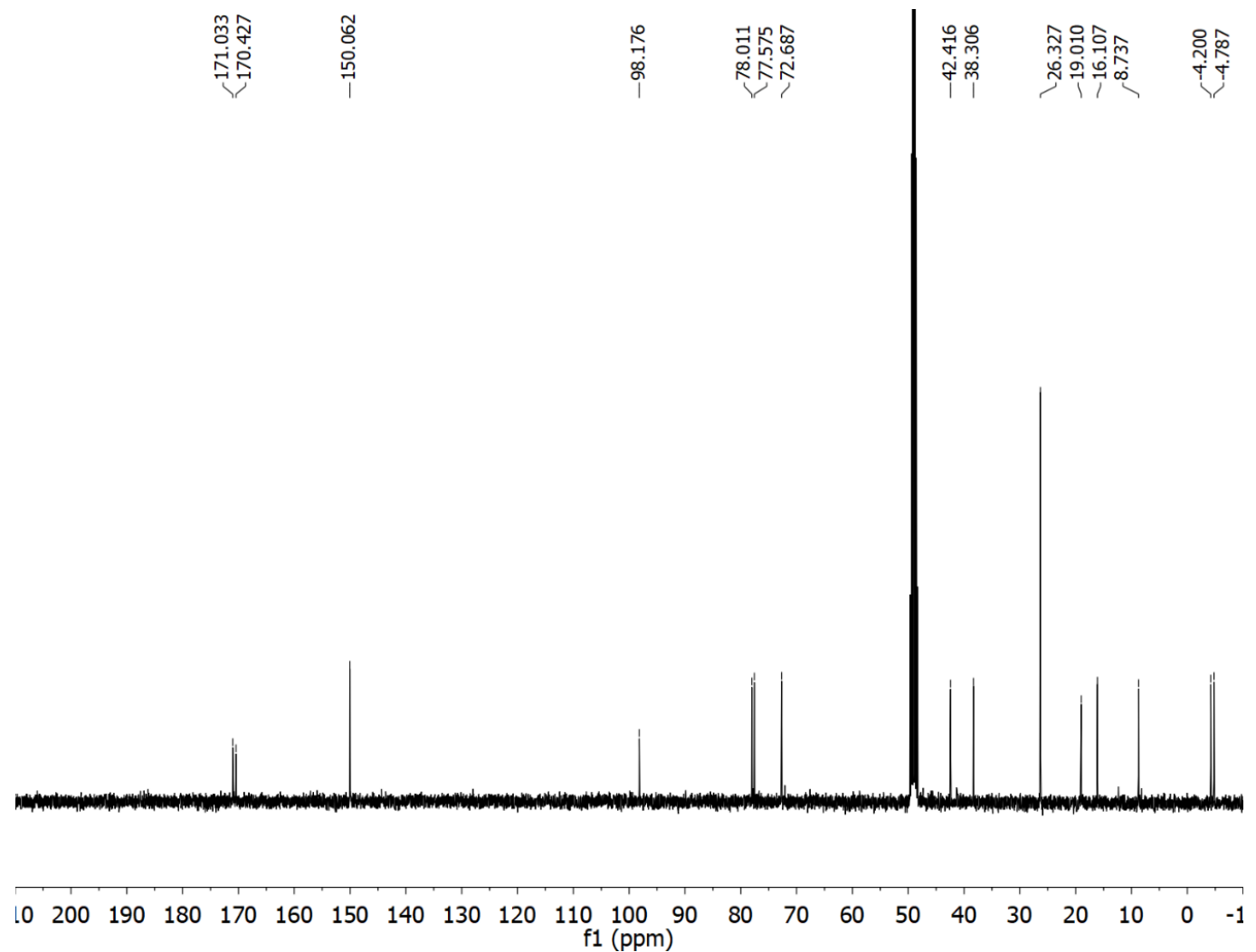


( $\left.{ }^{1} \mathrm{H} \mathrm{NMR}, \mathrm{CDCl}_{3}, 400 \mathrm{MHz}\right)$
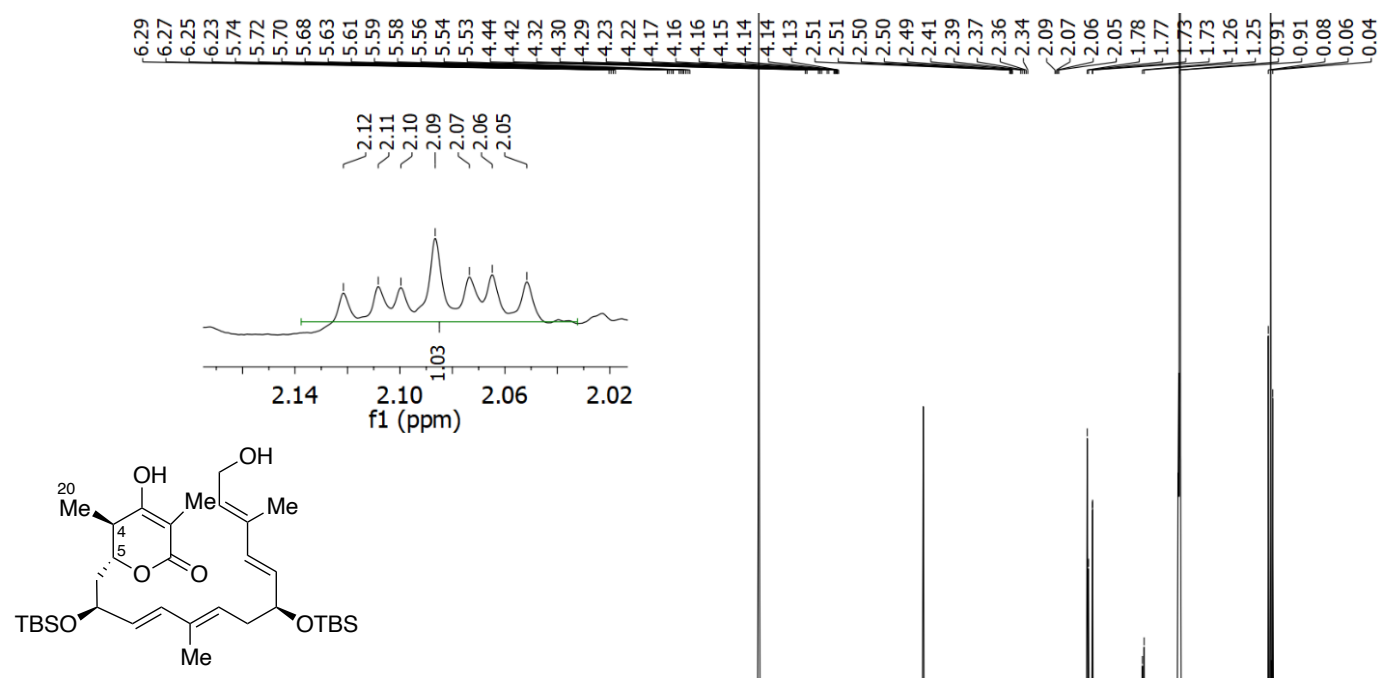

SI-4

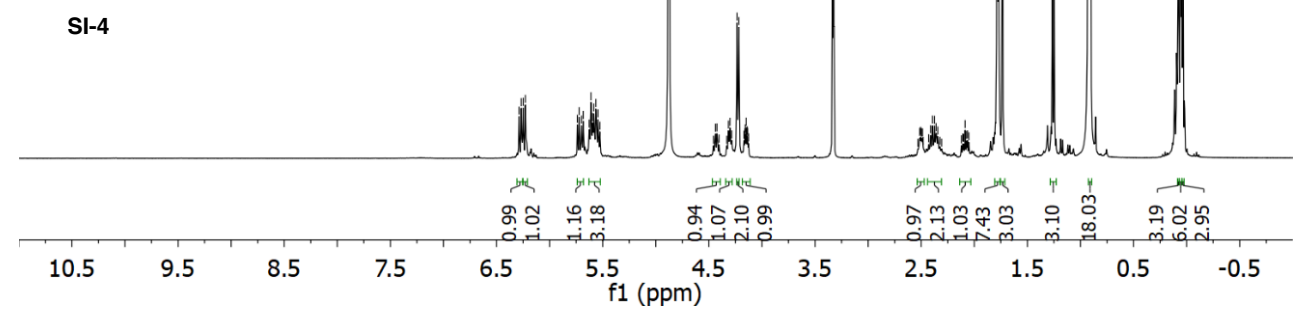

$\left({ }^{13} \mathrm{C} \mathrm{NMR}, \mathrm{CDCl}_{3}, 100 \mathrm{MHz}\right)$

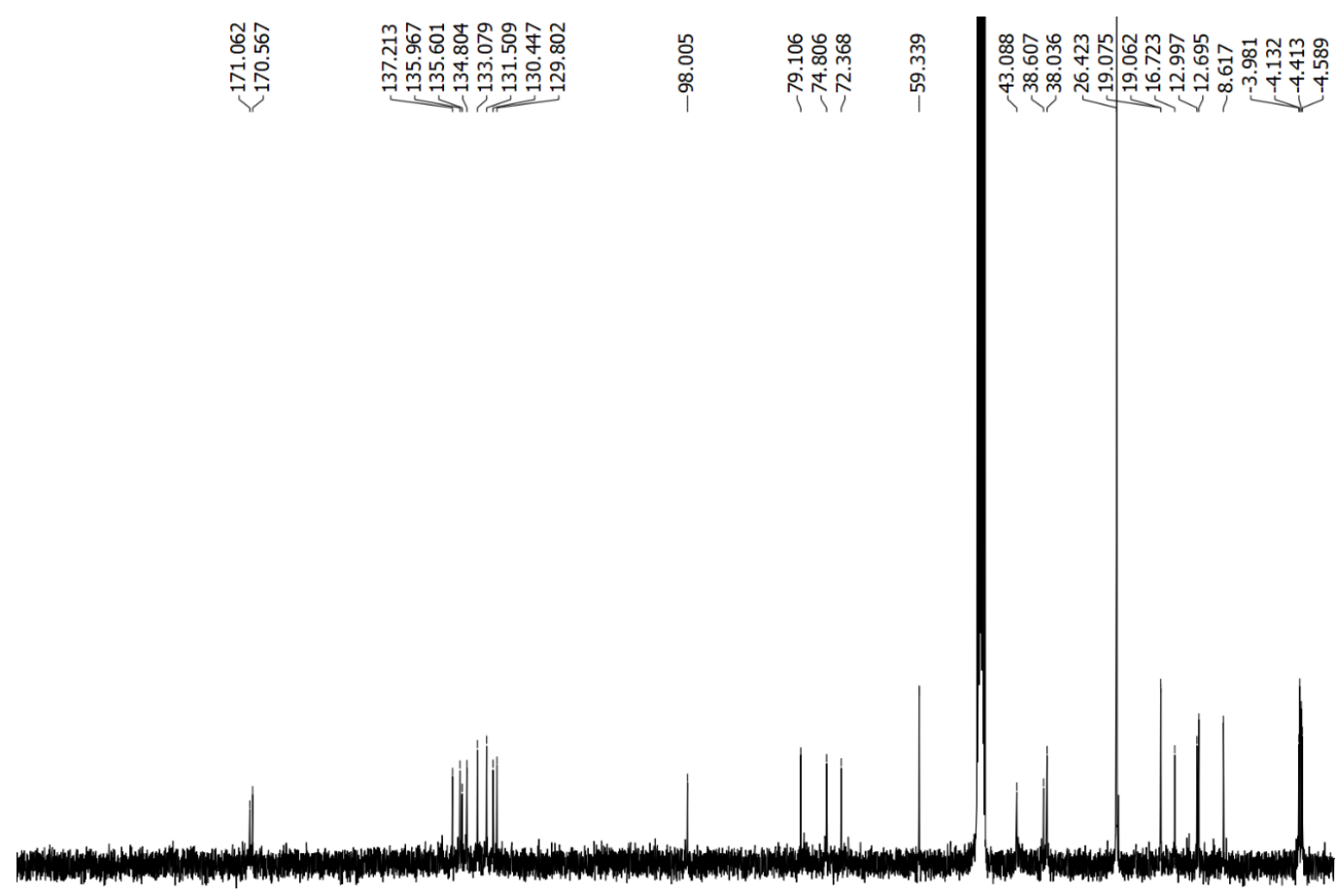

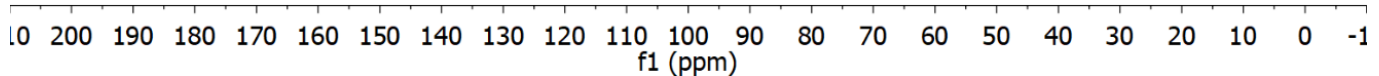


$\left({ }^{1} \mathrm{H} \mathrm{NMR}, \mathrm{CDCl}_{3}, 400 \mathrm{MHz}\right)$

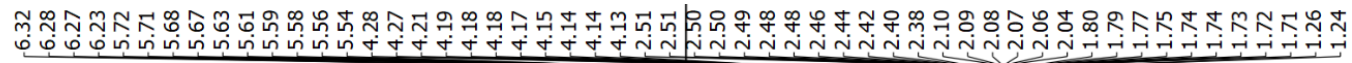

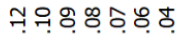

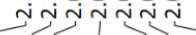

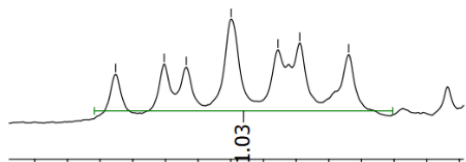

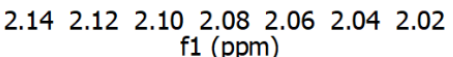
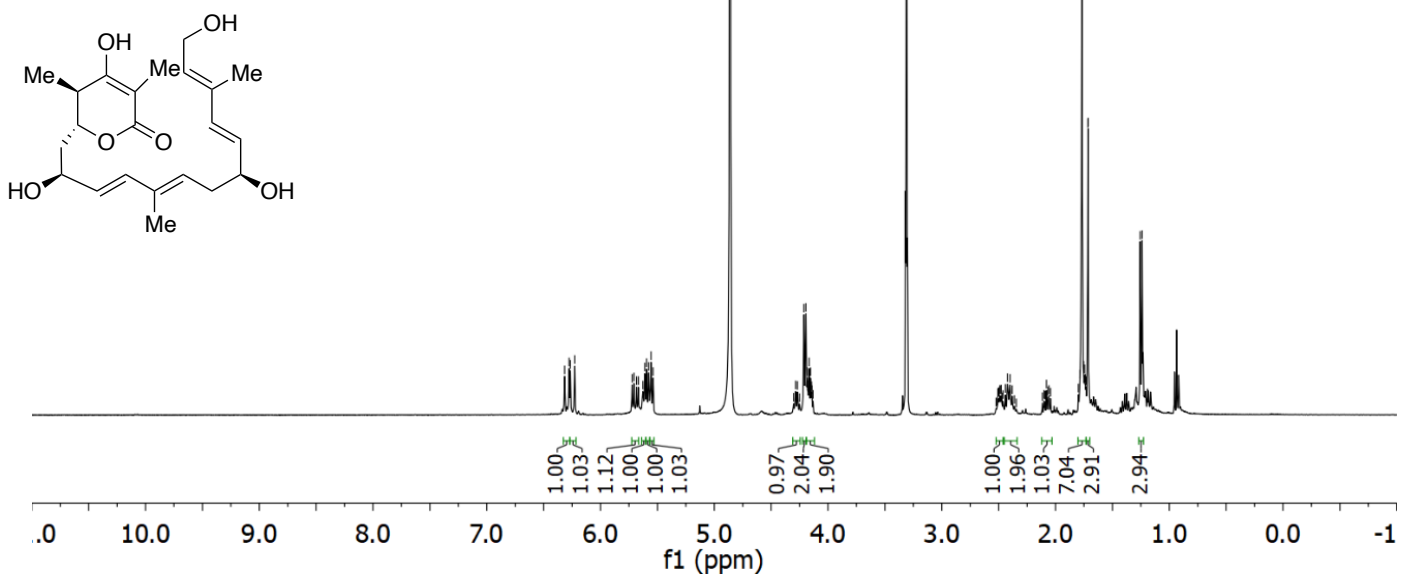

$\left({ }^{13} \mathrm{C} \mathrm{NMR}, \mathrm{CDCl}_{3}, 100 \mathrm{MHz}\right)$

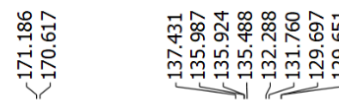

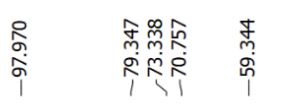

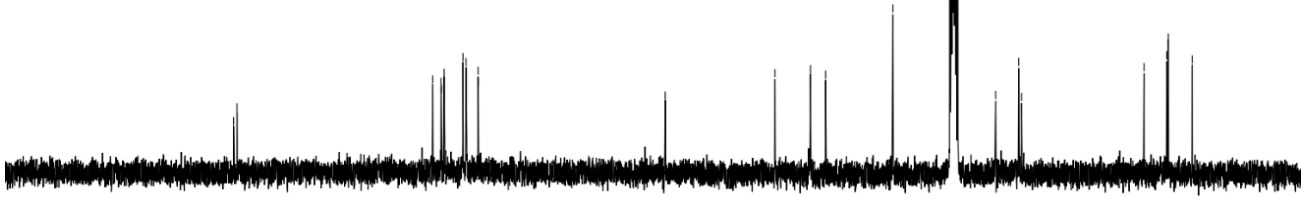

\begin{tabular}{llllllllllllllllllllllllllll}
\hline 0 & 200 & 190 & 180 & 170 & 160 & 150 & 140 & 130 & 120 & 110 & 100 & 90 & 80 & 70 & 60 & 50 & 40 & 30 & 20 & 10 & 0 & -1
\end{tabular} 
$\left({ }^{1} \mathrm{H} \mathrm{NMR}, \mathrm{CDCl}_{3}, 400 \mathrm{MHz}\right)$

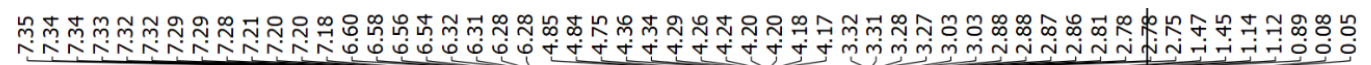

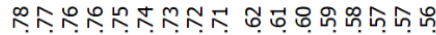

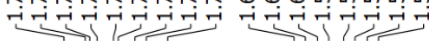

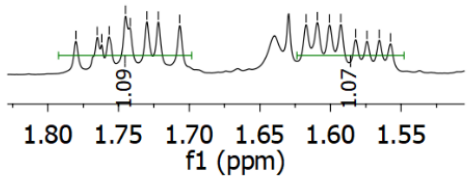

(

SI-5

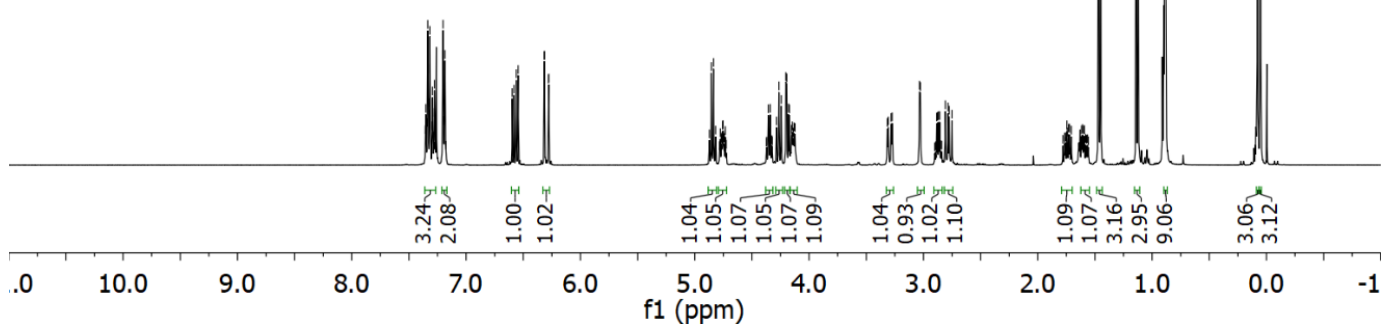

$\left({ }^{13} \mathrm{C} \mathrm{NMR}, \mathrm{CDCl}_{3}, 100 \mathrm{MHz}\right)$

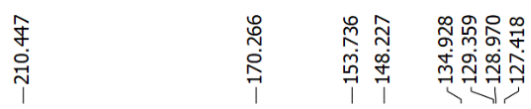

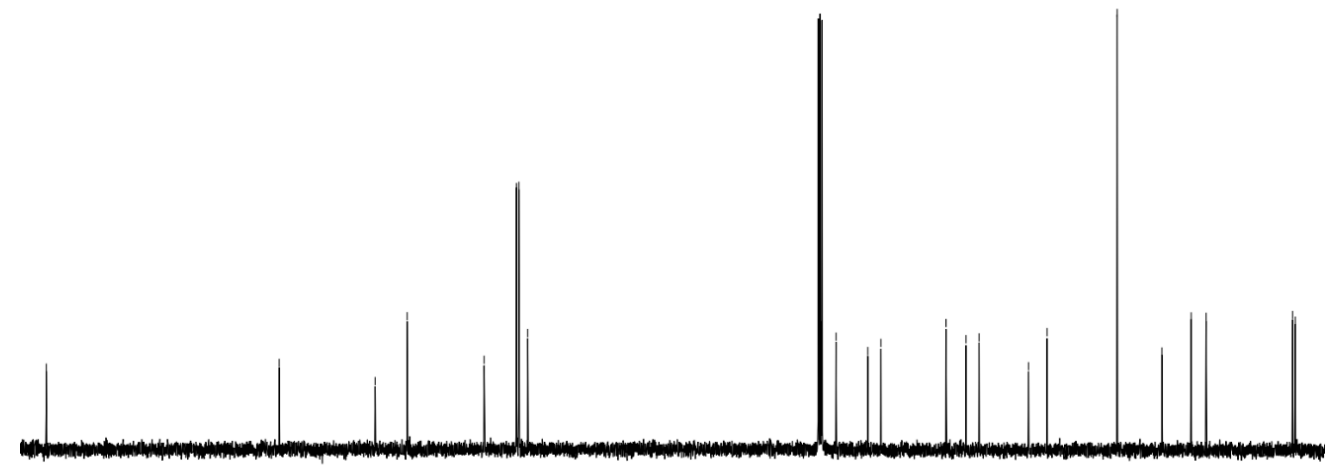

$\begin{array}{llllllllllllllllllllll}210 & 200 & 190 & 180 & 170 & 160 & 150 & 140 & 130 & 120 & 110 \\ \mathrm{f} 1(\mathrm{ppm}) & 100 & 90 & 80 & 70 & 60 & 50 & 40 & 30 & 20 & 10 & 0 & -1\end{array}$ 
$\left({ }^{1} \mathrm{H} \mathrm{NMR}, \mathrm{CDCl}_{3}, 400 \mathrm{MHz}\right)$

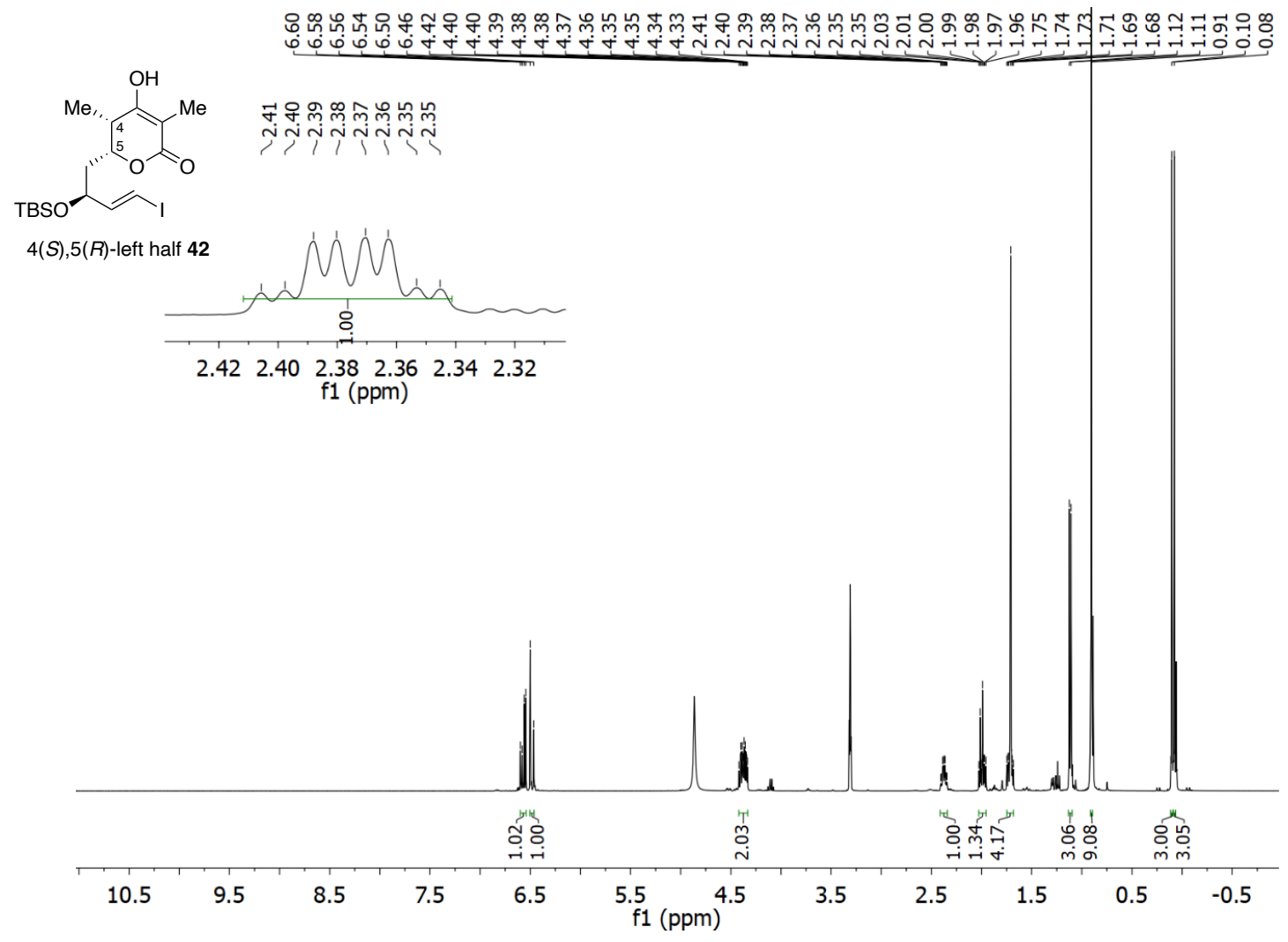

$\left({ }^{13} \mathrm{C} \mathrm{NMR}, \mathrm{CDCl}_{3}, 100 \mathrm{MHz}\right)$

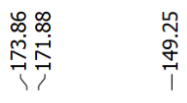


$\left({ }^{1} \mathrm{H} \mathrm{NMR}, \mathrm{CDCl}_{3}, 400 \mathrm{MHz}\right)$

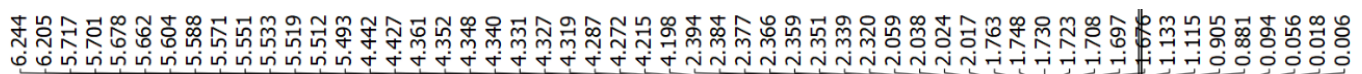

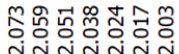
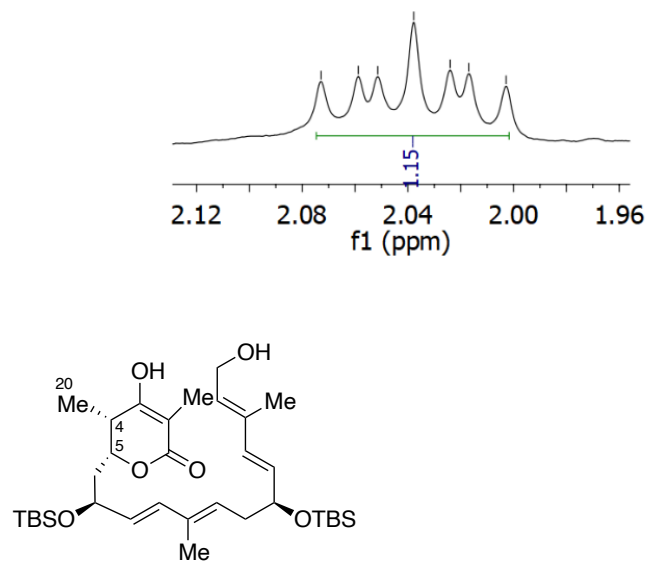

SI-6

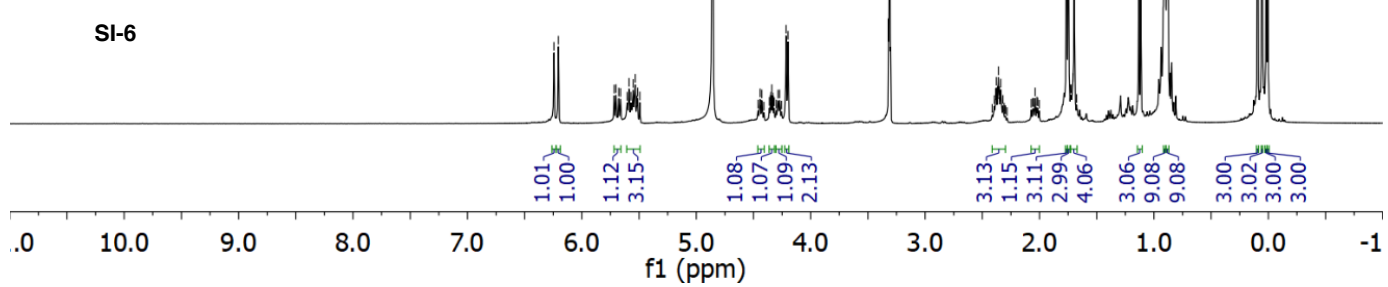

$\left({ }^{13} \mathrm{C} \mathrm{NMR}, \mathrm{CDCl}_{3}, 100 \mathrm{MHz}\right)$

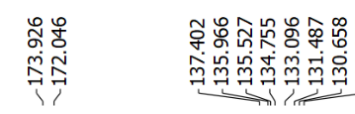

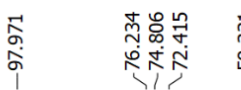

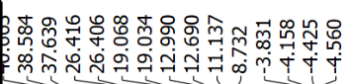

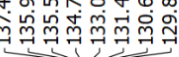

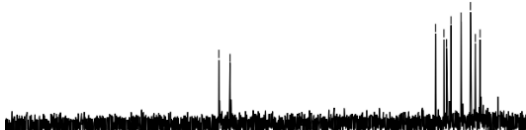

$\begin{array}{lllllllllllllllllllll}200 & 190 & 180 & 170 & 160 & 150 & 140 & 130 & 120 & 110 \quad \begin{array}{l}100 \\ \mathrm{f} 1\end{array}(\mathrm{ppm}) & 90 & 80 & 70 & 60 & 50 & 40 & 30 & 20 & 10 & 0 & -1\end{array}$ 
$\left({ }^{1} \mathrm{H} \mathrm{NMR}, \mathrm{CDCl}_{3}, 400 \mathrm{MHz}\right)$

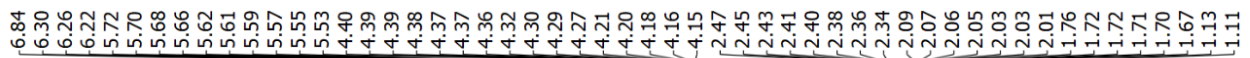
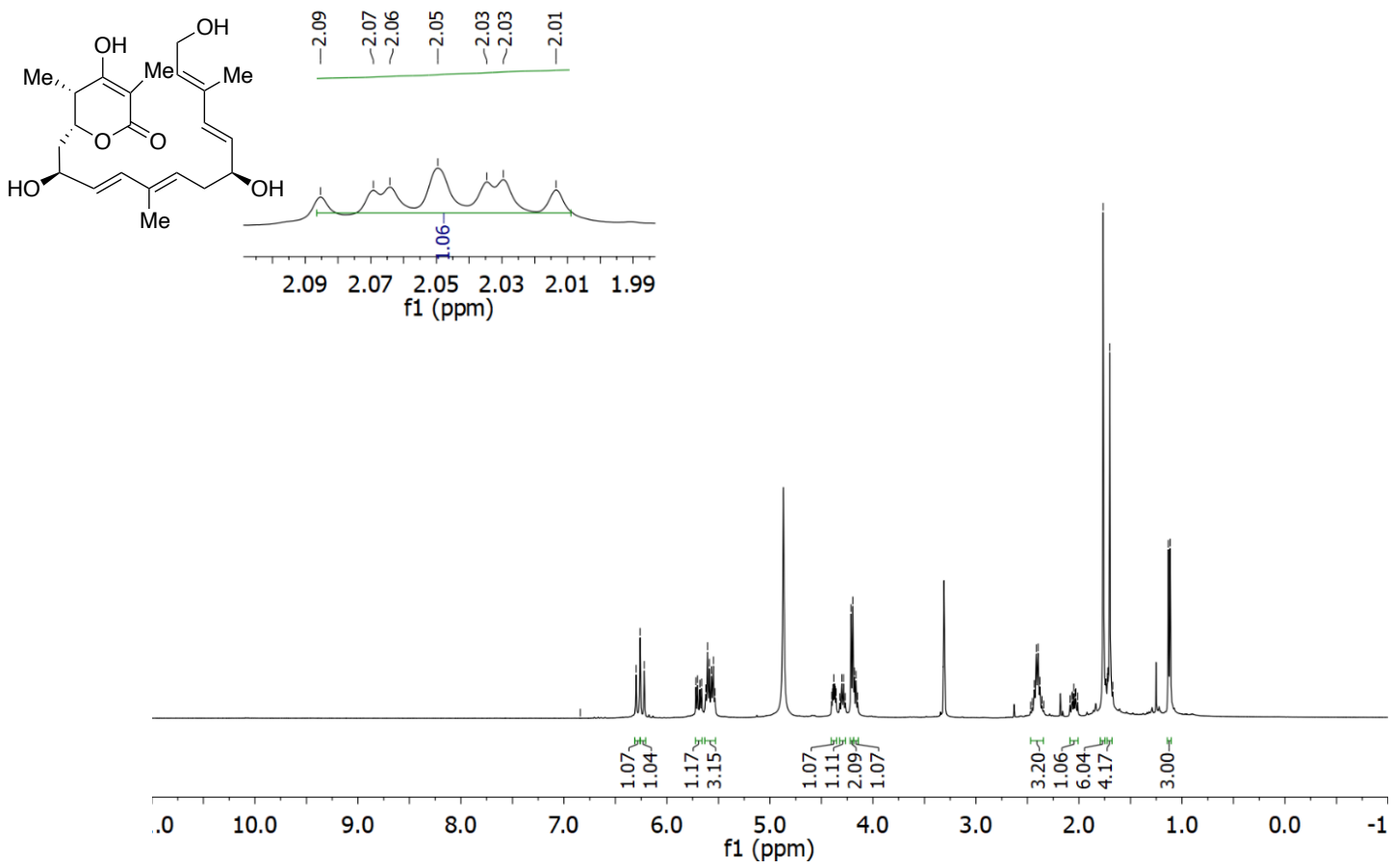

$\left({ }^{13} \mathrm{C} \mathrm{NMR}, \mathrm{CDCl}_{3}, 100 \mathrm{MHz}\right)$

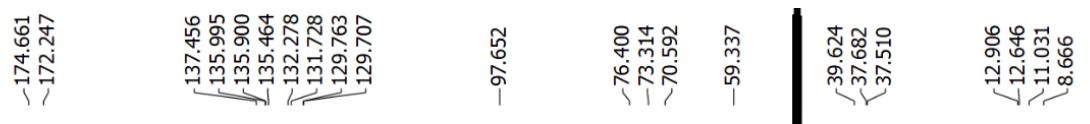

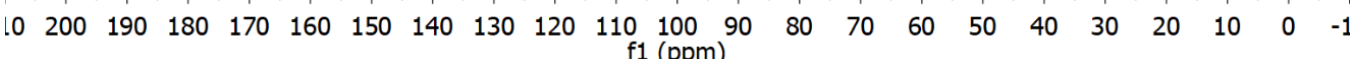


$\left({ }^{1} \mathrm{H} \mathrm{NMR}, \mathrm{CDCl}_{3}, 400 \mathrm{MHz}\right)$

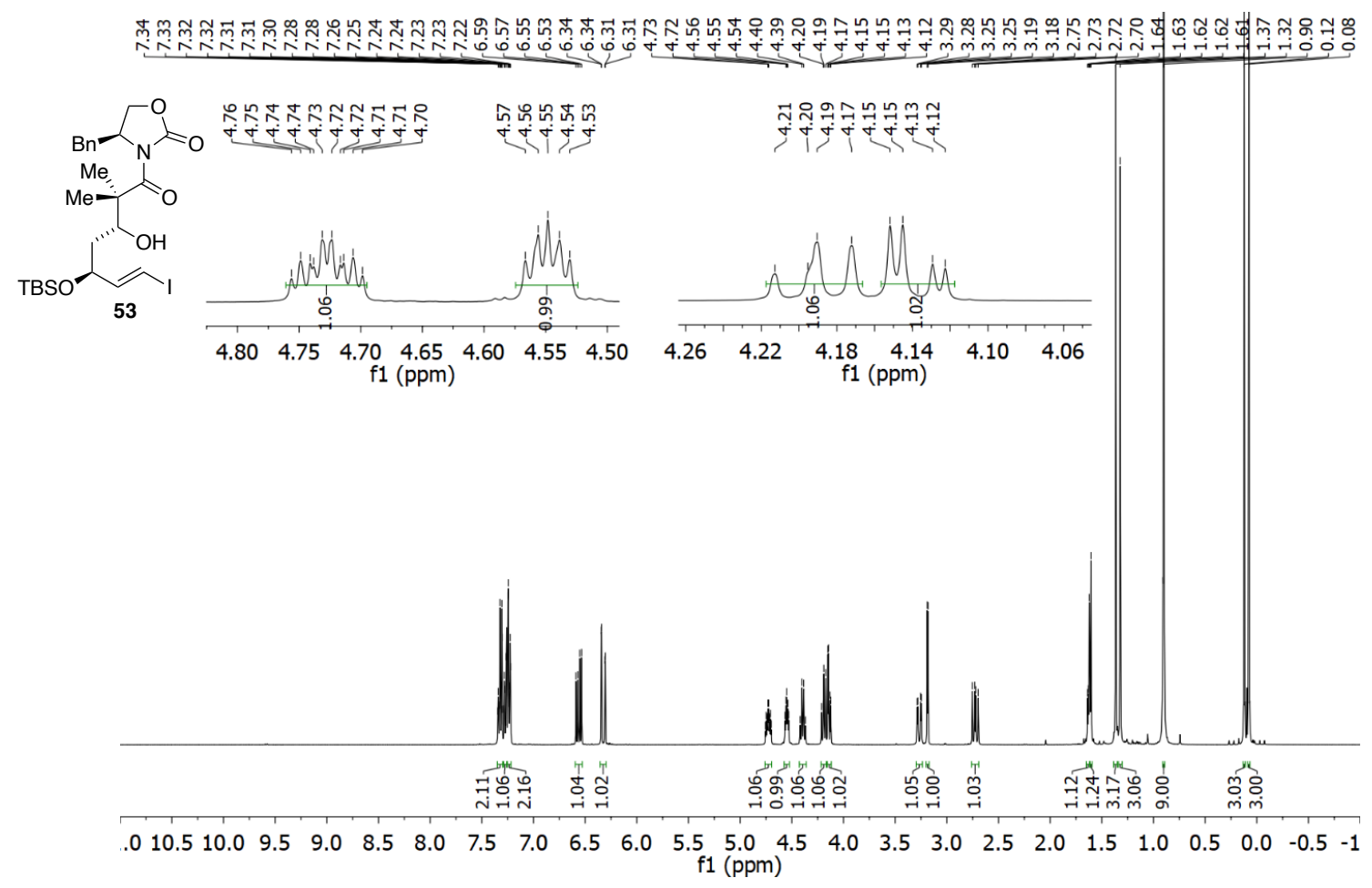

$\left({ }^{13} \mathrm{C} \mathrm{NMR}, \mathrm{CDCl}_{3}, 100 \mathrm{MHz}\right)$
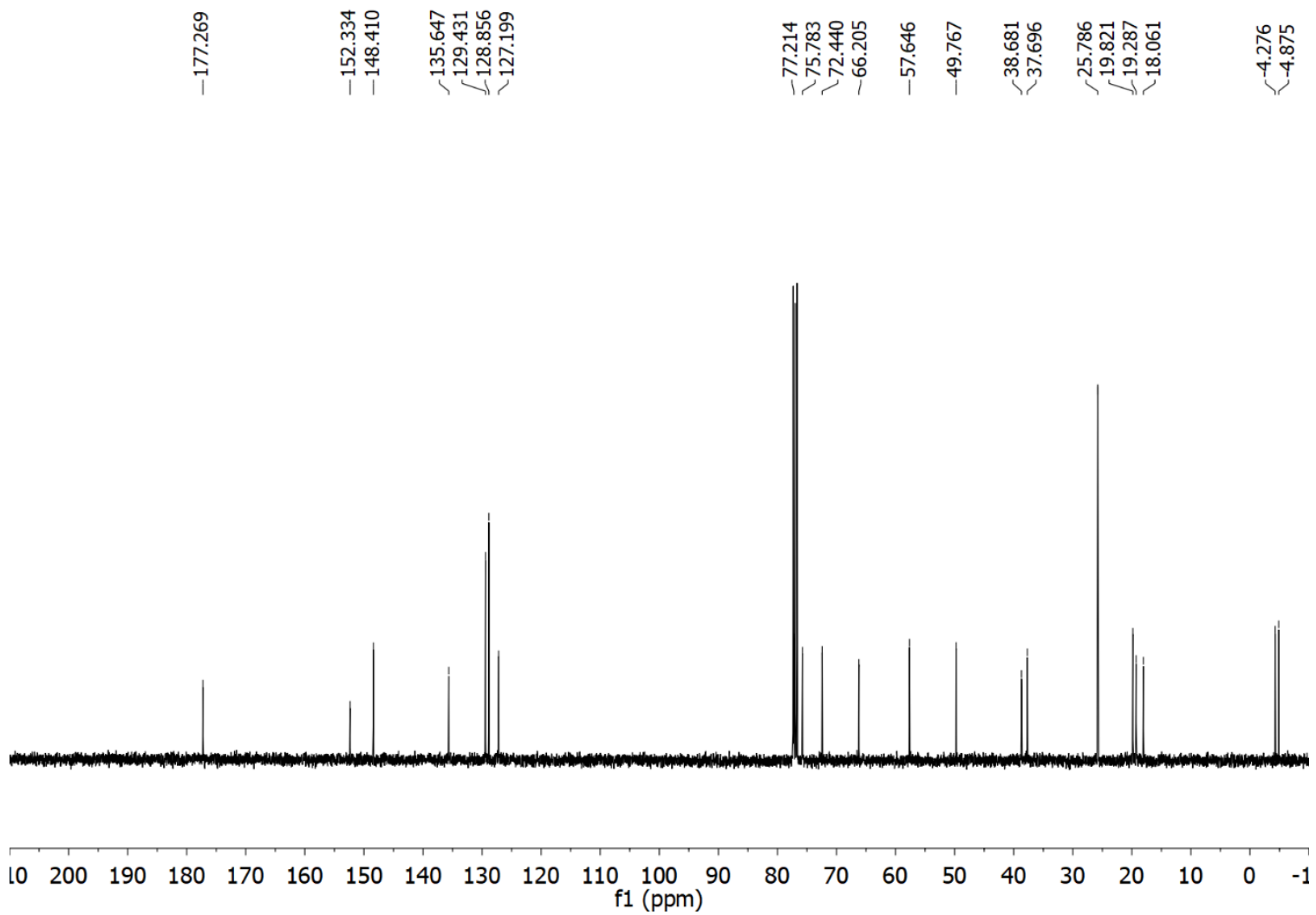
( $\left.{ }^{1} \mathrm{H} \mathrm{NMR}, \mathrm{CDCl}_{3}, 400 \mathrm{MHz}\right)$

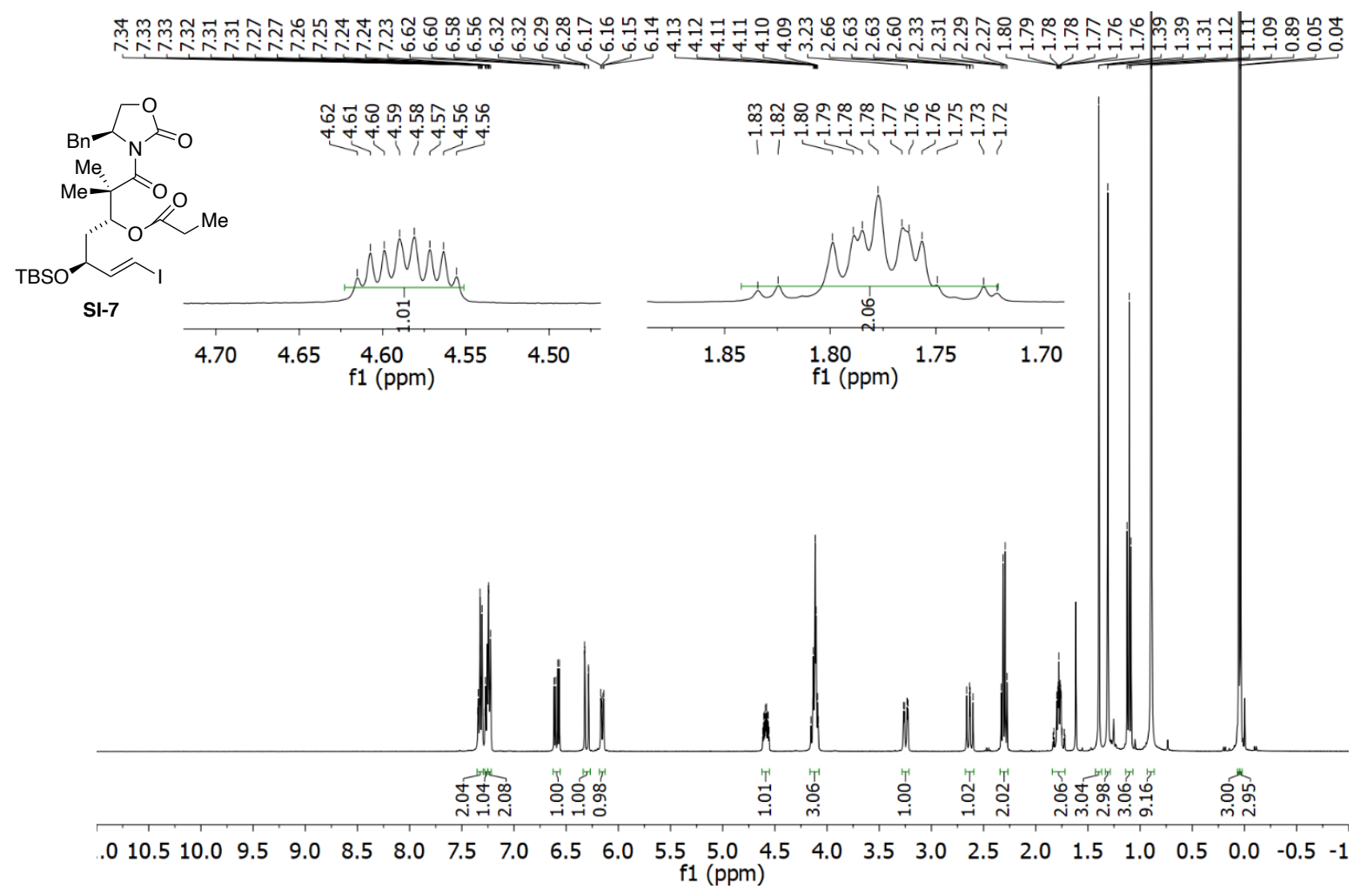

$\left({ }^{13} \mathrm{C} \mathrm{NMR}, \mathrm{CDCl}_{3}, 100 \mathrm{MHz}\right)$

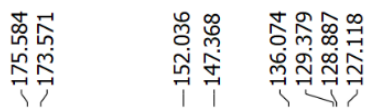

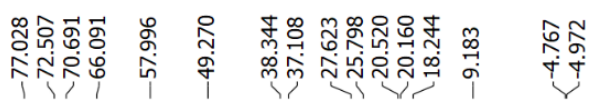

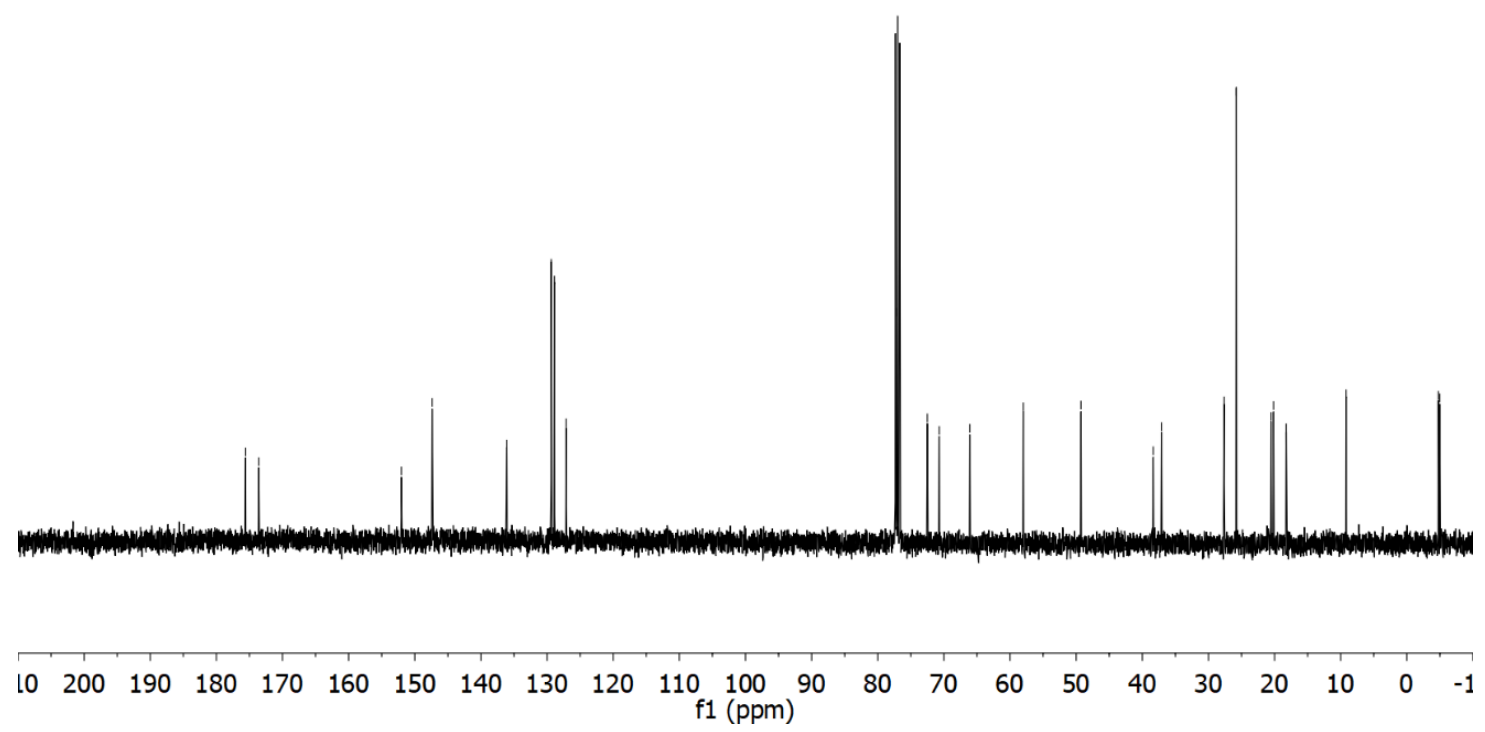


( $\left.{ }^{1} \mathrm{H} \mathrm{NMR}, \mathrm{CDCl}_{3}, 400 \mathrm{MHz}\right)$
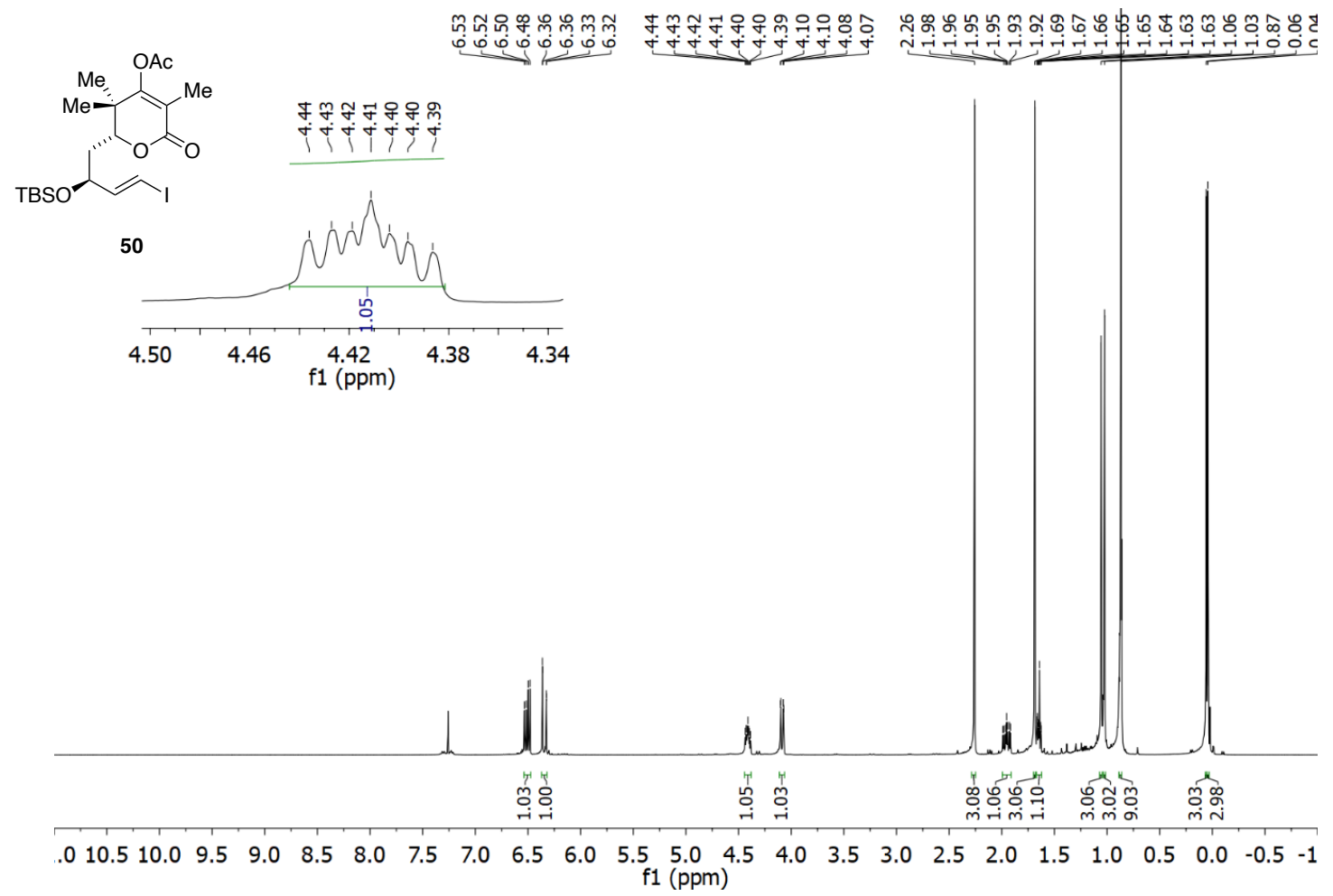

$\left({ }^{13} \mathrm{C} \mathrm{NMR}, \mathrm{CDCl}_{3}, 100 \mathrm{MHz}\right)$
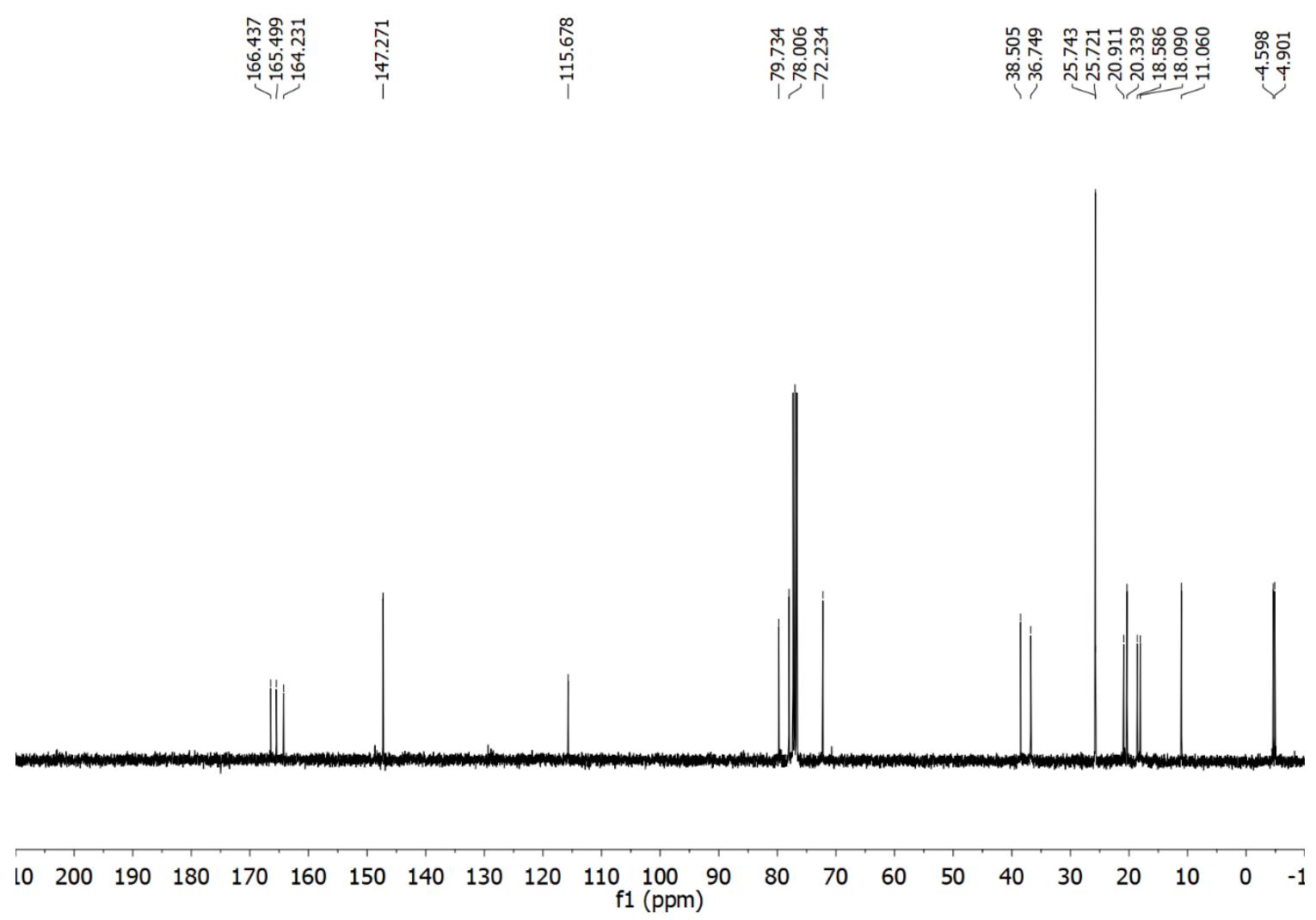
$\left({ }^{1} \mathrm{H} \mathrm{NMR}, \mathrm{CDCl}_{3}, 400 \mathrm{MHz}\right)$

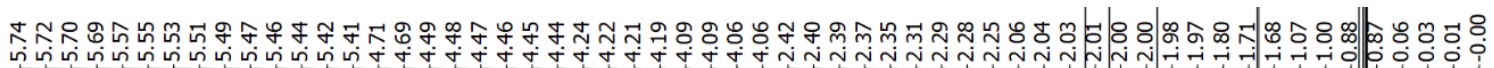

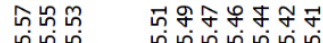

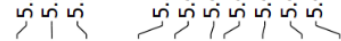

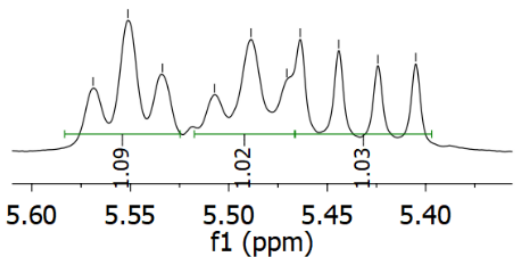

Me

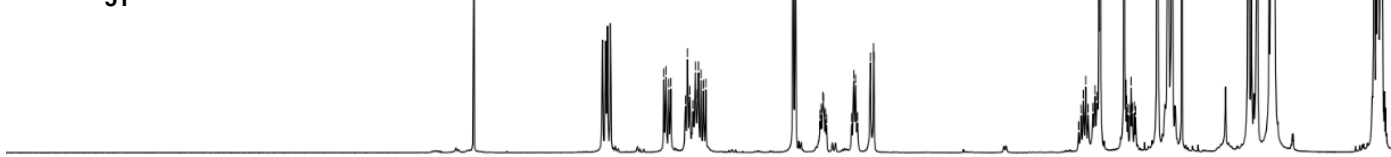

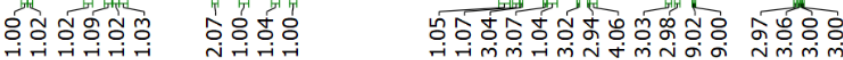

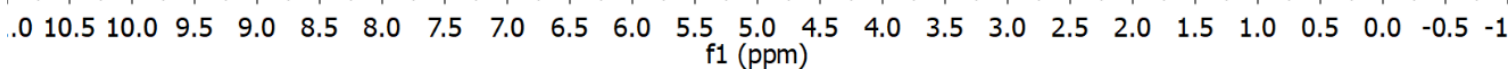

$\left({ }^{13} \mathrm{C} \mathrm{NMR}, \mathrm{CDCl}_{3}, 100 \mathrm{MHz}\right)$

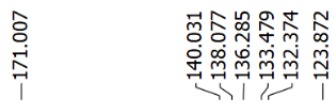

$\begin{array}{ll}\overrightarrow{0} & \stackrel{+}{1} \\ \stackrel{0}{i} & \vdots\end{array}$

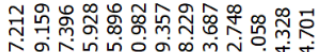

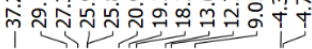

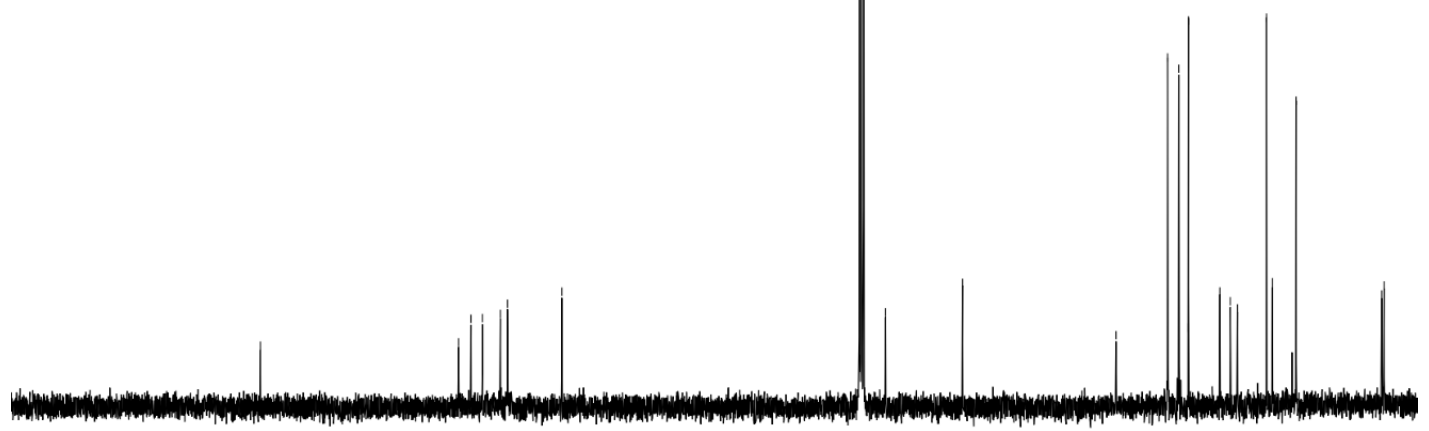

l0 $20 \begin{array}{lllllllllllllllllllll}190 & 180 & 170 & 160 & 150 & 140 & 130 & 120 & 110 \\ \mathrm{f} 1(\mathrm{ppm}) & 100 & 80 & 70 & 60 & 50 & 40 & 30 & 20 & 10 & 0 & -1\end{array}$ 
$\left({ }^{1} \mathrm{H} \mathrm{NMR}, \mathrm{CDCl}_{3}, 400 \mathrm{MHz}\right)$

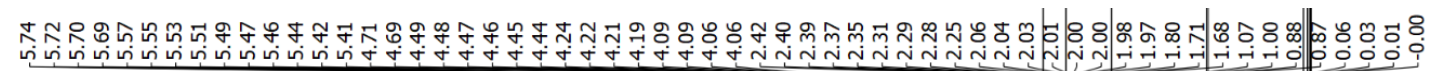

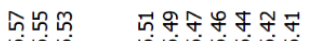

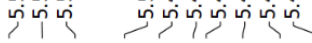
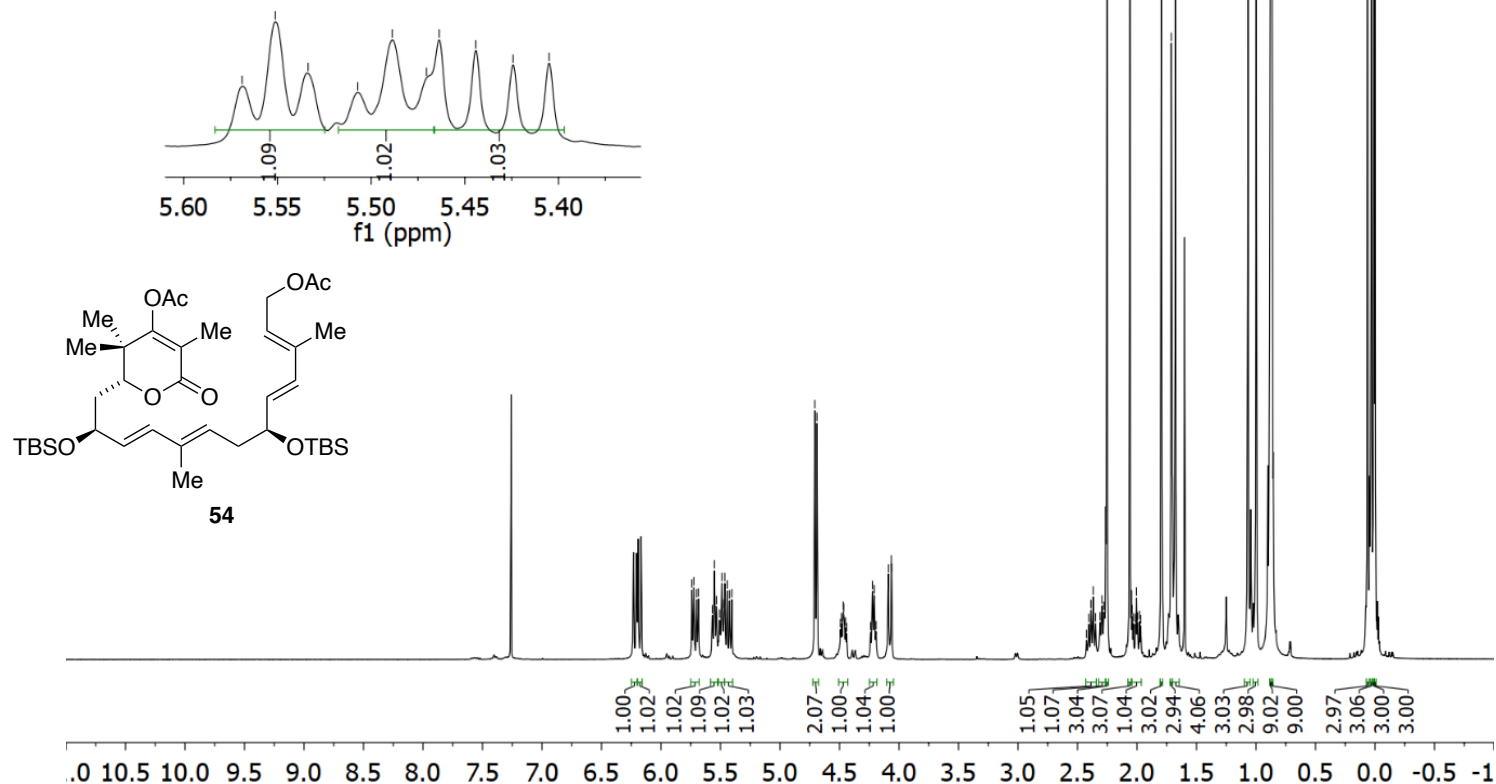

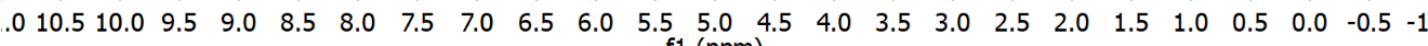

$\left({ }^{13} \mathrm{C} \mathrm{NMR}, \mathrm{CDCl}_{3}, 100 \mathrm{MHz}\right)$

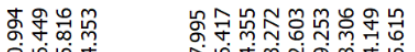

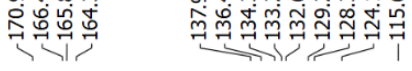

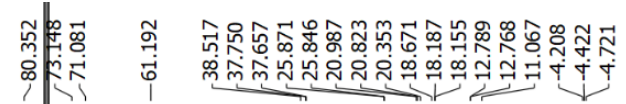

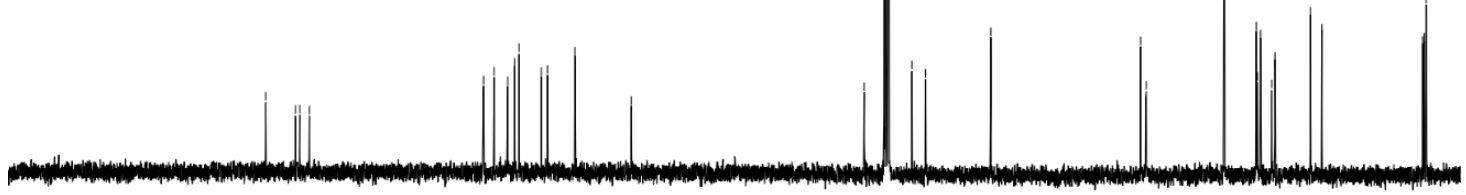

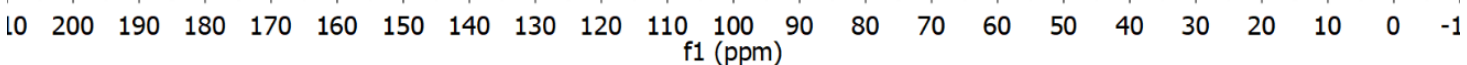


$\left({ }^{1} \mathrm{H} \mathrm{NMR}, \mathrm{CDCl}_{3}, 400 \mathrm{MHz}\right)$

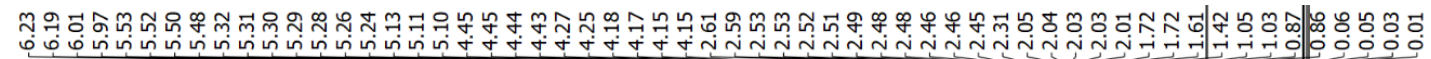

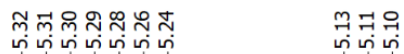

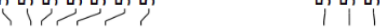
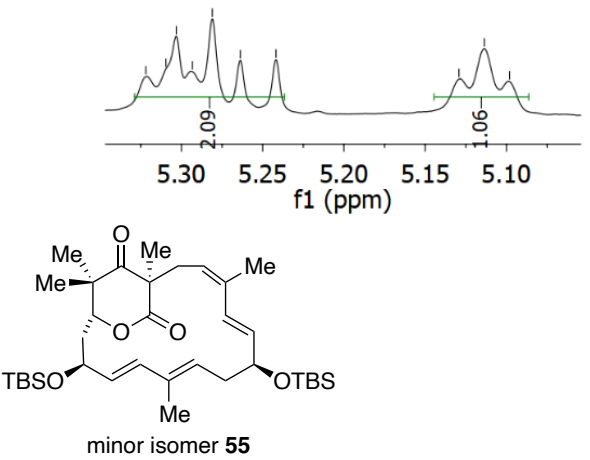

minor isomer $\mathbf{5 5}$

$\begin{array}{llllllllllllllllllllllllllll}.0 & 10.5 & 10.0 & 9.5 & 9.0 & 8.5 & 8.0 & 7.5 & 7.0 & 6.5 & 6.0 & 5.5 & 5.0 & 4.5 & 4.0 & 3.5 & 3.0 & 2.5 & 2.0 & 1.5 & 1.0 & 0.5 & 0.0 & -0.5 & -1\end{array}$

$\left({ }^{13} \mathrm{C} \mathrm{NMR}, \mathrm{CDCl}_{3}, 100 \mathrm{MHz}\right)$
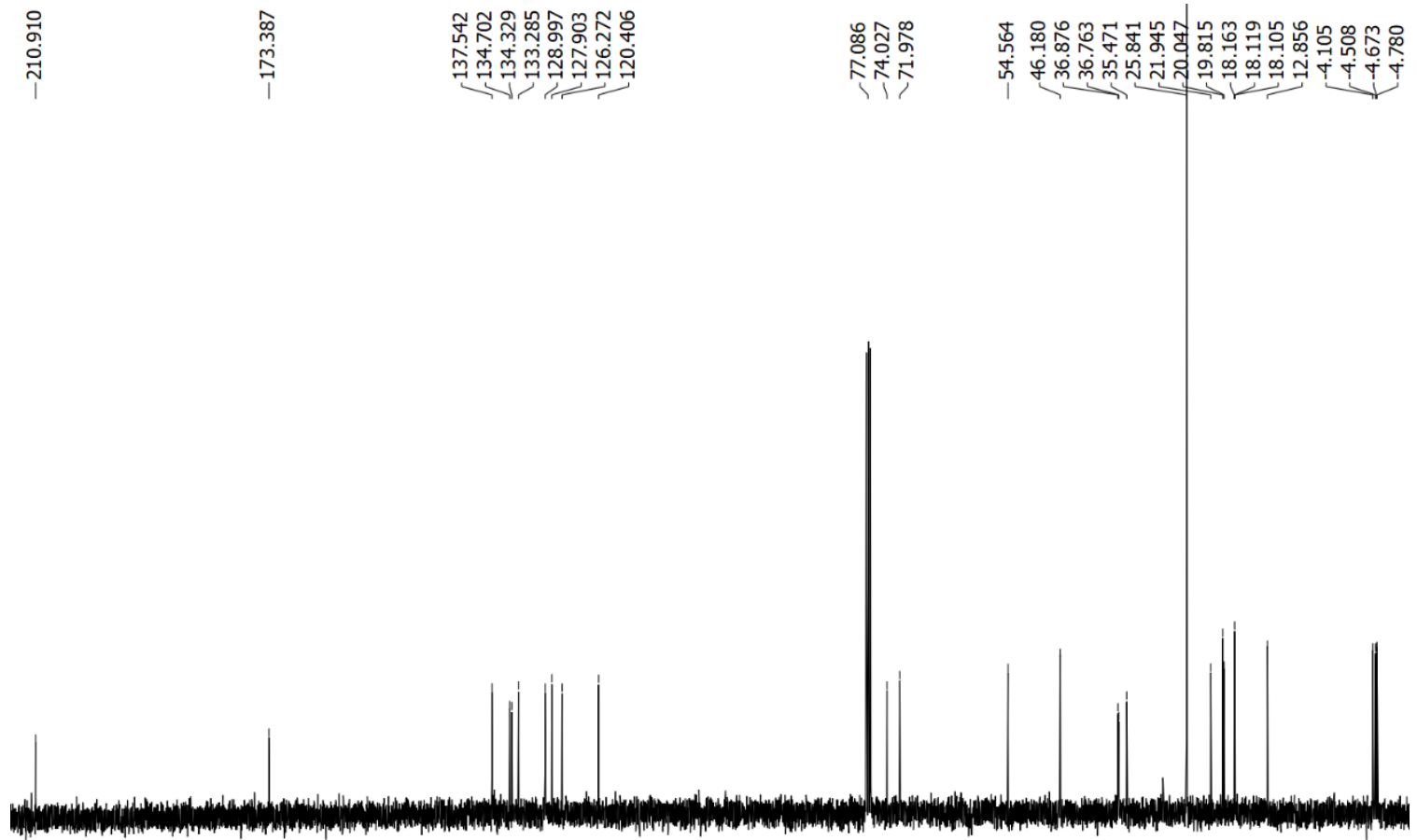

$\begin{array}{llllllllllllllllllllll}210 & 200 & 190 & 180 & 170 & 160 & 150 & 140 & 130 & 120 & \underset{f 1}{110} \underset{(\mathrm{ppm})}{100} & 90 & 80 & 70 & 60 & 50 & 40 & 30 & 20 & 10 & 0 & -1\end{array}$ 
$\left({ }^{1} \mathrm{H} \mathrm{NMR}, \mathrm{CDCl}_{3}, 400 \mathrm{MHz}\right)$

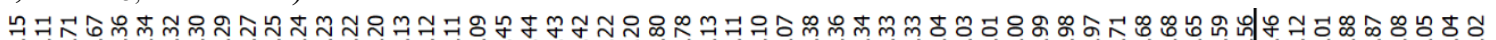
ö

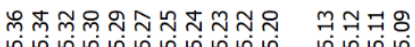

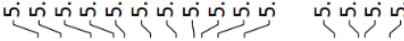

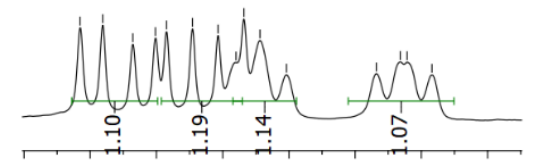

$5.405 .35 \quad 5.305 .25 \quad 5.205 .15 \quad 5.105 .05$

f1 (ppm)
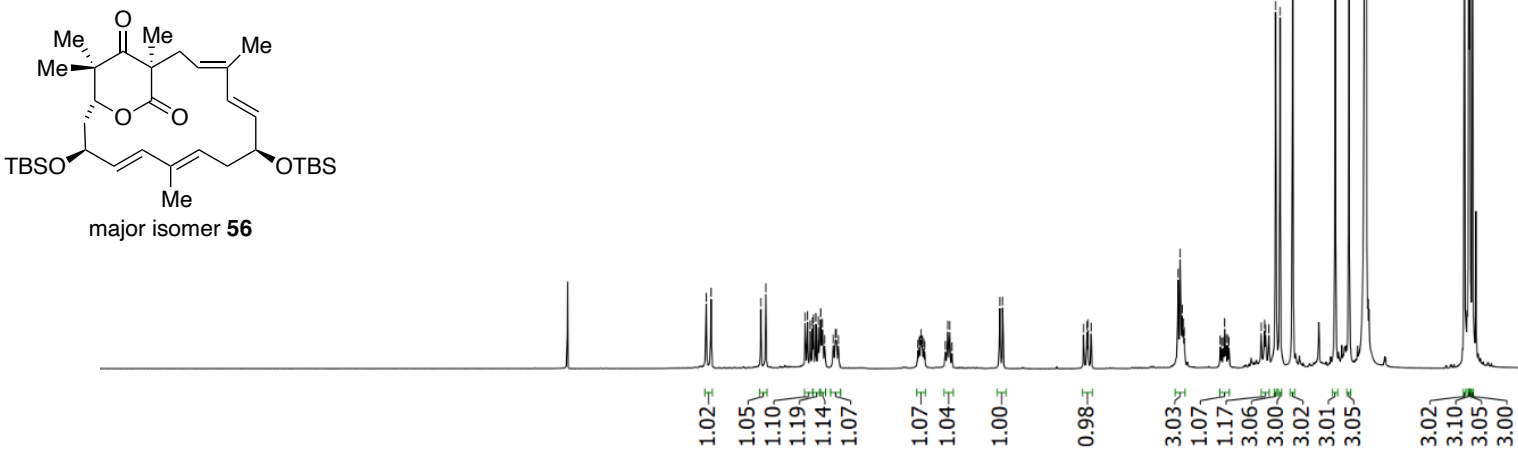

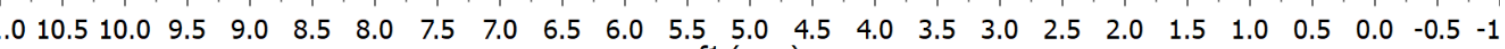
f1 (ppm)

$\left({ }^{13} \mathrm{C} \mathrm{NMR}, \mathrm{CDCl}_{3}, 100 \mathrm{MHz}\right)$

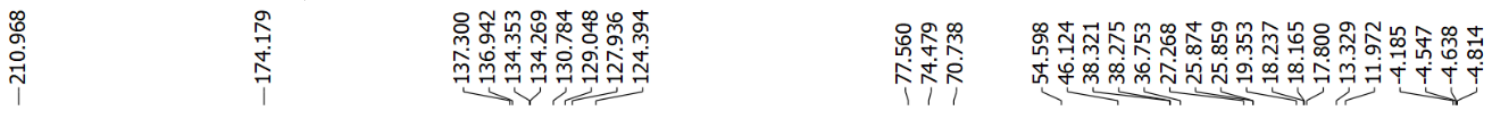

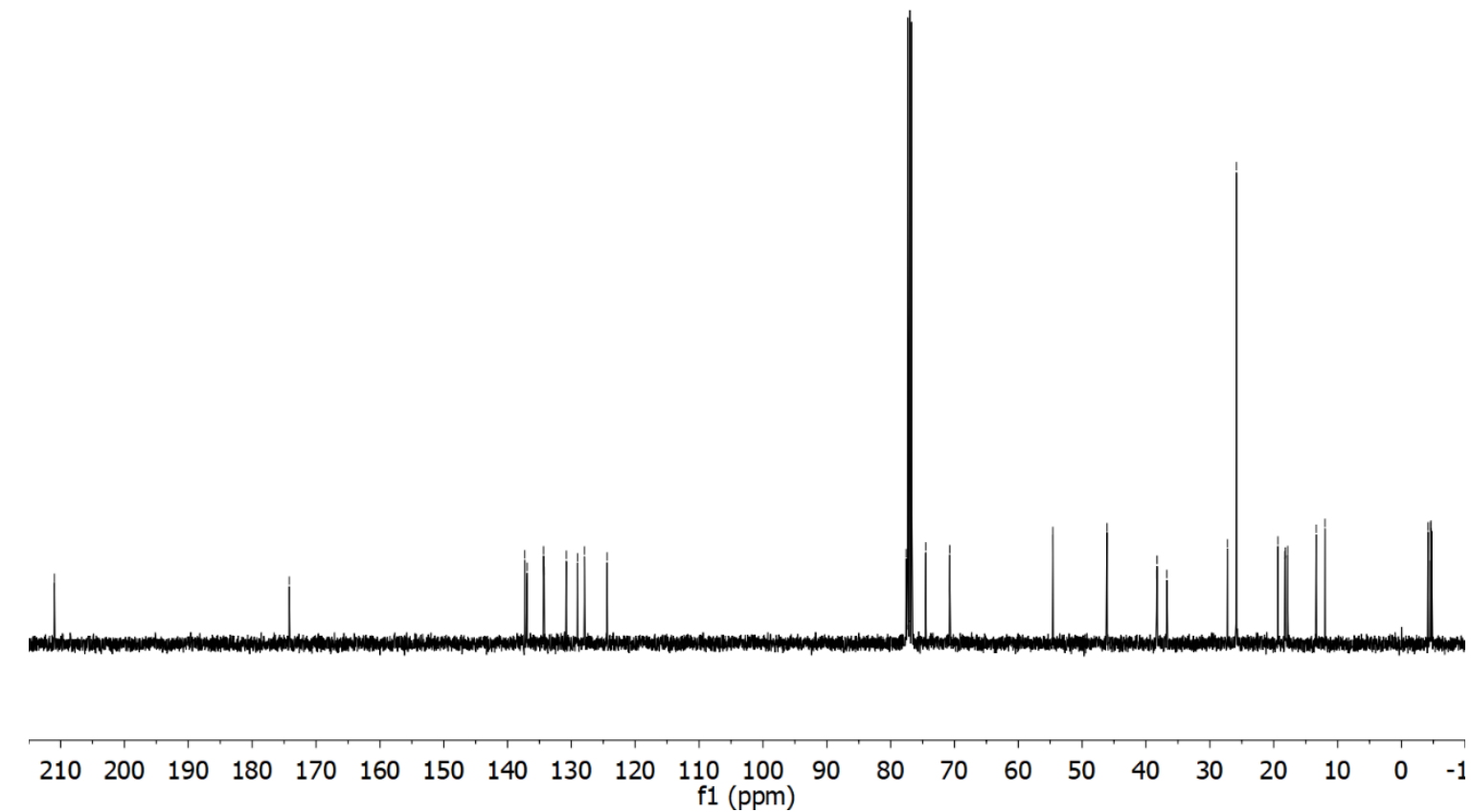


$\left({ }^{1} \mathrm{H} \mathrm{NMR}, \mathrm{CDCl}_{3}, 400 \mathrm{MHz}\right)$
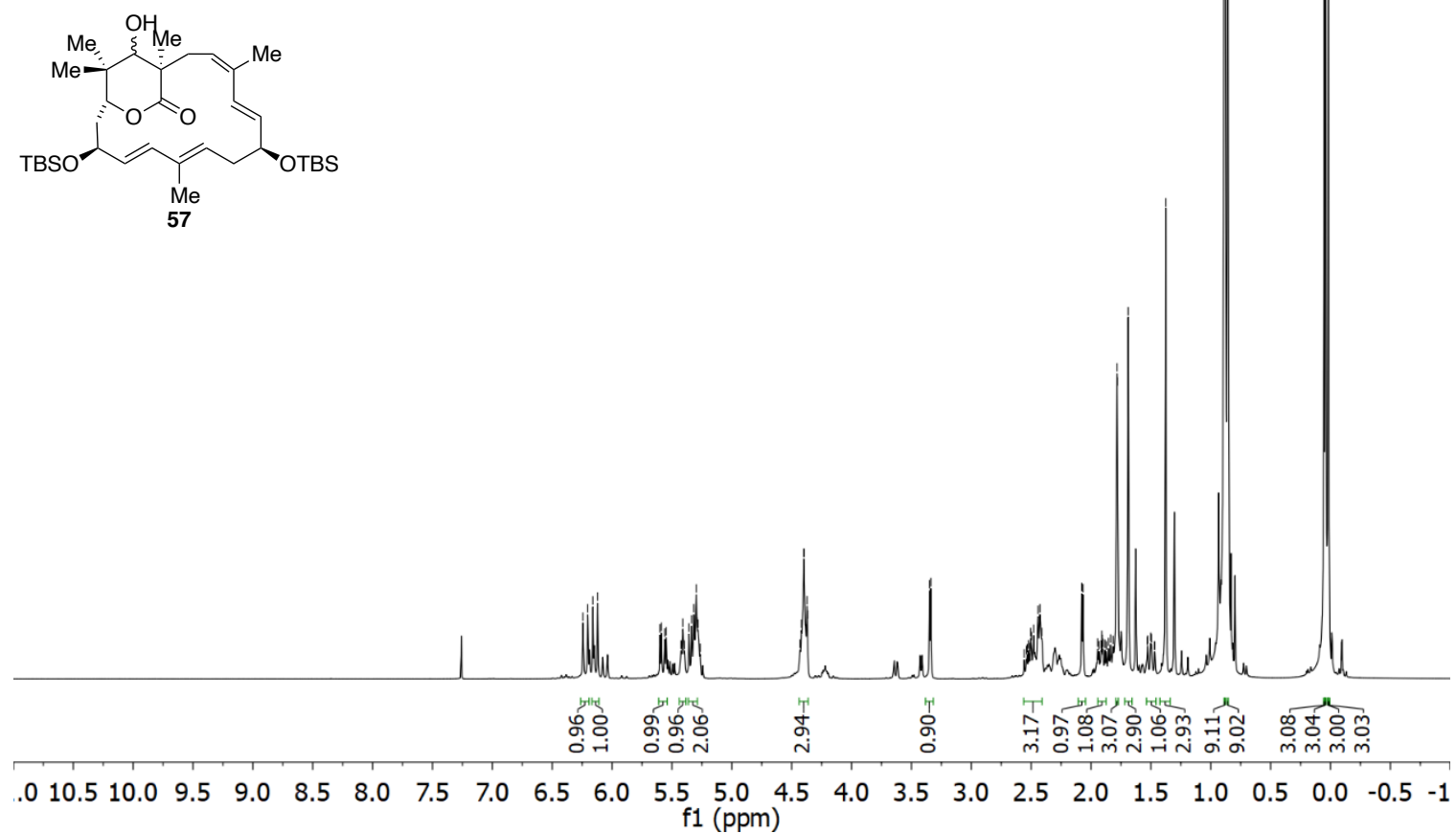

$\left({ }^{13} \mathrm{C} \mathrm{NMR}, \mathrm{CDCl}_{3}, 100 \mathrm{MHz}\right)$

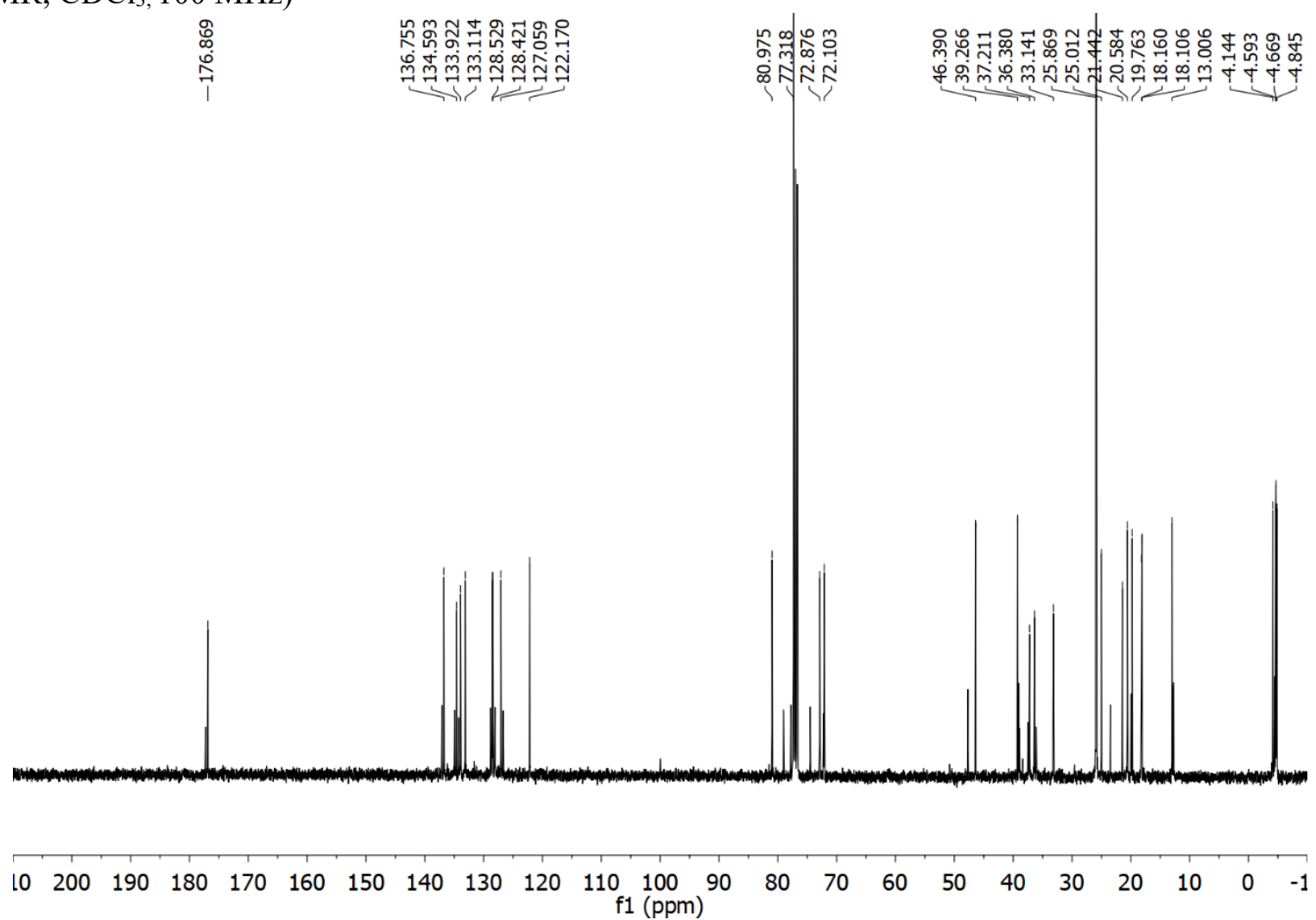


( ${ }^{1} \mathrm{H} \mathrm{NMR,} \mathrm{CDCl}_{3}, 400 \mathrm{MHz}$ )

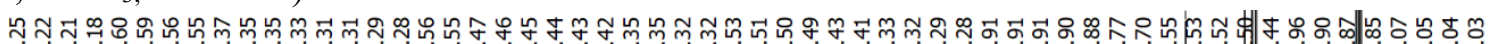

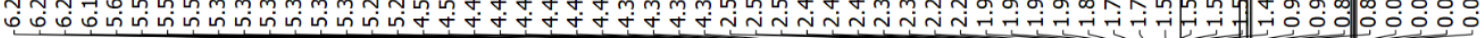
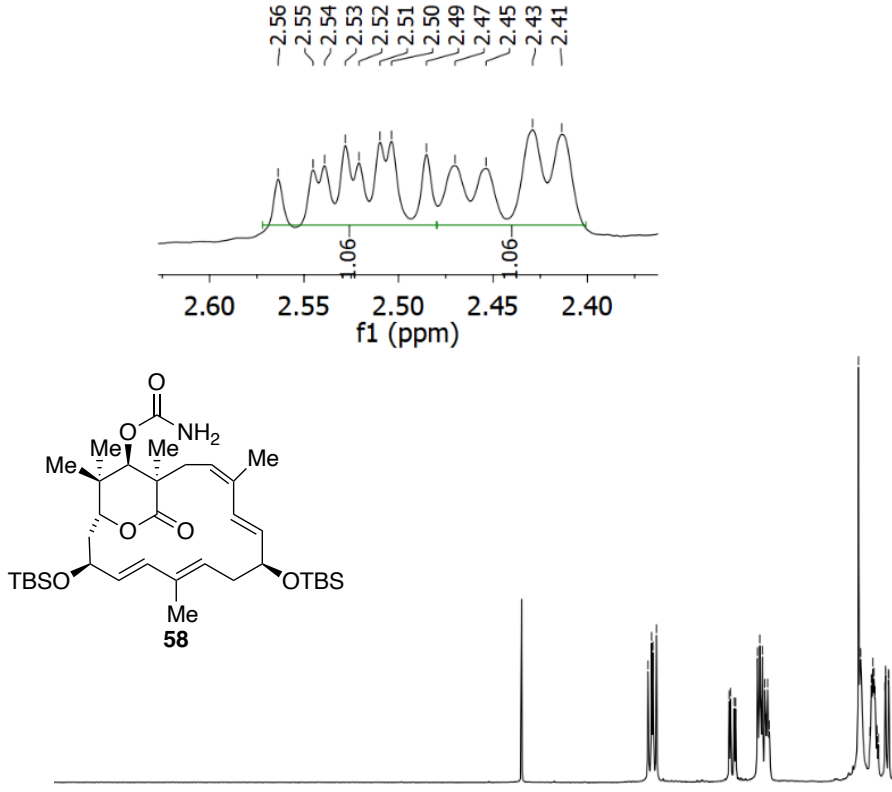

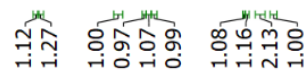
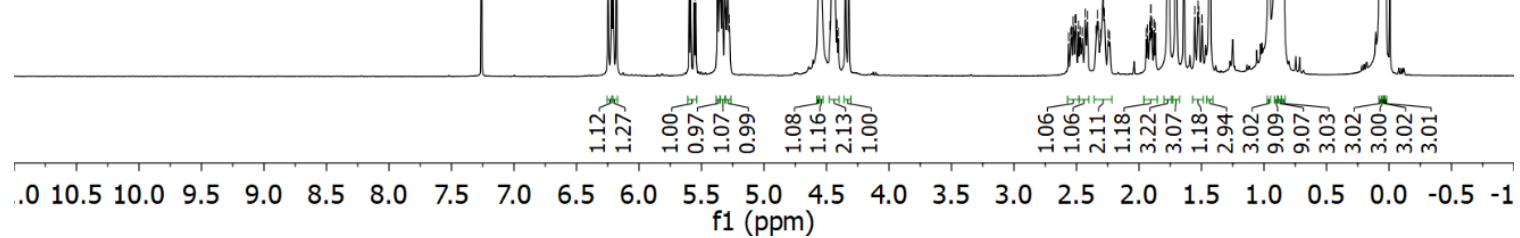

$\left({ }^{13} \mathrm{C} \mathrm{NMR}, \mathrm{CDCl}_{3}, 100 \mathrm{MHz}\right)$

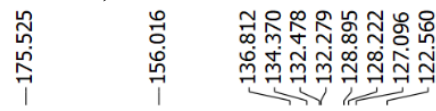

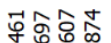

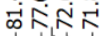
iyit

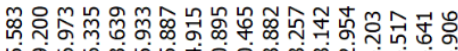

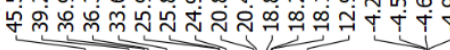

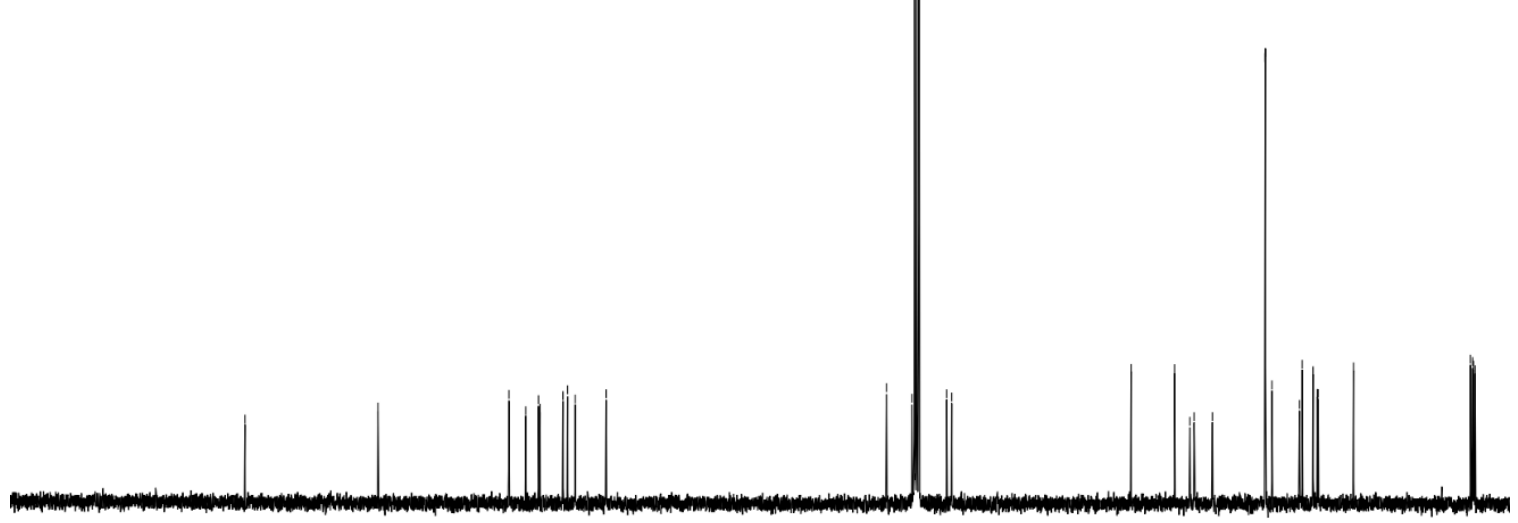
11000

$\begin{array}{lllllll}50 & 40 & 30 & 20 & 10 & 0 & -1\end{array}$ 
$\left({ }^{1} \mathrm{H} \mathrm{NMR}, \mathrm{CDCl}_{3}, 400 \mathrm{MHz}\right)$

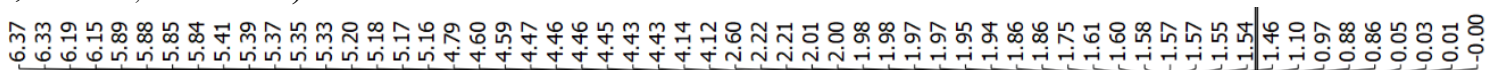

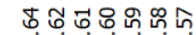

ํํำกำ
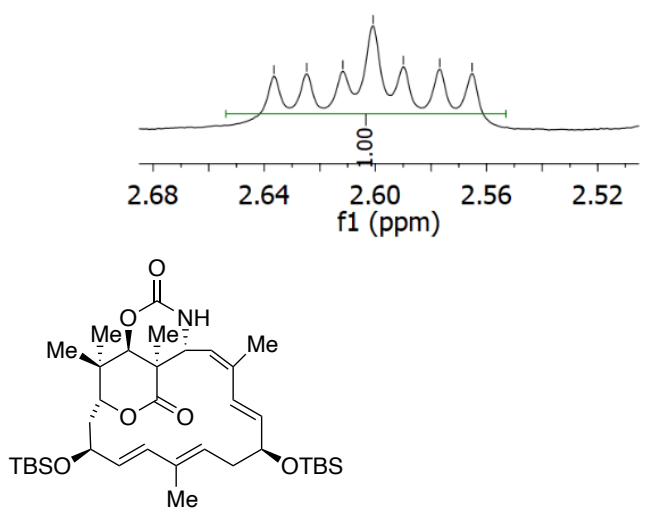

59

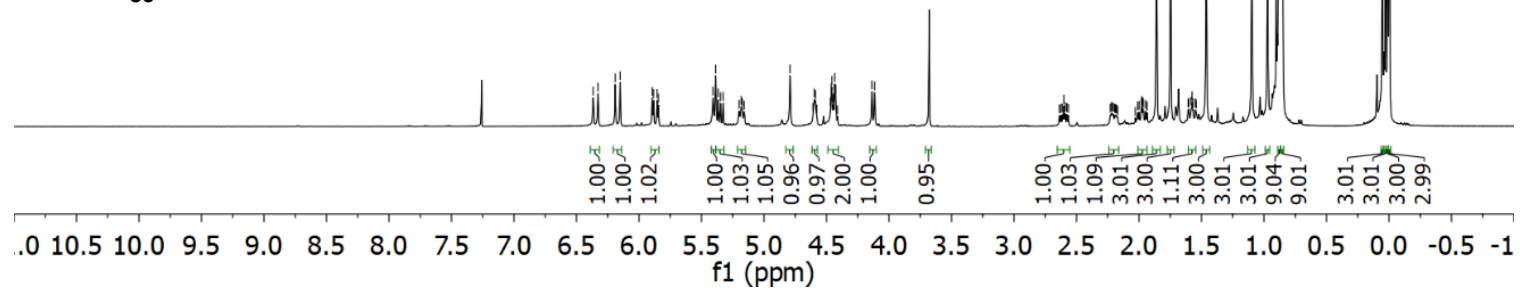

$\left({ }^{13} \mathrm{C} \mathrm{NMR}, \mathrm{CDCl}_{3}, 100 \mathrm{MHz}\right)$

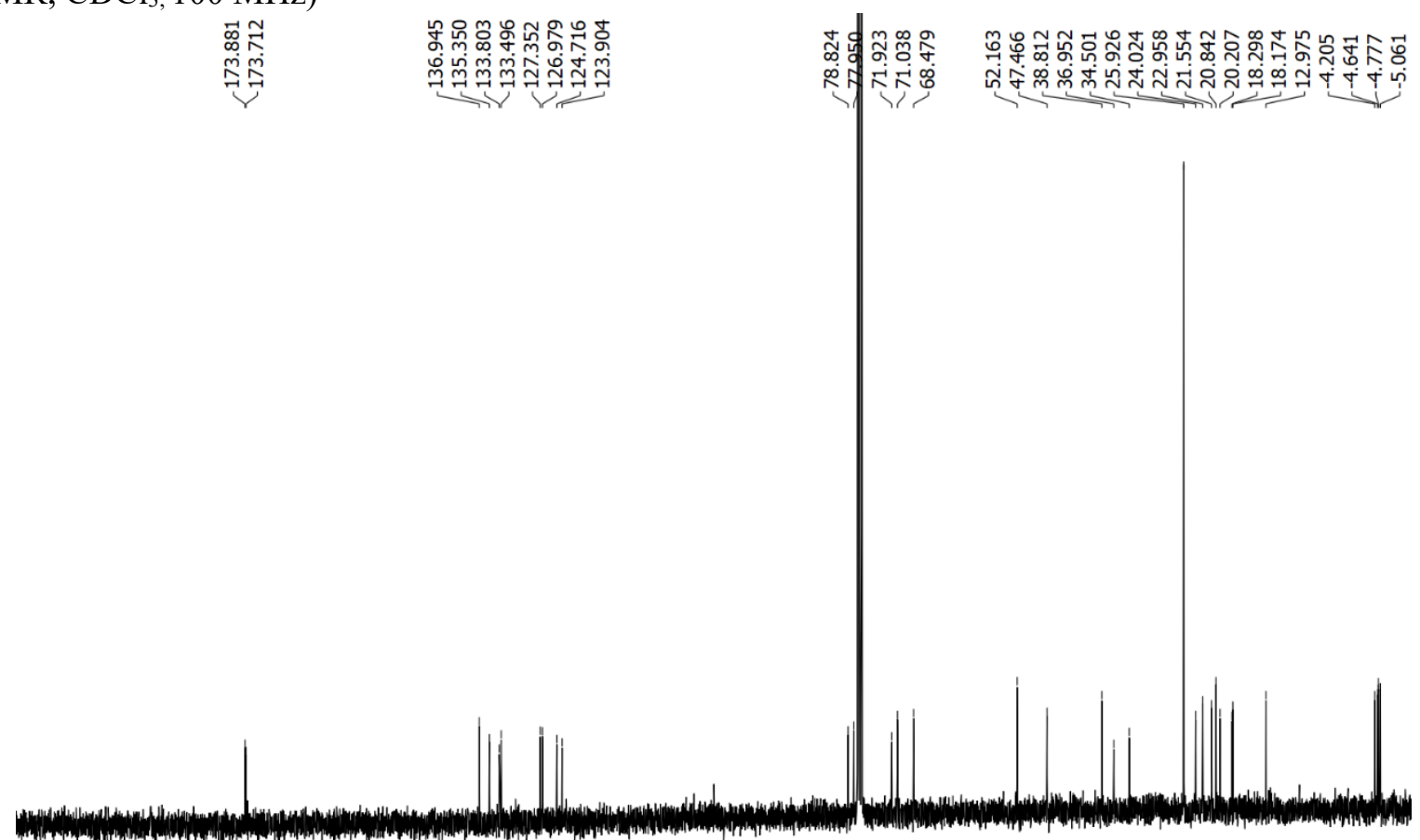

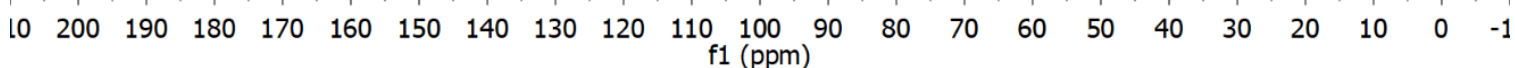




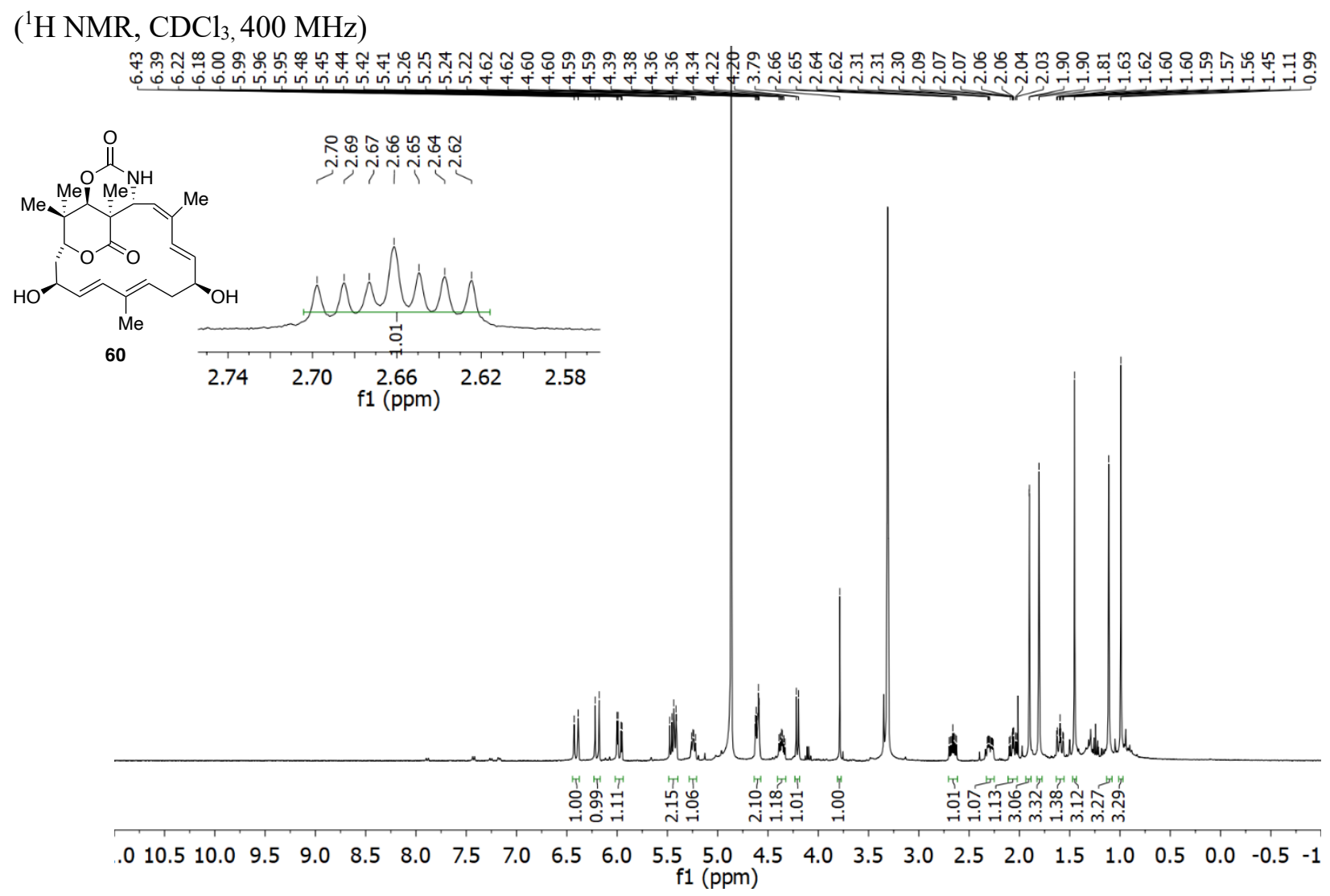

$\left({ }^{13} \mathrm{C} \mathrm{NMR}, \mathrm{CDCl}_{3}, 100 \mathrm{MHz}\right)$
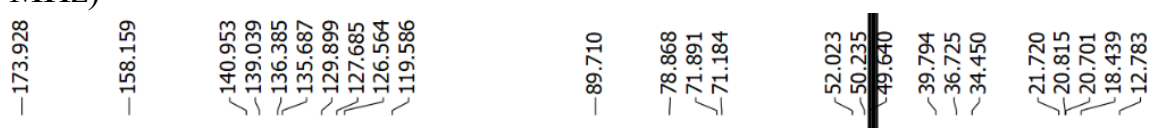

$\begin{array}{llllllllllllllllllllllllll}10 & 200 & 190 & 180 & 170 & 160 & 150 & 140 & 130 & 120 & 110 & 100 & 90 & 80 & 70 & 60 & 50 & 40 & 30 & 20 & 10 & 0 & -1\end{array}$ 
$\left({ }^{1} \mathrm{H} \mathrm{NMR}, \mathrm{CDCl}_{3}, 400 \mathrm{MHz}\right)$

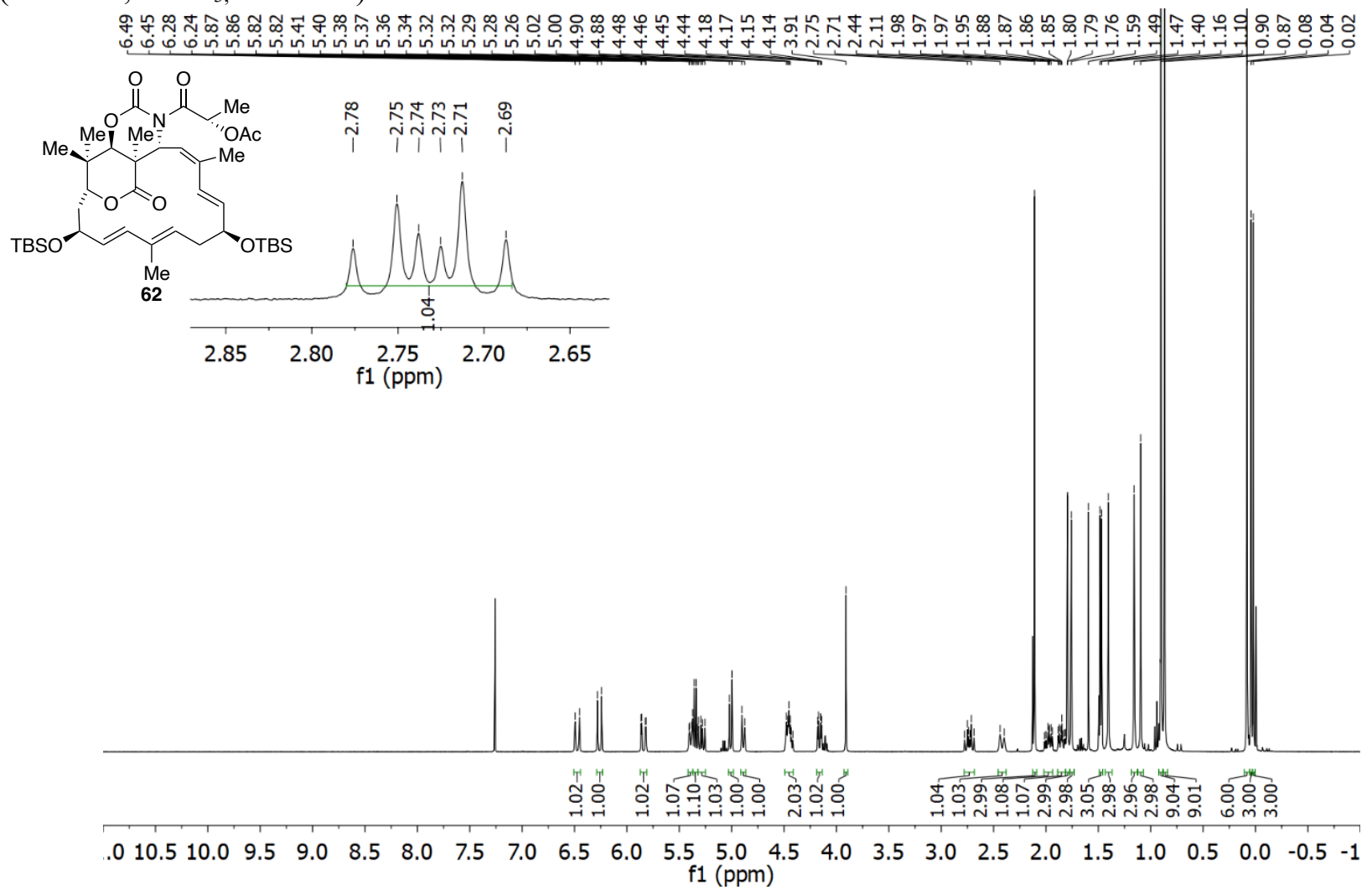

$\left({ }^{13} \mathrm{C} \mathrm{NMR}, \mathrm{CDCl}_{3}, 100 \mathrm{MHz}\right)$

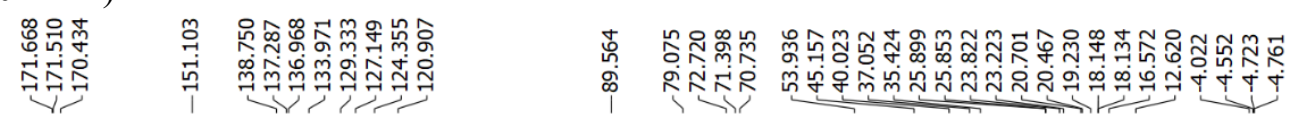

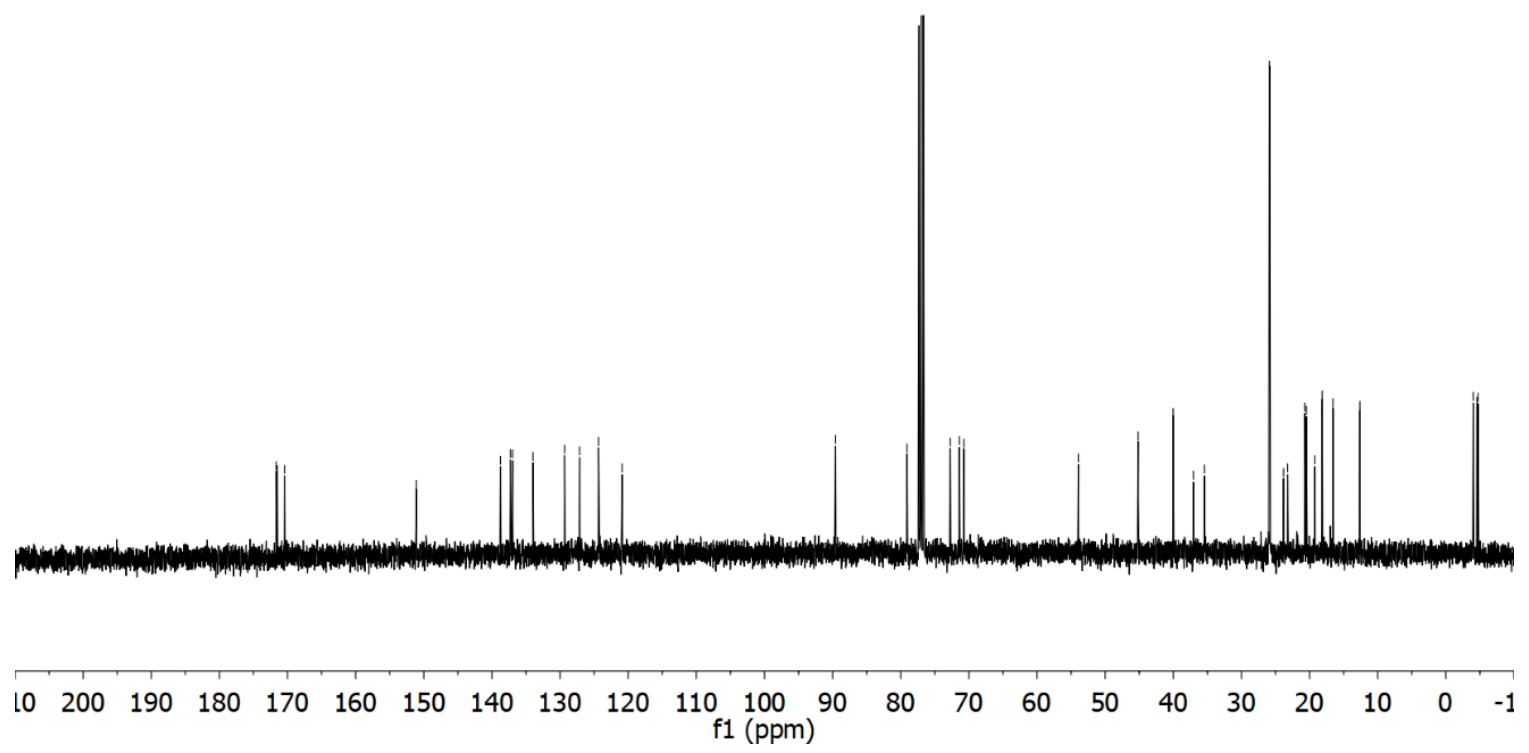


$\left({ }^{1} \mathrm{H} \mathrm{NMR}, \mathrm{CDCl}_{3}, 400 \mathrm{MHz}\right)$

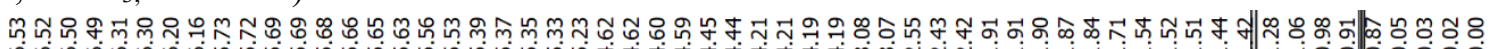

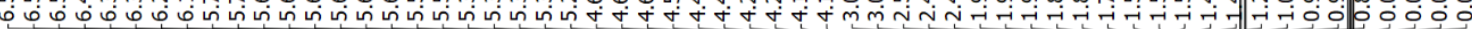
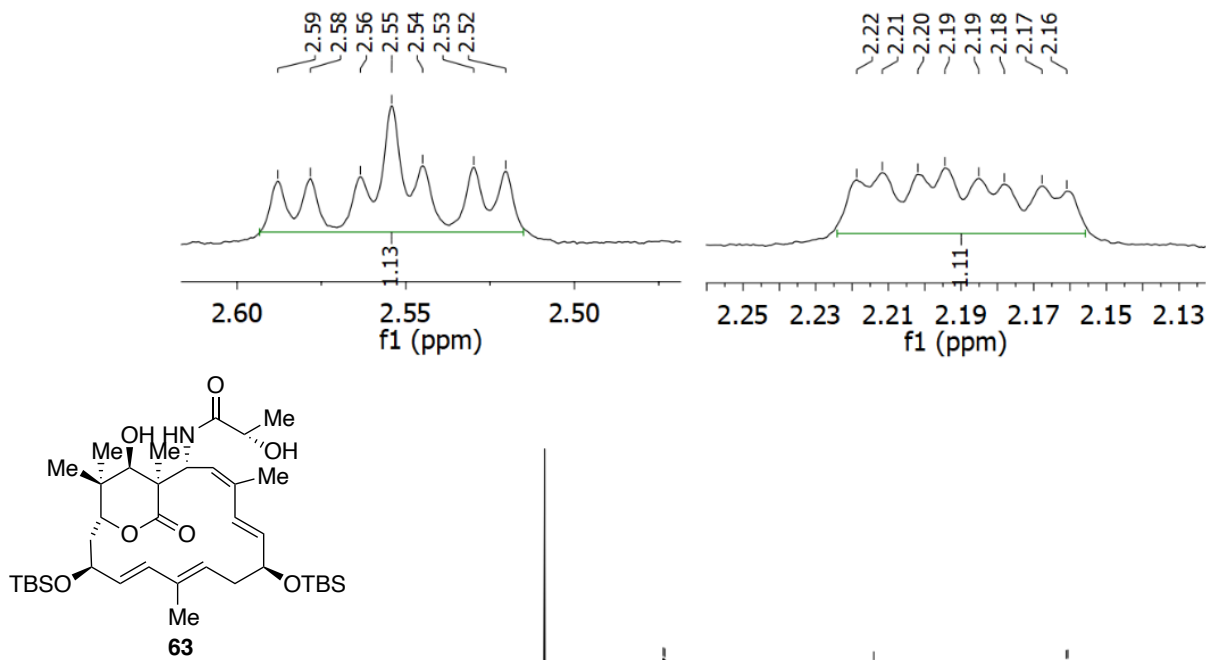

$1(\mathrm{ppm})$

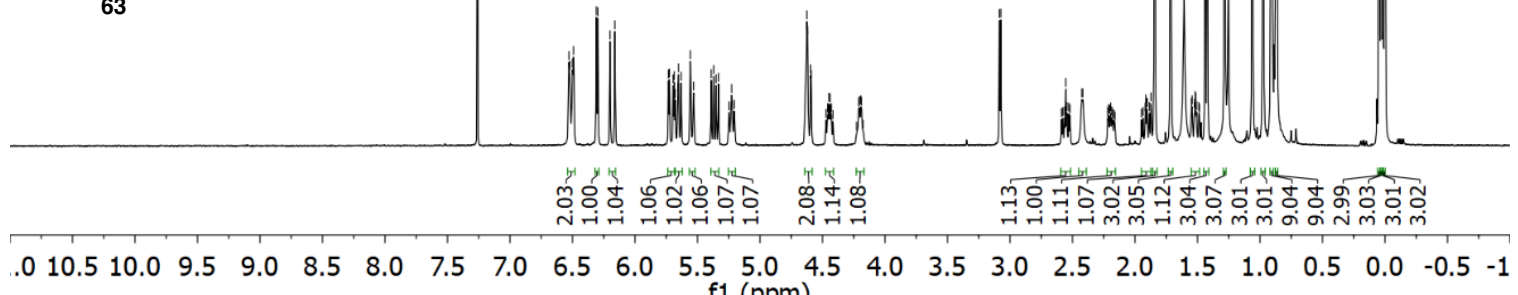

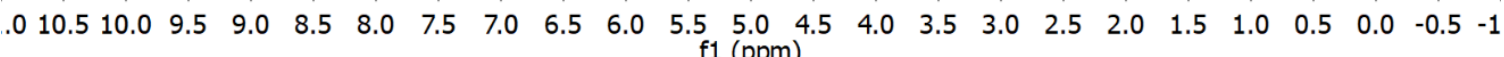

$\left({ }^{13} \mathrm{C} \mathrm{NMR}, \mathrm{CDCl}_{3}, 100 \mathrm{MHz}\right)$

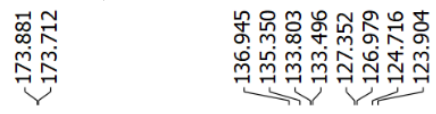

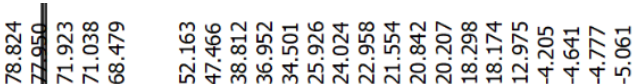

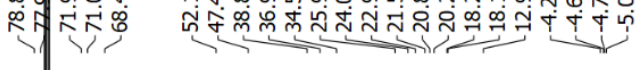

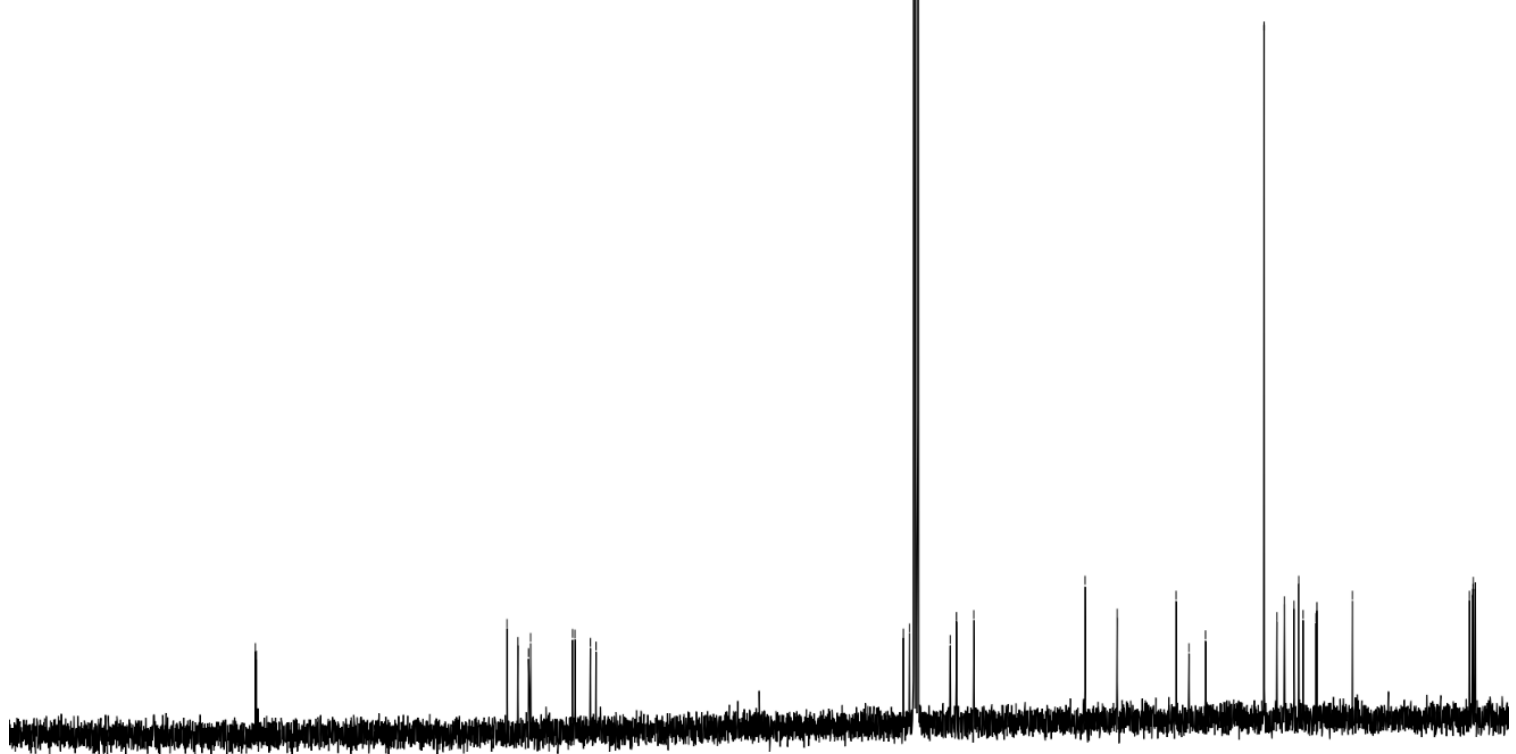

$\begin{array}{lllllllllllllllllllllll}10 & 200 & 190 & 180 & 170 & 160 & 150 & 140 & 130 & 120 & 110 \underset{\mathrm{f} 1(\mathrm{ppm})}{100} & 90 & 80 & 70 & 60 & 50 & 40 & 30 & 20 & 10 & 0 & -1\end{array}$ 
( $\left.{ }^{1} \mathrm{H} \mathrm{NMR}, \mathrm{CDCl}_{3}, 400 \mathrm{MHz}\right)$

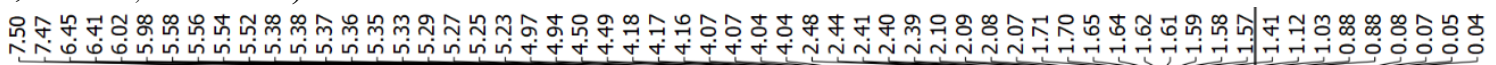

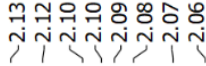
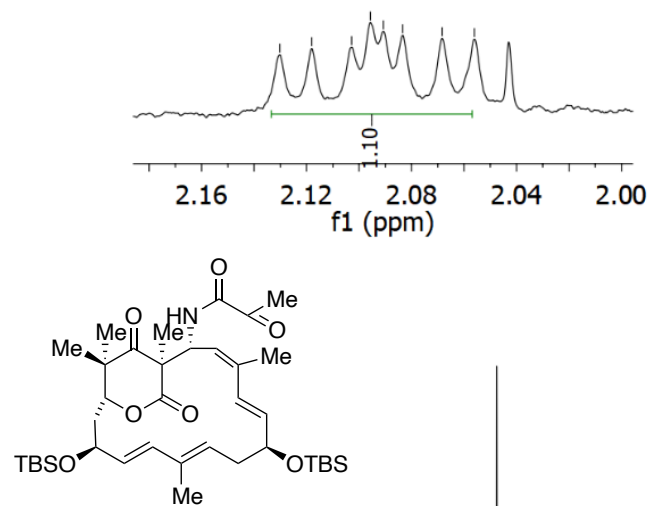

64

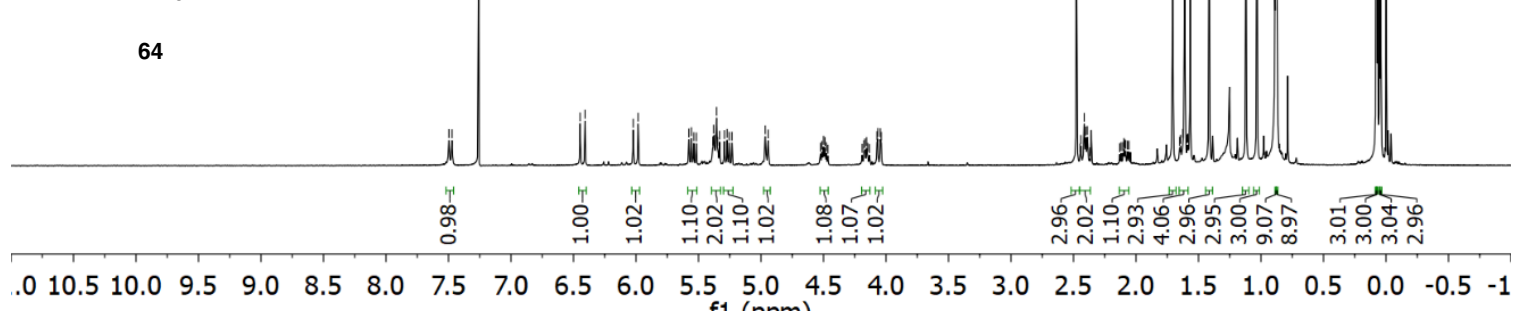

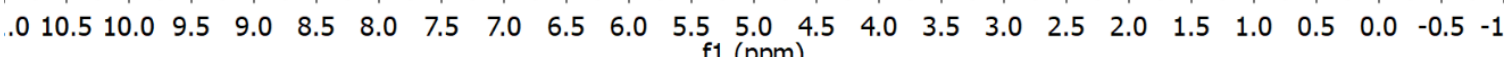

$\left({ }^{13} \mathrm{C} \mathrm{NMR}, \mathrm{CDCl}_{3}, 100 \mathrm{MHz}\right)$

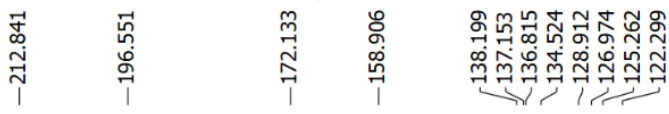

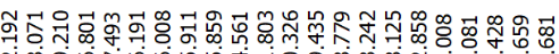

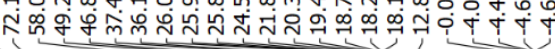

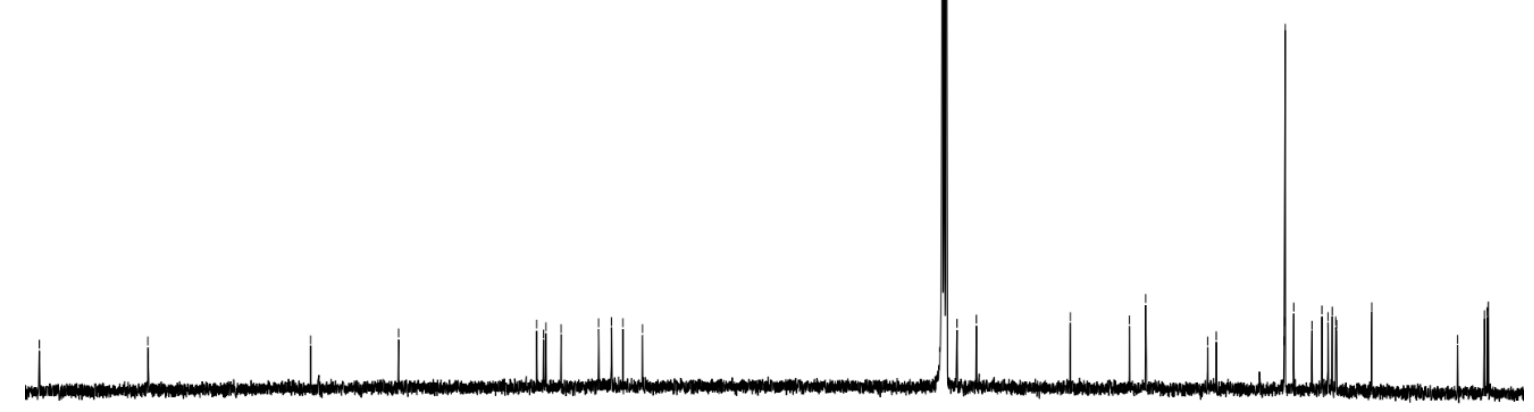

$\begin{array}{lllllllllllllllllllllll}210 & 200 & 190 & 180 & 170 & 160 & 150 & 140 & 130 & 120 & \begin{array}{c}110 \\ \mathrm{f} 1\end{array}\left(\begin{array}{llllll}(\mathrm{ppm}) \\ (100\end{array}\right. & 90 & 80 & 70 & 60 & 50 & 40 & 30 & 20 & 10 & 0 & -1\end{array}$ 
( ${ }^{1} \mathrm{H} \mathrm{NMR,} \mathrm{CDCl}_{3}, 400 \mathrm{MHz}$ )

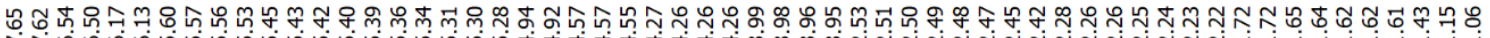

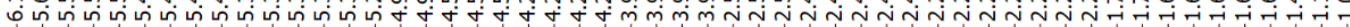

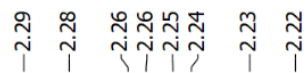
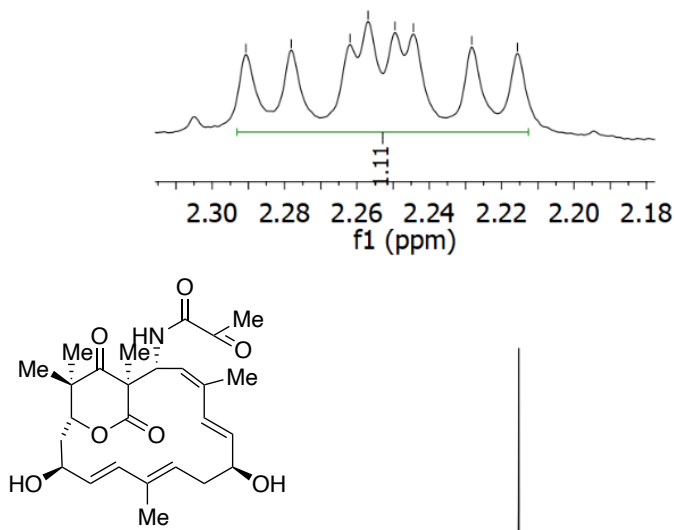

65

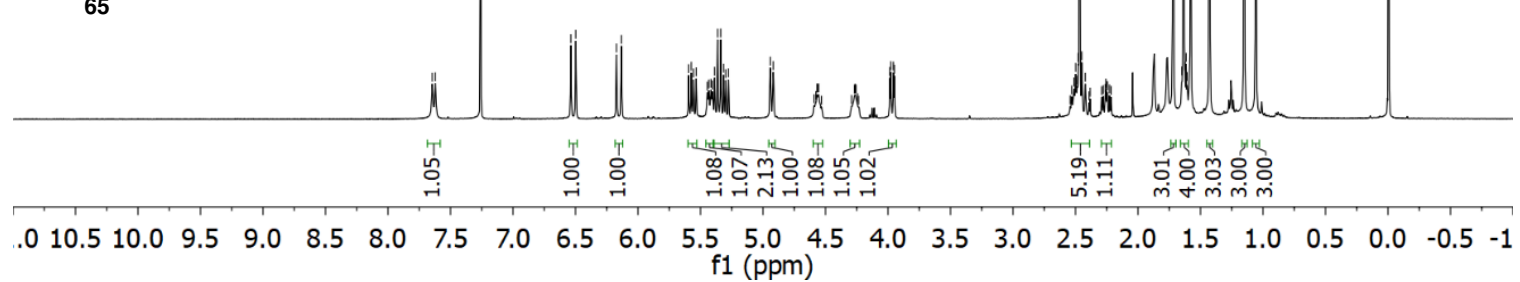

$\left({ }^{13} \mathrm{C} \mathrm{NMR}, \mathrm{CDCl}_{3}, 100 \mathrm{MHz}\right)$

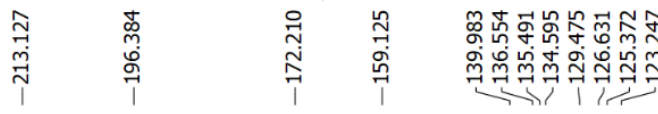

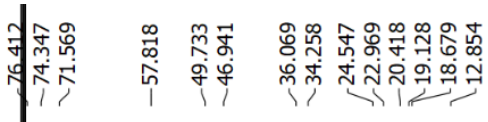

$\begin{array}{lllllllllllllllllllllll}210 & 200 & 190 & 180 & 170 & 160 & 150 & 140 & 130 & 120 & \begin{array}{c}110 \\ \mathrm{f} 1(\mathrm{ppm})\end{array} & \begin{array}{l}100 \\ (\mathrm{pm})\end{array} & 80 & 70 & 60 & 50 & 40 & 30 & 20 & 10 & 0 & -1\end{array}$ 
$\left({ }^{1} \mathrm{H} \mathrm{NMR}, \mathrm{CDCl}_{3}, 400 \mathrm{MHz}\right)$

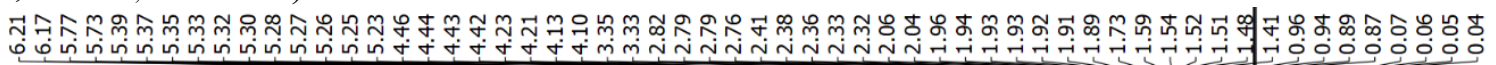

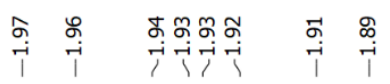
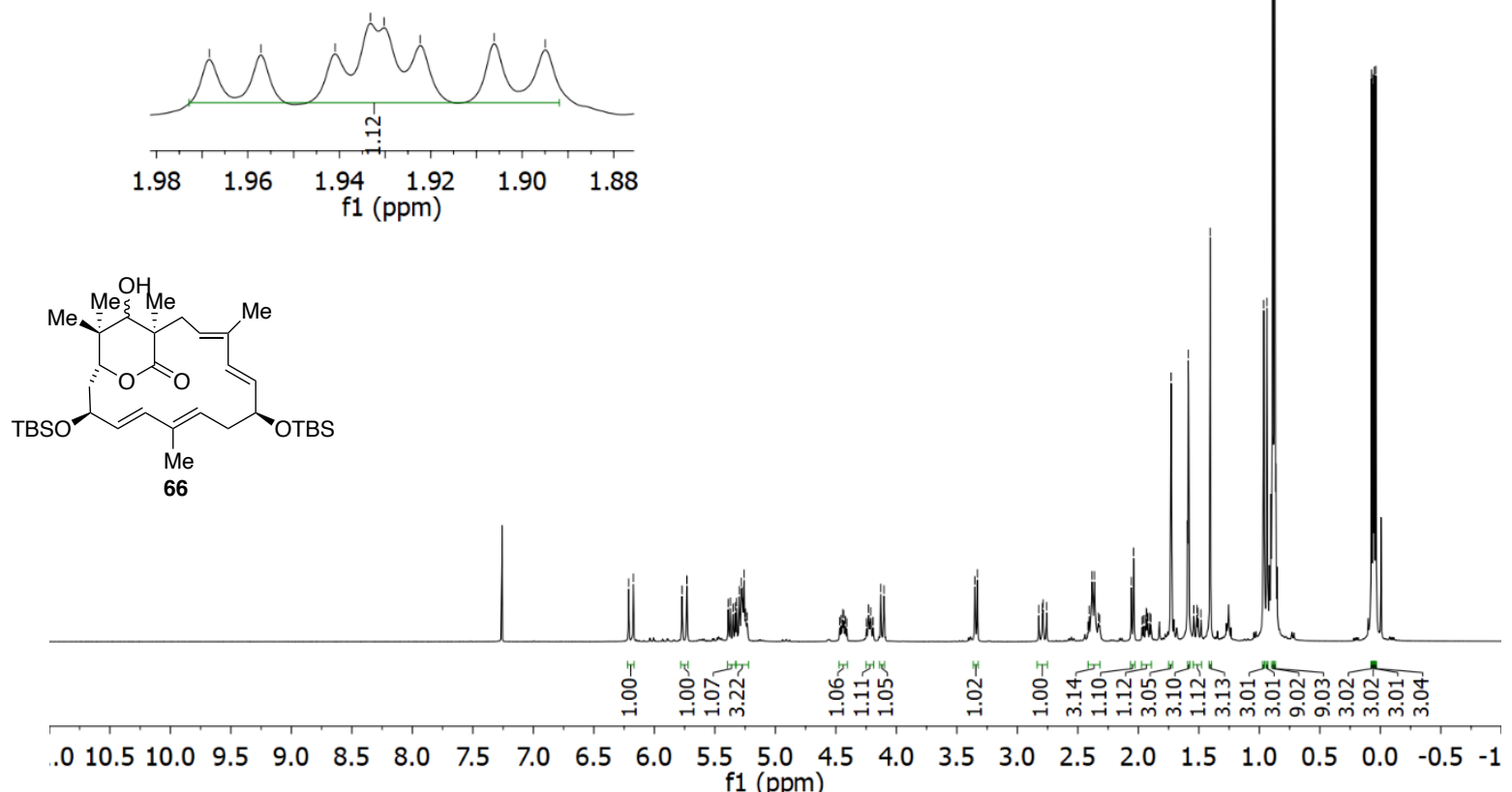

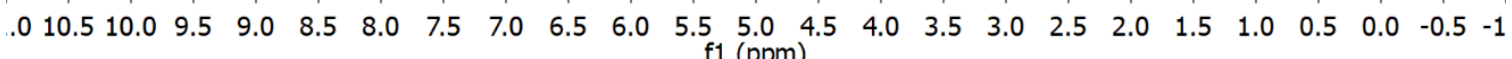

$\left({ }^{13} \mathrm{C} \mathrm{NMR}, \mathrm{CDCl}_{3}, 100 \mathrm{MHz}\right)$

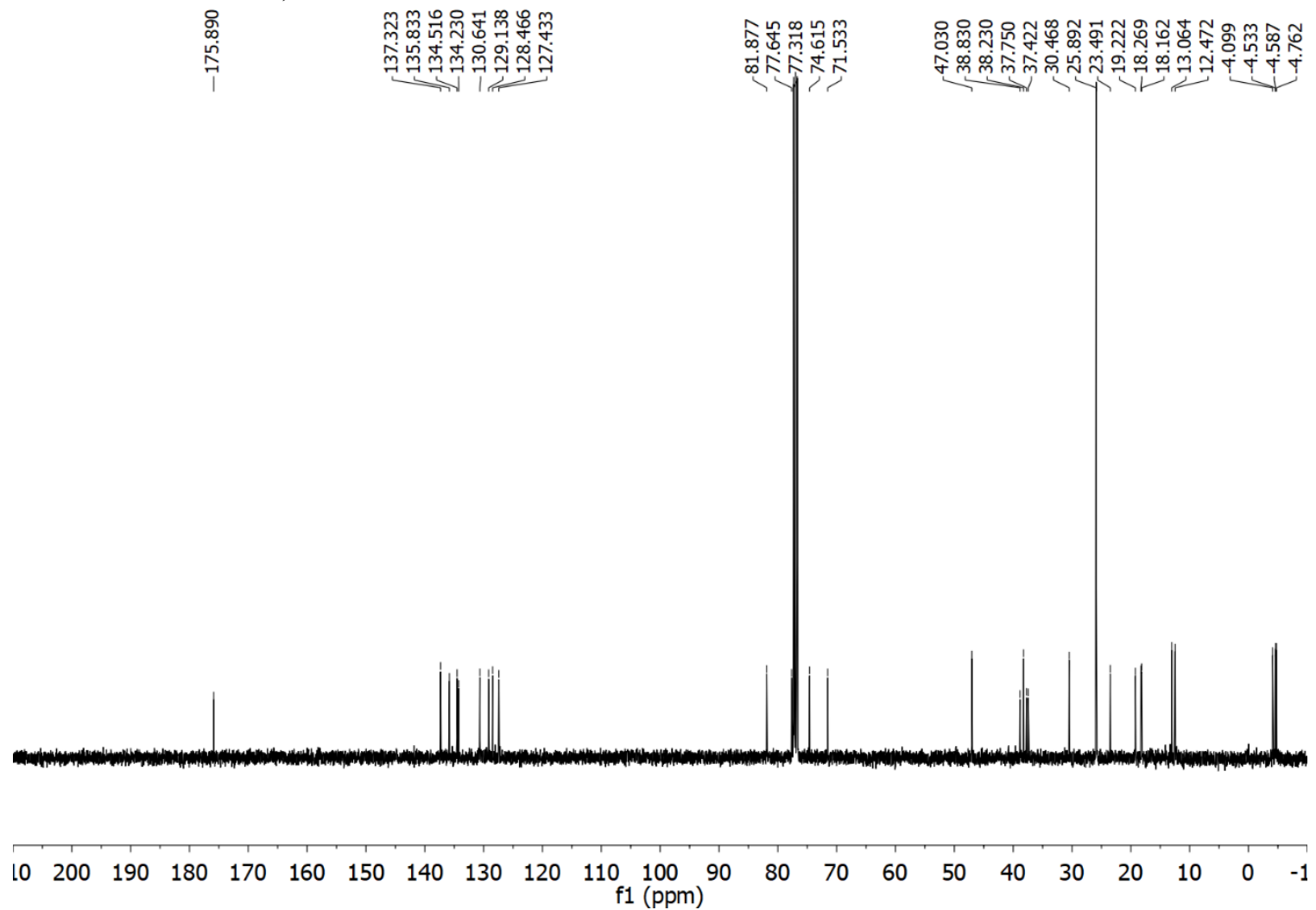


$\left({ }^{1} \mathrm{H} \mathrm{NMR}, \mathrm{CDCl}_{3}, 400 \mathrm{MHz}\right)$

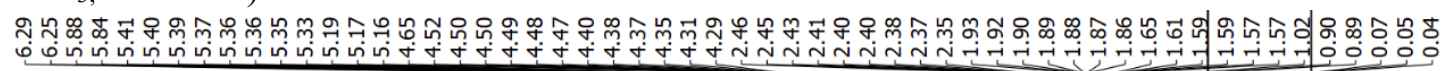
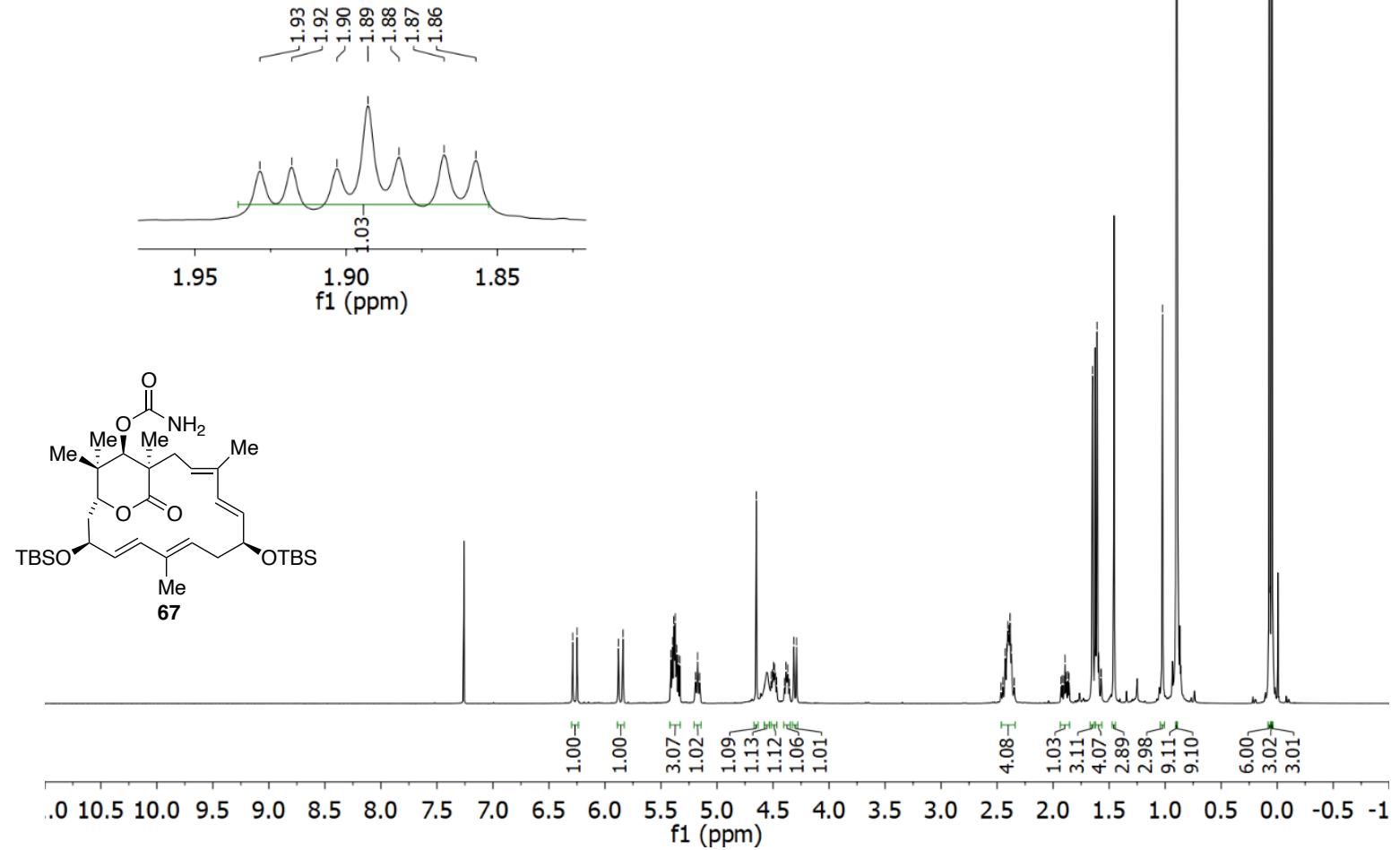

$\left({ }^{13} \mathrm{C} \mathrm{NMR}, \mathrm{CDCl}_{3}, 100 \mathrm{MHz}\right)$
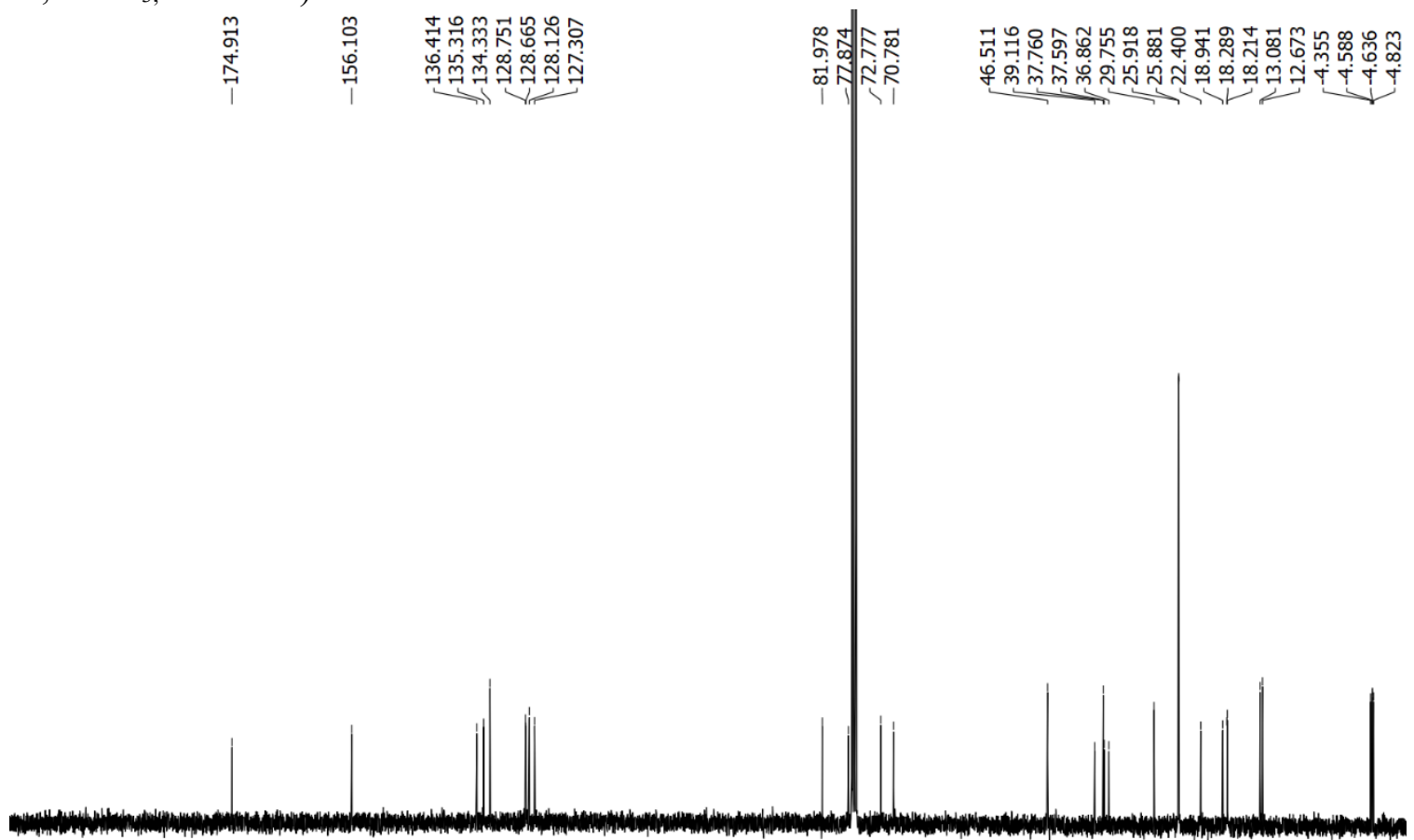

$\begin{array}{lllllllllllllllllllllll}10 & 200 & 190 & 180 & 170 & 160 & 150 & 140 & 130 & 120 & 110 & 100 & 90 & 80 & 70 & 60 & 50 & 40 & 30 & 20 & 10 & 0 & -1\end{array}$ 
( $\left.{ }^{1} \mathrm{H} \mathrm{NMR,} \mathrm{CDCl}_{3}, 400 \mathrm{MHz}\right)$

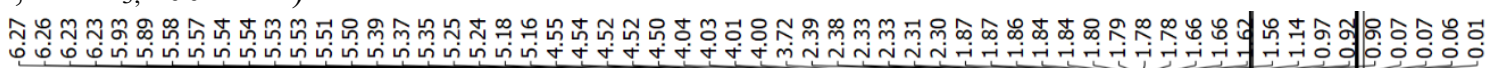

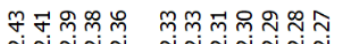

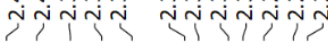
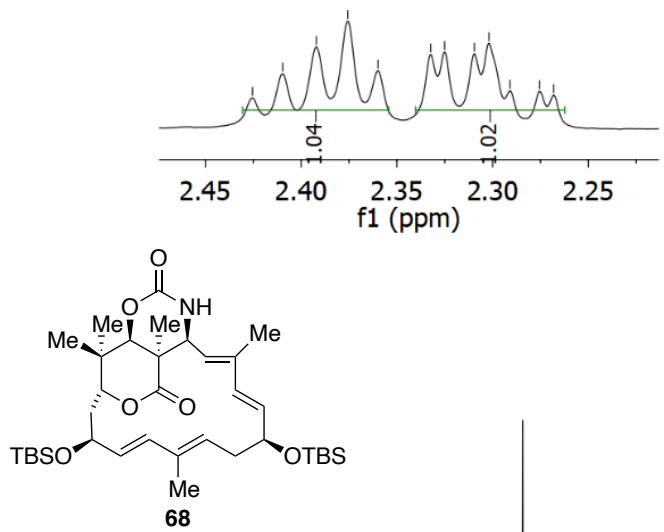

68

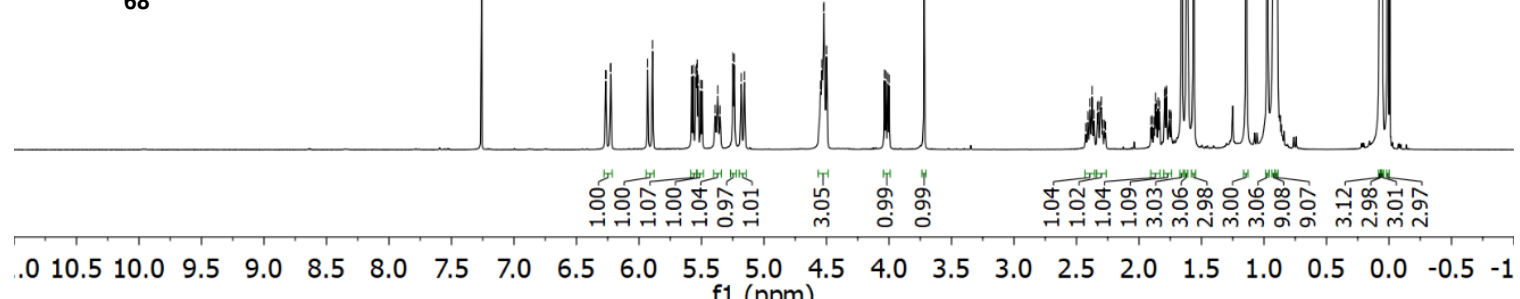

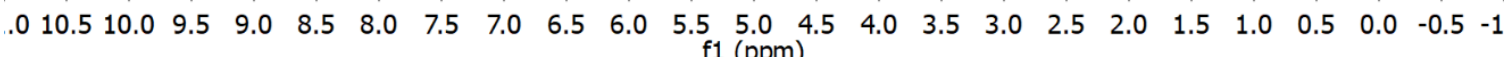

$\left({ }^{13} \mathrm{C} \mathrm{NMR}, \mathrm{CDCl}_{3}, 100 \mathrm{MHz}\right)$

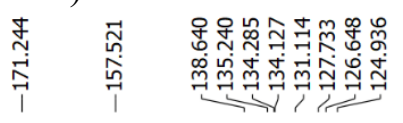
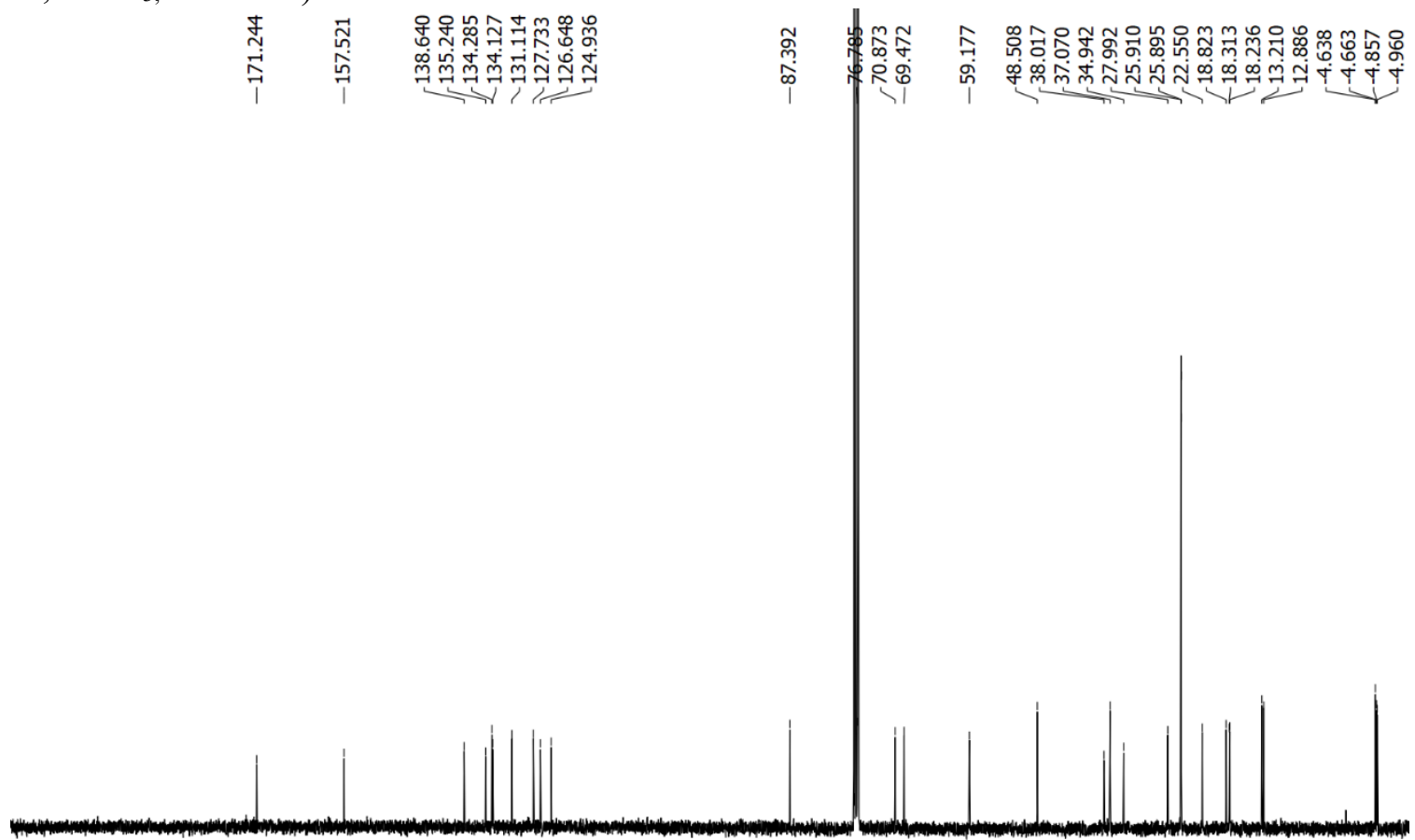

$\begin{array}{lllllllll}200 & 190 & 180 & 170 & 160 & 150 & 140 & 130 & 120\end{array}$

11010090 
( ${ }^{1} \mathrm{H} \mathrm{NMR}, \mathrm{CDCl}_{3}, 400 \mathrm{MHz}$ )

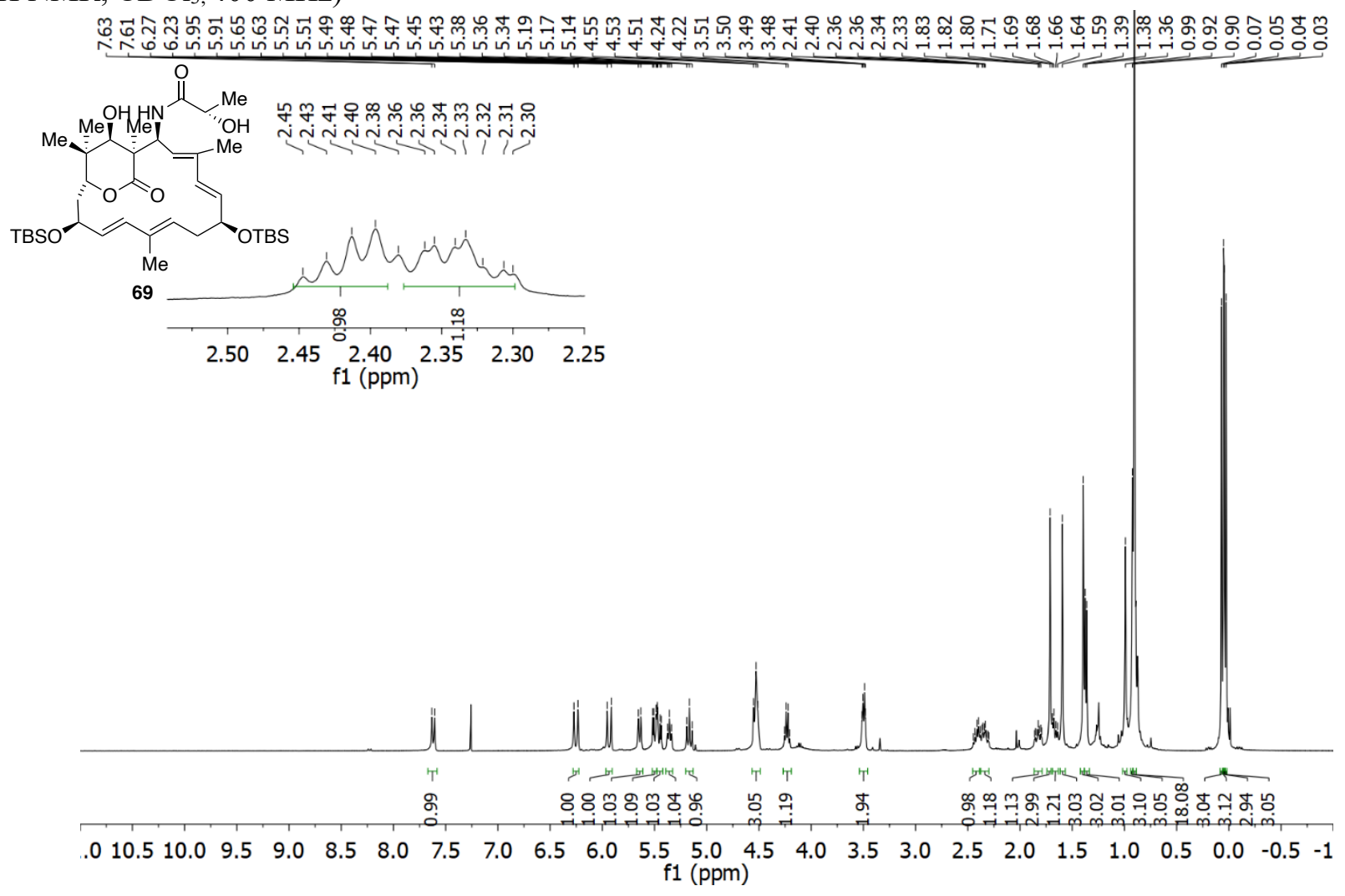

$\left({ }^{13} \mathrm{C} \mathrm{NMR}, \mathrm{CDCl}_{3}, 100 \mathrm{MHz}\right)$

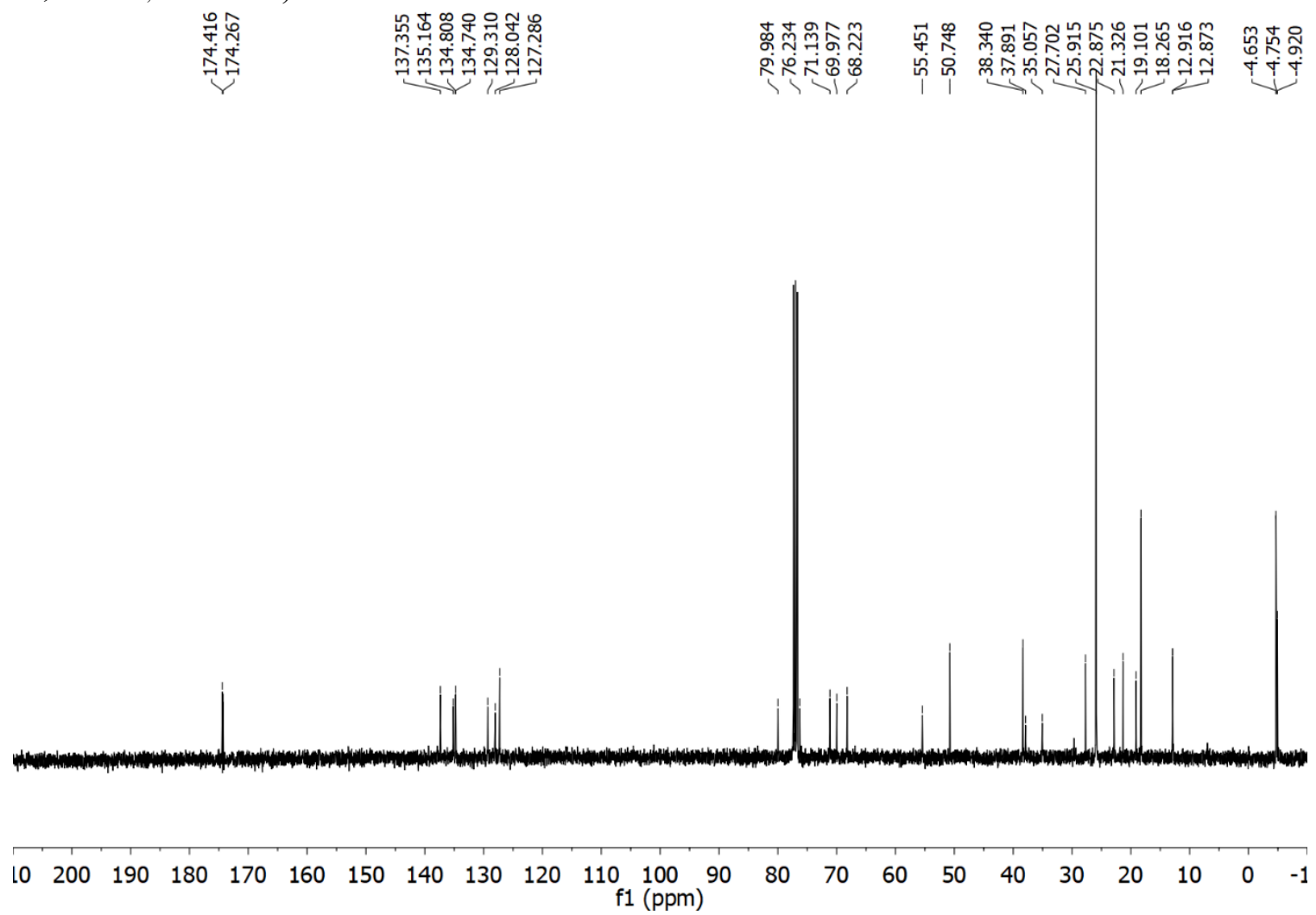


$\left({ }^{1} \mathrm{H} \mathrm{NMR}, \mathrm{CDCl}_{3}, 400 \mathrm{MHz}\right)$

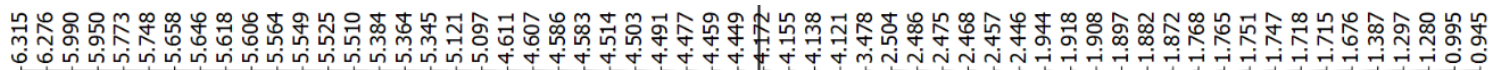

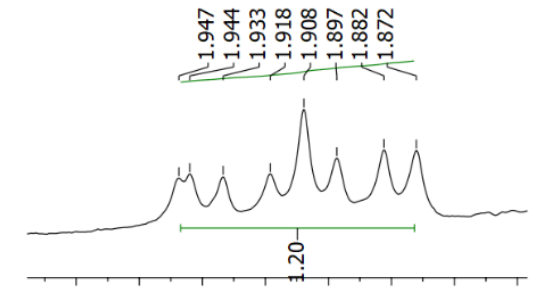

1.981 .961 .941 .921 .901 .881 .861 .84 f1 (ppm)
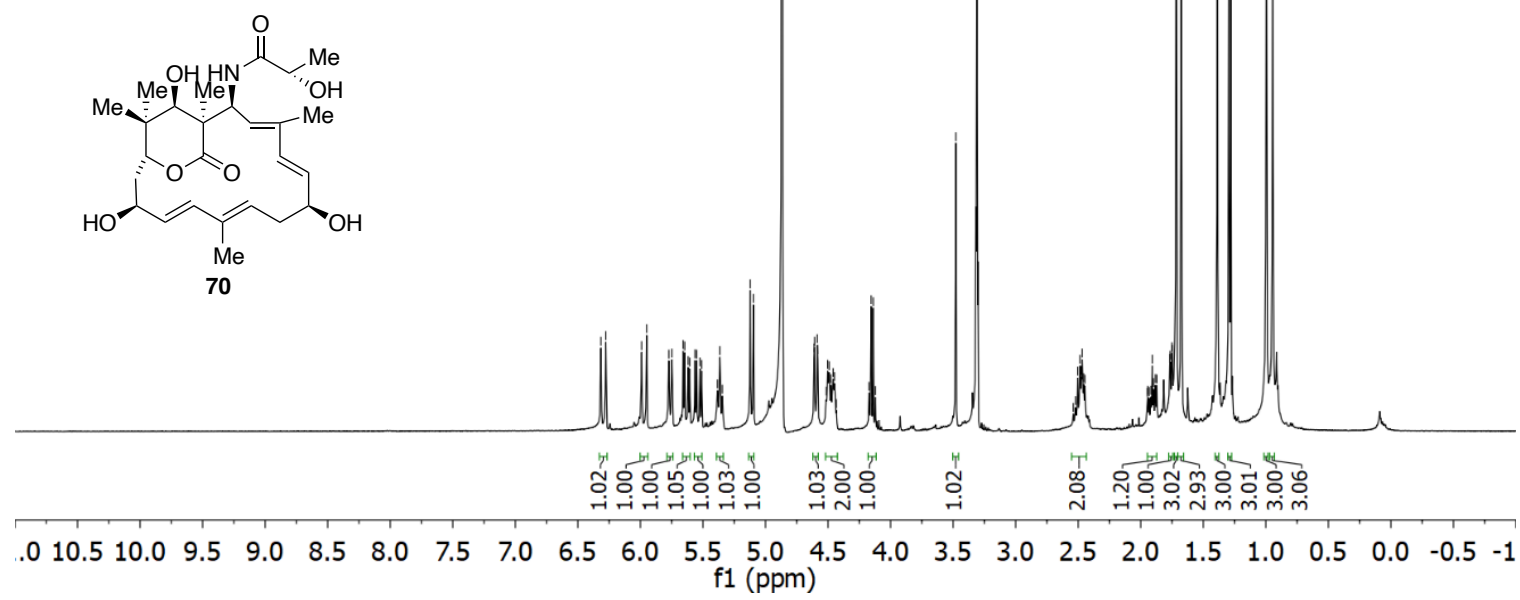

$\left({ }^{13} \mathrm{C} \mathrm{NMR}, \mathrm{CDCl}_{3}, 100 \mathrm{MHz}\right)$

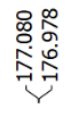

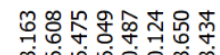

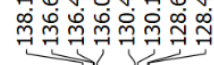

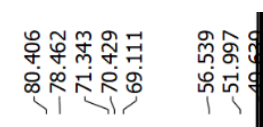

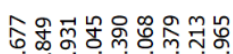

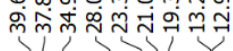


$\left({ }^{1} \mathrm{H} \mathrm{NMR}, \mathrm{CDCl}_{3}, 400 \mathrm{MHz}\right)$

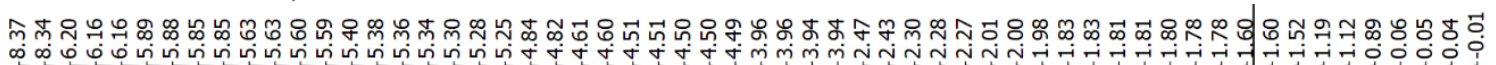

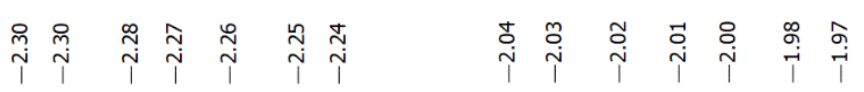
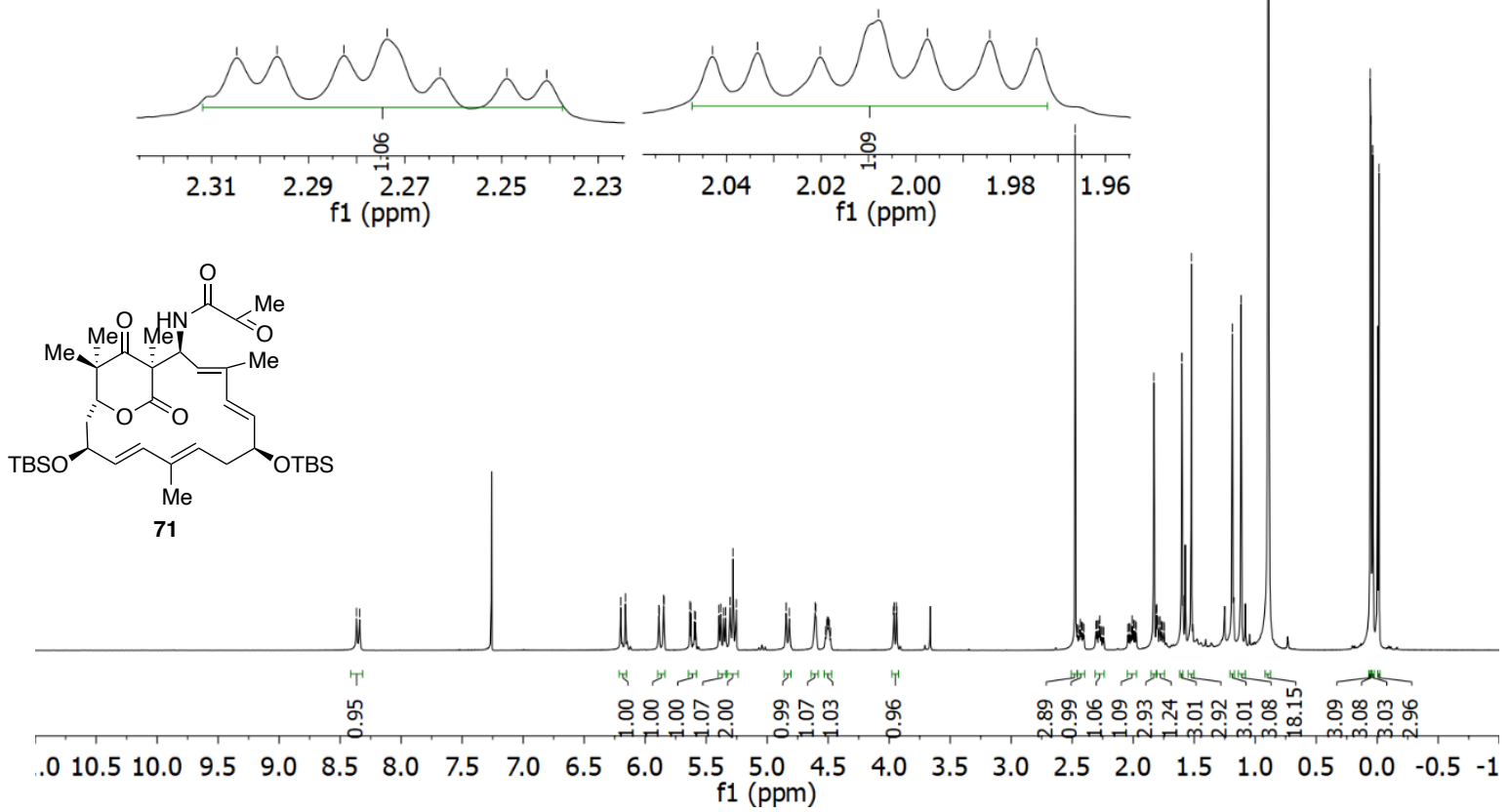

$\left({ }^{13} \mathrm{C} \mathrm{NMR}, \mathrm{CDCl}_{3}, 100 \mathrm{MHz}\right)$

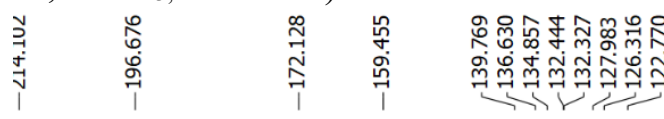

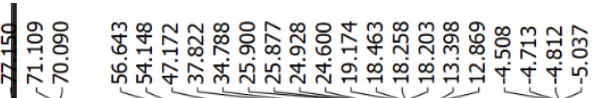

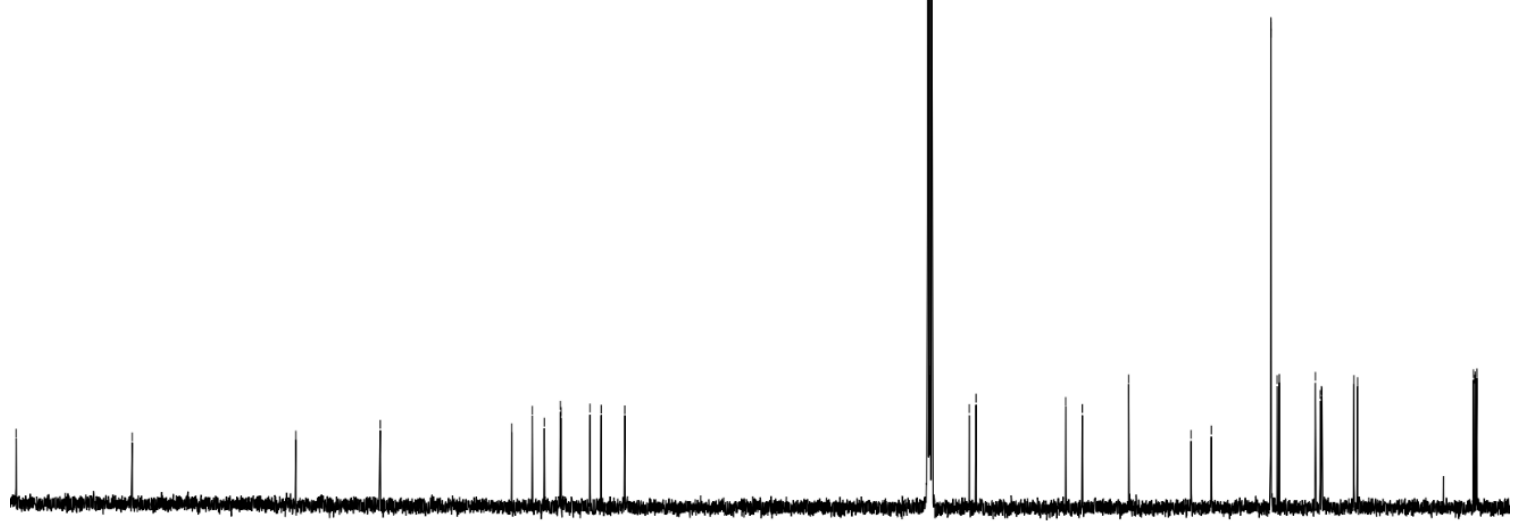

$\begin{array}{llllllllllllllllllllllll}210 & 200 & 190 & 180 & 170 & 160 & 150 & 140 & 130 & 120 & \begin{array}{c}110 \\ \mathrm{f} 1(\mathrm{ppm})\end{array} & \begin{array}{ll}100 \\ (\mathrm{ppm}\end{array} & 80 & 70 & 60 & 50 & 40 & 30 & 20 & 10 & 0 & -1\end{array}$ 
( $\left.{ }^{1} \mathrm{H} \mathrm{NMR}, \mathrm{CDCl}_{3}, 400 \mathrm{MHz}\right)$

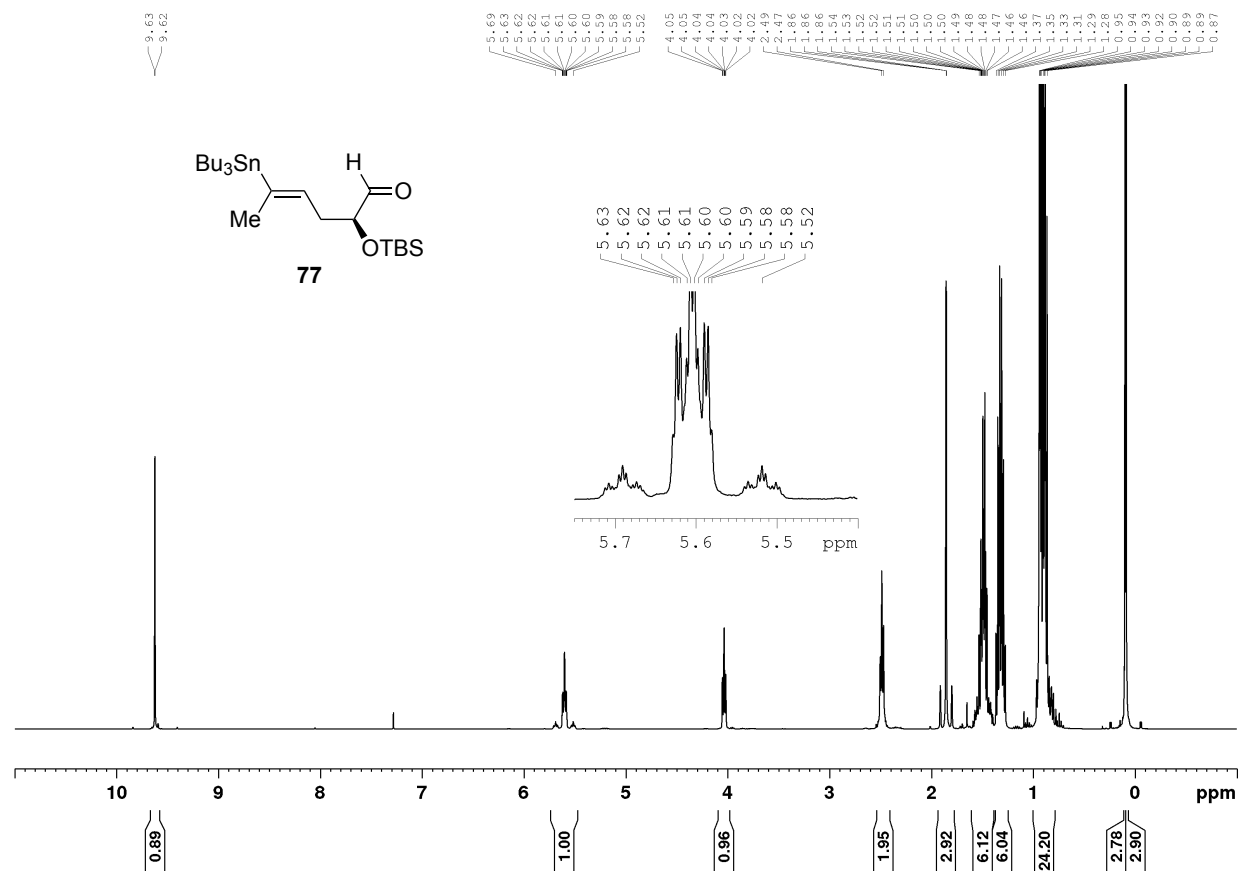

$\left({ }^{13} \mathrm{C} \mathrm{NMR}, \mathrm{CDCl}_{3}, 100 \mathrm{MHz}\right)$

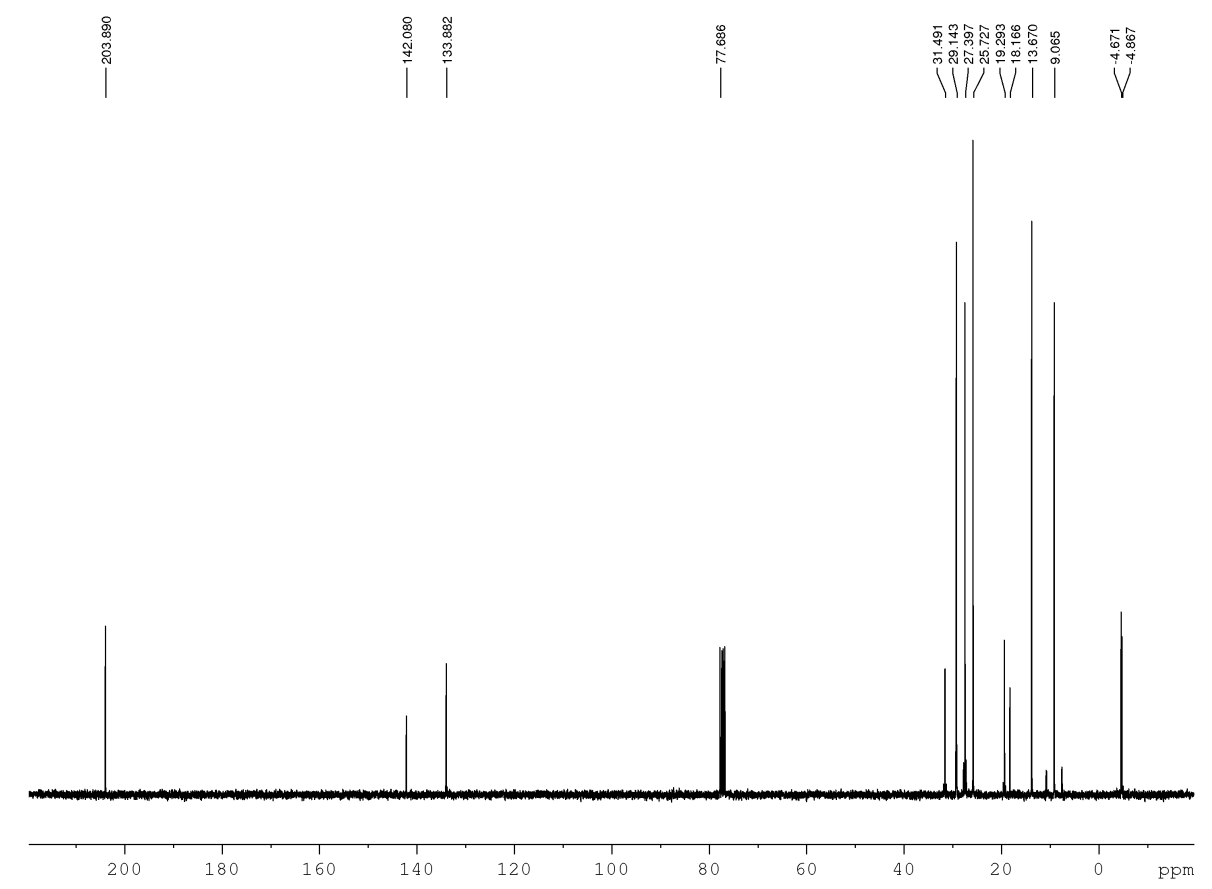


( $\left.{ }^{1} \mathrm{H} \mathrm{NMR}, \mathrm{CDCl}_{3}, 400 \mathrm{MHz}\right)$

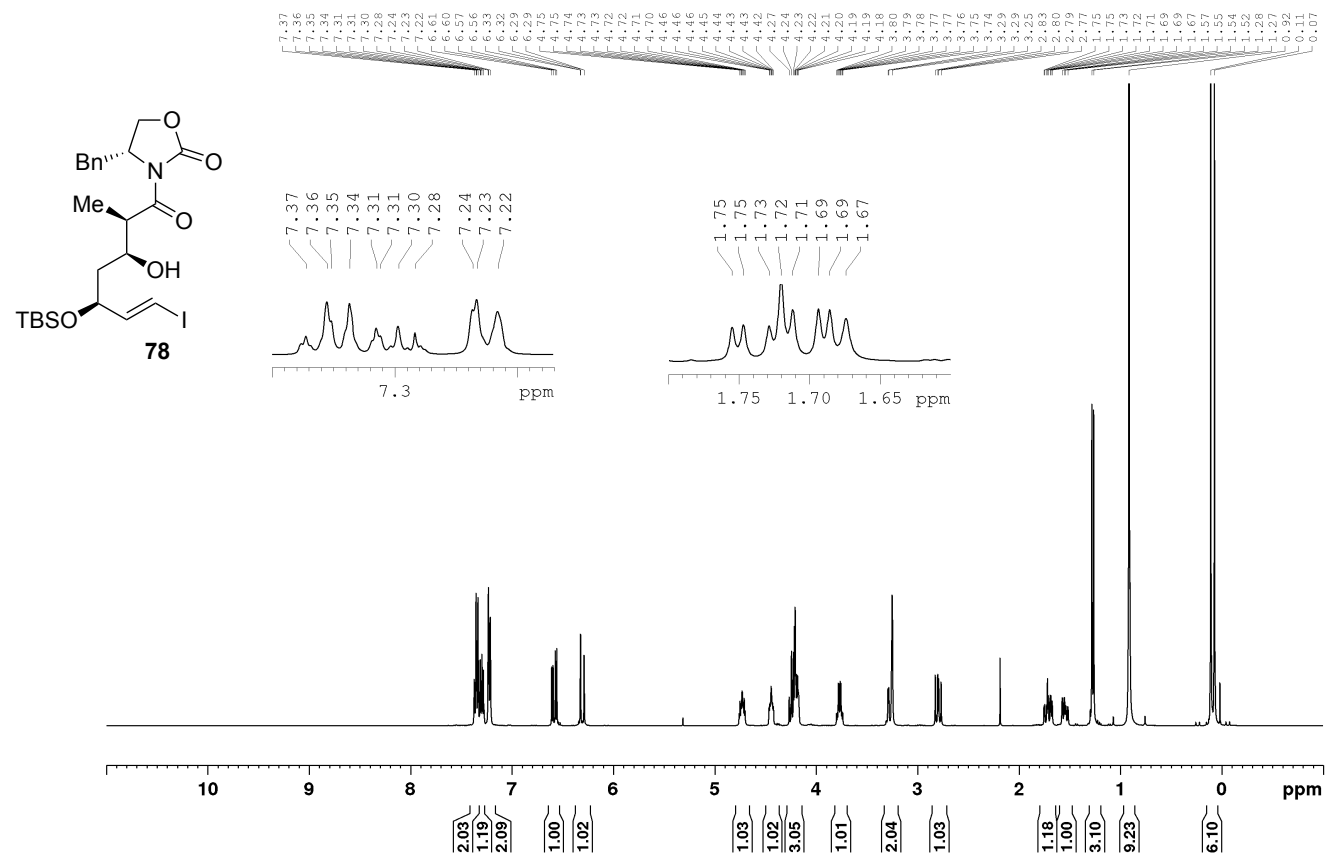

$\left({ }^{13} \mathrm{C} \mathrm{NMR}, \mathrm{CDCl}_{3}, 100 \mathrm{MHz}\right)$
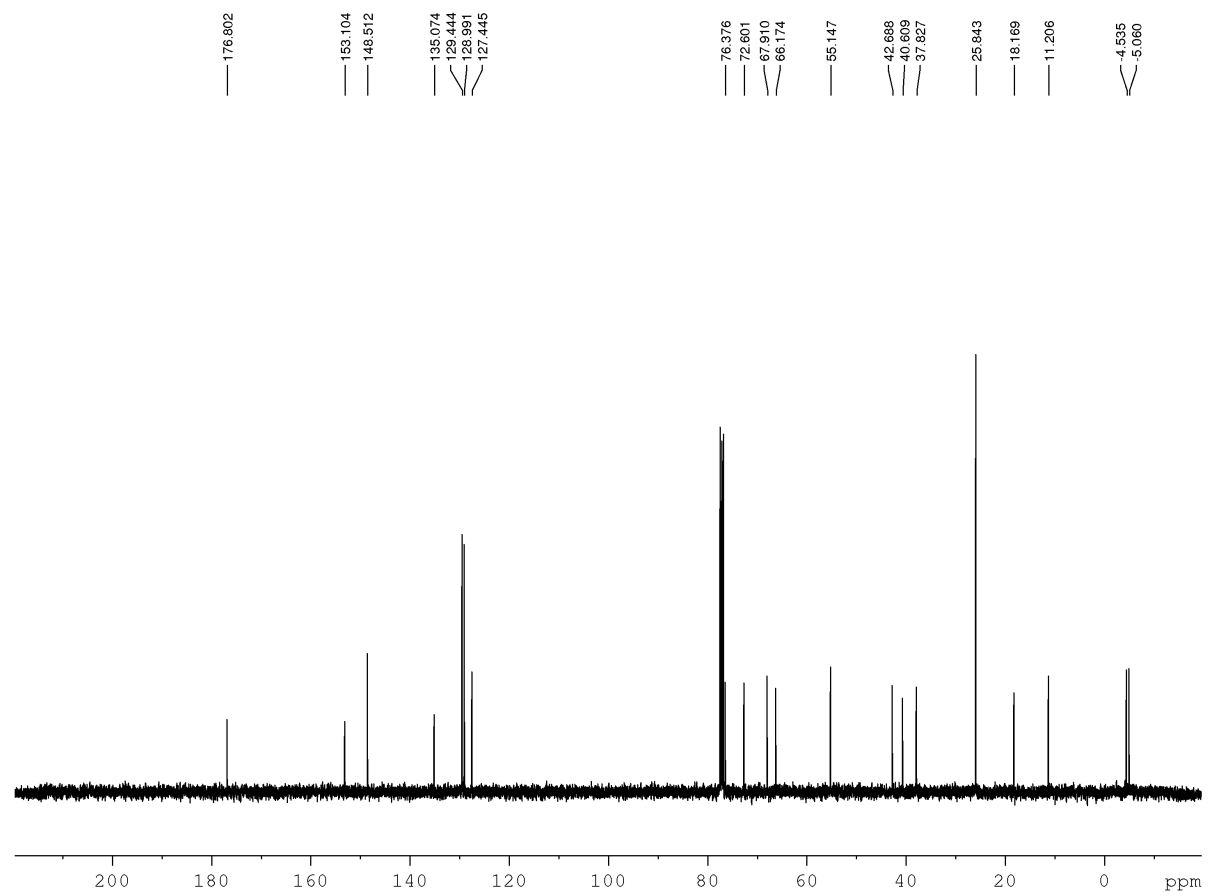
( $\left.{ }^{1} \mathrm{H} \mathrm{NMR}, \mathrm{CDCl}_{3}, 400 \mathrm{MHz}\right)$

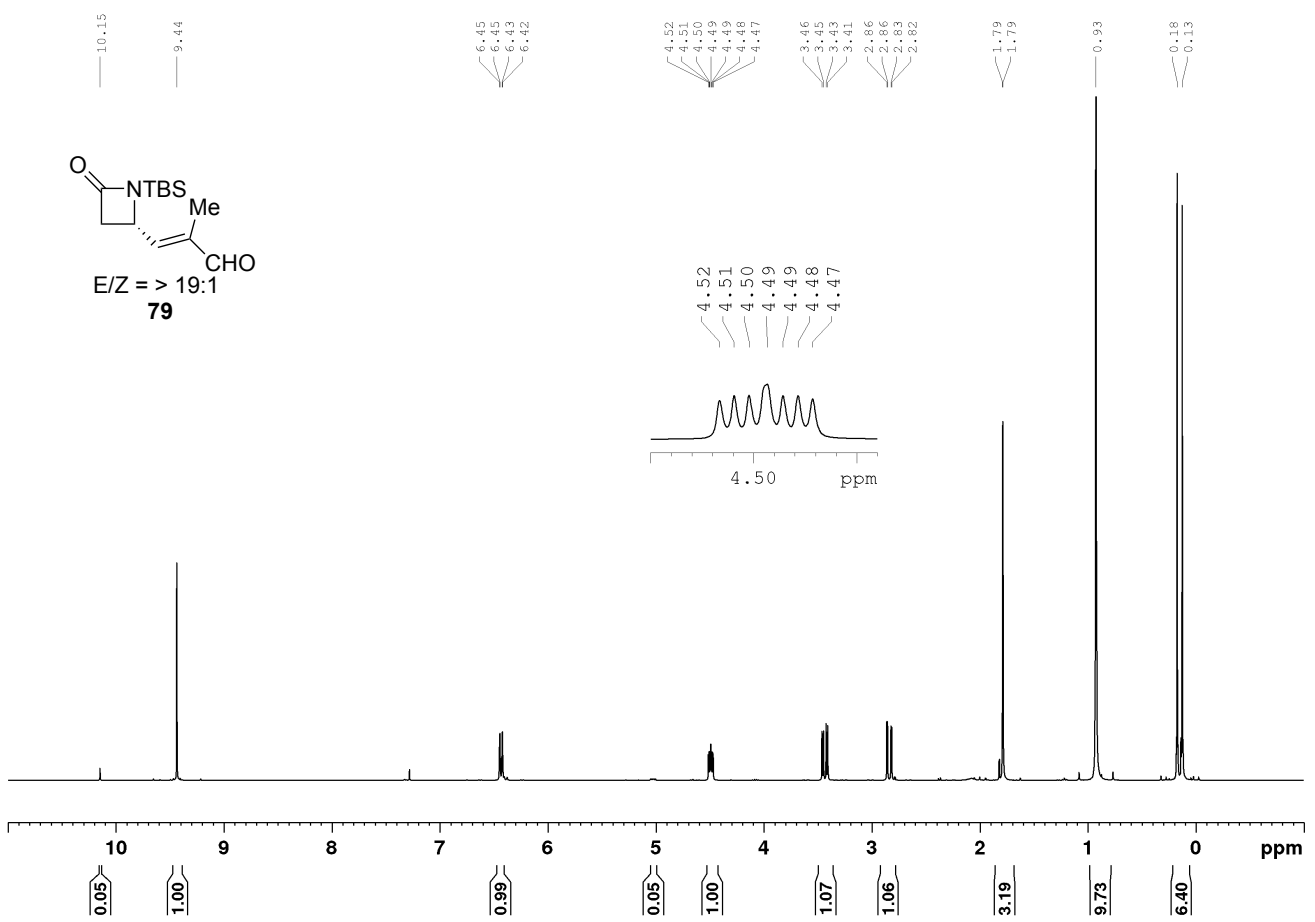

$\left({ }^{13} \mathrm{C} \mathrm{NMR}, \mathrm{CDCl}_{3}, 100 \mathrm{MHz}\right)$

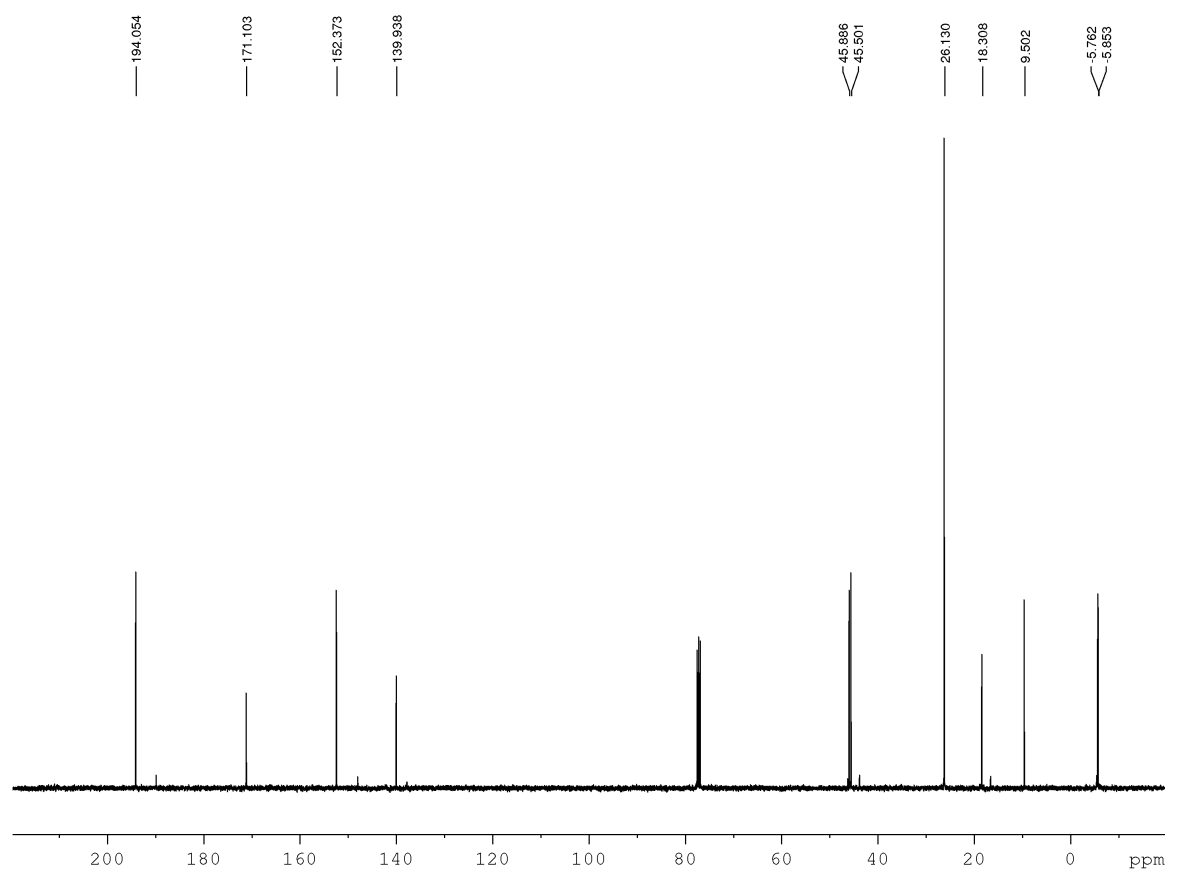


( $\left.{ }^{1} \mathrm{H} \mathrm{NMR}, \mathrm{CDCl}_{3}, 400 \mathrm{MHz}\right)$

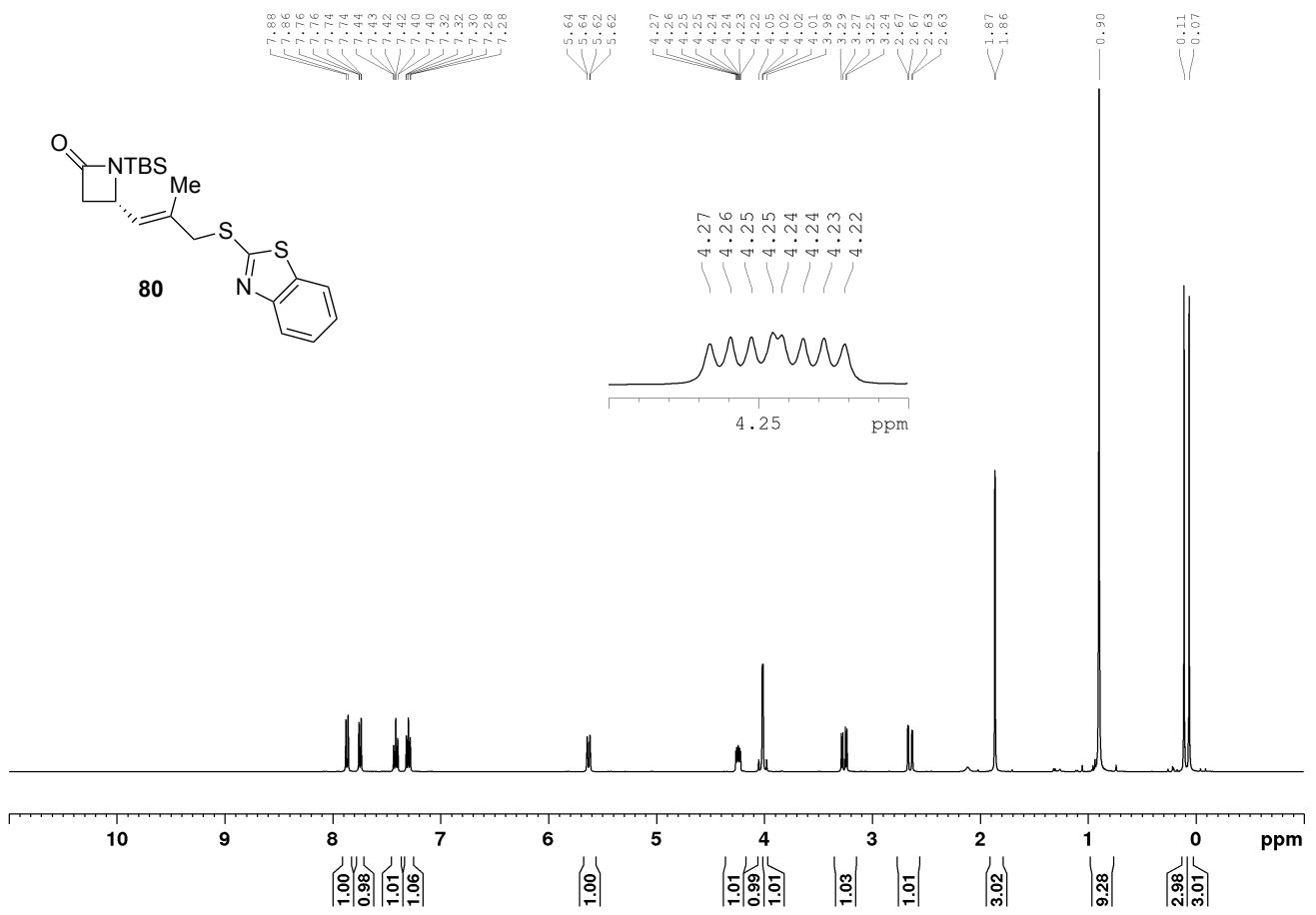

$\left({ }^{13} \mathrm{C} \mathrm{NMR}, \mathrm{CDCl}_{3}, 100 \mathrm{MHz}\right)$

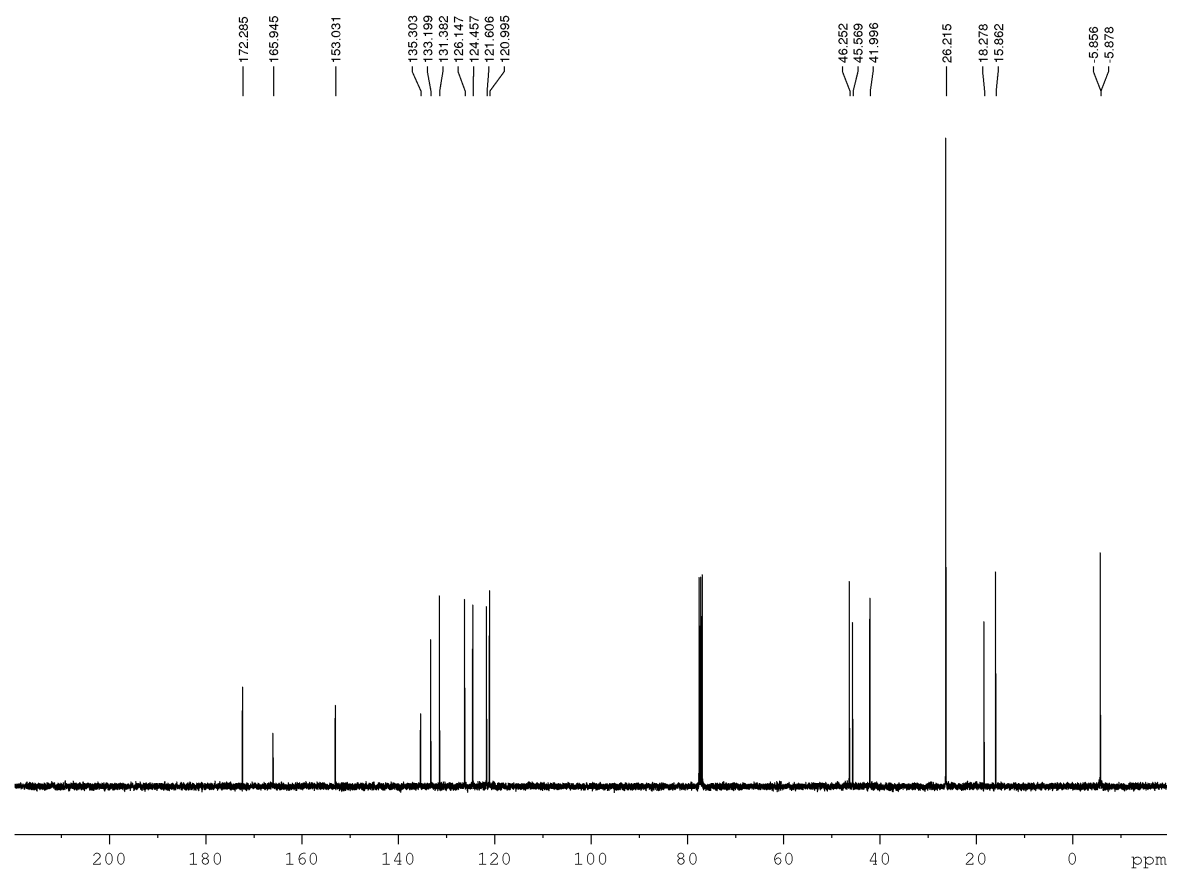


( $\left.{ }^{1} \mathrm{H} \mathrm{NMR}, \mathrm{CDCl}_{3}, 400 \mathrm{MHz}\right)$

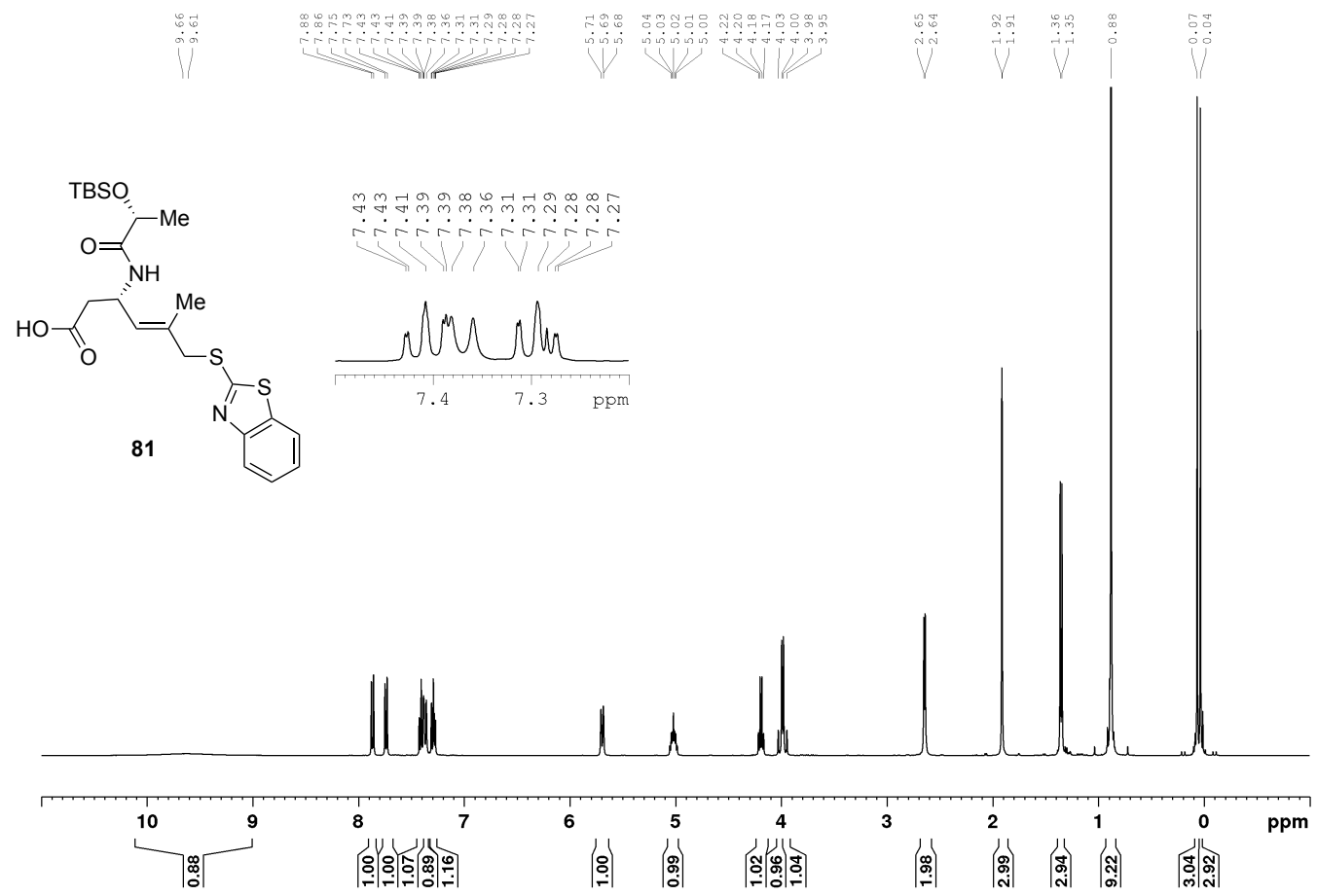

$\left({ }^{13} \mathrm{C} \mathrm{NMR}, \mathrm{CDCl}_{3}, 100 \mathrm{MHz}\right)$
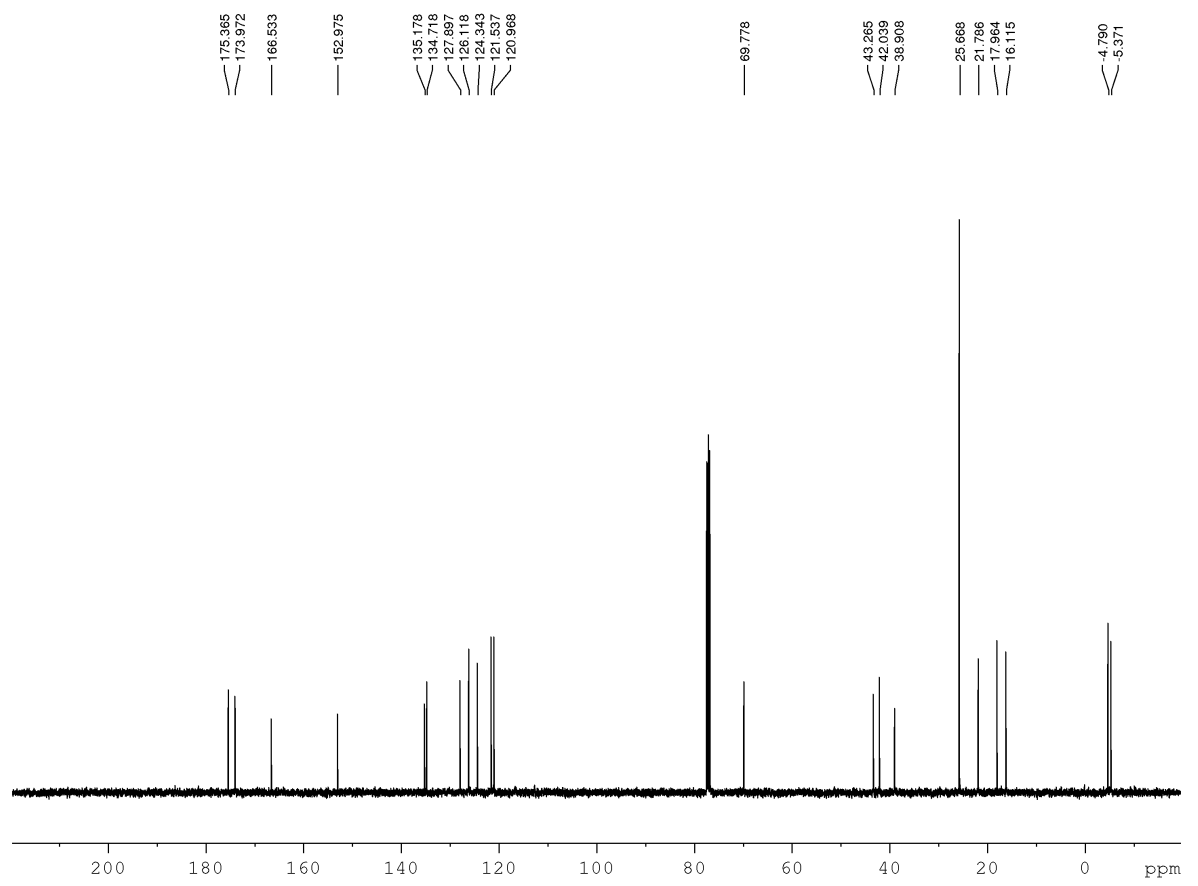
( $\left.{ }^{1} \mathrm{H} \mathrm{NMR}, \mathrm{CDCl}_{3}, 400 \mathrm{MHz}\right)$

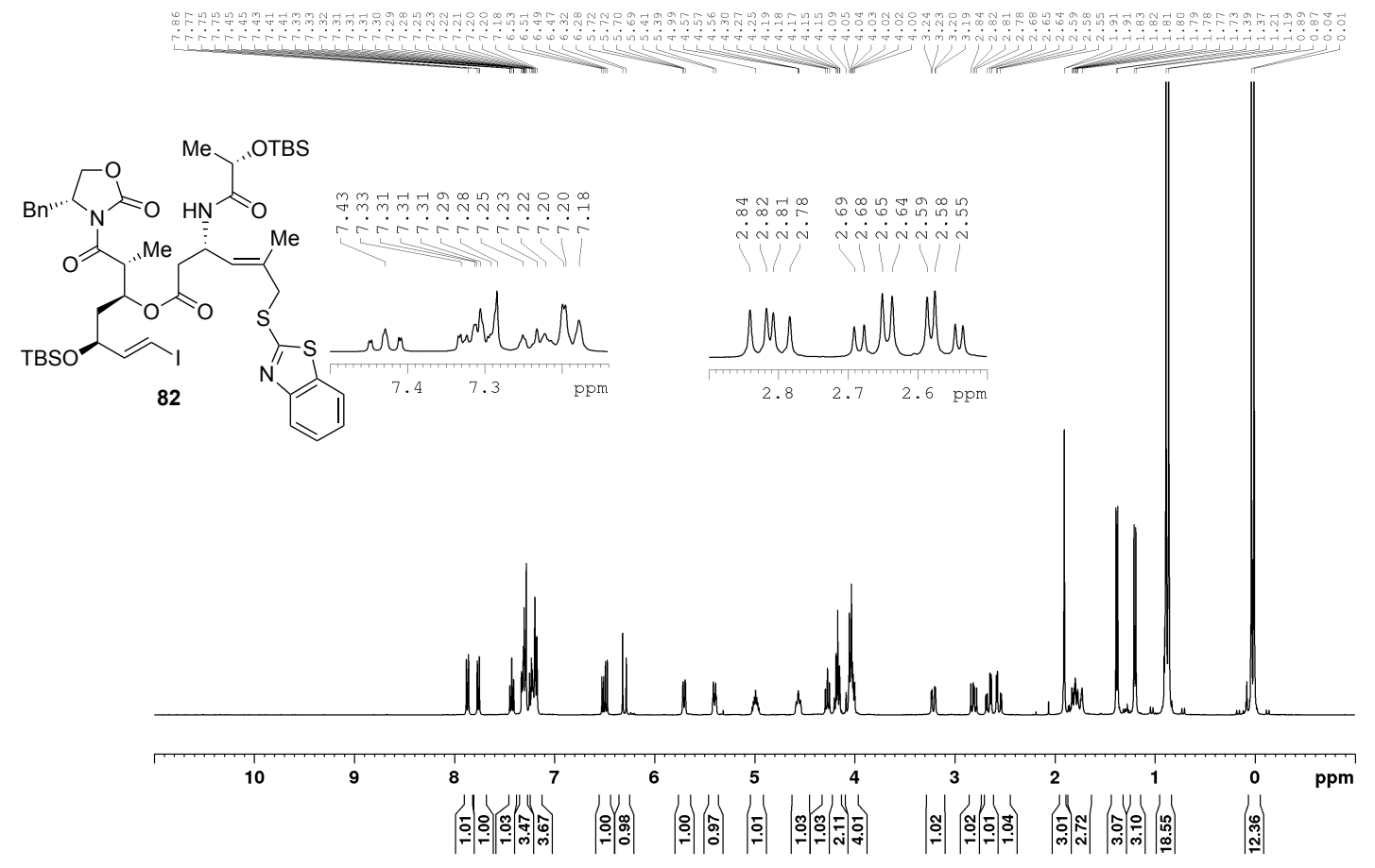

$\left({ }^{13} \mathrm{C}\right.$ NMR, $\left.\mathrm{CDCl}_{3}, 100 \mathrm{MHz}\right)$

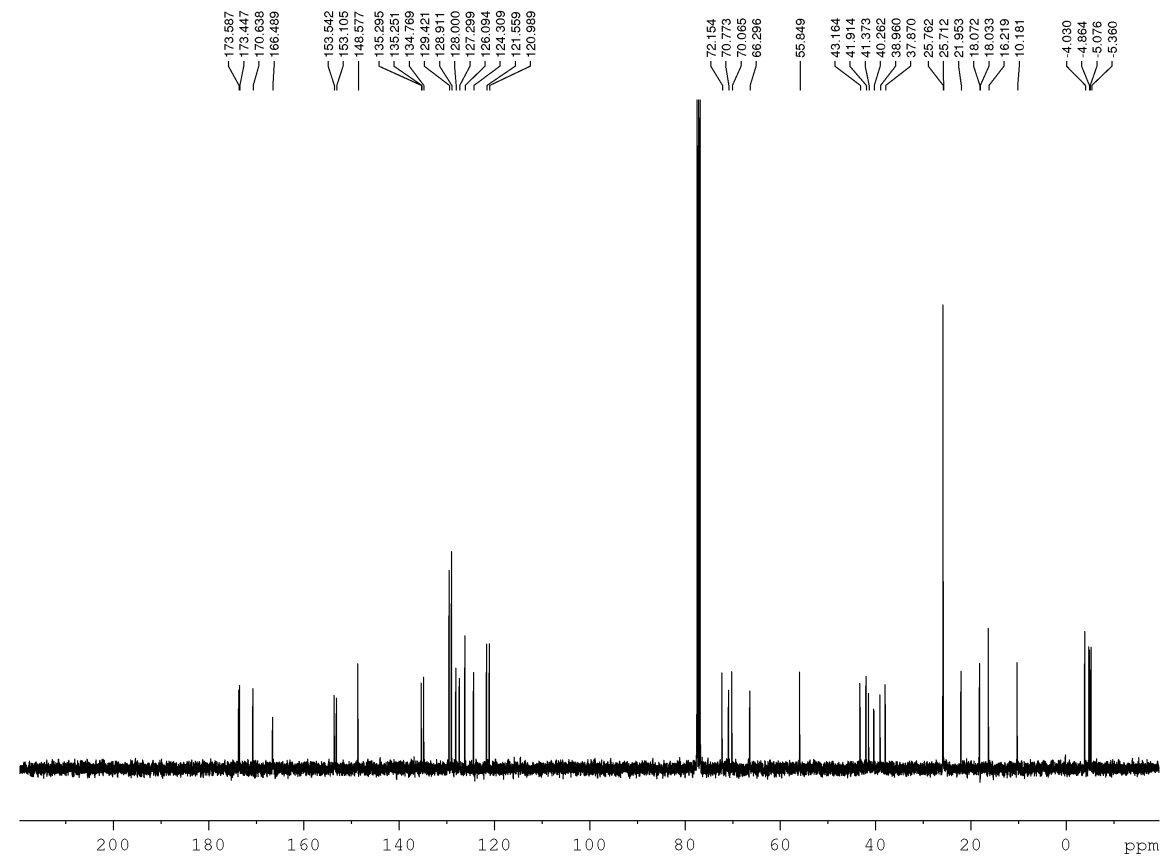


( $\left.{ }^{1} \mathrm{H} \mathrm{NMR}, \mathrm{CDCl}_{3}, 400 \mathrm{MHz}\right)$

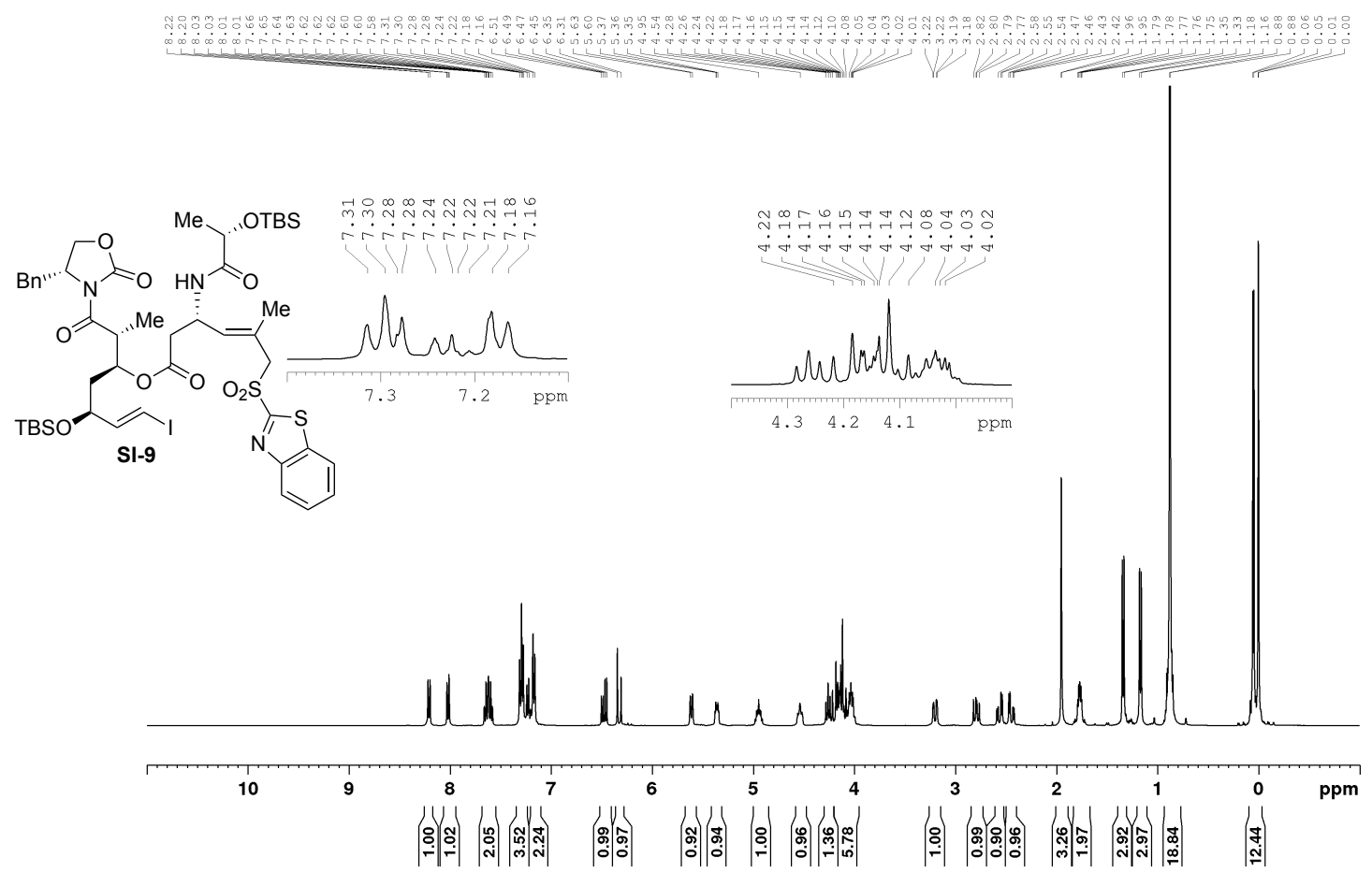

$\left({ }^{13} \mathrm{C} \mathrm{NMR}, \mathrm{CDCl}_{3}, 100 \mathrm{MHz}\right)$

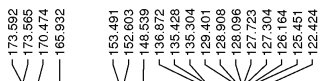

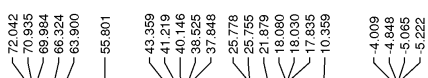

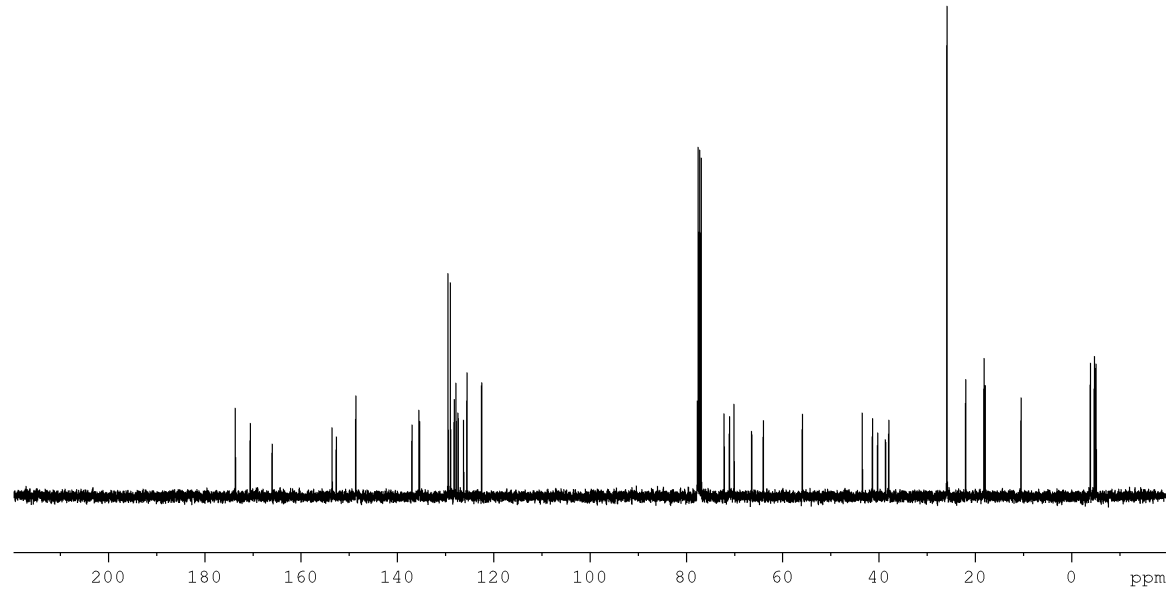


( $\left.{ }^{1} \mathrm{H} \mathrm{NMR}, \mathrm{CDCl}_{3}, 400 \mathrm{MHz}\right)$

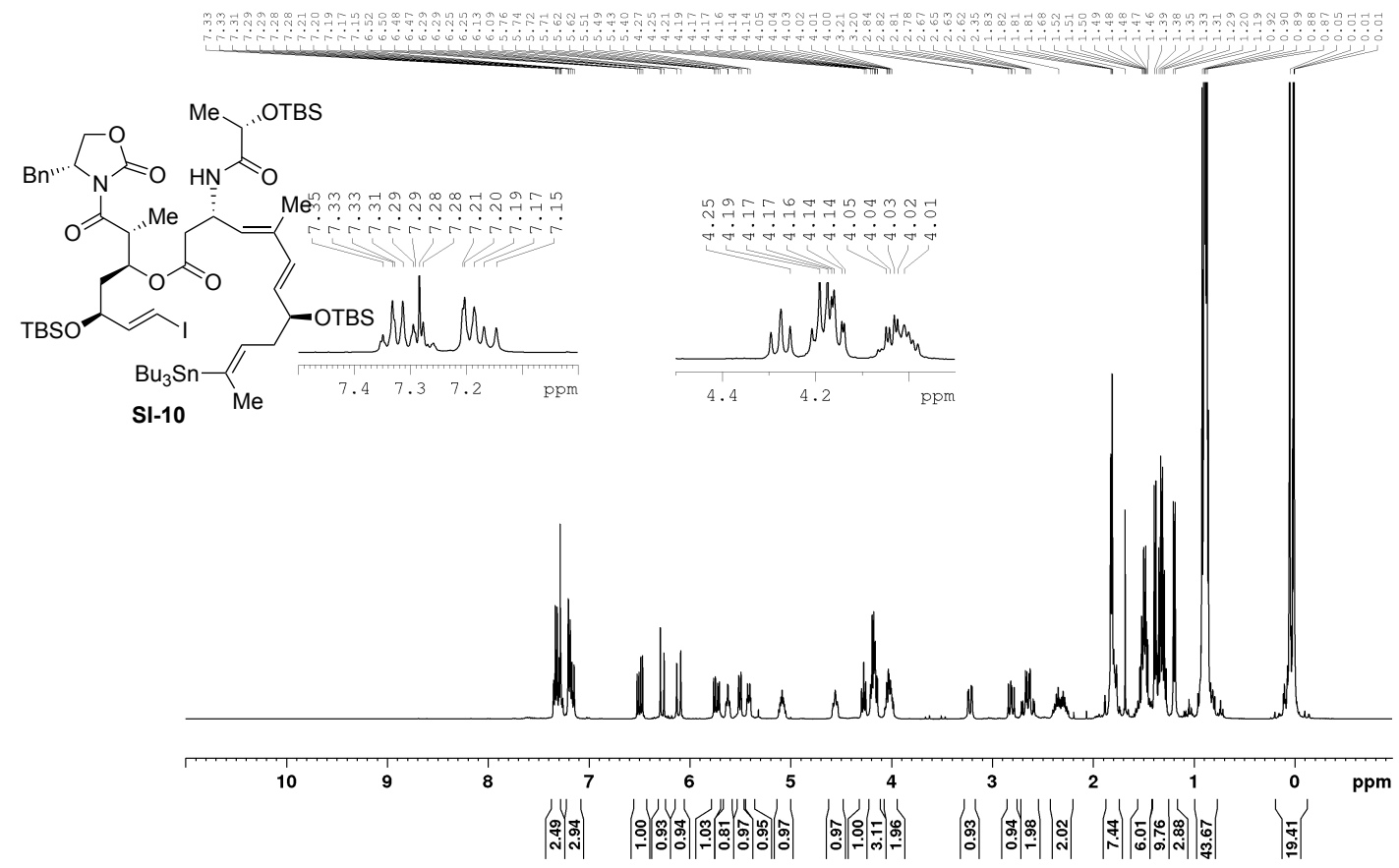

$\left({ }^{13} \mathrm{C} \mathrm{NMR}, \mathrm{CDCl}_{3}, 100 \mathrm{MHz}\right)$

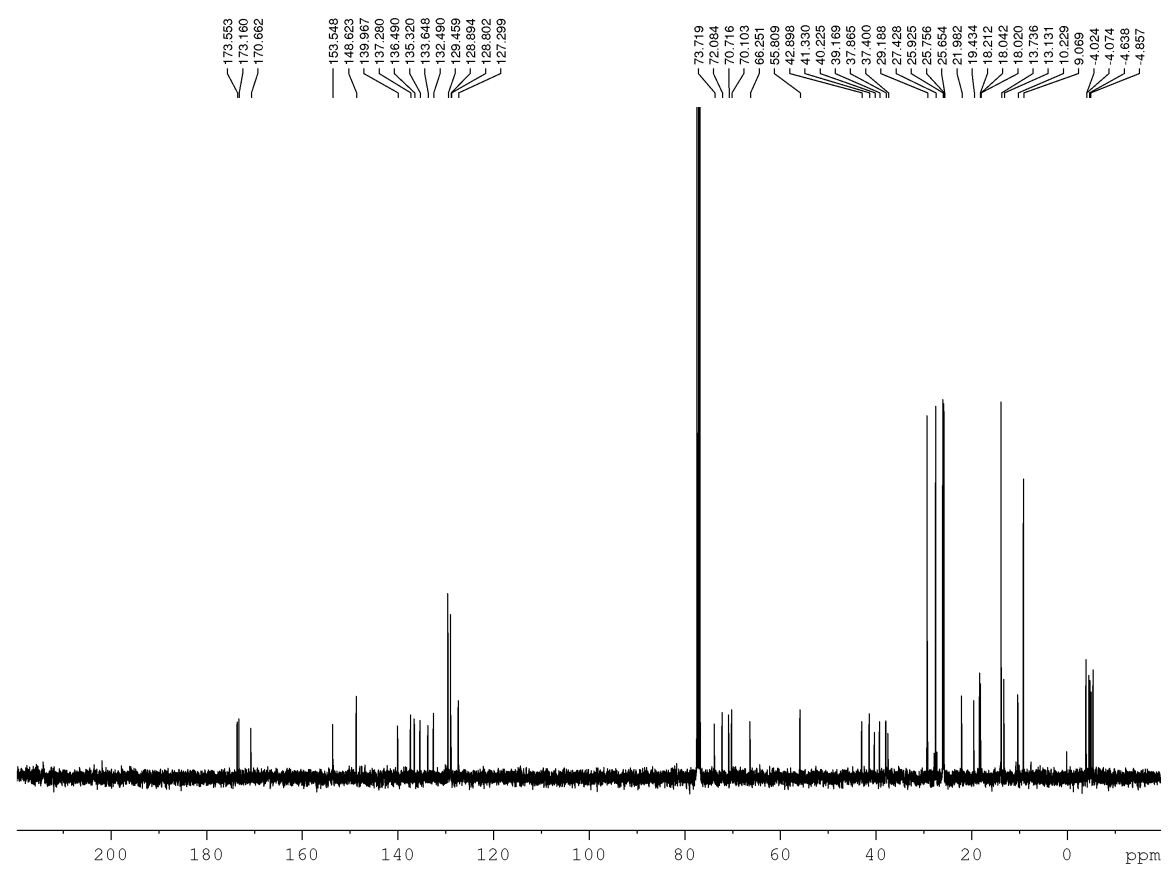


( $\left.{ }^{1} \mathrm{H} \mathrm{NMR}, \mathrm{CDCl}_{3}, 400 \mathrm{MHz}\right)$

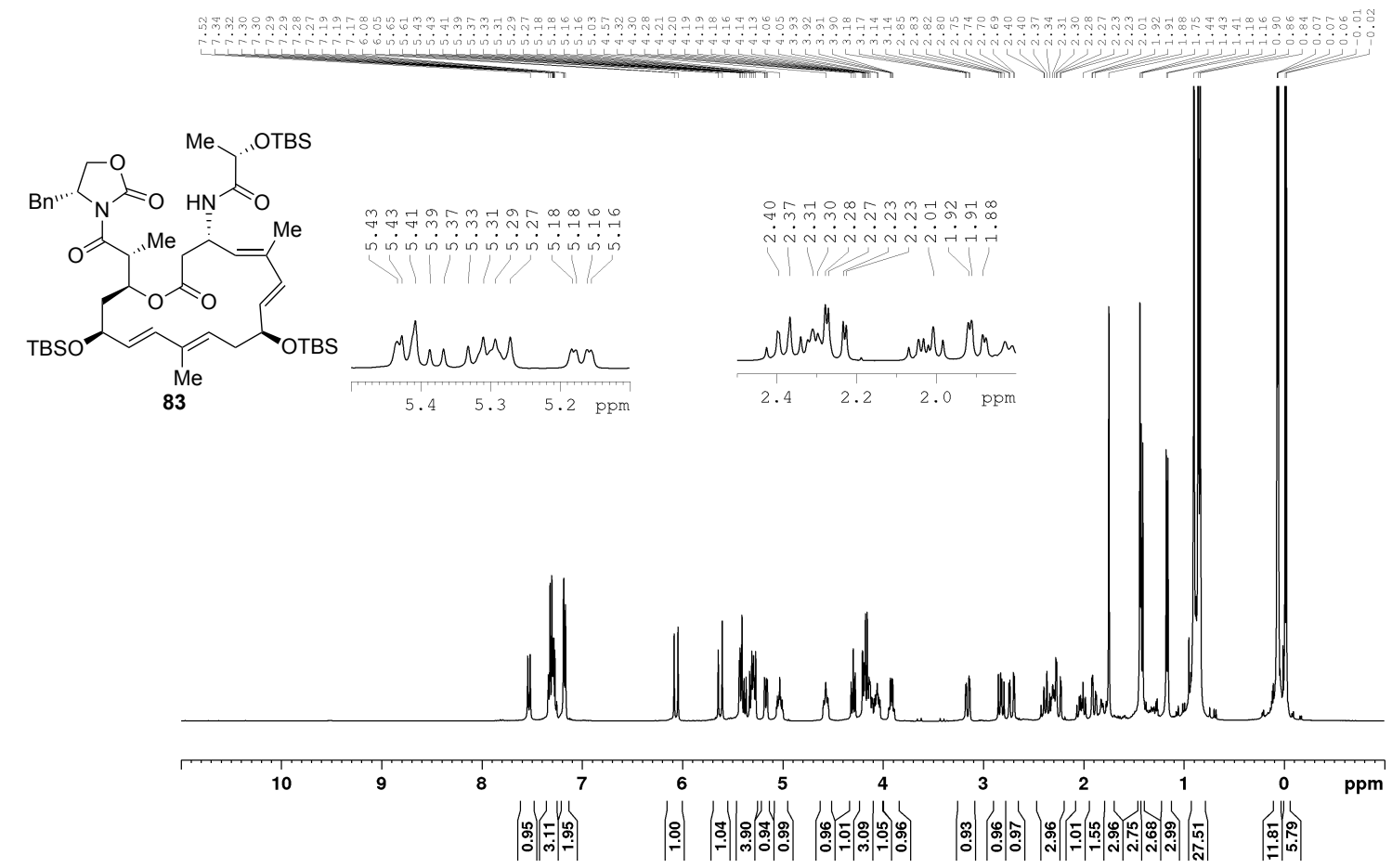

$\left({ }^{13} \mathrm{C} \mathrm{NMR}, \mathrm{CDCl}_{3}, 100 \mathrm{MHz}\right)$

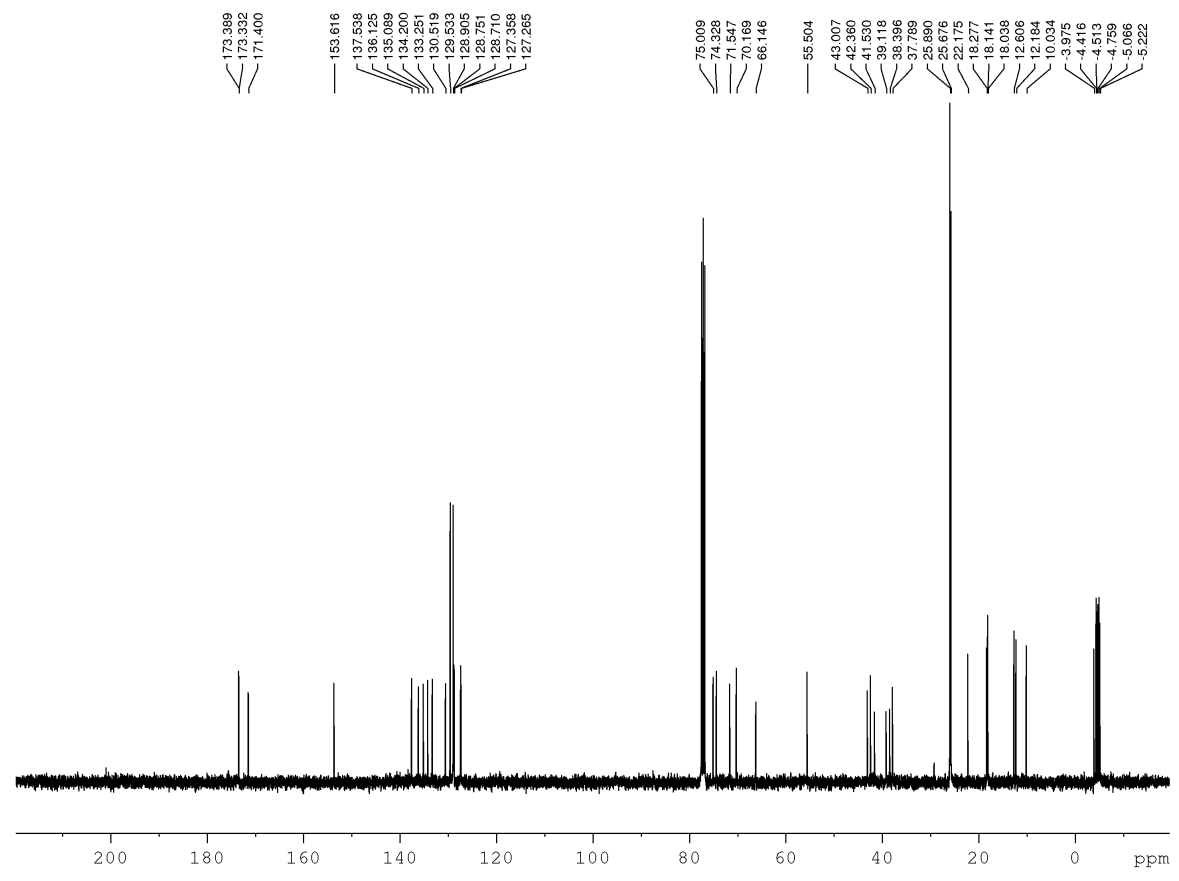


( $\left.{ }^{1} \mathrm{H} \mathrm{NMR}, \mathrm{CDCl}_{3}, 400 \mathrm{MHz}\right)$

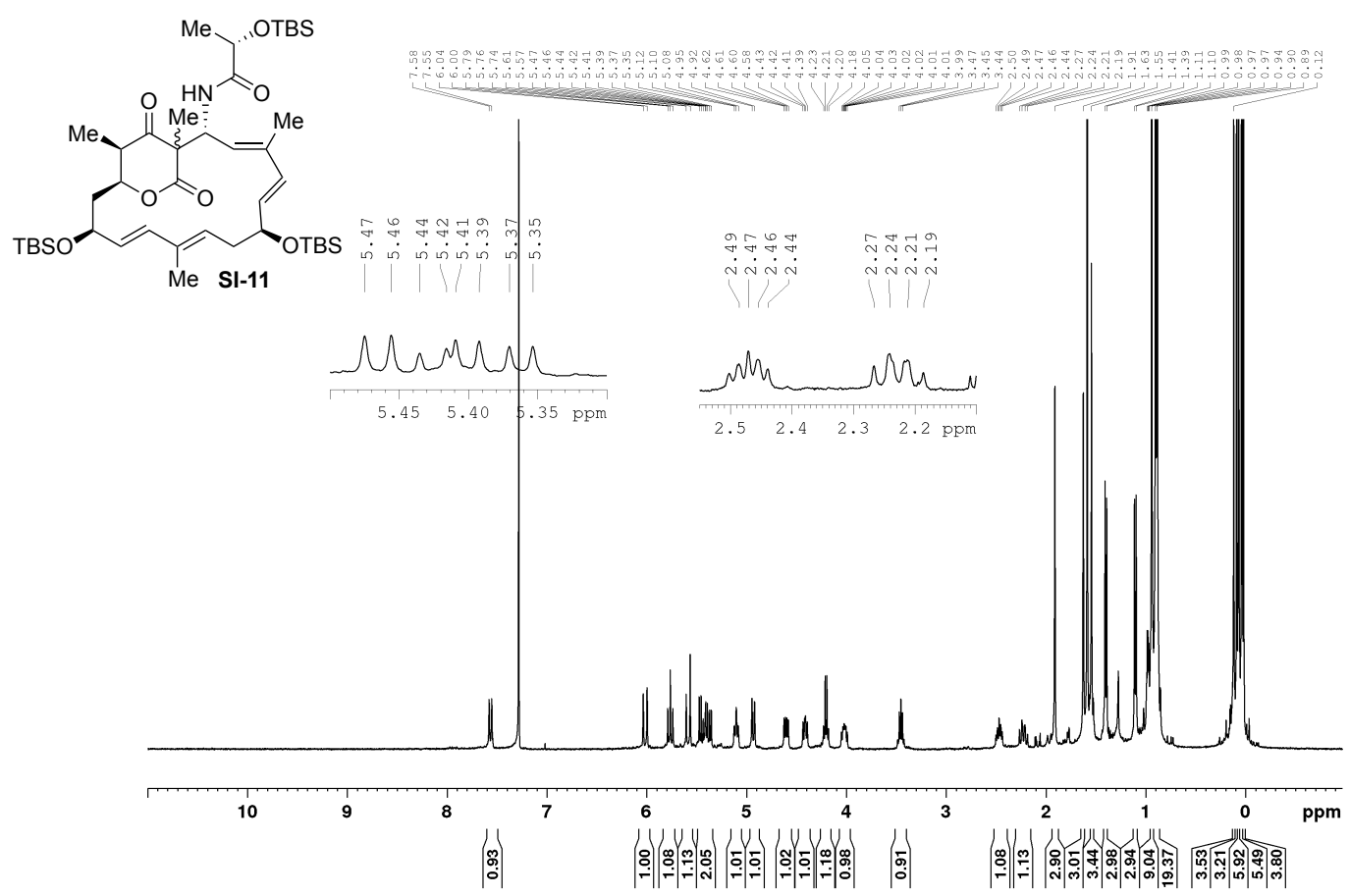

$\left({ }^{13} \mathrm{C} \mathrm{NMR}, \mathrm{CDCl}_{3}, 400 \mathrm{MHz}\right)$

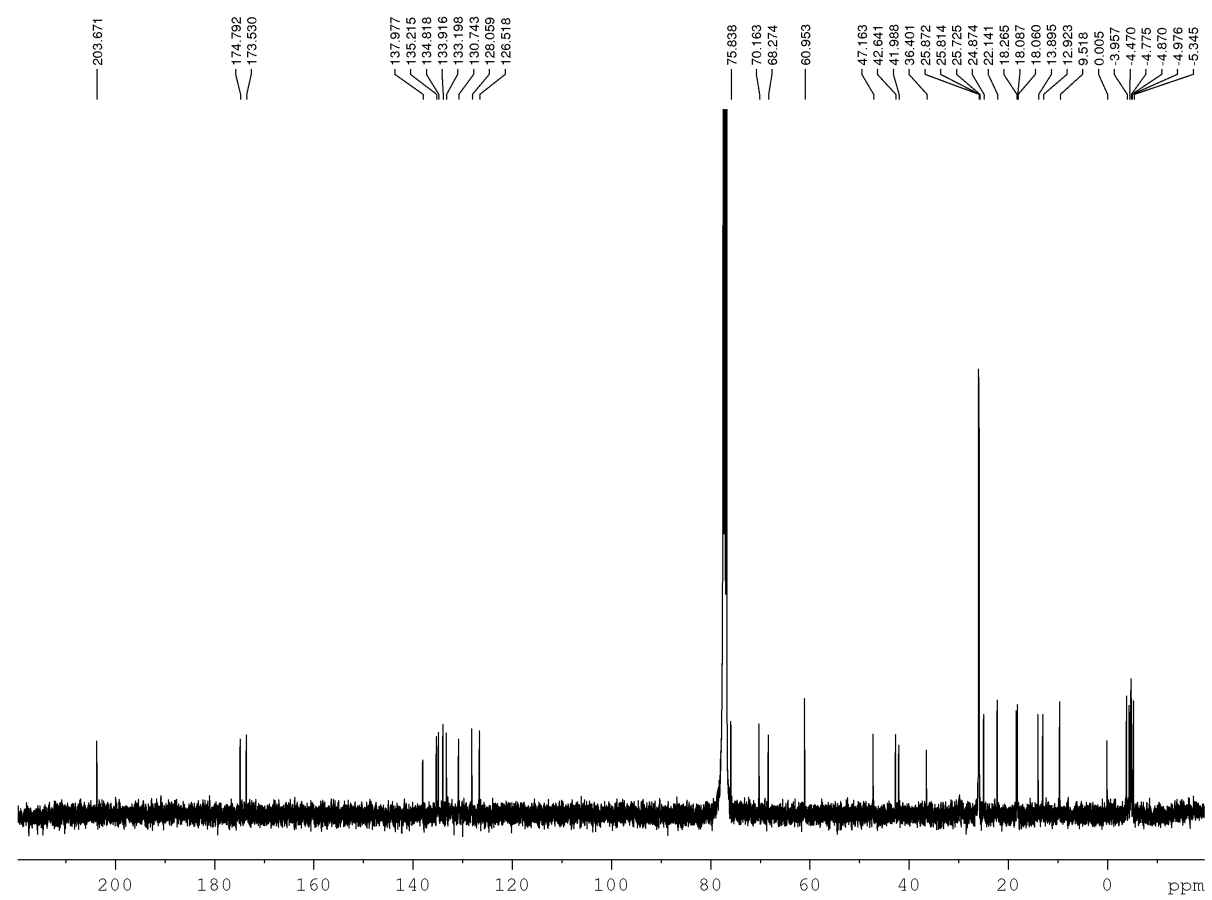


$\left({ }^{1} \mathrm{H} \mathrm{NMR}, \mathrm{CD}_{3} \mathrm{OD}, 400 \mathrm{MHz}\right)$

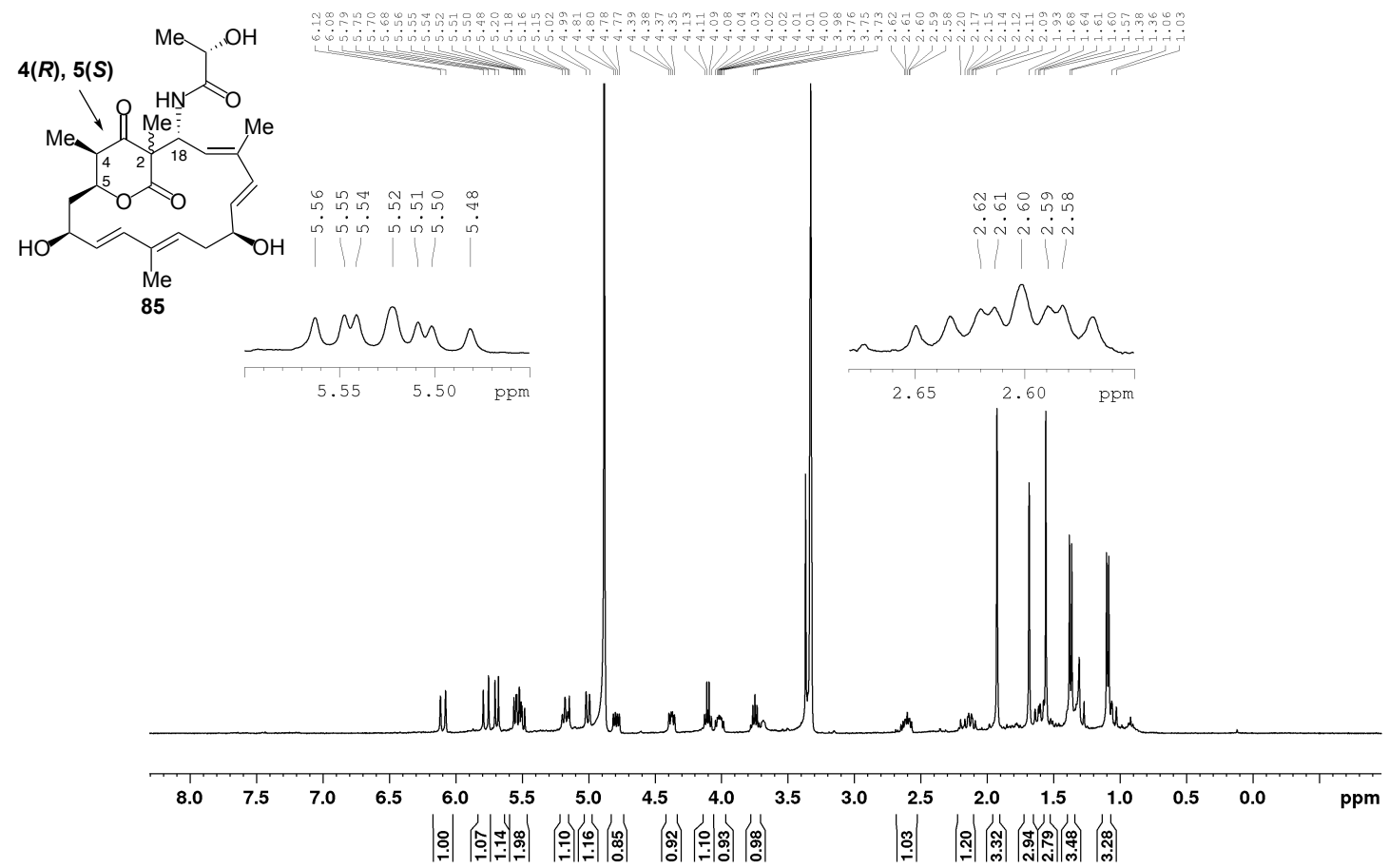

$\left({ }^{13} \mathrm{C} \mathrm{NMR}, \mathrm{CD}_{3} \mathrm{OD}, 400 \mathrm{MHz}\right)$

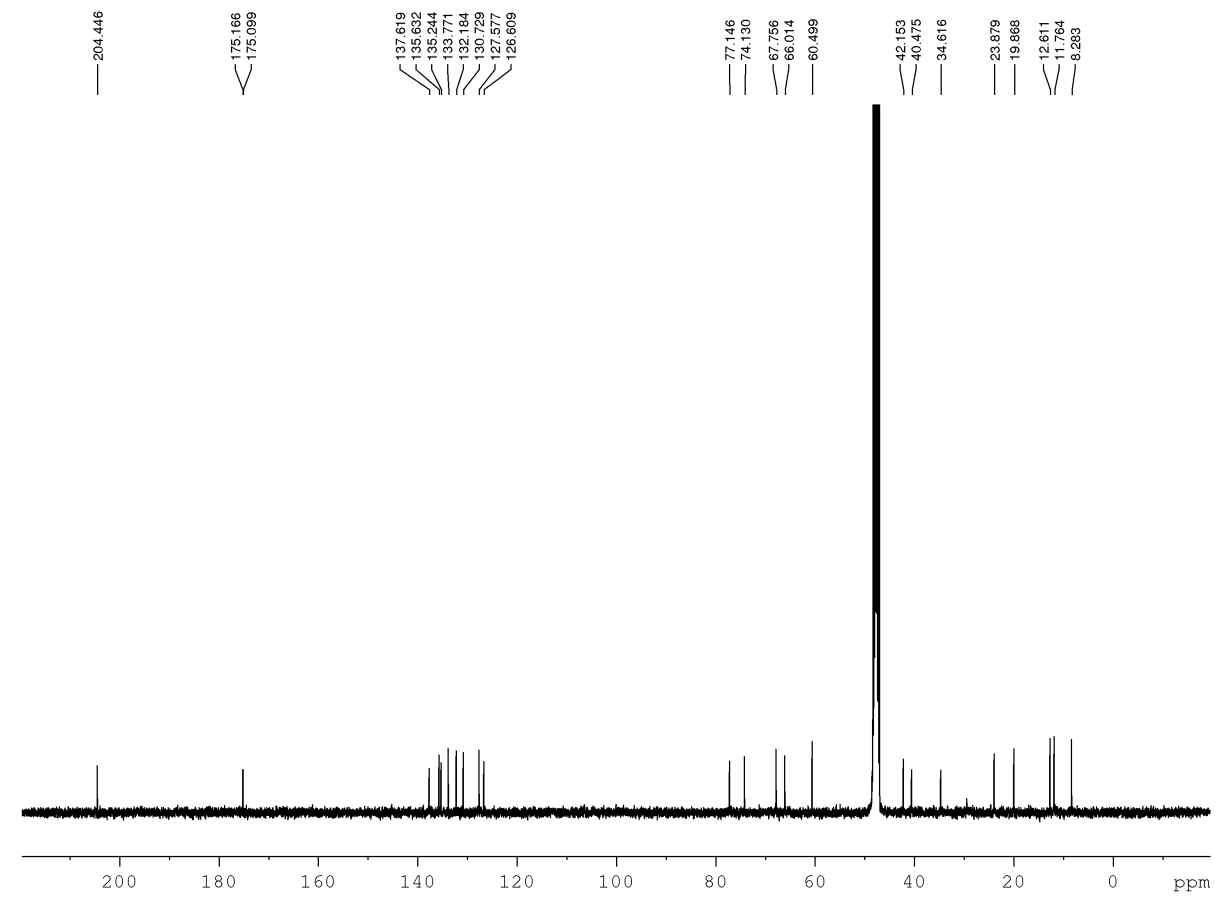


(COSY, $\left.\mathrm{CD}_{3} \mathrm{OD}, 400 \mathrm{MHz}\right)$

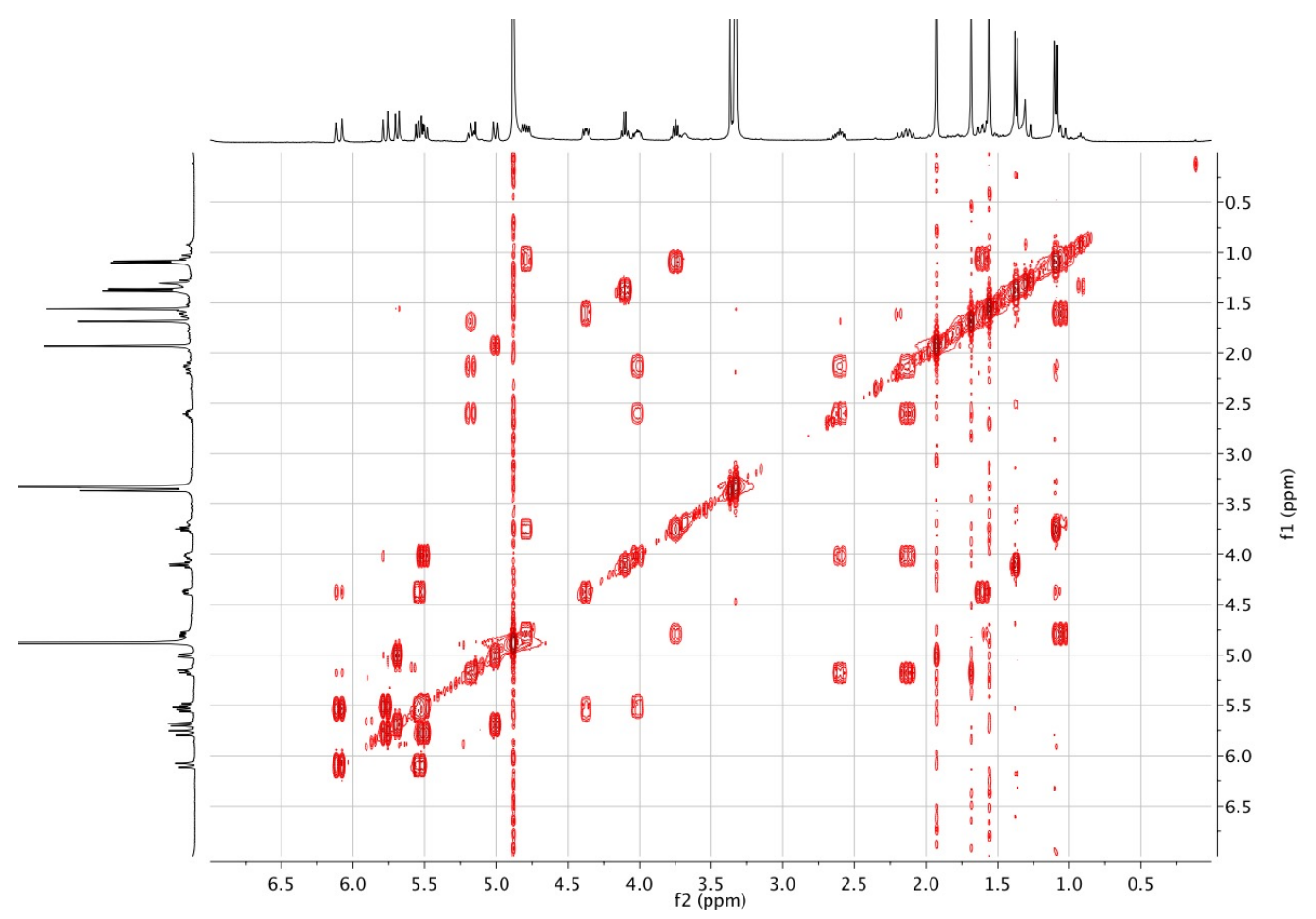

(NOESY, $\left.\mathrm{CD}_{3} \mathrm{OD}, 400 \mathrm{MHz}\right)$

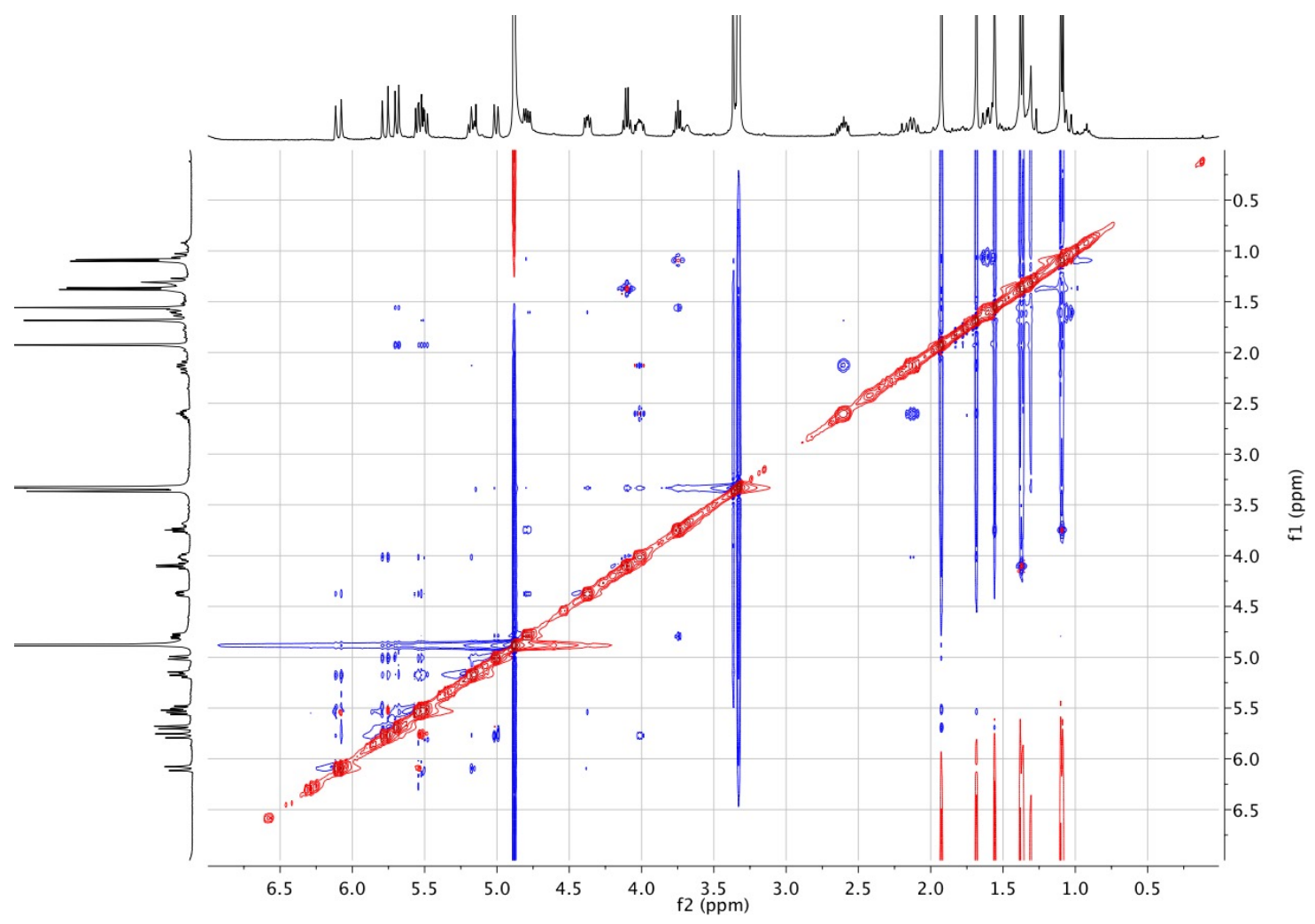


( $\left.{ }^{1} \mathrm{H} \mathrm{NMR}, \mathrm{CDCl}_{3}, 400 \mathrm{MHz}\right)$

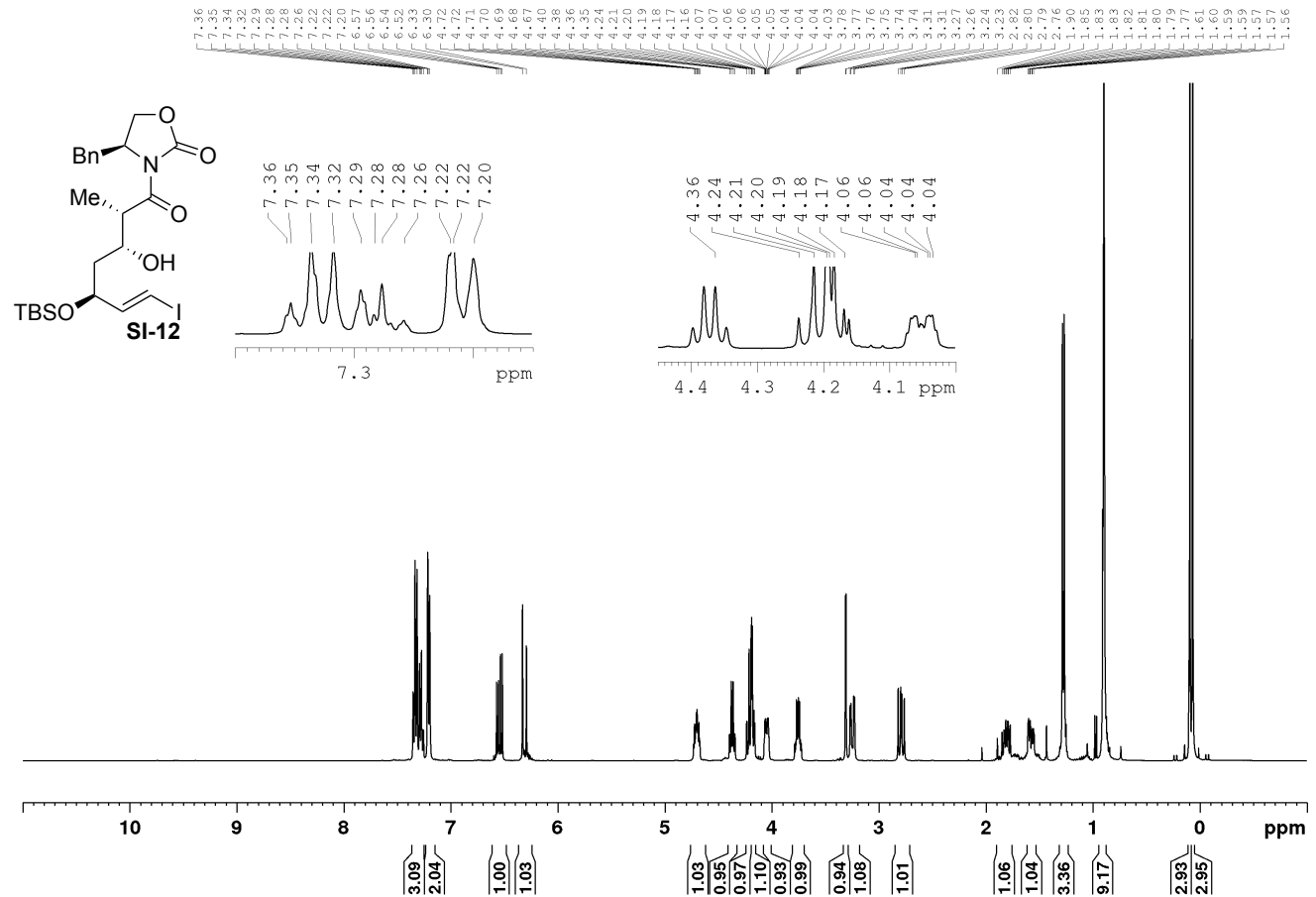

$\left({ }^{13} \mathrm{C} \mathrm{NMR}, \mathrm{CDCl}_{3}, 100 \mathrm{MHz}\right)$

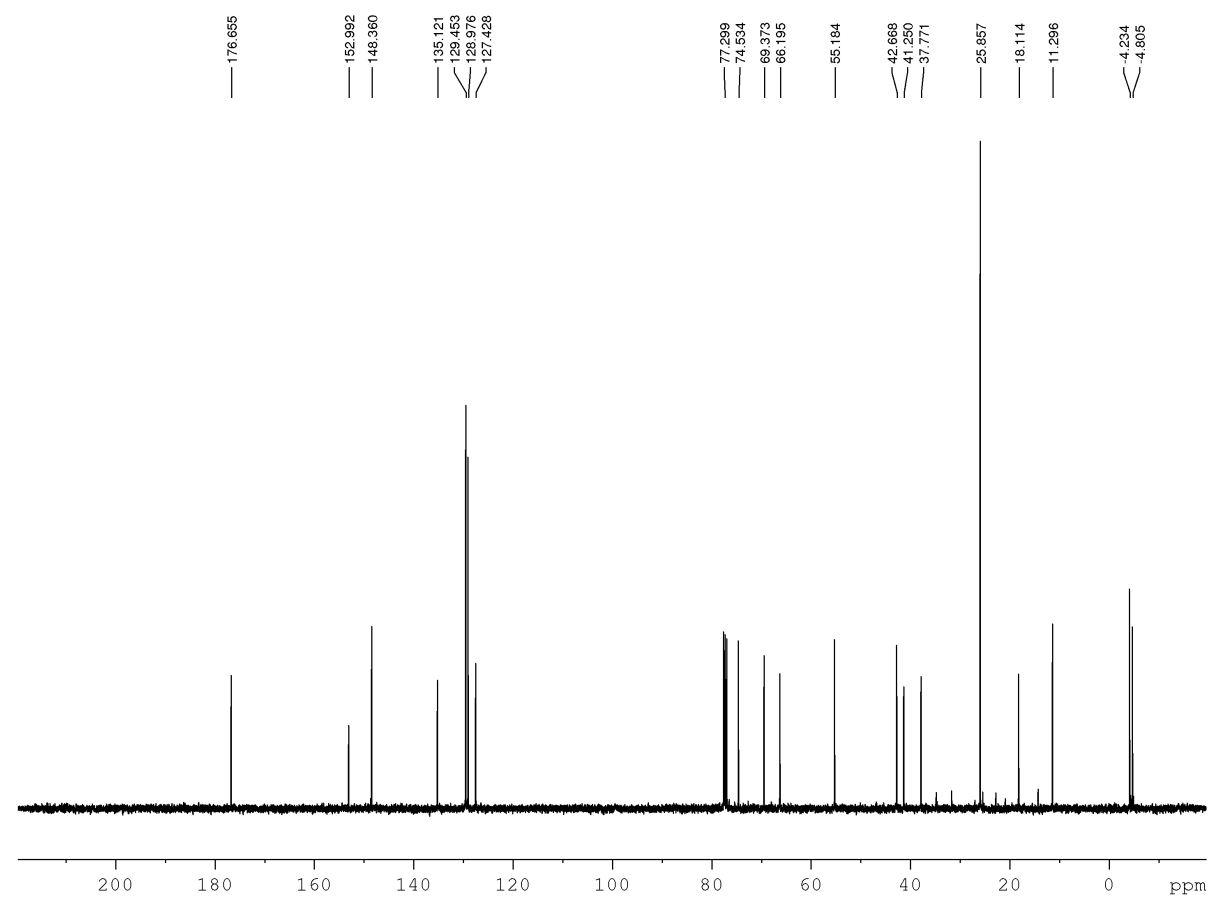


( $\left.{ }^{1} \mathrm{H} \mathrm{NMR}, \mathrm{CDCl}_{3}, 400 \mathrm{MHz}\right)$

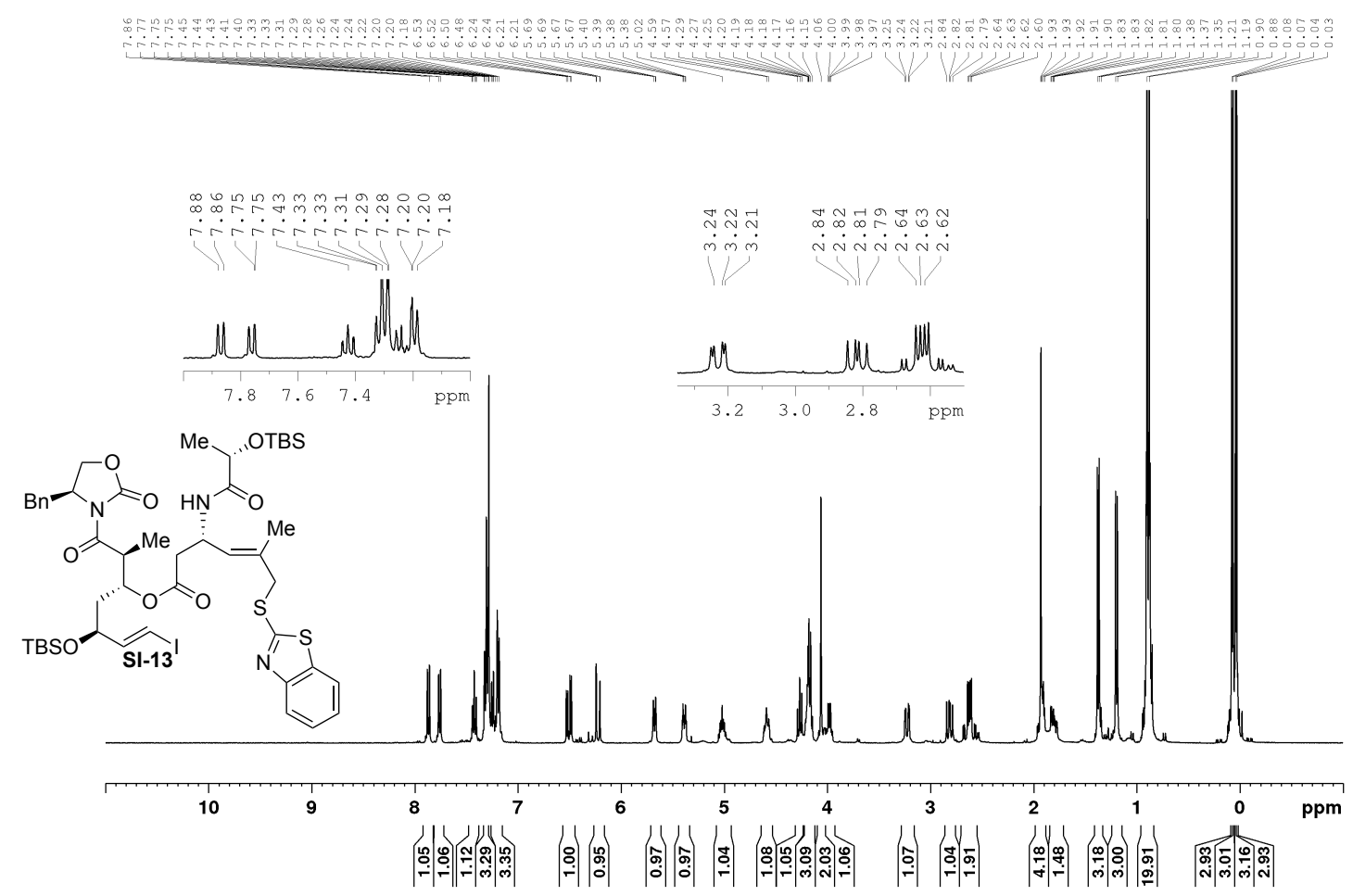

$\left({ }^{13} \mathrm{C} \mathrm{NMR}, \mathrm{CDCl}_{3}, 100 \mathrm{MHz}\right)$

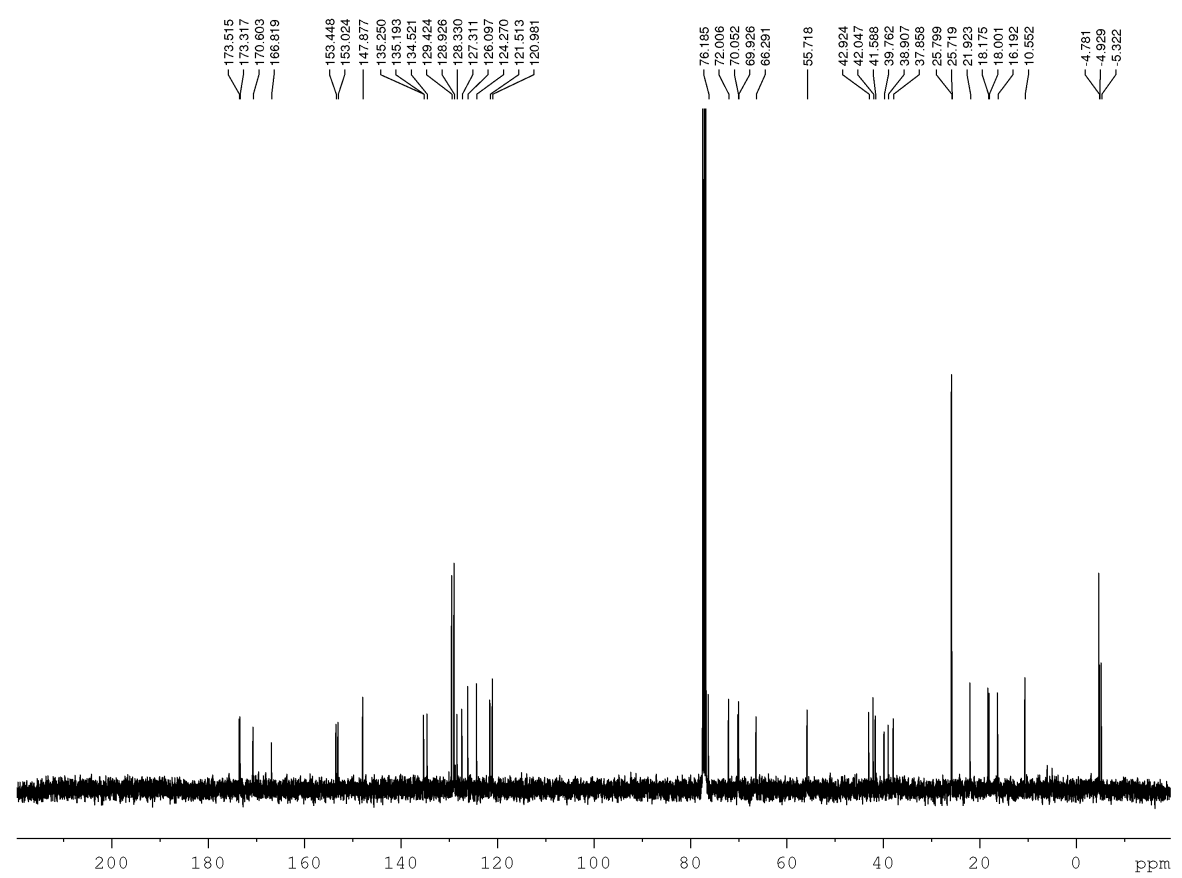


( $\left.{ }^{1} \mathrm{H} \mathrm{NMR}, \mathrm{CDCl}_{3}, 400 \mathrm{MHz}\right)$

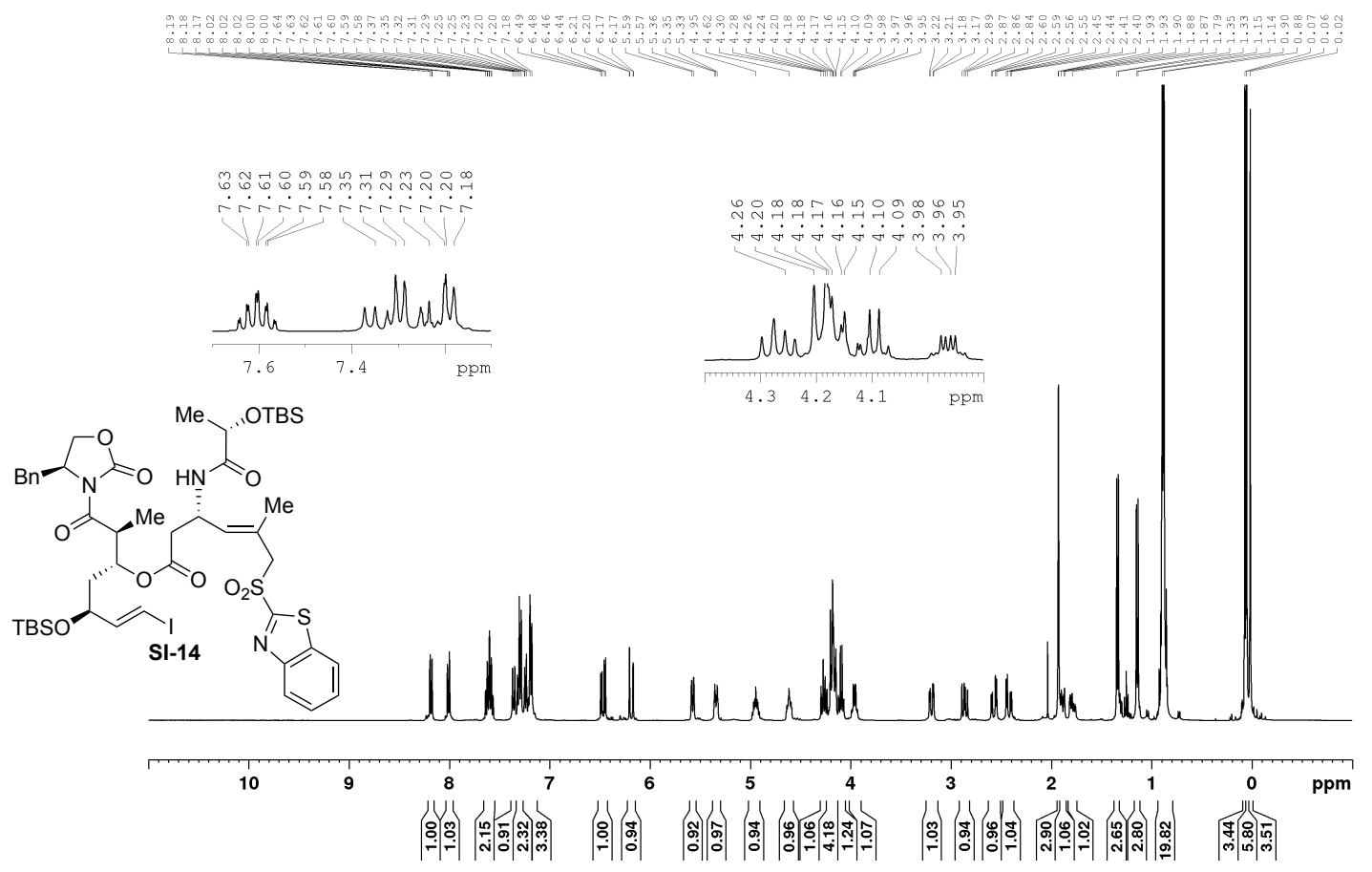

$\left({ }^{13} \mathrm{C} \mathrm{NMR}, \mathrm{CDCl}_{3}, 100 \mathrm{MHz}\right)$

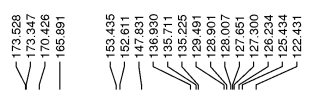

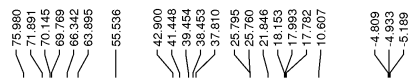

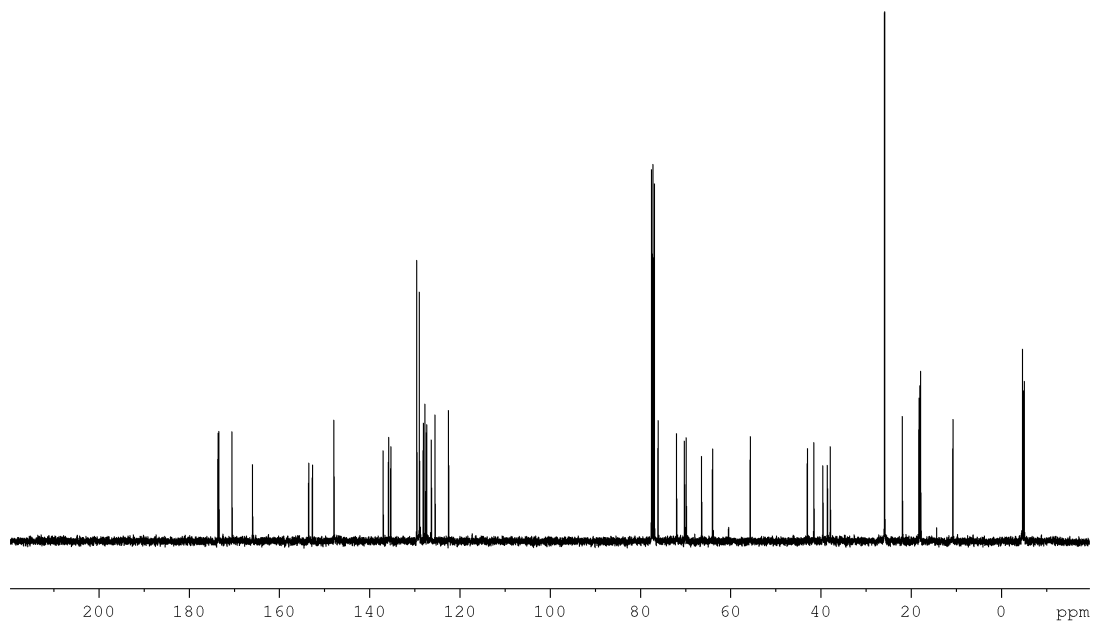


$\left({ }^{1} \mathrm{H} \mathrm{NMR}, \mathrm{CDCl}_{3}, 400 \mathrm{MHz}\right)$

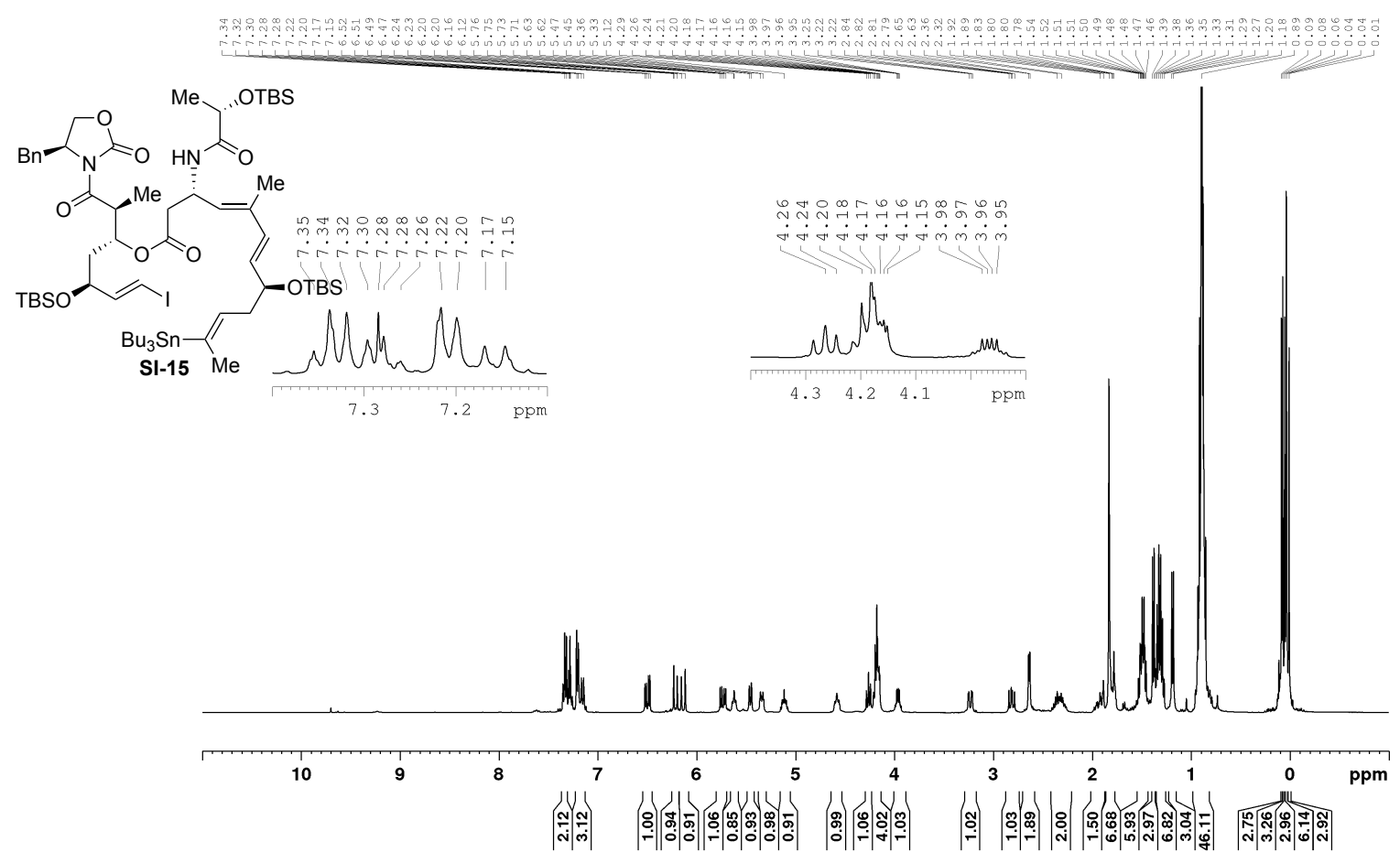

$\left({ }^{13} \mathrm{C} \mathrm{NMR}, \mathrm{CDCl}_{3}, 100 \mathrm{MHz}\right)$

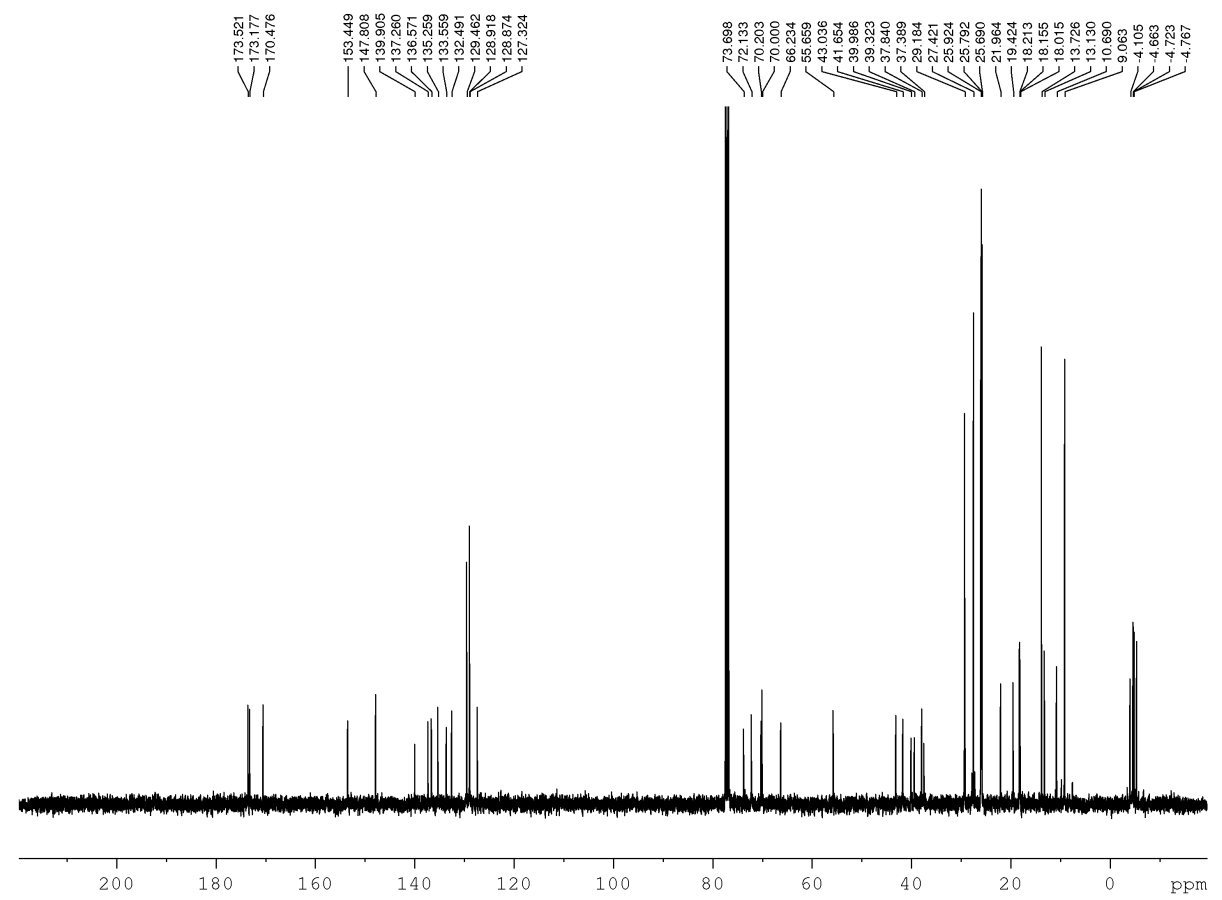


( $\left.{ }^{1} \mathrm{H} \mathrm{NMR}, \mathrm{CDCl}_{3}, 400 \mathrm{MHz}\right)$

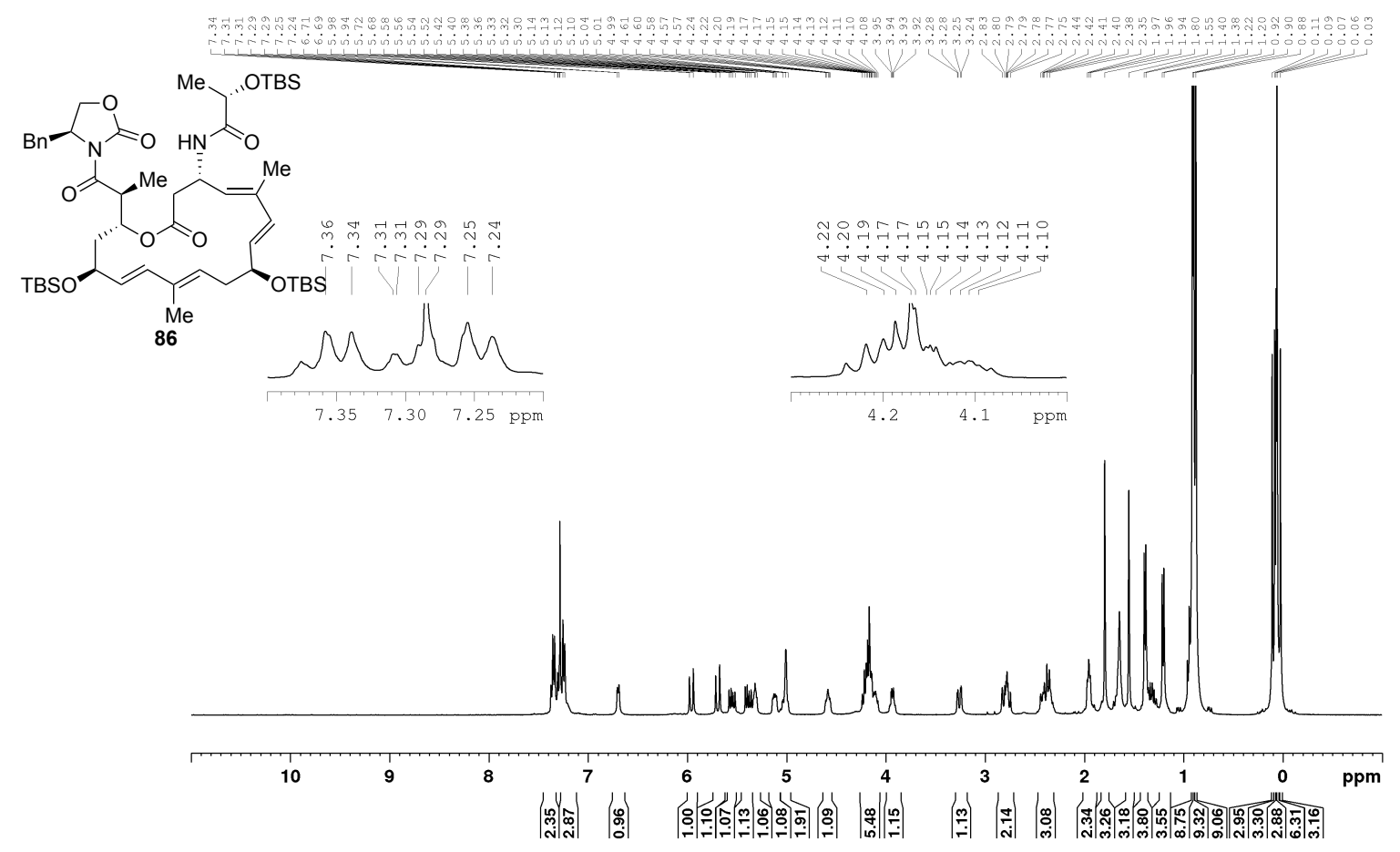

$\left({ }^{13} \mathrm{C}\right.$ NMR, $\left.\mathrm{CDCl}_{3}, 100 \mathrm{MHz}\right)$

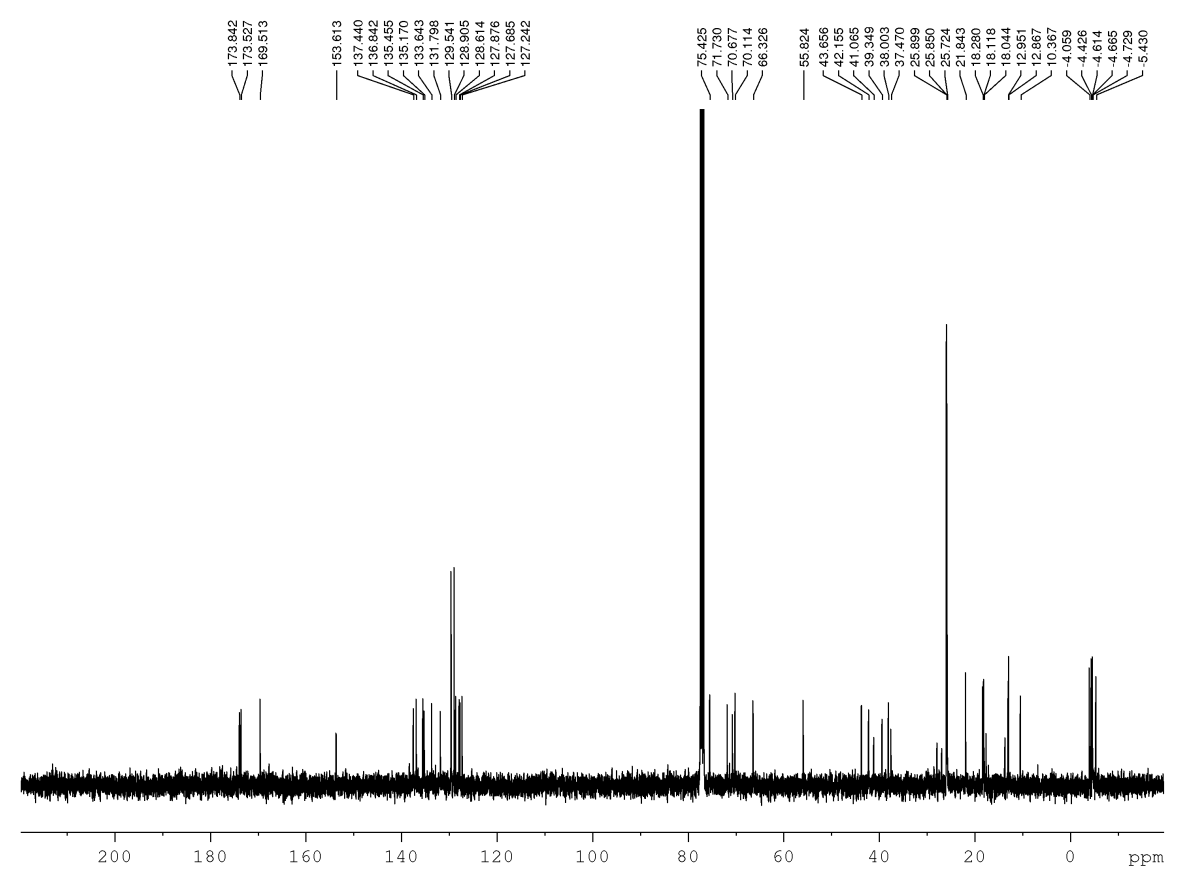


$\left({ }^{1} \mathrm{H} \mathrm{NMR}, \mathrm{CDCl}_{3}, 400 \mathrm{MHz}\right)$

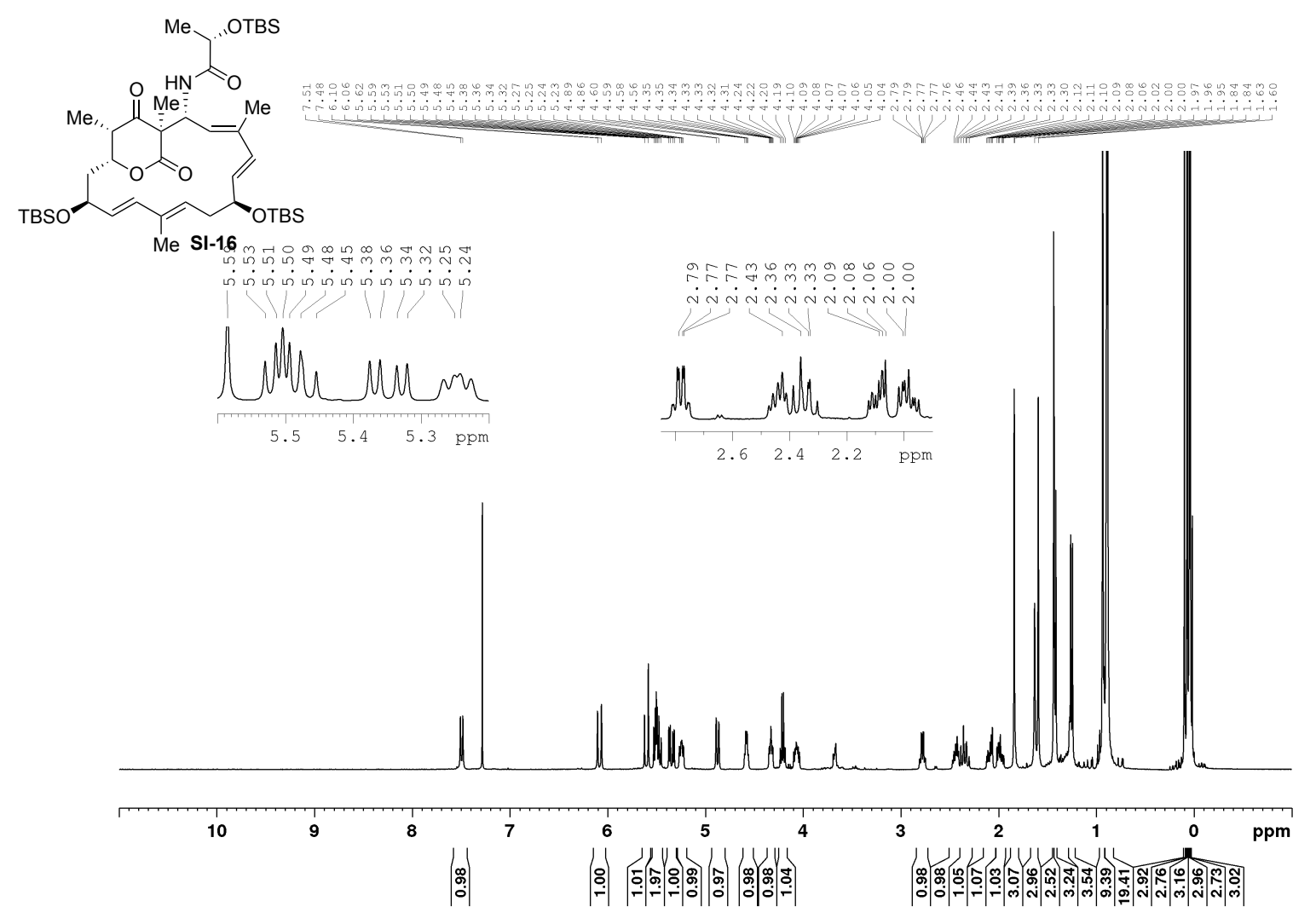

$\left({ }^{13} \mathrm{C} \mathrm{NMR}, \mathrm{CDCl}_{3}, 100 \mathrm{MHz}\right)$

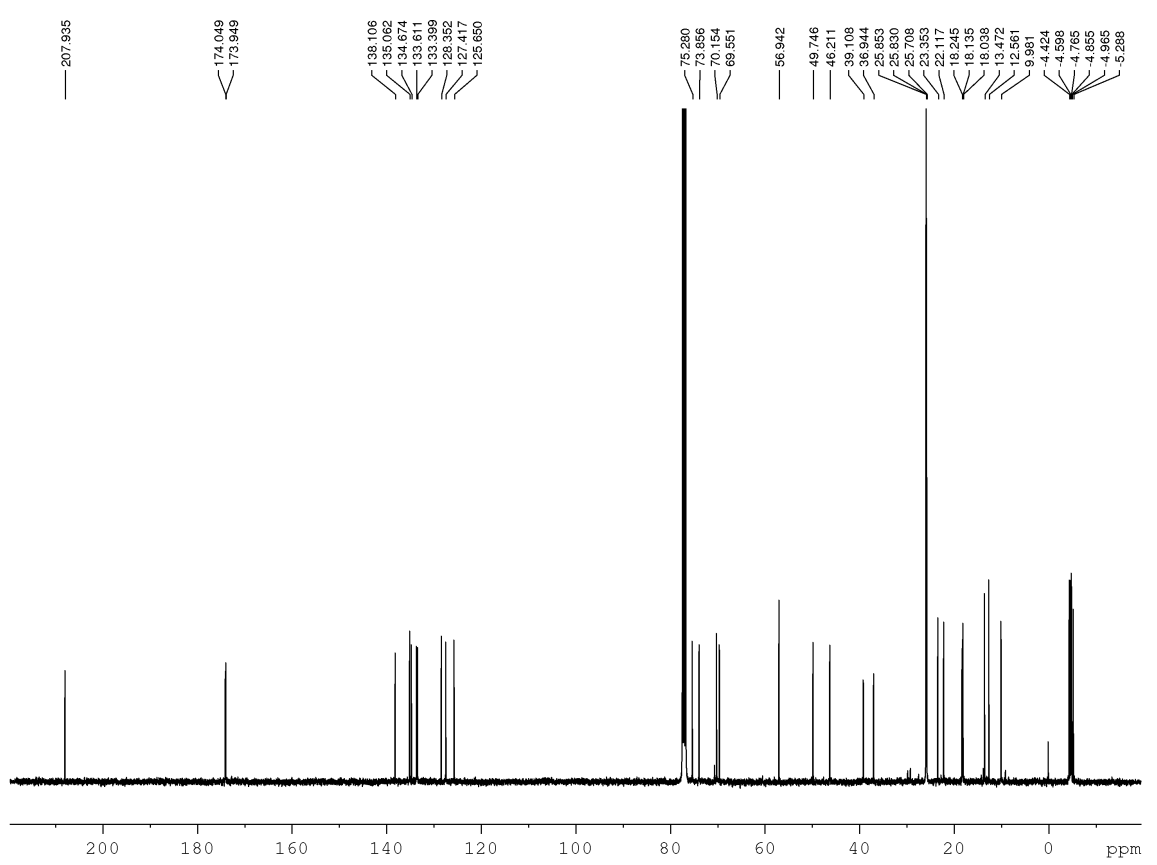


$\left({ }^{1} \mathrm{H} \mathrm{NMR}, \mathrm{CD}_{3} \mathrm{OD}, 400 \mathrm{MHz}\right)$

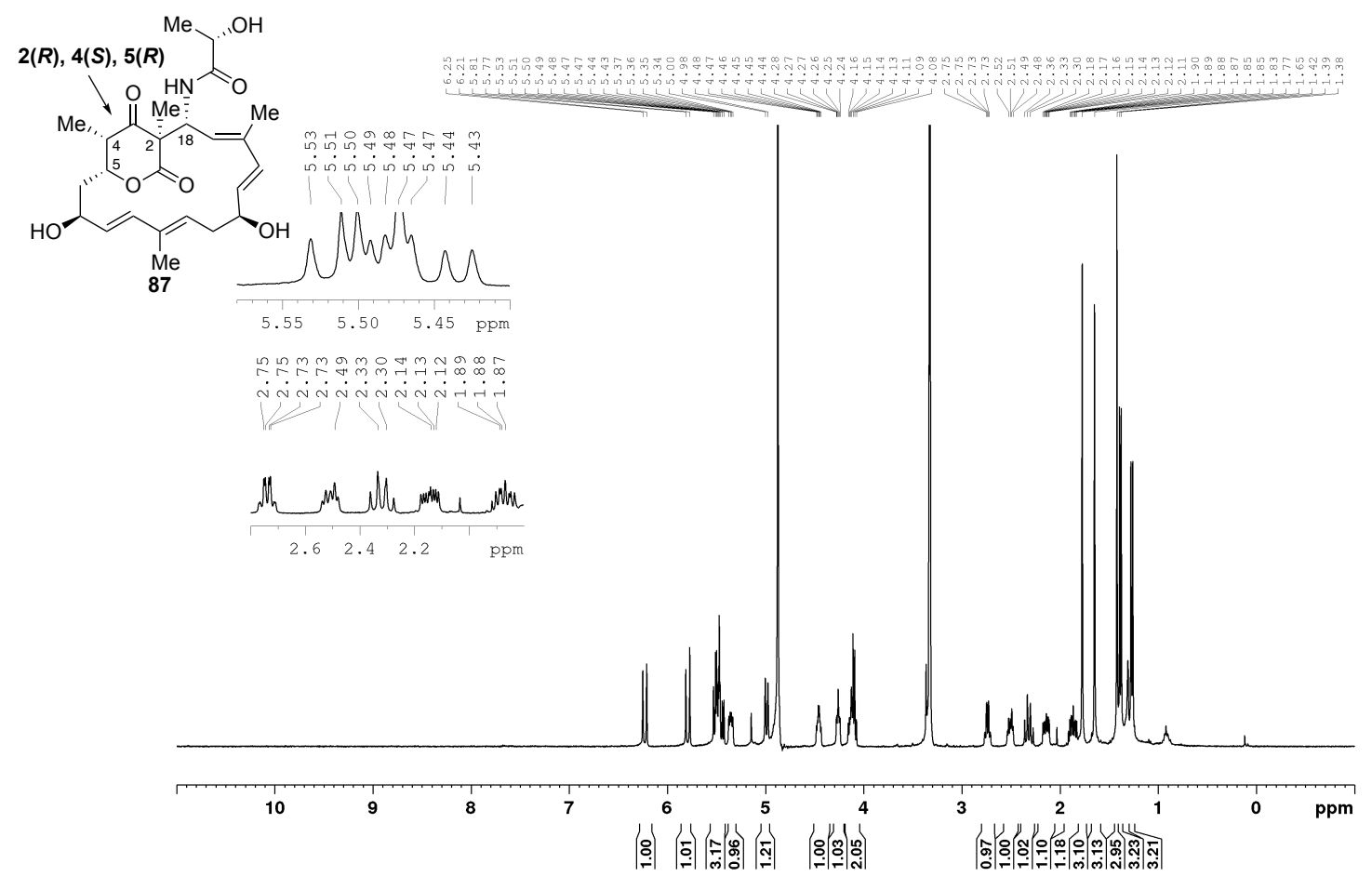

$\left({ }^{13} \mathrm{C} \mathrm{NMR}, \mathrm{CD}_{3} \mathrm{OD}, 100 \mathrm{MHz}\right)$
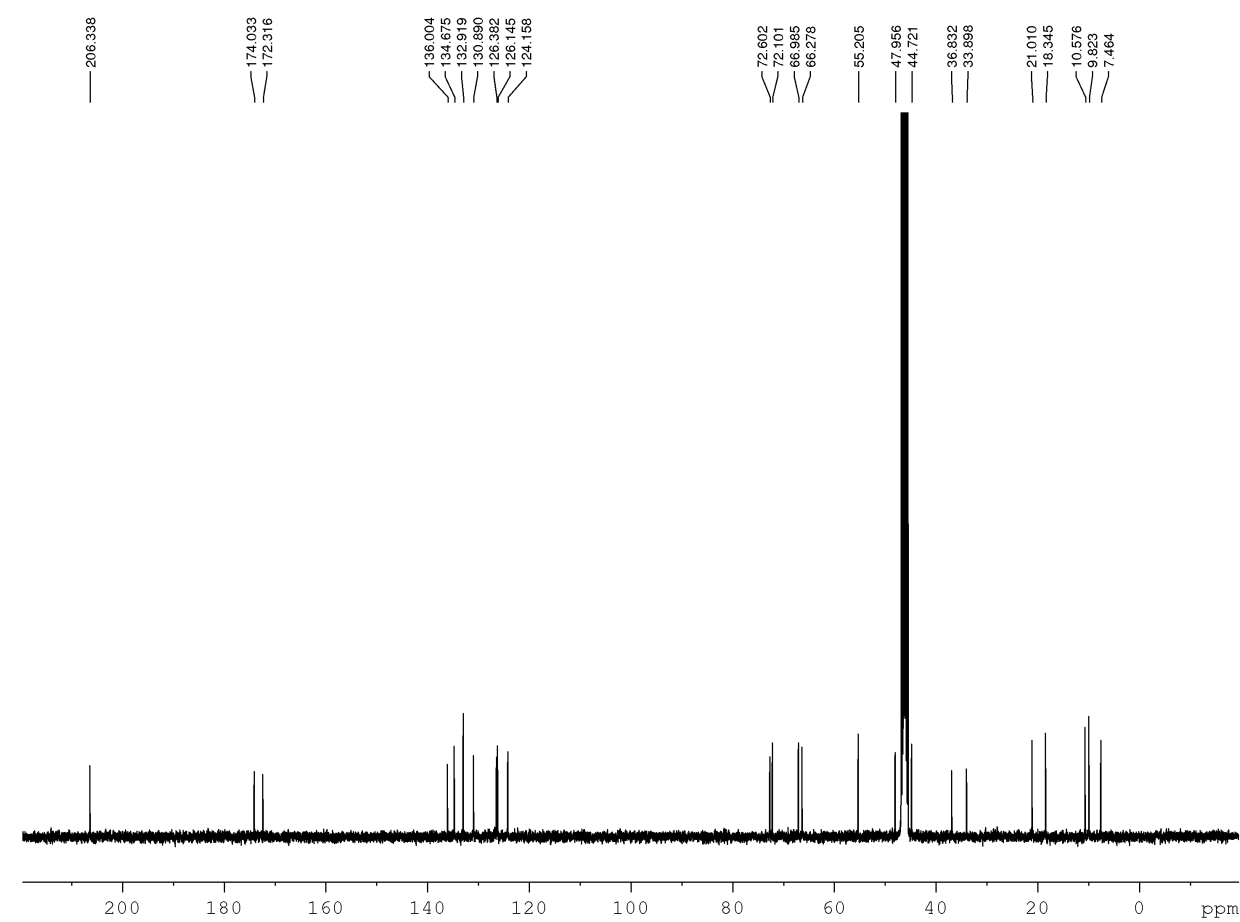
(COSY, $\left.\mathrm{CD}_{3} \mathrm{OD}, 400 \mathrm{MHz}\right)$

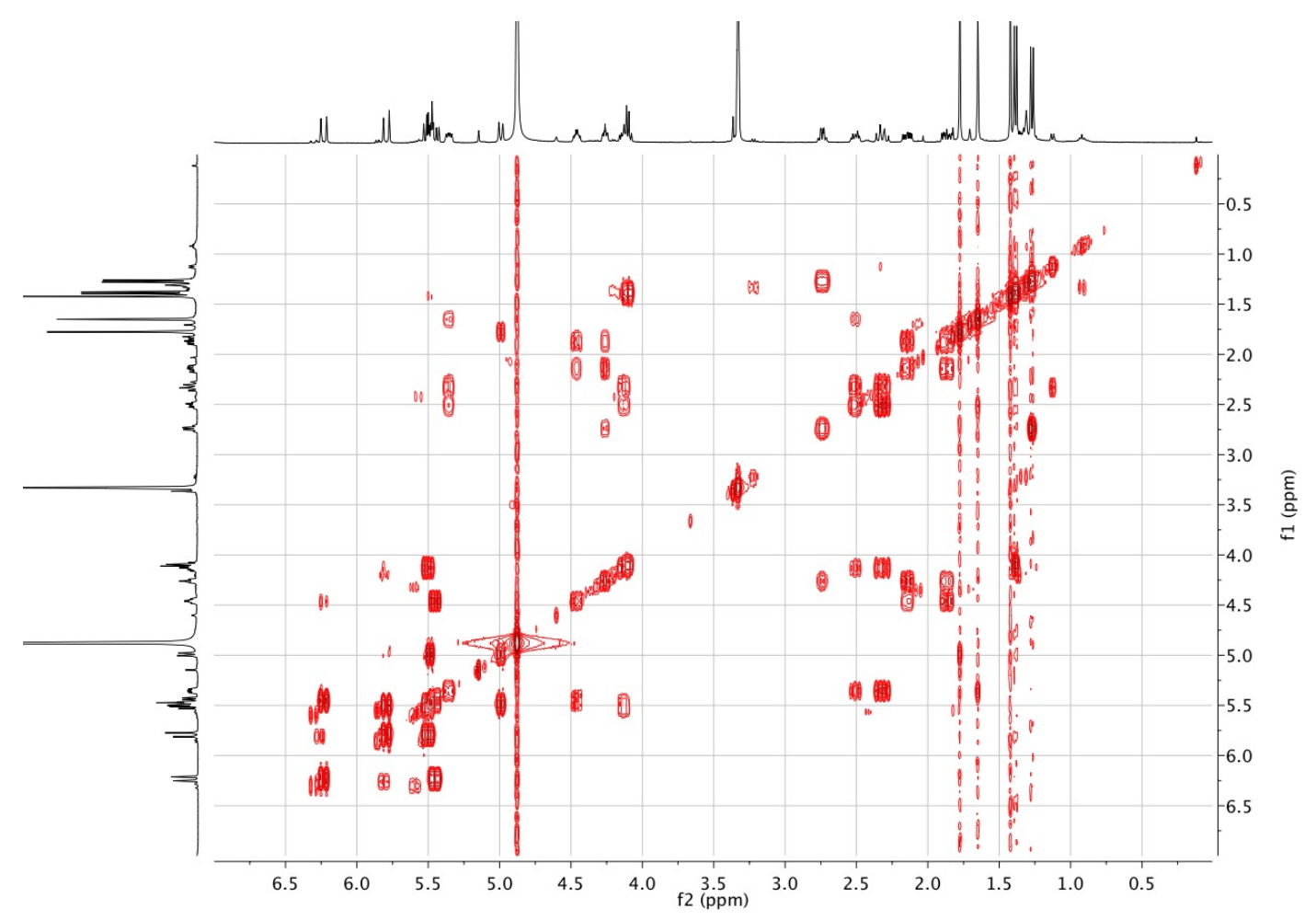

(NOESY, CD ${ }_{3} \mathrm{OD}, 400 \mathrm{MHz}$ )

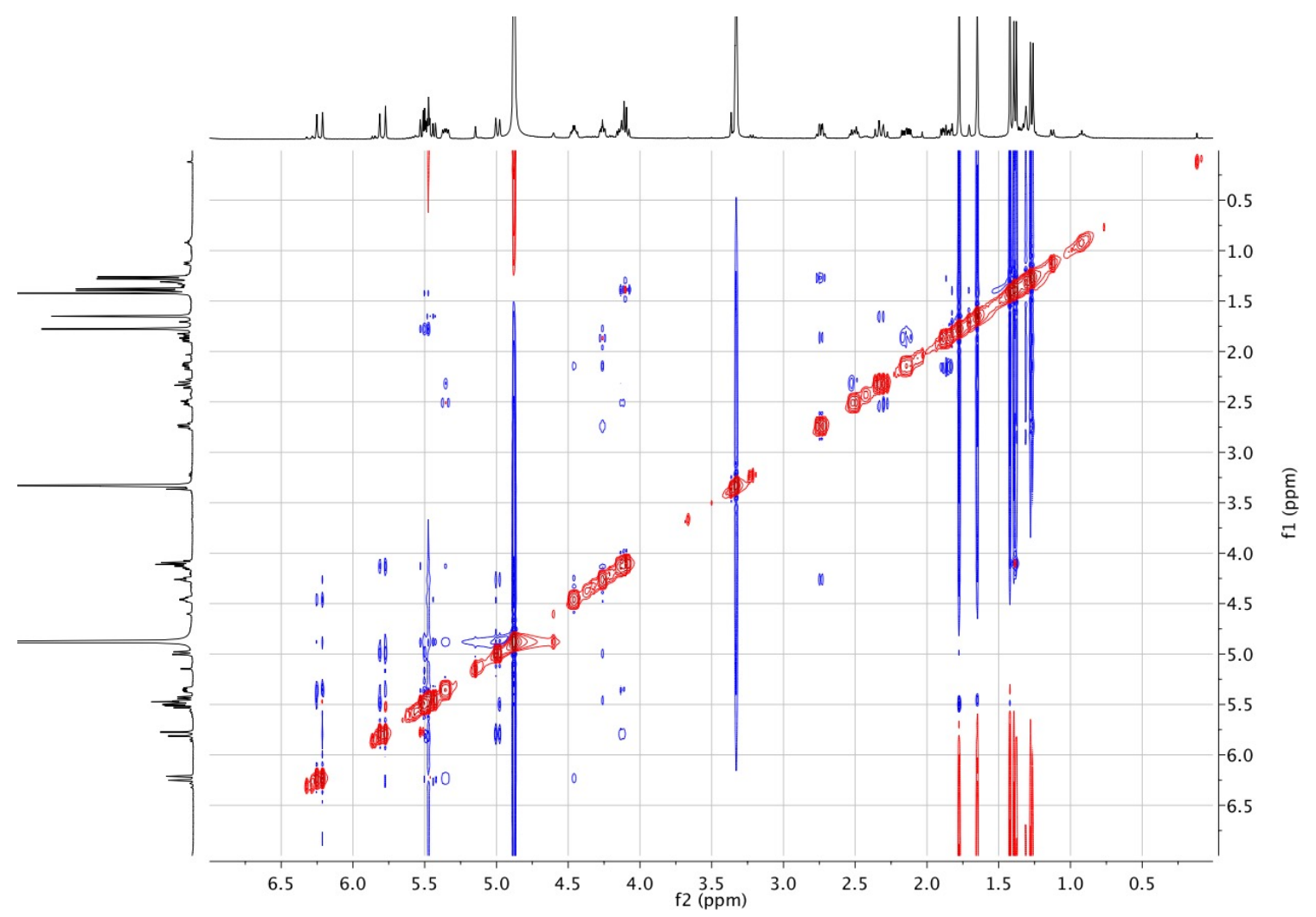


( $\left.{ }^{1} \mathrm{H} \mathrm{NMR}, \mathrm{CDCl}_{3}, 400 \mathrm{MHz}\right)$

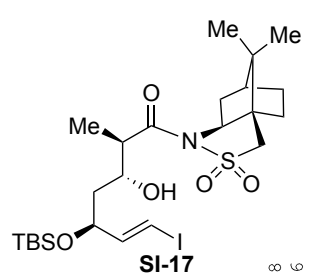

SI-17
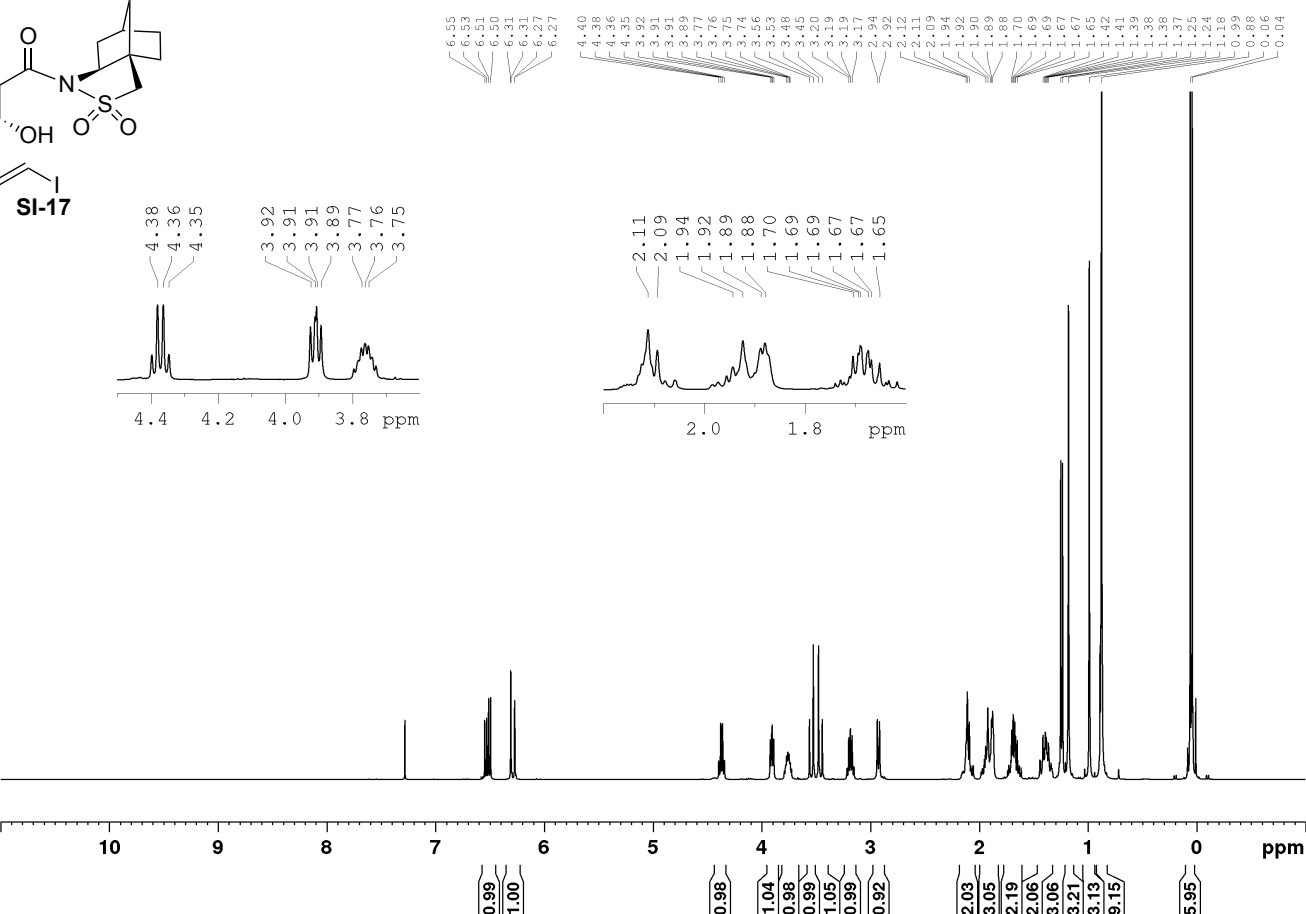

$\left({ }^{13} \mathrm{C} \mathrm{NMR}, \mathrm{CDCl}_{3}, 100 \mathrm{MHz}\right)$
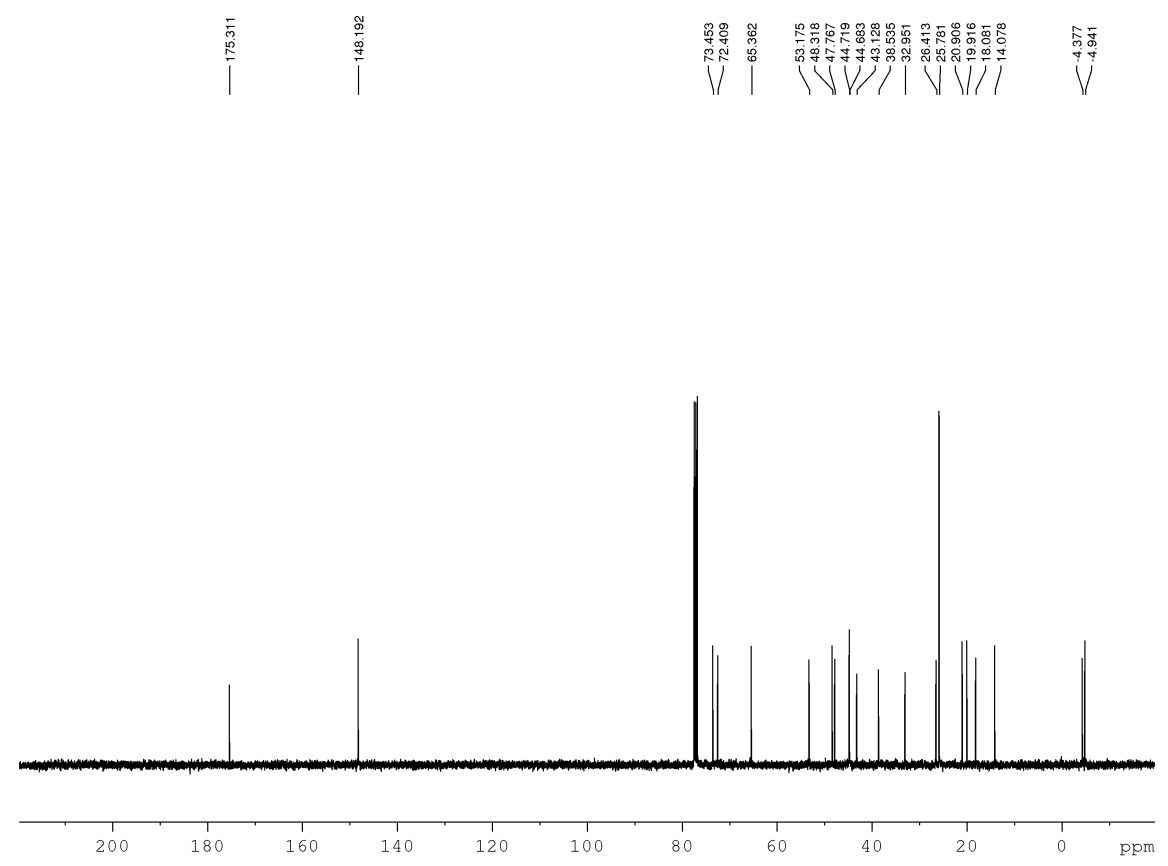
$\left({ }^{1} \mathrm{H} \mathrm{NMR}, \mathrm{CDCl}_{3}, 400 \mathrm{MHz}\right)$
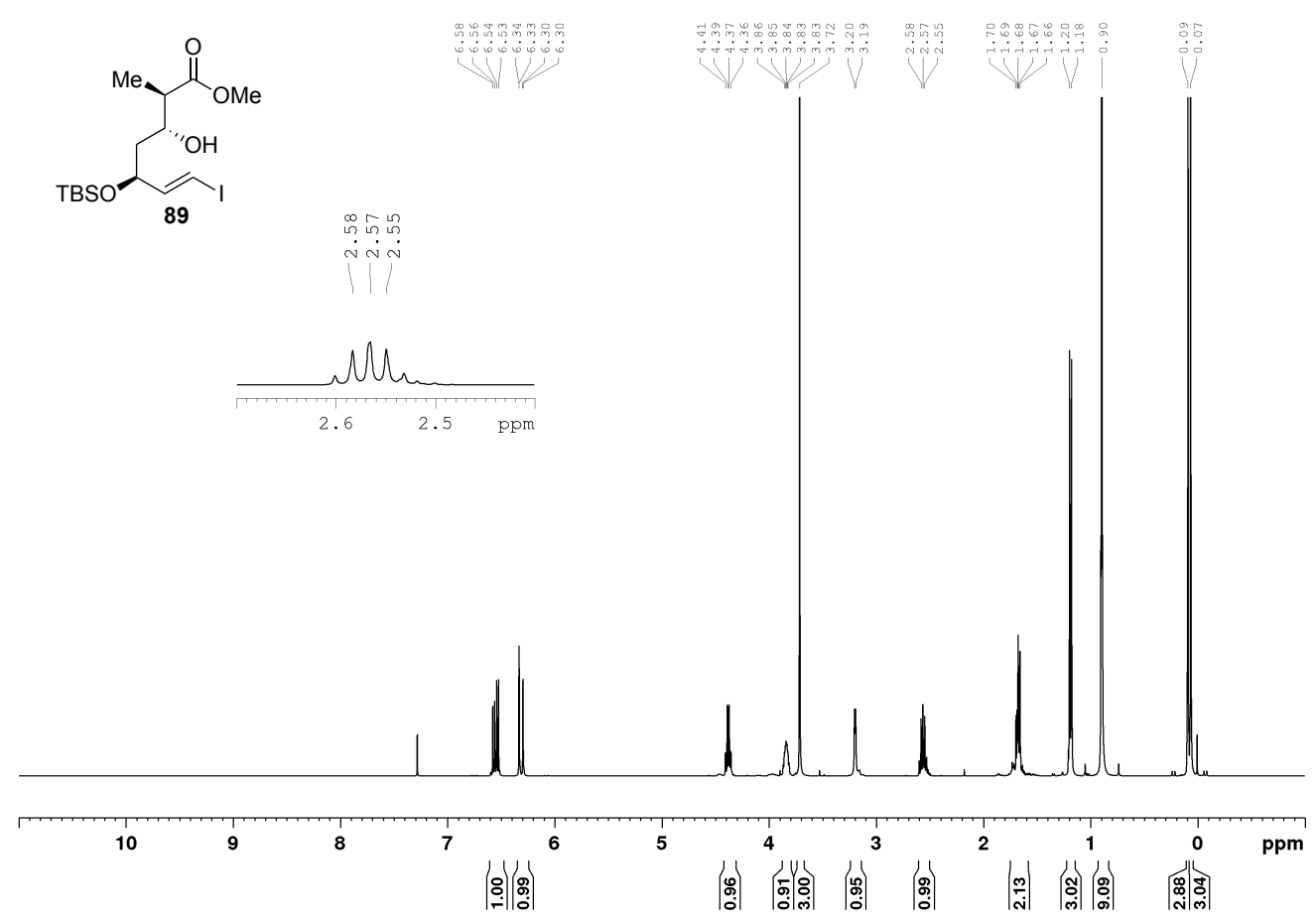

$\left({ }^{13} \mathrm{C} \mathrm{NMR}, \mathrm{CDCl}_{3}, 100 \mathrm{MHz}\right)$

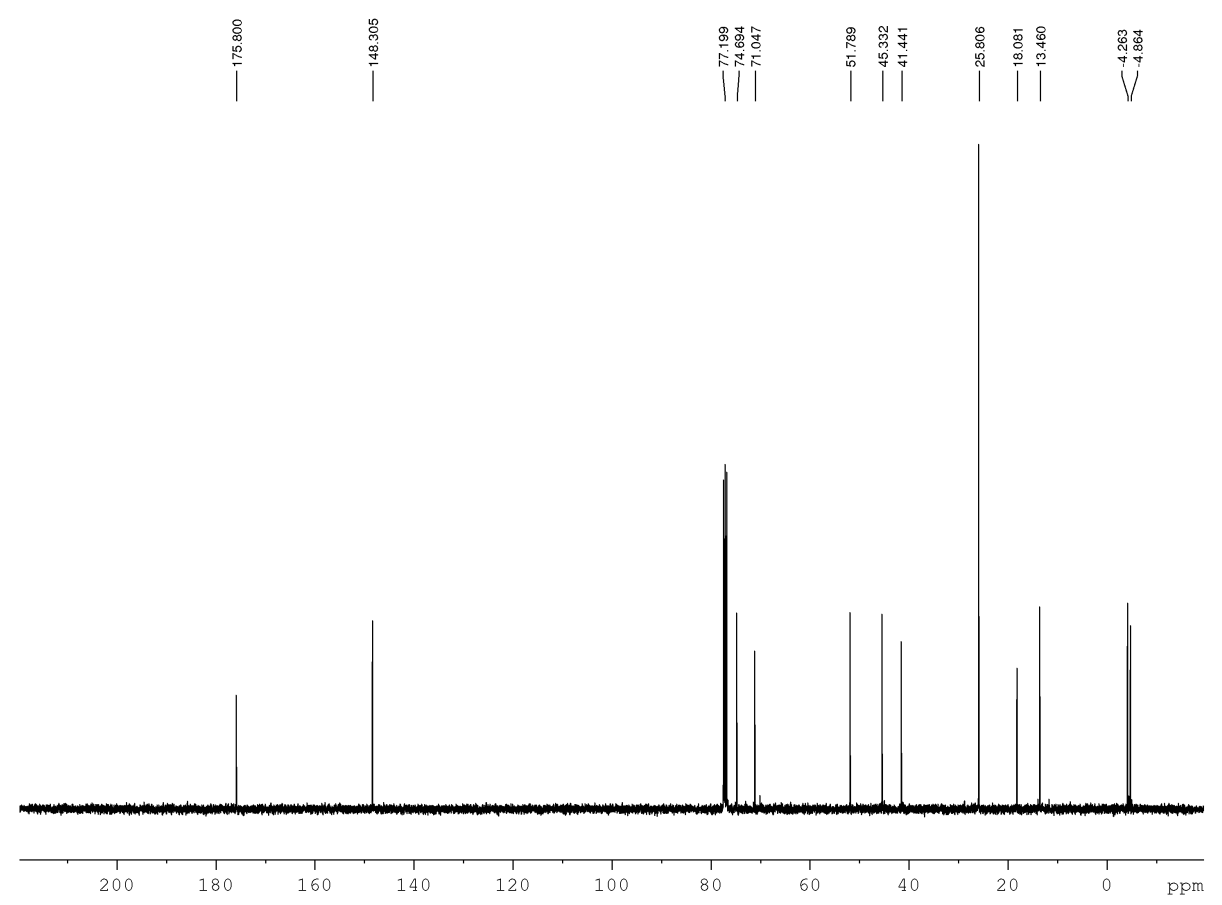


( $\left.{ }^{1} \mathrm{H} \mathrm{NMR}, \mathrm{CDCl}_{3}, 400 \mathrm{MHz}\right)$

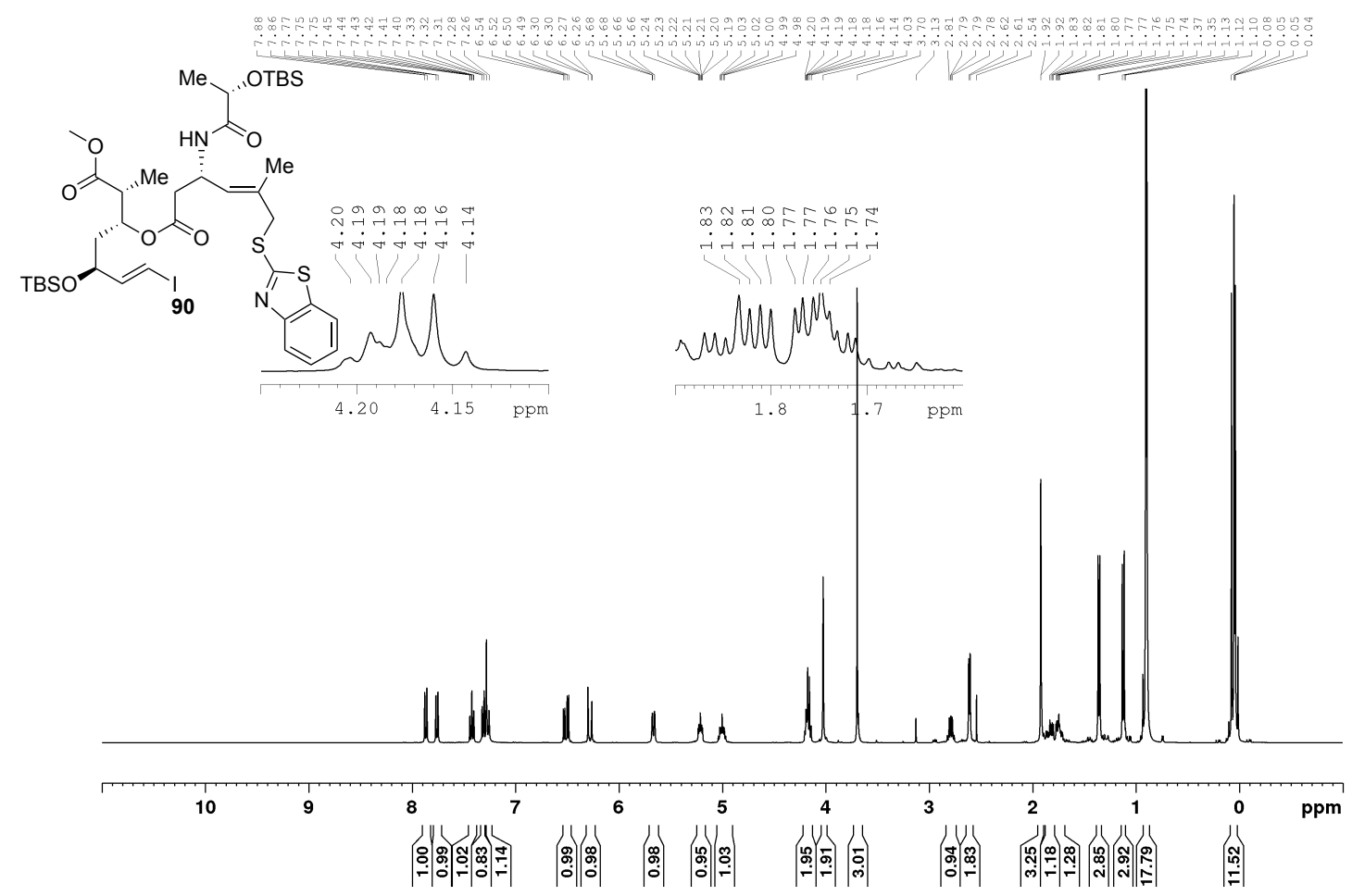

$\left({ }^{13} \mathrm{C} \mathrm{NMR}, \mathrm{CDCl}_{3}, 100 \mathrm{MHz}\right)$

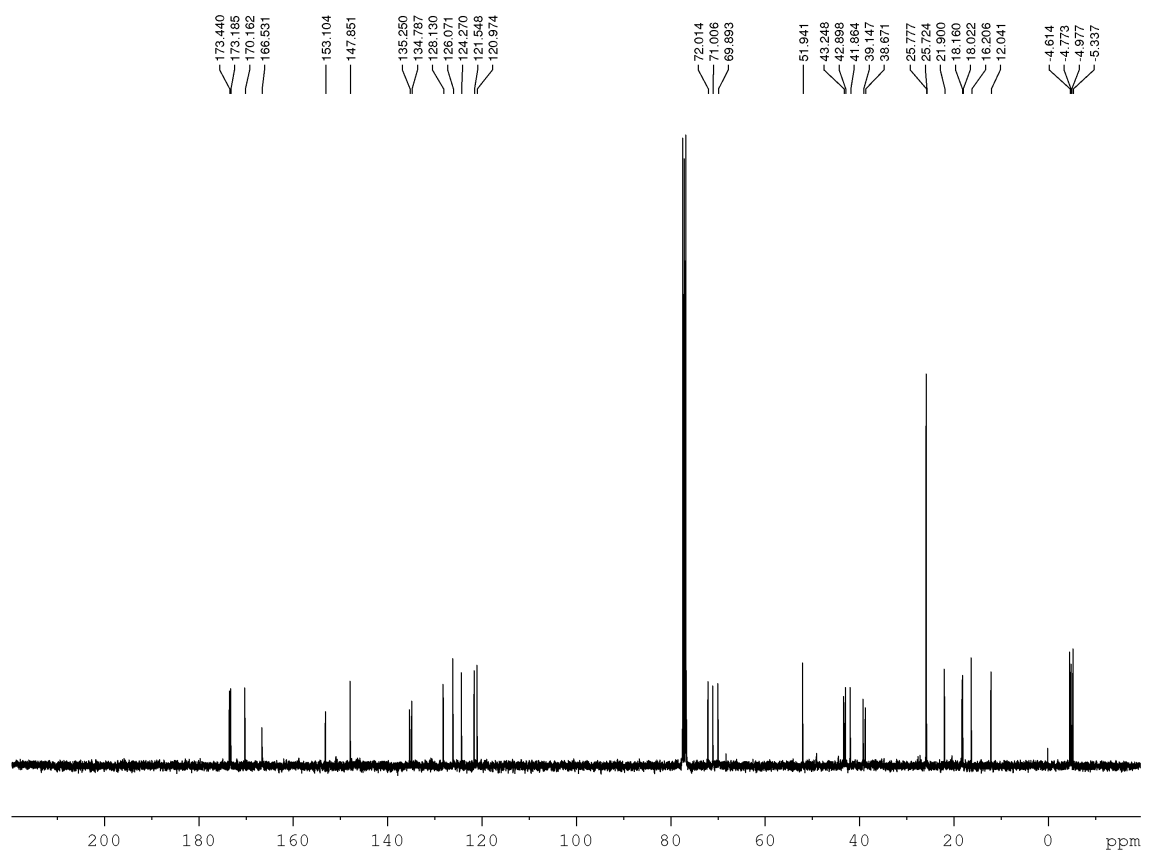


( $\left.{ }^{1} \mathrm{H} \mathrm{NMR}, \mathrm{CDCl}_{3}, 400 \mathrm{MHz}\right)$

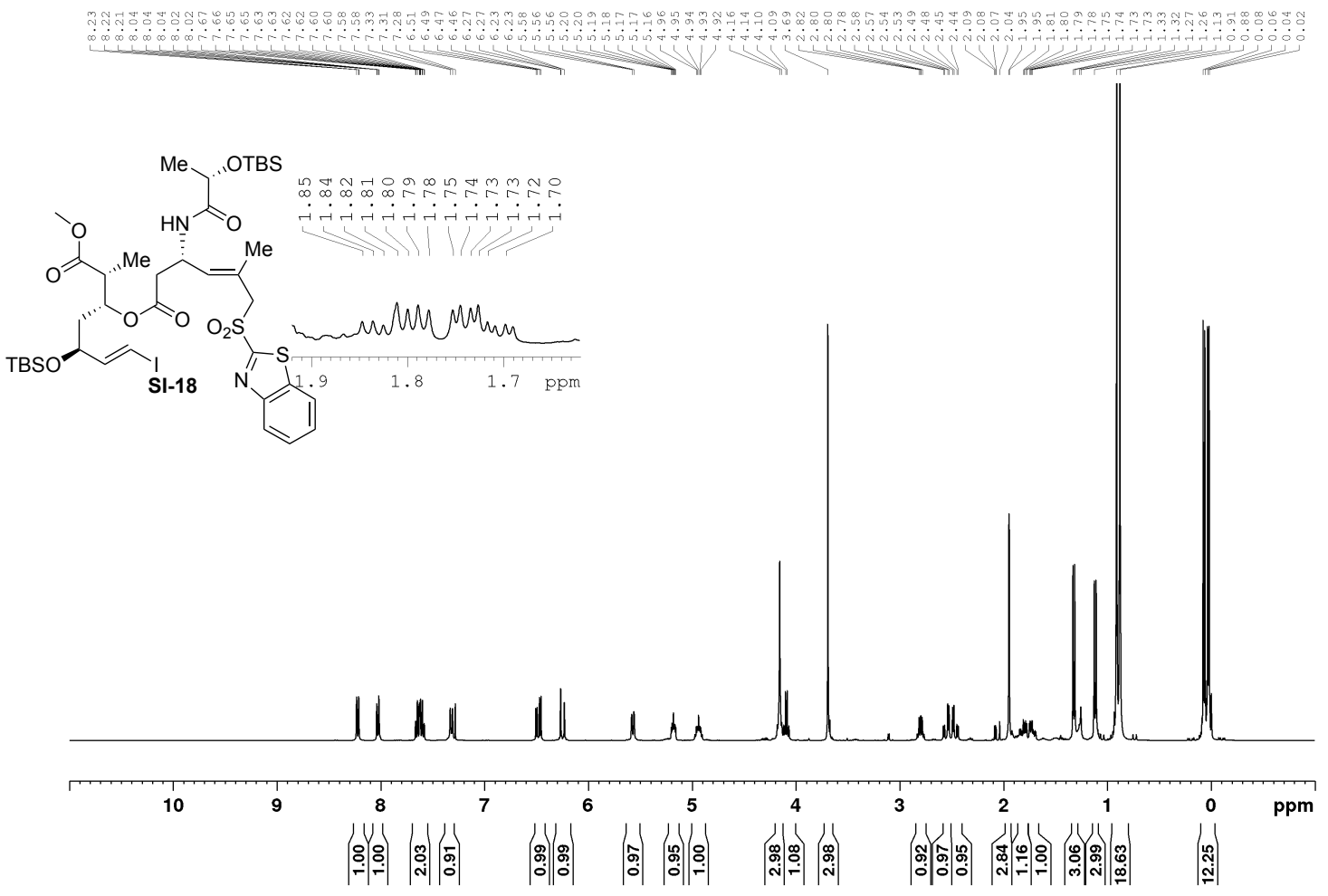

$\left({ }^{13} \mathrm{C} \mathrm{NMR}, \mathrm{CDCl}_{3}, 100 \mathrm{MHz}\right)$

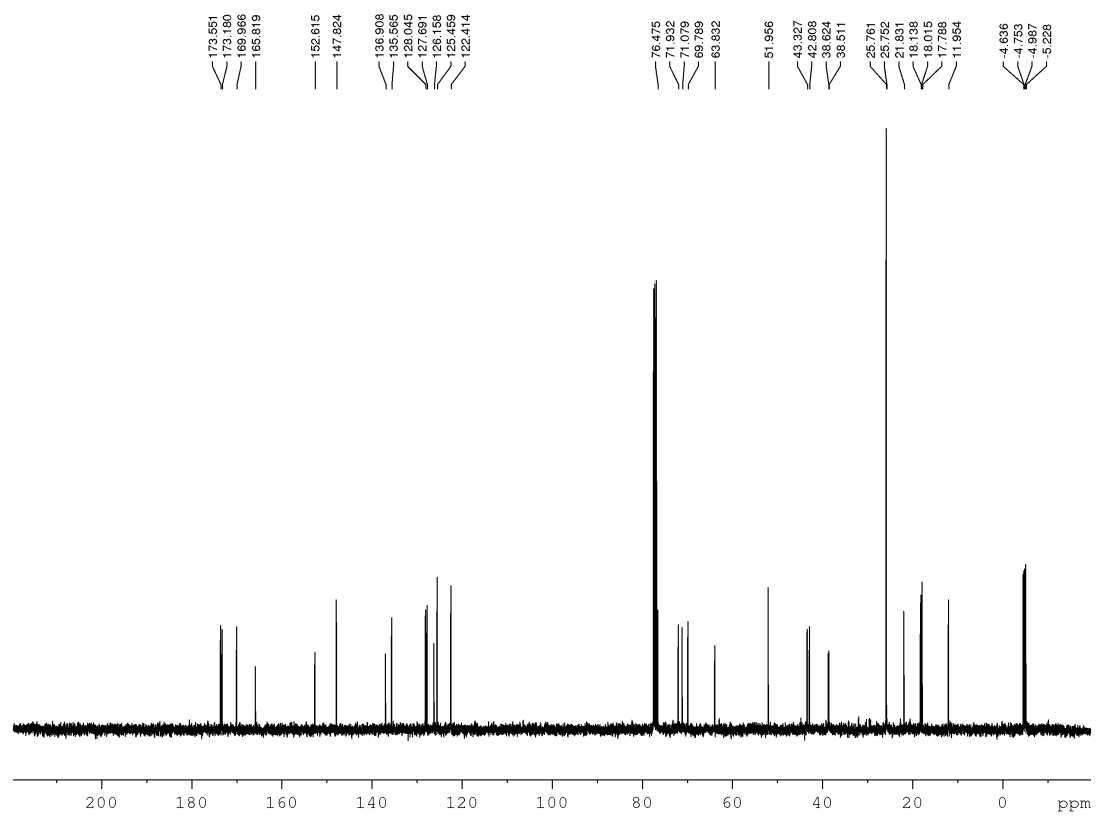


( $\left.{ }^{1} \mathrm{H} \mathrm{NMR}, \mathrm{CDCl}_{3}, 400 \mathrm{MHz}\right)$

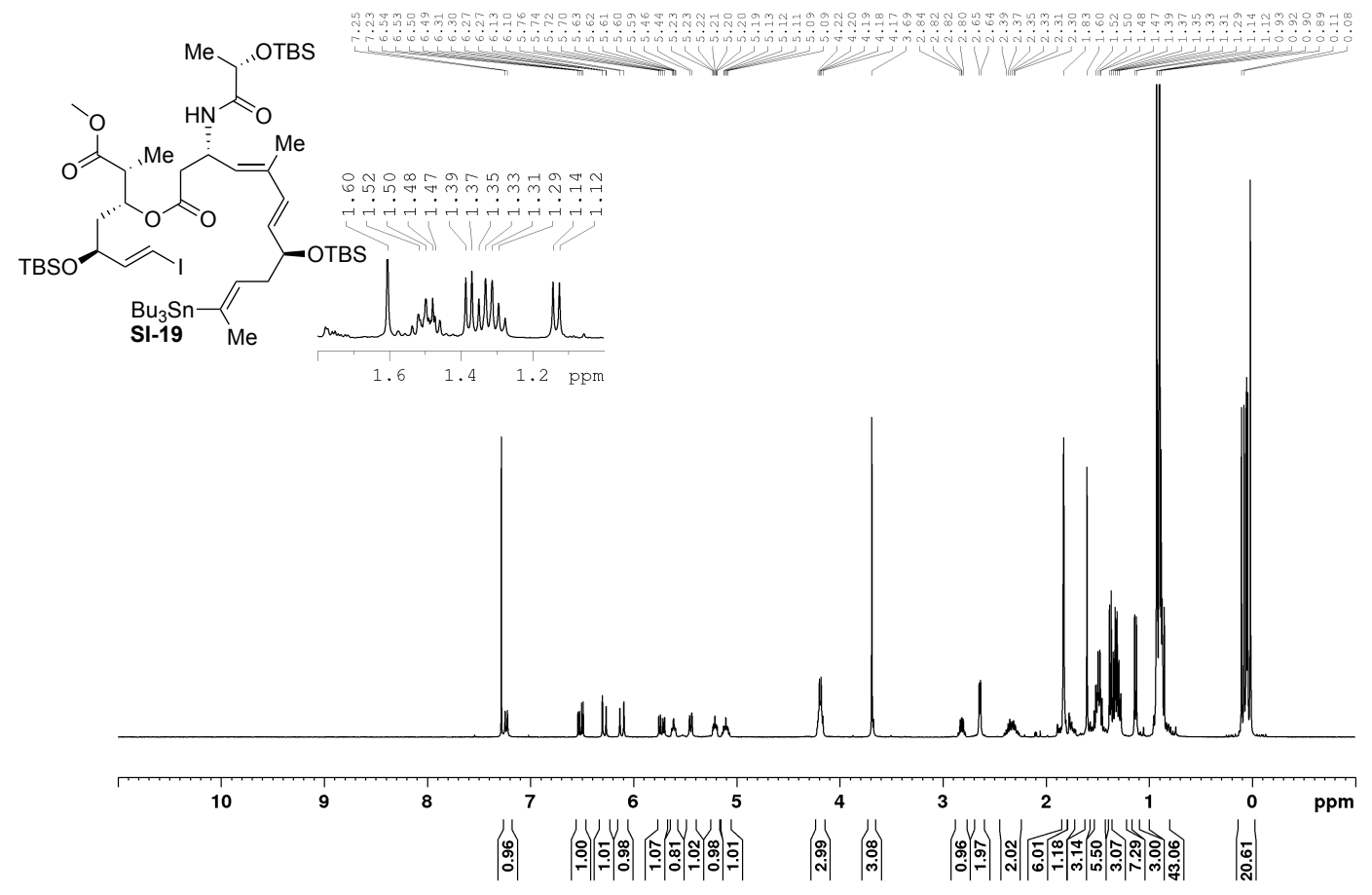

$\left({ }^{13} \mathrm{C}\right.$ NMR, $\left.\mathrm{CDCl}_{3}, 100 \mathrm{MHz}\right)$

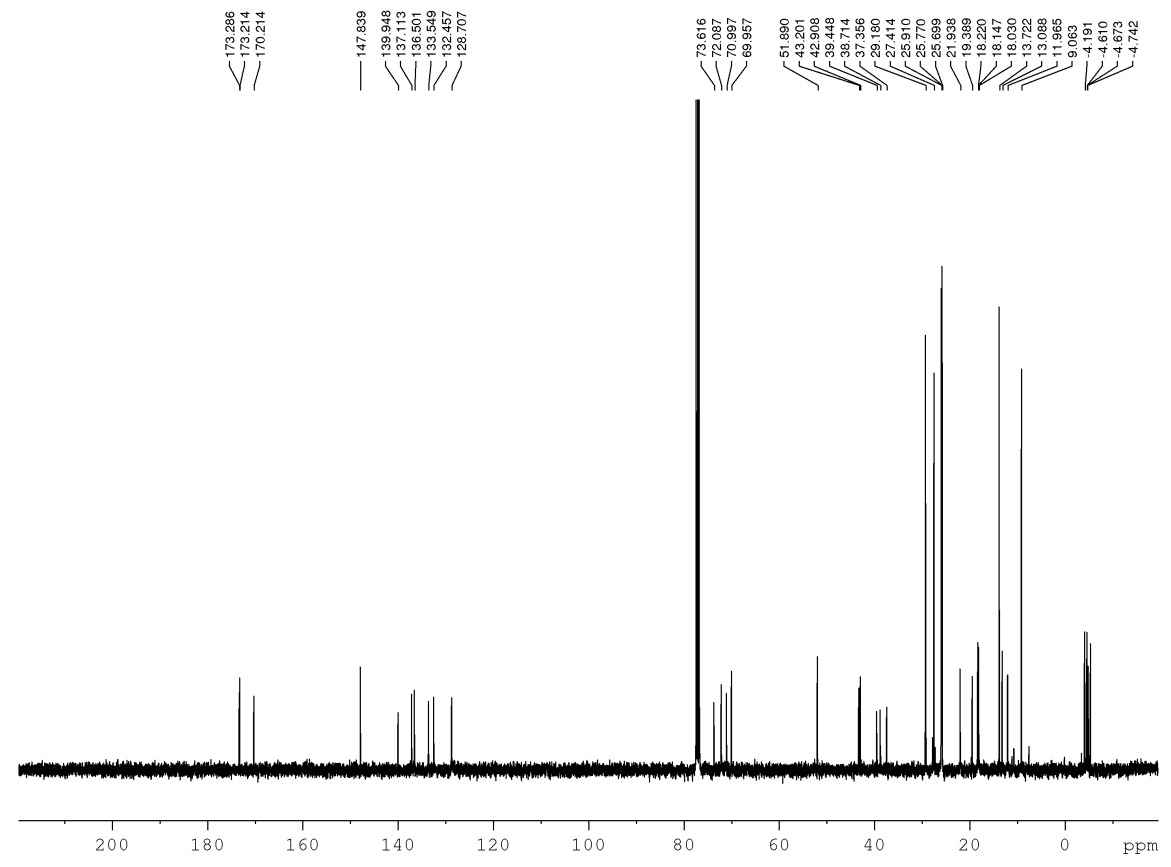


( $\left.{ }^{1} \mathrm{H} \mathrm{NMR}, \mathrm{CDCl}_{3}, 400 \mathrm{MHz}\right)$

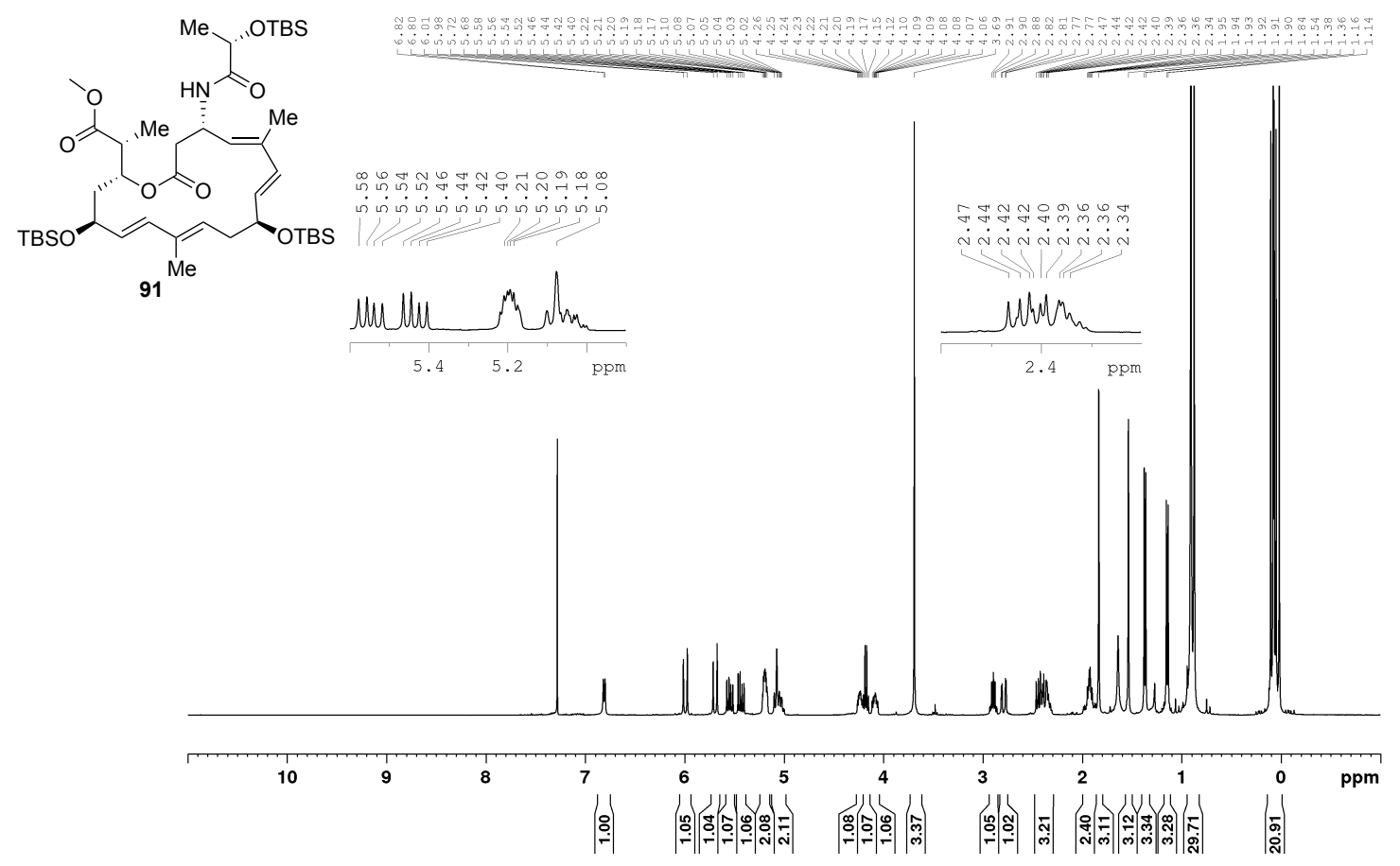

$\left({ }^{13} \mathrm{C} \mathrm{NMR}, \mathrm{CDCl}_{3}, 100 \mathrm{MHz}\right)$

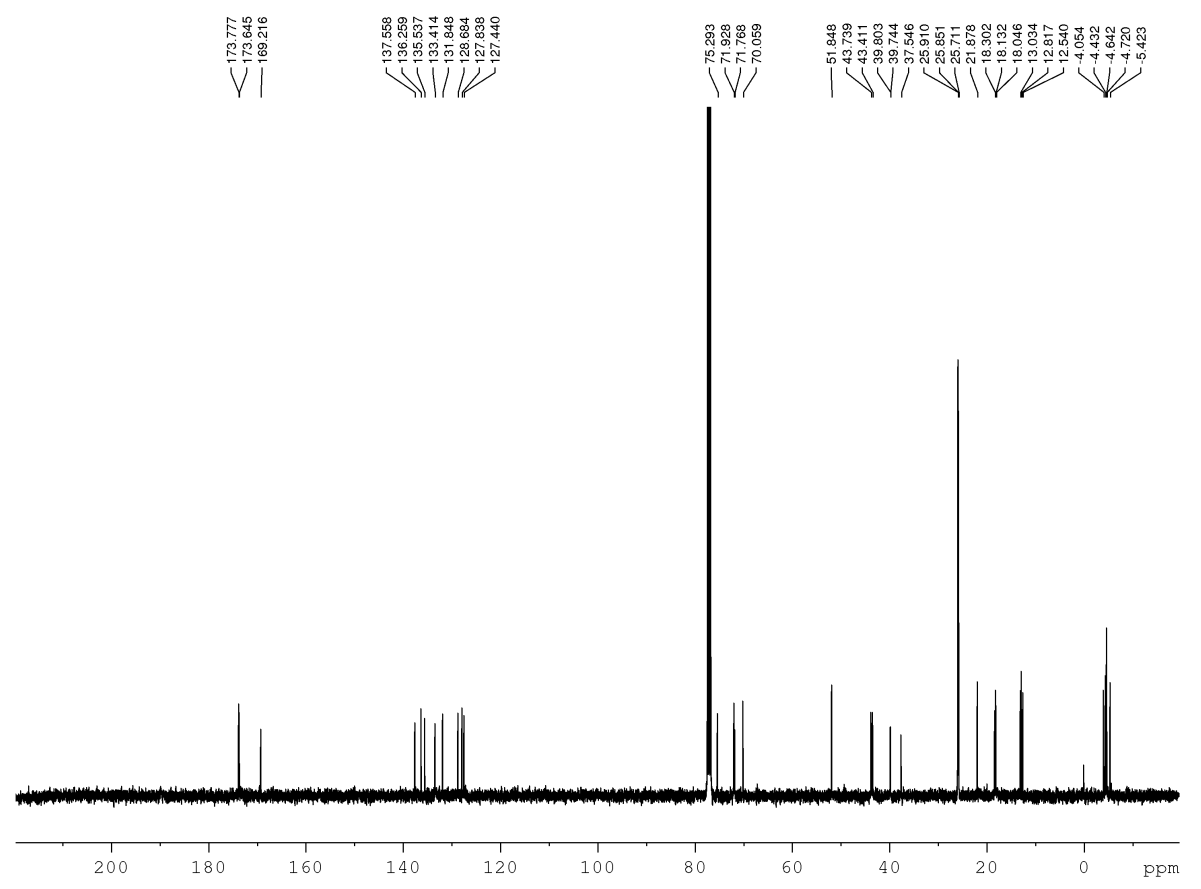


( ${ }^{1} \mathrm{H} \mathrm{NMR}, \mathrm{CDCl}_{3}, 400 \mathrm{MHz}$ )

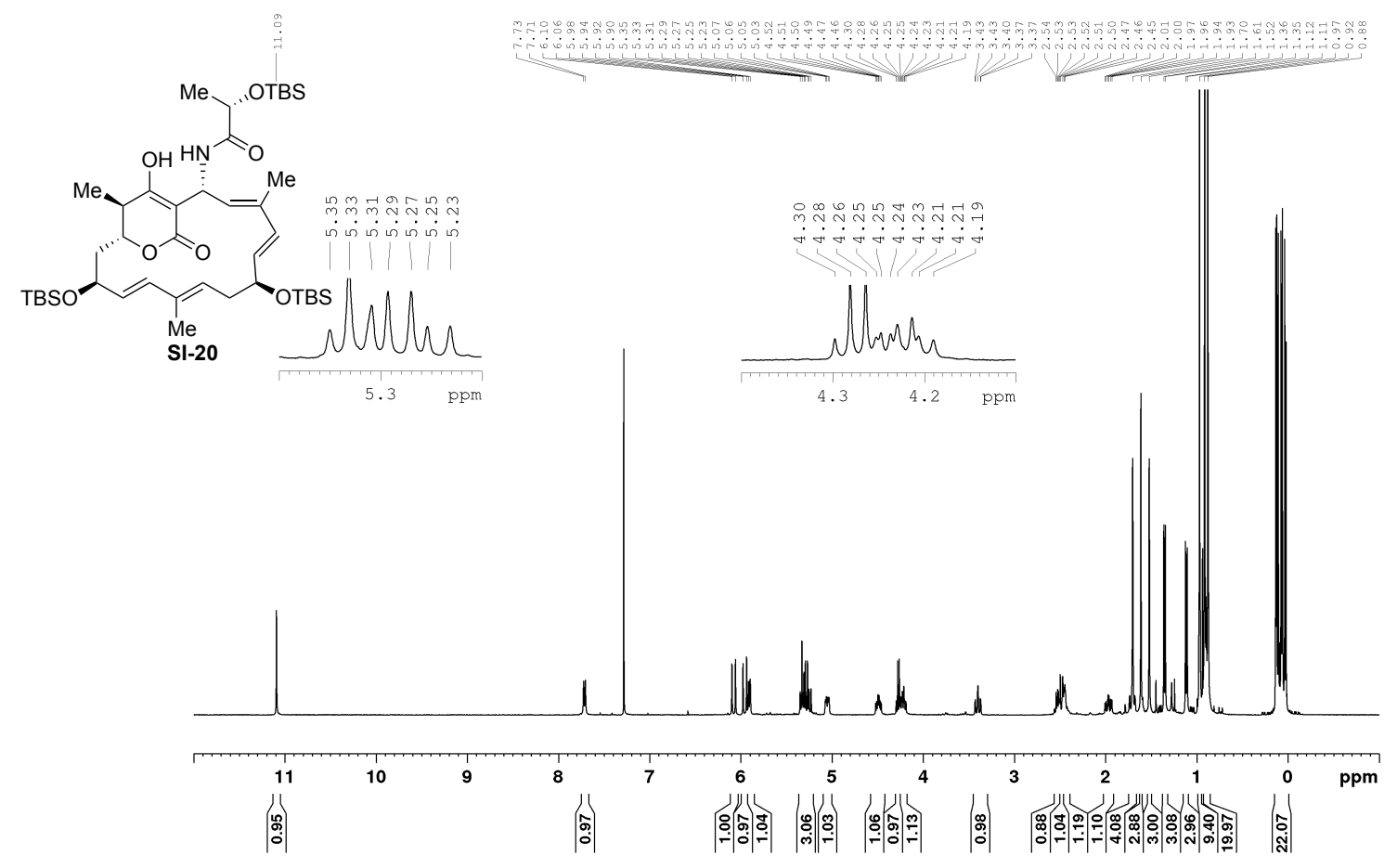

$\left({ }^{13} \mathrm{C} \mathrm{NMR}, \mathrm{CDCl}_{3}, 100 \mathrm{MHz}\right)$

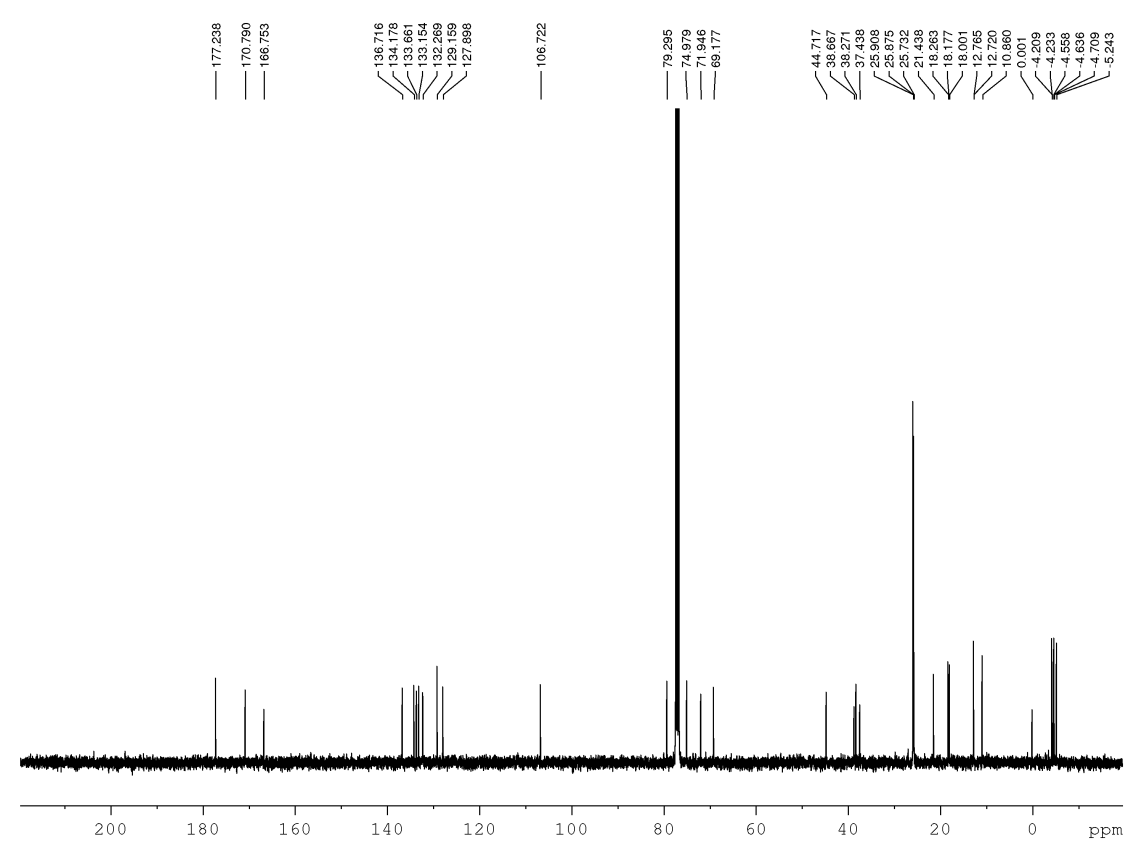


( $\left.{ }^{1} \mathrm{H} \mathrm{NMR}, \mathrm{CDCl}_{3}, 400 \mathrm{MHz}\right)$

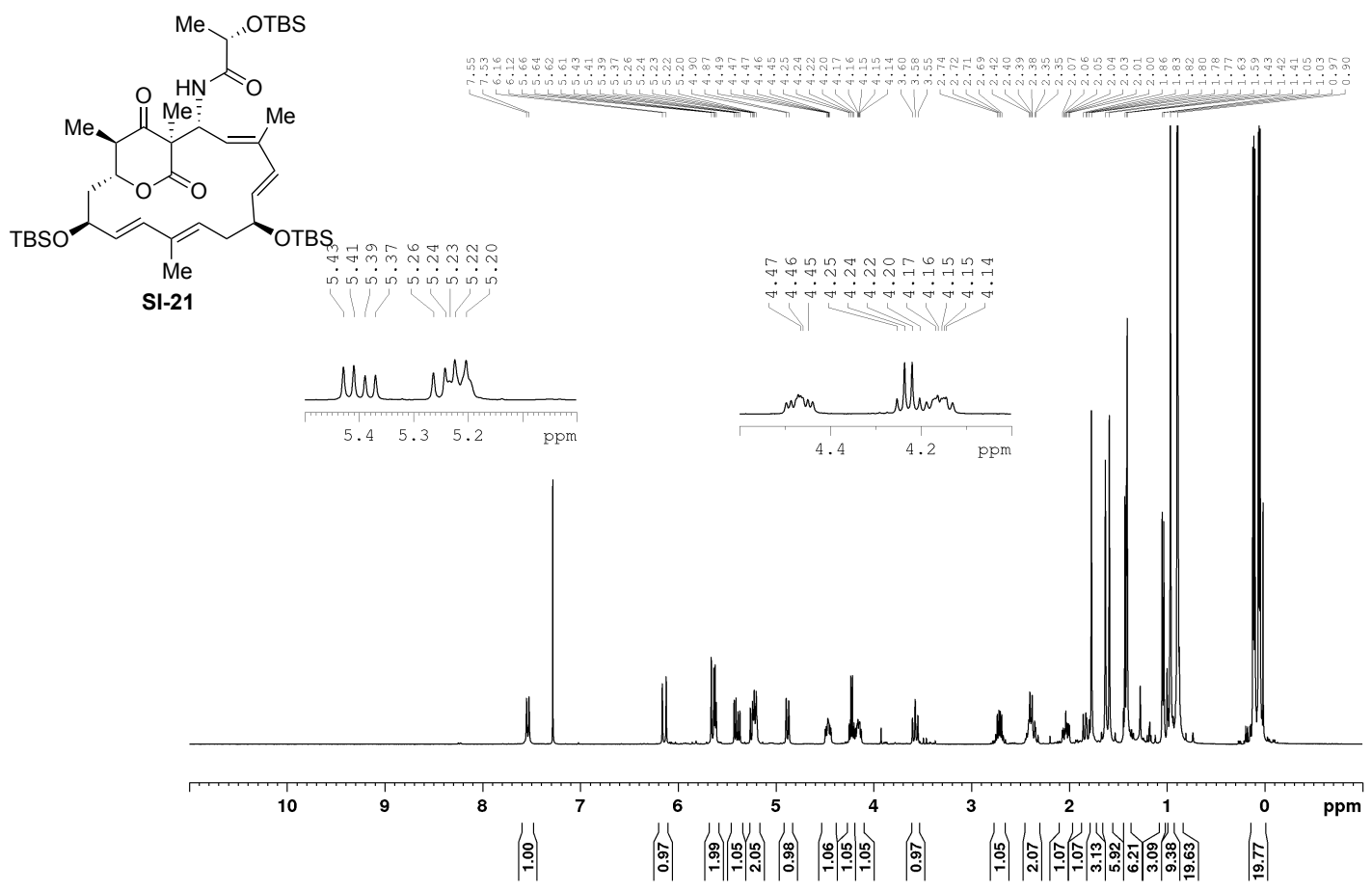

$\left({ }^{13} \mathrm{C} \mathrm{NMR}, \mathrm{CDCl}_{3}, 100 \mathrm{MHz}\right)$

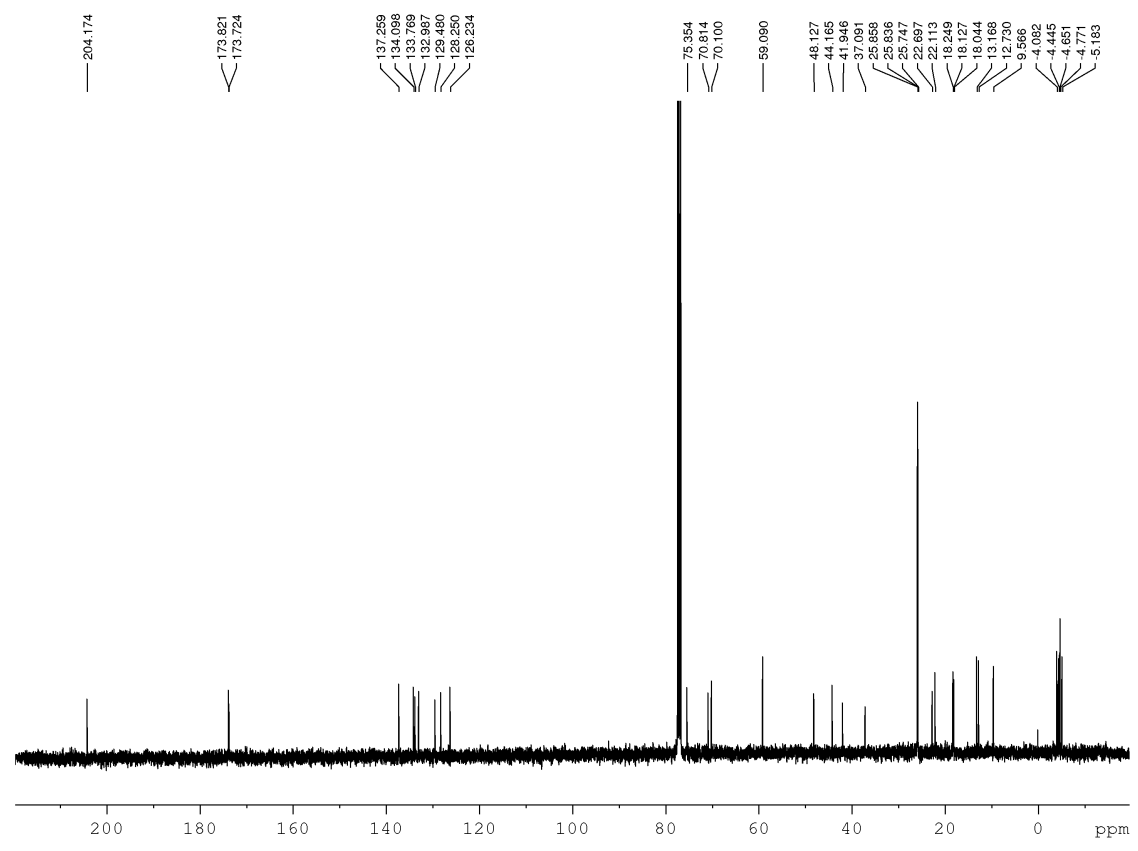


( $\left.{ }^{1} \mathrm{H} \mathrm{NMR}, \mathrm{CD}_{3} \mathrm{OD}, 400 \mathrm{MHz}\right)$

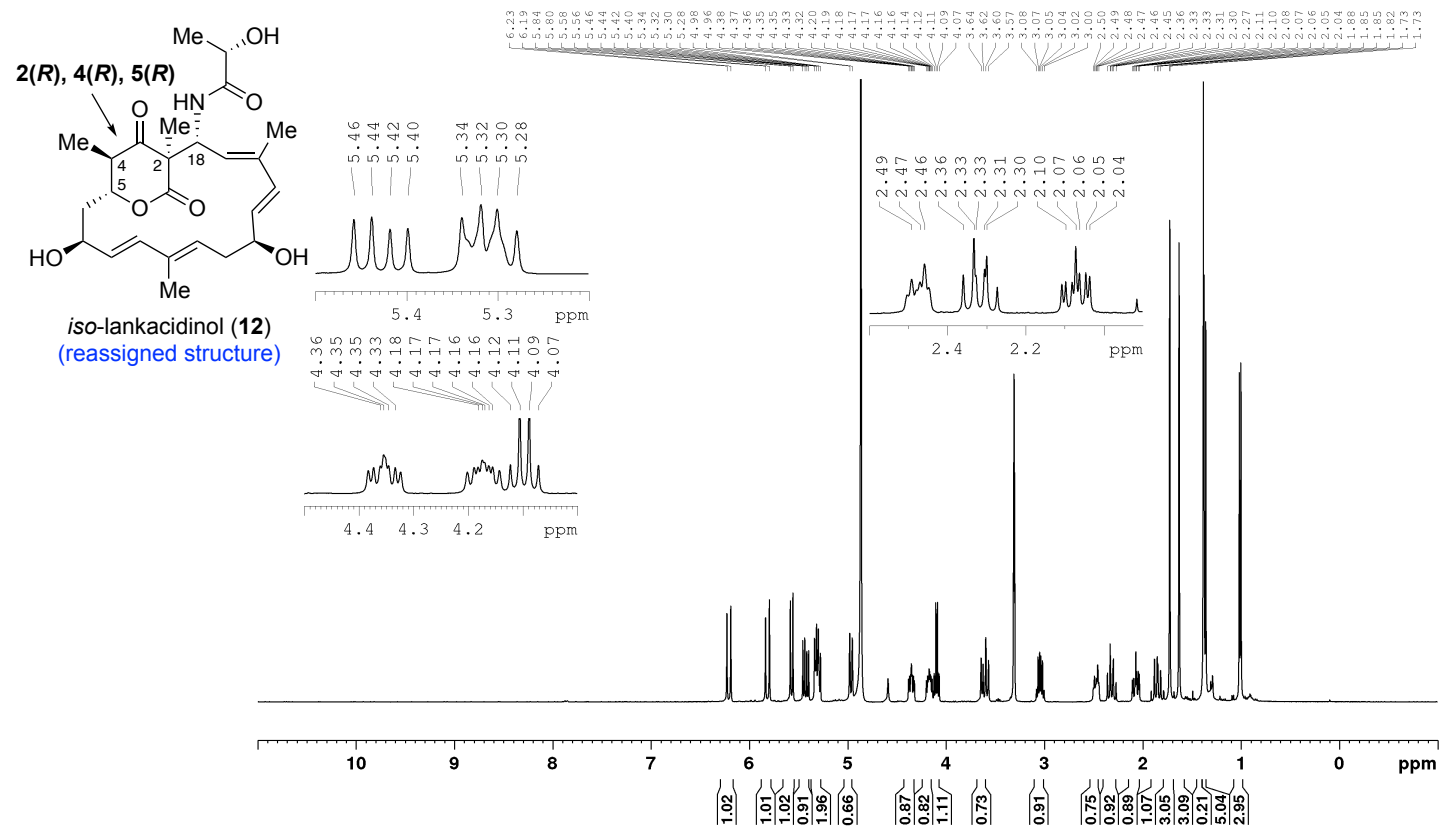

$\left({ }^{13} \mathrm{C}\right.$ NMR, $\left.\mathrm{CD}_{3} \mathrm{OD}, 100 \mathrm{MHz}\right)$

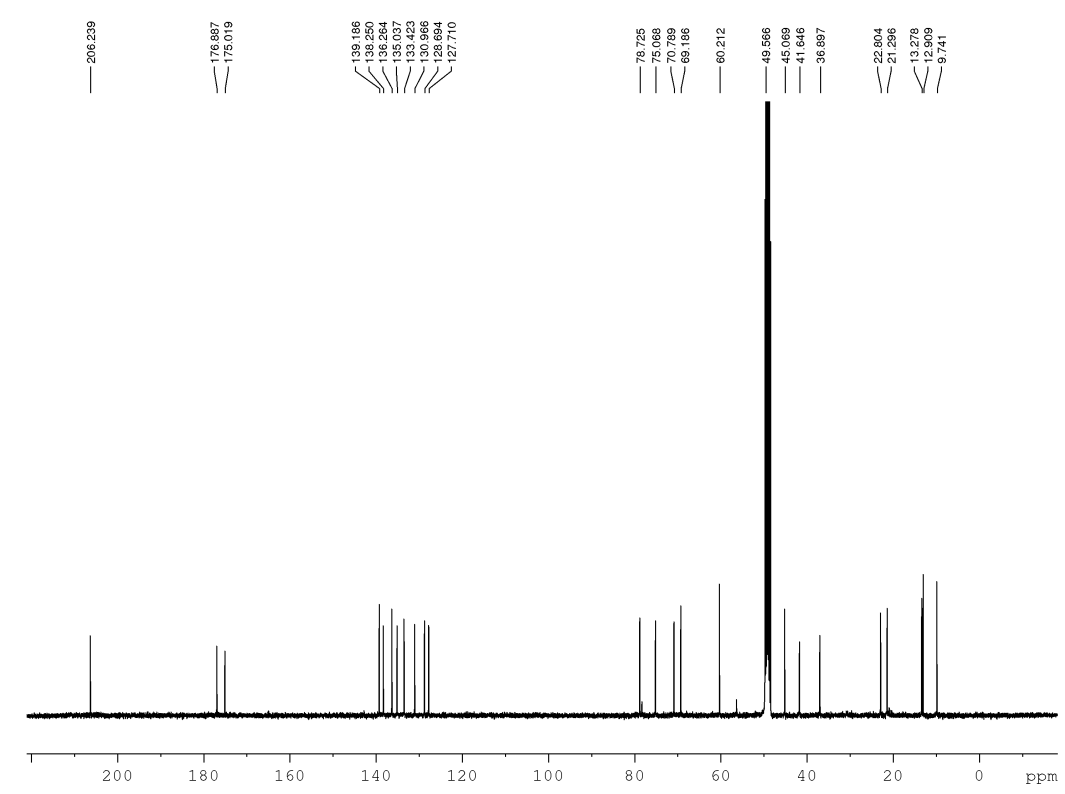


(COSY, $\left.\mathrm{CD}_{3} \mathrm{OD}, 400 \mathrm{MHz}\right)$

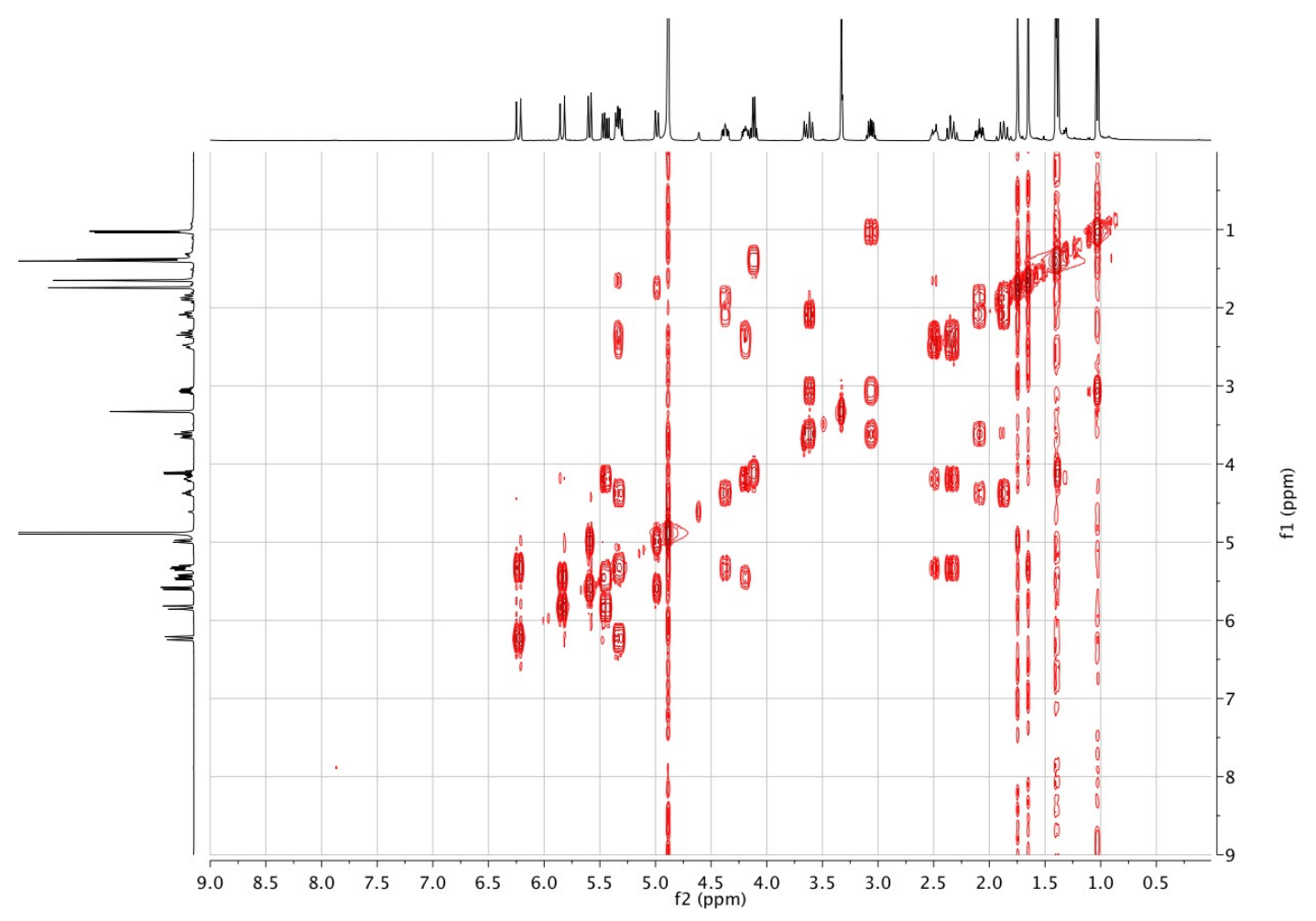

(NOESY, $\mathrm{CD}_{3} \mathrm{OD}, 400 \mathrm{MHz}$ )

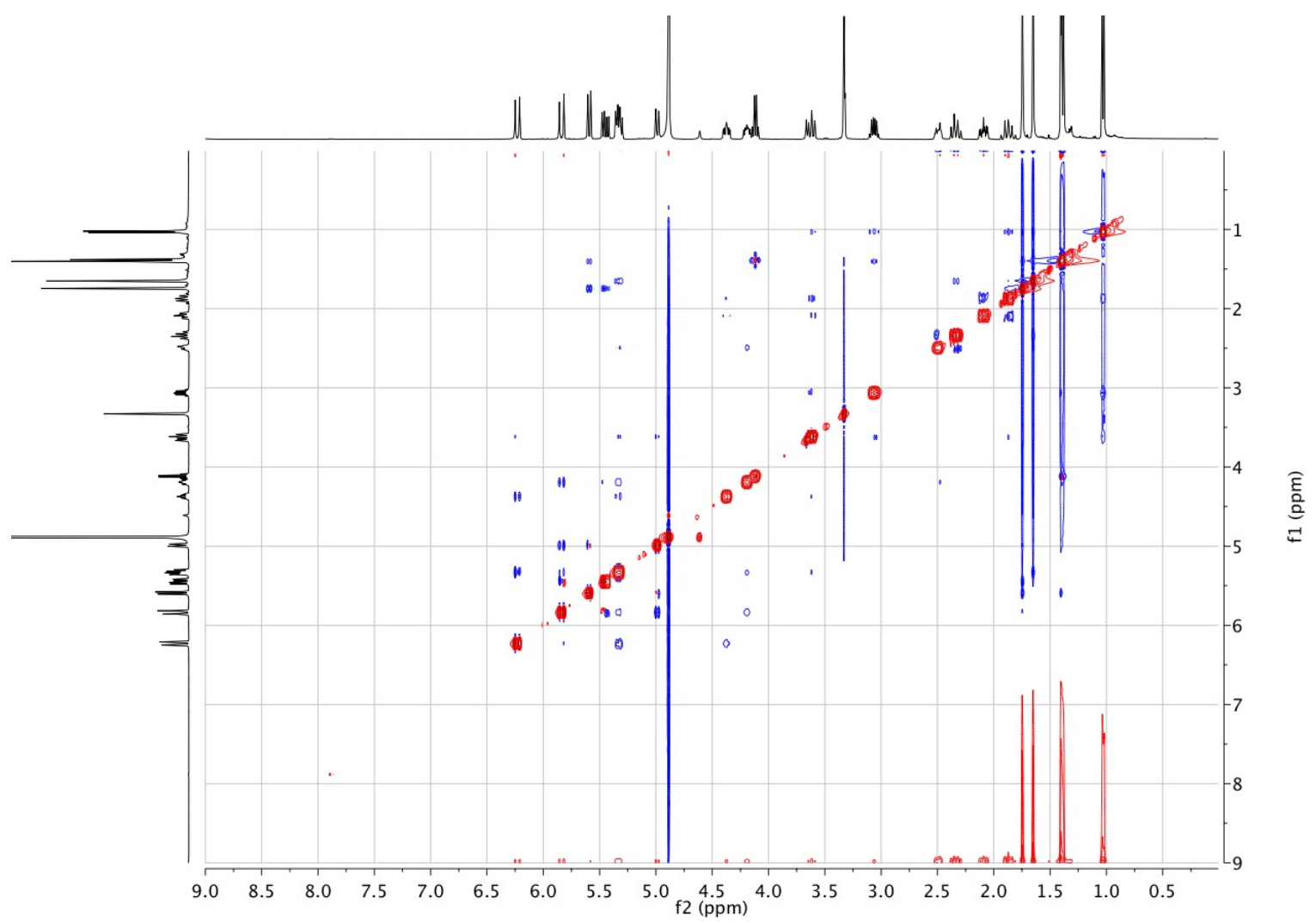




\section{X-Ray Crystallographic Information}

Compound 60 : CCDC 2008805
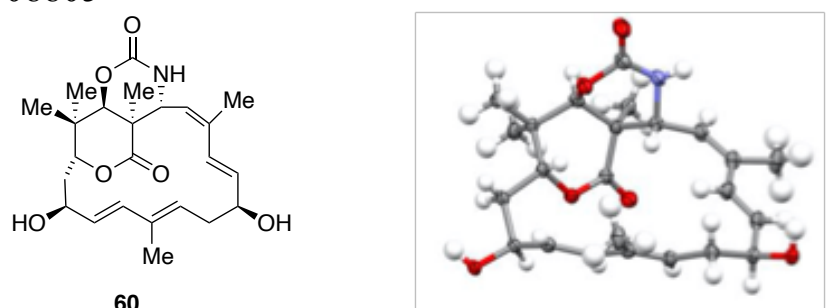

Table 1 Crystal data and structure refinement for Lankacidin.

Identification code

Lankacidin

Empirical formula

$\mathrm{C}_{24} \mathrm{H}_{33} \mathrm{NO}_{6}$

Formula weight

431.51

Temperature/K

125.0

Crystal system tetragonal

Space group

$\mathrm{P}_{3}$

$\mathrm{a} / \AA$

$8.3644(2)$

$\mathrm{b} / \AA$

$8.3644(2)$

$\mathrm{c} / \AA$

$32.7039(8)$

$\alpha /{ }^{\circ}$

90

$\beta /{ }^{\circ}$

90

$\gamma /{ }^{\circ}$

90

Volume $/ \AA^{3}$

2288.07(12)

Z

4

$\rho_{\text {calc }} / \mathrm{cm}^{3}$

1.253

$\mu / \mathrm{mm}^{-1}$

0.730

$\mathrm{F}(000)$

928.0

Crystal size $/ \mathrm{mm}^{3}$

$0.35 \times 0.3 \times 0.25$

Radiation

$\operatorname{CuK} \alpha(\lambda=1.54178)$

$2 \Theta$ range for data collection $/{ }^{\circ} 10.576$ to 159.176

Index ranges $-10 \leq \mathrm{h} \leq 10,-10 \leq \mathrm{k} \leq 10,-41 \leq 1 \leq 41$

Reflections collected 81813

Independent reflections

$4951\left[\mathrm{R}_{\text {int }}=0.0307, \mathrm{R}_{\text {sigma }}=0.0117\right]$

Data/restraints/parameters $4951 / 1 / 295$

Goodness-of-fit on $\mathrm{F}^{2}$

1.040

Final $\mathrm{R}$ indexes $[\mathrm{I}>=2 \sigma(\mathrm{I})]$

$\mathrm{R}_{1}=0.0291, \mathrm{wR}_{2}=0.0801$

Final $\mathrm{R}$ indexes [all data] $\quad \mathrm{R}_{1}=0.0294, \mathrm{wR}_{2}=0.0802$

Largest diff. peak/hole / e $\AA^{-3} 0.26 /-0.16$

Flack parameter

$0.005(30)$ 
Table 2 Fractional Atomic Coordinates $\left(\times 10^{4}\right)$ and Equivalent Isotropic Displacement Parameters $\left(\AA^{2} \times 10^{3}\right)$ for Lankacidin. $U_{\text {eq }}$ is defined as $1 / 3$ of of the trace of the orthogonalised $\mathrm{U}_{\mathrm{IJ}}$ tensor.

\begin{tabular}{|c|c|c|c|c|}
\hline Atom & $x$ & $y$ & $z$ & $\mathrm{U}(\mathbf{e q})$ \\
\hline $\mathrm{O}_{1}$ & $6272.2(15)$ & $4514.9(16)$ & $4770.8(4)$ & $24.7(3)$ \\
\hline $\mathrm{O}_{2}$ & $3715.4(16)$ & $4636.1(18)$ & 4908.3(4) & $32.6(3)$ \\
\hline $\mathrm{O}_{3}$ & $9833.7(17)$ & $3927.2(16)$ & $3864.6(4)$ & $25.9(3)$ \\
\hline $\mathrm{O}_{4}$ & $2648.2(17)$ & 12920.1(16) & $4217.0(4)$ & $29.2(3)$ \\
\hline $\mathrm{O}_{5}$ & $8242.4(18)$ & $8455.6(19)$ & $6136.8(5)$ & $37.5(3)$ \\
\hline $\mathrm{O}_{6}$ & $8059.8(15)$ & $6587.3(16)$ & $5649.7(4)$ & $27.5(3)$ \\
\hline $\mathrm{N}_{1}$ & $5835(2)$ & $7932(2)$ & $5839.0(5)$ & $28.7(3)$ \\
\hline $\mathrm{C}_{1}$ & $5065(2)$ & $4829(2)$ & $5030.2(5)$ & $24.4(3)$ \\
\hline $\mathrm{C}_{2}$ & $5392(2)$ & $5411(2)$ & $5466.0(5)$ & $22.6(3)$ \\
\hline $\mathrm{C}_{3}$ & $7135(2)$ & $5125(2)$ & $5613.8(5)$ & $22.7(3)$ \\
\hline $\mathrm{C}_{4}$ & $8119(2)$ & $4031(2)$ & $5334.1(5)$ & $22.5(3)$ \\
\hline $\mathrm{C}_{5}$ & $7929(2)$ & $4724(2)$ & $4903.3(5)$ & $22.1(3)$ \\
\hline $\mathrm{C}_{6}$ & $9007(2)$ & $3993(2)$ & $4579.6(5)$ & $23.3(3)$ \\
\hline $\mathrm{C}_{7}$ & $8635(2)$ & $4524(2)$ & $4139.7(5)$ & $22.0(3)$ \\
\hline $\mathrm{C}_{8}$ & $8526(2)$ & $6308(2)$ & $4105.8(5)$ & $23.0(3)$ \\
\hline $\mathrm{C}_{9}$ & $7119(2)$ & $7067(2)$ & $4088.5(5)$ & $23.3(3)$ \\
\hline $\mathrm{C}_{10}$ & $6865(2)$ & $8792(2)$ & $4094.8(5)$ & $23.9(3)$ \\
\hline $\mathrm{C}_{11}$ & $5356(2)$ & $9342(2)$ & 4109.1(5) & $25.1(3)$ \\
\hline $\mathrm{C}_{12}$ & $4871(2)$ & $11067(2)$ & $4155.5(6)$ & $28.0(4)$ \\
\hline $\mathrm{C}_{13}$ & $3093(2)$ & $11260(2)$ & $4238.5(5)$ & $26.3(4)$ \\
\hline $\mathrm{C}_{14}$ & $2497(2)$ & $10523(2)$ & $4629.8(6)$ & $30.0(4)$ \\
\hline $\mathrm{C}_{15}$ & $3295(2)$ & $9579(2)$ & $4886.9(6)$ & $29.3(4)$ \\
\hline $\mathrm{C}_{16}$ & $2583(2)$ & $8796(2)$ & $5246.3(6)$ & $28.4(4)$ \\
\hline $\mathrm{C}_{17}$ & $3354(2)$ & $7753(2)$ & $5485.4(6)$ & $27.1(4)$ \\
\hline $\mathrm{C}_{18}$ & $5082(2)$ & $7272(2)$ & $5470.4(6)$ & $23.5(3)$ \\
\hline $\mathrm{C}_{19}$ & $7407(2)$ & $7732(2)$ & $5899.7(6)$ & $27.7(4)$ \\
\hline $\mathrm{C}_{20}$ & $4244(2)$ & $4539(2)$ & $5758.2(6)$ & $26.6(3)$ \\
\hline $\mathrm{C}_{21}$ & $9886(2)$ & $4049(2)$ & $5462.1(6)$ & $28.2(4)$ \\
\hline $\mathrm{C}_{22}$ & $7482(2)$ & $2308(2)$ & $5368.0(6)$ & $28.2(4)$ \\
\hline $\mathrm{C}_{23}$ & $8293(2)$ & $9872(2)$ & $4094.9(8)$ & $34.4(4)$ \\
\hline $\mathrm{C}_{24}$ & $865(2)$ & $9192(3)$ & $5345.5(8)$ & $40.1(5)$ \\
\hline
\end{tabular}


Table 3 Anisotropic Displacement Parameters $\left(\AA^{2} \times 10^{3}\right)$ for Lankacidin. The Anisotropic displacement factor exponent takes the form: $-2 \pi^{2}\left[h^{2} a^{* 2} U_{11}+2 h k a * b * U_{12}+\ldots\right]$.

\begin{tabular}{|c|c|c|c|c|c|c|}
\hline Atom & $\mathbf{U}_{11}$ & $\mathbf{U}_{22}$ & $\mathbf{U}_{33}$ & $\mathbf{U}_{23}$ & $\mathbf{U}_{13}$ & $\mathbf{U}_{12}$ \\
\hline $\mathrm{O}_{1}$ & $21.3(6)$ & $32.2(6)$ & $20.6(5)$ & $-0.7(5)$ & $-0.2(4)$ & $2.1(5)$ \\
\hline $\mathrm{O}_{2}$ & $23.4(6)$ & $42.4(8)$ & $32.0(7)$ & $-8.4(6)$ & $-2.7(5)$ & $1.0(5)$ \\
\hline $\mathrm{O}_{3}$ & $27.3(6)$ & $28.6(6)$ & $22.0(6)$ & $-1.0(5)$ & $3.9(5)$ & $9.0(5)$ \\
\hline $\mathrm{O}_{4}$ & $32.4(7)$ & $26.6(6)$ & $28.7(7)$ & $-1.2(5)$ & $-3.3(5)$ & $10.8(5)$ \\
\hline $\mathrm{O}_{5}$ & $31.4(7)$ & $37.6(7)$ & $43.6(9)$ & $-14.7(6)$ & $-2.2(6)$ & $-0.1(6)$ \\
\hline $\mathrm{O}_{6}$ & $23.0(6)$ & $28.2(6)$ & $31.2(6)$ & $-7.2(5)$ & $2.1(5)$ & $-1.4(5)$ \\
\hline $\mathrm{N}_{1}$ & $28.1(8)$ & $31.1(8)$ & $26.8(8)$ & $-7.5(6)$ & $1.0(6)$ & $3.2(6)$ \\
\hline $\mathrm{C}_{1}$ & $22.6(8)$ & $27.3(8)$ & $23.2(8)$ & $0.2(6)$ & $0.3(6)$ & $1.7(6)$ \\
\hline $\mathrm{C}_{2}$ & $20.5(7)$ & $24.9(8)$ & $22.4(7)$ & $0.8(6)$ & $3.1(6)$ & $3.0(6)$ \\
\hline $\mathrm{C}_{3}$ & $21.9(7)$ & $24.4(8)$ & $21.7(8)$ & $-1.9(6)$ & $1.3(6)$ & $-2.3(6)$ \\
\hline $\mathrm{C}_{4}$ & $19.6(7)$ & $25.1(8)$ & $22.6(8)$ & $-0.2(6)$ & $0.4(6)$ & $2.2(6)$ \\
\hline $\mathrm{C}_{5}$ & $19.4(7)$ & $24.8(7)$ & $22.0(8)$ & $-1.8(6)$ & $0.4(6)$ & $1.5(6)$ \\
\hline $\mathrm{C}_{6}$ & $24.0(8)$ & $22.6(7)$ & $23.3(8)$ & $-0.5(6)$ & $2.2(6)$ & $5.2(6)$ \\
\hline $\mathrm{C}_{7}$ & $22.9(7)$ & $22.4(8)$ & $20.8(7)$ & $-1.5(6)$ & $2.8(6)$ & $3.3(6)$ \\
\hline $\mathrm{C}_{8}$ & $25.2(8)$ & $22.3(8)$ & $21.6(8)$ & $-0.3(6)$ & $2.0(6)$ & $1.2(6)$ \\
\hline $\mathrm{C}_{9}$ & $26.6(8)$ & $22.8(8)$ & $20.4(8)$ & $-0.3(6)$ & $-0.1(6)$ & $1.2(6)$ \\
\hline $\mathrm{C}_{10}$ & $27.9(8)$ & $22.8(8)$ & $21.0(8)$ & $0.0(6)$ & $0.5(6)$ & $2.9(6)$ \\
\hline $\mathrm{C}_{11}$ & $28.0(8)$ & $23.7(8)$ & $23.6(8)$ & $0.5(6)$ & $0.9(7)$ & $3.3(6)$ \\
\hline $\mathrm{C}_{12}$ & $31.0(9)$ & $23.4(8)$ & $29.5(8)$ & $1.9(7)$ & $3.8(7)$ & $6.6(7)$ \\
\hline $\mathrm{C}_{13}$ & $27.6(8)$ & $25.2(8)$ & $26.0(8)$ & $-0.6(7)$ & $-3.6(6)$ & 7.1(7) \\
\hline $\mathrm{C}_{14}$ & $23.5(8)$ & $31.3(9)$ & $35.2(10)$ & $1.3(7)$ & $1.9(7)$ & $4.8(7)$ \\
\hline $\mathrm{C}_{15}$ & $23.2(8)$ & $32.2(9)$ & $32.3(9)$ & $2.3(8)$ & $1.5(7)$ & $4.1(7)$ \\
\hline $\mathrm{C}_{16}$ & $23.6(8)$ & $28.0(8)$ & $33.7(9)$ & $0.4(7)$ & $2.1(7)$ & $2.6(7)$ \\
\hline $\mathrm{C}_{17}$ & $25.7(8)$ & $27.2(8)$ & $28.4(8)$ & $-0.8(7)$ & $6.1(7)$ & $3.5(6)$ \\
\hline $\mathrm{C}_{18}$ & $22.6(8)$ & $25.1(8)$ & $22.8(8)$ & $-1.2(6)$ & $1.3(6)$ & $2.3(6)$ \\
\hline $\mathrm{C}_{19}$ & $27.3(9)$ & $27.8(8)$ & $28.0(8)$ & $-4.9(7)$ & $2.8(7)$ & $-1.3(7)$ \\
\hline $\mathrm{C}_{20}$ & $21.9(8)$ & $29.4(8)$ & $28.5(8)$ & $5.8(7)$ & $3.0(7)$ & $0.1(6)$ \\
\hline $\mathrm{C}_{21}$ & $21.3(8)$ & $37.9(9)$ & $25.3(8)$ & $-2.0(7)$ & $-0.8(6)$ & $4.1(7)$ \\
\hline $\mathrm{C}_{22}$ & $30.4(9)$ & $24.1(8)$ & $30.0(9)$ & $4.0(7)$ & $2.9(7)$ & $4.4(7)$ \\
\hline $\mathrm{C}_{23}$ & $28.4(9)$ & $22.6(8)$ & $52.4(12)$ & $-2.3(8)$ & $2.2(8)$ & $2.3(7)$ \\
\hline $\mathrm{C}_{24}$ & $24.7(9)$ & $39.9(11)$ & $55.8(13)$ & $11.4(10)$ & $9.8(9)$ & $8.5(8)$ \\
\hline
\end{tabular}

Table 4 Bond Lengths for Lankacidin. 


$\begin{array}{llllll}\mathrm{O}_{1} & \mathrm{C}_{1} & 1.344(2) & \mathrm{C}_{4} & \mathrm{C}_{22} & 1.541(2) \\ \mathrm{O}_{1} & \mathrm{C}_{5} & 1.462(2) & \mathrm{C}_{5} & \mathrm{C}_{6} & 1.519(2) \\ \mathrm{O}_{2} & \mathrm{C}_{1} & 1.208(2) & \mathrm{C}_{6} & \mathrm{C}_{7} & 1.537(2) \\ \mathrm{O}_{3} & \mathrm{C}_{7} & 1.437(2) & \mathrm{C}_{7} & \mathrm{C}_{8} & 1.499(2) \\ \mathrm{O}_{4} & \mathrm{C}_{13} & 1.439(2) & \mathrm{C}_{8} & \mathrm{C}_{9} & 1.338(2) \\ \mathrm{O}_{5} & \mathrm{C}_{19} & 1.207(2) & \mathrm{C}_{9} & \mathrm{C}_{10} & 1.459(2) \\ \mathrm{O}_{6} & \mathrm{C}_{3} & 1.452(2) & \mathrm{C}_{10} & \mathrm{C}_{11} & 1.344(3) \\ \mathrm{O}_{6} & \mathrm{C}_{19} & 1.372(2) & \mathrm{C}_{10} & \mathrm{C}_{23} & 1.497(3) \\ \mathrm{N}_{1} & \mathrm{C}_{18} & 1.468(2) & \mathrm{C}_{11} & \mathrm{C}_{12} & 1.507(2) \\ \mathrm{N}_{1} & \mathrm{C}_{19} & 1.340(3) & \mathrm{C}_{12} & \mathrm{C}_{13} & 1.520(3) \\ \mathrm{C}_{1} & \mathrm{C}_{2} & 1.531(2) & \mathrm{C}_{13} & \mathrm{C}_{14} & 1.506(3) \\ \mathrm{C}_{2} & \mathrm{C}_{3} & 1.555(2) & \mathrm{C}_{14} & \mathrm{C}_{15} & 1.333(3) \\ \mathrm{C}_{2} & \mathrm{C}_{18} & 1.578(2) & \mathrm{C}_{15} & \mathrm{C}_{16} & 1.471(3) \\ \mathrm{C}_{2} & \mathrm{C}_{20} & 1.538(2) & \mathrm{C}_{16} & \mathrm{C}_{17} & 1.337(3) \\ \mathrm{C}_{3} & \mathrm{C}_{4} & 1.533(2) & \mathrm{C}_{16} & \mathrm{C}_{24} & 1.510(3) \\ \mathrm{C}_{4} & \mathrm{C}_{5} & 1.532(2) & \mathrm{C}_{17} & \mathrm{C}_{18} & 1.502(2) \\ \mathrm{C}_{4} & \mathrm{C}_{21} & 1.536(2) & & & \end{array}$

Table 5 Bond Angles for Lankacidin.

\begin{tabular}{|c|c|c|c|c|c|c|c|}
\hline tom & & & & Atom & & m & \\
\hline $\mathrm{C}_{1}$ & $\mathrm{O}_{1}$ & $\mathrm{C}_{5}$ & $120.10(13)$ & $\mathrm{O}_{3}$ & $\mathrm{C}_{7}$ & $\mathrm{C}_{6}$ & \\
\hline 10 & $\mathrm{O}_{6}$ & $C$ & & $\mathrm{O}_{3}$ & C & & \\
\hline 9 & $\mathrm{~N}_{1}$ & $\mathrm{C}_{18}$ & 6) & $\mathrm{C}_{8}$ & $\mathrm{C}_{7}$ & $\mathrm{C}_{6}$ & \\
\hline 1 & $\mathrm{C}_{1}$ & $\mathrm{C}_{2}$ & & $\mathrm{C}_{9}$ & $\mathrm{C}_{8}$ & $\mathrm{C}_{7}$ & \\
\hline 2 & $\mathrm{C}_{1}$ & $\mathrm{O}_{1}$ & & $\mathrm{C}_{8}$ & $\mathrm{C}_{9}$ & -10 & \\
\hline & $\mathrm{C}_{1}$ & $\mathrm{C}_{2}$ & & $\mathrm{C}_{9}$ & $C_{10}$ & & \\
\hline & $\mathrm{C}_{2}$ & $\mathrm{C}_{3}$ & & $\mathrm{C}_{11}$ & $\mathrm{C}$ & & \\
\hline & $\mathrm{C}_{2}$ & $\mathrm{C}_{18}$ & & $\mathrm{C}_{11}$ & $\mathrm{C}_{10}$ & 0 & \\
\hline & $\mathrm{C}_{2}$ & $\mathrm{C}_{20}$ & 10 & $\mathrm{C}_{10}$ & $\mathrm{C}_{11}$ & $\mathrm{C}_{12}$ & \\
\hline & $\mathrm{C}_{2}$ & $\mathrm{C}_{18}$ & & $\mathrm{C}_{11}$ & 12 & $\mathrm{C}_{13}$ & 16 \\
\hline & $\mathrm{C}_{2}$ & $\mathrm{C}_{3}$ & & $\mathrm{O}_{4}$ & $\mathrm{C}_{13}$ & $\mathrm{C}_{12}$ & \\
\hline 20 & $\mathrm{C}_{2}$ & $\mathrm{C}_{18}$ & & $\mathrm{O}_{4}$ & $\mathrm{C}_{13}$ & & 11 \\
\hline & $\mathrm{C}_{3}$ & $\mathrm{C}_{2}$ & 4) & $\mathrm{C}_{14}$ & $\mathrm{C}_{13}$ & $\mathrm{C}_{12}$ & 15) \\
\hline & $\mathrm{C}_{3}$ & $\mathrm{C}_{4}$ & 105 & $\mathrm{C}_{15}$ & $\mathrm{C}_{14}$ & $\mathrm{C}_{13}$ & 127 \\
\hline & & & & $\mathrm{C}_{14}$ & $\mathrm{C}_{15}$ & -10 & \\
\hline$C_{3}$ & $\mathrm{C}_{4}$ & $\mathrm{C}_{21}$ & $110.37(14)$ & $\mathrm{C}_{15}$ & $\mathrm{C}_{16}$ & $\mathrm{C}_{24}$ & $117.34\left(1^{7}\right.$ \\
\hline
\end{tabular}




$\begin{array}{llllllll}\mathrm{C}_{3} & \mathrm{C}_{4} & \mathrm{C}_{22} & 109.23(14) & \mathrm{C}_{17} & \mathrm{C}_{16} & \mathrm{C}_{15} & 124.23(17) \\ \mathrm{C}_{5} & \mathrm{C}_{4} & \mathrm{C}_{3} & 105.51(13) & \mathrm{C}_{17} & \mathrm{C}_{16} & \mathrm{C}_{24} & 118.43(18) \\ \mathrm{C}_{5} & \mathrm{C}_{4} & \mathrm{C}_{21} & 110.32(14) & \mathrm{C}_{16} & \mathrm{C}_{17} & \mathrm{C}_{18} & 128.30(17) \\ \mathrm{C}_{5} & \mathrm{C}_{4} & \mathrm{C}_{22} & 112.57(15) & \mathrm{N}_{1} & \mathrm{C}_{18} & \mathrm{C}_{2} & 107.95(15) \\ \mathrm{C}_{21} & \mathrm{C}_{4} & \mathrm{C}_{22} & 108.81(15) & \mathrm{N}_{1} & \mathrm{C}_{18} & \mathrm{C}_{17} & 106.59(14) \\ \mathrm{O}_{1} & \mathrm{C}_{5} & \mathrm{C}_{4} & 109.02(13) & \mathrm{C}_{17} & \mathrm{C}_{18} & \mathrm{C}_{2} & 114.99(15) \\ \mathrm{O}_{1} & \mathrm{C}_{5} & \mathrm{C}_{6} & 107.94(13) & \mathrm{O}_{5} & \mathrm{C}_{19} & \mathrm{O}_{6} & 120.14(17) \\ \mathrm{C}_{6} & \mathrm{C}_{5} & \mathrm{C}_{4} & 115.28(14) & \mathrm{O}_{5} & \mathrm{C}_{19} & \mathrm{~N}_{1} & 126.94(18) \\ \mathrm{C}_{5} & \mathrm{C}_{6} & \mathrm{C}_{7} & 114.57(14) & \mathrm{N}_{1} & \mathrm{C}_{19} & \mathrm{O}_{6} & 112.90(16)\end{array}$

Table 6 Hydrogen Bonds for Lankacidin.

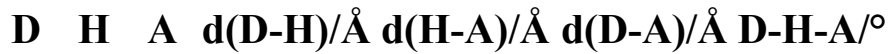

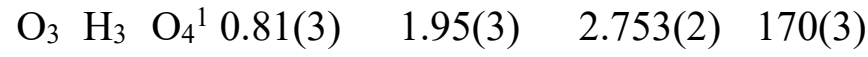
$\mathrm{O}_{4} \mathrm{H}_{4} \mathrm{O}_{2}^{2} 0.88(4) \quad 1.95(4) \quad 2.823(2) \quad 171(3)$

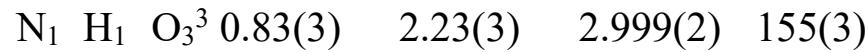

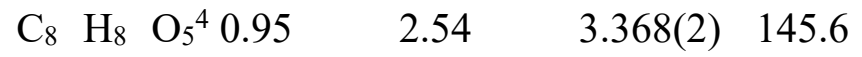
$\mathrm{C}_{13} \mathrm{H}_{13} \mathrm{O}_{5}^{5} 1.00 \quad 2.53 \quad 3.453(2) \quad 153.0$
${ }^{1} 1+\mathrm{X},-1+\mathrm{Y},+\mathrm{Z} ;{ }^{2}+\mathrm{X}, 1+\mathrm{Y},+\mathrm{Z} ;{ }^{3}+\mathrm{Y}, 2-\mathrm{X}, 1 / 4+\mathrm{Z} ;{ }^{4} 2-\mathrm{Y},+\mathrm{X},-1 / 4+Z ;{ }^{5} 1-\mathrm{Y},+\mathrm{X},-1 / 4+\mathrm{Z}$

Table 7 Hydrogen Atom Coordinates $\left(\AA \times 10^{4}\right)$ and Isotropic Displacement Parameters $\left(\AA^{2} \times 10^{3}\right)$ for Lankacidin.

\begin{tabular}{lllll}
\multicolumn{1}{r}{ Atom } & \multicolumn{1}{c}{$\boldsymbol{x}$} & $\boldsymbol{z}$ & $\mathbf{U}(\mathbf{e q})$ \\
$\mathrm{H}_{3}$ & $10600(40)$ & $3570(30)$ & $3989(10)$ & 39 \\
$\mathrm{H}_{4}$ & $3060(40)$ & $13370(40)$ & $4435(11)$ & $43(8)$ \\
$\mathrm{H}_{1}$ & $5390(30)$ & $8740(30)$ & $5925(9)$ & 34 \\
$\mathrm{H}_{3 \mathrm{~A}}$ & 7085.9 & 4615.78 & 5890.18 & 27 \\
$\mathrm{H}_{5}$ & 8157.53 & 5896.02 & 4916.43 & 26 \\
$\mathrm{H}_{6 \mathrm{~A}}$ & 8914.13 & 2814.35 & 4594.9 & 28 \\
$\mathrm{H}_{6 \mathrm{~B}}$ & 10128.32 & 4276.38 & 4643.43 & 28 \\
$\mathrm{H}_{7}$ & 7580.81 & 4059.01 & 4058.58 & 26 \\
$\mathrm{H}_{8}$ & 9483.54 & 6917.3 & 4096.3 & 28 \\
$\mathrm{H}_{9}$ & 6191.51 & 6415.33 & 4070.25 & 28 \\
$\mathrm{H}_{11}$ & 4521.82 & 8575.05 & 4088.19 & 30 \\
$\mathrm{H}_{12 \mathrm{~A}}$ & 5149.96 & 11653.9 & 3902.41 & 34
\end{tabular}




$\begin{array}{lllll}\mathrm{H}_{12 \mathrm{~B}} & 5481.97 & 11549.54 & 4383.56 & 34 \\ \mathrm{H}_{13} & 2520.8 & 10706.68 & 4010.35 & 32 \\ \mathrm{H}_{14} & 1422.62 & 10760 & 4702.24 & 36 \\ \mathrm{H}_{15} & 4397.63 & 9400.13 & 4834.6 & 35 \\ \mathrm{H}_{17} & 2727.49 & 7252.47 & 5691.18 & 33 \\ \mathrm{H}_{18} & 5591.22 & 7761.12 & 5223.6 & 28 \\ \mathrm{H}_{20 \mathrm{~A}} & 3159.03 & 4566.03 & 5645.67 & 40 \\ \mathrm{H}_{20 \mathrm{~B}} & 4255.8 & 5068.85 & 6025.44 & 40 \\ \mathrm{H}_{20 \mathrm{C}} & 4587.91 & 3425.26 & 5789.79 & 40 \\ \mathrm{H}_{21 \mathrm{~A}} & 10478 & 3258.22 & 5301.16 & 42 \\ \mathrm{H}_{21 \mathrm{~B}} & 9971.73 & 3786.58 & 5753.3 & 42 \\ \mathrm{H}_{21 \mathrm{C}} & 10334.59 & 5114.82 & 5413.54 & 42 \\ \mathrm{H}_{22 \mathrm{~A}} & 6354.17 & 2283.33 & 5287.05 & 42 \\ \mathrm{H}_{22 \mathrm{~B}} & 7585.11 & 1937.5 & 5651.07 & 42 \\ \mathrm{H}_{22 \mathrm{C}} & 8101.15 & 1607.18 & 5187.5 & 42 \\ \mathrm{H}_{23 \mathrm{~A}} & 8818.56 & 9823.22 & 4362.45 & 52 \\ \mathrm{H}_{23 \mathrm{~B}} & 7950.99 & 10971.48 & 4040 & 52 \\ \mathrm{H}_{23 \mathrm{C}} & 9043.58 & 9526.69 & 3882.46 & 52 \\ \mathrm{H}_{24 \mathrm{~A}} & 173.93 & 8832.95 & 5121.68 & 60 \\ \mathrm{H}_{24 \mathrm{~B}} & 752.23 & 10350.52 & 5380.38 & 60 \\ \mathrm{H}_{24 \mathrm{C}} & 553.49 & 8650.42 & 5599.01 & 60 \\ & & & & \end{array}$

\section{Experimental}

Single crystals of $\mathrm{C}_{24} \mathrm{H}_{33} \mathrm{NO}_{6}$ Lankacidin were obtain via vapor diffusion between acetone and pentane. A suitable crystal was selected and measured on a D8 Venture diffractometer. The crystal was kept at $125.0 \mathrm{~K}$ during data collection. Using Apex3 [1], the structure was solved with the ShelXT [2] structure solution program using intrinsic phasing. Using Olex2 [3] refined with the XL [4] refinement package using least squares minimization. The absolute structure was determined using anomalous dispersion. The Flack parameter [5] was determined from 2389 selected quotients during refinement using XL.

1. Bruker-AXS (2016). APEX3. Version 2016.9-0. Madison, Wisconsin

2. Sheldrick, G.M. (2015). Acta Cryst. A71, 3-8.

3. Dolomanov, O.V., Bourhis, L.J., Gildea, R.J, Howard, J.A.K. \& Puschmann, H. (2009), J. Appl. Cryst. 42, 339-341.

4. Sheldrick, G.M. (2008). Acta Cryst. A64, 112-122.

5. Parsons, S., Flack, H. D., \& Wagner, T. (2013).69(Pt 3), 249-259

Crystal structure determination of [Lankacidin] 
Crystal Data for $\mathrm{C}_{24} \mathrm{H}_{33} \mathrm{NO}_{6}(M=431.51 \mathrm{~g} / \mathrm{mol})$ : tetragonal, space group $\mathrm{P}_{3}$ (no. 78), $a=$ 8.3644(2) $\AA, c=32.7039(8) \AA, V=2288.07(12) \AA^{3}, Z=4, T=125.0 \mathrm{~K}, \mu(\mathrm{CuK \alpha})=0.730 \mathrm{~mm}^{-1}$, Dcalc $=1.253 \mathrm{~g} / \mathrm{cm}^{3}, 81813$ reflections measured $\left(10.576^{\circ} \leq 2 \Theta \leq 159.176^{\circ}\right), 4951$ unique $\left(R_{\text {int }}\right.$ $=0.0307, \mathrm{R}_{\text {sigma }}=0.0117$ ) which were used in all calculations. The final $R_{1}$ was 0.0291 (I $>$ $2 \sigma(\mathrm{I}))$ and $w R_{2}$ was 0.0802 (all data).

\section{Refinement model description}

Details:

1. Fixed Uiso

At 1.2 times of:

All C $(\mathrm{H})$ groups, All $\mathrm{C}(\mathrm{H}, \mathrm{H})$ groups, All $\mathrm{N}(\mathrm{H})$ groups

At 1.5 times of:

All $\mathrm{C}(\mathrm{H}, \mathrm{H}, \mathrm{H})$ groups, All $\mathrm{O}(\mathrm{H})$ groups

2.a Ternary $\mathrm{CH}$ refined with riding coordinates:

$\mathrm{C} 3(\mathrm{H} 3 \mathrm{~A}), \mathrm{C} 5(\mathrm{H} 5), \mathrm{C} 7(\mathrm{H} 7), \mathrm{C} 13(\mathrm{H} 13), \mathrm{C} 18(\mathrm{H} 18)$

2.b Secondary $\mathrm{CH} 2$ refined with riding coordinates:

$\mathrm{C} 6(\mathrm{H} 6 \mathrm{~A}, \mathrm{H} 6 \mathrm{~B}), \mathrm{C} 12(\mathrm{H} 12 \mathrm{~A}, \mathrm{H} 12 \mathrm{~B})$

2. C Aromatic/amide $\mathrm{H}$ refined with riding coordinates:

$\mathrm{C} 8(\mathrm{H} 8), \mathrm{C} 9(\mathrm{H} 9), \mathrm{C} 11(\mathrm{H} 11), \mathrm{C} 14(\mathrm{H} 14), \mathrm{C} 15(\mathrm{H} 15), \mathrm{C} 17$ (H17)

2.d Idealised Me refined as rotating group:

C20 (H20A, H20B, H20C), C21 (H21A, H21B, H21C), C22 (H22A, H22B, H22C),

C23 (H23A, H23B,

$\mathrm{H} 23 \mathrm{C}), \mathrm{C} 24(\mathrm{H} 24 \mathrm{~A}, \mathrm{H} 24 \mathrm{~B}, \mathrm{H} 24 \mathrm{C})$

Compound 70 : CCDC 2008806
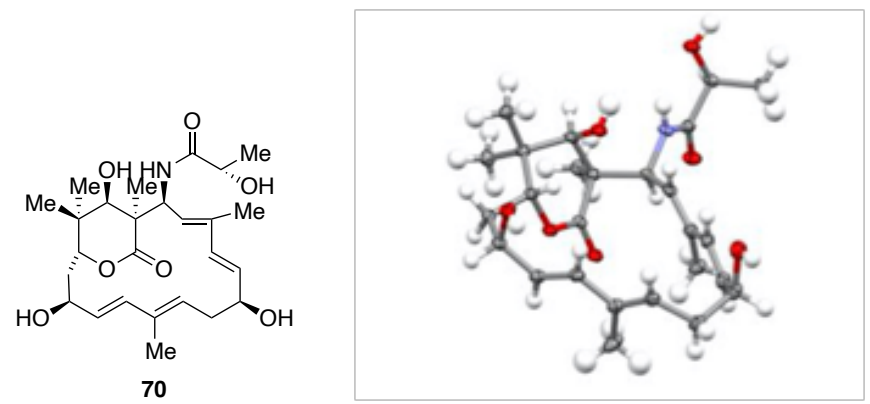

Table 1. Crystal data and structure refinement for Seiple004_UCSF.

Identification code

Empirical formula

Formula weight

Temperature

Wavelength

Crystal system

Space group
Seiple004_UCSF

C26 H39 N O7

477.58

100(2) K

$1.54184 \AA$

Monoclinic

P 21 


$\begin{array}{lll}\text { Unit cell dimensions } & \mathrm{a}=6.36890(10) \AA & \alpha=90^{\circ} . \\ & \mathrm{b}=17.2690(2) \AA & \beta=93.0650(10)^{\circ} . \\ & \mathrm{c}=11.54750(10) \AA & \gamma=90^{\circ} . \\ \text { Volume } & 1268.23(3) \AA^{3} & \\ Z & 2 & 1.251 \mathrm{Mg} / \mathrm{m}^{3} \\ \text { Density (calculated) } & 0.736 \mathrm{~mm}^{-1} \\ \text { Absorption coefficient } & 516 \\ \text { F(000) } & 0.180 \mathrm{x} 0.170 \mathrm{x} 0.100 \mathrm{~mm}^{3} \\ \text { Crystal size } & 3.833 \text { to } 74.493^{\circ} . \\ \text { Theta range for data collection } & -7<=\mathrm{h}<=7,-21<=\mathrm{k}<=21,-14<=1<=14 \\ \text { Index ranges } & 32281 \\ \text { Reflections collected } & 5146[\mathrm{R}(\mathrm{int})=0.0874] \\ \text { Independent reflections } & 100.0 \% \\ \text { Completeness to theta }=74.000^{\circ} & \text { Semi-empirical from equivalents } \\ \text { Absorption correction } & 1.00000 \text { and } 0.62071 \\ \text { Max. and min. transmission } & \text { Full-matrix least-squares on } \mathrm{F}^{2} \\ \text { Refinement method } & 5146 / 1 / 334 \\ \text { Data / restraints / parameters } & 1.080 \\ \text { Goodness-of-fit on F } & \mathrm{R} 1=0.0373, \mathrm{wR} 2=0.1043 \\ \text { Final R indices [I }>2 \text { sigma(I)] } & \mathrm{R} 1=0.0379, \mathrm{wR} 2=0.1048 \\ \text { R indices (all data) } & 0.08(11) \\ \text { Absolute structure parameter } & 0.0022(8) \\ \text { Extinction coefficient } & 0.225 \text { and }-0.236 \mathrm{e} . \AA^{-3} & \\ \text { Largest diff. peak and hole } & & \\ & & \end{array}$

Table 2. Atomic coordinates ( $\left.\times 10^{4}\right)$ and equivalent isotropic displacement parameters $\left(\AA^{2} \times 10^{3}\right)$ for seiple004_ucsf. U(eq) is defined as one third of the trace of the orthogonalized $\mathrm{U}^{\mathrm{ij}}$ tensor.

\begin{tabular}{lcccc}
\hline & $\mathrm{x}$ & $\mathrm{y}$ & $\mathrm{z}$ & $\mathrm{U}(\mathrm{eq})$ \\
\hline $\mathrm{C}(1)$ & & & & \\
$\mathrm{C}(2)$ & $8353(4)$ & $4278(2)$ & $1741(2)$ & $29(1)$ \\
$\mathrm{C}(3)$ & $8046(3)$ & $5123(1)$ & $2076(2)$ & $20(1)$ \\
$\mathrm{C}(4)$ & $6057(3)$ & $5188(1)$ & $2760(2)$ & $19(1)$ \\
$\mathrm{C}(5)$ & $4496(3)$ & $5230(1)$ & $4653(2)$ & $19(1)$ \\
$\mathrm{C}(6)$ & $4100(3)$ & $6044(1)$ & $5228(2)$ & $18(1)$ \\
$\mathrm{C}(7)$ & $3495(4)$ & $6602(1)$ & $4216(2)$ & $23(1)$ \\
& $5969(3)$ & $6363(1)$ & $5995(2)$ & $20(1)$
\end{tabular}




\begin{tabular}{|c|c|c|c|c|}
\hline$C(8)$ & $5276(3)$ & $6916(1)$ & $6969(2)$ & $20(1)$ \\
\hline$C(9)$ & $4117(4)$ & $7645(1)$ & $6526(2)$ & $24(1)$ \\
\hline $\mathrm{C}(10)$ & $7267(4)$ & $7165(2)$ & $7680(2)$ & $25(1)$ \\
\hline $\mathrm{C}(11)$ & $3896(3)$ & $6420(1)$ & $7718(2)$ & $19(1)$ \\
\hline $\mathrm{C}(12)$ & $2154(3)$ & $5928(1)$ & $5908(2)$ & $19(1)$ \\
\hline $\mathrm{C}(13)$ & 2949(3) & $6815(1)$ & $8748(2)$ & $20(1)$ \\
\hline $\mathrm{C}(14)$ & $2505(4)$ & $6257(1)$ & $9739(2)$ & $21(1)$ \\
\hline$C(15)$ & 1293(4) & $5550(1)$ & $9355(2)$ & $21(1)$ \\
\hline$C(16)$ & 2013(3) & $4826(1)$ & $9458(2)$ & $21(1)$ \\
\hline $\mathrm{C}(17)$ & $912(4)$ & $4116(1)$ & $9074(2)$ & $23(1)$ \\
\hline $\mathrm{C}(18)$ & $-1225(4)$ & $4195(2)$ & $8472(3)$ & $35(1)$ \\
\hline$C(19)$ & 1907(4) & $3443(1)$ & $9260(2)$ & $24(1)$ \\
\hline$C(20)$ & $1226(4)$ & $2650(1)$ & $8862(2)$ & $26(1)$ \\
\hline $\mathrm{C}(21)$ & $2555(4)$ & $2366(1)$ & $7857(2)$ & $25(1)$ \\
\hline$C(22)$ & $2205(4)$ & $2889(1)$ & $6831(2)$ & $23(1)$ \\
\hline $\mathrm{C}(23)$ & $3582(4)$ & $3412(1)$ & $6496(2)$ & $22(1)$ \\
\hline $\mathrm{C}(24)$ & $3186(4)$ & 3993(1) & $5581(2)$ & $21(1)$ \\
\hline$C(25)$ & $1152(4)$ & $3932(2)$ & $4866(2)$ & $25(1)$ \\
\hline$C(26)$ & $4649(3)$ & $4548(1)$ & $5468(2)$ & $19(1)$ \\
\hline $\mathrm{N}(1)$ & $6305(3)$ & $5260(1)$ & $3910(2)$ & $18(1)$ \\
\hline $\mathrm{O}(1)$ & $9784(3)$ & $5411(1)$ & $2764(1)$ & $23(1)$ \\
\hline $\mathrm{O}(2)$ & $4320(3)$ & $5142(1)$ & $2220(1)$ & $25(1)$ \\
\hline $\mathrm{O}(3)$ & $7066(3)$ & $5743(1)$ & $6567(2)$ & $22(1)$ \\
\hline $\mathrm{O}(4)$ & $2070(2)$ & $6127(1)$ & 7014(1) & $21(1)$ \\
\hline $\mathrm{O}(5)$ & $534(2)$ & $5669(1)$ & $5428(1)$ & $24(1)$ \\
\hline $\mathrm{O}(6)$ & $4509(3)$ & $6076(1)$ & 10294(1) & $22(1)$ \\
\hline $\mathrm{O}(7)$ & $4729(3)$ & $2353(1)$ & $8217(2)$ & $27(1)$ \\
\hline
\end{tabular}

Table 3. Bond lengths $[\AA]$ and angles $\left[{ }^{\circ}\right]$ for seiple004_ucsf.

\begin{tabular}{ll}
\hline $\mathrm{C}(1)-\mathrm{C}(2)$ & $1.524(3)$ \\
$\mathrm{C}(1)-\mathrm{H}(1 \mathrm{~B})$ & 0.9800 \\
$\mathrm{C}(1)-\mathrm{H}(1 \mathrm{C})$ & 0.9800 \\
$\mathrm{C}(1)-\mathrm{H}(1 \mathrm{D})$ & 0.9800 \\
$\mathrm{C}(2)-\mathrm{O}(1)$ & $1.418(3)$ \\
$\mathrm{C}(2)-\mathrm{C}(3)$ & $1.532(3)$
\end{tabular}




\begin{tabular}{|c|c|}
\hline $\mathrm{C}(2)-\mathrm{H}(2)$ & 1.0000 \\
\hline $\mathrm{C}(3)-\mathrm{O}(2)$ & $1.243(3)$ \\
\hline $\mathrm{C}(3)-\mathrm{N}(1)$ & $1.335(3)$ \\
\hline $\mathrm{C}(4)-\mathrm{N}(1)$ & $1.474(3)$ \\
\hline$C(4)-C(26)$ & $1.507(3)$ \\
\hline$C(4)-C(5)$ & $1.580(3)$ \\
\hline $\mathrm{C}(4)-\mathrm{H}(4)$ & 1.0000 \\
\hline$C(5)-C(12)$ & $1.516(3)$ \\
\hline$C(5)-C(7)$ & $1.547(3)$ \\
\hline$C(5)-C(6)$ & $1.547(3)$ \\
\hline $\mathrm{C}(6)-\mathrm{H}(6 \mathrm{~A})$ & 0.9800 \\
\hline $\mathrm{C}(6)-\mathrm{H}(6 \mathrm{~B})$ & 0.9800 \\
\hline $\mathrm{C}(6)-\mathrm{H}(6 \mathrm{C})$ & 0.9800 \\
\hline $\mathrm{C}(7)-\mathrm{O}(3)$ & $1.422(3)$ \\
\hline$C(7)-C(8)$ & $1.558(3)$ \\
\hline $\mathrm{C}(7)-\mathrm{H}(7 \mathrm{~A})$ & 1.0000 \\
\hline$C(8)-C(11)$ & $1.529(3)$ \\
\hline $\mathrm{C}(8)-\mathrm{C}(9)$ & $1.532(3)$ \\
\hline$C(8)-C(10)$ & $1.534(3)$ \\
\hline $\mathrm{C}(9)-\mathrm{H}(9 \mathrm{~A})$ & 0.9800 \\
\hline $\mathrm{C}(9)-\mathrm{H}(9 \mathrm{~B})$ & 0.9800 \\
\hline $\mathrm{C}(9)-\mathrm{H}(9 \mathrm{C})$ & 0.9800 \\
\hline $\mathrm{C}(10)-\mathrm{H}(10 \mathrm{~A})$ & 0.9800 \\
\hline $\mathrm{C}(10)-\mathrm{H}(10 \mathrm{~B})$ & 0.9800 \\
\hline $\mathrm{C}(10)-\mathrm{H}(10 \mathrm{C})$ & 0.9800 \\
\hline $\mathrm{C}(11)-\mathrm{O}(4)$ & $1.473(2)$ \\
\hline $\mathrm{C}(11)-\mathrm{C}(13)$ & $1.524(3)$ \\
\hline $\mathrm{C}(11)-\mathrm{H}(11)$ & 1.0000 \\
\hline $\mathrm{C}(12)-\mathrm{O}(5)$ & $1.230(3)$ \\
\hline $\mathrm{C}(12)-\mathrm{O}(4)$ & $1.326(3)$ \\
\hline$C(13)-C(14)$ & $1.533(3)$ \\
\hline $\mathrm{C}(13)-\mathrm{H}(13 \mathrm{~A})$ & 0.9900 \\
\hline $\mathrm{C}(13)-\mathrm{H}(13 \mathrm{~B})$ & 0.9900 \\
\hline $\mathrm{C}(14)-\mathrm{O}(6)$ & $1.432(3)$ \\
\hline$C(14)-C(15)$ & $1.499(3)$ \\
\hline $\mathrm{C}(14)-\mathrm{H}(14)$ & 1.0000 \\
\hline
\end{tabular}




\begin{tabular}{|c|c|}
\hline$C(15)-C(16)$ & $1.334(3)$ \\
\hline $\mathrm{C}(15)-\mathrm{H}(15)$ & 0.9500 \\
\hline$C(16)-C(17)$ & $1.469(3)$ \\
\hline $\mathrm{C}(16)-\mathrm{H}(16)$ & 0.9500 \\
\hline$C(17)-C(19)$ & $1.337(4)$ \\
\hline$C(17)-C(18)$ & $1.501(3)$ \\
\hline $\mathrm{C}(18)-\mathrm{H}(18 \mathrm{~A})$ & 0.9800 \\
\hline $\mathrm{C}(18)-\mathrm{H}(18 \mathrm{~B})$ & 0.9800 \\
\hline $\mathrm{C}(18)-\mathrm{H}(18 \mathrm{C})$ & 0.9800 \\
\hline$C(19)-C(20)$ & $1.500(3)$ \\
\hline C(19)-H(19) & 0.9500 \\
\hline $\mathrm{C}(20)-\mathrm{C}(21)$ & $1.552(3)$ \\
\hline $\mathrm{C}(20)-\mathrm{H}(20 \mathrm{~A})$ & 0.9900 \\
\hline $\mathrm{C}(20)-\mathrm{H}(20 \mathrm{~B})$ & 0.9900 \\
\hline $\mathrm{C}(21)-\mathrm{O}(7)$ & $1.424(3)$ \\
\hline$C(21)-C(22)$ & $1.498(3)$ \\
\hline $\mathrm{C}(21)-\mathrm{H}(21)$ & 1.0000 \\
\hline $\mathrm{C}(22)-\mathrm{C}(23)$ & $1.331(4)$ \\
\hline $\mathrm{C}(22)-\mathrm{H}(22)$ & 0.9500 \\
\hline$C(23)-C(24)$ & $1.469(3)$ \\
\hline $\mathrm{C}(23)-\mathrm{H}(23)$ & 0.9500 \\
\hline$C(24)-C(26)$ & $1.348(3)$ \\
\hline$C(24)-C(25)$ & $1.502(3)$ \\
\hline $\mathrm{C}(25)-\mathrm{H}(25 \mathrm{~A})$ & 0.9800 \\
\hline $\mathrm{C}(25)-\mathrm{H}(25 \mathrm{~B})$ & 0.9800 \\
\hline $\mathrm{C}(25)-\mathrm{H}(25 \mathrm{C})$ & 0.9800 \\
\hline $\mathrm{C}(26)-\mathrm{H}(26)$ & 0.9500 \\
\hline $\mathrm{N}(1)-\mathrm{H}(1 \mathrm{~A})$ & $0.90(4)$ \\
\hline $\mathrm{O}(1)-\mathrm{H}(1)$ & $0.85(4)$ \\
\hline $\mathrm{O}(3)-\mathrm{H}(3)$ & $0.80(4)$ \\
\hline $\mathrm{O}(6)-\mathrm{H}(6)$ & $0.95(4)$ \\
\hline $\mathrm{O}(7)-\mathrm{H}(7)$ & $0.86(4)$ \\
\hline $\mathrm{C}(2)-\mathrm{C}(1)-\mathrm{H}(1 \mathrm{~B})$ & 109.5 \\
\hline $\mathrm{C}(2)-\mathrm{C}(1)-\mathrm{H}(1 \mathrm{C})$ & 109.5 \\
\hline $\mathrm{H}(1 \mathrm{~B})-\mathrm{C}(1)-\mathrm{H}(1 \mathrm{C})$ & 109.5 \\
\hline
\end{tabular}




\begin{tabular}{|c|c|}
\hline $\mathrm{C}(2)-\mathrm{C}(1)-\mathrm{H}(1 \mathrm{D})$ & 109.5 \\
\hline $\mathrm{H}(1 \mathrm{~B})-\mathrm{C}(1)-\mathrm{H}(1 \mathrm{D})$ & 109.5 \\
\hline $\mathrm{H}(1 \mathrm{C})-\mathrm{C}(1)-\mathrm{H}(1 \mathrm{D})$ & 109.5 \\
\hline $\mathrm{O}(1)-\mathrm{C}(2)-\mathrm{C}(1)$ & $111.8(2)$ \\
\hline $\mathrm{O}(1)-\mathrm{C}(2)-\mathrm{C}(3)$ & $109.14(17)$ \\
\hline$C(1)-C(2)-C(3)$ & $108.75(19)$ \\
\hline $\mathrm{O}(1)-\mathrm{C}(2)-\mathrm{H}(2)$ & 109.0 \\
\hline $\mathrm{C}(1)-\mathrm{C}(2)-\mathrm{H}(2)$ & 109.0 \\
\hline $\mathrm{C}(3)-\mathrm{C}(2)-\mathrm{H}(2)$ & 109.0 \\
\hline $\mathrm{O}(2)-\mathrm{C}(3)-\mathrm{N}(1)$ & $124.0(2)$ \\
\hline $\mathrm{O}(2)-\mathrm{C}(3)-\mathrm{C}(2)$ & $118.36(19)$ \\
\hline $\mathrm{N}(1)-\mathrm{C}(3)-\mathrm{C}(2)$ & $117.55(19)$ \\
\hline $\mathrm{N}(1)-\mathrm{C}(4)-\mathrm{C}(26)$ & $111.40(17)$ \\
\hline$N(1)-C(4)-C(5)$ & $111.09(18)$ \\
\hline$C(26)-C(4)-C(5)$ & $116.08(17)$ \\
\hline $\mathrm{N}(1)-\mathrm{C}(4)-\mathrm{H}(4)$ & 105.8 \\
\hline $\mathrm{C}(26)-\mathrm{C}(4)-\mathrm{H}(4)$ & 105.8 \\
\hline $\mathrm{C}(5)-\mathrm{C}(4)-\mathrm{H}(4)$ & 105.8 \\
\hline$C(12)-C(5)-C(7)$ & $112.05(18)$ \\
\hline$C(12)-C(5)-C(6)$ & $107.19(17)$ \\
\hline$C(7)-C(5)-C(6)$ & $111.31(18)$ \\
\hline$C(12)-C(5)-C(4)$ & $104.71(17)$ \\
\hline$C(7)-C(5)-C(4)$ & $114.98(17)$ \\
\hline$C(6)-C(5)-C(4)$ & $106.02(17)$ \\
\hline$C(5)-C(6)-H(6 A)$ & 109.5 \\
\hline$C(5)-C(6)-H(6 B)$ & 109.5 \\
\hline$H(6 A)-C(6)-H(6 B)$ & 109.5 \\
\hline$C(5)-C(6)-H(6 C)$ & 109.5 \\
\hline $\mathrm{H}(6 \mathrm{~A})-\mathrm{C}(6)-\mathrm{H}(6 \mathrm{C})$ & 109.5 \\
\hline $\mathrm{H}(6 \mathrm{~B})-\mathrm{C}(6)-\mathrm{H}(6 \mathrm{C})$ & 109.5 \\
\hline $\mathrm{O}(3)-\mathrm{C}(7)-\mathrm{C}(5)$ & 109.99(17) \\
\hline $\mathrm{O}(3)-\mathrm{C}(7)-\mathrm{C}(8)$ & $106.13(17)$ \\
\hline$C(5)-C(7)-C(8)$ & $113.15(18)$ \\
\hline $\mathrm{O}(3)-\mathrm{C}(7)-\mathrm{H}(7 \mathrm{~A})$ & 109.2 \\
\hline $\mathrm{C}(5)-\mathrm{C}(7)-\mathrm{H}(7 \mathrm{~A})$ & 109.2 \\
\hline $\mathrm{C}(8)-\mathrm{C}(7)-\mathrm{H}(7 \mathrm{~A})$ & 109.2 \\
\hline
\end{tabular}




\begin{tabular}{|c|c|}
\hline$C(11)-C(8)-C(9)$ & $111.62(18)$ \\
\hline$C(11)-C(8)-C(10)$ & $109.73(18)$ \\
\hline$C(9)-C(8)-C(10)$ & $108.55(19)$ \\
\hline$C(11)-C(8)-C(7)$ & $104.95(18)$ \\
\hline$C(9)-C(8)-C(7)$ & $114.38(19)$ \\
\hline$C(10)-C(8)-C(7)$ & $107.46(18)$ \\
\hline $\mathrm{C}(8)-\mathrm{C}(9)-\mathrm{H}(9 \mathrm{~A})$ & 109.5 \\
\hline $\mathrm{C}(8)-\mathrm{C}(9)-\mathrm{H}(9 \mathrm{~B})$ & 109.5 \\
\hline $\mathrm{H}(9 \mathrm{~A})-\mathrm{C}(9)-\mathrm{H}(9 \mathrm{~B})$ & 109.5 \\
\hline $\mathrm{C}(8)-\mathrm{C}(9)-\mathrm{H}(9 \mathrm{C})$ & 109.5 \\
\hline $\mathrm{H}(9 \mathrm{~A})-\mathrm{C}(9)-\mathrm{H}(9 \mathrm{C})$ & 109.5 \\
\hline $\mathrm{H}(9 \mathrm{~B})-\mathrm{C}(9)-\mathrm{H}(9 \mathrm{C})$ & 109.5 \\
\hline $\mathrm{C}(8)-\mathrm{C}(10)-\mathrm{H}(10 \mathrm{~A})$ & 109.5 \\
\hline $\mathrm{C}(8)-\mathrm{C}(10)-\mathrm{H}(10 \mathrm{~B})$ & 109.5 \\
\hline $\mathrm{H}(10 \mathrm{~A})-\mathrm{C}(10)-\mathrm{H}(10 \mathrm{~B})$ & 109.5 \\
\hline $\mathrm{C}(8)-\mathrm{C}(10)-\mathrm{H}(10 \mathrm{C})$ & 109.5 \\
\hline $\mathrm{H}(10 \mathrm{~A})-\mathrm{C}(10)-\mathrm{H}(10 \mathrm{C})$ & 109.5 \\
\hline $\mathrm{H}(10 \mathrm{~B})-\mathrm{C}(10)-\mathrm{H}(10 \mathrm{C})$ & 109.5 \\
\hline $\mathrm{O}(4)-\mathrm{C}(11)-\mathrm{C}(13)$ & $104.52(17)$ \\
\hline $\mathrm{O}(4)-\mathrm{C}(11)-\mathrm{C}(8)$ & 109.97(17) \\
\hline $\mathrm{C}(13)-\mathrm{C}(11)-\mathrm{C}(8)$ & $116.99(19)$ \\
\hline $\mathrm{O}(4)-\mathrm{C}(11)-\mathrm{H}(11)$ & 108.4 \\
\hline $\mathrm{C}(13)-\mathrm{C}(11)-\mathrm{H}(11)$ & 108.4 \\
\hline $\mathrm{C}(8)-\mathrm{C}(11)-\mathrm{H}(11)$ & 108.4 \\
\hline $\mathrm{O}(5)-\mathrm{C}(12)-\mathrm{O}(4)$ & $116.9(2)$ \\
\hline $\mathrm{O}(5)-\mathrm{C}(12)-\mathrm{C}(5)$ & $120.3(2)$ \\
\hline $\mathrm{O}(4)-\mathrm{C}(12)-\mathrm{C}(5)$ & $122.80(19)$ \\
\hline $\mathrm{C}(11)-\mathrm{C}(13)-\mathrm{C}(14)$ & $113.41(19)$ \\
\hline $\mathrm{C}(11)-\mathrm{C}(13)-\mathrm{H}(13 \mathrm{~A})$ & 108.9 \\
\hline $\mathrm{C}(14)-\mathrm{C}(13)-\mathrm{H}(13 \mathrm{~A})$ & 108.9 \\
\hline $\mathrm{C}(11)-\mathrm{C}(13)-\mathrm{H}(13 \mathrm{~B})$ & 108.9 \\
\hline $\mathrm{C}(14)-\mathrm{C}(13)-\mathrm{H}(13 \mathrm{~B})$ & 108.9 \\
\hline $\mathrm{H}(13 \mathrm{~A})-\mathrm{C}(13)-\mathrm{H}(13 \mathrm{~B})$ & 107.7 \\
\hline $\mathrm{O}(6)-\mathrm{C}(14)-\mathrm{C}(15)$ & $112.73(19)$ \\
\hline $\mathrm{O}(6)-\mathrm{C}(14)-\mathrm{C}(13)$ & $105.99(18)$ \\
\hline$C(15)-C(14)-C(13)$ & $113.84(18)$ \\
\hline
\end{tabular}




\begin{tabular}{|c|c|}
\hline $\mathrm{O}(6)-\mathrm{C}(14)-\mathrm{H}(14)$ & 108.0 \\
\hline $\mathrm{C}(15)-\mathrm{C}(14)-\mathrm{H}(14)$ & 108.0 \\
\hline $\mathrm{C}(13)-\mathrm{C}(14)-\mathrm{H}(14)$ & 108.0 \\
\hline$C(16)-C(15)-C(14)$ & $124.6(2)$ \\
\hline $\mathrm{C}(16)-\mathrm{C}(15)-\mathrm{H}(15)$ & 117.7 \\
\hline $\mathrm{C}(14)-\mathrm{C}(15)-\mathrm{H}(15)$ & 117.7 \\
\hline$C(15)-C(16)-C(17)$ & $126.8(2)$ \\
\hline $\mathrm{C}(15)-\mathrm{C}(16)-\mathrm{H}(16)$ & 116.6 \\
\hline $\mathrm{C}(17)-\mathrm{C}(16)-\mathrm{H}(16)$ & 116.6 \\
\hline$C(19)-C(17)-C(16)$ & $117.6(2)$ \\
\hline$C(19)-C(17)-C(18)$ & $124.3(2)$ \\
\hline$C(16)-C(17)-C(18)$ & $118.1(2)$ \\
\hline $\mathrm{C}(17)-\mathrm{C}(18)-\mathrm{H}(18 \mathrm{~A})$ & 109.5 \\
\hline $\mathrm{C}(17)-\mathrm{C}(18)-\mathrm{H}(18 \mathrm{~B})$ & 109.5 \\
\hline $\mathrm{H}(18 \mathrm{~A})-\mathrm{C}(18)-\mathrm{H}(18 \mathrm{~B})$ & 109.5 \\
\hline $\mathrm{C}(17)-\mathrm{C}(18)-\mathrm{H}(18 \mathrm{C})$ & 109.5 \\
\hline $\mathrm{H}(18 \mathrm{~A})-\mathrm{C}(18)-\mathrm{H}(18 \mathrm{C})$ & 109.5 \\
\hline $\mathrm{H}(18 \mathrm{~B})-\mathrm{C}(18)-\mathrm{H}(18 \mathrm{C})$ & 109.5 \\
\hline $\mathrm{C}(17)-\mathrm{C}(19)-\mathrm{C}(20)$ & $128.2(2)$ \\
\hline $\mathrm{C}(17)-\mathrm{C}(19)-\mathrm{H}(19)$ & 115.9 \\
\hline $\mathrm{C}(20)-\mathrm{C}(19)-\mathrm{H}(19)$ & 115.9 \\
\hline$C(19)-C(20)-C(21)$ & $111.0(2)$ \\
\hline $\mathrm{C}(19)-\mathrm{C}(20)-\mathrm{H}(20 \mathrm{~A})$ & 109.4 \\
\hline $\mathrm{C}(21)-\mathrm{C}(20)-\mathrm{H}(20 \mathrm{~A})$ & 109.4 \\
\hline $\mathrm{C}(19)-\mathrm{C}(20)-\mathrm{H}(20 \mathrm{~B})$ & 109.4 \\
\hline $\mathrm{C}(21)-\mathrm{C}(20)-\mathrm{H}(20 \mathrm{~B})$ & 109.4 \\
\hline $\mathrm{H}(20 \mathrm{~A})-\mathrm{C}(20)-\mathrm{H}(20 \mathrm{~B})$ & 108.0 \\
\hline $\mathrm{O}(7)-\mathrm{C}(21)-\mathrm{C}(22)$ & $110.0(2)$ \\
\hline $\mathrm{O}(7)-\mathrm{C}(21)-\mathrm{C}(20)$ & $110.31(19)$ \\
\hline $\mathrm{C}(22)-\mathrm{C}(21)-\mathrm{C}(20)$ & $109.6(2)$ \\
\hline $\mathrm{O}(7)-\mathrm{C}(21)-\mathrm{H}(21)$ & 109.0 \\
\hline $\mathrm{C}(22)-\mathrm{C}(21)-\mathrm{H}(21)$ & 109.0 \\
\hline $\mathrm{C}(20)-\mathrm{C}(21)-\mathrm{H}(21)$ & 109.0 \\
\hline $\mathrm{C}(23)-\mathrm{C}(22)-\mathrm{C}(21)$ & $124.6(2)$ \\
\hline $\mathrm{C}(23)-\mathrm{C}(22)-\mathrm{H}(22)$ & 117.7 \\
\hline $\mathrm{C}(21)-\mathrm{C}(22)-\mathrm{H}(22)$ & 117.7 \\
\hline
\end{tabular}




\begin{tabular}{ll}
$\mathrm{C}(22)-\mathrm{C}(23)-\mathrm{C}(24)$ & $125.6(2)$ \\
$\mathrm{C}(22)-\mathrm{C}(23)-\mathrm{H}(23)$ & 117.2 \\
$\mathrm{C}(24)-\mathrm{C}(23)-\mathrm{H}(23)$ & 117.2 \\
$\mathrm{C}(26)-\mathrm{C}(24)-\mathrm{C}(23)$ & $117.5(2)$ \\
$\mathrm{C}(26)-\mathrm{C}(24)-\mathrm{C}(25)$ & $125.3(2)$ \\
$\mathrm{C}(23)-\mathrm{C}(24)-\mathrm{C}(25)$ & $117.2(2)$ \\
$\mathrm{C}(24)-\mathrm{C}(25)-\mathrm{H}(25 \mathrm{~A})$ & 109.5 \\
$\mathrm{C}(24)-\mathrm{C}(25)-\mathrm{H}(25 \mathrm{~B})$ & 109.5 \\
$\mathrm{H}(25 \mathrm{~A})-\mathrm{C}(25)-\mathrm{H}(25 \mathrm{~B})$ & 109.5 \\
$\mathrm{C}(24)-\mathrm{C}(25)-\mathrm{H}(25 \mathrm{C})$ & 109.5 \\
$\mathrm{H}(25 \mathrm{~A})-\mathrm{C}(25)-\mathrm{H}(25 \mathrm{C})$ & 109.5 \\
$\mathrm{H}(25 \mathrm{~B})-\mathrm{C}(25)-\mathrm{H}(25 \mathrm{C})$ & 109.5 \\
$\mathrm{C}(24)-\mathrm{C}(26)-\mathrm{C}(4)$ & $126.44(19)$ \\
$\mathrm{C}(24)-\mathrm{C}(26)-\mathrm{H}(26)$ & 116.8 \\
$\mathrm{C}(4)-\mathrm{C}(26)-\mathrm{H}(26)$ & 116.8 \\
$\mathrm{C}(3)-\mathrm{N}(1)-\mathrm{C}(4)$ & $121.41(18)$ \\
$\mathrm{C}(3)-\mathrm{N}(1)-\mathrm{H}(1 \mathrm{~A})$ & $119(2)$ \\
$\mathrm{C}(4)-\mathrm{N}(1)-\mathrm{H}(1 \mathrm{~A})$ & $119(2)$ \\
$\mathrm{C}(2)-\mathrm{O}(1)-\mathrm{H}(1)$ & $106(2)$ \\
$\mathrm{C}(7)-\mathrm{O}(3)-\mathrm{H}(3)$ & $108(3)$ \\
$\mathrm{C}(12)-\mathrm{O}(4)-\mathrm{C}(11)$ & $123.27(17)$ \\
$\mathrm{C}(14)-\mathrm{O}(6)-\mathrm{H}(6)$ & $110(2)$ \\
$\mathrm{C}(21)-\mathrm{O}(7)-\mathrm{H}(7)$ & $104(2)$ \\
\hline & \\
&
\end{tabular}

Symmetry transformations used to generate equivalent atoms: 
Table 4. Anisotropic displacement parameters $\left(\AA^{2} \times 10^{3}\right)$ for seiple004_ucsf. The anisotropic displacement factor exponent takes the form: $-2 \pi^{2}\left[h^{2} a^{* 2} U^{11}+\ldots+2 h k a^{*} b^{*} U^{12}\right]$

\begin{tabular}{|c|c|c|c|c|c|c|}
\hline & $\mathrm{U}^{11}$ & $\mathrm{U}^{22}$ & $\mathrm{U}^{33}$ & $\mathrm{U}^{23}$ & $\mathrm{U}^{13}$ & $\mathrm{U}^{12}$ \\
\hline $\mathrm{C}(1)$ & $28(1)$ & $28(1)$ & $33(1)$ & $-5(1)$ & $8(1)$ & $-2(1)$ \\
\hline $\mathrm{C}(2)$ & $16(1)$ & $24(1)$ & $20(1)$ & $0(1)$ & $1(1)$ & $-1(1)$ \\
\hline$C(3)$ & $17(1)$ & $19(1)$ & $22(1)$ & $1(1)$ & $-1(1)$ & $-1(1)$ \\
\hline$C(4)$ & $14(1)$ & $21(1)$ & $21(1)$ & $0(1)$ & $2(1)$ & $2(1)$ \\
\hline $\mathrm{C}(5)$ & $13(1)$ & $19(1)$ & $22(1)$ & $3(1)$ & $1(1)$ & $2(1)$ \\
\hline$C(6)$ & $23(1)$ & $23(1)$ & $23(1)$ & $4(1)$ & $-1(1)$ & $3(1)$ \\
\hline$C(7)$ & $12(1)$ & $21(1)$ & $27(1)$ & $3(1)$ & $1(1)$ & $2(1)$ \\
\hline $\mathrm{C}(8)$ & $15(1)$ & $19(1)$ & $26(1)$ & $2(1)$ & $-2(1)$ & $1(1)$ \\
\hline $\mathrm{C}(9)$ & $25(1)$ & $20(1)$ & $29(1)$ & $2(1)$ & $-1(1)$ & $3(1)$ \\
\hline $\mathrm{C}(10)$ & $17(1)$ & $25(1)$ & $33(1)$ & $1(1)$ & $-2(1)$ & $-3(1)$ \\
\hline $\mathrm{C}(11)$ & $13(1)$ & $20(1)$ & $24(1)$ & $-1(1)$ & $-2(1)$ & $-1(1)$ \\
\hline $\mathrm{C}(12)$ & $14(1)$ & $20(1)$ & $24(1)$ & $0(1)$ & $0(1)$ & $3(1)$ \\
\hline $\mathrm{C}(13)$ & $16(1)$ & $19(1)$ & $26(1)$ & $-2(1)$ & $-1(1)$ & $2(1)$ \\
\hline$C(14)$ & $17(1)$ & $22(1)$ & $23(1)$ & $-2(1)$ & $-1(1)$ & $2(1)$ \\
\hline $\mathrm{C}(15)$ & $16(1)$ & $24(1)$ & $24(1)$ & $1(1)$ & $0(1)$ & $-1(1)$ \\
\hline$C(16)$ & $16(1)$ & $25(1)$ & $22(1)$ & $0(1)$ & $-1(1)$ & $-1(1)$ \\
\hline $\mathrm{C}(17)$ & $20(1)$ & $25(1)$ & $24(1)$ & $2(1)$ & $-1(1)$ & $-2(1)$ \\
\hline $\mathrm{C}(18)$ & $29(1)$ & $27(1)$ & $47(2)$ & $5(1)$ & $-15(1)$ & $-6(1)$ \\
\hline$C(19)$ & $21(1)$ & $26(1)$ & $24(1)$ & $0(1)$ & $2(1)$ & $-3(1)$ \\
\hline $\mathrm{C}(20)$ & $27(1)$ & $21(1)$ & $29(1)$ & $4(1)$ & $0(1)$ & $-4(1)$ \\
\hline $\mathrm{C}(21)$ & $30(1)$ & $18(1)$ & $26(1)$ & $2(1)$ & $-3(1)$ & $-2(1)$ \\
\hline $\mathrm{C}(22)$ & $25(1)$ & $19(1)$ & $25(1)$ & $1(1)$ & $-3(1)$ & $1(1)$ \\
\hline $\mathrm{C}(23)$ & $21(1)$ & $18(1)$ & $26(1)$ & $-1(1)$ & $-2(1)$ & $3(1)$ \\
\hline $\mathrm{C}(24)$ & $19(1)$ & $21(1)$ & $22(1)$ & $-1(1)$ & $0(1)$ & $2(1)$ \\
\hline$C(25)$ & $23(1)$ & $24(1)$ & $28(1)$ & $4(1)$ & $-5(1)$ & $-3(1)$ \\
\hline$C(26)$ & $16(1)$ & $20(1)$ & $22(1)$ & $-1(1)$ & $-1(1)$ & $3(1)$ \\
\hline $\mathrm{N}(1)$ & $13(1)$ & $22(1)$ & $20(1)$ & $1(1)$ & $1(1)$ & $2(1)$ \\
\hline $\mathrm{O}(1)$ & $14(1)$ & $30(1)$ & $26(1)$ & $-2(1)$ & $1(1)$ & $-1(1)$ \\
\hline $\mathrm{O}(2)$ & $17(1)$ & $34(1)$ & $23(1)$ & $1(1)$ & $-2(1)$ & $0(1)$ \\
\hline $\mathrm{O}(3)$ & $13(1)$ & $22(1)$ & $31(1)$ & $4(1)$ & $-1(1)$ & $3(1)$ \\
\hline $\mathrm{O}(4)$ & $13(1)$ & $26(1)$ & $22(1)$ & $-3(1)$ & $-1(1)$ & $-2(1)$ \\
\hline
\end{tabular}




\begin{tabular}{lllllll}
$\mathrm{O}(5)$ & $13(1)$ & $32(1)$ & $26(1)$ & $-6(1)$ & $-1(1)$ & $1(1)$ \\
$\mathrm{O}(6)$ & $19(1)$ & $22(1)$ & $24(1)$ & $0(1)$ & $-6(1)$ & $-1(1)$ \\
$\mathrm{O}(7)$ & $29(1)$ & $23(1)$ & $30(1)$ & $6(1)$ & $-2(1)$ & $5(1)$ \\
\hline
\end{tabular}

Table 5. Hydrogen coordinates ( $\left.\times 10^{4}\right)$ and isotropic displacement parameters $\left(\AA^{2} \times 10^{3}\right)$ for seiple004_ucsf.

\begin{tabular}{|c|c|c|c|c|}
\hline & $\mathrm{x}$ & $y$ & $\mathrm{z}$ & $\mathrm{U}(\mathrm{eq})$ \\
\hline $\mathrm{H}(1 \mathrm{~B})$ & 9585 & 4233 & 1272 & 44 \\
\hline $\mathrm{H}(1 \mathrm{C})$ & 7103 & 4094 & 1291 & 44 \\
\hline $\mathrm{H}(1 \mathrm{D})$ & 8568 & 3964 & 2444 & 44 \\
\hline $\mathrm{H}(2)$ & 7862 & 5442 & 1355 & 24 \\
\hline $\mathrm{H}(4)$ & 3238 & 5129 & 4119 & 22 \\
\hline $\mathrm{H}(6 \mathrm{~A})$ & 2253 & 6401 & 3776 & 35 \\
\hline $\mathrm{H}(6 \mathrm{~B})$ & 4669 & 6643 & 3705 & 35 \\
\hline $\mathrm{H}(6 \mathrm{C})$ & 3178 & 7114 & 4527 & 35 \\
\hline $\mathrm{H}(7 \mathrm{~A})$ & 6950 & 6645 & 5495 & 24 \\
\hline $\mathrm{H}(9 \mathrm{~A})$ & 2698 & 7505 & 6228 & 37 \\
\hline $\mathrm{H}(9 \mathrm{~B})$ & 4889 & 7880 & 5903 & 37 \\
\hline $\mathrm{H}(9 \mathrm{C})$ & 4024 & 8016 & 7163 & 37 \\
\hline $\mathrm{H}(10 \mathrm{~A})$ & 6901 & 7556 & 8251 & 38 \\
\hline $\mathrm{H}(10 \mathrm{~B})$ & 8280 & 7384 & 7161 & 38 \\
\hline $\mathrm{H}(10 \mathrm{C})$ & 7890 & 6714 & 8082 & 38 \\
\hline $\mathrm{H}(11)$ & 4744 & 5967 & 8012 & 23 \\
\hline $\mathrm{H}(13 \mathrm{~A})$ & 1618 & 7071 & 8481 & 24 \\
\hline $\mathrm{H}(13 \mathrm{~B})$ & 3927 & 7223 & 9048 & 24 \\
\hline $\mathrm{H}(14)$ & 1663 & 6542 & 10309 & 25 \\
\hline $\mathrm{H}(15)$ & -85 & 5622 & 9016 & 26 \\
\hline $\mathrm{H}(16)$ & 3379 & 4764 & 9817 & 25 \\
\hline $\mathrm{H}(18 \mathrm{~A})$ & -2138 & 4496 & 8960 & 52 \\
\hline $\mathrm{H}(18 \mathrm{~B})$ & -1832 & 3680 & 8333 & 52 \\
\hline $\mathrm{H}(18 \mathrm{C})$ & -1100 & 4462 & 7730 & 52 \\
\hline $\mathrm{H}(19)$ & 3208 & 3469 & 9700 & 28 \\
\hline $\mathrm{H}(20 \mathrm{~A})$ & -277 & 2663 & 8596 & 31 \\
\hline
\end{tabular}




\begin{tabular}{lclll}
$\mathrm{H}(20 \mathrm{~B})$ & 1385 & 2283 & 9519 & 31 \\
$\mathrm{H}(21)$ & 2101 & 1829 & 7633 & 30 \\
$\mathrm{H}(22)$ & 909 & 2845 & 6389 & 28 \\
$\mathrm{H}(23)$ & 4937 & 3410 & 6882 & 26 \\
$\mathrm{H}(25 \mathrm{~A})$ & -24 & 4045 & 5354 & 38 \\
$\mathrm{H}(25 \mathrm{~B})$ & 1000 & 3407 & 4552 & 38 \\
$\mathrm{H}(25 \mathrm{C})$ & 1151 & 4305 & 4227 & 38 \\
$\mathrm{H}(26)$ & 5901 & 4504 & 5949 & 23 \\
$\mathrm{H}(1)$ & $10880(60)$ & $5250(20)$ & $2450(30)$ & $33(9)$ \\
$\mathrm{H}(1 \mathrm{~A})$ & $7610(60)$ & $5310(20)$ & $4240(30)$ & $29(8)$ \\
$\mathrm{H}(3)$ & $8220(70)$ & $5720(30)$ & $6320(30)$ & $46(11)$ \\
$\mathrm{H}(6)$ & $4340(50)$ & $5710(20)$ & $10890(30)$ & $32(8)$ \\
$\mathrm{H}(7)$ & $4840(50)$ & $1960(20)$ & $8680(30)$ & $31(8)$ \\
\hline
\end{tabular}

Table 6. Torsion angles $\left[^{\circ}\right]$ for seiple004_ucsf.

\begin{tabular}{lc}
\hline $\mathrm{O}(1)-\mathrm{C}(2)-\mathrm{C}(3)-\mathrm{O}(2)$ & $162.9(2)$ \\
$\mathrm{C}(1)-\mathrm{C}(2)-\mathrm{C}(3)-\mathrm{O}(2)$ & $-74.9(3)$ \\
$\mathrm{O}(1)-\mathrm{C}(2)-\mathrm{C}(3)-\mathrm{N}(1)$ & $-19.6(3)$ \\
$\mathrm{C}(1)-\mathrm{C}(2)-\mathrm{C}(3)-\mathrm{N}(1)$ & $102.7(2)$ \\
$\mathrm{N}(1)-\mathrm{C}(4)-\mathrm{C}(5)-\mathrm{C}(12)$ & $177.38(17)$ \\
$\mathrm{C}(26)-\mathrm{C}(4)-\mathrm{C}(5)-\mathrm{C}(12)$ & $-54.0(2)$ \\
$\mathrm{N}(1)-\mathrm{C}(4)-\mathrm{C}(5)-\mathrm{C}(7)$ & $-59.2(2)$ \\
$\mathrm{C}(26)-\mathrm{C}(4)-\mathrm{C}(5)-\mathrm{C}(7)$ & $69.4(2)$ \\
$\mathrm{N}(1)-\mathrm{C}(4)-\mathrm{C}(5)-\mathrm{C}(6)$ & $64.2(2)$ \\
$\mathrm{C}(26)-\mathrm{C}(4)-\mathrm{C}(5)-\mathrm{C}(6)$ & $-167.16(18)$ \\
$\mathrm{C}(12)-\mathrm{C}(5)-\mathrm{C}(7)-\mathrm{O}(3)$ & $85.2(2)$ \\
$\mathrm{C}(6)-\mathrm{C}(5)-\mathrm{C}(7)-\mathrm{O}(3)$ & $-154.77(18)$ \\
$\mathrm{C}(4)-\mathrm{C}(5)-\mathrm{C}(7)-\mathrm{O}(3)$ & $-34.2(2)$ \\
$\mathrm{C}(12)-\mathrm{C}(5)-\mathrm{C}(7)-\mathrm{C}(8)$ & $-33.3(2)$ \\
$\mathrm{C}(6)-\mathrm{C}(5)-\mathrm{C}(7)-\mathrm{C}(8)$ & $86.7(2)$ \\
$\mathrm{C}(4)-\mathrm{C}(5)-\mathrm{C}(7)-\mathrm{C}(8)$ & $-152.69(18)$ \\
$\mathrm{O}(3)-\mathrm{C}(7)-\mathrm{C}(8)-\mathrm{C}(11)$ & $-59.3(2)$ \\
$\mathrm{C}(5)-\mathrm{C}(7)-\mathrm{C}(8)-\mathrm{C}(11)$ & $61.4(2)$ \\
$\mathrm{O}(3)-\mathrm{C}(7)-\mathrm{C}(8)-\mathrm{C}(9)$ & $178.03(18)$ \\
$\mathrm{C}(5)-\mathrm{C}(7)-\mathrm{C}(8)-\mathrm{C}(9)$ & $-61.3(2)$ \\
&
\end{tabular}




\begin{tabular}{|c|c|}
\hline $\mathrm{O}(3)-\mathrm{C}(7)-\mathrm{C}(8)-\mathrm{C}(10)$ & $57.5(2)$ \\
\hline$C(5)-C(7)-C(8)-C(10)$ & $178.16(18)$ \\
\hline $\mathrm{C}(9)-\mathrm{C}(8)-\mathrm{C}(11)-\mathrm{O}(4)$ & $64.2(2)$ \\
\hline $\mathrm{C}(10)-\mathrm{C}(8)-\mathrm{C}(11)-\mathrm{O}(4)$ & $-175.38(18)$ \\
\hline $\mathrm{C}(7)-\mathrm{C}(8)-\mathrm{C}(11)-\mathrm{O}(4)$ & $-60.2(2)$ \\
\hline $\mathrm{C}(9)-\mathrm{C}(8)-\mathrm{C}(11)-\mathrm{C}(13)$ & $-54.7(3)$ \\
\hline$C(10)-C(8)-C(11)-C(13)$ & $65.6(2)$ \\
\hline $\mathrm{C}(7)-\mathrm{C}(8)-\mathrm{C}(11)-\mathrm{C}(13)$ & $-179.16(18)$ \\
\hline $\mathrm{C}(7)-\mathrm{C}(5)-\mathrm{C}(12)-\mathrm{O}(5)$ & $-179.2(2)$ \\
\hline $\mathrm{C}(6)-\mathrm{C}(5)-\mathrm{C}(12)-\mathrm{O}(5)$ & $58.4(3)$ \\
\hline $\mathrm{C}(4)-\mathrm{C}(5)-\mathrm{C}(12)-\mathrm{O}(5)$ & $-53.9(3)$ \\
\hline $\mathrm{C}(7)-\mathrm{C}(5)-\mathrm{C}(12)-\mathrm{O}(4)$ & $3.8(3)$ \\
\hline $\mathrm{C}(6)-\mathrm{C}(5)-\mathrm{C}(12)-\mathrm{O}(4)$ & $-118.6(2)$ \\
\hline $\mathrm{C}(4)-\mathrm{C}(5)-\mathrm{C}(12)-\mathrm{O}(4)$ & $129.0(2)$ \\
\hline $\mathrm{O}(4)-\mathrm{C}(11)-\mathrm{C}(13)-\mathrm{C}(14)$ & $85.1(2)$ \\
\hline $\mathrm{C}(8)-\mathrm{C}(11)-\mathrm{C}(13)-\mathrm{C}(14)$ & $-153.04(19)$ \\
\hline $\mathrm{C}(11)-\mathrm{C}(13)-\mathrm{C}(14)-\mathrm{O}(6)$ & $74.3(2)$ \\
\hline$C(11)-C(13)-C(14)-C(15)$ & $-50.2(3)$ \\
\hline $\mathrm{O}(6)-\mathrm{C}(14)-\mathrm{C}(15)-\mathrm{C}(16)$ & $-3.2(3)$ \\
\hline $\mathrm{C}(13)-\mathrm{C}(14)-\mathrm{C}(15)-\mathrm{C}(16)$ & $117.6(2)$ \\
\hline $\mathrm{C}(14)-\mathrm{C}(15)-\mathrm{C}(16)-\mathrm{C}(17)$ & $-178.5(2)$ \\
\hline $\mathrm{C}(15)-\mathrm{C}(16)-\mathrm{C}(17)-\mathrm{C}(19)$ & $-179.8(2)$ \\
\hline$C(15)-C(16)-C(17)-C(18)$ & $1.7(4)$ \\
\hline$C(16)-C(17)-C(19)-C(20)$ & $-174.0(2)$ \\
\hline $\mathrm{C}(18)-\mathrm{C}(17)-\mathrm{C}(19)-\mathrm{C}(20)$ & $4.4(4)$ \\
\hline $\mathrm{C}(17)-\mathrm{C}(19)-\mathrm{C}(20)-\mathrm{C}(21)$ & $106.4(3)$ \\
\hline $\mathrm{C}(19)-\mathrm{C}(20)-\mathrm{C}(21)-\mathrm{O}(7)$ & $58.3(3)$ \\
\hline$C(19)-C(20)-C(21)-C(22)$ & $-62.9(3)$ \\
\hline $\mathrm{O}(7)-\mathrm{C}(21)-\mathrm{C}(22)-\mathrm{C}(23)$ & $-16.0(3)$ \\
\hline $\mathrm{C}(20)-\mathrm{C}(21)-\mathrm{C}(22)-\mathrm{C}(23)$ & $105.4(3)$ \\
\hline$C(21)-C(22)-C(23)-C(24)$ & $-172.4(2)$ \\
\hline $\mathrm{C}(22)-\mathrm{C}(23)-\mathrm{C}(24)-\mathrm{C}(26)$ & $170.7(2)$ \\
\hline $\mathrm{C}(22)-\mathrm{C}(23)-\mathrm{C}(24)-\mathrm{C}(25)$ & $-7.8(3)$ \\
\hline $\mathrm{C}(23)-\mathrm{C}(24)-\mathrm{C}(26)-\mathrm{C}(4)$ & $-175.2(2)$ \\
\hline$C(25)-C(24)-C(26)-C(4)$ & $3.1(4)$ \\
\hline $\mathrm{N}(1)-\mathrm{C}(4)-\mathrm{C}(26)-\mathrm{C}(24)$ & $-125.2(2)$ \\
\hline
\end{tabular}




$\begin{array}{lc}\mathrm{C}(5)-\mathrm{C}(4)-\mathrm{C}(26)-\mathrm{C}(24) & 106.4(3) \\ \mathrm{O}(2)-\mathrm{C}(3)-\mathrm{N}(1)-\mathrm{C}(4) & 5.0(4) \\ \mathrm{C}(2)-\mathrm{C}(3)-\mathrm{N}(1)-\mathrm{C}(4) & -172.36(19) \\ \mathrm{C}(26)-\mathrm{C}(4)-\mathrm{N}(1)-\mathrm{C}(3) & 116.7(2) \\ \mathrm{C}(5)-\mathrm{C}(4)-\mathrm{N}(1)-\mathrm{C}(3) & -112.2(2) \\ \mathrm{O}(5)-\mathrm{C}(12)-\mathrm{O}(4)-\mathrm{C}(11) & 177.8(2) \\ \mathrm{C}(5)-\mathrm{C}(12)-\mathrm{O}(4)-\mathrm{C}(11) & -5.0(3) \\ \mathrm{C}(13)-\mathrm{C}(11)-\mathrm{O}(4)-\mathrm{C}(12) & 161.63(19) \\ \mathrm{C}(8)-\mathrm{C}(11)-\mathrm{O}(4)-\mathrm{C}(12) & 35.3(3)\end{array}$

Symmetry transformations used to generate equivalent atoms:

Compound 28 : CCDC 1965635
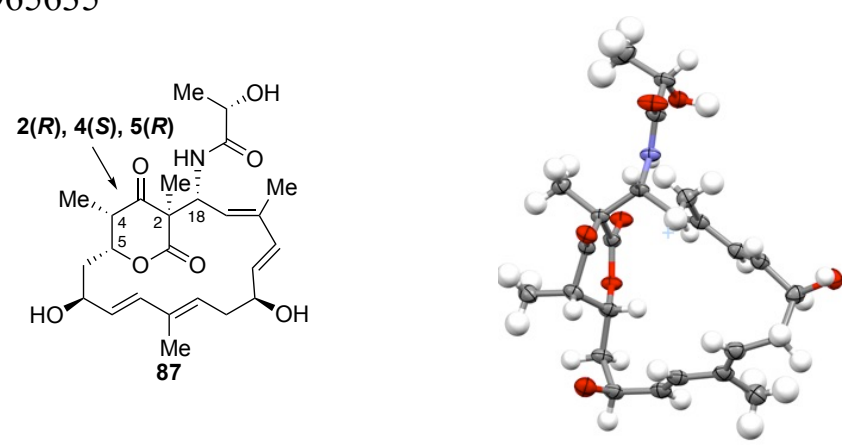

Table 1. Crystal data and structure refinement for Seiple002_UCSF.

Identification code

Empirical formula

Formula weight

Temperature

Wavelength

Crystal system

Space group

Unit cell dimensions

Volume

Z

Density (calculated)

Absorption coefficient

$\mathrm{F}(000)$
Seiple002_UCSF

C25 H35 N O7

461.54

100(2) K

$1.54184 \AA$

Monoclinic

P 21

$\mathrm{a}=6.41830(10) \AA$

$\alpha=90^{\circ}$.

$\mathrm{b}=12.4198(2) \AA$

$\beta=95.841(2)^{\circ}$.

$\mathrm{c}=15.6914(3) \AA$

$\gamma=90^{\circ}$.

1244.33(4) $\AA^{3}$

2

$1.232 \mathrm{Mg} / \mathrm{m}^{3}$

$0.735 \mathrm{~mm}^{-1}$

496 
Crystal size

Theta range for data collection

Index ranges

Reflections collected

Independent reflections

Completeness to theta $=74.000^{\circ}$

Absorption correction

Max. and min. transmission

Refinement method

Data / restraints / parameters

Goodness-of-fit on $\mathrm{F}^{2}$

Final $R$ indices [I $>2 \operatorname{sigma}(\mathrm{I})]$

$\mathrm{R}$ indices (all data)

Absolute structure parameter

Extinction coefficient

Largest diff. peak and hole
$0.290 \times 0.250 \times 0.050 \mathrm{~mm}^{3}$

2.831 to $74.433^{\circ}$.

$-8<=\mathrm{h}<=8,-15<=\mathrm{k}<=15,-19<=\mathrm{l}<=19$

25675

$5068[\mathrm{R}($ int $)=0.0662]$

$100.0 \%$

Semi-empirical from equivalents

1.00000 and 0.64489

Full-matrix least-squares on $\mathrm{F}^{2}$

$5068 / 1 / 315$

1.094

$\mathrm{R} 1=0.0527, \mathrm{wR} 2=0.1431$

$\mathrm{R} 1=0.0576, \mathrm{wR} 2=0.1491$

$-0.01(13)$

n/a

0.365 and -0.302 e. $\AA^{-3}$

Table 2. Atomic coordinates $\left(\mathrm{x} 10^{4}\right)$ and equivalent isotropic displacement parameters $\left(\AA^{2} \times 10^{3}\right)$ for seiple002_ucsf. $U(\mathrm{eq})$ is defined as one third of the trace of the orthogonalized $U^{\mathrm{ij}}$ tensor.

\begin{tabular}{|c|c|c|c|c|}
\hline & $\mathrm{x}$ & $\mathrm{y}$ & $\mathrm{z}$ & $\mathrm{U}(\mathrm{eq})$ \\
\hline $\mathrm{C}(1)$ & $194(7)$ & $829(4)$ & $8226(4)$ & $54(1)$ \\
\hline $\mathrm{C}(2)$ & $1420(6)$ & $1277(3)$ & $7537(3)$ & $38(1)$ \\
\hline $\mathrm{C}(3)$ & $799(6)$ & $2445(3)$ & $7373(2)$ & $35(1)$ \\
\hline$C(4)$ & $1670(5)$ & $4335(3)$ & $7633(2)$ & $29(1)$ \\
\hline$C(5)$ & $2023(5)$ & $4905(3)$ & $8514(2)$ & $29(1)$ \\
\hline$C(6)$ & $732(6)$ & $4340(3)$ & $9161(2)$ & $35(1)$ \\
\hline$C(7)$ & $4344(5)$ & $4855(3)$ & $8849(2)$ & $29(1)$ \\
\hline $\mathrm{C}(8)$ & $4671(5)$ & $6795(2)$ & $8669(2)$ & $31(1)$ \\
\hline $\mathrm{C}(9)$ & $2413(5)$ & $6938(3)$ & $8866(2)$ & $34(1)$ \\
\hline$C(10)$ & $2172(6)$ & $6937(4)$ & $9827(3)$ & $42(1)$ \\
\hline $\mathrm{C}(11)$ & $1157(5)$ & $6052(3)$ & $8396(2)$ & $30(1)$ \\
\hline$C(12)$ & $6223(6)$ & $7624(3)$ & $9076(2)$ & $34(1)$ \\
\hline$C(13)$ & $6169(6)$ & $8711(3)$ & $8606(2)$ & $37(1)$ \\
\hline$C(14)$ & $6773(6)$ & 8591(3) & $7720(2)$ & $39(1)$ \\
\hline$C(15)$ & $5520(6)$ & $8682(3)$ & $7006(3)$ & $41(1)$ \\
\hline
\end{tabular}




\begin{tabular}{lllll}
$\mathrm{C}(16)$ & $6026(6)$ & $8503(3)$ & $6129(2)$ & $39(1)$ \\
$\mathrm{C}(17)$ & $8331(7)$ & $8446(4)$ & $5998(3)$ & $50(1)$ \\
$\mathrm{C}(18)$ & $4509(7)$ & $8376(3)$ & $5501(3)$ & $42(1)$ \\
$\mathrm{C}(19)$ & $4660(7)$ & $8056(3)$ & $4590(3)$ & $44(1)$ \\
$\mathrm{C}(20)$ & $4680(6)$ & $6818(3)$ & $4459(2)$ & $38(1)$ \\
$\mathrm{C}(21)$ & $3087(6)$ & $6281(3)$ & $4943(2)$ & $34(1)$ \\
$\mathrm{C}(22)$ & $3582(5)$ & $5752(3)$ & $5675(2)$ & $32(1)$ \\
$\mathrm{C}(23)$ & $2125(5)$ & $5284(3)$ & $6224(2)$ & $31(1)$ \\
$\mathrm{C}(24)$ & $-177(6)$ & $5318(3)$ & $5926(2)$ & $38(1)$ \\
$\mathrm{C}(25)$ & $2913(5)$ & $4841(3)$ & $6977(2)$ & $30(1)$ \\
$\mathrm{N}(1)$ & $2141(5)$ & $3186(2)$ & $7712(2)$ & $31(1)$ \\
$\mathrm{O}(1)$ & $1158(2)$ & $7801(2)$ & $37(1)$ \\
$\mathrm{O}(2)$ & $3583(4)$ & $2677(2)$ & $6970(2)$ & $54(1)$ \\
$\mathrm{O}(3)$ & $-891(5)$ & $3995(2)$ & $9002(2)$ & $36(1)$ \\
$\mathrm{O}(4)$ & $5193(4)$ & $5753(2)$ & $8993(2)$ & $32(1)$ \\
$\mathrm{O}(5)$ & $5485(3)$ & $6217(2)$ & $7954(2)$ & $37(1)$ \\
$\mathrm{O}(6)$ & $-465(4)$ & $8170(2)$ & $8650(2)$ & $46(1)$ \\
$\mathrm{O}(7)$ & $4145(5)$ & $6570(2)$ & $3561(2)$ & $38(1)$ \\
\hline
\end{tabular}

Table 3. Bond lengths $[\AA]$ and angles $\left[{ }^{\circ}\right]$ for seiple 002 ucsf.

\begin{tabular}{ll}
\hline $\mathrm{C}(1)-\mathrm{C}(2)$ & $1.507(6)$ \\
$\mathrm{C}(1)-\mathrm{H}(1 \mathrm{~B})$ & 0.9800 \\
$\mathrm{C}(1)-\mathrm{H}(1 \mathrm{C})$ & 0.9800 \\
$\mathrm{C}(1)-\mathrm{H}(1 \mathrm{D})$ & 0.9800 \\
$\mathrm{C}(2)-\mathrm{O}(1)$ & $1.415(5)$ \\
$\mathrm{C}(2)-\mathrm{C}(3)$ & $1.519(5)$ \\
$\mathrm{C}(2)-\mathrm{H}(2)$ & 1.0000 \\
$\mathrm{C}(3)-\mathrm{O}(2)$ & $1.233(5)$ \\
$\mathrm{C}(3)-\mathrm{N}(1)$ & $1.333(5)$ \\
$\mathrm{C}(4)-\mathrm{N}(1)$ & $1.462(4)$ \\
$\mathrm{C}(4)-\mathrm{C}(25)$ & $1.502(5)$ \\
$\mathrm{C}(4)-\mathrm{C}(5)$ & $1.550(4)$ \\
$\mathrm{C}(4)-\mathrm{H}(4)$ & 1.0000 \\
$\mathrm{C}(5)-\mathrm{C}(7)$ & $1.529(4)$ \\
$\mathrm{C}(5)-\mathrm{C}(11)$ & $1.534(4)$
\end{tabular}




\begin{tabular}{|c|c|}
\hline$C(5)-C(6)$ & $1.543(5)$ \\
\hline $\mathrm{C}(6)-\mathrm{H}(6 \mathrm{~A})$ & 0.9800 \\
\hline $\mathrm{C}(6)-\mathrm{H}(6 \mathrm{~B})$ & 0.9800 \\
\hline $\mathrm{C}(6)-\mathrm{H}(6 \mathrm{C})$ & 0.9800 \\
\hline $\mathrm{C}(7)-\mathrm{O}(3)$ & $1.213(4)$ \\
\hline $\mathrm{C}(7)-\mathrm{O}(4)$ & $1.340(4)$ \\
\hline $\mathrm{C}(8)-\mathrm{O}(4)$ & $1.467(4)$ \\
\hline $\mathrm{C}(8)-\mathrm{C}(9)$ & $1.522(5)$ \\
\hline$C(8)-C(12)$ & $1.526(4)$ \\
\hline $\mathrm{C}(8)-\mathrm{H}(8)$ & 1.0000 \\
\hline$C(9)-C(11)$ & $1.510(5)$ \\
\hline$C(9)-C(10)$ & $1.532(5)$ \\
\hline $\mathrm{C}(9)-\mathrm{H}(9)$ & 1.0000 \\
\hline $\mathrm{C}(10)-\mathrm{H}(10 \mathrm{~A})$ & 0.9800 \\
\hline $\mathrm{C}(10)-\mathrm{H}(10 \mathrm{~B})$ & 0.9800 \\
\hline $\mathrm{C}(10)-\mathrm{H}(10 \mathrm{C})$ & 0.9800 \\
\hline $\mathrm{C}(11)-\mathrm{O}(5)$ & $1.209(4)$ \\
\hline$C(12)-C(13)$ & $1.536(5)$ \\
\hline $\mathrm{C}(12)-\mathrm{H}(12 \mathrm{~A})$ & 0.9900 \\
\hline $\mathrm{C}(12)-\mathrm{H}(12 \mathrm{~B})$ & 0.9900 \\
\hline $\mathrm{C}(13)-\mathrm{O}(6)$ & $1.426(5)$ \\
\hline$C(13)-C(14)$ & $1.488(6)$ \\
\hline $\mathrm{C}(13)-\mathrm{H}(13)$ & 1.0000 \\
\hline$C(14)-C(15)$ & $1.316(6)$ \\
\hline $\mathrm{C}(14)-\mathrm{H}(14)$ & 0.9500 \\
\hline$C(15)-C(16)$ & $1.462(6)$ \\
\hline $\mathrm{C}(15)-\mathrm{H}(15)$ & 0.9500 \\
\hline$C(16)-C(18)$ & $1.323(6)$ \\
\hline$C(16)-C(17)$ & $1.516(6)$ \\
\hline $\mathrm{C}(17)-\mathrm{H}(17 \mathrm{~A})$ & 0.9800 \\
\hline $\mathrm{C}(17)-\mathrm{H}(17 \mathrm{~B})$ & 0.9800 \\
\hline $\mathrm{C}(17)-\mathrm{H}(17 \mathrm{C})$ & 0.9800 \\
\hline $\mathrm{C}(18)-\mathrm{C}(19)$ & $1.496(6)$ \\
\hline C(18)-H(18) & 0.9500 \\
\hline$C(19)-C(20)$ & $1.553(5)$ \\
\hline C(19)-H(19A) & 0.9900 \\
\hline
\end{tabular}




\begin{tabular}{|c|c|}
\hline $\mathrm{C}(19)-\mathrm{H}(19 \mathrm{~B})$ & 0.9900 \\
\hline $\mathrm{C}(20)-\mathrm{O}(7)$ & $1.436(4)$ \\
\hline$C(20)-C(21)$ & $1.492(5)$ \\
\hline $\mathrm{C}(20)-\mathrm{H}(20)$ & 1.0000 \\
\hline$C(21)-C(22)$ & $1.333(5)$ \\
\hline $\mathrm{C}(21)-\mathrm{H}(21)$ & 0.9500 \\
\hline$C(22)-C(23)$ & $1.455(5)$ \\
\hline $\mathrm{C}(22)-\mathrm{H}(22)$ & 0.9500 \\
\hline$C(23)-C(25)$ & $1.354(5)$ \\
\hline$C(23)-C(24)$ & $1.504(5)$ \\
\hline $\mathrm{C}(24)-\mathrm{H}(24 \mathrm{~A})$ & 0.9800 \\
\hline $\mathrm{C}(24)-\mathrm{H}(24 \mathrm{~B})$ & 0.9800 \\
\hline $\mathrm{C}(24)-\mathrm{H}(24 \mathrm{C})$ & 0.9800 \\
\hline $\mathrm{C}(25)-\mathrm{H}(25)$ & 0.9500 \\
\hline $\mathrm{N}(1)-\mathrm{H}(1 \mathrm{~A})$ & $0.86(4)$ \\
\hline $\mathrm{O}(1)-\mathrm{H}(1)$ & $0.92(7)$ \\
\hline $\mathrm{O}(7)-\mathrm{H}(7)$ & $0.91(5)$ \\
\hline $\mathrm{C}(2)-\mathrm{C}(1)-\mathrm{H}(1 \mathrm{~B})$ & 109.5 \\
\hline $\mathrm{C}(2)-\mathrm{C}(1)-\mathrm{H}(1 \mathrm{C})$ & 109.5 \\
\hline $\mathrm{H}(1 \mathrm{~B})-\mathrm{C}(1)-\mathrm{H}(1 \mathrm{C})$ & 109.5 \\
\hline $\mathrm{C}(2)-\mathrm{C}(1)-\mathrm{H}(1 \mathrm{D})$ & 109.5 \\
\hline $\mathrm{H}(1 \mathrm{~B})-\mathrm{C}(1)-\mathrm{H}(1 \mathrm{D})$ & 109.5 \\
\hline $\mathrm{H}(1 \mathrm{C})-\mathrm{C}(1)-\mathrm{H}(1 \mathrm{D})$ & 109.5 \\
\hline $\mathrm{O}(1)-\mathrm{C}(2)-\mathrm{C}(1)$ & $108.7(3)$ \\
\hline $\mathrm{O}(1)-\mathrm{C}(2)-\mathrm{C}(3)$ & $112.4(3)$ \\
\hline $\mathrm{C}(1)-\mathrm{C}(2)-\mathrm{C}(3)$ & 109.1(3) \\
\hline $\mathrm{O}(1)-\mathrm{C}(2)-\mathrm{H}(2)$ & 108.9 \\
\hline $\mathrm{C}(1)-\mathrm{C}(2)-\mathrm{H}(2)$ & 108.9 \\
\hline $\mathrm{C}(3)-\mathrm{C}(2)-\mathrm{H}(2)$ & 108.9 \\
\hline $\mathrm{O}(2)-\mathrm{C}(3)-\mathrm{N}(1)$ & $122.8(3)$ \\
\hline $\mathrm{O}(2)-\mathrm{C}(3)-\mathrm{C}(2)$ & $120.8(3)$ \\
\hline $\mathrm{N}(1)-\mathrm{C}(3)-\mathrm{C}(2)$ & $116.4(3)$ \\
\hline $\mathrm{N}(1)-\mathrm{C}(4)-\mathrm{C}(25)$ & $110.3(3)$ \\
\hline $\mathrm{N}(1)-\mathrm{C}(4)-\mathrm{C}(5)$ & 111.1(3) \\
\hline$C(25)-C(4)-C(5)$ & $112.4(3)$ \\
\hline
\end{tabular}




\begin{tabular}{|c|c|}
\hline $\mathrm{N}(1)-\mathrm{C}(4)-\mathrm{H}(4)$ & 107.6 \\
\hline $\mathrm{C}(25)-\mathrm{C}(4)-\mathrm{H}(4)$ & 107.6 \\
\hline $\mathrm{C}(5)-\mathrm{C}(4)-\mathrm{H}(4)$ & 107.6 \\
\hline$C(7)-C(5)-C(11)$ & $114.0(3)$ \\
\hline$C(7)-C(5)-C(6)$ & $109.0(3)$ \\
\hline$C(11)-C(5)-C(6)$ & $106.9(3)$ \\
\hline$C(7)-C(5)-C(4)$ & $109.7(3)$ \\
\hline$C(11)-C(5)-C(4)$ & 107.3(3) \\
\hline$C(6)-C(5)-C(4)$ & $109.8(3)$ \\
\hline $\mathrm{C}(5)-\mathrm{C}(6)-\mathrm{H}(6 \mathrm{~A})$ & 109.5 \\
\hline $\mathrm{C}(5)-\mathrm{C}(6)-\mathrm{H}(6 \mathrm{~B})$ & 109.5 \\
\hline $\mathrm{H}(6 \mathrm{~A})-\mathrm{C}(6)-\mathrm{H}(6 \mathrm{~B})$ & 109.5 \\
\hline $\mathrm{C}(5)-\mathrm{C}(6)-\mathrm{H}(6 \mathrm{C})$ & 109.5 \\
\hline $\mathrm{H}(6 \mathrm{~A})-\mathrm{C}(6)-\mathrm{H}(6 \mathrm{C})$ & 109.5 \\
\hline $\mathrm{H}(6 \mathrm{~B})-\mathrm{C}(6)-\mathrm{H}(6 \mathrm{C})$ & 109.5 \\
\hline $\mathrm{O}(3)-\mathrm{C}(7)-\mathrm{O}(4)$ & $118.2(3)$ \\
\hline $\mathrm{O}(3)-\mathrm{C}(7)-\mathrm{C}(5)$ & $120.4(3)$ \\
\hline $\mathrm{O}(4)-\mathrm{C}(7)-\mathrm{C}(5)$ & $121.4(3)$ \\
\hline $\mathrm{O}(4)-\mathrm{C}(8)-\mathrm{C}(9)$ & $110.2(3)$ \\
\hline $\mathrm{O}(4)-\mathrm{C}(8)-\mathrm{C}(12)$ & $104.8(2)$ \\
\hline $\mathrm{C}(9)-\mathrm{C}(8)-\mathrm{C}(12)$ & $115.5(3)$ \\
\hline $\mathrm{O}(4)-\mathrm{C}(8)-\mathrm{H}(8)$ & 108.7 \\
\hline $\mathrm{C}(9)-\mathrm{C}(8)-\mathrm{H}(8)$ & 108.7 \\
\hline $\mathrm{C}(12)-\mathrm{C}(8)-\mathrm{H}(8)$ & 108.7 \\
\hline $\mathrm{C}(11)-\mathrm{C}(9)-\mathrm{C}(8)$ & $106.8(3)$ \\
\hline$C(11)-C(9)-C(10)$ & $112.1(3)$ \\
\hline $\mathrm{C}(8)-\mathrm{C}(9)-\mathrm{C}(10)$ & 113.1(3) \\
\hline $\mathrm{C}(11)-\mathrm{C}(9)-\mathrm{H}(9)$ & 108.2 \\
\hline $\mathrm{C}(8)-\mathrm{C}(9)-\mathrm{H}(9)$ & 108.2 \\
\hline $\mathrm{C}(10)-\mathrm{C}(9)-\mathrm{H}(9)$ & 108.2 \\
\hline $\mathrm{C}(9)-\mathrm{C}(10)-\mathrm{H}(10 \mathrm{~A})$ & 109.5 \\
\hline $\mathrm{C}(9)-\mathrm{C}(10)-\mathrm{H}(10 \mathrm{~B})$ & 109.5 \\
\hline $\mathrm{H}(10 \mathrm{~A})-\mathrm{C}(10)-\mathrm{H}(10 \mathrm{~B})$ & 109.5 \\
\hline $\mathrm{C}(9)-\mathrm{C}(10)-\mathrm{H}(10 \mathrm{C})$ & 109.5 \\
\hline $\mathrm{H}(10 \mathrm{~A})-\mathrm{C}(10)-\mathrm{H}(10 \mathrm{C})$ & 109.5 \\
\hline $\mathrm{H}(10 \mathrm{~B})-\mathrm{C}(10)-\mathrm{H}(10 \mathrm{C})$ & 109.5 \\
\hline
\end{tabular}




\begin{tabular}{|c|c|}
\hline $\mathrm{O}(5)-\mathrm{C}(11)-\mathrm{C}(9)$ & $122.9(3)$ \\
\hline $\mathrm{O}(5)-\mathrm{C}(11)-\mathrm{C}(5)$ & $120.4(3)$ \\
\hline $\mathrm{C}(9)-\mathrm{C}(11)-\mathrm{C}(5)$ & $116.7(3)$ \\
\hline $\mathrm{C}(8)-\mathrm{C}(12)-\mathrm{C}(13)$ & $114.2(3)$ \\
\hline $\mathrm{C}(8)-\mathrm{C}(12)-\mathrm{H}(12 \mathrm{~A})$ & 108.7 \\
\hline $\mathrm{C}(13)-\mathrm{C}(12)-\mathrm{H}(12 \mathrm{~A})$ & 108.7 \\
\hline $\mathrm{C}(8)-\mathrm{C}(12)-\mathrm{H}(12 \mathrm{~B})$ & 108.7 \\
\hline $\mathrm{C}(13)-\mathrm{C}(12)-\mathrm{H}(12 \mathrm{~B})$ & 108.7 \\
\hline $\mathrm{H}(12 \mathrm{~A})-\mathrm{C}(12)-\mathrm{H}(12 \mathrm{~B})$ & 107.6 \\
\hline $\mathrm{O}(6)-\mathrm{C}(13)-\mathrm{C}(14)$ & $114.2(3)$ \\
\hline $\mathrm{O}(6)-\mathrm{C}(13)-\mathrm{C}(12)$ & $107.7(3)$ \\
\hline$C(14)-C(13)-C(12)$ & $111.4(3)$ \\
\hline $\mathrm{O}(6)-\mathrm{C}(13)-\mathrm{H}(13)$ & 107.8 \\
\hline $\mathrm{C}(14)-\mathrm{C}(13)-\mathrm{H}(13)$ & 107.8 \\
\hline $\mathrm{C}(12)-\mathrm{C}(13)-\mathrm{H}(13)$ & 107.8 \\
\hline$C(15)-C(14)-C(13)$ & $126.3(4)$ \\
\hline $\mathrm{C}(15)-\mathrm{C}(14)-\mathrm{H}(14)$ & 116.8 \\
\hline $\mathrm{C}(13)-\mathrm{C}(14)-\mathrm{H}(14)$ & 116.8 \\
\hline$C(14)-C(15)-C(16)$ & $127.8(4)$ \\
\hline $\mathrm{C}(14)-\mathrm{C}(15)-\mathrm{H}(15)$ & 116.1 \\
\hline $\mathrm{C}(16)-\mathrm{C}(15)-\mathrm{H}(15)$ & 116.1 \\
\hline $\mathrm{C}(18)-\mathrm{C}(16)-\mathrm{C}(15)$ & $120.1(4)$ \\
\hline $\mathrm{C}(18)-\mathrm{C}(16)-\mathrm{C}(17)$ & $123.3(4)$ \\
\hline$C(15)-C(16)-C(17)$ & $116.6(3)$ \\
\hline $\mathrm{C}(16)-\mathrm{C}(17)-\mathrm{H}(17 \mathrm{~A})$ & 109.5 \\
\hline $\mathrm{C}(16)-\mathrm{C}(17)-\mathrm{H}(17 \mathrm{~B})$ & 109.5 \\
\hline $\mathrm{H}(17 \mathrm{~A})-\mathrm{C}(17)-\mathrm{H}(17 \mathrm{~B})$ & 109.5 \\
\hline $\mathrm{C}(16)-\mathrm{C}(17)-\mathrm{H}(17 \mathrm{C})$ & 109.5 \\
\hline $\mathrm{H}(17 \mathrm{~A})-\mathrm{C}(17)-\mathrm{H}(17 \mathrm{C})$ & 109.5 \\
\hline $\mathrm{H}(17 \mathrm{~B})-\mathrm{C}(17)-\mathrm{H}(17 \mathrm{C})$ & 109.5 \\
\hline$C(16)-C(18)-C(19)$ & $129.0(4)$ \\
\hline $\mathrm{C}(16)-\mathrm{C}(18)-\mathrm{H}(18)$ & 115.5 \\
\hline $\mathrm{C}(19)-\mathrm{C}(18)-\mathrm{H}(18)$ & 115.5 \\
\hline$C(18)-C(19)-C(20)$ & $113.1(3)$ \\
\hline $\mathrm{C}(18)-\mathrm{C}(19)-\mathrm{H}(19 \mathrm{~A})$ & 109.0 \\
\hline$C(20)-C(19)-H(19 A)$ & 109.0 \\
\hline
\end{tabular}




$\begin{array}{ll}\mathrm{C}(18)-\mathrm{C}(19)-\mathrm{H}(19 \mathrm{~B}) & 109.0 \\ \mathrm{C}(20)-\mathrm{C}(19)-\mathrm{H}(19 \mathrm{~B}) & 109.0 \\ \mathrm{H}(19 \mathrm{~A})-\mathrm{C}(19)-\mathrm{H}(19 \mathrm{~B}) & 107.8 \\ \mathrm{O}(7)-\mathrm{C}(20)-\mathrm{C}(21) & 111.3(3) \\ \mathrm{O}(7)-\mathrm{C}(20)-\mathrm{C}(19) & 109.9(3) \\ \mathrm{C}(21)-\mathrm{C}(20)-\mathrm{C}(19) & 111.1(3) \\ \mathrm{O}(7)-\mathrm{C}(20)-\mathrm{H}(20) & 108.1 \\ \mathrm{C}(21)-\mathrm{C}(20)-\mathrm{H}(20) & 108.1 \\ \mathrm{C}(19)-\mathrm{C}(20)-\mathrm{H}(20) & 108.1 \\ \mathrm{C}(22)-\mathrm{C}(21)-\mathrm{C}(20) & 122.9(3) \\ \mathrm{C}(22)-\mathrm{C}(21)-\mathrm{H}(21) & 118.5 \\ \mathrm{C}(20)-\mathrm{C}(21)-\mathrm{H}(21) & 118.5 \\ \mathrm{C}(21)-\mathrm{C}(22)-\mathrm{C}(23) & 126.6(3) \\ \mathrm{C}(21)-\mathrm{C}(22)-\mathrm{H}(22) & 116.7 \\ \mathrm{C}(23)-\mathrm{C}(22)-\mathrm{H}(22) & 116.7 \\ \mathrm{C}(25)-\mathrm{C}(23)-\mathrm{C}(22) & 118.3(3) \\ \mathrm{C}(25)-\mathrm{C}(23)-\mathrm{C}(24) & 123.4(3) \\ \mathrm{C}(22)-\mathrm{C}(23)-\mathrm{C}(24) & 118.4(3) \\ \mathrm{C}(23)-\mathrm{C}(24)-\mathrm{H}(24 \mathrm{~A}) & 109.5 \\ \mathrm{C}(23)-\mathrm{C}(24)-\mathrm{H}(24 \mathrm{~B}) & 109.5 \\ \mathrm{H}(24 \mathrm{~A})-\mathrm{C}(24)-\mathrm{H}(24 \mathrm{~B}) & 109.5 \\ \mathrm{C}(23)-\mathrm{C}(24)-\mathrm{H}(24 \mathrm{C}) & 109.5 \\ \mathrm{H}(24 \mathrm{~A})-\mathrm{C}(24)-\mathrm{H}(24 \mathrm{C}) & 109.5 \\ \mathrm{H}(24 \mathrm{~B})-\mathrm{C}(24)-\mathrm{H}(24 \mathrm{C}) & 109.5 \\ \mathrm{C}(23)-\mathrm{C}(25)-\mathrm{C}(4) & 126.2(3) \\ \mathrm{C}(23)-\mathrm{C}(25)-\mathrm{H}(25) & 116.9 \\ \mathrm{C}(4)-\mathrm{C}(25)-\mathrm{H}(25) & 116.9 \\ \mathrm{C}(3)-\mathrm{N}(1)-\mathrm{C}(4) & 121.4(3) \\ \mathrm{C}(3)-\mathrm{N}(1)-\mathrm{H}(1 \mathrm{~A}) & 119(3) \\ \mathrm{C}(4)-\mathrm{N}(1)-\mathrm{H}(1 \mathrm{~A}) & 120(3) \\ \mathrm{C}(2)-\mathrm{O}(1)-\mathrm{H}(1) & 102(4) \\ \mathrm{C}(7)-\mathrm{O}(4)-\mathrm{C}(8) & 120.4(2) \\ & 105(3) \\ & \end{array}$

Symmetry transformations used to generate equivalent atoms: 
Table 4. Anisotropic displacement parameters $\left(\AA^{2} \times 10^{3}\right)$ for seiple002_ucsf. The anisotropic displacement factor exponent takes the form: $-2 \pi^{2}\left[\mathrm{~h}^{2} \mathrm{a}^{* 2} \mathrm{U}^{11}+\ldots+2 \mathrm{hk} \mathrm{a} \mathrm{a}^{*} \mathrm{U}^{12}\right]$

\begin{tabular}{|c|c|c|c|c|c|c|}
\hline & $\mathrm{U}^{11}$ & $\mathrm{U}^{22}$ & $\mathrm{U}^{33}$ & $U^{23}$ & $\mathrm{U}^{13}$ & $\mathrm{U}^{12}$ \\
\hline $\mathrm{C}(1)$ & $50(2)$ & $34(2)$ & $81(3)$ & $17(2)$ & $18(2)$ & 1(2) \\
\hline $\mathrm{C}(2)$ & $46(2)$ & $21(2)$ & $48(2)$ & $-3(1)$ & $4(2)$ & $-4(1)$ \\
\hline$C(3)$ & $41(2)$ & $24(2)$ & $37(2)$ & $1(1)$ & $0(1)$ & $-4(1)$ \\
\hline $\mathrm{C}(4)$ & $32(2)$ & $19(1)$ & $36(2)$ & $3(1)$ & $0(1)$ & $-1(1)$ \\
\hline $\mathrm{C}(5)$ & $32(2)$ & $19(1)$ & $34(2)$ & $1(1)$ & $2(1)$ & $0(1)$ \\
\hline $\mathrm{C}(6)$ & $37(2)$ & $31(2)$ & $39(2)$ & $6(1)$ & $4(1)$ & $0(1)$ \\
\hline$C(7)$ & $35(2)$ & $20(1)$ & $33(2)$ & $0(1)$ & $3(1)$ & $1(1)$ \\
\hline $\mathrm{C}(8)$ & $38(2)$ & $17(2)$ & $37(2)$ & $4(1)$ & $2(1)$ & $2(1)$ \\
\hline $\mathrm{C}(9)$ & $38(2)$ & $22(2)$ & $40(2)$ & $-2(1)$ & $-1(1)$ & $5(1)$ \\
\hline $\mathrm{C}(10)$ & $36(2)$ & $41(2)$ & $47(2)$ & $-11(2)$ & $5(1)$ & $0(2)$ \\
\hline $\mathrm{C}(11)$ & $34(2)$ & $23(2)$ & $33(2)$ & $1(1)$ & $6(1)$ & $3(1)$ \\
\hline $\mathrm{C}(12)$ & $39(2)$ & $23(2)$ & $39(2)$ & $0(1)$ & $-2(1)$ & $-3(1)$ \\
\hline $\mathrm{C}(13)$ & $47(2)$ & $21(2)$ & $42(2)$ & $0(1)$ & $2(2)$ & $-3(1)$ \\
\hline$C(14)$ & $48(2)$ & $23(2)$ & $46(2)$ & $-1(1)$ & $5(2)$ & $-4(1)$ \\
\hline$C(15)$ & $49(2)$ & $24(2)$ & $50(2)$ & $0(2)$ & $4(2)$ & $-7(1)$ \\
\hline$C(16)$ & $54(2)$ & $22(2)$ & $40(2)$ & $5(1)$ & $3(2)$ & $-5(1)$ \\
\hline $\mathrm{C}(17)$ & $55(2)$ & $46(2)$ & $49(2)$ & $-4(2)$ & $7(2)$ & $-2(2)$ \\
\hline $\mathrm{C}(18)$ & $50(2)$ & $31(2)$ & $45(2)$ & $3(2)$ & $5(2)$ & $-3(2)$ \\
\hline$C(19)$ & $58(2)$ & $33(2)$ & $41(2)$ & $6(2)$ & $2(2)$ & $-7(2)$ \\
\hline$C(20)$ & $41(2)$ & $37(2)$ & $34(2)$ & $7(2)$ & $2(1)$ & $-2(2)$ \\
\hline $\mathrm{C}(21)$ & $40(2)$ & $25(2)$ & $37(2)$ & $-2(1)$ & $2(1)$ & $-1(1)$ \\
\hline $\mathrm{C}(22)$ & $36(2)$ & $24(2)$ & $36(2)$ & $0(1)$ & $2(1)$ & $2(1)$ \\
\hline$C(23)$ & $36(2)$ & $20(1)$ & $37(2)$ & $-1(1)$ & $0(1)$ & $2(1)$ \\
\hline $\mathrm{C}(24)$ & $39(2)$ & $36(2)$ & $39(2)$ & $5(2)$ & $2(1)$ & $2(1)$ \\
\hline$C(25)$ & $33(2)$ & 21(1) & $35(2)$ & $-2(1)$ & $4(1)$ & $3(1)$ \\
\hline $\mathrm{N}(1)$ & $33(1)$ & $19(1)$ & $41(2)$ & $2(1)$ & $0(1)$ & $-1(1)$ \\
\hline $\mathrm{O}(1)$ & $47(1)$ & $22(1)$ & $44(1)$ & $5(1)$ & $10(1)$ & $1(1)$ \\
\hline $\mathrm{O}(2)$ & $50(2)$ & $29(1)$ & $78(2)$ & $2(1)$ & $-19(1)$ & $-6(1)$ \\
\hline $\mathrm{O}(3)$ & $35(1)$ & $21(1)$ & $49(1)$ & $3(1)$ & $-4(1)$ & $1(1)$ \\
\hline $\mathrm{O}(4)$ & $32(1)$ & $18(1)$ & $44(1)$ & $2(1)$ & $-1(1)$ & $1(1)$ \\
\hline $\mathrm{O}(5)$ & $37(1)$ & $28(1)$ & $44(1)$ & $-1(1)$ & $-3(1)$ & $7(1)$ \\
\hline
\end{tabular}




\begin{tabular}{lllllll}
$\mathrm{O}(6)$ & $58(2)$ & $25(1)$ & $56(2)$ & $5(1)$ & $14(1)$ & $7(1)$ \\
$\mathrm{O}(7)$ & $43(1)$ & $36(1)$ & $35(1)$ & $2(1)$ & $6(1)$ & $7(1)$ \\
\hline
\end{tabular}

Table 5. Hydrogen coordinates $\left(\mathrm{x} 10^{4}\right)$ and isotropic displacement parameters $\left(\AA^{2} \times 10^{3}\right)$ for seiple002_ucsf.

\begin{tabular}{|c|c|c|c|c|}
\hline & $\mathrm{x}$ & $\mathrm{y}$ & $\mathrm{z}$ & $\mathrm{U}(\mathrm{eq})$ \\
\hline $\mathrm{H}(1 \mathrm{~B})$ & 440 & 52 & 8279 & 81 \\
\hline $\mathrm{H}(1 \mathrm{C})$ & -1302 & 963 & 8074 & 81 \\
\hline $\mathrm{H}(1 \mathrm{D})$ & 647 & 1180 & 8772 & 81 \\
\hline $\mathrm{H}(2)$ & 1068 & 856 & 6998 & 46 \\
\hline $\mathrm{H}(4)$ & 153 & 4408 & 7422 & 35 \\
\hline $\mathrm{H}(6 \mathrm{~A})$ & 873 & 4737 & 9703 & 53 \\
\hline $\mathrm{H}(6 \mathrm{~B})$ & 1247 & 3603 & 9259 & 53 \\
\hline $\mathrm{H}(6 \mathrm{C})$ & -746 & 4320 & 8930 & 53 \\
\hline $\mathrm{H}(8)$ & 4710 & 6813 & 8034 & 37 \\
\hline $\mathrm{H}(9)$ & 1894 & 7645 & 8623 & 40 \\
\hline $\mathrm{H}(10 \mathrm{~A})$ & 693 & 6842 & 9914 & 62 \\
\hline $\mathrm{H}(10 \mathrm{~B})$ & 2676 & 7622 & 10079 & 62 \\
\hline $\mathrm{H}(10 \mathrm{C})$ & 2993 & 6344 & 10104 & 62 \\
\hline $\mathrm{H}(12 \mathrm{~A})$ & 7654 & 7323 & 9093 & 41 \\
\hline $\mathrm{H}(12 \mathrm{~B})$ & 5924 & 7749 & 9675 & 41 \\
\hline $\mathrm{H}(13)$ & 7218 & 9195 & 8927 & 44 \\
\hline $\mathrm{H}(14)$ & 8201 & 8434 & 7665 & 47 \\
\hline $\mathrm{H}(15)$ & 4117 & 8887 & 7067 & 49 \\
\hline $\mathrm{H}(17 \mathrm{~A})$ & 9017 & 9123 & 6185 & 75 \\
\hline $\mathrm{H}(17 \mathrm{~B})$ & 8482 & 8329 & 5390 & 75 \\
\hline $\mathrm{H}(17 \mathrm{C})$ & 8985 & 7848 & 6335 & 75 \\
\hline $\mathrm{H}(18)$ & 3132 & 8506 & 5649 & 50 \\
\hline $\mathrm{H}(19 \mathrm{~A})$ & 5957 & 8365 & 4399 & 53 \\
\hline $\mathrm{H}(19 \mathrm{~B})$ & 3458 & 8366 & 4227 & 53 \\
\hline $\mathrm{H}(20)$ & 6093 & 6542 & 4684 & 45 \\
\hline $\mathrm{H}(21)$ & 1656 & 6317 & 4720 & 41 \\
\hline $\mathrm{H}(22)$ & 5031 & 5674 & 5857 & 39 \\
\hline
\end{tabular}




\begin{tabular}{lllll}
$\mathrm{H}(24 \mathrm{~A})$ & -948 & 4890 & 6315 & 57 \\
$\mathrm{H}(24 \mathrm{~B})$ & -416 & 5021 & 5346 & 57 \\
$\mathrm{H}(24 \mathrm{C})$ & -669 & 6065 & 5924 & 57 \\
$\mathrm{H}(25)$ & 4392 & 4851 & 7103 & 35 \\
$\mathrm{H}(1)$ & $4170(90)$ & $1200(50)$ & $7290(40)$ & $62(16)$ \\
$\mathrm{H}(1 \mathrm{~A})$ & $3270(70)$ & $2980(40)$ & $8000(30)$ & $26(9)$ \\
$\mathrm{H}(7)$ & $3120(80)$ & $6890(40)$ & $3380(30)$ & $39(11)$ \\
\hline
\end{tabular}


Table 6. Torsion angles $\left[{ }^{\circ}\right]$ for seiple 002 ucsf.

\begin{tabular}{|c|c|}
\hline $\mathrm{O}(1)-\mathrm{C}(2)-\mathrm{C}(3)-\mathrm{O}(2)$ & $164.4(4)$ \\
\hline $\mathrm{C}(1)-\mathrm{C}(2)-\mathrm{C}(3)-\mathrm{O}(2)$ & $-75.1(5)$ \\
\hline $\mathrm{O}(1)-\mathrm{C}(2)-\mathrm{C}(3)-\mathrm{N}(1)$ & $-17.8(5)$ \\
\hline $\mathrm{C}(1)-\mathrm{C}(2)-\mathrm{C}(3)-\mathrm{N}(1)$ & $102.8(4)$ \\
\hline $\mathrm{N}(1)-\mathrm{C}(4)-\mathrm{C}(5)-\mathrm{C}(7)$ & $64.7(3)$ \\
\hline$C(25)-C(4)-C(5)-C(7)$ & $-59.4(3)$ \\
\hline $\mathrm{N}(1)-\mathrm{C}(4)-\mathrm{C}(5)-\mathrm{C}(11)$ & $-170.9(3)$ \\
\hline $\mathrm{C}(25)-\mathrm{C}(4)-\mathrm{C}(5)-\mathrm{C}(11)$ & $65.0(3)$ \\
\hline $\mathrm{N}(1)-\mathrm{C}(4)-\mathrm{C}(5)-\mathrm{C}(6)$ & $-55.1(3)$ \\
\hline$C(25)-C(4)-C(5)-C(6)$ & $-179.2(3)$ \\
\hline $\mathrm{C}(11)-\mathrm{C}(5)-\mathrm{C}(7)-\mathrm{O}(3)$ & $177.0(3)$ \\
\hline $\mathrm{C}(6)-\mathrm{C}(5)-\mathrm{C}(7)-\mathrm{O}(3)$ & $57.7(4)$ \\
\hline $\mathrm{C}(4)-\mathrm{C}(5)-\mathrm{C}(7)-\mathrm{O}(3)$ & $-62.6(4)$ \\
\hline $\mathrm{C}(11)-\mathrm{C}(5)-\mathrm{C}(7)-\mathrm{O}(4)$ & $-2.0(4)$ \\
\hline $\mathrm{C}(6)-\mathrm{C}(5)-\mathrm{C}(7)-\mathrm{O}(4)$ & $-121.3(3)$ \\
\hline $\mathrm{C}(4)-\mathrm{C}(5)-\mathrm{C}(7)-\mathrm{O}(4)$ & $118.4(3)$ \\
\hline $\mathrm{O}(4)-\mathrm{C}(8)-\mathrm{C}(9)-\mathrm{C}(11)$ & $-62.1(3)$ \\
\hline $\mathrm{C}(12)-\mathrm{C}(8)-\mathrm{C}(9)-\mathrm{C}(11)$ & 179.3(3) \\
\hline $\mathrm{O}(4)-\mathrm{C}(8)-\mathrm{C}(9)-\mathrm{C}(10)$ & $61.7(4)$ \\
\hline $\mathrm{C}(12)-\mathrm{C}(8)-\mathrm{C}(9)-\mathrm{C}(10)$ & $-56.9(4)$ \\
\hline $\mathrm{C}(8)-\mathrm{C}(9)-\mathrm{C}(11)-\mathrm{O}(5)$ & $-131.2(3)$ \\
\hline $\mathrm{C}(10)-\mathrm{C}(9)-\mathrm{C}(11)-\mathrm{O}(5)$ & $104.4(4)$ \\
\hline $\mathrm{C}(8)-\mathrm{C}(9)-\mathrm{C}(11)-\mathrm{C}(5)$ & $49.7(4)$ \\
\hline$C(10)-C(9)-C(11)-C(5)$ & $-74.8(4)$ \\
\hline $\mathrm{C}(7)-\mathrm{C}(5)-\mathrm{C}(11)-\mathrm{O}(5)$ & $162.2(3)$ \\
\hline $\mathrm{C}(6)-\mathrm{C}(5)-\mathrm{C}(11)-\mathrm{O}(5)$ & $-77.3(4)$ \\
\hline $\mathrm{C}(4)-\mathrm{C}(5)-\mathrm{C}(11)-\mathrm{O}(5)$ & $40.5(4)$ \\
\hline$C(7)-C(5)-C(11)-C(9)$ & $-18.6(4)$ \\
\hline $\mathrm{C}(6)-\mathrm{C}(5)-\mathrm{C}(11)-\mathrm{C}(9)$ & $101.9(3)$ \\
\hline$C(4)-C(5)-C(11)-C(9)$ & $-140.3(3)$ \\
\hline $\mathrm{O}(4)-\mathrm{C}(8)-\mathrm{C}(12)-\mathrm{C}(13)$ & $159.5(3)$ \\
\hline $\mathrm{C}(9)-\mathrm{C}(8)-\mathrm{C}(12)-\mathrm{C}(13)$ & $-78.9(4)$ \\
\hline $\mathrm{C}(8)-\mathrm{C}(12)-\mathrm{C}(13)-\mathrm{O}(6)$ & $63.0(4)$ \\
\hline $\mathrm{C}(8)-\mathrm{C}(12)-\mathrm{C}(13)-\mathrm{C}(14)$ & $-62.9(4)$ \\
\hline
\end{tabular}




$\begin{array}{lc}\mathrm{O}(6)-\mathrm{C}(13)-\mathrm{C}(14)-\mathrm{C}(15) & -12.9(5) \\ \mathrm{C}(12)-\mathrm{C}(13)-\mathrm{C}(14)-\mathrm{C}(15) & 109.4(4) \\ \mathrm{C}(13)-\mathrm{C}(14)-\mathrm{C}(15)-\mathrm{C}(16) & -175.8(3) \\ \mathrm{C}(14)-\mathrm{C}(15)-\mathrm{C}(16)-\mathrm{C}(18) & 163.9(4) \\ \mathrm{C}(14)-\mathrm{C}(15)-\mathrm{C}(16)-\mathrm{C}(17) & -14.5(6) \\ \mathrm{C}(15)-\mathrm{C}(16)-\mathrm{C}(18)-\mathrm{C}(19) & -171.8(4) \\ \mathrm{C}(17)-\mathrm{C}(16)-\mathrm{C}(18)-\mathrm{C}(19) & 6.5(6) \\ \mathrm{C}(16)-\mathrm{C}(18)-\mathrm{C}(19)-\mathrm{C}(20) & 86.4(5) \\ \mathrm{C}(18)-\mathrm{C}(19)-\mathrm{C}(20)-\mathrm{O}(7) & 167.0(3) \\ \mathrm{C}(18)-\mathrm{C}(19)-\mathrm{C}(20)-\mathrm{C}(21) & 43.4(5) \\ \mathrm{O}(7)-\mathrm{C}(20)-\mathrm{C}(21)-\mathrm{C}(22) & 133.8(4) \\ \mathrm{C}(19)-\mathrm{C}(20)-\mathrm{C}(21)-\mathrm{C}(22) & -103.4(4) \\ \mathrm{C}(20)-\mathrm{C}(21)-\mathrm{C}(22)-\mathrm{C}(23) & 174.6(3) \\ \mathrm{C}(21)-\mathrm{C}(22)-\mathrm{C}(23)-\mathrm{C}(25) & -175.4(3) \\ \mathrm{C}(21)-\mathrm{C}(22)-\mathrm{C}(23)-\mathrm{C}(24) & 4.5(5) \\ \mathrm{C}(22)-\mathrm{C}(23)-\mathrm{C}(25)-\mathrm{C}(4) & 179.0(3) \\ \mathrm{C}(24)-\mathrm{C}(23)-\mathrm{C}(25)-\mathrm{C}(4) & -0.8(5) \\ \mathrm{N}(1)-\mathrm{C}(4)-\mathrm{C}(25)-\mathrm{C}(23) & 115.1(4) \\ \mathrm{C}(5)-\mathrm{C}(4)-\mathrm{C}(25)-\mathrm{C}(23) & -120.3(4) \\ \mathrm{O}(2)-\mathrm{C}(3)-\mathrm{N}(1)-\mathrm{C}(4) & 0.7(6) \\ \mathrm{C}(2)-\mathrm{C}(3)-\mathrm{N}(1)-\mathrm{C}(4) & -177.1(3) \\ \mathrm{C}(25)-\mathrm{C}(4)-\mathrm{N}(1)-\mathrm{C}(3) & -104.6(4) \\ \mathrm{C}(5)-\mathrm{C}(4)-\mathrm{N}(1)-\mathrm{C}(3) & 130.1(3) \\ \mathrm{O}(3)-\mathrm{C}(7)-\mathrm{O}(4)-\mathrm{C}(8) & 168.3(3) \\ \mathrm{C}(5)-\mathrm{C}(7)-\mathrm{O}(4)-\mathrm{C}(8) & -12.7(4) \\ \mathrm{C}(9)-\mathrm{C}(8)-\mathrm{O}(4)-\mathrm{C}(7) & 46.3(4) \\ \mathrm{C}(12)-\mathrm{C}(8)-\mathrm{O}(4)-\mathrm{C}(7) & \\ & \\ & \end{array}$

Symmetry transformations used to generate equivalent atoms: 
Compound 33 : CCDC 1965636
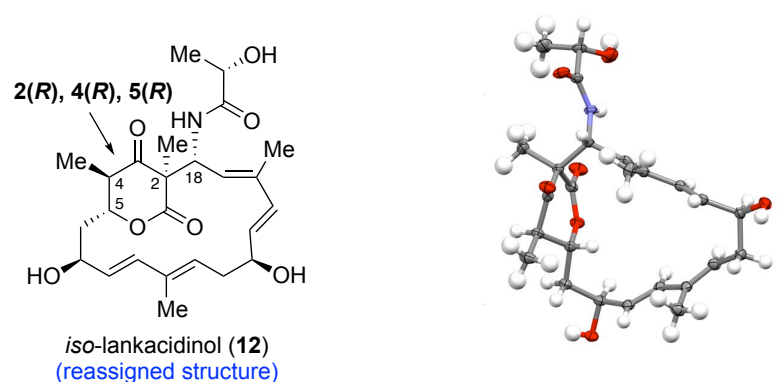

Table 1. Crystal data and structure refinement for Seiple003_UCSF.

Identification code

Empirical formula

Formula weight

Temperature

Wavelength

Crystal system

Space group

Unit cell dimensions

Volume

Z

Density (calculated)

Absorption coefficient

$\mathrm{F}(000)$

Crystal size

Theta range for data collection

Index ranges

Reflections collected

Independent reflections

Completeness to theta $=68.000^{\circ}$

Absorption correction

Max. and min. transmission

Refinement method

Data / restraints / parameters

Goodness-of-fit on $\mathrm{F}^{2}$

Final R indices [I $>2 \operatorname{sigma}(\mathrm{I})]$
Seiple003_UCSF

C28 H41 N O8

519.62

100(2) K

$1.54184 \AA$

Monoclinic

P 21

$\mathrm{a}=8.47060(10) \AA$ $\alpha=90^{\circ}$.

$\mathrm{b}=9.76130(10) \AA$ $\beta=96.5080(10)^{\circ}$.

$\mathrm{c}=17.6922(2) \AA$ $\gamma=90^{\circ}$.

1453.44(3) $\AA^{3}$

2

$1.187 \mathrm{Mg} / \mathrm{m}^{3}$

$0.709 \mathrm{~mm}^{-1}$

560

$0.300 \times 0.230 \times 0.110 \mathrm{~mm}^{3}$

5.032 to $68.229^{\circ}$.

$-10<=\mathrm{h}<=10,-11<=\mathrm{k}<=11,-21<=\mathrm{l}<=21$

38233

$5244[\mathrm{R}(\mathrm{int})=0.0809]$

$100.0 \%$

Semi-empirical from equivalents

1.00000 and 0.83468

Full-matrix least-squares on $\mathrm{F}^{2}$

$5244 / 1 / 357$

1.103

$\mathrm{R} 1=0.0396, w \mathrm{R} 2=0.1124$ 
$\mathrm{R}$ indices (all data)

Absolute structure parameter

Extinction coefficient

Largest diff. peak and hole
$\mathrm{R} 1=0.0402, \mathrm{wR} 2=0.1136$

$0.07(14)$

$\mathrm{n} / \mathrm{a}$

0.174 and -0.210 e. $\AA^{-3}$

Table 2. Atomic coordinates ( $\left.\times 10^{4}\right)$ and equivalent isotropic displacement parameters $\left(\AA^{2} \times 10^{3}\right)$ for seiple 003 ucsf. $\mathrm{U}(\mathrm{eq})$ is defined as one third of the trace of the orthogonalized $\mathrm{U}^{\mathrm{ij}}$ tensor.

\begin{tabular}{|c|c|c|c|c|}
\hline & $\mathrm{x}$ & $\mathrm{y}$ & $\mathrm{z}$ & $\mathrm{U}(\mathrm{eq})$ \\
\hline $\mathrm{C}(1)$ & $1370(4)$ & $6336(4)$ & $5681(2)$ & $39(1)$ \\
\hline $\mathrm{C}(2)$ & $322(3)$ & $6517(3)$ & $4930(1)$ & $26(1)$ \\
\hline$C(3)$ & $1257(3)$ & $7261(3)$ & $4367(1)$ & $22(1)$ \\
\hline$C(4)$ & $2959(3)$ & $6962(3)$ & $3337(1)$ & $19(1)$ \\
\hline$C(5)$ & $4754(3)$ & $6692(3)$ & $3619(1)$ & $20(1)$ \\
\hline$C(6)$ & $5153(3)$ & $7077(4)$ & $4462(1)$ & $32(1)$ \\
\hline$C(7)$ & $5809(3)$ & 7611(3) & $3189(1)$ & $22(1)$ \\
\hline $\mathrm{C}(8)$ & $7370(3)$ & $6996(3)$ & $3030(1)$ & $22(1)$ \\
\hline$C(9)$ & $8321(3)$ & 7998(3) & $2604(2)$ & $32(1)$ \\
\hline$C(10)$ & $6937(3)$ & $5670(3)$ & $2595(1)$ & $21(1)$ \\
\hline $\mathrm{C}(11)$ & $5090(3)$ & $5168(3)$ & $3533(1)$ & $23(1)$ \\
\hline $\mathrm{C}(12)$ & $8368(3)$ & $4873(3)$ & $2387(2)$ & $24(1)$ \\
\hline$C(13)$ & $7963(3)$ & $3697(3)$ & $1823(1)$ & $24(1)$ \\
\hline$C(14)$ & $7160(3)$ & $4224(3)$ & $1085(1)$ & $22(1)$ \\
\hline$C(15)$ & $5662(3)$ & $3936(3)$ & $815(1)$ & $22(1)$ \\
\hline$C(16)$ & $4750(3)$ & $4565(3)$ & $153(1)$ & $22(1)$ \\
\hline$C(17)$ & $5588(3)$ & $5595(3)$ & $-290(2)$ & $29(1)$ \\
\hline$C(18)$ & $3198(3)$ & $4272(3)$ & $9(1)$ & $24(1)$ \\
\hline$C(19)$ & 1990(3) & 4981(3) & $-541(1)$ & $25(1)$ \\
\hline$C(20)$ & $642(3)$ & $5568(3)$ & $-119(1)$ & $22(1)$ \\
\hline $\mathrm{C}(21)$ & $1264(3)$ & $6517(3)$ & $516(1)$ & $22(1)$ \\
\hline$C(22)$ & $1565(3)$ & $6106(3)$ & $1237(1)$ & $20(1)$ \\
\hline$C(23)$ & $2162(3)$ & $6944(3)$ & $1895(1)$ & $21(1)$ \\
\hline$C(24)$ & $2361(3)$ & $8450(3)$ & $1775(2)$ & $29(1)$ \\
\hline$C(25)$ & $2459(3)$ & $6314(3)$ & $2575(1)$ & $19(1)$ \\
\hline$C(26)$ & $3424(3)$ & $2037(3)$ & $2333(2)$ & $32(1)$ \\
\hline $\mathrm{C}(27)$ & $2506(3)$ & 1863(3) & $2997(2)$ & $28(1)$ \\
\hline
\end{tabular}




\begin{tabular}{lllll}
$\mathrm{C}(28)$ & $3325(3)$ & $1231(3)$ & $3702(2)$ & $30(1)$ \\
$\mathrm{N}(1)$ & $1931(2)$ & $6454(2)$ & $3884(1)$ & $21(1)$ \\
$\mathrm{O}(1)$ & $-226(3)$ & $5270(2)$ & $4598(1)$ & $36(1)$ \\
$\mathrm{O}(2)$ & $1408(2)$ & $8522(2)$ & $4388(1)$ & $31(1)$ \\
$\mathrm{O}(3)$ & $5402(2)$ & $8767(2)$ & $3005(1)$ & $30(1)$ \\
$\mathrm{O}(4)$ & $4436(2)$ & $4324(2)$ & $3882(1)$ & $33(1)$ \\
$\mathrm{O}(5)$ & $6096(2)$ & $4725(2)$ & $3052(1)$ & $25(1)$ \\
$\mathrm{O}(6)$ & $9418(2)$ & $3047(2)$ & $1671(1)$ & $28(1)$ \\
$\mathrm{O}(7)$ & $-533(2)$ & $6223(2)$ & $-637(1)$ & $26(1)$ \\
$\mathrm{O}(8)$ & $1140(3)$ & $2234(4)$ & $2976(1)$ & $66(1)$ \\
\hline
\end{tabular}

Table 3. Bond lengths $[\AA]$ and angles $\left[{ }^{\circ}\right]$ for seiple 003 ucsf.

\begin{tabular}{ll}
\hline $\mathrm{C}(1)-\mathrm{C}(2)$ & $1.522(4)$ \\
$\mathrm{C}(1)-\mathrm{H}(1 \mathrm{~B})$ & 0.9800 \\
$\mathrm{C}(1)-\mathrm{H}(1 \mathrm{C})$ & 0.9800 \\
$\mathrm{C}(1)-\mathrm{H}(1 \mathrm{D})$ & 0.9800 \\
$\mathrm{C}(2)-\mathrm{O}(1)$ & $1.407(4)$ \\
$\mathrm{C}(2)-\mathrm{C}(3)$ & $1.525(3)$ \\
$\mathrm{C}(2)-\mathrm{H}(2)$ & 1.0000 \\
$\mathrm{C}(3)-\mathrm{O}(2)$ & $1.238(4)$ \\
$\mathrm{C}(3)-\mathrm{N}(1)$ & $1.337(3)$ \\
$\mathrm{C}(4)-\mathrm{N}(1)$ & $1.460(3)$ \\
$\mathrm{C}(4)-\mathrm{C}(25)$ & $1.506(3)$ \\
$\mathrm{C}(4)-\mathrm{C}(5)$ & $1.568(3)$ \\
$\mathrm{C}(4)-\mathrm{H}(4)$ & 1.0000 \\
$\mathrm{C}(5)-\mathrm{C}(11)$ & $1.526(4)$ \\
$\mathrm{C}(5)-\mathrm{C}(7)$ & $1.529(3)$ \\
$\mathrm{C}(5)-\mathrm{C}(6)$ & $1.537(3)$ \\
$\mathrm{C}(6)-\mathrm{H}(6 \mathrm{~A})$ & 0.9800 \\
$\mathrm{C}(6)-\mathrm{H}(6 \mathrm{~B})$ & 0.9800 \\
$\mathrm{C}(6)-\mathrm{H}(6 \mathrm{C})$ & 0.9800 \\
$\mathrm{C}(7)-\mathrm{O}(3)$ & $1.214(3)$ \\
$\mathrm{C}(7)-\mathrm{C}(8)$ & $1.507(3)$ \\
$\mathrm{C}(8)-\mathrm{C}(9)$ & $1.521(4)$ \\
$\mathrm{C}(8)-\mathrm{C}(10)$ & $1.529(4)$ \\
&
\end{tabular}




\begin{tabular}{|c|c|}
\hline $\mathrm{C}(8)-\mathrm{H}(8)$ & 1.0000 \\
\hline $\mathrm{C}(9)-\mathrm{H}(9 \mathrm{~A})$ & 0.9800 \\
\hline $\mathrm{C}(9)-\mathrm{H}(9 \mathrm{~B})$ & 0.9800 \\
\hline $\mathrm{C}(9)-\mathrm{H}(9 \mathrm{C})$ & 0.9800 \\
\hline $\mathrm{C}(10)-\mathrm{O}(5)$ & $1.463(3)$ \\
\hline$C(10)-C(12)$ & $1.520(3)$ \\
\hline $\mathrm{C}(10)-\mathrm{H}(10)$ & 1.0000 \\
\hline $\mathrm{C}(11)-\mathrm{O}(4)$ & $1.203(3)$ \\
\hline $\mathrm{C}(11)-\mathrm{O}(5)$ & $1.343(3)$ \\
\hline$C(12)-C(13)$ & $1.534(4)$ \\
\hline $\mathrm{C}(12)-\mathrm{H}(12 \mathrm{~A})$ & 0.9900 \\
\hline $\mathrm{C}(12)-\mathrm{H}(12 \mathrm{~B})$ & 0.9900 \\
\hline $\mathrm{C}(13)-\mathrm{O}(6)$ & $1.438(3)$ \\
\hline$C(13)-C(14)$ & $1.494(4)$ \\
\hline $\mathrm{C}(13)-\mathrm{H}(13)$ & 1.0000 \\
\hline$C(14)-C(15)$ & $1.334(4)$ \\
\hline $\mathrm{C}(14)-\mathrm{H}(14)$ & 0.9500 \\
\hline$C(15)-C(16)$ & $1.463(4)$ \\
\hline $\mathrm{C}(15)-\mathrm{H}(15)$ & 0.9500 \\
\hline$C(16)-C(18)$ & $1.342(4)$ \\
\hline$C(16)-C(17)$ & $1.501(4)$ \\
\hline $\mathrm{C}(17)-\mathrm{H}(17 \mathrm{~A})$ & 0.9800 \\
\hline C(17)-H(17B) & 0.9800 \\
\hline C(17)-H(17C) & 0.9800 \\
\hline $\mathrm{C}(18)-\mathrm{C}(19)$ & $1.499(4)$ \\
\hline $\mathrm{C}(18)-\mathrm{H}(18)$ & 0.9500 \\
\hline$C(19)-C(20)$ & $1.543(3)$ \\
\hline C(19)-H(19A) & 0.9900 \\
\hline C(19)-H(19B) & 0.9900 \\
\hline $\mathrm{C}(20)-\mathrm{O}(7)$ & $1.425(3)$ \\
\hline$C(20)-C(21)$ & $1.504(4)$ \\
\hline $\mathrm{C}(20)-\mathrm{H}(20)$ & 1.0000 \\
\hline$C(21)-C(22)$ & $1.335(3)$ \\
\hline $\mathrm{C}(21)-\mathrm{H}(21)$ & 0.9500 \\
\hline$C(22)-C(23)$ & $1.465(3)$ \\
\hline $\mathrm{C}(22)-\mathrm{H}(22)$ & 0.9500 \\
\hline
\end{tabular}




\begin{tabular}{|c|c|}
\hline$C(23)-C(25)$ & $1.348(3)$ \\
\hline$C(23)-C(24)$ & $1.498(4)$ \\
\hline $\mathrm{C}(24)-\mathrm{H}(24 \mathrm{~A})$ & 0.9800 \\
\hline $\mathrm{C}(24)-\mathrm{H}(24 \mathrm{~B})$ & 0.9800 \\
\hline $\mathrm{C}(24)-\mathrm{H}(24 \mathrm{C})$ & 0.9800 \\
\hline $\mathrm{C}(25)-\mathrm{H}(25)$ & 0.9500 \\
\hline$C(26)-C(27)$ & $1.490(4)$ \\
\hline $\mathrm{C}(26)-\mathrm{H}(26 \mathrm{~A})$ & 0.9800 \\
\hline $\mathrm{C}(26)-\mathrm{H}(26 \mathrm{~B})$ & 0.9800 \\
\hline $\mathrm{C}(26)-\mathrm{H}(26 \mathrm{C})$ & 0.9800 \\
\hline $\mathrm{C}(27)-\mathrm{O}(8)$ & $1.209(4)$ \\
\hline$C(27)-C(28)$ & $1.491(4)$ \\
\hline $\mathrm{C}(28)-\mathrm{H}(28 \mathrm{~A})$ & 0.9800 \\
\hline $\mathrm{C}(28)-\mathrm{H}(28 \mathrm{~B})$ & 0.9800 \\
\hline $\mathrm{C}(28)-\mathrm{H}(28 \mathrm{C})$ & 0.9800 \\
\hline $\mathrm{N}(1)-\mathrm{H}(1 \mathrm{~A})$ & $0.89(4)$ \\
\hline $\mathrm{O}(1)-\mathrm{H}(1)$ & $0.86(5)$ \\
\hline $\mathrm{O}(6)-\mathrm{H}(6)$ & $0.91(4)$ \\
\hline $\mathrm{O}(7)-\mathrm{H}(7)$ & $0.88(4)$ \\
\hline $\mathrm{C}(2)-\mathrm{C}(1)-\mathrm{H}(1 \mathrm{~B})$ & 109.5 \\
\hline $\mathrm{C}(2)-\mathrm{C}(1)-\mathrm{H}(1 \mathrm{C})$ & 109.5 \\
\hline $\mathrm{H}(1 \mathrm{~B})-\mathrm{C}(1)-\mathrm{H}(1 \mathrm{C})$ & 109.5 \\
\hline $\mathrm{C}(2)-\mathrm{C}(1)-\mathrm{H}(1 \mathrm{D})$ & 109.5 \\
\hline $\mathrm{H}(1 \mathrm{~B})-\mathrm{C}(1)-\mathrm{H}(1 \mathrm{D})$ & 109.5 \\
\hline $\mathrm{H}(1 \mathrm{C})-\mathrm{C}(1)-\mathrm{H}(1 \mathrm{D})$ & 109.5 \\
\hline $\mathrm{O}(1)-\mathrm{C}(2)-\mathrm{C}(1)$ & 113.3(3) \\
\hline $\mathrm{O}(1)-\mathrm{C}(2)-\mathrm{C}(3)$ & $108.1(2)$ \\
\hline $\mathrm{C}(1)-\mathrm{C}(2)-\mathrm{C}(3)$ & $109.3(2)$ \\
\hline $\mathrm{O}(1)-\mathrm{C}(2)-\mathrm{H}(2)$ & 108.7 \\
\hline $\mathrm{C}(1)-\mathrm{C}(2)-\mathrm{H}(2)$ & 108.7 \\
\hline $\mathrm{C}(3)-\mathrm{C}(2)-\mathrm{H}(2)$ & 108.7 \\
\hline $\mathrm{O}(2)-\mathrm{C}(3)-\mathrm{N}(1)$ & $123.6(2)$ \\
\hline $\mathrm{O}(2)-\mathrm{C}(3)-\mathrm{C}(2)$ & $121.0(2)$ \\
\hline $\mathrm{N}(1)-\mathrm{C}(3)-\mathrm{C}(2)$ & $115.3(2)$ \\
\hline $\mathrm{N}(1)-\mathrm{C}(4)-\mathrm{C}(25)$ & $108.78(19)$ \\
\hline
\end{tabular}




\begin{tabular}{|c|c|}
\hline $\mathrm{N}(1)-\mathrm{C}(4)-\mathrm{C}(5)$ & $111.24(19)$ \\
\hline$C(25)-C(4)-C(5)$ & $112.24(19)$ \\
\hline $\mathrm{N}(1)-\mathrm{C}(4)-\mathrm{H}(4)$ & 108.1 \\
\hline $\mathrm{C}(25)-\mathrm{C}(4)-\mathrm{H}(4)$ & 108.1 \\
\hline $\mathrm{C}(5)-\mathrm{C}(4)-\mathrm{H}(4)$ & 108.1 \\
\hline $\mathrm{C}(11)-\mathrm{C}(5)-\mathrm{C}(7)$ & $113.4(2)$ \\
\hline $\mathrm{C}(11)-\mathrm{C}(5)-\mathrm{C}(6)$ & $108.2(2)$ \\
\hline$C(7)-C(5)-C(6)$ & $105.2(2)$ \\
\hline $\mathrm{C}(11)-\mathrm{C}(5)-\mathrm{C}(4)$ & $108.5(2)$ \\
\hline$C(7)-C(5)-C(4)$ & $110.1(2)$ \\
\hline$C(6)-C(5)-C(4)$ & $111.47(19)$ \\
\hline $\mathrm{C}(5)-\mathrm{C}(6)-\mathrm{H}(6 \mathrm{~A})$ & 109.5 \\
\hline $\mathrm{C}(5)-\mathrm{C}(6)-\mathrm{H}(6 \mathrm{~B})$ & 109.5 \\
\hline $\mathrm{H}(6 \mathrm{~A})-\mathrm{C}(6)-\mathrm{H}(6 \mathrm{~B})$ & 109.5 \\
\hline $\mathrm{C}(5)-\mathrm{C}(6)-\mathrm{H}(6 \mathrm{C})$ & 109.5 \\
\hline $\mathrm{H}(6 \mathrm{~A})-\mathrm{C}(6)-\mathrm{H}(6 \mathrm{C})$ & 109.5 \\
\hline $\mathrm{H}(6 \mathrm{~B})-\mathrm{C}(6)-\mathrm{H}(6 \mathrm{C})$ & 109.5 \\
\hline $\mathrm{O}(3)-\mathrm{C}(7)-\mathrm{C}(8)$ & $123.3(2)$ \\
\hline $\mathrm{O}(3)-\mathrm{C}(7)-\mathrm{C}(5)$ & $121.0(2)$ \\
\hline$C(8)-C(7)-C(5)$ & $115.7(2)$ \\
\hline $\mathrm{C}(7)-\mathrm{C}(8)-\mathrm{C}(9)$ & $111.2(2)$ \\
\hline $\mathrm{C}(7)-\mathrm{C}(8)-\mathrm{C}(10)$ & $105.4(2)$ \\
\hline $\mathrm{C}(9)-\mathrm{C}(8)-\mathrm{C}(10)$ & $113.9(2)$ \\
\hline $\mathrm{C}(7)-\mathrm{C}(8)-\mathrm{H}(8)$ & 108.7 \\
\hline $\mathrm{C}(9)-\mathrm{C}(8)-\mathrm{H}(8)$ & 108.7 \\
\hline $\mathrm{C}(10)-\mathrm{C}(8)-\mathrm{H}(8)$ & 108.7 \\
\hline $\mathrm{C}(8)-\mathrm{C}(9)-\mathrm{H}(9 \mathrm{~A})$ & 109.5 \\
\hline $\mathrm{C}(8)-\mathrm{C}(9)-\mathrm{H}(9 \mathrm{~B})$ & 109.5 \\
\hline $\mathrm{H}(9 \mathrm{~A})-\mathrm{C}(9)-\mathrm{H}(9 \mathrm{~B})$ & 109.5 \\
\hline $\mathrm{C}(8)-\mathrm{C}(9)-\mathrm{H}(9 \mathrm{C})$ & 109.5 \\
\hline $\mathrm{H}(9 \mathrm{~A})-\mathrm{C}(9)-\mathrm{H}(9 \mathrm{C})$ & 109.5 \\
\hline $\mathrm{H}(9 \mathrm{~B})-\mathrm{C}(9)-\mathrm{H}(9 \mathrm{C})$ & 109.5 \\
\hline $\mathrm{O}(5)-\mathrm{C}(10)-\mathrm{C}(12)$ & $105.2(2)$ \\
\hline $\mathrm{O}(5)-\mathrm{C}(10)-\mathrm{C}(8)$ & $111.06(19)$ \\
\hline $\mathrm{C}(12)-\mathrm{C}(10)-\mathrm{C}(8)$ & $113.7(2)$ \\
\hline $\mathrm{O}(5)-\mathrm{C}(10)-\mathrm{H}(10)$ & 108.9 \\
\hline
\end{tabular}




\begin{tabular}{|c|c|}
\hline $\mathrm{C}(12)-\mathrm{C}(10)-\mathrm{H}(10)$ & 108.9 \\
\hline $\mathrm{C}(8)-\mathrm{C}(10)-\mathrm{H}(10)$ & 108.9 \\
\hline $\mathrm{O}(4)-\mathrm{C}(11)-\mathrm{O}(5)$ & $117.9(3)$ \\
\hline $\mathrm{O}(4)-\mathrm{C}(11)-\mathrm{C}(5)$ & $121.0(2)$ \\
\hline $\mathrm{O}(5)-\mathrm{C}(11)-\mathrm{C}(5)$ & $121.1(2)$ \\
\hline$C(10)-C(12)-C(13)$ & $114.5(2)$ \\
\hline $\mathrm{C}(10)-\mathrm{C}(12)-\mathrm{H}(12 \mathrm{~A})$ & 108.6 \\
\hline $\mathrm{C}(13)-\mathrm{C}(12)-\mathrm{H}(12 \mathrm{~A})$ & 108.6 \\
\hline $\mathrm{C}(10)-\mathrm{C}(12)-\mathrm{H}(12 \mathrm{~B})$ & 108.6 \\
\hline $\mathrm{C}(13)-\mathrm{C}(12)-\mathrm{H}(12 \mathrm{~B})$ & 108.6 \\
\hline $\mathrm{H}(12 \mathrm{~A})-\mathrm{C}(12)-\mathrm{H}(12 \mathrm{~B})$ & 107.6 \\
\hline $\mathrm{O}(6)-\mathrm{C}(13)-\mathrm{C}(14)$ & $107.6(2)$ \\
\hline $\mathrm{O}(6)-\mathrm{C}(13)-\mathrm{C}(12)$ & $108.6(2)$ \\
\hline$C(14)-C(13)-C(12)$ & $110.9(2)$ \\
\hline $\mathrm{O}(6)-\mathrm{C}(13)-\mathrm{H}(13)$ & 109.9 \\
\hline $\mathrm{C}(14)-\mathrm{C}(13)-\mathrm{H}(13)$ & 109.9 \\
\hline $\mathrm{C}(12)-\mathrm{C}(13)-\mathrm{H}(13)$ & 109.9 \\
\hline$C(15)-C(14)-C(13)$ & $124.1(2)$ \\
\hline $\mathrm{C}(15)-\mathrm{C}(14)-\mathrm{H}(14)$ & 117.9 \\
\hline $\mathrm{C}(13)-\mathrm{C}(14)-\mathrm{H}(14)$ & 117.9 \\
\hline $\mathrm{C}(14)-\mathrm{C}(15)-\mathrm{C}(16)$ & $126.3(2)$ \\
\hline $\mathrm{C}(14)-\mathrm{C}(15)-\mathrm{H}(15)$ & 116.8 \\
\hline $\mathrm{C}(16)-\mathrm{C}(15)-\mathrm{H}(15)$ & 116.8 \\
\hline $\mathrm{C}(18)-\mathrm{C}(16)-\mathrm{C}(15)$ & $118.6(2)$ \\
\hline$C(18)-C(16)-C(17)$ & $123.7(2)$ \\
\hline$C(15)-C(16)-C(17)$ & $117.4(2)$ \\
\hline $\mathrm{C}(16)-\mathrm{C}(17)-\mathrm{H}(17 \mathrm{~A})$ & 109.5 \\
\hline $\mathrm{C}(16)-\mathrm{C}(17)-\mathrm{H}(17 \mathrm{~B})$ & 109.5 \\
\hline $\mathrm{H}(17 \mathrm{~A})-\mathrm{C}(17)-\mathrm{H}(17 \mathrm{~B})$ & 109.5 \\
\hline $\mathrm{C}(16)-\mathrm{C}(17)-\mathrm{H}(17 \mathrm{C})$ & 109.5 \\
\hline $\mathrm{H}(17 \mathrm{~A})-\mathrm{C}(17)-\mathrm{H}(17 \mathrm{C})$ & 109.5 \\
\hline $\mathrm{H}(17 \mathrm{~B})-\mathrm{C}(17)-\mathrm{H}(17 \mathrm{C})$ & 109.5 \\
\hline$C(16)-C(18)-C(19)$ & $127.4(2)$ \\
\hline $\mathrm{C}(16)-\mathrm{C}(18)-\mathrm{H}(18)$ & 116.3 \\
\hline $\mathrm{C}(19)-\mathrm{C}(18)-\mathrm{H}(18)$ & 116.3 \\
\hline$C(18)-C(19)-C(20)$ & $110.2(2)$ \\
\hline
\end{tabular}




$\begin{array}{ll}\mathrm{C}(18)-\mathrm{C}(19)-\mathrm{H}(19 \mathrm{~A}) & 109.6 \\ \mathrm{C}(20)-\mathrm{C}(19)-\mathrm{H}(19 \mathrm{~A}) & 109.6 \\ \mathrm{C}(18)-\mathrm{C}(19)-\mathrm{H}(19 \mathrm{~B}) & 109.6 \\ \mathrm{C}(20)-\mathrm{C}(19)-\mathrm{H}(19 B) & 109.6 \\ \mathrm{H}(19 \mathrm{~A})-\mathrm{C}(19)-\mathrm{H}(19 \mathrm{~B}) & 108.1 \\ \mathrm{O}(7)-\mathrm{C}(20)-\mathrm{C}(21) & 111.3(2) \\ \mathrm{O}(7)-\mathrm{C}(20)-\mathrm{C}(19) & 110.92(19) \\ \mathrm{C}(21)-\mathrm{C}(20)-\mathrm{C}(19) & 112.0(2) \\ \mathrm{O}(7)-\mathrm{C}(20)-\mathrm{H}(20) & 107.5 \\ \mathrm{C}(21)-\mathrm{C}(20)-\mathrm{H}(20) & 107.5 \\ \mathrm{C}(19)-\mathrm{C}(20)-\mathrm{H}(20) & 107.5 \\ \mathrm{C}(22)-\mathrm{C}(21)-\mathrm{C}(20) & 122.8(3) \\ \mathrm{C}(22)-\mathrm{C}(21)-\mathrm{H}(21) & 118.6 \\ \mathrm{C}(20)-\mathrm{C}(21)-\mathrm{H}(21) & 118.6 \\ \mathrm{C}(21)-\mathrm{C}(22)-\mathrm{C}(23) & 127.2(3) \\ \mathrm{C}(21)-\mathrm{C}(22)-\mathrm{H}(22) & 116.4 \\ \mathrm{C}(23)-\mathrm{C}(22)-\mathrm{H}(22) & 116.4 \\ \mathrm{C}(25)-\mathrm{C}(23)-\mathrm{C}(22) & 117.9(2) \\ \mathrm{C}(25)-\mathrm{C}(23)-\mathrm{C}(24) & 124.1(2) \\ \mathrm{C}(22)-\mathrm{C}(23)-\mathrm{C}(24) & 118.0(2) \\ \mathrm{C}(23)-\mathrm{C}(24)-\mathrm{H}(24 \mathrm{~A}) & 109.5 \\ \mathrm{C}(23)-\mathrm{C}(24)-\mathrm{H}(24 \mathrm{~B}) & 109.5 \\ \mathrm{H}(24 \mathrm{~A})-\mathrm{C}(24)-\mathrm{H}(24 \mathrm{~B}) & 109.5 \\ \mathrm{C}(23)-\mathrm{C}(24)-\mathrm{H}(24 \mathrm{C}) & 109.5 \\ \mathrm{H}(24 \mathrm{~A})-\mathrm{C}(24)-\mathrm{H}(24 \mathrm{C}) & 109.5 \\ \mathrm{H}(24 \mathrm{~B})-\mathrm{C}(24)-\mathrm{H}(24 \mathrm{C}) & 109.5 \\ \mathrm{C}(23)-\mathrm{C}(25)-\mathrm{C}(4) & 127.7(2) \\ \mathrm{C}(23)-\mathrm{C}(25)-\mathrm{H}(25) & 116.1 \\ \mathrm{C}(4)-\mathrm{C}(25)-\mathrm{H}(25) & 116.1 \\ \mathrm{C}(27)-\mathrm{C}(26)-\mathrm{H}(26 \mathrm{~A}) & 109.5 \\ \mathrm{C}(27)-\mathrm{C}(26)-\mathrm{H}(26 \mathrm{~B}) & 109.5 \\ \mathrm{H}(26 \mathrm{~A})-\mathrm{C}(26)-\mathrm{H}(26 \mathrm{~B}) & 109.5 \\ \mathrm{C}(27)-\mathrm{C}(26)-\mathrm{H}(26 \mathrm{C}) & 109.5 \\ & \end{array}$




$\begin{array}{ll}\mathrm{O}(8)-\mathrm{C}(27)-\mathrm{C}(28) & 120.2(3) \\ \mathrm{C}(26)-\mathrm{C}(27)-\mathrm{C}(28) & 118.2(2) \\ \mathrm{C}(27)-\mathrm{C}(28)-\mathrm{H}(28 \mathrm{~A}) & 109.5 \\ \mathrm{C}(27)-\mathrm{C}(28)-\mathrm{H}(28 \mathrm{~B}) & 109.5 \\ \mathrm{H}(28 \mathrm{~A})-\mathrm{C}(28)-\mathrm{H}(28 \mathrm{~B}) & 109.5 \\ \mathrm{C}(27)-\mathrm{C}(28)-\mathrm{H}(28 \mathrm{C}) & 109.5 \\ \mathrm{H}(28 \mathrm{~A})-\mathrm{C}(28)-\mathrm{H}(28 \mathrm{C}) & 109.5 \\ \mathrm{H}(28 \mathrm{~B})-\mathrm{C}(28)-\mathrm{H}(28 \mathrm{C}) & 109.5 \\ \mathrm{C}(3)-\mathrm{N}(1)-\mathrm{C}(4) & 123.6(2) \\ \mathrm{C}(3)-\mathrm{N}(1)-\mathrm{H}(1 \mathrm{~A}) & 115(2) \\ \mathrm{C}(4)-\mathrm{N}(1)-\mathrm{H}(1 \mathrm{~A}) & 121(2) \\ \mathrm{C}(2)-\mathrm{O}(1)-\mathrm{H}(1) & 112(3) \\ \mathrm{C}(11)-\mathrm{O}(5)-\mathrm{C}(10) & 122.1(2) \\ \mathrm{C}(13)-\mathrm{O}(6)-\mathrm{H}(6) & 106(2) \\ \mathrm{C}(20)-\mathrm{O}(7)-\mathrm{H}(7) & 108(2)\end{array}$

Symmetry transformations used to generate equivalent atoms:

Table 4. Anisotropic displacement parameters $\left(\AA^{2} \times 10^{3}\right)$ for seiple003_ucsf. The anisotropic displacement factor exponent takes the form: $-2 \pi^{2}\left[h^{2} a^{* 2} U^{11}+\ldots+2 h k a^{*} b^{*} U^{12}\right]$

\begin{tabular}{lcccccc}
\hline & $\mathrm{U}^{11}$ & $\mathrm{U}^{22}$ & $\mathrm{U}^{33}$ & $\mathrm{U}^{23}$ & $\mathrm{U}^{13}$ & $\mathrm{U}^{12}$ \\
\hline $\mathrm{C}(1)$ & $39(2)$ & $56(2)$ & $22(1)$ & $7(1)$ & $8(1)$ & $-4(2)$ \\
$\mathrm{C}(2)$ & $26(1)$ & $32(2)$ & $22(1)$ & $1(1)$ & $11(1)$ & $-2(1)$ \\
$\mathrm{C}(3)$ & $19(1)$ & $29(2)$ & $20(1)$ & $-1(1)$ & $7(1)$ & $-1(1)$ \\
$\mathrm{C}(4)$ & $17(1)$ & $24(1)$ & $17(1)$ & $1(1)$ & $7(1)$ & $0(1)$ \\
$\mathrm{C}(5)$ & $18(1)$ & $27(1)$ & $17(1)$ & $-1(1)$ & $5(1)$ & $0(1)$ \\
$\mathrm{C}(6)$ & $24(1)$ & $54(2)$ & $19(1)$ & $-4(1)$ & $4(1)$ & $-4(1)$ \\
$\mathrm{C}(7)$ & $20(1)$ & $26(1)$ & $18(1)$ & $-3(1)$ & $3(1)$ & $-2(1)$ \\
$\mathrm{C}(8)$ & $17(1)$ & $27(1)$ & $24(1)$ & $-2(1)$ & $6(1)$ & $-2(1)$ \\
$\mathrm{C}(9)$ & $26(1)$ & $29(2)$ & $43(2)$ & $-1(1)$ & $17(1)$ & $-2(1)$ \\
$\mathrm{C}(10)$ & $19(1)$ & $26(1)$ & $20(1)$ & $0(1)$ & $5(1)$ & $-1(1)$ \\
$\mathrm{C}(11)$ & $18(1)$ & $27(1)$ & $23(1)$ & $4(1)$ & $4(1)$ & $1(1)$ \\
$\mathrm{C}(12)$ & $17(1)$ & $30(2)$ & $25(1)$ & $-3(1)$ & $4(1)$ & $1(1)$ \\
$\mathrm{C}(13)$ & $18(1)$ & $28(1)$ & $27(1)$ & $-2(1)$ & $7(1)$ & $2(1)$
\end{tabular}




\begin{tabular}{lllllll}
$\mathrm{C}(14)$ & $21(1)$ & $24(1)$ & $24(1)$ & $-4(1)$ & $7(1)$ & $2(1)$ \\
$\mathrm{C}(15)$ & $23(1)$ & $23(1)$ & $22(1)$ & $-2(1)$ & $7(1)$ & $1(1)$ \\
$\mathrm{C}(16)$ & $22(1)$ & $24(1)$ & $21(1)$ & $-2(1)$ & $5(1)$ & $4(1)$ \\
$\mathrm{C}(17)$ & $26(1)$ & $34(2)$ & $28(1)$ & $6(1)$ & $6(1)$ & $1(1)$ \\
$\mathrm{C}(18)$ & $23(1)$ & $27(1)$ & $23(1)$ & $1(1)$ & $6(1)$ & $1(1)$ \\
$\mathrm{C}(19)$ & $20(1)$ & $36(2)$ & $20(1)$ & $0(1)$ & $2(1)$ & $1(1)$ \\
$\mathrm{C}(20)$ & $16(1)$ & $32(1)$ & $18(1)$ & $1(1)$ & $1(1)$ & $-1(1)$ \\
$\mathrm{C}(21)$ & $18(1)$ & $28(1)$ & $21(1)$ & $2(1)$ & $4(1)$ & $-1(1)$ \\
$\mathrm{C}(22)$ & $18(1)$ & $23(1)$ & $21(1)$ & $1(1)$ & $5(1)$ & $0(1)$ \\
$\mathrm{C}(23)$ & $18(1)$ & $25(1)$ & $20(1)$ & $1(1)$ & $6(1)$ & $1(1)$ \\
$\mathrm{C}(24)$ & $38(2)$ & $24(2)$ & $23(1)$ & $1(1)$ & $-1(1)$ & $-4(1)$ \\
$\mathrm{C}(25)$ & $16(1)$ & $21(1)$ & $21(1)$ & $0(1)$ & $7(1)$ & $0(1)$ \\
$\mathrm{C}(26)$ & $32(1)$ & $41(2)$ & $23(1)$ & $-2(1)$ & $8(1)$ & $-6(1)$ \\
$\mathrm{C}(27)$ & $26(1)$ & $36(2)$ & $23(1)$ & $-1(1)$ & $4(1)$ & $2(1)$ \\
$\mathrm{C}(28)$ & $34(1)$ & $29(2)$ & $26(1)$ & $2(1)$ & $2(1)$ & $0(1)$ \\
$\mathrm{N}(1)$ & $20(1)$ & $23(1)$ & $19(1)$ & $0(1)$ & $8(1)$ & $-1(1)$ \\
$\mathrm{O}(1)$ & $47(1)$ & $36(1)$ & $28(1)$ & $0(1)$ & $17(1)$ & $-16(1)$ \\
$\mathrm{O}(2)$ & $38(1)$ & $28(1)$ & $32(1)$ & $-5(1)$ & $20(1)$ & $0(1)$ \\
$\mathrm{O}(3)$ & $26(1)$ & $23(1)$ & $42(1)$ & $2(1)$ & $12(1)$ & $0(1)$ \\
$\mathrm{O}(4)$ & $31(1)$ & $30(1)$ & $41(1)$ & $13(1)$ & $15(1)$ & $3(1)$ \\
$\mathrm{O}(5)$ & $24(1)$ & $22(1)$ & $31(1)$ & $0(1)$ & $9(1)$ & $-1(1)$ \\
$\mathrm{O}(6)$ & $20(1)$ & $34(1)$ & $28(1)$ & $-5(1)$ & $3(1)$ & $10(1)$ \\
$\mathrm{O}(7)$ & $18(1)$ & $37(1)$ & $22(1)$ & $5(1)$ & $1(1)$ & $-1(1)$ \\
$\mathrm{O}(8)$ & $33(1)$ & $131(3)$ & $36(1)$ & $22(2)$ & $10(1)$ & $35(2)$ \\
\hline & & & & \\
\end{tabular}

Table 5. Hydrogen coordinates ( $\left.\times 10^{4}\right)$ and isotropic displacement parameters $\left(\AA^{2} \times 10^{3}\right)$ for seiple003_ucsf.

\begin{tabular}{lrrrc}
\hline & $x$ & $y$ & $z$ & $U(e q)$ \\
\hline$H(1 B)$ & 751 & 5913 & 6054 & 58 \\
$H(1 C)$ & 1761 & 7232 & 5869 & 58 \\
$H(1 D)$ & 2274 & 5746 & 5603 & 58 \\
$H(2)$ & -616 & 7090 & 5021 & 31 \\
$\mathrm{H}(4)$ & 2800 & 7974 & 3286 & 23
\end{tabular}




\begin{tabular}{|c|c|c|c|c|}
\hline $\mathrm{H}(6 \mathrm{~A})$ & 4614 & 6445 & 4778 & 48 \\
\hline $\mathrm{H}(6 \mathrm{~B})$ & 4796 & 8015 & 4543 & 48 \\
\hline $\mathrm{H}(6 \mathrm{C})$ & 6304 & 7017 & 4602 & 48 \\
\hline $\mathrm{H}(8)$ & 8001 & 6761 & 3526 & 27 \\
\hline $\mathrm{H}(9 \mathrm{~A})$ & 9400 & 7643 & 2590 & 48 \\
\hline $\mathrm{H}(9 \mathrm{~B})$ & 8371 & 8886 & 2864 & 48 \\
\hline $\mathrm{H}(9 \mathrm{C})$ & 7806 & 8110 & 2083 & 48 \\
\hline $\mathrm{H}(10)$ & 6234 & 5896 & 2119 & 26 \\
\hline $\mathrm{H}(12 \mathrm{~A})$ & 8945 & 4492 & 2858 & 28 \\
\hline $\mathrm{H}(12 \mathrm{~B})$ & 9094 & 5516 & 2165 & 28 \\
\hline $\mathrm{H}(13)$ & 7263 & 3017 & 2045 & 28 \\
\hline $\mathrm{H}(14)$ & 7753 & 4801 & 789 & 27 \\
\hline $\mathrm{H}(15)$ & 5136 & 3257 & 1078 & 27 \\
\hline $\mathrm{H}(17 \mathrm{~A})$ & 5752 & 6442 & 6 & 44 \\
\hline $\mathrm{H}(17 \mathrm{~B})$ & 4941 & 5790 & -772 & 44 \\
\hline $\mathrm{H}(17 \mathrm{C})$ & 6619 & 5226 & -392 & 44 \\
\hline $\mathrm{H}(18)$ & 2821 & 3532 & 288 & 29 \\
\hline $\mathrm{H}(19 \mathrm{~A})$ & 1543 & 4323 & -934 & 30 \\
\hline $\mathrm{H}(19 \mathrm{~B})$ & 2506 & 5732 & -798 & 30 \\
\hline $\mathrm{H}(20)$ & 119 & 4780 & 114 & 27 \\
\hline $\mathrm{H}(21)$ & 1451 & 7448 & 398 & 27 \\
\hline $\mathrm{H}(22)$ & 1370 & 5168 & 1335 & 24 \\
\hline $\mathrm{H}(24 \mathrm{~A})$ & 2553 & 8910 & 2270 & 43 \\
\hline $\mathrm{H}(24 \mathrm{~B})$ & 1394 & 8820 & 1491 & 43 \\
\hline $\mathrm{H}(24 \mathrm{C})$ & 3266 & 8606 & 1487 & 43 \\
\hline $\mathrm{H}(25)$ & 2338 & 5346 & 2573 & 23 \\
\hline $\mathrm{H}(26 \mathrm{~A})$ & 2689 & 2202 & 1873 & 48 \\
\hline $\mathrm{H}(26 \mathrm{~B})$ & 4041 & 1205 & 2266 & 48 \\
\hline $\mathrm{H}(26 \mathrm{C})$ & 4147 & 2819 & 2423 & 48 \\
\hline $\mathrm{H}(28 \mathrm{~A})$ & 4237 & 1797 & 3896 & 45 \\
\hline $\mathrm{H}(28 \mathrm{~B})$ & 3693 & 311 & 3586 & 45 \\
\hline $\mathrm{H}(28 \mathrm{C})$ & 2583 & 1169 & 4087 & 45 \\
\hline $\mathrm{H}(1)$ & $-550(50)$ & $4720(50)$ & $4930(30)$ & $50(12)$ \\
\hline $\mathrm{H}(1 \mathrm{~A})$ & $1760(40)$ & $5560(40)$ & $3940(20)$ & $30(8)$ \\
\hline $\mathrm{H}(6)$ & $9940(50)$ & $2820(40)$ & $2130(20)$ & $41(10)$ \\
\hline
\end{tabular}


Table 6. Torsion angles $\left[{ }^{\circ}\right]$ for seiple 003 _ucsf.

\begin{tabular}{lc}
\hline $\mathrm{O}(1)-\mathrm{C}(2)-\mathrm{C}(3)-\mathrm{O}(2)$ & $155.3(3)$ \\
$\mathrm{C}(1)-\mathrm{C}(2)-\mathrm{C}(3)-\mathrm{O}(2)$ & $-80.9(3)$ \\
$\mathrm{O}(1)-\mathrm{C}(2)-\mathrm{C}(3)-\mathrm{N}(1)$ & $-27.1(3)$ \\
$\mathrm{C}(1)-\mathrm{C}(2)-\mathrm{C}(3)-\mathrm{N}(1)$ & $96.7(3)$ \\
$\mathrm{N}(1)-\mathrm{C}(4)-\mathrm{C}(5)-\mathrm{C}(11)$ & $73.7(2)$ \\
$\mathrm{C}(25)-\mathrm{C}(4)-\mathrm{C}(5)-\mathrm{C}(11)$ & $-48.4(3)$ \\
$\mathrm{N}(1)-\mathrm{C}(4)-\mathrm{C}(5)-\mathrm{C}(7)$ & $-161.7(2)$ \\
$\mathrm{C}(25)-\mathrm{C}(4)-\mathrm{C}(5)-\mathrm{C}(7)$ & $76.1(3)$ \\
$\mathrm{N}(1)-\mathrm{C}(4)-\mathrm{C}(5)-\mathrm{C}(6)$ & $-45.3(3)$ \\
$\mathrm{C}(25)-\mathrm{C}(4)-\mathrm{C}(5)-\mathrm{C}(6)$ & $-167.5(2)$ \\
$\mathrm{C}(11)-\mathrm{C}(5)-\mathrm{C}(7)-\mathrm{O}(3)$ & $157.3(2)$ \\
$\mathrm{C}(6)-\mathrm{C}(5)-\mathrm{C}(7)-\mathrm{O}(3)$ & $-84.6(3)$ \\
$\mathrm{C}(4)-\mathrm{C}(5)-\mathrm{C}(7)-\mathrm{O}(3)$ & $35.6(3)$ \\
$\mathrm{C}(11)-\mathrm{C}(5)-\mathrm{C}(7)-\mathrm{C}(8)$ & $-23.9(3)$ \\
$\mathrm{C}(6)-\mathrm{C}(5)-\mathrm{C}(7)-\mathrm{C}(8)$ & $94.2(3)$ \\
$\mathrm{C}(4)-\mathrm{C}(5)-\mathrm{C}(7)-\mathrm{C}(8)$ & $-145.6(2)$ \\
$\mathrm{O}(3)-\mathrm{C}(7)-\mathrm{C}(8)-\mathrm{C}(9)$ & $-1.1(4)$ \\
$\mathrm{C}(5)-\mathrm{C}(7)-\mathrm{C}(8)-\mathrm{C}(9)$ & $-179.8(2)$ \\
$\mathrm{O}(3)-\mathrm{C}(7)-\mathrm{C}(8)-\mathrm{C}(10)$ & $-124.9(3)$ \\
$\mathrm{C}(5)-\mathrm{C}(7)-\mathrm{C}(8)-\mathrm{C}(10)$ & $56.3(3)$ \\
$\mathrm{C}(7)-\mathrm{C}(8)-\mathrm{C}(10)-\mathrm{O}(5)$ & $-168.8(2)$ \\
$\mathrm{C}(9)-\mathrm{C}(8)-\mathrm{C}(10)-\mathrm{O}(5)$ & $-170.8(3)$ \\
$\mathrm{C}(7)-\mathrm{C}(8)-\mathrm{C}(10)-\mathrm{C}(12)$ & $-177.1(2)$ \\
$\mathrm{C}(9)-\mathrm{C}(8)-\mathrm{C}(10)-\mathrm{C}(12)$ & $-179.2(2)$ \\
$\mathrm{C}(7)-\mathrm{C}(5)-\mathrm{C}(11)-\mathrm{O}(4)$ & $58.6(3)$ \\
$\mathrm{C}(6)-\mathrm{C}(5)-\mathrm{C}(11)-\mathrm{O}(4)$ & $174.5(2)$ \\
$\mathrm{C}(4)-\mathrm{C}(5)-\mathrm{C}(11)-\mathrm{O}(4)$ & $58.2(3)$ \\
$\mathrm{C}(7)-\mathrm{C}(5)-\mathrm{C}(11)-\mathrm{O}(5)$ & $-62.8(3)$ \\
$\mathrm{C}(6)-\mathrm{C}(5)-\mathrm{C}(11)-\mathrm{O}(5)$ & $-73)$ \\
$\mathrm{C}(4)-\mathrm{C}(5)-\mathrm{C}(11)-\mathrm{O}(5)$ & \\
$\mathrm{O}(5)-\mathrm{C}(10)-\mathrm{C}(12)-\mathrm{C}(13)$ & \\
$\mathrm{C}(8)-\mathrm{C}(10)-\mathrm{C}(12)-\mathrm{C}(13)$ & \\
& \\
&
\end{tabular}




$\begin{array}{lc}\mathrm{C}(10)-\mathrm{C}(12)-\mathrm{C}(13)-\mathrm{O}(6) & 179.3(2) \\ \mathrm{C}(10)-\mathrm{C}(12)-\mathrm{C}(13)-\mathrm{C}(14) & 61.2(3) \\ \mathrm{O}(6)-\mathrm{C}(13)-\mathrm{C}(14)-\mathrm{C}(15) & 126.6(3) \\ \mathrm{C}(12)-\mathrm{C}(13)-\mathrm{C}(14)-\mathrm{C}(15) & -114.7(3) \\ \mathrm{C}(13)-\mathrm{C}(14)-\mathrm{C}(15)-\mathrm{C}(16) & 171.1(2) \\ \mathrm{C}(14)-\mathrm{C}(15)-\mathrm{C}(16)-\mathrm{C}(18) & -172.4(3) \\ \mathrm{C}(14)-\mathrm{C}(15)-\mathrm{C}(16)-\mathrm{C}(17) & 2.7(4) \\ \mathrm{C}(15)-\mathrm{C}(16)-\mathrm{C}(18)-\mathrm{C}(19) & 168.0(2) \\ \mathrm{C}(17)-\mathrm{C}(16)-\mathrm{C}(18)-\mathrm{C}(19) & -6.8(4) \\ \mathrm{C}(16)-\mathrm{C}(18)-\mathrm{C}(19)-\mathrm{C}(20) & -120.9(3) \\ \mathrm{C}(18)-\mathrm{C}(19)-\mathrm{C}(20)-\mathrm{O}(7) & -178.9(2) \\ \mathrm{C}(18)-\mathrm{C}(19)-\mathrm{C}(20)-\mathrm{C}(21) & 56.1(3) \\ \mathrm{O}(7)-\mathrm{C}(20)-\mathrm{C}(21)-\mathrm{C}(22) & 139.5(2) \\ \mathrm{C}(19)-\mathrm{C}(20)-\mathrm{C}(21)-\mathrm{C}(22) & -95.7(3) \\ \mathrm{C}(20)-\mathrm{C}(21)-\mathrm{C}(22)-\mathrm{C}(23) & -179.7(2) \\ \mathrm{C}(21)-\mathrm{C}(22)-\mathrm{C}(23)-\mathrm{C}(25) & -176.9(2) \\ \mathrm{C}(21)-\mathrm{C}(22)-\mathrm{C}(23)-\mathrm{C}(24) & 4.8(4) \\ \mathrm{C}(22)-\mathrm{C}(23)-\mathrm{C}(25)-\mathrm{C}(4) & -175.2(2) \\ \mathrm{C}(24)-\mathrm{C}(23)-\mathrm{C}(25)-\mathrm{C}(4) & 3.0(4) \\ \mathrm{N}(1)-\mathrm{C}(4)-\mathrm{C}(25)-\mathrm{C}(23) & 130.2(3) \\ \mathrm{C}(5)-\mathrm{C}(4)-\mathrm{C}(25)-\mathrm{C}(23) & -106.3(3) \\ \mathrm{O}(2)-\mathrm{C}(3)-\mathrm{N}(1)-\mathrm{C}(4) & 1.7(4) \\ \mathrm{C}(2)-\mathrm{C}(3)-\mathrm{N}(1)-\mathrm{C}(4) & -175.8(2) \\ \mathrm{C}(25)-\mathrm{C}(4)-\mathrm{N}(1)-\mathrm{C}(3) & -133.7(2) \\ \mathrm{C}(5)-\mathrm{C}(4)-\mathrm{N}(1)-\mathrm{C}(3) & 102.2(3) \\ \mathrm{O}(4)-\mathrm{C}(11)-\mathrm{O}(5)-\mathrm{C}(10) & 179.4(2) \\ \mathrm{C}(5)-\mathrm{C}(11)-\mathrm{O}(5)-\mathrm{C}(10) & \\ \mathrm{C}(12)-\mathrm{C}(10)-\mathrm{O}(5)-\mathrm{C}(11) & \\ \mathrm{C}(8)-\mathrm{C}(10)-\mathrm{O}(5)-\mathrm{C}(11) & \\ & \\ & \\ & \end{array}$

Symmetry transformations used to generate equivalent atoms: 


\section{Biological Studies}

\section{Antimicrobial evaluation of 2,18-seco-lankacidinol B and derivatives}

Compounds were evaluated by Micromyx LLC for Minimum Inhibitory Concentration (MIC) activity against a variety of Gram-negative and Gram-positive pathogens, using the broth microdilution method as recommended by the Clinical and Laboratory Standards Institute (CLSI). Pre-weighed vials of the test agents were stored at $-20^{\circ} \mathrm{C}$ until testing. On the day of the assay, the compounds were dissolved in $100 \%$ DMSO (Sigma 472301, Lot No. SHBH5551V) to a stock concentration of $6,464 \mu \mathrm{g} / \mathrm{mL}$. The concentration range tested for each of the compounds was $64-0.06 \mu \mathrm{g} / \mathrm{mL}$. Levofloxacin was used as the quality control agent in this study.

\section{Test Organisms}

Test organisms consisted of reference strains from the American Type Culture Collection (ATCC; Manassas, VA). Organisms were initially received at Micromyx and were streaked for isolation. Colonies were picked by sterile swab and suspended in the appropriate broth containing cryoprotectant. The suspensions were aliquoted into cryogenic vials and maintained at $-80^{\circ} \mathrm{C}$.

Prior to testing, all isolates except for $\mathrm{H}$. influenzae were streaked from frozen vials onto Trypticase Soy agar plates with 5\% sheep blood (BBL Ref. No. 221261, Lot 7292618) and incubated overnight at $35^{\circ} \mathrm{C}$. The S. pneumoniae strain was incubated in the presence of $3 \% \mathrm{CO}_{2}$.

H. influenzae was streaked onto chocolate agar (BBL Ref. No. 221267, Lot 7299878) and incubated overnight at $35^{\circ} \mathrm{C}$ in the presence of $3 \% \mathrm{CO}_{2}$.

\section{Test Media}

The medium employed for testing in the broth microdilution MIC assay for all organisms except S. pneumoniae and H. influenzae was cation-adjusted Mueller Hinton broth (MHBII; Becton Dickenson 212322; Lot 7143896) prepared according to CLSI guidelines. S. pneumoniae was tested in MHBII supplemented with 3\% lysed horse blood (LHB; Hemostat, Lot 399694) and H. influenzae was tested in Haemophilus Test Medium (Remel Ref. No. R112380, Lot 106403).

\section{Broth Microdilution Susceptibility Testing}

The MIC assay method followed the procedure described by the Clinical and Laboratory Standards Institute $^{7,8}$ and employed automated liquid handlers (Multidrop 384, Labsystems, Helsinki, Finland; Biomek 2000 and Biomek FX, Beckman Coulter, Fullerton CA) to conduct serial dilutions and liquid transfers. The wells in columns 2-12 in a standard 96-well microdilution plate (Costar) were filled with 150 $\mu \mathrm{L}$ of the appropriate solvent (DMSO for the test agents and water for levofloxacin). These would become the 'mother plates' from which 'daughter' or test plates would be prepared. The drugs $(300 \mu \mathrm{L}$ at $101 \mathrm{X}$ the desired top concentration in the test plates) were dispensed into the appropriate well in Column 1 of the mother plates. The Biomek 2000 was used to make serial 2-fold dilutions through Column 11 in the "mother plate". The wells of Column 12 contained no drug and were the organism growth control wells.

The daughter plates were loaded with $190 \mu \mathrm{L}$ per well of MHBII using the Multidrop 384. Plates for testing of S. pneumoniae were loaded with MHBII + LHB and those for testing of H. influenzae with HTM using a multi-channel pipet. The daughter plates were prepared on the Biomek FX instrument which transferred $2 \mu \mathrm{L}$ of $101 \mathrm{X}$ drug solution from each well of a mother plate to the corresponding well of each daughter plate in a single step. The wells of the daughter plates ultimately contained $190 \mu \mathrm{L}$ of medium, $2 \mu \mathrm{L}$ of drug solution, and $10 \mu \mathrm{L}$ of bacterial inoculum prepared in broth.

A standardized inoculum of each organism was prepared per CLSI methods ${ }^{7,8}$. Suspensions were prepared in MHBII to equal a turbidity of a $0.5 \mathrm{McF}$ arland standard. The $0.5 \mathrm{McF}$ arland suspensions were diluted 
1:20 in the appropriate media. The inoculum for each organism was dispensed into sterile reservoirs divided by length (Beckman Coulter), and the Biomek 2000 was used to inoculate the plates. Daughter plates were placed on the Biomek 2000 work surface in reverse orientation so that inoculation took place from low to high drug concentration. The Biomek 2000 delivered $10 \mu \mathrm{L}$ of standardized inoculum into each well. This yielded a final cell density in the daughter plates of approximately $5 \times 105 \mathrm{CFU} / \mathrm{mL}$.

Plates were stacked 3-4 high, covered with a lid on the top plate, placed in plastic bags and incubated for approximately 20 hours at $35^{\circ} \mathrm{C}$. The microplates were viewed from the bottom using a plate viewer. An un-inoculated solubility control plate was observed for evidence of drug precipitation. The MIC was read and recorded as the lowest concentration of drug that inhibited visible growth of the organism.

\section{In vitro translation assays}

The ability of iso-lankacidinols to inhibit the $70 \mathrm{~S} \mathrm{E}$. coli ribosome was first screened using the PURExpress ${ }^{\circledR}$ In Vitro Protein Synthesis Kit (E6800, NEB), murine RNAse inhibitor (M0314, NEB), and $6.66 \mathrm{ng} / \mu \mathrm{l}$ of template DNA encoding the fluorescent protein mEGFP. The volume of the reaction mixture was scaled down 5-fold from the NEB protocol for a final reaction volume of $5 \mu \mathrm{L}$. Analogs were screened at a final concentration of $10 \mu \mathrm{M}$ in $10 \%$ DMSO. Translation reactions were carried out in triplicate at $37^{\circ} \mathrm{C}$ for 1 hour, then transferred to a $0^{\circ} \mathrm{C}$ metal block. To assist in the transfer of reactions to 96 -well half-area Non-Binding Surface (NBS) microplates (Corning 3993) for final measurements, the reaction volume was increased to $50 \mu \mathrm{L}$ by adding buffer $(20 \mathrm{mM}$ Tris- $\mathrm{HCl} \mathrm{pH} 7.5,60 \mathrm{mM} \mathrm{NH} 4 \mathrm{Cl}, 6 \mathrm{mM} \mathrm{MgCl}, 0.5 \mathrm{mM}$ EDTA). Using a Cytation 5 plate reader (BioTek), translated mEGFP was excited at $485 \mathrm{~nm}$; its emission was recorded at $535 \mathrm{~nm}$. For comparison of analog activities across multiple initial screens, fluorescence readouts were normalized to the blank. Data were analyzed using Excel. 


\section{References}

1. J. Suffert, D. Toussaint, J. Org. Chem. 1996, 60, 3550-3553.

2. K. C. Nicolaou, K. C. Fylaktakidou, H. Monenschein, Y. Li, B. Weyershausen, H. J. Mitchell, H.x. Wei, P. Guntupalli, D. Hepworth, K. Sugita, J. Am. Chem. Soc. 2003, 125, 15433-15442.

3. I. Paterson, T. Paquet, Org. Lett. 2010, 12, 2158-2161.

4. a) J. S. Clark, R. Metternich, V. J. Novack, G. S. Sheppard, D. A. Evans, J. Am. Chem. Soc. 1990, 112, 866-868; b) D. A. Evans, H. P. Ng, J. S. Clark, D. L. Rieger, Tetrahedron 1992, 48, $2127-$ 2142.

5. J. Srogl, G. D. Allred, L. S. Liebeskind, J. Am. Chem. Soc. 1997, 119, 12376-12377. 